REPORT S OF THE OAK RIDGE DOSE RECONSTRU CTION, V OI. 1 T he R eport of Project T ask $1 \bullet$ July 1999

lodine-131 $R$ eleases from $R$ adioactive $L$ anthanum Processing at the X-10 Site in $O$ ak R idge, T ennessee (1944-1956) an A ssessment of Q uantities $R$ eleased, $O$ ff-Site $R$ adiation D oses, and Potential Excess $R$ isks of $T$ hyroid $C$ ancer

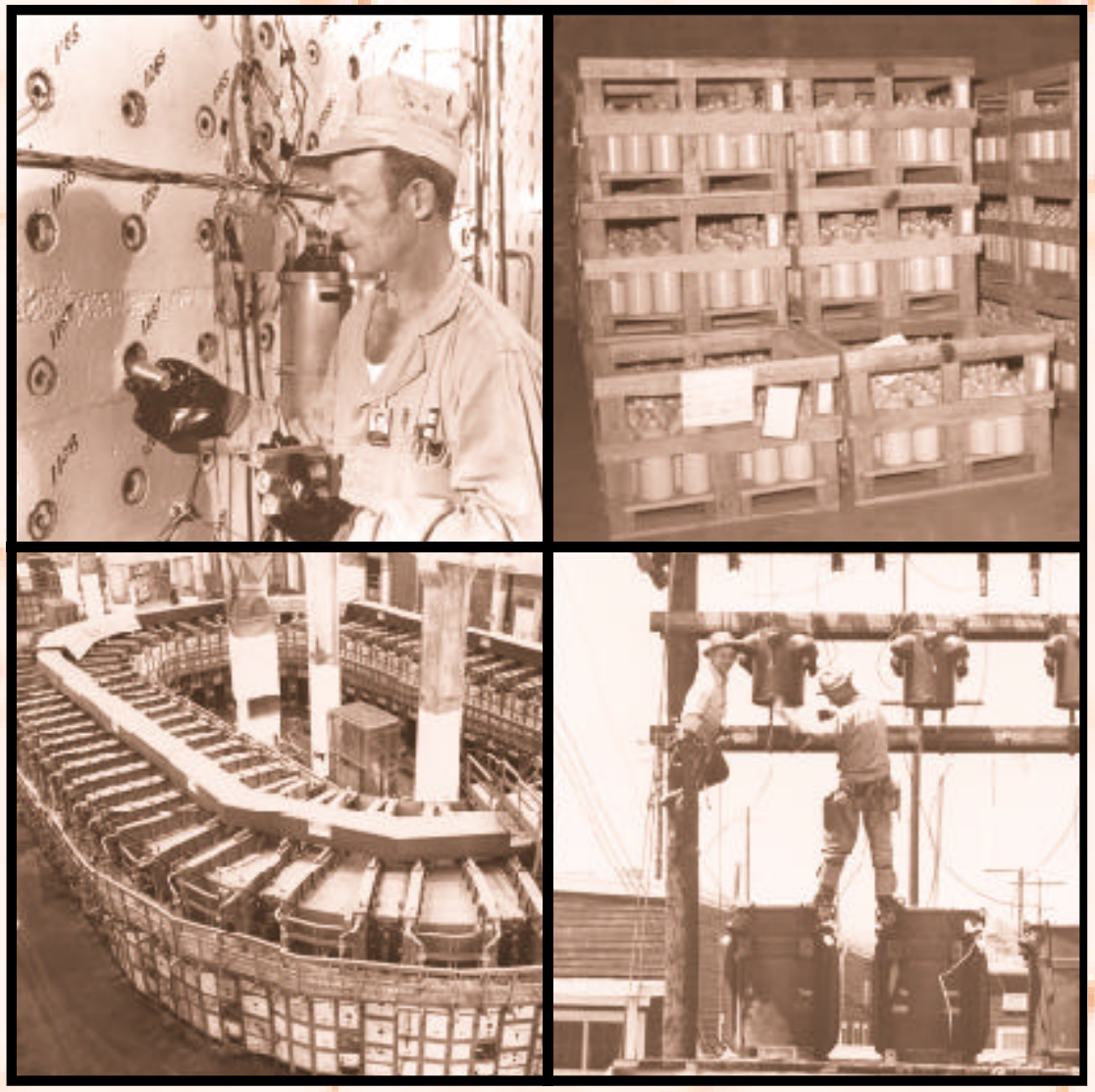

Submitted to the T ennessee D epartment of $\mathrm{H}$ ealth by

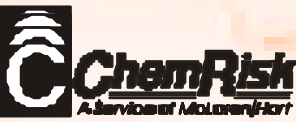




\author{
OAK RIDGE HEALTH STUDIES \\ OAK RIDGE DOSE RECONSTRUCTION
}

\author{
- TASK 1 REPORT -
}

\title{
IODINE-131 RELEASES FROM RADIOACTIVE LANTHANUM PROCESSING AT THE X-10 SITE IN OAK RIDGE, TENNESSEE (1944-1956) - AN ASSESSMENT OF QUANTITIES RELEASED, OFF-SITE RADIATION DOSES, AND POTENTIAL EXCESS RISKS OF THYROID CANCER
}

July 1999

Submitted to the Tennessee Department of Health by

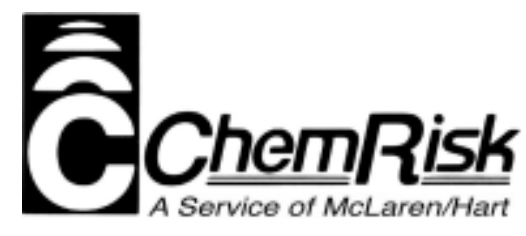


TASK 1 REPORT

July 1999

${ }^{131}$ I Releases from X-10 Radioactive Lanthanum Processing -

This page intentionally left blank. 
This document was prepared as a team effort by the following individuals:

\section{Project Manager}

Thomas E. Widner ${ }^{1}$

Task 1 Manager

F. Owen Hoffman ${ }^{2}$

\section{Principal Authors:}

A. Iulian Apostoaei ${ }^{2}$

Robert E. Burns ${ }^{3}$

F. Owen Hoffman

Talaat Ijaz ${ }^{4}$

Cathy J. Lewis ${ }^{2}$

Shyam K. Nair

Thomas E. Widner

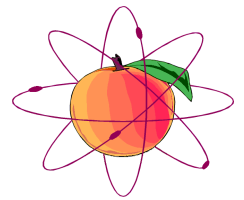

Associates

${ }^{1}$ ChemRisk® a Service of McLaren/Hart, Inc., 1320 Harbor Bay Parkway, Suite 100, Alameda, California 94502. (510) 521-5200. E-mail for the project team can be sent to: Tom_Widner@mclaren-hart.com.

2 SENES Oak Ridge, Inc., 102 Donner Drive, Oak Ridge, Tennessee 37830.

3 Shonka Research Associates, 4939 Lower Roswell Road, Suite 106, Marietta, Georgia 30068.

${ }_{5}^{4}$ ChemRisk, a Service of McLaren/Hart, Inc., 5900 Landerbrook Drive, Cleveland, Ohio, 44124.

${ }^{5}$ SENES Oak Ridge, Inc., 102 Donner Drive, Oak Ridge, Tennessee 37830. Dr. Nair is currently with The Cadmus Group, Inc. in Oak Ridge, Tennessee. 
TASK 1 REPORT

July 1999

${ }^{131}$ I Releases from X-10 Radioactive Lanthanum Processing -

This page intentionally left blank. 
TASK 1 REPORT

\section{TABLE OF CONTENTS}

EXECUTIVE SUMMARY ES-1

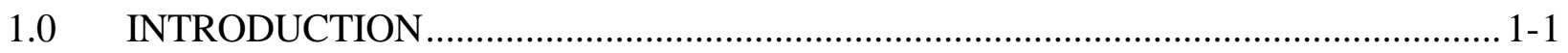

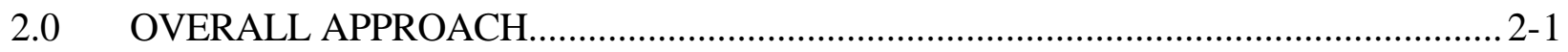

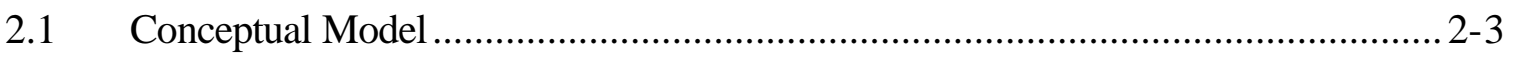

2.2 Description of Model Components ..................................................................... 2-4

2.3 Uncertainty and Sensitivity Analysis .................................................................... 2-6

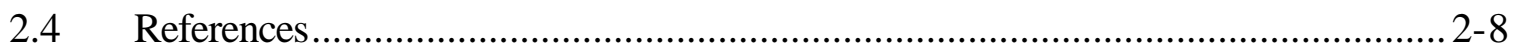

3.0 SOURCE TERM ASSESSMENT FOR IODINE-131 ............................................ 3-1

3.1 A Brief History of Radioactive Lanthanum (RaLa) Processing .................................. 3-1

3.1.1 RaLa Facilities at X-10 ……………………................................... 3-2

3.1.2 Key Sources of Radioiodine Releases.................................................... 3-8

3.1.3 Other Sources of Radioiodine at X-10 and Other Radionuclides ................. 3-9

3.2 Elements of the Source Term Assessment ............................................................ 3-11

3.3 Sources of Information Concerning X-10 RaLa Processing ………………............ 3-12

3.3.1 Slug Exposure and Discharge Reports ("Push Data")................................ 3-12

3.3.2 "100 Area" (Clinton Pile) Log Books......................................................... 3-12

3.3.3 Operations and Health Physics Log Books .............................................. 3-16

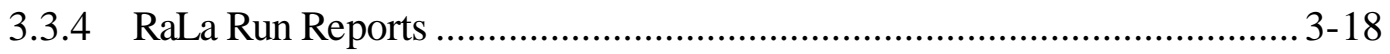

3.3.5 Analytical Reports of RaLa Runs ......................................................... 3-18

3.4 Chronology and Details of X-10 RaLa Runs ....................................................... 3-18

3.4.1 Start Times for Dissolving Batches.......................................................... 3-19

3.4.2 Numbers of Fuel Slugs Dissolved ........................................................ 3-20

3.4.3 Durations of Dissolving Batches............................................................... 3-21

3.4.4 Classification of Batches as to Likelihood of Direct Releases ...................... 3-22

3.5 Calculation of the I-131 Content of Fuel Slugs ………........................................ 3-24

3.5.1 Content of X-10 Slugs .................................................................... 3-24

3.5.2 Content of Hanford Slugs .................................................................... 3-27 
TASK 1 REPORT

July 1999

${ }^{131}$ I Emissions from X-10 Radioactive Lanthanum Processing -

Page TOC-2

Table of Contents

3.6 Correction for Delay between Reactor Shutdown and Dissolution ........................3-33

3.7 Estimation of the Species of Radioiodine Present in the Dissolver........................ 3-36

3.8 Estimation of Iodine Released to the Dissolver Off-gas Stream........................... 3-40

3.9 Estimation of the Magnitude of Direct Releases .............................................. 3-49

3.10 Iodine Removal in the Caustic Scrubber..................................................... 3-51

3.10.1 Initial Expert Opinion.................................................................. 3-51

3.10.2 Mathematical Modeling of the Caustic Scrubber ...................................... 3-51

3.10.3 Monitoring Study During Two RaLa Runs .......................................... 3-53

3.10.4 Building 205 Stack Monitoring ....................................................... 3-56

3.10.5 Expert Opinion Revisited.............................................................. 3-57

3.10.6 Experience with Scrubbers at Other Sites ........................................... 3-58

3.10.7 Representation of Scrubber Collection Efficiency ….............................. 3-58

3.11 Iodine Removal in the Exhaust Path ........................................................... 3-60

3.12 The Source Term Calculations ..................................................................... 3-61

3.13 Source Term Results for Annual Releases ...................................................... 3-66

3.14 Sensitivity Analysis for the Source Terms for Annual Releases ........................... 3-72

3.15 The Run 56 Accident of April 29, 1954 ...................................................... 3-75

3.15.1 Information about the Accident ............................................................ 3-75

3.15.2 Assessment of Iodine Releases from the Event ...................................3-76

3.16 Sensitivity Analysis for the 1954 Accident Source Term.....................................3-80

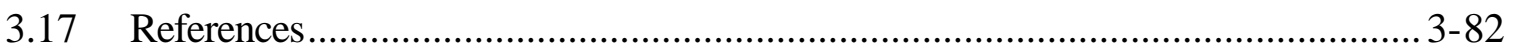

4.0 ATMOSPHERIC DISPERSION AND RADIOIODINE CHEMISTRY ........................4-1

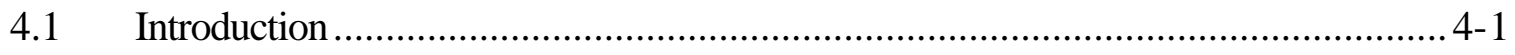

4.2 Atmospheric Chemistry ...................................................................... 4-2

4.2.1 Background ...................................................................... 4-2

4.2.2 Approach and Input Parameters ..................................................... 4-4

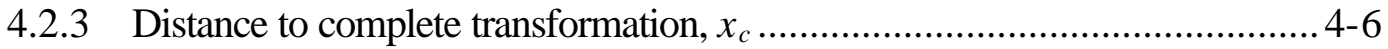


4.3 Atmospheric Dispersion Simulations ................................................................ 4-6

4.3.1 The SORAMI Model............................................................................ 4-6

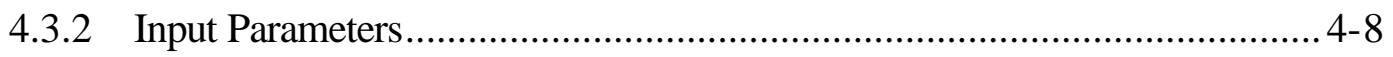

4.3.2.1 Routine releases ....................................................................... $4-8$

4.3.2.2 1954 Accident .......................................................................... 4- 14

4.3.3 Model Validation for Routine Releases ................................................... 4-18

4.3.3.1 Model validation without deposition, plume depletion, deposition and iodine chemistry ....

4.3.3.2 Model validation with plume depletion, deposition, and iodine chemistry

4.3.3.3 Verification for the use of Stack 3020 for all years of simulation.....4-32

4.3.3.4 Verification of the interpolation scheme

4.3.4 Model Validation for Short-Term Releases

4.4 Results.

4.4.1 Routine Releases.............................................................................. 4-35

4.4.2 1954 Accident .............................................................................

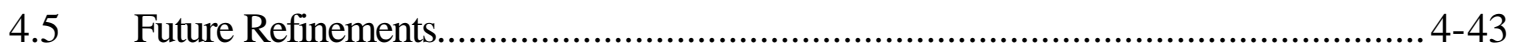

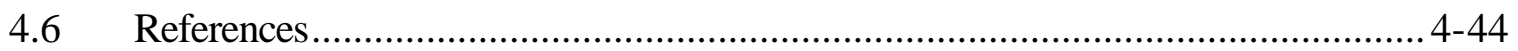

5.0 TRANSFER OF ${ }^{131}$ I FROM AIR TO VEGETATION ................................................... 5-1

5.1 Description of Processes and Model Assumptions …………………...................... 5-1

5.2 Modeling Approach.......................................................................................5-2

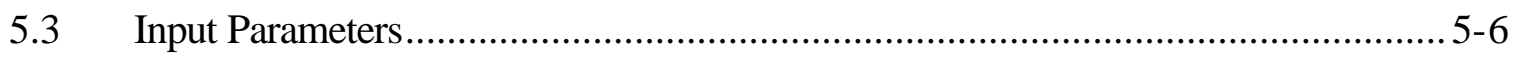

5.3.1 Dry Deposition.................................................................................... 5-6

5.3.2 Wet Deposition.................................................................................. $5-8$

5.3.2.1 Transfer to rain water ................................................................ $5-9$

5.3.2.2 Interception and initial retention by pasture vegetation.....................5-17

5.3.3 Weathering from Vegetative Surfaces ....................................................... 5-19

5.3.4 The Ratio between the Mass of a Dehydrated Vegetation Sample and its Fresh Mass. 
TASK 1 REPORT

July 1999

${ }^{131}$ I Emissions from X-10 Radioactive Lanthanum Processing -

Page TOC-4

Table of Contents

5.3.5 Summary of the Input Parameters ...................................................5-20

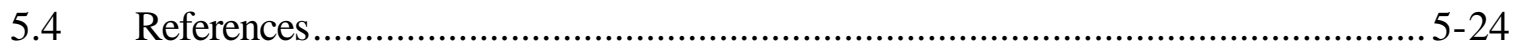

6.0 TRANSFER OF ${ }^{131}$ I FROM PASTURE TO VARIOUS FOOD PRODUCTS ................ 6-1

6.1 Description and Assumptions for Modeling the Transfer of ${ }^{131} \mathrm{I}$ from Pasture to Milk and Meat .............................................................................................. 6-1

6.1.1 Accidental Releases ....................................................................... 6-2

6.1.2 Routine Releases......................................................................... 6-3

6.2 Parameter Distributions .............................................................................. 6-4

6.2.1 Feed intake rates for cows and goats................................................. 6-4

6.2.2 Fraction of feed that is contaminated................................................ 6-7

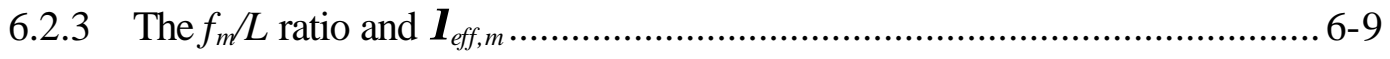

6.2.4 Transfer of Iodine from Feed to Milk of Cows.................................... 6-10

6.2.4.1 Review of Information....................................................... 6-10

6.2.4.2 Assignment of distributions ................................................ 6-16

6.2.5 Transfer of Iodine from Feed to Milk of Goats...................................... 6-19

6.2.6 Transfer of Iodine from Feed to Beef.............................................. 6-19

6.3 Validation of the Modeling Approaches Used to Estimate the ${ }^{131} \mathrm{I}$ Concentrations in Milk ............................................................................ 6-20

6.4 Predicted Milk Concentrations .................................................................. 6-23

6.5 Modeling the Transfer of ${ }^{131}$ I to Food Products other than

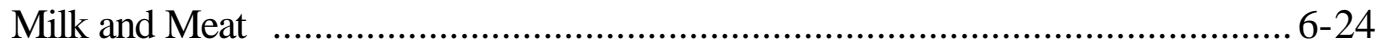

6.5.1 Accumulation of Iodine in Cottage Cheese ........................................ 6-24

6.5.2 Accumulation of Iodine in Eggs ....................................................... 6-28

6.5.3 Accumulation of Iodine in Mother's Milk .......................................... 6-28

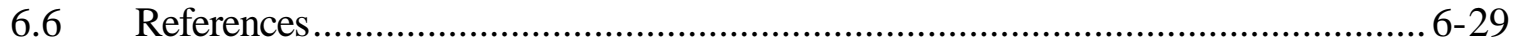

7.0 DISTRIBUTION SYSTEM FOR VARIOUS FOOD PRODUCTS ............................. 7-1

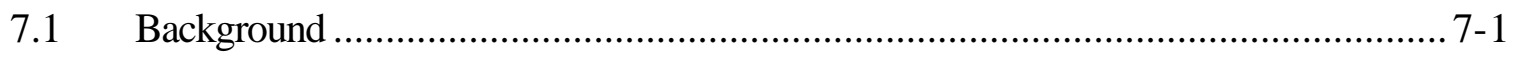

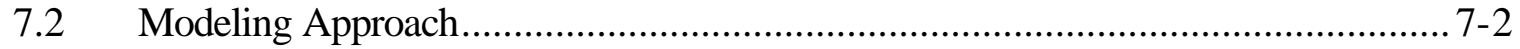


TASK 1 REPORT

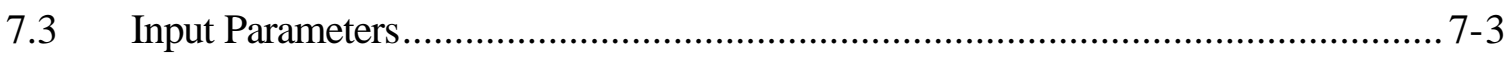

7.3.1 Holdup Time from Collection or Harvest to Consumption............................. 7-4

7.3.2 Fraction of Food that is Contaminated ....................................................... 7-7

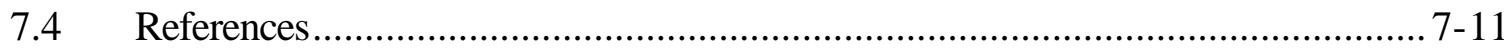

8.0 FOOD CONSUMPTION AND INHALATION PARAMETERS .................................... $8-1$

8.1 Food Consumption Patterns ............................................................................. 8-1

8.1.1 Milk Consumption............................................................................ 8-2

8.1.1.1 Consumption of Cow's Milk ............................................................ 8-2

8.1.1.2 Consumption of Goat's Milk ...................................................... 8-14

8.1.2 Consumption of Meat.................................................................. 8-18

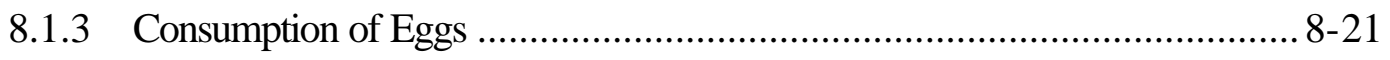

8.1.4 Consumption of Vegetables.................................................................. 8-26

8.1.5 Consumption of Cottage Cheese ………………………………....... 8-26

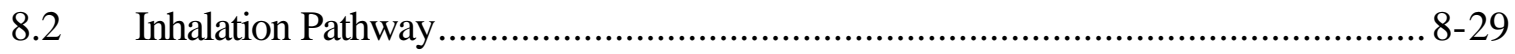

8.2.1 Description and Modeling Approach ..................................................... 8-29

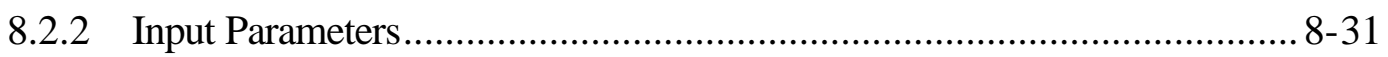

8.2.2.1 Fraction of the day spent outdoors................................................ 8-32

8.2.2.2 Indoor to outdoor concentration ratio ........................................... 8-34

8.2.2.3 Age-dependent breathing rate ...................................................... 8-36

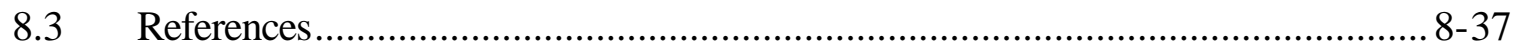

$9.0 \quad$ INTERNAL DOSIMETRY FOR IODINE-131 ……................................................

9.1 Biokinetics of ${ }^{131} \mathrm{I}$ in the Human Body ................................................................. 9-1

9.2 Fraction of energy absorbed in the thyroid …………….................................. 9-2

9.3 Modeling Approach.................................................................................... 9-2

9.3.1 Biokinetic Model Parameters......................................................................9-7

9.3.2 Fraction of ${ }^{131}$ I Deposited and Absorbed in the

Respiratory System.......................................................................... 9-11

$9.4 \quad$ Results and Conclusions .............................................................................. 9-16 
TASK 1 REPORT

July 1999

${ }^{131}$ I Emissions from X-10 Radioactive Lanthanum Processing -

Page TOC-6

Table of Contents

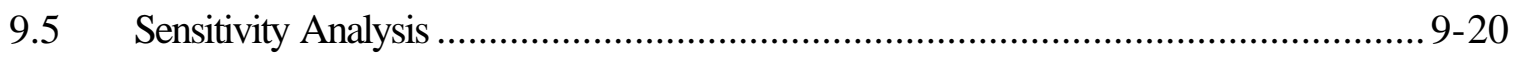

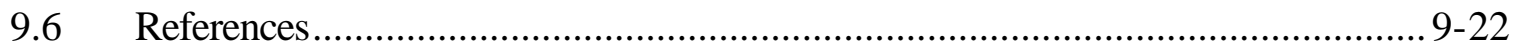

10.0 EXCESS LIFETIME RISK OF THYROID CANCER PER UNIT ABSORBED DOSE FOR CHILDREN EXPOSED TO ${ }^{131} \mathrm{I}$......................................................... 10-1

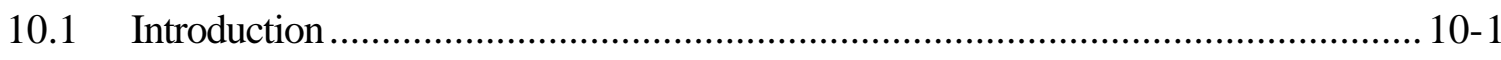

10.1.1 Radiation-induced thyroid cancer.................................................. 10-1

10.1.2 Non-Neoplastic Radiogenic Thyroid Disease ........................................ 10-6

10.1.3 Other Neoplasms .......................................................................... 10-6

10.2 Methodology Used for Estimation of Various Risk-Related Quantities .................. 10-7

10.2.1 The Lifetime Background Risk of Thyroid Cancer................................. 10-9

10.2.2 The Excess Relative and Excess Absolute Risk of Thyroid Cancer per Unit Dose ................................................................................... 10-11

10.2.3 Age and gender modifying factors ................................................ 10-15

10.2.4 Iodine-131 Effectiveness Factor ..................................................... 10-17

10.3 Estimates of ${ }^{131}$ I Risk Factors ................................................................. 10-20

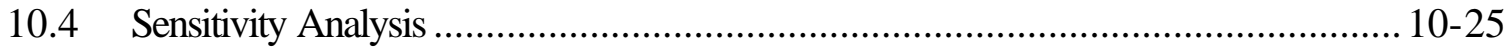

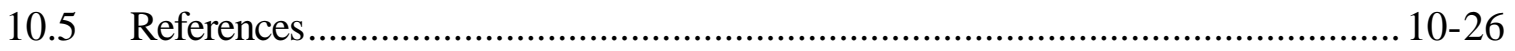

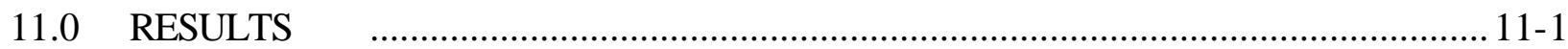

11.1 A Summary of Estimates of Historical Releases of ${ }^{131}$ I from X-10....................... 11-1

11.2 A Summary of Off-site Concentrations of ${ }^{131} \mathrm{I}$ in Environmental Media.

11.3 Estimates of Dose and Risk from the Combined Effect of Routine and Accidental Emissions from RaLa Processing at X-10 from 1944 to 1956

11.3.1 The importance of food type and source of milk ................................... 11-4

11.3.2 The effect of birth year and gender on dose and risk.............................. 11-6

11.3.3 Doses and risks to a female born in 1952 for different dietary sources of milk and different locations within a $38-\mathrm{km}$ radius of $\mathrm{X}-10$............... 11-10

11.3.4 Sensitivity analysis $11-26$ 
11.4 The April 29, 1954, Accident ....................................................................11-26

11.5 Influence of ${ }^{131} \mathrm{I}$ Deposited in Tennessee from the Atmospheric Testing of Nuclear Weapons in Nevada.......................................................................... 11-27

11.6 References............................................................................................... 11-34

12.0 ESTIMATION OF THE HEALTH IMPACT OF X-10 IODINE-131 RELEASES ........ 12-1

12.1 Estimation of the Number of Excess Thyroid Cancers within the Study Domain...... 12-1

12.1.1 Time- and space-averaged concentration of Iodine-131 in milk .................12-2

12.1.2 Milk production ...................................................................... $12-5$

12.1.2.1 Volume of milk.............................................................12-5

12.1.2.2 State milk production..................................................... 12-6

12.1.2.3 County milk production..................................................... 12-7

12.1.2.4 Fraction of fluid milk................................................... 12-10

12.1.2.5 Fraction of milk consumed by children under 15 years

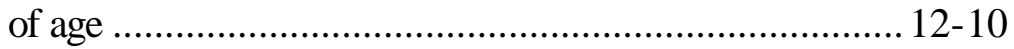

12.1.2.6 Fraction of milk produced within the area of interest........ 12-11

12.1.3 Dose and risk factors ....................................................................... 12-11

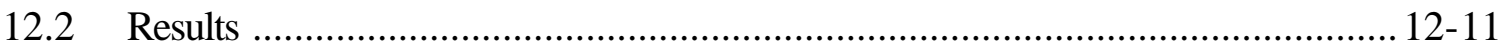

12.3 Sensitivity Analysis ................................................................................ 12-12

12.4 Estimation of the Incidence of Thyroid Cancer beyond $38 \mathrm{~km} . . . \ldots \ldots \ldots \ldots \ldots \ldots \ldots \ldots \ldots . . . . . .12-12$

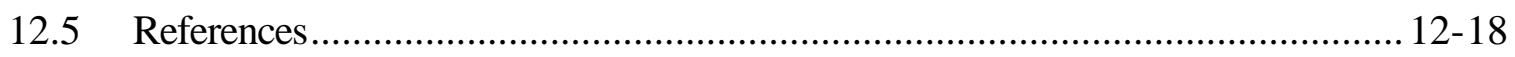

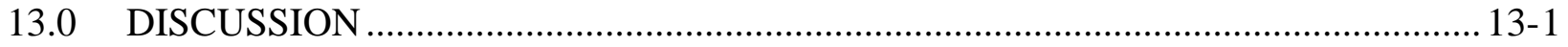

13.1 Differences in Individual Risk....................................................................... 13-1

13.2 Opportunities to Reduce Uncertainty in Dose and Risk Estimates .......................... 13-2

13.2.1 Reduce the scale of time averaging to account for releases occurring for each dissolution run ........................................................................... 13-2

13.2.2 Estimation of the transfer of ${ }^{131}$ I from pasture to milk ............................... 13-2

13.2.3 Assumptions about diet and consumption of milk....................................... 13-3

13.2.4 Organ burden versus absorbed dose......................................................13-3

13.2.5 Estimation of the dose-response relationship for ${ }^{131} \mathrm{I}$................................13-3 
TASK 1 REPORT

July 1999

${ }^{131}$ I Emissions from X-10 Radioactive Lanthanum Processing -

Page TOC-8

Table of Contents

13.2.6 Estimation of the total cases of radiogenic thyroid disease

$13-4$

13.2.7 Estimation of the probability of causation for diagnosed thyroid carcinomas.

13.3 Comparison with 1988-1995 Incidence Data for Thyroid Carcinoma $13-6$

13.4 Comparison with Dose Reconstruction Results for Atmospheric Releases of

${ }^{131}$ I at Other Sites.....

13.5 Comparison with Preliminary Results of Previous Iterations of the

Task 1 Report

13.6 References

14.0 CONCLUSIONS

14- 1

\section{APPENDICES (Volume 1A)}

The numerals that occur before the letters in the appendix titles indicate the section of the main report text to which they are related. Not all sections have appendices.

APPENDIX 2A: DETAILED MATHEMATICAL APPROACH......................................... 2A-1

APPENDIX 3A： SAMPLE “PUSH DATA” FOR CLINTON PILE SLUGS ........................ 3A-1

APPENDIX 3B: SAMPLE HANFORD SLUG IRRADIATION CORRESPONDENCE........3B-1

APPENDIX 3C： SAMPLE PAGES FROM 100 AREA (CLINTON PILE) LOG BOOKS.... 3C-1

APPENDIX 3D: SAMPLE PAGES FROM A CHEMICAL SEPARATIONS OPERATIONS LOG BOOK........................................................... 3D-1

APPENDIX 3E: $\quad$ A SAMPLE X-10 RALA RUN REPORT …............................................

APPENDIX 3F: A SAMPLE RALA RUN ANALYTICAL REPORT .................................3F-1

APPENDIX 3G: RALA DISSOLVING BATCHES CONDUCTED IN X-10'S BUILDING 706-D......................................................................... 3G-1

APPENDIX 3H: DETAILS OF BATCHES FOR WHICH NUMBERS OF SLUGS DISSOLVED WERE ESTIMATED $3 \mathrm{H}-1$

APPENDIX 3I: MEMO: FINAL INVENTORY VALUES FOR X-10 SLUGS 3I-1

APPENDIX 3J: MEMO: CHARACTERIZATION OF UNCERTAINTIES ASSOCIATED WITH THE CALCULATION OF DISCHARGE ${ }^{131}$ I INVENTORIES FOR X-SLUGS PUSHED FOR RALA PRODUCTION 
APPENDIX 3K: MEMO: INVENTORY CALCULATIONS FOR W-SLUG RALA RUNS

APPENDIX 3L: MEMO: TUBE FACTORS FOR THE HANFORD PRODUCTION REACTORS

APPENDIX 3M: MEMO: SLUG FACTORS FOR THE HANFORD PRODUCTION REACTORS

APPENDIX 3N： MEMO: DECAY TIMES FOR W-SLUGS ...................................... $3 \mathrm{~N}-1$

APPENDIX 30: DOCUMENTS PERTAINING TO THE 1954 RALA ACCIDENT ..............3O-1

APPENDIX 4A: AIR FLOW PATTERNS AT THE OAK RIDGE RESERVATION ............. 4A-1

APPENDIX 10A: THYROID CANCER INCIDENCE DATA …………………….............. 10A-1

APPENDIX 11A: RATIONALES FOR LOCATIONS AND EXPOSURE PATHWAYS ...... 11A-1

APPENDIX 11B: ESTIMATED IODINE-131 CONCENTRATIONS

IN ENVIRONMENTAL MEDIA $11 \mathrm{~B}-1$

APPENDIX 11C: DETAILED RESULTS OF THE DOSE RECONSTRUCTION

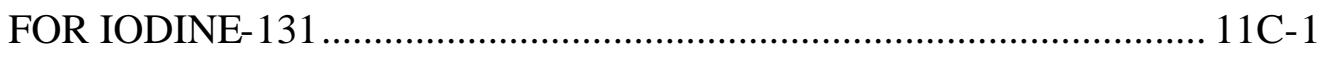

APPENDIX 12A: MILK PRODUCTION DATA ........................................................... 12A-1 


\section{LIST OF TABLES}

Table 3.1 Time History of Usage of X-10 Stacks for RaLa Process Exhausts .......................3-8

Table 3.2 Listing of X-10 Chemical Separations Operations Log Books ............................ 3-17

Table 3.3 Method of Assigning Start Times to Dissolving Batches Based on Operations Shifts

Table 3.4 Method for Estimation of Durations of Dissolving Batches ................................. 3-21

Table 3.5 Classification of Batches as to Apparent Opportunity for Direct Releases ............. 3-23

Table 3.6 Prevalences of the Classes of Dissolving Batches by Potential for Direct

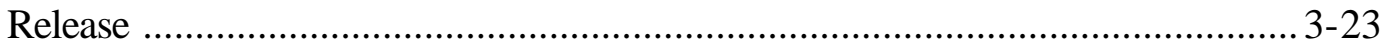

Table 3.7 Uncertainty Factors Applied to Inventories Calculated for X-10 Slugs ..................3-28

Table 3.8 Uncertainty Factors Applied to Inventories Calculated for Hanford Slugs..............3-33

Table 3.9 Estimated Decay Times for Sets of Hanford RaLa Slugs....................................3-34

Table 3.10 Coefficients for Decay Correction of the Total Iodine Content of Hanford Slugs ....3-36

Table 3.11 Documented Irradiation Durations for Hanford RaLa Slugs and Durations Assumed for Decay Correction of Total Iodine Inventories................................ 3-37

Table 3.12 RaLa Plant Model Input Parameters for the Dissolver and Dissolver Condenser

Table 3.13 Direct Release Percentages Assumed for each Batch Class ................................3-50

Table 3.14 Scrubber Model Inputs and Results for Nine Cases ..........................................3-53

Table 3.15 Annual Iodine Releases from RaLa Processing via the X-10 Stack .....................3-67

Table 3.16 Annual Iodine Releases from the RaLa Processing Building .............................. 3-67

Table 3.17 Iodine-131 Releases from the April 29, 1954 Accident During RaLa

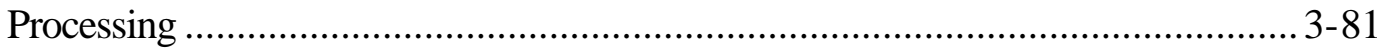

Table 4.1 Relative humidity for Oak Ridge and Hanford ............................................. 4-4

Table 4.2 Mixing height data used for analysis ....................................................... 4-11

Table $4.3 \quad$ Stack release parameters........................................................................ $4-12$

Table 4.4 Weather conditions between 4 and 8 p.m. on April 29, 1854, from the X-10 Health Physics Group Meteorological Station 012 4-15 
TASK 1 REPORT

Table 4.5 Meteorological data from strip-chart records for April 29, 1954 from X-10 Health Physics Group Meteorological Station 012 within Bethel Valley.....

Table 4.6 Amounts of ${ }^{131}$ I released from Bethel and Melton Valley Stacks between 1967 and 1969

Table 4.7 Measured annual average ground-level concentrations of ${ }^{131} \mathrm{I}$

Table 4.8 Comparison of the $\mathrm{P} / \mathrm{O}$ ratios of the annual average concentrations of ${ }^{131} \mathrm{I}$ from the SORAMI model and the public-domain model, ISC3-ST.

Table 4.9 The geometric mean P/O ratios of the predicted annual average concentrations of ${ }^{131}$ I from SORAMI with the inclusion of dry and wet deposition, plume depletion, and chemistry, and assuming that all of the releases between 1967 and 1969 occurred from Stack 3020, to the measured concentrations

Table 4.10 The geometric mean P/O ratios of the predicted annual average concentrations of ${ }^{131}$ I from SORAMI with the inclusion of dry and wet deposition, plume depletion, and chemistry, and assuming that all of the releases between 1967 and 1969 occurred from Stack 3020 and all of the Melton Valley releases between 1967 and 1969 occurred from Stack 7911, to the measured concentrations

Table 4.11 Results from the validation of SORAMI model for short-term (weekly) releases .... 4-35

Table 5.1 Selected values and distribution shapes for the total dry deposition velocity $V_{d} \ldots \ldots . .5-7$

Table 5.2 Selected values and distribution shapes for the mass interception factor $(r / Y)_{d r y}$ for dry deposition....................................................................................... 5-8

Table 5.3 Comparison between calculated and measured values for normalized

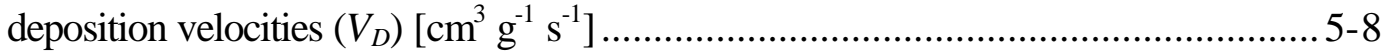

Table 5.4 Annual statistics of the 400 sets of a full year's hourly rainfall data using the Monte Carlo Markov Chain model, annual statistics from the monthly average data provided in Holland (1953), and annual statistics of the measured data from ATDD between 1986 and 1996.

Table 5.5 Monthly statistics of the 400 sets of a full year's hourly rainfall data using the Monte Carlo Markov Chain model, monthly average of measurements from Holland (1953), and monthly statistics of the measured data from ATDD between 1986 and 1996 
TASK 1 REPORT

July 1999

${ }^{131}$ I Emissions from X-10 Radioactive Lanthanum Processing -

Page TOC-12

Table of Contents

Table 5.6 Analysis of the rainfall data measured at ATDD between 1986 and 1992 ...............5-16

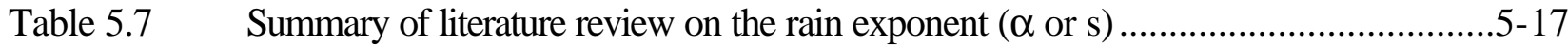

Table 5.8 Parameter distributions used for estimation of the transfer of ${ }^{131} \mathrm{I}$ from air to vegetation

Table 6.1 Parameter distributions used for the preliminary calculations of the transfer of

${ }^{131}$ I from pasture to milk and beef.

Table 6.2 Subjective cumulative probability levels for values of the milk transfer coefficient for ${ }^{131}$ I recommended by individual experts

Table 6.3 Summary of literature documenting the variability of feed-to-milk transfer coefficient $\left(F_{m}\right)$ for ${ }^{131} \mathrm{I}$ in dairy cows

Table 6.4 Locations of interest where concentrations of ${ }^{131} \mathrm{I}$ in milk were estimated and which are closest to the 8 milk sampling stations identified in Figure 6

Table 6.5 Predicted and measured average ${ }^{131} \mathrm{I}$ concentrations in milk $\left(\mathrm{Bq} \mathrm{L}^{-1}\right)$ for the selected periods of interest in 1962 and 1964

Table 6.6 Summary of milk-to-cheese transfer coefficients reported by different studies

Table 7.1 Parameter distributions used for the uncertainty analysis for various food products

Table 8.1 Values for parameters used in the cow's milk ingestion pathway for routine releases

Table 8.2 Values for parameters used in the cow's milk ingestion pathway for accidental releases

Table 8.3 Values for parameters used in the goat's milk ingestion pathway for routine and accidental releases.

Table 8.4 Values for parameters used in the meat ingestion pathway for routine releases

Table 8.5 Values for parameters used in the egg ingestion pathway for routine releases

Table 8.6 Values for parameters used in the egg pathway for the accidental release

Table 8.7 Values for parameters used in the vegetable ingestion pathway for the routine release

Table $8.8 \quad$ Values for parameters used in the vegetable ingestion pathway for the accidental release 
TASK 1 REPORT

Table 8.9 Values for parameters used in the cottage cheese ingestion pathway for the routine releases

Table 8.10 Values for parameters used in the cottage cheese ingestion pathway for the accidental release

Table 8.11 Summary of the input parameters used in the estimation of dose and excess lifetime risk due to inhalation of ${ }^{131} \mathrm{I}$......

Table 8.12 Age-dependent breathing rates for exposed individuals

Table 9.1 Distributions for the biological half-life $\left(T^{*}\right)$ and the fractional uptake $(a)$ based on the data from Dunning and Schwartz (1981).................................................... 9-8

Table 9.2 Thyroid mass (grams) obtained by ultrasonography (Bier, 1996)

Table 9.3 Age-specific dose factors $\left(\mathrm{Gy} \mathrm{Bq}^{-1}\right)$ for ingestion of ${ }^{131} \mathrm{I}$ derived for use in this study.....

Table 9.4 Dose to the thyroid of a fetus per unit activity of ${ }^{131} \mathrm{I}$ ingested by a pregnant woman $(\mathrm{NCI}, 1997)$......................................................................................... 9-19

Table 10.1 Results of epidemiological studies for exposure to gamma and $\mathrm{X}$ rays .................... 10-2

Table 10.2 Results of epidemiological studies for exposure to $\mathrm{X}$ rays ……………................... 10-3

Table 10.3 Results of epidemiological and experimental studies for exposure to ${ }^{131}$ I............... 10-4

Table 10.4 Background lifetime risk of cancer for all counties in the State of Tennessee other than Anderson, Roane, Loudon and Knox determined for an average life expectancy of 70 years $10-13$

Table 10.5 Pooled analysis of cohort studies of persons exposed before age 15 years: modifiers of excess relative risk per Gy $\left(\mathrm{ERR} \mathrm{Gy}{ }^{-1}\right)$

Table 10.6 Estimates of the excess lifetime risk per unit dose (risk factors) $\left[\mathrm{Gy}^{-1}\right]$, assuming an uncertain ${ }^{131}$ I effectiveness factor $\quad 10-20$

Table 10.7 Estimates of the excess lifetime risk per unit dose (risk factors) $\left[\mathrm{Gy}^{-1}\right]$, assuming that ${ }^{131} \mathrm{I}$ is equally effective to $\mathrm{X}$-rays and gamma rays in inducing thyroid cancer $(\delta=1.0) \quad 10-23$

Table 10.8 Comparison between the excess lifetime risk per unit dose (risk factors) obtained using two types of dose-response relationships (models). The risk factors are estimated for children of both genders (ages 0 to 14) exposed to ${ }^{131} \mathrm{I}$

Table 11.1 Locations of concern for which the analysis was conducted 
Table 11.2 Thyroid doses (cGy), excess lifetime risk of thyroid cancer, relative risk, and probability of causation for a female born in 1952 who lived in Solway, Tennessee, from 1952 to 1956

Table 11.3 Thyroid doses (cGy) from ingestion of commercial milk produced at Solway and contaminated with ${ }^{131} \mathrm{I}$ from routine releases at $\mathrm{X}-10$

Table 11.4 Excess Lifetime Risk of thyroid cancer from ingestion of local produce and locally produced commercial milk (diet 2) for individuals living in Solway.....

Table 11.5 Factors for estimating doses and risks for individuals of any birth year from those estimated for individuals born in 1952 .

Table 11.6 Factors for estimating doses for single exposure pathways from the estimated dose from diet 1 (backyard cow's milk and all other non-milk exposure pathways) for a female born in 1952 .

Table 11.7 Thyroid doses (cGy) to a female born in 1952 from a diet consisting of local produce and backyard cow's milk

Table 11.8 Thyroid doses (cGy) to a female born in 1952 consuming locally produced commercial milk

Table 11.9 Thyroid doses (cGy) to a female born in 1952 consuming goat's milk (diet 4)..... 11-20

Table 11.10 Excess Lifetime Risk for a female born in 1952 on a diet consisting of local produce and backyard cow's milk (diet1)......

Table 11.11 Excess Lifetime Risk for a female born in 1952 consuming locally produced commercial milk

Table 11.12 Excess Lifetime Risk for a female born in 1952 consuming goat's milk (diet 4)..... 11-25

Table 11.13 Thyroid doses and excess lifetime risk of thyroid cancer for females of various ages exposed to ${ }^{131}$ I released from X-10 during April 29, 1954 accident

Table 11.14 Thyroid dose and excess lifetime risk of thyroid cancer for females of different ages exposed to ${ }^{131}$ I released from X-10 during April 29, 1954 accident

Table 11.15 Estimates of average thyroid doses, with associated uncertainties from exposure to ${ }^{131} \mathrm{I}$ in fallout from atmospheric nuclear bomb testing in Nevada, for females born on January 1, 1952 in counties adjacent to X-10, shown by milk source...... 11-30 
Table 11.16 Thyroid doses and risks from combined exposure to ${ }^{131}$ I from NTS fallout and $\mathrm{X}-10$ releases (routine and accidental) for females born in 1952 and on a diet of backyard cow's milk

Table 11.17 Thyroid doses and risks from combined exposure to ${ }^{131} \mathrm{I}$ from NTS fallout and X-10 releases (routine and accidental) for females born in 1952 and on a diet of milk from commercial sources.

Table 11.18 Thyroid doses and risks from combined exposure to ${ }^{131} \mathrm{I}$ from NTS fallout and $\mathrm{X}-10$ releases (routine and accidental) for females born in 1952 on a diet of goat's milk

Table 13.1 Comparison of uncertainty statements for estimated doses from other ${ }^{131} \mathrm{I}$ dose reconstruction studies.

Table 13.2 Progress of Task 1 of the Oak Ridge Dose Reconstruction study during the three iterations.

Table 13.3 Summary of the changes in the major results during the three iterations of Task 1 of the Oak Ridge Dose Reconstruction 


\section{LIST OF FIGURES}

Photograph 1 Los Alamos Apparatus for Using RaLa to Test the Implosion Process....................3-3

Photograph 2 The Central Area of the X-10 Site .................................................................. 3-4

Photograph 3 Interior View of the Building 706-D RaLa Processing Facility...............................3-5

Photograph 4 The Building 706-D Dissolver and Associated Components................................ 3-6

Figure 1.1 The Oak Ridge Reservation (Oak Ridge, Tennessee), study domain, and locations of interest at which the consequences of ${ }^{131} \mathrm{I}$ releases between 1944 and 1956 from the RaLa processing facility at X-10 are studies.

Figure 2.1 Schematic of the processes addressed for the assessment of the health outcomes from exposure to ${ }^{131} \mathrm{I}$ released from the RaLa processing facility at X10

Figure 2.2 Monte-Carlo method for uncertainty propagation.

Figure 3.1 Radioactive Lanthanum (RaLa) Processing at the Oak Ridge, Tennessee Site; Facility and Process Overview

Figure 3.2 Calculated Average Iodine-131 Inventories of X-10 RaLa Slugs..... 3-26

Figure 3.3 Trend of I-131 Content of Hanford RaLa Slugs ..... 3-32

Figure 3.4 RaLa Process Components Included in the Plant Model. 3-41

Figure 3.5 Elemental Iodine Flow Rate from the RaLa Condenser as a Function of the Product of Uranium Dissolution Rate $\times$ Iodine Fraction (4-inch X-10 Slugs).

Figure 3.6 Elemental Iodine Flow Rate from the RaLa Condenser as a Function of the Product of Uranium Dissolution Rate $\times$ Iodine Fraction (8-inch Hanford Slugs)...... 3-48

Figure 3.7 Annual Elemental I-131 Releases from the X-10 Stack (Ci) .............................3-68

Figure 3.8 Annual Elemental I-131 Releases from the RaLa Process Building (Ci) ................3-68

Figure 3.9 Annual Organic I-131 Releases from the X-10 Stack (Ci)...............................3-69

Figure 3.10 Annual Organic I-131 Releases from the RaLa Process Building (Ci) .................. 3-69

Figure 3.11 Annual Particulate I-131 Releases from the X-10 Stack (Ci) ............................3-70

Figure 3.12 Annual Particulate I-131 Releases from the RaLa Process Building (Ci)............... 3-70

Figure 3.13 Annual Elemental F131 Releases from the X-10 Stack (Ci) with Operational

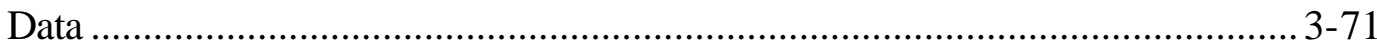


Figure 4.1 Map including the extent of contamination from the April 29, 1954 accident............ 4-17

Figure 4.2 Locations of the Health Physics Group monitoring stations.................................... 4-19

Figure 4.3 Elevations of Bethel and Melton Valley stacks...................................................... 4-22

Figure 4.4 The P/O ratios from the SORAMI Model for the case for which all of the ${ }^{131} \mathrm{I}$ assumed to be released in the organic form.

Figure 4.5 Measured concentrations and the $95 \%$ subjective confidence intervals of the predicted concentrations from the SORAMI model for 1967 for the case for which all of the ${ }^{131} \mathrm{I}$ assumed to be released in the organic form.

Figure 4.6 Measured concentrations and the 95\% subjective confidence intervals of the predicted concentrations from the SORAMI model for 1968 for the case for which all of the ${ }^{131} \mathrm{I}$ assumed to be released in the organic form.

Figure 4.7 Measured concentrations and the 95\% subjective confidence intervals of the predicted concentrations from the SORAMI model for 1969 for the case for which all of the ${ }^{131} \mathrm{I}$ assumed to be released in the organic form.

Figure 4.8 Contours for the lower bound estimates (2.5 percentile) of the $95 \%$ subjective confidence interval for the annual average, ground-level concentrations of total ${ }^{131} \mathrm{I}\left({ }^{131} \mathrm{I}\right.$ in all three chemical forms) in air

Figure 4.9 Contours for the upper bound estimates (97.5 percentile) of the $95 \%$ subjective confidence interval for the annual average, ground-level concentrations of total ${ }^{131} \mathrm{I}\left({ }^{131} \mathrm{I}\right.$ in all three chemical forms $)$ in air.

Figure 4.10 Contours for the lower bound estimates ( 2.5 percentile) of the $95 \%$ subjective confidence interval for the time-integrated, ground-level concentrations of total ${ }^{131} \mathrm{I}$ in air following the accident on April 29, 1954.

Figure 4.11 Contours for the central estimate (50th percentile) of the 95\% subjective confidence interval for the time-integrated, ground-level concentrations of ${ }^{131} \mathrm{I}$ in air following the accident on April 29, 1954

Figure 4.12 Contours for the upper bound estimates (97.5 percentile) of the $95 \%$ subjective confidence interval for the time-integrated, ground-level concentrations of total ${ }^{131} \mathrm{I}$ in air following the accident on April 29, 1954.

Figure 6.1 Fraction of the cow's feed that is fresh pasture $\left(p_{m}\right)$, in different grazing seasons ....... 6-8

Figure 6.2 Feed-to-milk transfer factors for ${ }^{131} \mathrm{I}$ administered to different breeds of cows dairy cows during 1962 to 1970 at the Univ. of Tennessee, Knoxville.

Figure 6.3 Effect of milk yield on ${ }^{131} \mathrm{I}$ transfer from feed to milk in different breeds of dairy cows.

Figure 6.4 Effect of milk yield on ${ }^{131}$ I transfer from feed to milk in dairy cows (all breeds)....... 6-15 
TASK 1 REPORT

July 1999

${ }^{131}$ I Emissions from X-10 Radioactive Lanthanum Processing -

Page TOC- 18

Table of Contents

Figure 6.5 Comparison between various studies that document variability of ${ }^{131} \mathrm{I}$ feed-to-

milk transfer coefficient

Figure 6.6 Locations of milk sampling stations around the X-10 facility.

Figure 6.7 Estimated lower limit (2.5 percentile) of the thirteen-year (1944-1956) average concentrations of ${ }^{131} \mathrm{I}$ in backyard cow milk $\left(\mathrm{Bq} \mathrm{L}^{-1}\right)$

Figure 6.8 Estimated upper limit (97.5 percentile) of the thirteen-year (1944-1956) average concentrations of ${ }^{131} \mathrm{I}$ in backyard cow milk $\left(\mathrm{Bq} \mathrm{L}^{-1}\right)$

Figure 6.9 Milk-to-cottage cheese transfer coefficient reported by different studies.

Figure $9.1 \quad$ Nuclear decay scheme for ${ }^{131} \mathrm{I}$

Figure 9.2 Two-compartment biokinetic model for iodine

Figure 9.3 Comparison of the thyroid masses obtained by autopsy investigation and by ultrasound investigations

Figure 9.4 Comparison of the thyroid masses from Bier (1996) and from Mogilev and Gomel Counties (Former Soviet Union) which were affected by the Chernobyl accident

Figure 9.5 Comparison of the thyroid masses from Bier (1996) and from Bryansk and Kiev Counties (Former Soviet Union) which were affected by the Chernobyl accident

Figure 9.6 Comparison of the thyroid mass from Bier (1996) and from Zhytomir County (Former Soviet Union) which were affected by the Chernobyl accident.

Figure 9.7 Comparison of the ingestion dose factors for ${ }^{131} \mathrm{I}$ calculated in this study to the most recent recommended ICRP (1989) values....

Figure 9.8 Contributors to the uncertainty in the ${ }^{131} \mathrm{I}$ ingestion dose factor for various age groups

Figure 10.1 Age-specific thyroid cancer rate for males and females in Tennessee (19881995 diagnosis period)

Figure 10.2 Background lifetime risk of thyroid cancer based on statistics for all counties in the State of Tennessee other than Anderson, Roane, Loudon, and Knox counties (1988-1995 diagnosis period).

Figure 10.3 Example of the effect of age at time of exposure on the annual risk of thyroid cancer for a single dose of $0.3 \mathrm{~Gy}$ (external exposure).

Figure 10.4 Discrete probability distribution function that describe the uncertainty in the reduction factor accounting for ${ }^{131}$ I effectiveness with respect to an acute dose of $\mathrm{X}$ rays or gamma rays 
Figure 10.5 Excess lifetime risk of thyroid cancer per Gy (estimated Risk Factors).

Figure 10.6 Comparison between different assumptions about ${ }^{131} \mathrm{I}$ effectiveness in estimation of the excess lifetime risks of thyroid cancer per Gy for females

Figure 11.1 Thyroid doses (cGy) from various exposure pathways and diets for a female born in 1952 who lived in Solway, Tennessee

Figure 11.2 Contours for the lower bound $\left(2.5^{\text {th }}\right.$ percentile) of the $95 \%$ subjective confidence interval of the dose to a female born in 1952 from a diet consisting of local produce and backyard cow's milk (diet 1).

Figure 11.3 Contours for the central estimate $\left(50^{\text {th }}\right.$ percentile $)$ of the $95 \%$ subjective confidence interval of the dose to a female born in 1952 from a diet consisting of local produce and backyard cow's milk (diet 1).

Figure 11.4 Contours for the upper bound $\left(97.5^{\text {th }}\right.$ percentile) of the $95 \%$ subjective confidence interval of the dose to a female born in 1952 from a diet consisting of local produce and backyard cow's milk (diet 1).

Figure 11.5 Contours for the lower bound $\left(2.5^{\text {th }}\right.$ percentile $)$ of the $95 \%$ subjective confidence interval of the excess lifetime risk of developing thyroid cancer for a female born in 1952 on a diet consisting of local produce and backyard cow's milk (diet 1)

Figure 11.6 Contours for the central estimate $\left(50^{\text {th }}\right.$ percentile) of the $95 \%$ subjective confidence interval of the excess lifetime risk of developing thyroid cancer for a female born in 1952 on a diet consisting of local produce and backyard cow's milk (diet 1)

Figure 11.7 Contours for the upper bound $\left(97.5^{\text {th }}\right.$ percentile) of the $95 \%$ subjective confidence interval of the excess lifetime risk of developing thyroid cancer for a female born in 1952 on a diet consisting of local produce and backyard cow's milk (diet 1)

Figure 12.1 Schematic showing the locations at which the ${ }^{131} \mathrm{I}$ concentrations in milk were calculated.......

Figure 12.2 The number of cows and the annual milk volumes for the State of Tennessee

Figure 12.3 Contributors to the uncertainty in the total number of cancers estimate ................. 12-13

Figure 12.4 Amount of ${ }^{131}$ I deposited within a circle of a given radius around X-10 ............. 12-15

Figure 12.5 Amount of ${ }^{131} \mathrm{I}$ deposited within a circle of a given radius around X-10 .............12-16

Figure 13.1 Comparison of the results produced in the three iterations of the Oak Ridge Dose Reconstruction, in terms of thyroid dose for a female born in 1944 drinking milk from commercial sources. 
TASK 1 REPORT

July 1999

${ }^{131}$ I Releases from X-10 Radioactive Lanthanum Processing -

Page TOC-20

This page intentionally left blank. 


\section{EXECUTIVE SUMMARY}

Oak Ridge National Laboratory (ORNL), originally known by the code name X-10, released radioactive iodine $\left({ }^{131} \mathrm{I}\right)$ to the air from 1944 through 1956 as it processed spent nuclear reactor fuel. The process recovered radioactive lanthanum (RaLa) to support weapons development at Los Alamos, for atmospheric radiation tracking, and for radiation warfare experiments. Iodine concentrates in the thyroid gland. Therefore, the health concerns stemming from exposure to ${ }^{131} \mathrm{I}$ include various diseases of the thyroid such as thyroid cancer and non-neoplastic abnormalities such as autoimmune hypothyroidism and Graves disease.

This study investigated the possible risks of thyroid cancer from the releases of ${ }^{131}$ I between 1944 and 1956 at 41 representative locations within 38 kilometers of ORNL. Communities within this region include Oak Ridge, Clinton, Oliver Springs, Kingston, Harriman, Lenoir City, Sweetwater, Maryville, and Knoxville. At each of these locations, the risks of developing thyroid cancer, the relative risk with respect to an unexposed population, and the probability of causation for diagnosed cases of thyroid cancer were determined for individuals of both genders and of various age groups at the time of exposure. The numbers of cancers that could have resulted from exposure to the Oak Ridge ${ }^{131} \mathrm{I}$ releases within $38-\mathrm{km}, 100-\mathrm{km}$, and $200-\mathrm{km}$ radii of the X-10 facility were also estimated. The overall impact of ${ }^{131} \mathrm{I}$ exposure from the combined contributions of X-10 release and fallout from atmospheric testing of nuclear weapons at the Nevada Test Site was also evaluated. The results of this study were found to be comparable to results from similar studies conducted at other sites in the United States. The probability of occurrence of non-neoplastic thyroid disease within $38-\mathrm{km}$ of X-10 has been discussed based on new evidence on the doses of radiation required to induce such diseases.

A number of recommendations have been made in this report that can be used in a follow-up work to reduce the uncertainty in dose and risk and to identify and eliminate any remaining sources of possible bias. Important components of the study, the processes addressed within each component, and intermediate results are summarized below, followed by a discussion of the estimated health impacts.

\section{Source Term}

Over the 13-year period of the RaLa operations at X-10, approximately 30,000 reactor fuel slugs were dissolved in about 731 batches during the process of separating over 19,000 TBq $(1 \mathrm{TBq}=$ $10^{12} \mathrm{~Bq}$; approximately $500,000 \mathrm{Ci}$ ) of radioactive barium as a source of ${ }^{140} \mathrm{La}$ for shipment to Los Alamos. The analysis of the source term (the amount of ${ }^{131} \mathrm{I}$ released to the atmosphere) involved estimation of the annual quantities of radioiodine released from vents and openings in Buildings 706-C and 706-D and from the X-10 stacks 3020 and 3039, as a result of routine normal and off-normal conditions. For a large accident that occurred on April 29, 1954, releases estimates were made for each half-hour period. 
The most important source of radioiodine releases from RaLa processing was exhaust ("off gas") from the slug dissolver. Volatile gases, which included radioiodine, were withdrawn from the dissolver by negative pressure, passed through a condenser and a chemical scrubber, and then routed through piping to the 200-foot tall 3020 stack. After March 1950, exhausts from the chemical scrubber were routed to a central treatment facility in which contaminated air was passed through an electrostatic precipitator and particulate filters prior to release up the 250-foot tall 3039 stack.

The iodine and barium contents of the irradiated fuel slugs from the X-10 Clinton Pile (Graphite Reactor) and the reactors at Hanford were estimated. For each of the 731 dissolving batches, the potential for "direct" releases of untreated exhaust to the atmosphere through building vents, windows, and other openings were evaluated. Because of the absence of monitoring data, expert opinion was extensively used to quantify the ${ }^{131} \mathrm{I}$ collection efficiency of the condenser and caustic scrubber for routine operations. Expert opinion was also used to quantify the potential degradation of collection efficiencies during the April 1954 accident.

Approximately 8,800 to $42,000 \mathrm{Ci}\left(0.3\right.$ to $\left.1.6 \mathrm{PBq} ; 1 \mathrm{PBq}=10^{15} \mathrm{~Bq}\right)$ of ${ }^{131} \mathrm{I}$ was released between 1944 and 1956, of which 6,300 to $36,000 \mathrm{Ci}(0.23$ to $1.3 \mathrm{PBq})$ was in the elemental (most environmentally reactive) form of iodine; the remainder was in the nonreactive volatile organic form. As shown in Tables ES-1, ES-2, and ES-3, the largest releases occurred between 1952 and 1956, when the freshly spent uranium fuel slugs came from Hanford reactors. The April 29, 1954, accident released 105 to $500 \mathrm{Ci}$ (3.9 to $21 \mathrm{TBq}$ ) over 2.5 hours $^{1}$, accounting for about $6.5 \%$ of the total releases for 1954 .

\section{Atmospheric Dispersion}

After being released into the atmosphere, ${ }^{131}$ I was transported by the prevailing winds. A fraction of the iodine released in the elemental (reactive) form was chemically transformed during transport to particulate and organic (nonreactive) forms within a few kilometers of the RaLa processing facility. The ground-level concentration of ${ }^{131} \mathrm{I}$ in air is affected by several factors including the distance of the location of interest from the RaLa processing facility, the dilution of the concentrations in the air during atmospheric dispersion or mixing, the depletion of iodine from air by the processes of wet and dry deposition, and the chemical form in which iodine is present.

Annual average ground-level concentrations of ${ }^{131} \mathrm{I}$ for the routine releases and time-integrated ground-level concentrations of ${ }^{131}$ I for the 1954 accident were estimated using SORAMI, a mathematical model that accounts for all of the relevant processes that are important during the transport of ${ }^{131} \mathrm{I}$ in the atmosphere. The model was benchmarked using another public-domain model. The model was validated using site-specific release and monitoring data, and the validation results indicate that the model predictions were within a factor of 2 of the annual average measurements.

\footnotetext{
${ }^{1}$ While the period of direct release from the RaLa dissolver appears to have lasted 30 minutes, it took some time for the building ventilation to clear out this airborne contamination. Releases were estimated for five half-hour periods, resulting in a total release duration of 2.5 hours.
} 
For the analysis of routine releases between 1944 and 1956, electronic data collected at X-10 from 1987 to 1996 were analyzed statistically to generate surrogate hourly meteorological data. For the April 29, 1954, accident, the SORAMI model was supplied with half-hourly meteorological data obtained from documented records of the specific meteorology prevailing at the time of the accident. Uncertainties in all input parameters to SORAMI were quantified before the concentrations were estimated for each of the 41 locations of interest.

\section{Transfer from Air to Vegetation}

Consumption of contaminated milk and meat from cattle grazing on pastureland contaminated by the deposition of ${ }^{131} \mathrm{I}$ from air is one of the most important pathways by which ${ }^{131} \mathrm{I}$ enters the human body. Once ${ }^{131} \mathrm{I}$ is transported to a given location, it is transferred from the atmosphere to vegetation and the ground surface by precipitation scavenging of the plume and by dry deposition processes. The rates of transfer from the atmosphere to vegetation surfaces are dependent on the chemical form of iodine in air; therefore, the total amount of ${ }^{131}$ I transferred was estimated by accounting for all three forms of iodine (elemental, particulate, and organic).

For routine releases, annual average concentrations of ${ }^{131} \mathrm{I}$ on vegetation were estimated using a constant rate of deposition of ${ }^{131}$ I from the air for a given year and the assumption that the annual average concentrations of ${ }^{131} \mathrm{I}$ in vegetation and in air were in equilibrium with each other. For the 1954 accident, deposition of ${ }^{131}$ I onto vegetation lasted for a period of 2.5 hours, but it remained on the vegetation for a longer period of time until the processes of removal from vegetation and natural radioactive decay eliminated it completely. A time-integrated concentration of ${ }^{131} \mathrm{I}$ on the vegetation was, therefore, estimated to account for the longer-term availability of contaminated feed to the cattle.

\section{Transfer from Pasture to Various Food Products}

Once ${ }^{131} \mathrm{I}$ is transferred to the surfaces of vegetation, it is available for ingestion by grazing animals. Once ingested, it is further transferred into milk, meat, cheese, and eggs. Estimates of the transfer of ${ }^{131} \mathrm{I}$ from pasture to milk and beef were based on information from available literature and on unpublished measurements of the transfer of ${ }^{131}$ I into the milk of various breeds of dairy cattle used on farms in East Tennessee during the 1950s and 60s. No significant difference was observed among the various breeds of cows; however, an inverse relationship was observed between ${ }^{131} \mathrm{I}$ transfer to milk and milk yield for those animals producing less than $10 \mathrm{~L}$ $\mathrm{d}^{-1}$. For this reason, a distinction was made between the transfer of ${ }^{131} \mathrm{I}$ into the milk for lowproducing "backyard" cows and the higher-producing cows belonging to commercial dairies. Estimates of ${ }^{131}$ I concentrations were also made for goat's milk, human breast milk, cottage cheese, eggs, and beef.

The modeling approach used in this study for estimating concentrations of ${ }^{131} \mathrm{I}$ in milk was validated. Average concentrations of ${ }^{131} \mathrm{I}$ measured in raw milk collected from locations around X-10 in 1962 and 1964 were found to lie well within the 95\% subjective confidence intervals of the average value predicted by the model for eight locations in the vicinity of the sampling stations. Radioiodine releases from ORNL during this period resulted from reactor operations and radioisotope production programs. 
The concentrations of ${ }^{131} \mathrm{I}$ in animal food products were estimated with mathematical models. For routine releases, the annual average concentration on pasture was related directly to the annual average concentration in animal food products. For the 1954 accident, time-integrated concentrations of ${ }^{131} \mathrm{I}$ in milk and meat were estimated based on the time-dependent concentration of ${ }^{131} \mathrm{I}$ in pasture grass.

\section{Distribution of Contaminated Food Products}

The distribution of food products from various producers to a potential consumer is a complex process that is difficult to reproduce with high accuracy. Contaminated foodstuffs produced in an affected area may be distributed to other areas not directly exposed by the radioactive plume. Conversely, individuals in the affected area may consume uncontaminated products imported from unaffected areas. In this analysis, food products produced at a given farm were generally assumed to be consumed locally or distributed to the local population for consumption. Milk was often exchanged between counties to cover consumption needs in milk-deficient areas. The effects of the distribution system for a given food type were considered by accounting for radioactive decay between the time of milking or harvest and the time of human consumption of the food product, for the fraction of the food consumed that originated from uncontaminated areas, and for the amount of radioiodine lost during food preparation. Eggs and cottage cheese were assumed to be produced and consumed locally, and only the reduction of contamination due to radioactive decay during the time between production and consumption was considered.

\section{Inhalation and Food Consumption}

Human exposure to ${ }^{131} \mathrm{I}$ is dependent on the concentration of ${ }^{131} \mathrm{I}$ in air and in food at a given location and on the rates of inhalation and food consumption. This study included a detailed investigation of inhalation and ingestion rates by gender and age. Different rates of inhalation and ingestion were considered for estimating exposures from the routine releases of ${ }^{131} \mathrm{I}$ and from the 1954 accident. Because of the importance of human exposure via the consumption of contaminated milk, this study focused heavily on the estimation of consumption rates of fresh milk for infants and children under the age of 10. These estimates included differences in the consumption of locally produced milk and milk obtained at school. The estimated rates for inhalation of contaminated air for infants, children, teenagers, and adults accounted for the amount of time spent indoors and the differences between the concentration of ${ }^{131} \mathrm{I}$ in air between the indoor and outdoor environments.

Consumption of meat, leafy vegetables, eggs, and cheese was also considered in addition to the ingestion of milk. In all, doses and risks were estimated for 11 individual exposure pathways: ingestion of backyard cow's milk, commercial milk, regionally mixed commercial milk, goat's milk, meat, leafy vegetables, eggs, cottage cheese, inhalation of contaminated air, prenatal exposure from ${ }^{131} \mathrm{I}$ ingested by mother, and ingestion of contaminated mother's milk during infancy. 
Individuals living near X-10 may have been exposed via more than one exposure pathway at a time. Three special exposure scenarios are designed to match the most likely dietary habits and lifestyles in the vicinity of the Oak Ridge Reservation. The first exposure scenario (called "diet 1") refers to individuals living in a "rural farm" setting. The intake for this exposure scenario is obtained from ingestion of backyard cow's milk, beef, leafy vegetables, eggs, and cheese and from inhalation. The second exposure scenario (called "diet 2") refers to individuals in a rural area who buy milk from a local dairy farm. They are also exposed to contaminated beef, leafy vegetables, eggs, cottage cheese, and air. The third scenario (called "diet 3") refers to individuals in a more "urban" setting, who buy milk and food products from the grocery store. The intake for this exposure scenario is obtained from ingestion of regionally averaged commercial milk and from inhalation. Given that the doses and risks from ingestion of goat milk are substantially larger than the doses and risks from any other exposure pathway, it is addressed separately under the "diet 4" scenario.

\section{Internal Dosimetry}

After it is inhaled or ingested, ${ }^{131} \mathrm{I}$ is absorbed into the bloodstream and then metabolized in a manner that is identical to the absorption and metabolism of stable iodine in the human body. The thyroid gland preferentially absorbs iodine from the extracellular fluid into the thyroid cells and follicles. Iodine is then used in the production of hormones essential for human metabolism. In this analysis, the absorbed thyroid dose per unit intake of ${ }^{131}$ I was estimated as a function of age. Since the uncertainty in the dose per unit intake is largely affected by the interindividual variability in the thyroid mass, this investigation employed the most recent information on thyroid volume as determined by ultrasonography. It was found that the mass of the thyroid was somewhat smaller than assumed in past studies. However, this finding was offset by the finding of a more rapid biological clearance rate from the thyroid, which resulted in a central estimate of the dose per unit intake that was similar to values recommended by the International Commission on Radiological Protection for newborn children up to children 15 years or age.

\section{Excess Lifetime Risk per Unit Absorbed Dose}

It is well established that X- and gamma irradiation of the thyroid at doses approaching $10 \mathrm{cGy}$ will result in increased incidences of thyroid carcinomas and adenomas in children exposed before the age of 15 . At higher doses, radiation might also induce non-neoplastic thyroid conditions such as autoimmune hypothyroidism, Hashimoto's thyroiditis, or Graves disease. The thyroid gland in children has one of the highest radiogenic risk coefficients of any organ. Fortunately, fatal thyroid cancers are rare (the 5-year survival rate is 95\%). The effectiveness of ${ }^{131} \mathrm{I}$ in producing thyroid cancers is a subject that is still under investigation with ongoing epidemiological studies at numerous other locations. The most convincing evidence of the link between ${ }^{131} \mathrm{I}$ exposure and thyroid cancer is still emerging from reports and preliminary results from the follow-up of children exposed to ${ }^{131}$ I from the 1986 Chernobyl accident. Additional supporting evidence exists from animal studies and from epidemiological investigations of Utah school children exposed to ${ }^{131}$ I from atmospheric weapons testing at the Nevada Test Site. 
In this study, a relative risk model was used to estimate the chance of acquiring a thyroid cancer from an absorbed dose of ${ }^{131} \mathrm{I}$. This model calculates the excess lifetime risk of thyroid cancer per unit dose as the product of the excess relative risk per unit absorbed dose and the background lifetime risk for an unexposed individual, including a series of modifying factors to account for differences in radiosensitivity by gender and age at time of exposure and for the effectiveness of

${ }^{131} \mathrm{I}$ in producing thyroid cancer compared with that of X-rays and gamma rays. The background lifetime risk of thyroid cancer was obtained from incidence data for the state of Tennessee, excluding the counties of Anderson, Roane, Loudon, and Knox, which were affected by releases of ${ }^{131}$ I from X-10.

The excess lifetime risk of thyroid cancer per cGy changes markedly depending on the gender of the individual and the age at time of exposure. For females exposed to 1 cGy before the age of 5 years, the excess lifetime risk ranged from about five chances in one hundred thousand to sixteen chances in ten thousand, with a central value of three chances in ten thousand. At the same dose, the risk to males in this age group would be about four times less than those for females. Females who were over the age of 20 at time of exposure to $1 \mathrm{cGy}$ would have had risks almost 80 times less, while males over the age of 20 would have had risks about 300 times less than females who were under the age of 5 years.

\section{Results}

Females born in 1952 who consumed goat's milk (diet 4) received the highest doses and have the highest risks of contacting thyroid cancer during their lifetime. The next highest dose results from the consumption of milk from a backyard cow, followed by milk from a local commercial dairy and milk that was regionally mixed. Since the concentration of ${ }^{131} \mathrm{I}$ in regionally mixed retail milk is about the same regardless of location within the 38-km domain, its importance with respect to the consumption of local produce or to inhalation varies from location to location.

Lower doses are obtained from inhalation or from the consumption of locally produced beef, cottage cheese, mother's milk (with the mother assumed to be on diet 1), or leafy vegetables. The doses from inhalation or from the consumption of one of these food types for a child under the age of 5 at the time of exposure are several hundred to more than 1000 times less important than the dose from the consumption of backyard cow's milk. The thyroid dose from prenatal exposure during the first part of 1952 (assuming the mother to be on diet 1) is about equal to the 5 -year total thyroid dose obtained from the consumption of beef or cottage cheese. Risks were estimated specifically for the four diet categories, each of which consists of a combination of pathways.

Among the 41 selected locations within the 38-km vicinity of the X-10 facility, the highest doses occurred at Gallaher Bend, located a little more than $6 \mathrm{~km}$ to the east of X-10, while the lowest doses occurred at Wartburg, located $27 \mathrm{~km}$ northwest of X-10. For example, at Gallaher Bend, thyroid doses ranged from about 4 to $250 \mathrm{cGy}$ for individuals of each gender born between 1940 and 1952 who consumed milk from a backyard cow (between one and five 8-ounce glasses each day) and who consumed food products from a local garden or farm. A similar group of individuals residing in Wartburg would have received doses ranging from about 0.08 to 6 cGy. 
Doses from the consumption of regionally mixed commercial retail milk ranged from about 0.3 to $10 \mathrm{cGy}$ for individuals born from 1940 to 1952 and did not vary by location.

Doses were calculated for males and females born in 1920, 1930, 1935, 1940, 1944, 1950, 1952, 1954, and 1956 who lived near X-10 during the period of radioactive lanthanum processing (1944-1956). These years of birth were chosen to allow comparison of doses and risks from ${ }^{131} \mathrm{I}$ intake by individuals of different ages at time of exposure. It was assumed that all members of the reference populations received no ${ }^{131}$ I exposure other than that from the X-10 RaLa releases that occurred from 1944 to 1956 . This being the case, it is not important where a person was born or where they lived before 1944 or after 1956. It is very important, however, where in the Oak Ridge area the person lived from 1944 through 1956. Assuming the same dietary sources for ingestion of ${ }^{131} \mathrm{I}$ at a specific location, differences in gender account for only minor differences in the estimation of the thyroid doses. More significant differences are determined by the year of birth, with the lowest doses being for individuals born in 1920, 1930, and 1956. These doses are about one-fourth to one-fifth of the largest doses received by individuals born between the years of 1944 and 1952. Individuals born in 1954 have about the same doses as those born in 1940, which are about 65\% of the doses for those born between 1944 and 1952.

The highest excess risk of developing thyroid cancer for a female born in 1952 on diet 1 occur at the agricultural communities of Bradbury and Gallaher Bend. For these locations, based on the assumed quantities of backyard cows' milk, beef, vegetables, eggs, and cheese consumed each day, the risk estimates are confidently above one chance in one thousand $\left(1 \times 10^{-3}\right)$ but less than 1 chance in ten $\left(1 \times 10^{-1}\right)$. In addition, at these locations, the central estimate of the probability of causation approaches or exceeds 50\% for females born in 1952 on diets 1, 2, and 4, meaning that a diagnosed thyroid cancer has more than an even chance of being due to exposure to ${ }^{131} \mathrm{I}$ released from X-10. The central estimate of risk for a female born in 1952 on diet 1 is likely to exceed 1 chance in $1,000\left(1 \times 10^{-3}\right)$ up to distances of $35 \mathrm{~km}$ to the southwest and more than 38 $\mathrm{km}$ to the northeast of $\mathrm{X}-10$. A risk of more than one chance in ten thousand $\left(1 \times 10^{-4}\right)$ is likely with a subjective confidence of over $50 \%$ at all locations in the $38-\mathrm{km}$ vicinity of $\mathrm{X}-10$.

Depending on the year of birth, the excess lifetime risk to females is 3 to 4 times larger than the risk to males. At a location such as Bradbury or Gallaher Bend, the lowest risk is for a male born in 1920, who has an excess lifetime risk of thyroid cancer almost 1000 times less than the highest risk for females born in 1952. A female born in 1920 has a risk about 350 times lower than that for a female born in 1952. Individuals of the same gender born in 1944 have about 50-60\% of the risk than those born in 1952 have, while individuals born in 1940 or 1956 have risks about 5 times lower than those for individuals born in 1952. However, a male born in 1940 or 1956 has a risk almost 20 times less than that of a female born in 1952.

The primary locations affected by the April 29, 1954, accident are those situated to the north and northwest of X-10, such as Jonesville, Norwood, East Fork Poplar Creek in Oak Ridge, Oliver Springs, and Wartburg. The excess lifetime risk of thyroid cancer to females on diet 1, exposed in their early childhood at either Jonesville, Norwood, East Fork Poplar Creek, or Oliver Springs ranged from a few chances in 10 million $\left(3\right.$ or $\left.4 \times 10^{-7}\right)$ to nearly one chance in thousand $\left(1 \times 10^{-3}\right)$. In general, the total doses and risks from exposure to the April 29, 1954, accident are much lower than those resulting from exposure to the accident and the routine emissions combined. 
Most of the uncertainty in the estimates of risk is associated with the uncertainty in the estimates of dose $(55 \%)$, followed by the uncertainty in the dose response for cohorts exposed to external sources of radiation. Uncertainty in the dose estimates are dominated by the uncertainty in the estimated concentrations of ${ }^{131} \mathrm{I}$ in milk (45\%), followed by the uncertainty in the internal dose conversion factor $(40 \%)$. The uncertainty in the estimates of milk concentrations is dominated by the uncertainty in the transfer of ${ }^{131}$ I from air to pasture $(71 \%)$. The uncertainty in the internal dose conversion factor is dominated by the uncertainty in determining the actual mass of the thyroid for any individual of a given gender and age.

Fallout from atmospheric testing of nuclear weapons at the Nevada Test Site (NTS) during 1952, 1953, 1955, and 1957 was a significant contributor to the total ${ }^{131}$ I exposure for individuals located within $38 \mathrm{~km}$ of the X-10 site. Beyond this distance, ${ }^{131} \mathrm{I}$ from NTS fallout was clearly the dominant source of exposure. For a female born in 1952 who consumed backyard cow's milk, central estimates of the thyroid dose from combined exposures from X-10 releases and NTS fallout within the $15 \mathrm{~km}$ vicinity of X-10 ranged from 25 to $30 \mathrm{cGy}$, with upper bounds of the $95 \%$ subjective confidence interval exceeding $200 \mathrm{cGy}$. At all locations within $15 \mathrm{~km}$ of X10 , the risk to a female born in 1952 on a rural diet 1 exceeds one chance in one thousand $(1 \times$ $\left.10^{-3}\right)$. The upper bound of the $95 \%$ subjective confidence interval for the excess lifetime risk of thyroid cancer exceeds several chances in one hundred $\left(6-9 \times 10^{-2}\right)$ up to more than one chance in ten $\left(1 \times 10^{-1}\right)$ at the communities of Bradbury and Gallaher Bend. The doses from the combined exposure to ${ }^{131}$ I released from X-10 and ${ }^{131}$ I deposited via NTS fallout are sufficiently high to have possibly manifested excess cases of non-neoplastic disease, namely autoimmune thyroiditis.

Number of Excess Cancers Expected in the 38-, 100-, and 200-km Vicinity of X-10

Within the $38-\mathrm{km}$ vicinity of $\mathrm{X}-10$, the $95 \%$ subjective confidence interval of the number of excess thyroid cancers resulting from consumption of cow's milk (commercial and backyard milk, combined), contaminated by ${ }^{131}$ I released from the RaLa processing facility between 1944 and 1956, range between 6 and 84, with a central estimate of 21. For the consumption of backyard cow's milk alone, the 95\% subjective confidence interval ranged from 1 to 33 excess cases of cancer, with a central estimate of 7. Commercial cow's milk contributed more to the number of excess cancers because of the larger quantity of commercial milk produced and the larger number of individuals exposed via its consumption.

The 95\% subjective confidence interval of the number of excess thyroid cancers from consumption of cow's milk contaminated by X-10 ${ }^{131}$ I releases (commercial and backyard milk, combined) range from 14 to 103 (central estimate of 35) within a 100-km vicinity of X-10, and from 25 to 149 (central estimate of 58) within a 200-km vicinity of X-10. These cancers are expected to manifest between 1950 and 2020, with the majority occurring after 1970. It should be noted that these calculations of possible numbers of excess thyroid cancers are based on the linear, no threshold hypothesis. Under that hypothesis, added cancer risk is assumed to be proportional to the collective radiation dose received. This holds whether one has large doses received by a few people or low doses received by a much larger population. However, available data cannot exclude the possibility that there is a low-level threshold for causation of cancer. If this is the case, then the values presented above are likely over-estimates of true incidence rates. 
These estimates were made using a baseline of thyroid cancer diagnoses within the regional population with the assumption that the individuals in this population were unexposed. Only about $28 \%$ of the total number of thyroid cancers in a population are diagnosed and reported. Therefore, it is likely that the total number of diagnosed and occult cases of thyroid cancer is about 3 to 4 times greater than the total estimate given in this study which are based on the incidence of thyroid cancer reported only through clinical diagnosis. The clinical significance of an excess incidence of benign nodules is not evaluated in this study, but it is noted that about $9 \%$ of benign nodules diagnosed through palpation and about $28 \%$ of those diagnosed through ultrasound will be surgically removed. 
TASK 1 REPORT

July 1999

${ }^{131}$ I Releases from X-10 Radioactive Lanthanum Processing Page ES-10

Executive Summary

This page intentionally left blank. 


\subsection{INTRODUCTION}

From 1944 to 1956, freshly irradiated uranium fuel slugs were dissolved at the X-10 site in Oak Ridge, Tennessee, for the purpose of separating radioactive barium $\left({ }^{140} \mathrm{Ba}\right)$ as a source of radioactive lanthanum $\left({ }^{140} \mathrm{La}\right)$ for Los Alamos weapons development and other research projects; ${ }^{140} \mathrm{La}$ is formed by the decay of ${ }^{140} \mathrm{Ba}$. This process involving the production of Radioactive Lanthanum has been historically referred to as "RaLa." Due to the 12.8-d half-life of the parent fission product ${ }^{140} \mathrm{Ba}$, the reactor fuel used for production of RaLa was processed shortly after removal from the reactor. As a result, ${ }^{131} \mathrm{I}$, a highly volatile and short-lived fission product (halflife of $8.04 \mathrm{~d}$ ), was released to the atmosphere.

This report presents the details of the assessment conducted to estimate the amounts of ${ }^{131}$ I that were released from the RaLa process into the atmosphere, transported off-site, and taken up into the thyroid glands of exposed individuals. The endpoints of the assessment are the absorbed radiation doses to the thyroids of males and females at various ages at the time of their exposure and their corresponding excess lifetime risks of developing thyroid cancer. The doses are defined by the energy deposited in the thyroid tissue from the decay of ${ }^{131} \mathrm{I}$ accumulated in the thyroid. The deposited energy induces modifications in the thyroid cells that may lead to thyroid cancer or other abnormalities. The risk values represent the probability (or the chance) that an exposed individual will contract a thyroid cancer during his or her remaining lifetime after exposure to ${ }^{131} \mathrm{I}$. The risks presented here represent the radiation-induced thyroid cancer incidence in excess of the natural background incidence of thyroid cancer. In addition, the total number of excess cases of thyroid cancer that could have resulted, to date, from exposures to releases of ${ }^{131}$ I have been estimated for regions of $38-\mathrm{km}$ and $200-\mathrm{km}$ radii around the RaLa processing facility.

Effects of exposure to ${ }^{131}$ I are estimated for the ingestion of cow's and goat's milk, cottage cheese, beef, leafy vegetables, eggs, and human breast milk, and for the direct inhalation of airborne ${ }^{131}$ I. For exposures to contaminated cow's and goat's milk, ingestion of commercial dairy milk, milk from a backyard cow, and goat's milk are considered separately. Thyroid doses and the associated risks are also estimated for individuals on three different types of combined diets and for a fetus exposed to ${ }^{131}$ I ingested or inhaled by the mother.

Forty-one locations of concern were selected in the vicinity of the Oak Ridge Reservation on the basis of the presence of population clusters, backyard cows or goats, cows contributing to the commercial supply of milk, and beef cattle during the 1944 to 1956 period (Figure 1.1). All locations of concern are within a radius of $38 \mathrm{~km}$ from the RaLa processing facility.

The methodology presented in this study allows a person of any age and either gender to obtain an individualized estimate of dose to the thyroid and excess lifetime risk of developing thyroid cancer. In all steps of this assessment, uncertainties have been identified, quantified, and propagated through to the estimates of doses to the thyroid glands and the corresponding risks of thyroid cancer for the exposed individuals. 
TASK 1 REPORT

July 1999

${ }^{131}$ I Releases from X-10 Radioactive Lanthanum Processing -

Page 1-2

This page intentionally left blank. 


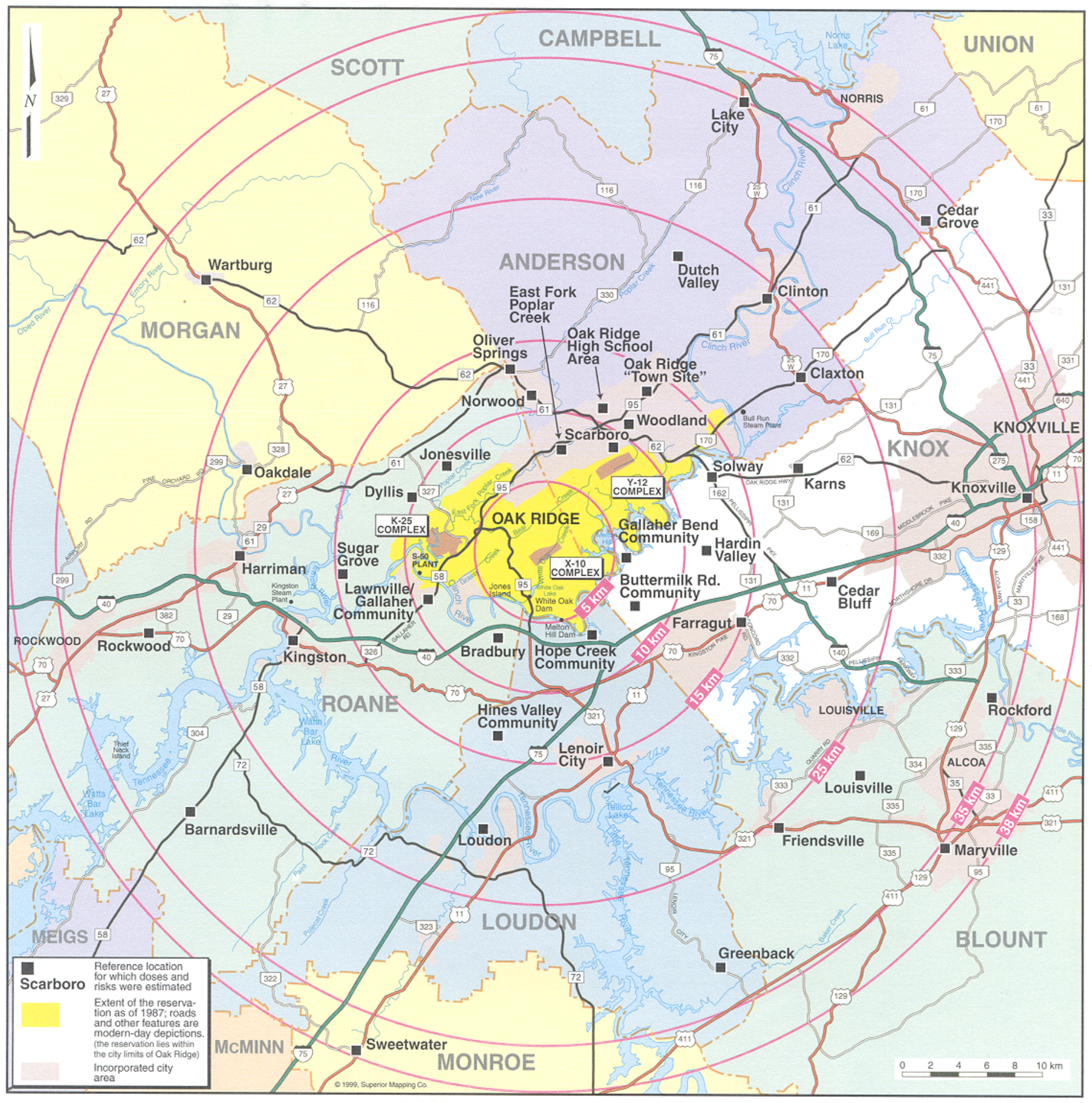

Figure 1.1 The Oak Ridge Reservation (Oak Ridge, Tennessee), study domain, and locations of interest at which the consequences of ${ }^{131}$ I releases between 1944 and 1956 from the RaLa processing facility at X-10 were studied. 
TASK 1 REPORT

July 1999

${ }^{131}$ I Releases from X-10 Radioactive Lanthanum Processing -

Page 1-4

Introduction

This page intentionally left blank. 


\subsection{OVERALL APPROACH}

During the period 1944 to 1956 , when the ${ }^{131}$ I releases occurred from RaLa processing, environmental monitoring and surveillance technology was not sufficiently developed to provide direct information about the concentrations of ${ }^{131} \mathrm{I}$ in air, vegetation, or milk. In addition, use of present-day measurements of longer-lived fission products to infer historical concentrations of the short-lived ${ }^{131} \mathrm{I}$ in air and agricultural food products is not practical because of the presence of weapons fallout and the release of trace quantities of longer-lived radionuclides from numerous other operations at the X-10 site (Bruce et al., 1993). As a result, the present Task 1 dose reconstruction is dependent on the use of mathematical models to estimate the release, transport, uptake, doses, and health effects of ${ }^{131} \mathrm{I}$. This section presents the modeling approach used in this study to estimate the doses and thyroid cancer risks to people exposed to ${ }^{131}$ I released from the X-10 facility. All available effluent and environmental data were used to calibrate and validate the models.

Two separate approaches are taken to assess the health impacts within a study domain of 38-km radius around the RaLa processing facility. The first approach represents the analysis of routine releases from 1944 to 1956. The second approach represents a detailed analysis of an April 29, 1954, accident. A schematic of the processes addressed for each of the approaches is presented in Figure 2.1. For the routine releases, the processes of atmospheric dispersion, deposition, and iodine chemistry are analyzed on an hourly basis, and the source terms, contamination of milk and the doses and risks to members of the public are assessed on the basis of annual averages. For the 2.5-hour accident in 1954, all analyses up to the estimation of the concentrations of ${ }^{131} \mathrm{I}$ in air, milk, and leafy vegetables are conducted with a half-hour time-step.

A distinction is made between the ingestion rates used to estimate the effects of routine releases and those used for the 1954 accidental release, even for individuals of the same age. For routine releases, the ingestion rates are defined as annual averages; for the 1954 accident, ingestion rates could show seasonal effects because of a much shorter time period of concern (approximately 3 to 6 weeks). Therefore, the ingestion rates for the 1954 accident have a larger range of possible values than the range of values specified for the annual average rates.

The dosimetry of ${ }^{131} \mathrm{I}$ in the human body and the dose-response relationship depend on the age of the individual; thus, they vary from one year to another for any individual. However, the uncertainty in internal dosimetry and in the dose-response relationships is attributable mainly to interindividual variability. The necessary parameters for the analyses of internal dosimetry and dose-to-risk are selected by accounting for interindividual variability, and this variability is assumed to be the same for the analyses of both routine and accidental releases.

The health impact of the releases from X-10 is assessed in terms of the excess lifetime risk of thyroid cancer for individuals exposed at various locations within $38 \mathrm{~km}$ of X-10.

In addition, the total number of excess cases of thyroid cancers expected within a $200-\mathrm{km}$ radius of the RaLa processing facility is discussed. 


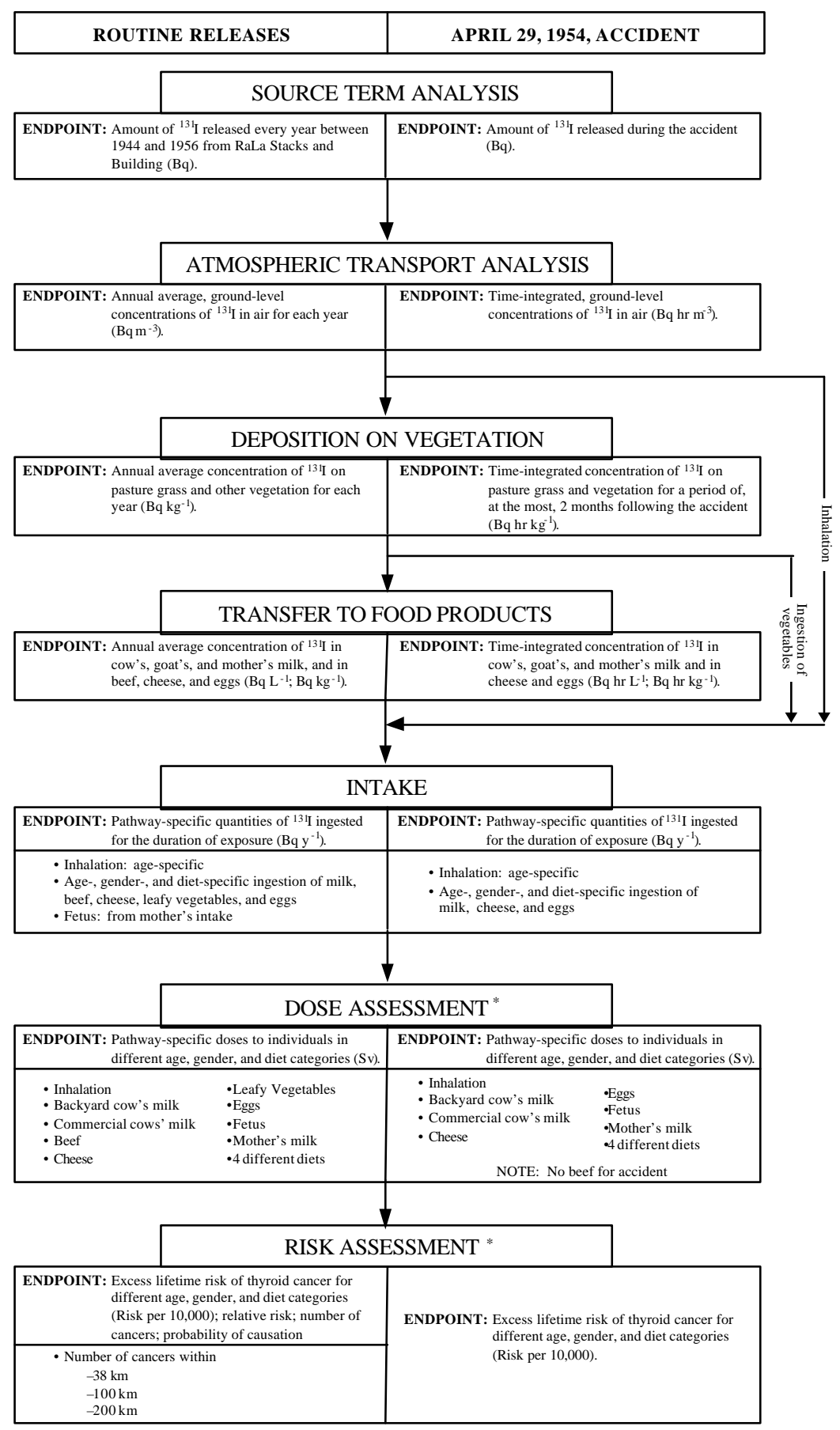

* Dose and risk assessments for the routine releases include contributions from the April 29, 1954 accident.

Figure 2.1: $\quad$ Schematic of the processes addressed for the assessment of health outcomes from exposure to ${ }^{131}$ I released from the RaLa processing facility at X-10 


\subsection{Conceptual Model}

The excess lifetime risk (ELR) of thyroid cancer from exposure to ${ }^{131} \mathrm{I}$ is defined as the probability of acquiring a radiation-induced thyroid cancer by an individual exposed to a single dose of ${ }^{131} \mathrm{I}\left(D_{i}\right)$ at a given age $(i)$. In this study, the excess lifetime risk of thyroid cancer is calculated for an individual exposed to ${ }^{131}$ I released from the X-10 facility as follows:

$$
E L R_{i}=R F_{i} \cdot D_{i}
$$

where

$$
\begin{aligned}
& R F_{i}=\quad \text { the risk factor, defined as the excess lifetime risk of thyroid cancer per unit } \\
& \text { dose from exposure at age " } i \text { " }\left[\mathrm{Gy}^{-1}\right] \text {; and } \\
& D_{i} \quad=\quad \text { the dose delivered to the thyroid from an exposure to }{ }^{131} \text { I at age }(i)[\mathrm{Gy}]
\end{aligned}
$$

\section{Routine Releases}

If an individual is exposed for a longer time period than one year, the total excess lifetime risk (TELR) of thyroid cancer from multiple exposures occurring over several years is given by

$$
T E L R=\sum_{i=N_{1}}^{N_{2}} R F_{i} \cdot D_{i}
$$

In the above equation, $N_{1}$ is the age at which exposure began and $N_{2}$ is the age at which the exposure ended. The historical releases of ${ }^{131} \mathrm{I}$ from X-10 occurred for 13 years, from 1944 to 1956. Therefore, it is reasonable to expect that individuals could have been exposed to ${ }^{131} \mathrm{I}$ for several years, if not the entire 13 years of the RaLa operation.

A process-based approach is used to estimate the excess lifetime risk (ELR) of thyroid cancer. The contributing processes include the following:

- the source term, or the annual average release of ${ }^{131} \mathrm{I}$ to the atmosphere;

- atmospheric transport, including the processes of dispersion, deposition, plume depletion, and radioiodine chemistry;

- transfer of ${ }^{131}$ I from air to vegetation;

- transfer of ${ }^{131}$ I from vegetation to food products;

- distribution and storage of food products;

- intake of ${ }^{131}$ I from ingestion of food and inhalation of air;

- dose to the thyroid per unit intake of ${ }^{131} \mathrm{I}$; and

- lifetime risk of thyroid cancer, considering the metabolism and dosimetry within the human body. 
The total excess lifetime risk to an individual exposed by more than one pathway is obtained by calculating the total intake of iodine from all applicable pathways. Appendix 2-A provides the methodologies to estimate the total excess lifetime risk of thyroid cancer and thyroid doses from individual pathways, starting with ${ }^{131} \mathrm{I}$ concentrations in air. Details of the underlying approaches and mathematical formulations used for the estimation of each component of this assessment are presented in individual sections of this report.

\section{Accident}

On April 29, 1954, an accident occurring at the RaLa dissolution plant resulted in the release of iodine to the atmosphere both from the stack and from the building itself. The duration of the accidental release was about 2.5 hours, starting after 4:30 p.m. The accident produced elevated concentrations of ${ }^{131} \mathrm{I}$ in the air for a short time after the accident and elevated concentrations of

${ }^{131} \mathrm{I}$ in various food items for a period of 1 to 2 months after the accident. The effect of this accidental release on members of the public is based on the total intake (Bq) resulting from inhalation of contaminated air and consumption of contaminated food items during the spring and summer of 1954. Exposure to ${ }^{131}$ I from the 1954 accident is investigated separately, as well as in combination with the exposure from routine releases in 1954.

The excess lifetime risk of thyroid cancer to an exposed individual from an accidental release can be directly calculated using Equation 2.1. The methodology leading to the estimate of exposure $\left(D_{i}\right)$ in Equation 2.1 is presented in Appendix 2-A.

\subsection{Description of Model Components}

\section{Source Term}

Assessment of the "source term" involves the estimation of the quantities of the various physicochemical forms of ${ }^{131}$ I released to the atmosphere from the dissolution of irradiated fuel slugs during the production of radioactive lanthanum (the RaLa process). The fuel slugs were obtained from the Clinton Pile (later referred to as the X-10 Graphite Reactor) until 1948-1949. After this time, fuel slugs were transported to the X-10 site from reactors at the Hanford Reservation. The assessment of the source term is described in detail in Section 3 of this report. Separate assessments were conducted for the estimation of annual average source terms for 1944 to 1956 and for the accidental release of April 29, 1954.

\section{Atmospheric Dispersion and Deposition}

The second component of the analysis includes the atmospheric dispersion, chemical transformations, wet and dry deposition, and removal (plume depletion) of ${ }^{131} \mathrm{I}$ during the transport of the contaminated plume from the X-10 stack to the locations of interest. The dispersion analysis is conducted on an hourly scale for the routine releases and on a half-hourly scale for the accidental releases. However, for the routine releases, the resulting air concentrations and deposition rates are presented as annual averages for each year of the RaLa operation, corresponding to the annual average source terms. For the accidental release of April 29, 1954, the estimates of atmospheric dispersion and deposition are presented as time-integrated concentrations of ${ }^{131} \mathrm{I}$ in air. This component of the assessment is described in detail in Section 4 of this report. 


\section{Exposure Pathways}

Starting with the concentration of ${ }^{131} \mathrm{I}$ in air, a number of different exposure pathways are considered. For exposure due to ingestion of milk and milk products, beef, leafy vegetables and eggs, the deposition of ${ }^{131}$ I to vegetation surfaces is first analyzed. The processes of wet and dry deposition are taken into account for the three chemical forms of ${ }^{131} \mathrm{I}$ : (a) highly reactive (elemental); (b) moderately reactive (particulate); and (c) poorly reactive (organic). The transfer from air to vegetation is described in Section 5 of this report for both routine releases and the accidental release in 1954.

Iodine-131 deposited on pasture grass is ingested by grazing animals and transferred to milk and animal muscle. The modeling approach used to estimate the contamination of milk, meat, and other food products, is described in Section 6 for both routine releases and the accidental release.

Changes in the contamination of food products during the distribution and storage of food between production and consumption are discussed in Section 7. An analysis of food ingestion rates for potentially exposed members of the public is presented in Section 8, along with the parameters used to evaluate exposure from inhalation of contaminated air.

\section{Thyroid Dose and the Risk of Thyroid Cancer}

To estimate the thyroid dose, a quantitative evaluation of the behavior of radioiodine in the human body is necessary. After ingestion or inhalation of contaminated food, iodine is absorbed into the blood stream and then accumulates in the thyroid gland. Section 9 describes the biological basis and the methodology for estimating the dose delivered to the thyroid by ${ }^{131}$ I accumulated in the interior of the gland. The dose to the thyroid from a unit intake of ${ }^{131} \mathrm{I}$ is called the "dose factor" or "dose coefficient." The dose factors in this study were estimated using the most recent data on the mass of the thyroid obtained by ultrasonography (Section 9).

Exposure of the thyroid gland to radiation results in an increased incidence of thyroid carcinomas and adenomas, especially if the exposed individuals are children under 15 years of age at the time of exposure. The relationship between exposure and thyroid cancer incidence is discussed in Section 10, and a set of "risk factors" is estimated. A "risk factor" is defined as the lifetime risk of thyroid cancer incidence from a unit dose to thyroid. Risk factors are estimated for different age groups and for both genders (Section 10).

\section{Results of the Analysis}

The estimates of doses and total excess lifetime risks of thyroid cancer for different classes of exposed individuals are presented in Section 11. An estimate of the total number of cancers that may have occurred to date in the vicinity of Oak Ridge is described in Section 12. 


\subsection{Uncertainty and Sensitivity Analysis}

Each of the components listed in Section 2.2 contains parameters whose values are known only imperfectly. To quantify the present state of knowledge, subjective probability distributions are assigned using professional judgment after review of the literature and consultation with outside experts (IAEA, 1989; NCRP, 1996). Whenever possible, distributions of values have been selected to reflect specific characteristics of the Oak Ridge site and of the relevant processes during the time period 1944 through 1956.

The uncertainty in the dose and risk estimates is obtained by propagating the uncertainties in each of the individual parameters (Figure 2.2). The Monte Carlo method is used for uncertainty propagation, employing the Latin Hypercube Sampling technique with a sample size of 400. The most important contributors to the uncertainty in dose and risk are identified through a sensitivity analysis. In this document, the sensitivity analysis expresses the relative contribution of the uncertainty of each input parameter to the uncertainty in each endpoint of the calculation. The analysis is based on a summation of the squares of the Spearman rank correlation coefficients ${ }^{1}$ (between each input parameter and the endpoint of interest), normalized to $100 \%$ (Decisioneering, 1993). Identification of important contributors to the overall uncertainty determines directions for further study and will allow efficient allocation of resources to any future refinements of the estimates of dose and risk to individuals who may have resided near the Oak Ridge Reservation.

A specific feature of the sensitivity analysis performed in this work is the treatment of the timedependent nature of the processes involved. The main endpoints of the calculations, namely the total excess lifetime risk (TELR) of thyroid cancer and the total dose $(T D)$, represent timeintegrated quantities. Their relationship to the time-dependent parameters is complex and difficult to analyze. The solution chosen for the sensitivity analysis was to investigate the relationship between the uncertainty in the above-mentioned endpoints and the uncertainty in the time-averaged values for the time-dependent parameters.

\footnotetext{
1 Spearman rank correlation coefficients are calculated between each input parameter and the output. Correlation coefficients provide a meaningful measure of the degree to which an input parameter and the result change together. A high correlation coefficient means that the input parameter has a significant impact on the result. A positive coefficient indicates that the result increases as the input parameter increases. For a negative coefficient, the result decreases as the input parameter increases. The larger the absolute value of the correlation coefficient, the stronger the relationship (Decisioneering, 1993).
} 

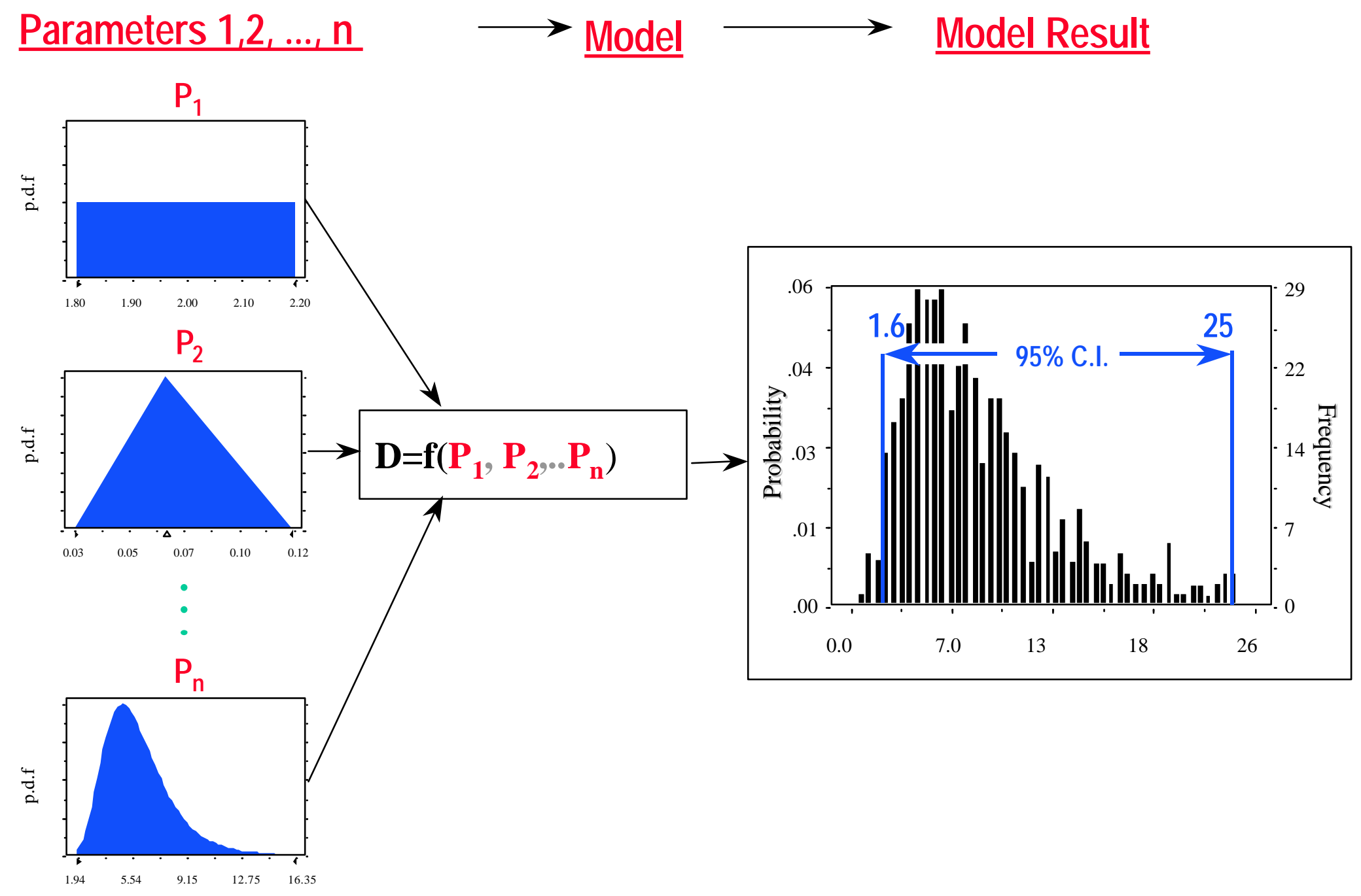

Figure 2.2: Monte-Carlo method for uncertainty propagation 


\subsection{References}

Bruce, G. M., Buddenbaum, J. E., Lamb, J. K., and Widner, T. E. Volume II - Part A of the Dose Reconstruction Feasibility Study. Tasks 1 \& 2: A Summary of Historical Activities on the Oak Ridge Reservation with Emphasis on Information Concerning Off-Site Emission of Hazardous Material. McLaren/Hart-ChemRisk for the Tennessee Department of Health. September 1993.

IAEA (International Atomic Energy Agency). Evaluating the Reliability of Predictions Made Using Environmental Transfer Models. Safety Series No. 100. IAEA, Vienna, Austria. 1989.

Decisioneering. "Crystal Ball® Version 3.0, Forecasting and Risk Analysis for Spreadsheet Users” 1380 Lawrence Street, Suite 520, Denver, Colorado 80204-9849. 1993.

NCRP (National Council on Radiation Protection and Measurements). A Guide for Uncertainty Analysis in Dose and Risk Assessments Related to Environmental Contamination. NCRP Commentary No. 14. NCRP. Bethesda, MD. 1996. (ChemRisk Repository No. 3092). 


\title{
3.0 SOURCE TERM ASSESSMENT FOR IODINE-131
}

Source term assessment involves the estimation of quantities and qualities of a contaminant released to the environment. In the case of $\mathrm{X}-10$ radioactive lanthanum (RaLa) processing, the analysis is focused on airborne releases of radioactive iodine-131 $\left({ }^{131} \mathrm{I}\right)$. In this source term analysis, annual quantities of radioiodine released from the building that housed the RaLa process and from the X-10 stacks are estimated, taking into account the expected physical and chemical forms of iodine that were likely present in the source material and available information concerning the behavior of the different species of radioiodine in process equipment. Releases from an accident involving RaLa processing on April 29, 1954 are also estimated. Attribution of radioiodine releases to specific physical/chemical forms is important because the different species are transferred from air to vegetation at different rates.

\subsection{A Brief History of Radioactive Lanthanum (RaLa) Processing}

The intense, 1.6 million electron volt $(\mathrm{MeV})$ gamma ray of ${ }^{140} \mathrm{La}$ made it useful for early atomic weapons development work. Lanthanum was used in testing of the implosion process in early weapon designs, for atmospheric radiation tracking tests, and for radiation warfare experiments. Large scale production of radioactive barium-lanthanum was requested by Los Alamos on 8 April 1944, reportedly in an urgent request from J. Robert Oppenheimer, the civilian head of the Los Alamos Scientific Laboratory weapon development program (Thompson, 1949). The primary application for radioactive lanthanum at Los Alamos, which is depicted in Photograph 1, was described as follows in 1972 (Glasstone and Redman, 1972):

\begin{abstract}
"The RaLa (pronounced "rah-lah") method ... was a kind of radiographic technique which made use of radioactive lanthanum (RaLa), mass number 140, as the source of radiation, namely 1.65- and 2.30-MeV gamma rays. The RaLa method was originally used to study the compression of a solid core (or ball) as a function of time in an implosion and also to determine the symmetry of the latter. The strong RaLa source (between 500 and 3000 curies) was placed at the center of a ball... The implosion system was then surrounded by a number of scintillation detectors to measure the gamma-ray transmission in various directions. The transmitted intensity is related to the integrated product of density and thickness of the material through which the gamma rays have passed. Because the RaLa source emitted radiation continuously, information could be obtained of both time-behavior and symmetry of the imploding system."
\end{abstract}

Radioactive lanthanum produced at Oak Ridge was used in a number of other applications, including:

! atmospheric tracking tests at Los Alamos in 1950, in which radioactive clouds were tracked as far as 70 miles downwind to study dispersion and fallout (USGAO 1993); 
! a radiation warfare test at Oak Ridge in 1948, in which radiation levels were measured at various distances (up to 1,000 feet) and three above-ground heights (up to 12 feet) from a 1,000 Ci source of metallic lanthanum (Roberson 1948, USGAO 1993); and

! "Operation Quick and Dirty" at the Pantex site in Texas in 1956, in which a 500-Ci La-140 source was placed in a device that contained high explosives and uranium, positioned in a soil-covered bunker, and exploded. Soil and radioactivity dispersal were studied (LANL 1956).

RaLa processing at Oak Ridge initially involved acid dissolution of aluminum-canned natural uranium fuel slugs from the Clinton Pile, later known as the Oak Ridge Graphite Reactor, followed by a series of separation and purification steps. Due to the 12.8-d half-life of the parent fission product ${ }^{140} \mathrm{Ba}$, the reactor fuel used for RaLa processing had to be fed to the process shortly after removal from the reactor. As a result, short-lived fission products such as ${ }^{131} \mathrm{I}$, which normally decay away when fuel is allowed to cool for longer periods before dissolution, were available for release.

Around 1948 the U.S. Atomic Energy Commission decided that fuel slugs from the Hanford, Washington reactors would be used permanently as the starting material for RaLa separation. This decision was made for economic reasons. Around 1950 the USAEC decided that it would not be practical to further alter the Building 706-D equipment at X-10 to increase production capacity. In 1954 the USAEC decided to build a new RaLa production facility at the Idaho Chemical Processing Plant, and X-10 was relieved of its RaLa production responsibilities at the end of calendar year 1956.

\subsubsection{RaLa Facilities at X-10}

A production process was developed and put into operation in Building 706-C, where existing laboratory facilities designed for small-scale (1-10 Ci) fission product separations were converted to barium-lanthanum production in five months. The first RaLa run was completed in September 1944. Within months, when routine shipments of 300-Ci batches were requested, the available facilities were deemed inadequate. A dedicated facility (Building 706-D) was designed for production of barium in batches up to 1,000 Ci in size. The key facilities and operations involved in X-10 RaLa processing are depicted in Figure 3.1. Relevant portions of the X-10 site around 1946 are shown in Photograph 2, and an interior view of Building 706-D is shown in Photograph 3. The RaLa slug dissolver vessel and related process components are shown in Photograph 4.

During the course of Building 706-D construction, the requirement for production of radioactive lanthanum was increased from 1,000 to 2,000 Ci per batch for shipment. Operations in the new facility began in May 1945. In the years that followed, the equipment designed for RaLa processing at Oak Ridge was operated far beyond its design capacity. Requested batch sizes increased to $10,000 \mathrm{Ci}$ in 1950 , and alterations to the final purification equipment were completed to allow processing of batches approaching 65,000 $\mathrm{Ci}$ (reported to have been shipped from X-10 in January 1954). Even though production levels were increased to over sixty times the design capacity of the plant, the primary dissolving and precipitation equipment was never changed (Larson, 1954). Over the 13-year period of the RaLa production operations at X-10, an estimated 28,558 reactor fuel slugs were dissolved in an estimated 733 batches in the process of separating over 500,000 Ci of radioactive barium as a source of ${ }^{140} \mathrm{La}$. 


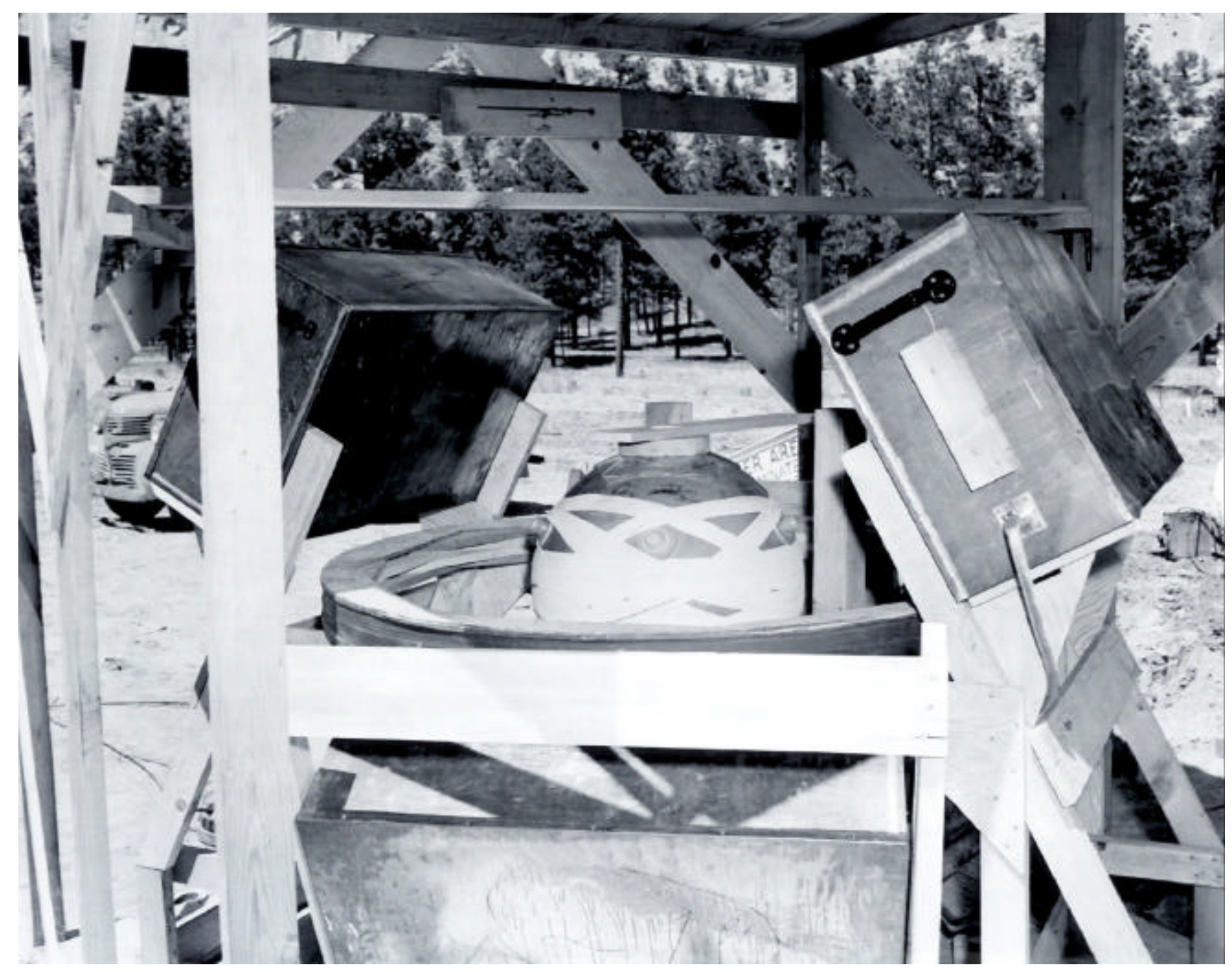

Photograph 1: Los Alamos Apparatus for Using RaLa to Test the Implosion Process Photo LANL-5386 courtesy of Los Alamos National Laboratory 


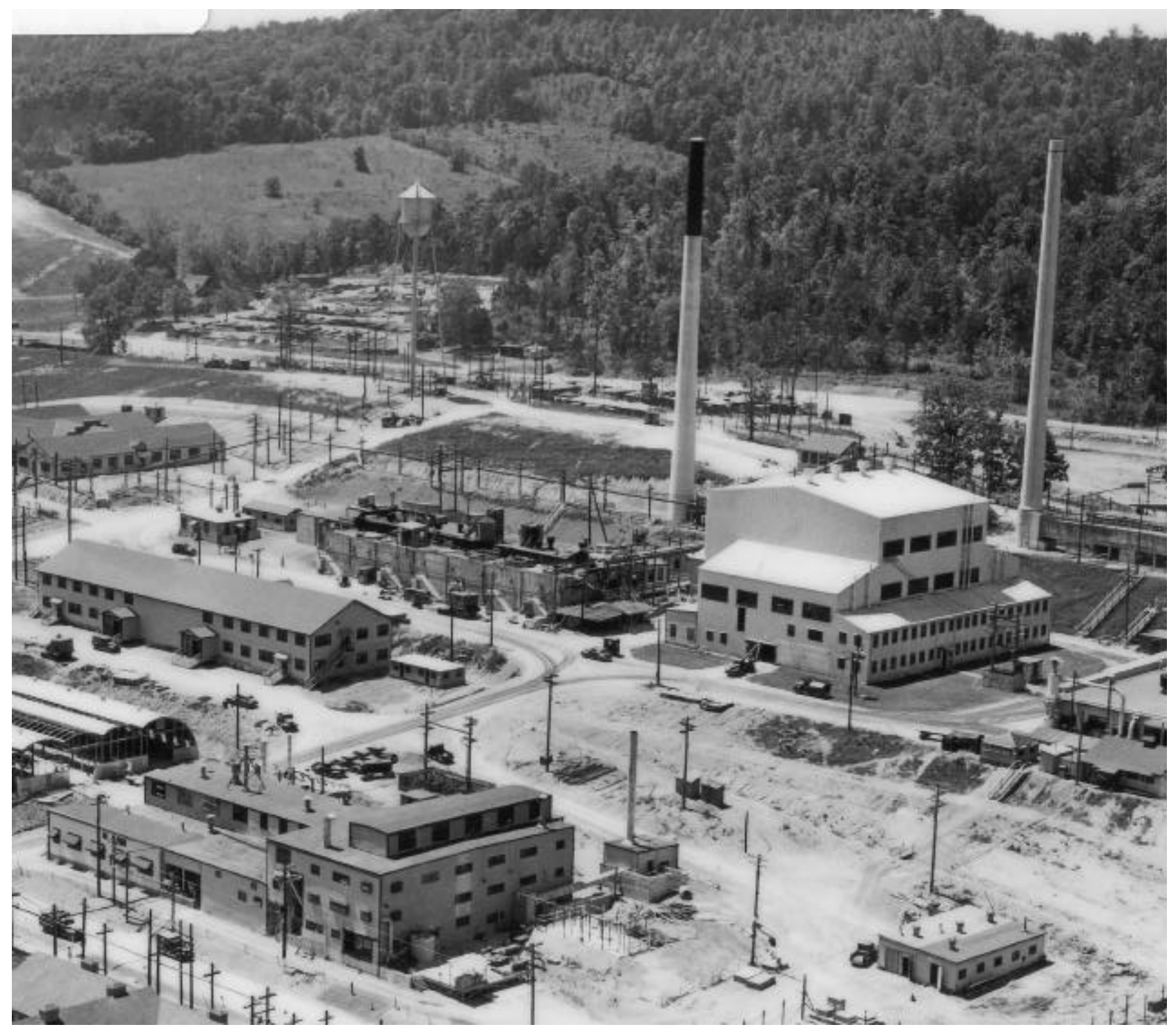

Photograph 2: The Central Area of the X-10 Site (circa 1946; view toward the northwest).

Photo ORO-3521 courtesy of U.S. Department of Energy 


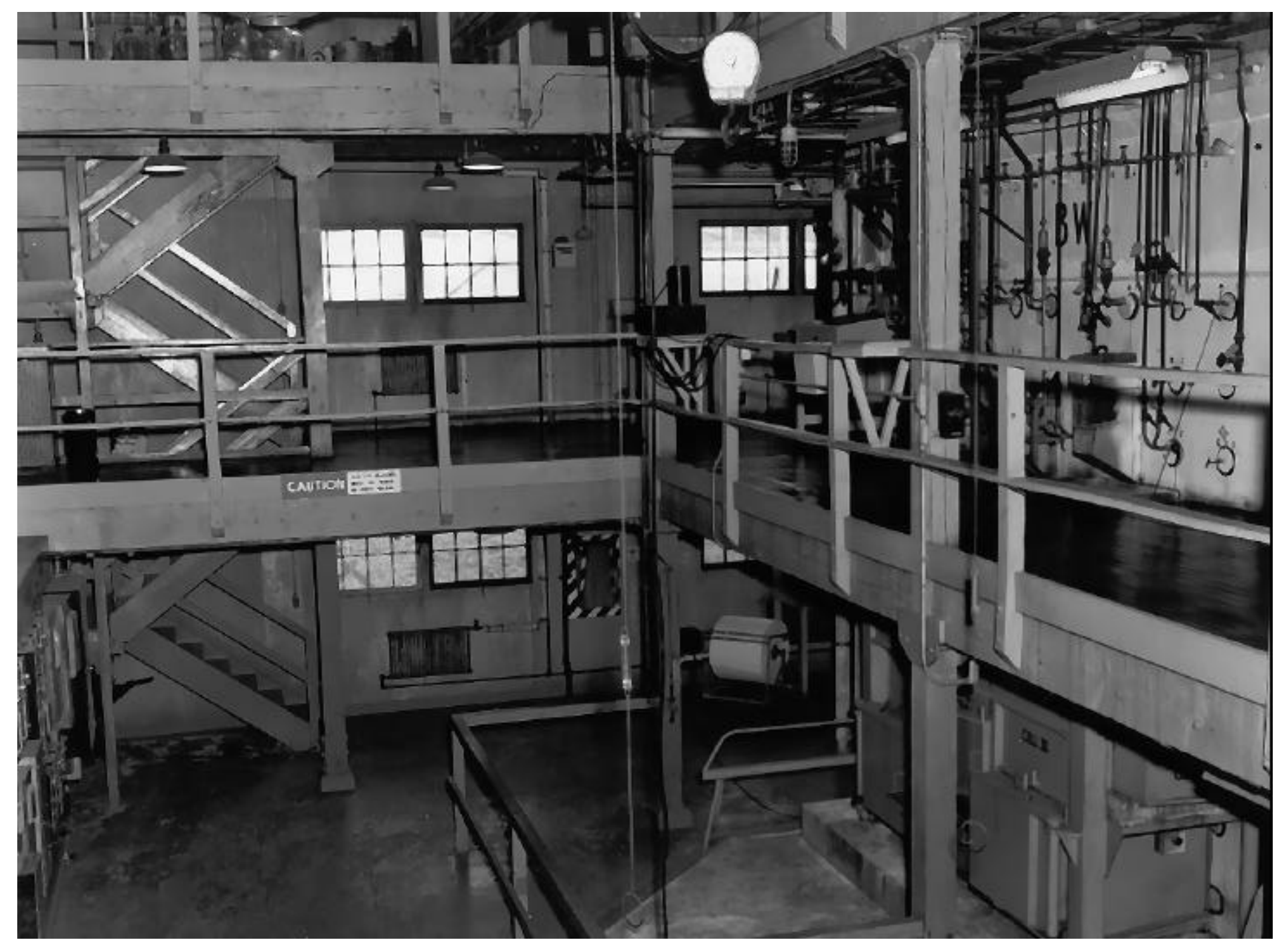

Photograph 3: Interior View of the Building 706-D RaLa Processing Facility ORNL-Photo-3688 courtesy of Oak Ridge National Laboratory 


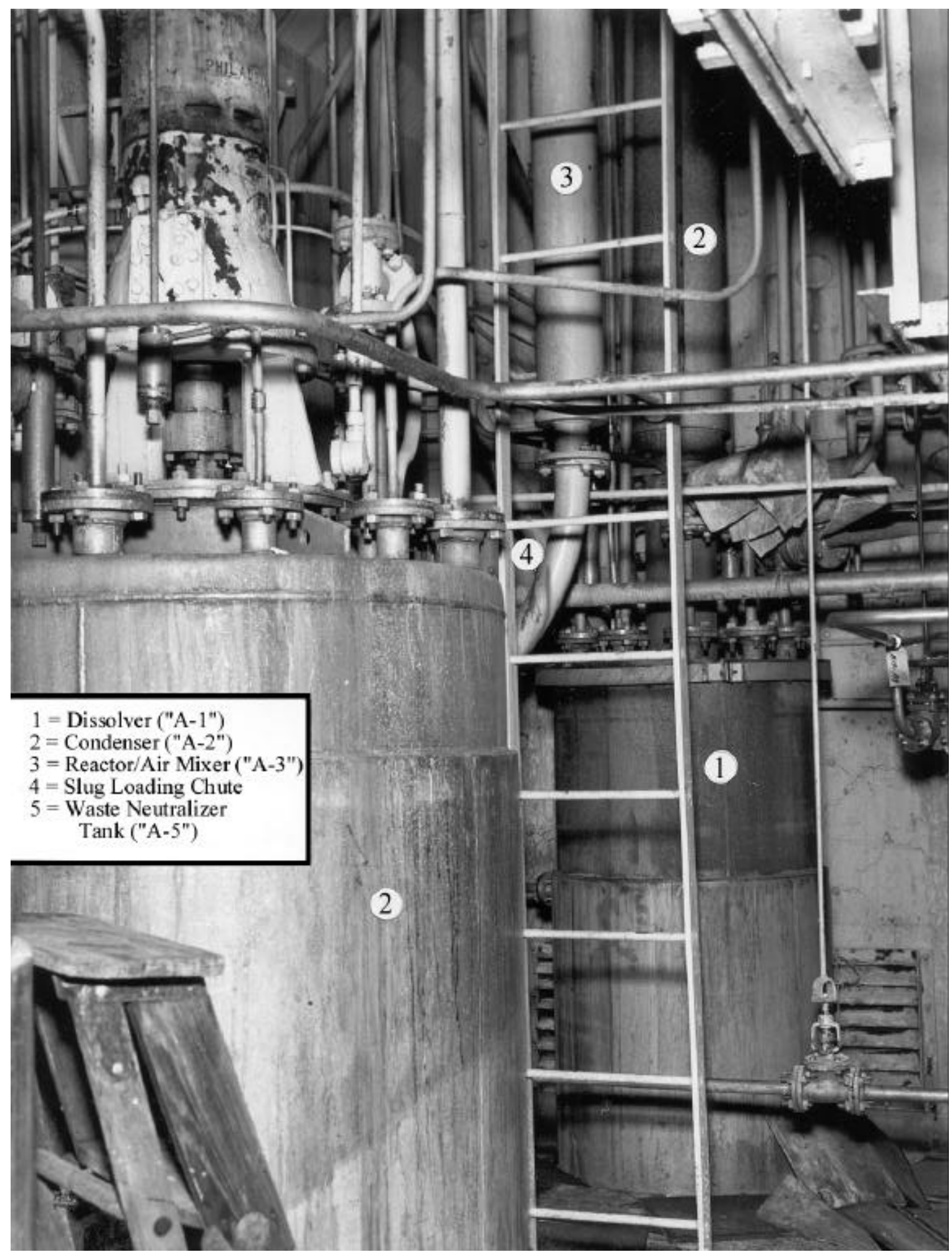

Photograph 4: The Building 706-D Dissolver and Associated Components

ORNL-Photo-6749 courtesy of Oak Ridge National Laboratory 


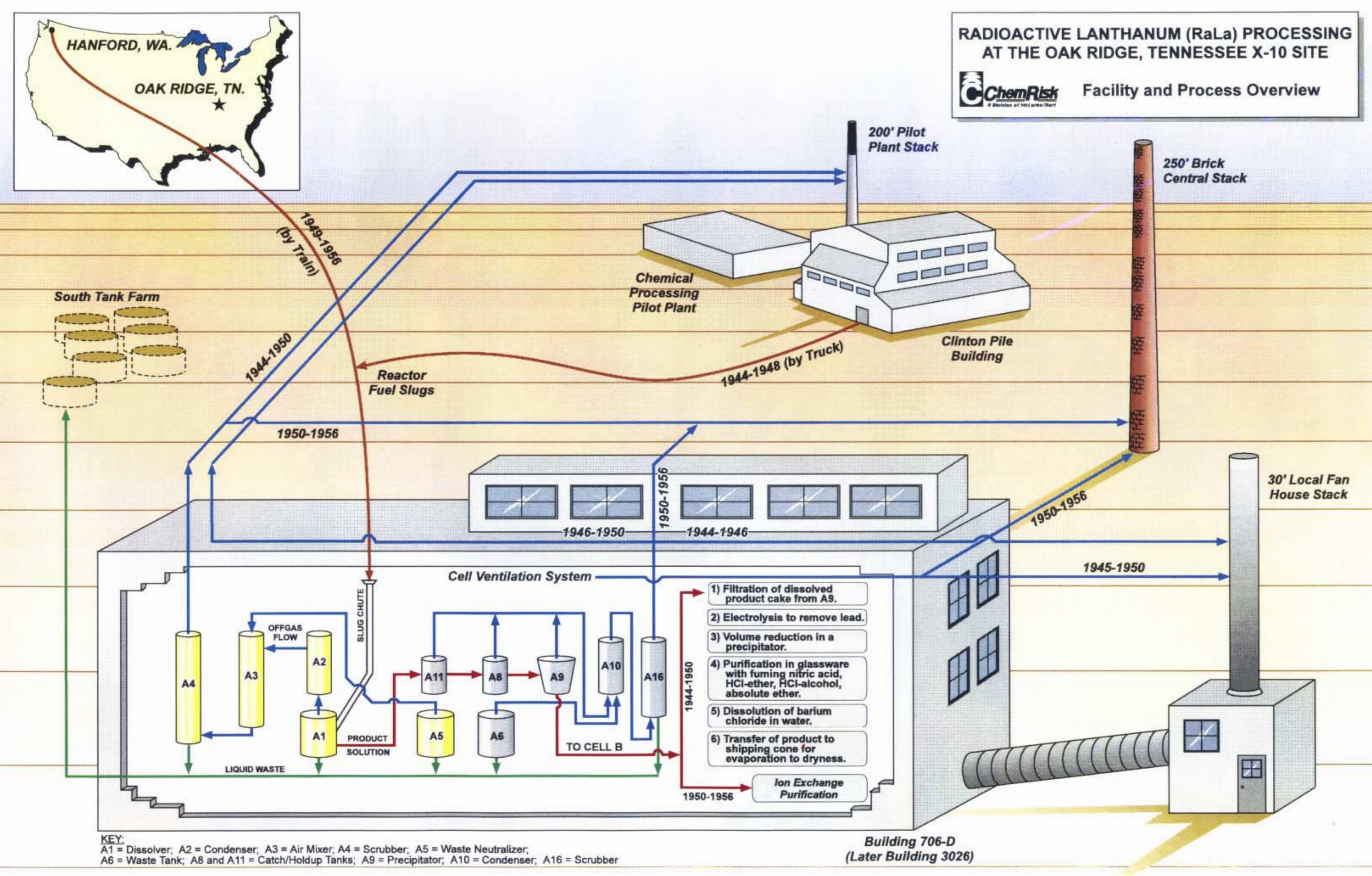




\subsubsection{Key Sources of Radioiodine Releases}

The most important source of radioiodine releases from RaLa processing was exhaust (off-gas) from the slug dissolver. In this 165-gallon, cylindrical, stainless steel tank, fuel slugs were dissolved in nitric acid to begin the chemical separation process. During the process of dissolution, some chemicals were volatilized and escaped from the dissolver in gaseous form. Volatile radioiodine and other gases were withdrawn from the dissolver by a vacuum supplied by the dissolver off-gas system; the gases were passed through a condenser and a chemical scrubber before passing through a length of piping to a stack at least 200 feet tall.

As shown in Figure 3.1, off-gas treatment features included a chemical scrubber for the dissolver and waste neutralizer tank and a second scrubber on other process vessels. Both scrubbers were located inside heavilyshielded rooms (known as cells) in the process building. Particle filtration devices were added in late 1948 after it was recognized that the process was a significant contributor to airborne radioactivity in the area of the Laboratory (Coughlen, 1950). RaLa exhausts were first routed to a central off-gas treatment system in March 1950. That central treatment facility passed contaminated air through a Cottrell electrostatic precipitator and particulate filters, then up a 250-foot brick stack. The utilization of the various release points for airborne effluents from RaLa processing over the years is summarized in Table 3.1.

Table 3.1: Time History of Usage of X-10 Stacks for RaLa Process Exhausts

\begin{tabular}{|c|c|c|c|}
\hline Source of Exhaust & $\begin{array}{c}\text { 30-ft } \\
\text { Local Stack }\end{array}$ & $\begin{array}{c}\text { 200-ft Pilot Plant } \\
\text { Stack (Stack } \\
\mathbf{3 0 2 0}\end{array}$ & $\begin{array}{c}\text { 250-ft Central Brick } \\
\text { Stack }^{\mathbf{1}} \text { (Stack 3039) }\end{array}$ \\
\hline \hline $\begin{array}{c}\text { Dissolver and Waste } \\
\text { Neutralizer Off-gas }\end{array}$ & $\begin{array}{c}\text { Not used for } \\
\text { this source }\end{array}$ & $1944-1950$ & $1950-1956$ \\
\hline $\begin{array}{c}\text { Off-gas from Other } \\
\text { RaLa Process Vessels }\end{array}$ & $1944-1946$ & $1946-1950$ & $1950-1956$ \\
\hline Cell Ventilation Exhaust & $1944-1950$ & $\begin{array}{c}\text { Not used for } \\
\text { this source. }\end{array}$ & $1950-1956$ \\
\hline
\end{tabular}

In RaLa processing, indications are that a large majority of the iodine that was evolved was evolved during the dissolution of uranium slugs. While a portion of the iodine remained in the dissolver solution and became a waste stream that was neutralized and disposed of at the X-10 tank farm, experts consulted indicate that

\footnotetext{
${ }^{1}$ Plans to connect RaLa off-gas systems to the central, "isotope area" waste gas decontamination system and its 250 foot stack were discussed in a March 1949 progress report (Winters,1949a). A May 1949 progress report projected operation of the systems in that configuration in late 1949 or early 1950 (Winters, 1949b). The central stack is shown under construction in January 1949 in ORNL Photo 4110 and complete in ORNL Photo 588-84 taken in 1950. The new system is documented in use during RaLa Run 40 in March 1950 (ORNL, 1950)
} 
evolution of iodine from this waste essentially stopped when the liquid was neutralized ${ }^{2}$. Because of this, many of the details about RaLa processing beyond the dissolution step, such as refinements to the methods and equipment used to separate and purify barium, are not of primary importance to the estimation of radioiodine releases.

\subsubsection{Other Sources of Radioiodine at X-10 and Other Radionuclides}

Radioactive lanthanum processing has not been the only source of radioiodine emissions at the X-10 site. In the Oak Ridge Dose Reconstruction Feasibility Study, the following sources of radionuclide releases at the X10 site were addressed in preliminary evaluations of potential off-site health effects (ChemRisk, 1993a):

- $\quad$ Radioactive lanthanum processing

- $\quad$ Thorex processing of short-decay irradiated thorium

- $\quad$ Chemical separation of plutonium from Clinton Pile fuel

- $\quad$ Graphite Reactor fuel slug ruptures

- $\quad$ Argon-41 in Graphite Reactor cooling air

- $\quad$ Tritium from isotope processing programs

The first four of these radionuclide sources were evaluated for releases of radioiodine in the Feasibility Study. In addition to these sources, operations were conducted at the X-10 site for production and distribution of radioactive isotopes of iodine for use in research and medicine. These isotope production operations were not separately screened, because they involved dissolution of a much smaller number of reactor fuel slugs than did RaLa processing and steps were taken to maximize the collection of the radioiodine that was, in those operations, the desired product. And while other nuclear material separation campaigns were undertaken at the X-10 site over the years that may have been sources of some radioiodine releases (ChemRisk, 1993b), each of the first four sources listed above stood out in the Feasibility Study evaluation because they exhibited one or more of the following characteristics:

1. They involved processing of large quantities of nuclear material,

2. They involved processes in which fission products were likely released with a much shorter decay or "cooling" period than is typically desired,

3. They were associated with documented cases of environmental contamination of the $\mathrm{X}-10$ site and (in some cases) surrounding areas, and/or

\footnotetext{
${ }^{2}$ Personal communications between Tom Widner and Bob Burns of the project team and Orlan Yarbro, Bill Burch, Leland Burger, and Bill Wichner, former and active ORNL and Hanford scientists with relevant chemical processing experience. 1996.
} 
4. They occurred in time periods before effluent treatment systems at the X-10 site had become well developed.

The RaLa processing operation stood out as the most important source of radioiodine emissions from the $\mathrm{X}-10$ site because of its processing of large quantities of short-decayed reactor fuel slugs, its considerable 13-year duration, and its initiation during a period when effluent treatment systems were minimal at first and not completely developed by program's end. While ${ }^{131}$ I releases from RaLa processing in 1952 as estimated in the Feasibility Study were only about a factor of three above those from early plutonium separation operations in 1944 and about a factor of six greater than those from Thorex short-decay runs in 1957, the plutonium separation only lasted one year and the Thorex processing 1.5 years, compared to RaLa's 13 years. While releases of ${ }^{129}$ I estimated in the Feasibility Study for RaLa processing in 1952 were $35 \%$ lower than those from early plutonium separation in 1944, quantities of ${ }^{129}$ I released from each of those operations were lower than the quantities of ${ }^{131}$ I released by factors of over 10 million, and that plutonium separation lasted one year.

Isotopes of iodine stood out among the radionuclides evaluated for RaLa processing in the Feasibility Study because of their volatility. It is unlikely that temperatures in RaLa dissolving were high enough to volatilize elements such as cesium and strontium, which would have been present in small quantities given the relatively low reactor power levels and irradiation durations experienced by the fuel slugs that were processed. The fraction of the radioiodine isotopes present in the dissolver that were assumed to be released to the environment was set at $80 \%$ in the Feasibility Study based on analyses of iodine release fractions at the Hanford plant performed as part of the Hanford dose reconstruction project. While detailed evaluation during this study has indicated that the release fraction for RaLa iodine may have been significantly lower than $80 \%$ during most processing, the estimated effective release fraction for iodine isotopes remains considerably higher than the release fraction of $0.1 \%$ that was used in the Feasibility Study for ${ }^{144} \mathrm{Ce},{ }^{137} \mathrm{Cs},{ }^{95} \mathrm{Zr},{ }^{95} \mathrm{Nb},{ }^{103} \mathrm{Ru},{ }^{106} \mathrm{Ru},{ }^{89} \mathrm{Sr}$, ${ }^{90} \mathrm{Sr},{ }^{140} \mathrm{Ba},{ }^{140} \mathrm{La},{ }^{239} \mathrm{Pu},{ }^{235} \mathrm{U}$, and ${ }^{238} \mathrm{U}$. This release fraction for radionuclides in particulate form was based on measured particulate emissions from RaLa processing at the Idaho Chemical Processing Plant during 1957 compared to the estimated radionuclide inventories in the Materials Testing Reactor fuel used as the barium source at that plant.

In the Feasibility Study, the highest screening index for radionuclides and carcinogenic chemicals was obtained for ${ }^{131} \mathrm{I}$. This indicated that ${ }^{131} \mathrm{I}$ warranted highest priority for detailed investigation. Screening indices for ${ }^{133} \mathrm{I}$ and ${ }^{137} \mathrm{Cs}$ were $60 \%$ and 6\%, respectively, of the result for ${ }^{131} \mathrm{I}$. Almost all of the ${ }^{137} \mathrm{Cs}$ total came via surface water and soil pathways associated with sources other than RaLa processing. All of the other radionuclides evaluated had screening indices lower than $2 \%$ of the value for ${ }^{131} \mathrm{I}$ (including ${ }^{129} \mathrm{I}$, at about $0.00001 \%$ of the ${ }^{131}$ I value). The indicated significance of ${ }^{133} \mathrm{I}$ in RaLa releases is attributable in part to the fact that it has a higher fission yield than ${ }^{131} \mathrm{I}$. The average ${ }^{133} \mathrm{I}$ content of RaLa slugs at the time of discharge from the X-10 Graphite Reactor was $9.2 \mathrm{Ci} / \mathrm{kg}$, compared to $4.1 \mathrm{Ci} / \mathrm{kg}$ for ${ }^{131} \mathrm{I}$ (see Appendix 3I). On the other hand, ${ }^{133} \mathrm{I}$ decays away with a half-life that is just over one-tenth of the half-life for ${ }^{131} \mathrm{I}\left(20.3 \mathrm{~h}\right.$ compared to $8.04 \mathrm{~d}$ for $\left.{ }^{131} \mathrm{I}\right)$, and the thyroid dose conversion factor for ${ }^{133} \mathrm{I}$ is $17 \%$ of the factor for ${ }^{131} \mathrm{I}$ (USEPA, 1988). And while 
${ }^{132} \mathrm{I}$ has a relatively high fission yield (it was present at 1.5 times the concentration of ${ }^{131} \mathrm{I}$ in X-10 slugs; see Appendix 3I), its potential significance in RaLa releases is also limited, especially for fuel slugs shipped from Hanford, bythe 2.3-hour half-life of that isotope and its relatively low dose conversion factor (less than $1 \%$ of that for $\left.{ }^{131} \mathrm{I}\right)$.

As stated in the Feasibility Study report, the relative importance of ${ }^{133} \mathrm{I}$ in that screening analysis was exaggerated by the fact that the radioactive decay of radionuclides between the time of release from the X-10 facility and the time of human intake was not taken into account. The entire screening index for ${ }^{133} \mathrm{I}$ was from exposure pathways that would involve significant decay in the environment, as they involved ingestion of milk, meat, and vegetables that had been contaminated by air releases. More direct pathways such as inhalation of or immersion in ${ }^{133}$ I were not included in the final Feasibility Study analysis because they yielded doses less than $1 \%$ of those from the air-pasture-cow milk pathway in preliminary screening. Actual off-site intakes of

${ }^{133}$ I were likely lower than indicated in the Feasibility Study by a factor of 10 or more, depending on the length of time assumed between release and consumption.

The considerations summarized above led the Task 1 project team to focus on ${ }^{131}$ I releases from RaLa processing in the evaluation described in this report. While it is true that radionuclides other than ${ }^{131} \mathrm{I}$ were released from X-10 RaLa processing, it is most likely that potential exposures to ${ }^{131} \mathrm{I}$ dominated the doses and health risks to an individual that lived in or near Oak Ridge during the period of interest. The pathway by which doses and health risks from RaLa releases could most likely have been enhanced by the presence of shorter-lived iodine isotopes (i.e., ${ }^{132} \mathrm{I}$ and ${ }^{133} \mathrm{I}$ ) is direct inhalation of contaminated air. Possible contributions from the shorter-lived isotopes to inhalation doses were not evaluated in this study. In this current assessment, inhalation doses were generally minor contributors to total doses to individuals. We consider it unlikely that consideration of the shorter-lived iodine isotopes would significantly elevate the relative importance of the inhalation pathway. An evaluation of possible additional doses from these isotopes would be a reasonable refinement to this assessment, with the level of detail that is warranted possible to be determined based on a preliminary screening calculation that could likely be performed with the information developed and assembled for this study.

\subsection{Elements of the Source Term Assessment}

The major steps in the estimation of radioiodine released from X-10 RaLa processing were as follows::

- $\quad$ Collection of necessary information about each RaLa production campaign;

- Collection of information concerning how the reactor fuel slugs used as RaLa feed material were irradiated in the X-10 or Hanford reactors (such as power levels, positions in the reactor, and exposure durations);

- Calculation of the quantities of radioiodine that were present in the fuel slugs at the times their irradiation ended and at the time of dissolving; and 
- $\quad$ Estimation of quantities of radioiodine that were released to the environment after the fuel slugs were dissolved for chemical separation of radioactive barium/lanthanum.

\subsection{Sources of Information Concerning X-10 RaLa Processing}

No one source of information contains all of the data necessary to estimate releases from X-10 RaLa runs. This section describes the information sources that proved to be most valuable in characterizing the RaLa runs that occurred at X-10 and in estimating off-site releases.

\subsubsection{Slug Exposure and Discharge Reports ("Push Data")}

Irradiated fuel slugs from the Clinton Pile were used as a source of barium/lanthanum until slugs shipped from Hanford became the feed source in late 1948. Oak Ridge fuel exposure and discharge records indicate:

- $\quad$ position in the pile (row or channel number)

- $\quad$ date each row was "charged" with new slugs

- $\quad$ date each row was discharged ("pushed" from the pile)

- $\quad$ number of days each group of slugs was exposed

- $\quad$ percent of maximum neutron flux (or "Position Factor")

- $\quad$ accumulated power $(\mathrm{kWh})$ within the last $40 \mathrm{~d}$, before the last $40 \mathrm{~d}$, and

- $\quad$ total number of slugs discharged from each row.

Samples of exposure and discharge reports for Oak Ridge slugs are shown in Appendix 3A. Of the $28 \mathrm{RaLa}$ runs in Building 706-D that used exclusively X-10 slugs, the project team has located complete push data for 21 runs, partial data for 2 runs, and no data for 5 runs. To-date, detailed records of fuel exposure data for Hanford slugs shipped to Oak Ridge have not been found for most RaLa runs. The exceptions are letters and/or teletypes that discuss individual sets of slugs that were being sent to Oak Ridge. Appendix 3B presents sample correspondence regarding Hanford slugs for Run 28 in November 1948 and Run 30 in February 1949.

\subsection{2 "100 Area" (Clinton Pile) Log Books}

Operators of the Clinton Pile maintained log books detailing operations on each shift, with each volume typically covering two to three months. Volume 1, which begins in 1943, is located in a display case in the Graphite Reactor historical display. The project team has found volumes 2 through 121 (through mid-1962) in boxes in the X-10 Records Center. 


\section{Key RaLa Process Components}

DISSOLVER (“A-1")- The dissolver was a 165-gallon, cylindrical, jacketed stainless steel tank mounted on a concrete pad. The inside of the tank was lined with baffles which protected the wall and service piping during slug loading. Slugs were loaded through a three-inch diameter pipe slug chute that extended from the third floor. The chute was curved to prevent direct "shine" of radiation from the dissolver to the third floor. A crash plate at the exit of the chute broke the fall of the slugs to prevent damage to the dissolver. The dissolver was serviced with vacuum from the A4-205 off-gas system through a condenser ("A-2") that was located on top of the dissolver.

CONDENSER ("A-2")- The reflux condenser was used to reduce the release of nitric acid vapors into the off-gas system during the dissolving of slugs. It was essentially an 8-foot by 1 -foot jacketed, stainless steel pipe with three stainless steel water cooling coils inside its top end. Lines supplied water to the condenser jacket and to the three cooling coils.

AIR MIXER or "REACTOR" (“A-3")- The reactor was a 6-inch by 8.5-foot stainless steel pipe connecting the condenser to the A-4 scrubber. Its purpose was to mix air (through a pinhole in A3 ) with oxides of nitrogen in the off-gas to facilitate dissolving of these oxides in the A-4 scrubber.

SCRUBBER ("A-4")- The dissolver scrubber was an 8-inch inside diameter by 9-foot stainless steel pipe packed with 1-inch steel Raschig rings. During slug dissolvings, a 4\% solution of $\mathrm{NaOH}$ trickled down over these rings while off-gas from the dissolver traveled up through the scrubber to a 2-inch pipe that led to an elevated stack.

SCRUBBER ("A-16")- This scrubber used $\mathrm{NH}_{3}$ and water to remove acid vapors and active gases from all tanks except the dissolver and neutralizer. It was a 8 -foot by 14 -inch inside diameter stainless steel pipe packed with 1-inch stainless steel Raschig rings. 


\section{Some Terminology of RaLa Processing}

To prepare radioactive barium/lanthanum for shipment to Los Alamos, uranium fuel slugs were loaded into the RaLa process dissolver. Acid was added, the dissolver was heated, and the slugs began to dissolve. When the specific gravity of the dissolver solution reached a certain value, the solution was diluted and cooled, and the dissolving reaction stopped. Each slug in the dissolver was at that point partially dissolved. The dissolver solution, representing a RaLa processing "batch," was then transferred to other vessels for separation and purification of the contained barium. More acid was added over the partially-dissolved slugs in the dissolver, and a new dissolving batch began. Dissolving batches continued until there was little uranium metal left in the dissolver. Slugs dissolved per batch ranged from 1 to 85 . If more barium was still needed, more slugs were added to the dissolver, and a series of dissolving batches were done with the new slugs. A small amount of uranium metal left in the dissolver when new slugs were added was called a "heel." The presence of the heel helped dissolving of the new slugs start faster. A "run" was a processing campaign, often lasting a week or more, that included between 2 and 22 dissolving batches and led to purification of a single product for shipment to Los Alamos. Each RaLa run was given a number (up to Run 68), and individual dissolving batches were assigned letters. For example, Run 68 included Batches 68-A, 68-B, 68-C, and 68-D. Each shipment was also assigned a number. Shipment numbers did not match run numbers, because some runs had multiple shipments and some runs failed and did not yield a product worth shipping to Los Alamos.

\section{The X-10 Radioactive Barium/Lanthanum Separation Process}

Slug Loading: Clinton Pile slugs were natural uranium slugs "canned" in aluminum. Lead slug carriers were used to transport slugs from their temporary storage place in the water-filled Clinton Pile canal. The carriers were placed over the slug loading chute on the 3rd floor of Building 706-D, and slugs were discharged one-by-one by means of two long handled cranks and dropped to the dissolver on the first floor.

Coating Removal: $136 \mathrm{lbs}$ of $1.37 \pm 0.02$ specific gravity nitric acid and $150 \mathrm{lbs}$ of $1.38 \pm 0.02$ specific gravity sodium hydroxide were added to the dissolver. The acid line was rinsed with $50 \mathrm{lbs}$ of water and the caustic line with $16 \mathrm{lbs}$ of water. With a vacuum of 4 to 8 inches on the dissolver, $156 \mathrm{lbs}$ of the $\mathrm{NaOH}$ were added to the dissolver. Steam was turned on to the dissolver jacket to achieve a reaction temperature of $100-110 \mathrm{C}$. Removal of aluminum coats was complete within 30 min. after reaching the reaction temperature. The aluminum solution was jetted to Tank W-11 at the X-10 tank farm, and the slugs were rinsed with water. 


\section{The X-10 RaLa Separation Process (continued)}

Dissolving: $390 \mathrm{lbs}$ of $1.37 \pm 0.02$ specific gravity nitric acid was added to the dissolver through the scale tank and funnel on the 3rd floor. The tank and line were rinsed with $8 \mathrm{lbs}$ of water. With 4 to 8 inches of vacuum on the dissolver, heat was applied to raise the solution to the reaction temperature of 105-110 C, which was maintained until the specific gravity of the solution reached 1.78. The uranyl nitrate hexahydrate solution was diluted with $170 \mathrm{lbs}$ of water while maintaining at least $80 \mathrm{C}$. The diluted UNH solution was then sparged for $15 \mathrm{~min}$. The first two batches of the run were transferred directly to the A-9 precipitator for extraction; all other batches were transferred to A-9 via A-11 to pick up a metal waste heel in A-11.

Extraction: $670 \mathrm{~mL}$ of $20 \%$ lead nitrate was added to the UNH solution in the A-9 extractor. The solution addition line was rinsed with $2 \mathrm{~L}$ of water, and $98 \mathrm{lbs}$ of water were added to the extractor. Over 30 min., $255 \mathrm{lbs}$ of $90 \%$ sulfuric acid was added while A-9 was kept at 80-90 C. Slow addition of the acid promoted growth of lead and barium sulfate crystals. The co-precipitate of lead and barium sulfate was allowed to settle for $8 \mathrm{hrs} ; 3 \mathrm{hrs}$ at $80-90 \mathrm{C}$ and $5 \mathrm{hrs}$ at $35 \mathrm{C}$. The precipitate was then washed with $30 \mathrm{lbs}$ of $25 \%$ sulfuric acid and four 33-lb portions of water. Lead/barium sulfate precipitate from multiple batches was accumulated in A-9 before metathesis and transfer to Cell B for purification.

Neutralization: The supernatant metal waste solution was decanted to catch tanks, sampled, and allowed to resettle. The waste was added to $2700 \mathrm{lbs}$ of carbophosphate solution in Tank A-5 for neutralization. The addition was done slowly to avoid rapid evolution of gases and release of airborne contamination to the cell.

Metathesis (double decomposition; barium sulfate " barium carbonate and potassium carbonate " potassium sulfate): $2.5 \mathrm{~L}$ of $40 \%$ potassium carbonate was added to the sulfate precipitate in the A9 precipitator. This solution was digested for $15 \mathrm{~min}$. at $85-95 \mathrm{C}$. The lead and barium carbonate formed was soluble in this potassium carbonate. While maintaining 85-95 C, $35 \mathrm{lbs}$ of water was added over a period of $30 \mathrm{~min}$. The diluted solution was digested for $30 \mathrm{~min}$. at the reaction temperature. The solution was then cooled to about $30 \mathrm{C}$ over a 30 -min. period to promote growth of lead/barium carbonate crystals. The agitation was stopped, and the precipitate allowed to settle for 2.5 hrs. Metathesis was then repeated, and the carbonate cake was washed with two 33-lb portions of water with an intermittent settling for $2.5 \mathrm{hrs}$.

Dissolution of Metathesis Cake: With the mechanical agitator on, $500 \mathrm{~mL}$ of $70 \%$ nitric acid was added to A-9 and the contents agitated for five minutes. The solution was then jetted and blown to Cell B for purification. 


\section{The X-10 RaLa Separation Process (Continued)}

Cell B Purification: The dissolved metathesis cake was passed through a "crud filter" to remove undesirable insolubles. The solution then entered an electrolytic cell, where the contaminating lead was removed as $\mathrm{PbO}_{2}$. The volume of the product solution was reduced in a precipitator, then transferred to a glassware reactor in a lead cubicle outside Cell B. Subsequent treatments with fuming nitric acid, $\mathrm{HCl}$-ether, $\mathrm{HCl}$-alcohol, and absolute ether removed contaminants $\mathrm{La}, \mathrm{Fe}, \mathrm{Ni}, \mathrm{Cr}$, $\mathrm{Pb}$, and $\mathrm{Sr}$. The final product, barium chloride, was then dissolved in water, sampled, and transferred to the shipping cone where it was evaporated to dryness prior to shipping. In early 1950, an ion exchange process replaced the glassware purification operations that were done near Cell B. This change was instrumental in allowing the size of Oak Ridge barium batches to increase to about $65,000 \mathrm{Ci}$.

Samples of pages from 100 Area log books are presented in Appendix 3C. The log books detail activities by the operators, such as checks of reactor systems, conduct of experiments, and loading and unloading (charging and discharging) of fuel slugs. Typically there were entries when slugs were discharged for RaLa production, with indications of which channels the slugs were pushed from and which buckets in the water-filled pile canal they were put in. In the back of each 100 Area log book are tables of Clinton Pile power levels and accumulated power for each shift for every day of its operation.

\subsubsection{Operations and Health Physics Log Books}

Chemical separation operations log books and 706-D health physics log books were valuable sources of information regarding the "charging" of fuel slugs to the RaLa dissolver, the timing of RaLa dissolving events, and the occurrence of off-normal events. A summary of the availability of chemical separations operations log books is presented in Table 3.2, and sample pages from an operations log book are included in Appendix 3D.

With entries made during each operating shift [day (8 a.m.-4 p.m.), "swing" (4 p.m.-12 midnight), and night (12 midnight-8 a.m.)], the operations log books recorded the status of the RaLa process equipment at the end of each shift (such as summaries of what each process vessel contained, and what stage of processing was active), listings of accomplishments during the shift, documentation of any problems with RaLa processing or radioactive contamination in the building, and instructions to the crew coming on duty. From entries in the operations log books, it is often possible to determine when RaLa dissolvings began. Exact times are rarely given, but it is often stated when a batch had been started, completed, or was in progress. 
Table 3.2: Listing of X-10 Chemical Separations Operations Log Books

\begin{tabular}{|c|c|c|c|c|}
\hline Number & Description & Start Date & End Date & Runs Included \\
\hline$\overline{C \text { CL-884 }}$ & $\overline{706 \mathrm{D} \text { Shift Log, Volume } 1}$ & $4 / 2 / 1945$ & $5 / 21 / 1945$ & Dummy Runs $1-5$ \\
\hline CL-915 & Log \#2 from 706D & $5 / 21 / 1945$ & $8 / 7 / 1945$ & 1, 2, Dummy Run, 3, 4 \\
\hline Cl-1024 & 706D Ops Log \#3 & $8 / 7 / 1945$ & $11 / 5 / 1945$ & 4,5, HP Prep. \\
\hline CL-1098 & 706-D Log Book \#4 & $11 / 6 / 1945$ & $1 / 20 / 1946$ & $6,7,8$ \\
\hline CL-1154 & 706-D Shift Log \#5 & $\sim 1 / 21 / 46$ & $4 / 1 / 46$ & 9 \\
\hline CL-1202 & 706-D Shift Log \#6 & $4 / 2 / 1946$ & $8 / 14 / 1946$ & $10,11,12,13$ (partial) \\
\hline not found & 706-D Shift Log \#7 & $8 / 14 / 1946$ & $\sim 12 / 10 / 46$ & 13 (partial), 14 (partial)? \\
\hline CL-1555 & 706-D Shift Log \#8 & $12 / 11 / 1946$ & $3 / 2 / 47$ & 14 (partial), $15,15 \mathrm{~A}$ (partial) \\
\hline CL-1690 & 706-D Shift Log \#9 & $3 / 4 / 1947$ & $5 / 30 / 1947$ & $15 \mathrm{~A}$ (partial), 16,17 \\
\hline CL-1691 & 706-D Shift Log \#10 & $6 / 2 / 1947$ & $\sim 9 / 12 / 47$ & $18,19,20$ \\
\hline CL-1702 & 706-D Shift Log \#11 & 9/13/1947 & $12 / 15 / 1947$ & $21,22,22 \mathrm{~A}$ \\
\hline not found & 706-D Shift Log \#12 & $12 / 15 / 1947$ & $\sim$ March 1948 & $23-24 ?$ \\
\hline not found & 706-D Shift Log \#13 & $\sim$ March 1948 & - June 1948 & $25-26 ?$ \\
\hline not found & 706-D Shift Log \#14 & - June 1948 & $10 / 22 / 1948$ & $27 ?$ \\
\hline A-7881 & 706-D Shift Log \#15 & $10 / 22 / 1948$ & $2 / 25 / 1949$ & $28,29,30,30 \mathrm{~A}$ (partial) \\
\hline A-37 Part I & 706-D Log Book \#16 & $2 / 25 / 1949$ & $8 / 16 / 1949$ & 30A (partial), 31 thru 35 \\
\hline A-37 Part II & 706-D Log Book \#17 & $8 / 16 / 1949$ & $2 / 28 / 1950$ & 35 thru 39 \\
\hline A-82 & Chemical Separations Log \#18 & $2 / 28 / 1950$ & $7 / 9 / 1950$ & $40,41,42$ \\
\hline $\mathrm{A}-122$ & Chemical Separations Log \#19 & $7 / 10 / 1950$ & $12 / 22 / 1950$ & Dummy Runs \\
\hline $\mathrm{A}-205$ & Chemical Separations Log \#20 & $12 / 23 / 1950$ & $5 / 16 / 1951$ & 43,44 \\
\hline A-240 & Chemical Separations Log \#21 & $5 / 17 / 1951$ & $10 / 10 / 1951$ & 44,45 \\
\hline A-302 & Chemical Separations Log \#22 & $10 / 10 / 1951$ & $3 / 11 / 1952$ & 46, Dummy \#7 \\
\hline A-328 & Chemical Separations Log \#23 & $3 / 11 / 1952$ & $8 / 12 / 1952$ & Dummy Runs 8-11, Runs 47-49 \\
\hline A-379 & Chemical Separations Log \#24 & $8 / 13 / 1952$ & $1 / 10 / 1953$ & 50,51 \\
\hline $\mathrm{A}-401$ & Chemical Separations Log \#25 & $1 / 10 / 1953$ & $5 / 27 / 1953$ & 52 (partial) \\
\hline A-436 & Chemical Separations Log \#26 & $5 / 27 / 1953$ & $10 / 20 / 1953$ & 52 (partial), $52 \mathrm{~A}, 53$ \\
\hline $\mathrm{A}-453$ & Chemical Separations Log \#27 & $10 / 21 / 1953$ & $3 / 1 / 1954$ & 54, Dummy Run, 55, 55A \\
\hline not found & Chemical Separations Log \#28 & $\sim 3 / 1 / 54$ & $\sim 6 / 1 / 54$ & 56 \\
\hline A-616 & Chemical Separations Log \#29 & $6 / 1 / 1954$ & $11 / 16 / 1954$ & 57,58 \\
\hline A-664 & Chemical Separations Log \#30 & $11 / 16 / 1954$ & $4 / 5 / 1955$ & 59 \\
\hline A-704 & Chemical Separations Log \#31 & $4 / 5 / 1955$ & $8 / 4 / 1955$ & 60,61 \\
\hline A-7879 & Chemical Separations Log \#32 & $8 / 5 / 55$ & $12 / 6 / 1955$ & 62,63 \\
\hline not found & Chemical Separations Log \#33? & $12 / 6 / 1955$ & $\sim$ April 1956 & $64-65 ?$ \\
\hline not found & Chemical Separations Log \#34? & $\sim$ April 1956 & August 1956 & $66 ?$ \\
\hline not found & Chemical Separations Log \#35? & $\sim$ August 1956 & ح December 1956 & $67-68 ?$ \\
\hline
\end{tabular}


For some RaLa runs, operations log books have not been located, but health physics log books are available. The health physics log books contain similar information regarding the timing of transfers of slugs from the pile building, addition of slugs to the RaLa dissolver, and initiation or completion of dissolving batches.

\subsection{4 $\quad \underline{\text { RaLa Run Reports }}$}

Much information concerning the conduct of RaLa runs is in run reports that document many of the processing campaigns. The run reports typically contain the following information:

- $\quad$ documentation of the number of slugs dissolved, and in how many batches

- $\quad$ descriptions of how the steps of processing progressed

- descriptions of any incidents, equipment failures, or difficulties encountered

- $\quad$ material accountability figures for each processing step

A sample of a typical RaLa run report is presented in Appendix 3E.

\subsubsection{Analytical Reports of RaLa Runs}

For many RaLa runs, reports have been located that provide the results of analyses of samples taken during radioactive lanthanum processing. These analytical reports typically document the number of fuel slugs dissolved during each batch of a RaLa run. A sample analytical report is presented in Appendix 3F.

The numbers of slugs dissolved were estimated based on laboratory analyses of samples taken from the uranium solution present in the dissolver after a dissolving was complete. As such, these values were in terms of "effective" slugs dissolved, and did not always match the number of slugs actually added to the dissolver for a series of dissolvings. In a dissolving batch, all of the slugs present were partially dissolved. The numbers of effective slugs dissolved indicate the numbers of slugs equivalent to the quantity of uranium brought into solution from the partially dissolved slugs.

\subsection{Chronology and Details of X-10 RaLa Runs}

There were approximately $80 \mathrm{RaLa}$ production runs performed at Oak Ridge. Several runs were redone (e.g., Runs 15 and 15A, 30 and 30A). Operations also included some special preparations (e.g.,for X-10 radiological warfare experiments), and several "dress rehearsals" and "dummy" runs. Run numbers started at 1 when operations in Building 706-D started in 1945.

Available data concerning the dissolving batches that were part of X-10 RaLa runs in Building 706-D are summarized in Appendix 3G. The table in Appendix 3G includes the following data for each dissolving batch: 
- the batch name;

- $\quad$ the date when dissolving started;

- the estimated time that dissolving started, as a specific time or an operations shift;

- $\quad$ number of slugs dissolved (X-10, 4-inch Hanford, and/or 8-inch Hanford slugs);

- the duration of dissolving determined or estimated, in hours; and

- a classification of each batch based on the potential for releases of iodine from pathways that bypassed the caustic scrubber and the normal path to an elevated stack.

\subsubsection{Start Times for Dissolving Batches}

The dates and times of day when RaLa dissolving batches occurred are of interest if releases are to be matched with meteorological conditions representative of the time of year and time of day when the releases actually occurred. This is particularly important for the Oak Ridge area, where nocturnal inversions and low wind speed conditions are quite common. While the air dispersion assessment described in this report dealt with routine releases from RaLa processing on an annual average basis, the start times for individual RaLa batches have been documented to the extent possible, to support modeling of releases from the individual dissolving batches as a possible future refinement.

In many cases, specific start times were not documented for RaLa dissolving batches. When specific start times were documented, they were preserved for use in the Task 1 assessment. In many cases, only the date and shift were documented, or the shift and some indication of whether dissolving started near the beginning, middle, or end of the shift. When only shift information was available to indicate when a dissolving batch was started, start times were assigned as shown in Table 3.3.

For batches with start times linked only to whole shifts or said to have started in the middle of a shift, the midpoint hours of the operations shifts (i.e., hours 4, 12, or 20) were specified as reasonable start times. For batches determined to have started near the beginnings or ends of shifts, start times were set to the first or last hours of the shift, respectively.

Start times for dissolving batches were specified by the project team to the nearest whole hour. For some runs, operations log books were not located. For batches for which a specific start date could not be determined, the month could be determined in each case. For these cases, a date within that month was assigned so that meteorological data representative of the time of year could be selected should dispersion modeling for individual batches be undertaken. For batches for which start times were not available, start times were set to Hour 1 of the documented or assigned start date so that dispersion would likely not be overestimated. 
Table 3.3: Method of Assigning Start Times to Dissolving Batches Based on Operations Shifts

\begin{tabular}{|l|c|}
\hline \multicolumn{1}{|c|}{$\begin{array}{c}\text { Operations Shift when Dissolving } \\
\text { Started }\end{array}$} & $\begin{array}{c}\text { Start Time Assigned (hour of the day, } \\
\mathbf{1} \text { - 24) }\end{array}$ \\
\hline \hline Night Shift $\quad$ (12 Midnight to 8 a.m.) & 4 \\
\hline Day Shift $\quad$ (4 a.m. to 4 p.m.) & 12 \\
\hline Swing Shift & 20 \\
\hline Night Shift, Beginning & 1 \\
\hline Night Shift, Middle & 4 \\
\hline Night Shift, End & 8 \\
\hline Day Shift, Beginning & 9 \\
\hline Day Shift, Middle & 12 \\
\hline Day Shift, End & 16 \\
\hline Swing Shift, Beginning & 17 \\
\hline Swing Shift, Middle & 20 \\
\hline Swing Shift, End & 24 \\
\hline
\end{tabular}

\subsubsection{Numbers of Fuel Slugs Dissolved}

For many RaLa dissolving batches, specific numbers of slugs dissolved were documented. In other cases, less complete information was available, and the project team estimated numbers of slugs dissolved based on the available information. In some cases, the total number of slugs dissolved over a whole run was specified. The project team apportioned these totals to the individual dissolving batches using methods such as 1) according to documented quantities of barium attributed to each batch, 2) according to typical numbers of slugs per batch from nearby batches, or 3) evenly across the documented number of batches. The specifics of each case in which slugs dissolved per batch were estimated are given in Appendix $3 \mathrm{H}$.

For some batches, no analytical results were located by the project team. In these cases, the numbers of slugs physically dropped to the dissolver were determined from documents such as the Chemical Separations Operations log books. These totals were then apportioned to the individual dissolving batches evenly or in accordance with barium analyses or dissolving rates from nearby batches. 


\subsubsection{Durations of Dissolving Batches}

Durations of dissolving batches are important for several reasons. First, when coupled with a start time, they determine the lengths of time over which the most significant iodine releases would have occurred during a RaLa run. In addition, for a given number of fuel slugs determined or estimated to have been dissolved in a RaLa batch in this study, the duration of dissolving determines the rate of uranium dissolution, which in turn determines the rate of iodine evolution from the dissolved metal.

Specific durations of dissolving batches were documented for some of the early RaLa runs, but this practice was apparently discontinued after the first several years of processing. When specific durations were recorded for batches, they were preserved. In many cases, however, it is only possible to ascertain from the log books whether a dissolving batch was completed during the same shift or a certain number of shifts later. When only shift information was available to estimate durations of dissolving, the lengths of dissolving periods were estimated as shown in Table 3.4. The rationale for specification of these durations is as follows. Based on review of operations log books, approximately 4 hours was the minimum reasonable duration for completion of a dissolving batch. This is why 4 hours is the lower bound for the cases in which dissolving was completed during the "same shift" or "one shift later." For the remaining cases, in which dissolving was completed $n$ shifts later, the lower bounds of the duration ranges (in hours) were calculated as $[(n-1) \times 8]+1$. To illustrate, if a batch was completed six shifts later, the duration would have had to included at a minimum five complete eight-hour shifts, plus a certain amount of time during the shift during which dissolving started and during the shift in which dissolving ended. This amount of time is represented by the 1 hour that is added to $(n-1)$ shifts. The upper bounds of the dissolving batch durations were calculated as $[(n+1) \times 8]$. To illustrate, if a batch was completed six shifts later, at most it could have included seven eight-hour shifts.

Table 3.4: Method for Estimation of Durations of Dissolving Batches

\begin{tabular}{|l|c|}
\hline \multicolumn{1}{|c|}{$\begin{array}{c}\text { Shift During Which Dissolving } \\
\text { Ended }\end{array}$} & $\begin{array}{c}\text { Range of Possible Durations } \\
\text { (hours) }\end{array}$ \\
\hline \hline Same shift as when started & 4 to 8 \\
\hline One shift later & 4 to 16 \\
\hline Two shifts later & 9 to 24 \\
\hline Three shifts later & 17 to 32 \\
\hline Four shifts later & 25 to 40 \\
\hline Six shifts later & 41 to 56 \\
\hline Seven shifts later & 49 to 64 \\
\hline Eight shifts later & 57 to 72 \\
\hline Nine shifts later & 65 to 80 \\
\hline
\end{tabular}


For the Task 1 uncertainty analysis, the duration of each batch estimated as shown above is specified as a uniform distribution of discrete, whole number values between the range limits specified above. This is done so that the results could be cleanly matched with meteorological data representative of specific hours of the day should impacts of releases be assessed on an individual basis as a possible future refinement.

\subsubsection{Classification of Batches as to Likelihood of Direct Releases}

It has been said that the only predictable thing about the X-10 barium/lanthanum separation process was that it was unpredictable. Extreme pressure to produce larger and larger quantities of RaLa product, use of equipment far beyond its design capacity, equipment failure, and human error led to many occurrences over the period from 1944 through 1956 when things didn't go according to procedure or according to expectations. Based on review of available chemical separations operations logs and health physics log books, it is evident that some of the occurrences led to releases of radioactivity into Building 706-D. It is also likely that, under some conditions, iodine was released to the building and then to the environment via paths that would have bypassed the off-gas treatment system and its caustic scrubber. It is important that these release pathways not be ignored. "Direct releases" to the environment were not diminished by the caustic scrubber. The radioiodine released directly to the environment would have contained much more of the highly reactive elemental form of iodine; this is the form that is most efficiently transferred from air to pasture vegetation (see Sections 4 and 5).

Based on the information found in RaLa operations log books and other documents, each of the 733 dissolving batches was classified as to its relative potential for releases of iodine to the environment via paths that bypassed the caustic scrubber and the normal path to an elevated stack. The dissolving batches were classified as shown in Table 3.5, with prevalences of the classes as shown in Table 3.6. 
Table 3.5: Classification of Batches as to Apparent Opportunity for Direct Releases

\begin{tabular}{|c|l|}
\hline $\begin{array}{c}\text { Direct } \\
\text { Release Class }\end{array}$ & \multicolumn{1}{c|}{ Criteria for Selection } \\
\hline \hline 1 & $\begin{array}{l}\text { Operations and/or Health Physics log books are available, and provide no evidence of } \\
\text { significant off-gas leakage beyond that of normal operations. }\end{array}$ \\
\hline 2 & $\begin{array}{l}\text { Available records provide evidence of conditions conducive to leakage of process off-gas } \\
\text { (such as documentation of the inability to maintain sufficient negative pressure on the } \\
\text { dissolver or presence of airborne contamination in the building). }\end{array}$ \\
\hline 3 & $\begin{array}{l}\text { Available records provide evidence of significant leakage of radioactive materials from the } \\
\text { RaLa processing equipment (such as the need for operators to evacuate the building or wear } \\
\text { respirators in the course of their normal duties). }\end{array}$ \\
\hline 4 & $\begin{array}{l}\text { The records that have been located by the project team provide insufficient information } \\
\text { upon which to judge the extent to which direct releases were experienced (no operations log } \\
\text { books or equivalent records are available). }\end{array}$ \\
\hline
\end{tabular}

Table 3.6: Prevalences of the Classes of Dissolving Batches by Potential for Direct Release

\begin{tabular}{|c|c|c|c|c|c|}
\hline \multirow{2}{*}{$\begin{array}{c}\text { Calendar } \\
\text { Year }\end{array}$} & \multicolumn{4}{|c|}{ Number of Cases of Direct Release Class } & \multirow{2}{*}{$\begin{array}{c}\text { Total Number of } \\
\text { Dissolving Batches }\end{array}$} \\
\cline { 2 - 5 } & $\mathbf{1}$ & $\mathbf{2}$ & $\mathbf{3}$ & $\mathbf{4}$ & 42 (all in Building 706-C) \\
\hline \hline 1944 & 42 & 0 & 0 & 0 & 185 (90 in Building 706-C) \\
\hline 1945 & 170 & 10 & 5 & 0 & 97 \\
\hline 1946 & 66 & 14 & 0 & 17 & 136 \\
\hline 1947 & 130 & 5 & 1 & 0 & 77 \\
\hline 1948 & 17 & 0 & 0 & 60 & 36 \\
\hline 1949 & 33 & 3 & 0 & 0 & 17 \\
\hline 1950 & 16 & 1 & 0 & 0 & 9 \\
\hline 1951 & 9 & 0 & 0 & 0 & 38 \\
\hline 1952 & 38 & 0 & 0 & 0 & 22 \\
\hline 1953 & 20 & 2 & 0 & 0 & 28 (29 with 56D) \\
\hline 1954 & 17 & 3 & 0 & 8 & 24 \\
\hline 1955 & 19 & 2 & 0 & 0 & 732 (733 with 56D) \\
\hline 1956 & 24 & 0 & 0 & 0 & \\
\hline \hline Total & 600 & 40 & 6 & 85 & \\
\hline
\end{tabular}

${ }^{1}$ Due to an accident during Run 56 in April 1954, Batch 56-D is treated as a special case, and is not assigned a direct release class here. 


\subsection{Calculation of the I-131 Content of Fuel Slugs}

Because reactor fuel slugs from X-10 and Hanford reactors were used as sources of radioactive barium and lanthanum, methods were developed for estimation of the ${ }^{131}$ I content of slugs from each source. This section describes the methodologies used in this assessment for estimation of fuel slug inventories.

\subsubsection{Content of X-10 Slugs}

The iodine and barium content of natural uranium fuel slugs from X-10's Clinton Pile were estimated using the ORIGEN2.1 computer code (RSIC, 1992). In the calculations, the 1.175-kg natural uranium slugs were irradiated in eight-hour increments from the time they were inserted into the reactor until they were discharged, at peak neutron flux levels corresponding to the documented operating power levels for each eight-hour shift of Clinton Pile operations. The ORIGEN2.1 calculations used the CANDUNAU ${ }^{1}$ cross-section and fission product yield library file that was distributed with the code. Inventories were calculated for seven iodine isotopes, total iodine, barium-140, and total barium. Total iodine was included, in part, because ${ }^{131}$ I does not behave differently than stable iodine in chemical reactions. The key application for this information is in the assessment of the efficiency of the caustic scrubber, where the presence of much stable iodine could decrease the collection efficiency for ${ }^{131}$ I should the capacity of the scrubber fluid be depleted. For the Building 706-D caustic scrubber, in which the caustic scrubbing solution was not recirculated, calculations described in Section 3.10 indicated that depletion of the capacity of the scrubber was not a factor that needed to be further considered in this assessment.

The inventory calculations proceeded as follows:

- $\quad$ Established the operating power level for the pile for each eight-hour shift from 7/27/44 to $12 / 5 / 47$.

- $\quad$ Established the charge and discharge dates for all slugs pushed for each RaLa run.

- $\quad$ For each charge and discharge date, established the shift on which the slugs were charged to the Clinton Pile or pushed from the pile.

- $\quad$ Calculated the peak slug inventory (the highest inventory expected in any single slug) for each group of slugs pushed for each RaLa run.

- $\quad$ Established the position factors for each slug in each group of slugs pushed. These factors indicate the relative inventories of slugs at various positions in the pile compared to that of the peak slug.

\footnotetext{
${ }^{1}$ Developed for CANDU reactors using NAtural Uranium fuel.
} 
- $\quad$ Summed the total inventory of selected isotopes for each group pushed for each run (most importantly in terms of grams of total iodine and curies of ${ }^{131} \mathrm{I}$ ).

- $\quad$ Divided the totals for each run by the number of slugs pushed and their mass to get the average content per slug and per kilogram of uranium.

- $\quad$ Computed the average content of each isotope per slug and per kilogram of uranium over all RaLa runs in which X slugs were used and for which push data had been obtained.

The calculated average ${ }^{131}$ I inventories of Clinton Pile slugs used in RaLa Runs 1 through 22 are shown in Figure 3.2. The iodine inventories do not vary significantly across the RaLa runs that used X-10 slugs; the coefficient of variation of the values depicted in Figure 3.2 is $7.3 \%$. This is in stark contrast to the inventories of Hanford RaLa slugs, which will be shown in the following section contained increasingly higher quantities of iodine as the years of RaLa processing progressed from 1948 to 1956 (see Section 3.5.2). Based on the ORIGEN2.1 calculations for all RaLa runs that used Clinton Pile slugs, the ${ }^{131} \mathrm{I}$ content of X-10 slugs is represented by the following weighted probability distribution:

- $\quad$ Range: 3.3 to 3.8 curies per kilogram of uranium (3.9 to 4.5 $\mathrm{Ci}$ per slug)

Probability: 0.095 (i.e., 2 out of 21 runs)

- $\quad$ Range: 3.8 to 4.6 curies per kilogram of uranium (4.5 to 5.4 $\mathrm{Ci}$ per slug)

Probability: 0.905 (i.e., 19 out of 21 runs)

Within each stated range, each value is considered to be equally likely- uniform distributions were used. The total iodine content of the dissolved X-10 uranium was represented by the following weighted probability distribution:

- $\quad$ Range: 100 to $120 \mathrm{Fg}$ per kilogram of uranium (120 to $140 \mathrm{Fg}$ per slug)

Probability: 0.095 (i.e., 2 out of 21 runs)

- $\quad$ Range: 120 to $220 \mathrm{Fg}$ per kilogram of uranium (140 to $250 \mathrm{Fg}$ per slug)

Probability: 0.857 (i.e., 18 out of 21 runs)

- $\quad$ Range: 220 to $290 \mathrm{Fg}$ per kilogram of uranium (250 to $340 \mathrm{Fg}$ per slug)

Probability: 0.048 (i.e., 1 out of 21 runs) 
TASK 1 REPORT

July 1999

Page 3-26
${ }^{131}$ I Releases from X-10 Radioactive Lanthanum ProcessingSource Term Assessment

Figure 3.2: Calculated Average Iodine-131 Inventories of X-10 RaLa Slugs

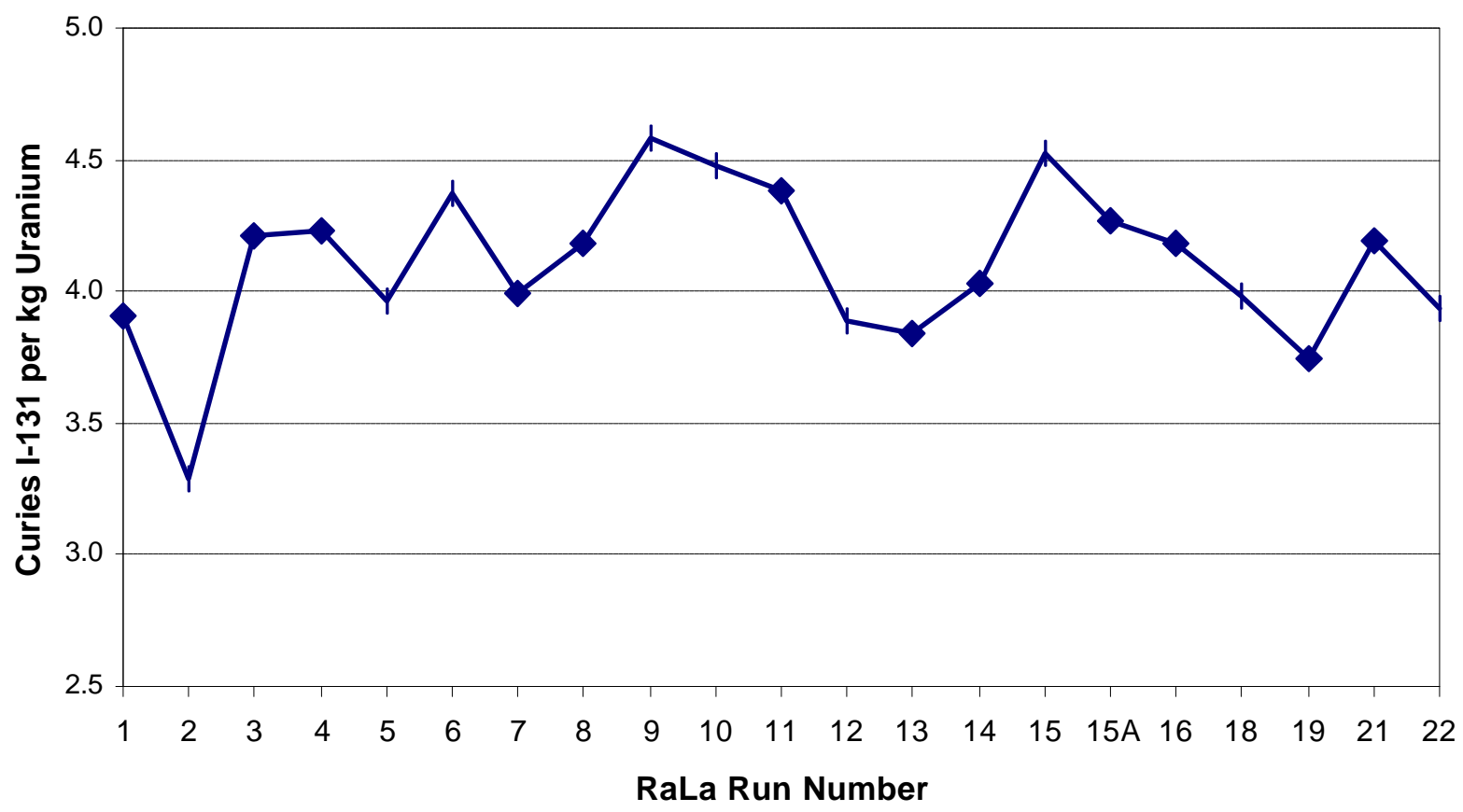


These ranges are assumed to be uniformly distributed, and represent the iodine inventories at the time of reactor shutdown after irradiation of the slugs for each run.

Some uncertain parameters were involved in the calculations of the iodine content of X-10 RaLa slugs. In the uncertainty analysis for the Task 1 source term, five multiplicative uncertainty factors (described in Table 3.7) are applied to the inventory values for X-10 slugs to reflect the level of knowledge about these parameters and the inventory results. In each of the 400 realizations of Monte Carlo simulation used for propagation of uncertainty in the source term results, values are selected from the distribution for calculated total iodine inventory, the distribution for ${ }^{131}$ I inventory, and from each of the five uncertainty factor distributions described in Table 3.7.

Further details of the inventory calculations for X-10 slugs and associated uncertain parameters can be found in Appendices 3I and 3J.

\subsubsection{Content of Hanford Slugs}

The iodine and barium contents of natural uranium fuel slugs from the Hanford, Washington reactors were estimated using the ORIGEN2.1 computer code. Inventories were calculated for each RaLa run for I-131, $\mathrm{Ba}-140$, and total iodine content at the time of reactor shutdown. The inventory values for I-131 and Ba-140 were given in terms of curies of radioactivity per unit mass of uranium shipped (in kilograms). Total iodine values were given in terms of mass of total iodine (grams) per unit mass of uranium. The calculations accounted for the fact that only the center-most slugs were used from each tube pushed.

Two sets of factors are relevant to calculation of Hanford slug inventories- "tube factors" and "slug factors." Tube factors are measures of the relative power in a given reactor fuel channel (tube) with respect to the average for all tubes in a given reactor; tube factors account for radial variations in power. Axial power variations (along the length of a tube) are described by slug factors, which are fractions of the total power for a tube produced by a specific slug in that tube. Characterization of tube factors and slug factors was important so that inventories calculated for certain slugs in a reactor could be related to other slugs at other positions in the reactor. Details regarding characterization of tube factors and slug factors are provided in Appendices $3 \mathrm{~L}$ and $3 \mathrm{M}$.

The following parameters were uncertain in the inventory calculations for the Hanford fuel slugs used in some X-10 RaLa runs:

- Reactor of origin ("source" reactor)

- $\quad$ Length of irradiation time

- Tube factor 
TASK 1 REPORT

July 1999

${ }^{131}$ I Releases from X-10 Radioactive Lanthanum Processing-

Page 3-28

Source Term Assessment

Table 3.7: Uncertainty Factors Applied to Inventories Calculated for X-10 Slugs

\begin{tabular}{|c|c|}
\hline $\begin{array}{l}\text { Uncertain } \\
\text { Parameter } \\
\end{array}$ & Multiplicative Uncertainty Factor Applied \\
\hline $\begin{array}{c}\text { Mass of Clinton Pile } \\
\text { Slugs }\end{array}$ & $\begin{array}{l}\text { Normal distribution; mean }=1 \text {, standard deviation }=0.6 \% \\
\text { Rationale: From ORNL Central Files Memorandum } 52-6-10 \text {, the original specifications } \\
\text { for X-10 slugs was length }=4.000 \pm 0.010 \text { inches and diameter }=1.100 \pm 0.002 \text { inches. } \\
\text { Those tolerances result in a maximum dispersion in the slug mass of } \pm 0.6 \% \text {. }\end{array}$ \\
\hline $\begin{array}{l}\text { Physical constants } \\
\text { used } \\
\text { in the ORIGEN code }\end{array}$ & $\begin{array}{l}\text { Normal distribution; mean }=1 \text {, standard deviation }=5 \% \\
\text { Rationale: Formal calculations performed under the Hanford Environmental Dose } \\
\text { Reconstruction study to assess the uncertainties associated with using the ORIGEN2 } \\
\text { code showed the maximum errors to be within } \pm 5 \% \text { for the radionuclides considered in } \\
\text { this study (Heeb, 1991). }\end{array}$ \\
\hline Clinton Pile Power & $\begin{array}{l}\text { Normal distribution; mean }=1 \text {, standard deviation }=3 \% \\
\text { Rationale: Clinton Pile power for each operations shift was determined based on the } \\
\text { thrice-daily accumulated power (kilowatt-hour) values that pile operators recorded from } \\
\text { the accumulated kWh meter in the control room. The accumulated power over a shift } \\
\text { was divided by the nominal shift length of eight hours to determine power generation } \\
(\mathrm{kW}) \text { for each shift. Uncertainty is associated with the actual length of the time interval } \\
\text { between readings; an estimated uncertainty of } 15 \text { minutes corresponds to a dispersion } \\
\text { of } \pm 3 \% \text { about an } 8.0 \text { hour interval. The project team believes it is unnecessary to } \\
\text { expand this uncertainty to reflect variability in the pile's galvanometer (power } \\
\text { measurement) circuit itself, because the circuit was quite stable, calibrated at least } \\
\text { monthly, and very consistent on a day-to-day basis. }\end{array}$ \\
\hline $\begin{array}{l}\text { Power-to-peak-flux } \\
\text { conversion factor }\end{array}$ & $\begin{array}{l}\text { Normal distribution; mean }=1 \text {, standard deviation }=5 \% \\
\text { Rationale: Based on a } 4 \% \text { bias between results of Monte Carlo N-Particle (MCNP) } \\
\text { modeling of the Clinton Pile and physical measurements made by Clinton Pile physicists } \\
\text { in 1945, plus the 1.3\% precision of the MCNP calculations performed to yield the fuel- } \\
\text { to-air flux ratio for the Clinton Pile. }\end{array}$ \\
\hline Relative flux values & $\begin{array}{l}\text { Normal distribution; mean }=1 \text {, standard deviation }=5 \% \\
\text { Rationale: Based on a 5\% ripple seen in the silver wire measurements performed in } 1945 \\
\text { (Jones et. al, 1945). It is reasonable to assume that the observed variability in the silver } \\
\text { wire measurements was less than 5\%, hence overall error associated with the counting } \\
\text { of the activated silver segments would have been within } \pm 5 \% \text {. }\end{array}$ \\
\hline
\end{tabular}


For cases where all the above parameters were known (Runs 28-31 and 40-42), the inventory results were the product of the following:

- $\quad$ The ORIGEN results (average inventory values for all tubes in the reactor),

- a "saturation factor" (the ratio of inventory for discontinuous reactor operation to that from continuous operation; ranged 0.92 to 0.93 for ${ }^{131} \mathrm{I}$ ),

- a correction factor to reflect the fact that the center 38 four-inch slugs or the center 18 eightinch slugs were shipped to Oak Ridge (the factor was 0.749 for four-inch slugs and 0.718 for eight-inch slugs), and

- the tube factor,

- $\quad$ divided by the mass of the 38 four-inch slugs (all of these cases involved four-inch Hanford slugs).

In cases where the irradiation time was unknown, ORIGEN results for the upper and lower bounds of irradiation time were sampled in a Monte Carlo simulation. Based on review of the data for 15 runs for which the irradiation times are known, the upper bound used was 100 days, while the lower bound was 50 days prior to 1951 and 14 days from 1951 on.

In cases where the tube factor was unknown, possible tube factors were sampled from a Beta distribution between estimated lower and upper bounds. Based on review of tube factors that are documented, lower bounds for tube factors ranged from 1.2 to 1.3, while upper bounds ranged from 1.2 to 1.6. Beta distributions were used because they effectively represented empirical tube factor data, namely observed tube factors documented for Hanford slugs pushed from 11 fuel channels in 1949 and 1950. The Beta distribution is a very flexible distribution often used to represent variability over a fixed range. The distribution parameters were adjusted until the distribution best matched the known data. Appendix 3L contains additional details regarding tube factors for Hanford slugs.

There were 40 RaLa runs that used fuel slugs from Hanford piles (Runs 28 through 68, with the exception of Run 43, which used X-10 slugs). The Hanford plutonium production piles that could have supplied RaLa slugs to Oak Ridge were called the $\mathrm{B}, \mathrm{C}, \mathrm{D}, \mathrm{DR}, \mathrm{F}$, and $\mathrm{H}$ piles. The $\mathrm{KW}$ and $\mathrm{KE}$ reactors were of different design, and were used for tritium production; it is unlikely that they supplied RaLa slugs.

For 20 of the Hanford slug runs, the project team was able to identify the specific reactor from which the slugs originated based on teletypes from Hanford to X-10, Hanford reactor operations monthly reports (Barnard, 1949) and summaries of Hanford dissolving data (Jaech, undated). For eight runs, inventory distributions were calculated that represented averages over the possible source reactors. Distributions of ${ }^{131}$ I and total iodine were prepared and used in the source term calculations for each individual RaLa run that involved Hanford slugs.

For 12 runs, all occurring after 1952, the project team calculated distributions of iodine inventories for the $\mathrm{H}$ (most likely) pile and the $\mathrm{C}$ (highest power) pile and weighted inventories are estimated based on these values and a subjective representation of the probability that slugs came from the $\mathrm{H}$ pile, the $\mathrm{C}$ pile, or one of the other 
piles (i.e., B, D, DR, or F). The choice of the H pile as most likely was based on the fact that, starting with Run 51 (September 1952), in the majority of cases in which the source of RaLa slugs was determined, it was the $\mathrm{H}$ reactor. The $\mathrm{H}$ reactor was likely also an attractive option because of its higher power relative to the other piles (second only to C) and its physical location- it was roughly 15 miles closer to Richland, from where slugs shipments departed.

Distributions of potential iodine inventories for the cases when the specific pile of origin was not known were developed as follows:

- $\quad$ For Runs 39 and 44 through 50, the specific reactors of origin were not known, but there was overlap in the power levels of the possible reactors (that is, their power levels were similar). These runs occurred before the $\mathrm{C}$ pile became operational and the power level of the $\mathrm{H}$ pile was significantly increased. Inventory values for these cases reflect the average across possible source reactors at the time.

- For Runs 52, 54, 56, 58, and 60 through 67, the specific reactors of origin were not known and there was not overlap in the power levels of the possible source reactors. For these cases, ORIGEN calculations were performed for the $\mathrm{H}$ pile (the most likely source of RaLa slugs) and the $\mathrm{C}$ pile (the pile from the group with the highest power level). While iodine inventories were not calculated for the other four piles that could have supplied RaLa slugs, due to schedule and resource limitations, these calculations could be performed as a future refinement. Weighted distributions of possible iodine inventories are calculated for each run as follows:

1. Subjective weighted probability is assigned to the following cases of potential RaLa slug origin: $\mathrm{H}$ reactor (66\% weighted probability), $\mathrm{C}$ reactor (17\%), and "other reactors" $(17 \%)$. These probabilities are based on observation of the piles of origin for the six runs after Run 50 for which slug source is known. Of these six runs, four (66\%) used slugs from the $\mathrm{H}$ pile and one each used slugs from the $\mathrm{C}$ and $\mathrm{F}$ piles (17\% each case).

2. For each realization of Monte Carlo simulation used to propagate uncertainties, a slug source is selected in accordance with these probabilities.

3. When the $\mathrm{H}$ pile case is selected, the inventory distribution calculated for the $\mathrm{H}$ pile for that run is sampled. When the $\mathrm{C}$ pile case is selected, the inventory distribution calculated for the $\mathrm{C}$ pile for that run is sampled. If the "other reactors" case is selected, the inventory distribution for the $\mathrm{H}$ reactor is sampled, and the result is multiplied times a value sampled from a distribution between 0.70 and 0.75 . This latter distribution accounts for the fact that the "other" reactors (B, D, DR, and F) were operated at power levels between $25 \%$ and $30 \%$ lower than the $\mathrm{H}$ reactor during the period of interest. 
4. Across 400 realizations of Monte Carlo simulation, the contributions of the three slug-source cases are summed, resulting in weighted distributions of possible iodine inventories for the Hanford slugs that reflects the calculated inventories and the subjective probability of each potential slug source. ${ }^{1}$

The average ${ }^{131}$ I content of Hanford slugs used for RaLa processing varied from 1948 through 1956 (Runs 28 through 58) as shown in Figure 3.3. The peak value of $160 \mathrm{Ci} / \mathrm{kg}$ estimated for Run 66 is over three times the $51 \mathrm{Ci} / \mathrm{kg}$ average over the first five Hanford-slug runs. Because the iodine content of Hanford slugs varied so significantly over time, run-specific distributions of ${ }^{131}$ I and total iodine content were applied for each dissolving batch.

Two multiplicative uncertainty factors are applied to the calculated inventory values for Hanford RaLa slugs. These uncertainty factors are described in Table 3.8. For the two RaLa runs in which mixtures of X-10 and Hanford slugs were dissolved (Runs 30 and 30A in 1949), the five inventory uncertainty factors for X-10 slugs were applied, because in every case X-10 slugs significantly outnumbered Hanford slugs.

Additional details of the inventory calculations for Hanford slugs are presented in Appendix 3K.

${ }^{1}$ For Run 54, the only run that used both 4-inch and 8-inch Hanford slugs, calculated iodine inventory distributions for the 4-inch slugs from the $\mathrm{H}$ and $\mathrm{C}$ piles were used in this weighting method, as the 4-inch slugs contained slightly $(4 \%)$ higher iodine- 131 content per unit mass than the 8 -inch slugs. 
TASK 1 REPORT

July 1999

Page 3-32
${ }^{131}$ I Releases from X-10 Radioactive Lanthanum ProcessingSource Term Assessment

Figure 3.3: Trend of Iodine-131 Content of Hanford RaLa Slugs

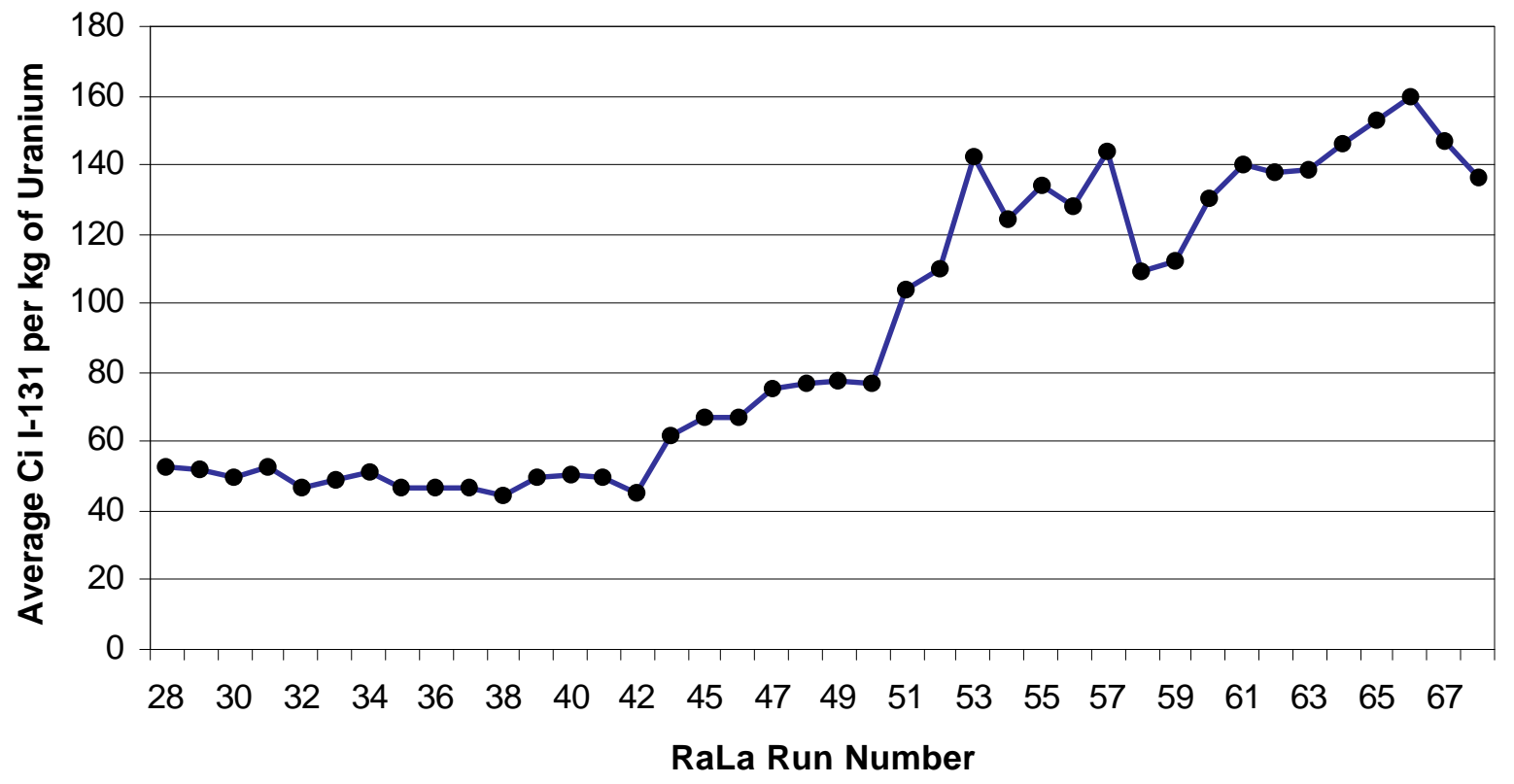


Table 3.8: Uncertainty Factors Applied to Inventories Calculated for Hanford Slugs

\begin{tabular}{|c|l|}
\hline \multicolumn{1}{|c|}{ Uncertain Parameter } & \multicolumn{1}{c|}{ Multiplicative Uncertainty Factor Applied } \\
\hline \hline $\begin{array}{l}\text { Combined Uncertainty from } \\
\text { Physical Constants used in } \\
\text { ORIGEN (reaction cross-sections } \\
\text { and fission product yield values) } \\
\text { and Actual Reactor Power } \\
\text { Variations (degree of saturation). }\end{array}$ & $\begin{array}{l}\text { Normal distribution; mean }=1 \text {, standard deviation }=5 \% \\
\text { Environmental Dose Reconstruction study to assess the } \\
\text { uncertainties associated with using the ORIGEN2 code showed the } \\
\text { maximum errors to be within } \pm 5 \% \text { for the radionuclides considered } \\
\text { in this study (Heeb, 1991). Given the maximum 5\% dispersion from } \\
\text { the HEDR assessment and the small variability seen in the } \\
\text { saturation factors computed for the Hanford RaLa slugs, an } \\
\text { uncertainty of } \pm 5 \% \text { was selected to account for combined } \\
\text { uncertainties from physical constants and reactor power variation. }\end{array}$ \\
\hline $\begin{array}{c}\text { Noncertainty in Recorded } \\
\text { Reactor Power Levels and } \\
\text { Asserted Tube Factors }\end{array}$ & $\begin{array}{l}\text { Normal distribution; mean }=1, \text { standard deviation }=5 \% \\
\text { Rationale: The HEDR study (Heeb and Bates, 1994) used a value of } \\
\text { likely an overstatement given that pile power was recorded to much } \\
\text { greater precision, hence an uncertainty of } \pm 5 \% \text { is considered } \\
\text { appropriate for the combined uncertainties in pile power and } \\
\text { asserted tube factors. }\end{array}$ \\
\hline
\end{tabular}

\subsection{Correction for Delay between Reactor Shutdown and Dissolution}

A certain amount of time passed between the point when the $\mathrm{X}-10$ and Hanford reactors were powered down for slug discharge and the time that dissolving of the slugs occurred at X-10. It is important to account for this delay in the source term assessment because of the 8.04-day half-life of iodine-131. With X-10 slugs, a RaLa run included multiple dissolving batches. The pile was shut down for discharge of slugs for each dissolver "charge," and then restarted until slugs were needed for the next charge. Delay times as short as a few hours were possible with X-10 slugs. For this analysis, the delay time between end of irradiation and dissolution of $\mathrm{X}-10$ slugs (which is essentially the time of iodine evolution) is assumed to follow a uniform distribution between 2 hours and 14 hours. This distribution is based on detailed information concerning the timing of slug discharges from the Clinton Pile (Section 3.3.2) and the initiation of RaLa dissolving batches (Section 3.3.3 and 3.4.1). The upper end is to reflect cases where equipment malfunctions or operational difficulties delayed dissolving batches. As applied in this analysis, the decay time sampled from the uniform distribution described above represents decay between the time of reactor shutdown and the time of dissolution/iodine evolution, which for a given molecule of ${ }^{131} \mathrm{I}$ could have extended beyond the start of dissolution by as much as the estimated duration of the dissolving period.

Hanford slugs contained significantly higher levels of fission products than X-10 slugs, so fewer slugs were needed for a given quantity of product, and fewer dissolving batches per run were necessary. There was, however, a delay between shutdown of the reactor and actual discharge of the slugs and a relatively long delay 
time associated with the transport of the slugs from Hanford to Oak Ridge by express train. Documentation has been found of at least one case where slugs were delayed by bad weather.

The times of reactor shutdown are known for nine sets of Hanford RaLa slugs. There are another seven sets of Hanford RaLa slugs for which the dates the slugs were discharged from their reactor are known, but the times of reactor shutdown are not. Based on review of the data for the sets of slugs with known shutdown times, a nominal interval of 12 hours is assumed between reactor shutdown and discharge of slugs. Decay times estimated for the 16 sets of Hanford RaLa slugs are shown in Table 3.9; additional details of these data can be found in Appendix 3-N. In this assessment, the delay time between end of irradiation and dissolution of Hanford slugs is assumed to follow a triangular distribution between 5 days and 11 days, with 6 days being most likely. This range reflects the fact that dissolving batches for Hanfordslug RaLa runs often extended over several days, and its upper end reflects cases where weather or other factors delayed delivery of slugs from Hanford and some cases where malfunctions or operational difficulties delayed dissolving of the slugs.

Table 3.9: Estimated Decay Times for Sets of Hanford RaLa Slugs

\begin{tabular}{|c|lr|}
\hline Decay Time (days, to nearest $1 / 2 \mathrm{~d}$ ) & \multicolumn{2}{|c|}{ Number of Occurrences } \\
\hline \hline 5.0 & 1 & (Run 37) \\
\hline 5.5 & 5 & (Runs 30, 35, 57-59) \\
\hline 6.0 & 5 & (Runs 31-34 and 36) \\
\hline 6.5 & 1 & (Run 29) \\
\hline 7.0 & 2 & (Runs 51 and 68) \\
\hline 7.5 & 1 & (Run 53) \\
\hline 11.0 & 1 & (Run 55) \\
\hline
\end{tabular}

The I-131 content of X-10 and Hanford slugs is corrected for decay between reactor shutdown and dissolving based on simple radioactive decay:

$$
\mathrm{A}_{\mathrm{t}}=\mathrm{A}_{0} \mathrm{e}^{-\lambda \mathrm{t}}
$$

where: $\quad \mathrm{A}_{\mathrm{t}}=$ activity at time $\mathrm{t}$

$\mathrm{A}_{0}=$ activity at time 0

$\lambda=$ decay constant (equals $0.693 / \mathrm{T}_{1 / 2}$, where $\mathrm{T}_{1 / 2}$ is the radioactive decay half-life, $\mathrm{d} ; 8.04$ for I-131)

$\mathrm{t}=$ elapsed time, $\mathrm{d}$. 
The decay correction of total iodine content of fuel slugs is more involved. The presence of stable fission product ${ }^{127}$ I makes it necessary to establish decay expressions that are a function of irradiation time. Unlike ${ }^{131}$ I content, which would reach an equilibrium between formation and decay, the ${ }^{127} I$ content of the reactor slugs continues to increase with increased irradiation time. Concurrently, the fraction of total iodine that ${ }^{131}$ I comprises decreases as a function of time.

The following equation, which was determined by fitting total iodine results calculated by ORIGEN2.1 as a function of time after irradiation, based on 100-day irradiation time, is used to decay grams of total iodine in X-10 slugs. The fit was excellent, with agreement to a few hundredths of a percent.

$$
f(t)^{\prime} a e^{b t} \% c e^{d t}
$$

where: $f(t)$ is the fraction remaining at time $\mathrm{t}$;

$\mathrm{a}, \mathrm{b}, \mathrm{c}$ and $\mathrm{d}$ are constant coefficients; and $\mathrm{t}$ is the elapsed time in hours.

For total iodine decay of X-10 slugs, the coefficients are:

$$
\begin{array}{ll}
a= & 0.91771 \\
b= & -8.39947 \times 10^{-4} \\
c= & 0.08229 \\
d= & -4.59712 \times 10^{-2}
\end{array}
$$

This expression is valid for elapsed times after reactor shutdown from 0 to 44.0 hours. For decay correction of total iodine in Hanford slugs, the above equation is used with a different set of coefficients. The coefficients vary depending on the length of the irradiation period, as shown in Table 3.10. 
Table 3.10: Coefficients for Decay Correction of the Total Iodine Content of Hanford Slugs

\begin{tabular}{|c|c|c|c|}
\hline \multirow{2}{*}{ Coefficient } & \multicolumn{3}{|c|}{ Irradiation Duration } \\
\cline { 2 - 4 } & 14 days & $\mathbf{5 0}$ days & 100 days \\
\hline \hline $\mathrm{a}$ & 0.6810 & 0.7971 & 0.8741 \\
\hline $\mathrm{b}$ & -0.0362 & -0.0203 & -0.0104 \\
\hline $\mathrm{c}$ & 0.3190 & 0.2029 & 0.1259 \\
\hline $\mathrm{d}$ & -0.4477 & -0.4749 & -0.4433 \\
\hline
\end{tabular}

In this assessment, the coefficient sets are selected based on documented irradiation durations when possible. Irradiation durations have been identified for 15 sets of Hanford RaLa slugs. For these cases, the set of coefficients for the decay equation closest to the actual irradiation duration is used. With runs for which irradiation durations have not been determined, the irradiation period for the closest preceding run with a known value is noted; the coefficient set closest to this estimated irradiation duration is then used. Documented irradiation durations and values used for selection of coefficient sets for the decay correction of total iodine inventories are shown in Table 3.11. Additional detail regarding decay times and decay correction for Hanford slugs can be found in Appendix 3N.

\subsection{Estimation of the Species of Radioiodine Present in the Dissolver}

This section and the sections that follow discuss the behavior of radioiodine in the RaLa process equipment, particularly with regard to how much of the iodine present in the dissolved fuel slugs was likely released to the environment. While the project team was quite successful in locating records describing the details of RaLa processing and related reactor operations, very few measurements were made of iodine concentrations in RaLa process fluids in releases from RaLa processing. Some information has, however, been identified that is relevant to the behavior of radioiodine in RaLa processing at X-10 and to the fraction of the iodine present in the dissolver that was likely to have been released. This information comes from the following sources:

- $\quad$ expert opinion,

- $\quad$ mathematical modeling of the RaLa plant process,

- $\quad$ several reports of effluent and waste monitoring at X-10, and

- $\quad$ reports of experience with nuclear material processing at other sites. 
Table 3.11: Documented Irradiation Durations for Hanford RaLa Slugs and Durations Assumed for Decay Correction of Total Iodine Inventories

\begin{tabular}{|c|c|c|}
\hline \multirow{2}{*}{$\begin{array}{c}\text { Run } \\
\text { Number }\end{array}$} & \multicolumn{2}{|c|}{ Irradiation Duration (days) } \\
\cline { 2 - 3 } & Known & $\begin{array}{c}\text { Used for Decay } \\
\text { Calcs. }\end{array}$ \\
\hline \hline 28 & 87 & 100 \\
\hline 29 & NA $^{11}$ & 100 \\
\hline 30 & NA & 100 \\
\hline 31 & $69 \& 46$ & 50 \\
\hline 32 & NA & 50 \\
\hline 33 & NA & 50 \\
\hline 34 & NA & 50 \\
\hline 35 & NA & 50 \\
\hline 36 & NA & 50 \\
\hline 37 & NA & 50 \\
\hline 38 & 98 & 100 \\
\hline 39 & NA & 100 \\
\hline 40 & 99 & 100 \\
\hline 41 & 90 & 50 \\
\hline 42 & 50 & 50 \\
\hline $44^{2}$ & NA & 50 \\
\hline 45 & NA & 50 \\
\hline 46 & NA & NA \\
\hline 47 & NA & 50 \\
\hline 48 & NA & 50 \\
\hline
\end{tabular}

\begin{tabular}{|c|c|c|}
\hline \multirow{2}{*}{$\begin{array}{c}\text { Run } \\
\text { Number }\end{array}$} & \multicolumn{2}{|c|}{ Irradiation Duration (days) } \\
\cline { 2 - 3 } & Known & $\begin{array}{c}\text { Used for Decay } \\
\text { Calcs. }\end{array}$ \\
\hline \hline 49 & NA & 50 \\
\hline 50 & NA & 50 \\
\hline 51 & 302 & 100 \\
\hline 52 & NA & 100 \\
\hline 53 & 40 & 50 \\
\hline 54 & NA & 50 \\
\hline 55 & 63 & 50 \\
\hline 56 & NA & 50 \\
\hline 57 & 33 & 50 \\
\hline 58 & 21 & 14 \\
\hline 59 & 14 & 14 \\
\hline 60 & NA & 14 \\
\hline 61 & NA & 14 \\
\hline 62 & NA & 14 \\
\hline 63 & NA & 14 \\
\hline 64 & NA & \\
\hline 65 & NA & 14 \\
\hline 66 & NA & NA \\
\hline 67 & 43 & 14 \\
\hline 68 & & 50 \\
\hline
\end{tabular}

${ }^{1} \mathrm{NA}=$ Not Available (not determined from available records).

${ }^{2}$ Run 43 was a low-level break-in run that used only X-10 slugs. 
A number of past RaLa engineers and operators and iodine behavior experts have been interviewed as sources of information regarding the X-10 facilities and processes and the manners in which iodine was likely to have behaved under the conditions at the time. Individuals who have been interviewed include:

Orlan Yarbro . . . . . . . . . F Former X-10 fuel reprocessing specialist, iodine behavior expert

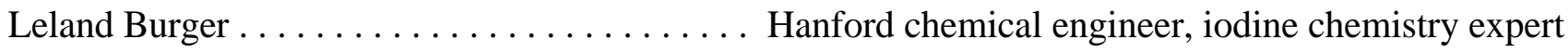
Cal Heeb . . . . . . . . . . . Hanford Environmental Dose Reconstruction source term investigator Fred Weber . . . . . . . . Univ. of Tennessee chemical engineer with fuel reprocessing expertise William Burch . . . . . . . . . . . . . . . X-10 chemical technology staff member Bob Wichner $\ldots \ldots \ldots \ldots \ldots \ldots \ldots \ldots \ldots \ldots \ldots \ldots \ldots \ldots \ldots$. $10 \ldots \ldots$ chemical processing specialist

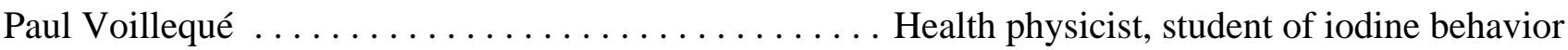
Stanley Rimshaw $\ldots \ldots \ldots \ldots \ldots \ldots \ldots \ldots \ldots \ldots \ldots \ldots \ldots \ldots \ldots$ Former RaLa process operator

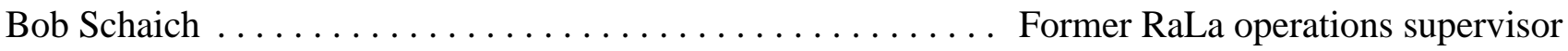

The group came to the following conclusions. First, elemental iodine is removed very efficiently by caustic scrubbers. Organic and particulate forms of iodine are very poorly removed. Even though most of the iodine present in dissolved fuel slugs was likely in the form of elemental iodine, a majority of routine iodine releases past the caustic scrubber were likely in the form of organic iodides. If scrubber was operated with water instead of caustic solution, iodine capture would have occurred at reduced efficiency. While there was general agreement that less than five percent of the iodine available in the dissolved fuel was likely released to the environment when the caustic scrubber was functional, it was thought that release fractions could be higher for "upsets" such as accidents and off-normal operating conditions.

A meeting was held in December 1995 between project team members and Orlan Yarbro, Bob Wichner, Frank Gifford, and Paul Voillequé to discuss release forms and fractions of ${ }^{131} \mathrm{I}$ and the atmospheric chemistry of radioiodine. Based upon the opinions of this group, the following summary statistics were used as partial basis for the Task 1 source term assessment:

- $\quad$ The radioiodine present in the dissolver solution was likely 93.9 to $98.9 \%$ elemental, 0.5 to $4 \%$ volatile organic, 0.5 to $2 \%$ nonvolatile organic, and less than $0.1 \%$ particulate (most likely soluble entrained iodine).

- $\quad$ Between 70 and $90 \%$ of total iodine is released from the dissolver to the dissolver off-gas stream. The nonvolatile heavy organic fraction remains in solution.

- In the caustic scrubber, iodine capture efficiencies would be expected to be approximately $99 \%$ for elemental, 1 to $10 \%$ for volatile organic, and 1 to $10 \%$ for particulate. 
Upon closer examination of the summary of this meeting and interview notes, it is clear that the $0.5 \%$ to $4 \%$ range was not meant to represent the range for RaLa iodine. Rather, the upper value is typical for chemical separation operations such as Purex, that used recycled acid and solvent extraction processes. RaLa did not use recycled reagents or organic solvents, thus involved very small quantities of organics. Most organic iodine that appeared in the RaLa off-gas was likely formed by reactions of iodine with traces of oils, greases, solvents, impurities in water, or carbon impurities in the uranium (Burger, 1991; Burger interview; Yarbro interview). The radiation field assists by providing free radicals. A volatile organic iodine fraction of around $0.5 \%$, the lower bound of the range previously stated, would be typical for RaLa processing (Yarbro, 12/95 mtg). Bill Burch agrees that it should be down in the 1\% or less range (Personal communication, 8/98).

Based on the above information, the fraction of iodine in the RaLa dissolver that was in volatile organic form is represented as a log-triangular distribution between $0.1 \%$ and $4 \%$, with $0.5 \%$ most likely. This distribution is specified based on the expert opinion summarized above and the lack of relevant monitoring data from the $\mathrm{X}-10$ processing. While the log-triangular distribution specified emphasizes values between $0.1 \%$ and $0.5 \%$, the upper bound is maintained at $4 \%$ in light of the lack of specific measurements in the $\mathrm{X}-10$ dissolver.

The following method is used to determine the fraction of each radioiodine species in the RaLa dissolver:

1. The percentage of particulate/aerosol-associated iodine is sampled from a loguniform distribution between 0.01 and 0.1 percent.

2. The percentage of volatile organic iodine is sampled from a log-triangular distribution between 0.1 and 4.0 percent, with 0.5 percent most likely.

3. The percentage of nonvolatile organic iodine is sampled from a uniform distribution between 0.5 and 2.0 percent.

4. The percentage of elemental iodine is determined as 100 minus the sum of the percentages of volatile organic, nonvolatile organic, and particulate iodine present in the dissolver.

Using this method, the amount of iodine available in the dissolver is apportioned to the identified species in a manner that approximates the desired distribution and in which the percentages add up to 100 in each Monte Carlo realization. 


\subsection{Estimation of Iodine Released to the Dissolver Off-gas Stream}

As described above, the initial belief of the experts consulted was that between 70 and $90 \%$ of total iodine present in the dissolver was released to the dissolver off-gas stream, while the nonvolatile heavy organic fraction remained in solution. To confirm and/or refine this understanding of iodine releases from the dissolver, a mathematical model of the RaLa process was developed. The model, which was specified by Orlan Yarbro, addresses flows between the RaLa dissolver, condenser, reactor (an air mixer), and caustic scrubber as depicted in Figure 3.4.

For this assessment, the RaLa plant model was used to estimate flow rates of elemental iodine from the dissolver condenser for a number of cases that span the range of dissolving rates that were experienced in processing of X-10 and Hanford fuel slugs. Based on plant model results for these cases, a set of correlation equations were developed that allow one to estimate the elemental iodine release rate from the condenser for a RaLa dissolving batch based on the rate at which uranium was dissolved and the iodine content of the dissolved uranium slugs. These correlation equations are then used for the remainder of the 733 dissolving batches to estimate iodine release rates from the condenser based on the dissolution rate and iodine content data that can readily be determined or estimated for each dissolving batch. Use of this approach, which is described in more detail in the text that follows, eliminates the need to run the plant model for each of the 733 individual dissolving batches.

\section{$\underline{\text { Reactions of the RaLa Dissolver }}$}

The dissolution of metallic uranium by nitric acid produced a series of species which constituted the flow rates that are modeled.

$$
\mathrm{U}+6 \mathrm{HNO}_{3} " \mathrm{UO}_{2}\left(\mathrm{NO}_{3}\right)_{2}+3 \mathrm{H}_{2} \mathrm{O}+\mathrm{NO}+3 \mathrm{NO}_{2}
$$

The formation of $\mathrm{NO}$ and $\mathrm{NO}_{2}$ is based on the above reaction. The rate of production is based on the stoichiometric relationship and the rate at which the uranium was dissolved. The water flow rate is based on the fraction of water in the gas over the dissolver solution. The mass of air into the dissolver is based on inleakage and on the flow of air from the sparger. The sparger was only operational during the last 15 minutes of the dissolution cycle and was not a contributor to the flow of air into the dissolver. 
Figure 3.4: RaLa Process Components Included in the Plant Model

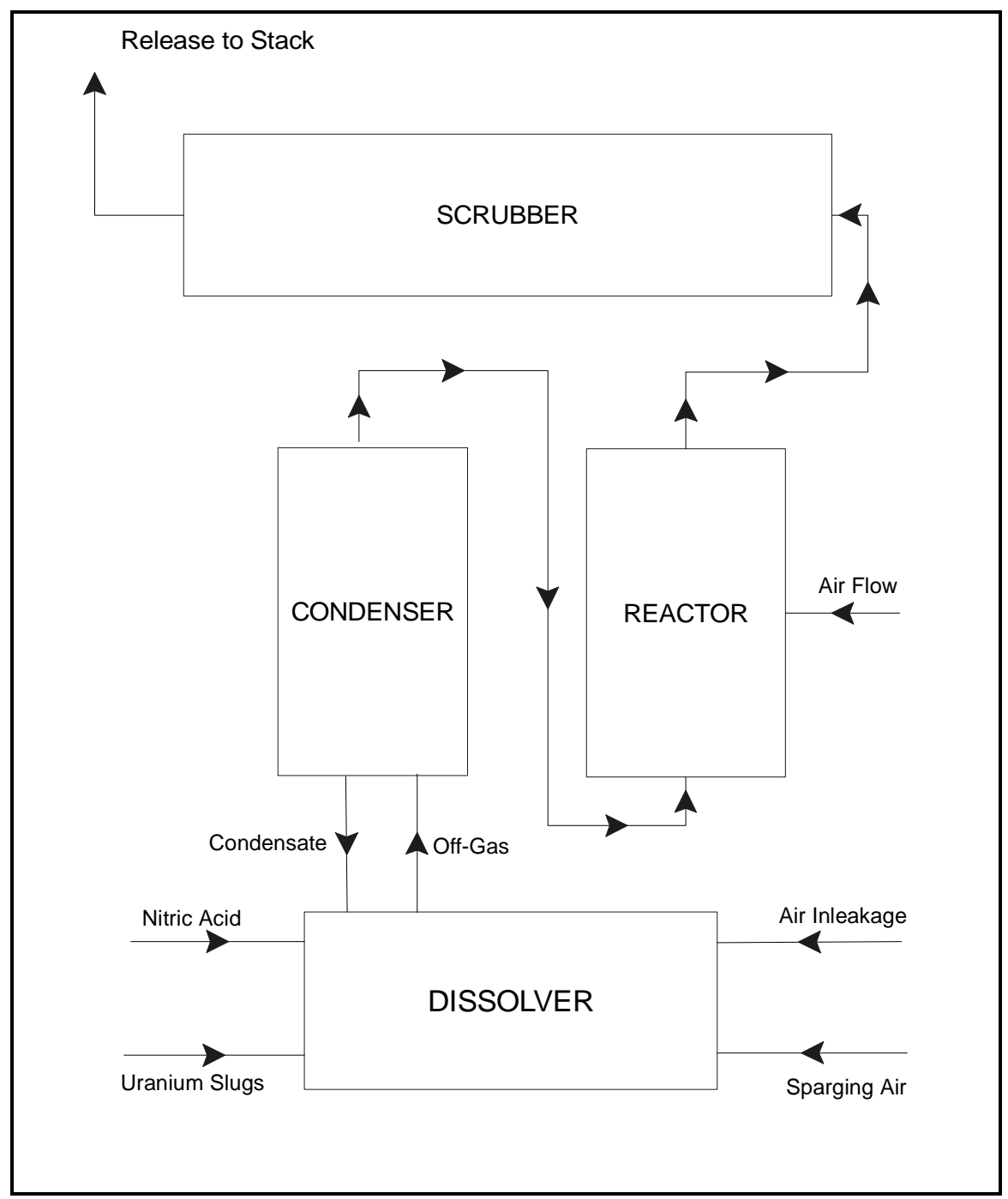

The dissolution of uranium in nitric acid led to the release of the fission products, including ${ }^{131} \mathrm{I}$, from the interstitial voids in the metal. The release of iodine is directly a function of the rate at which the metal was dissolved. The elemental iodine flow rate is based on the fraction of iodine in the dissolver solution as a function of total mass in the dissolver. This fraction is multiplied by the total mass flow exiting the dissolver into the condenser to generate an iodine mass flow rate $(\mathrm{kg} / \mathrm{min})$. Both the total iodine and total mass of the solution are calculated as a function of time; as the uranium slugs dissolved, the mass of elemental iodine increased. However, volatile iodine was also being removed as part of the flow of gases from the dissolver to the condenser. In addition, the condensate from the condenser, which consisted of acid, iodine, water and $\mathrm{NO}_{2}$, provided a positive term to the total mass balance in the dissolver. The concentration of iodine in the dissolver is given by: 


$$
C_{I} \text { (dissolver) }{ }^{\prime} \frac{\left.M_{I} \text { (dissolver }\right)}{\left.M_{T} \text { (dissolver }\right)}
$$

where:

$$
\begin{array}{ll}
\mathrm{C}_{\mathrm{I}}(\text { dissolver }) & =\mathrm{kg} \text { of iodine per } \mathrm{kg} \text { of dissolver solution } \\
\left.\mathrm{M}_{\mathrm{I}} \text { (dissolver }\right) & =\mathrm{kg} \text { iodine in dissolver solution } \\
\mathrm{M}_{\mathrm{T}}(\text { dissolver }) & =\text { total mass of solution in dissolver }(\mathrm{kg}) .
\end{array}
$$

The concentration of iodine in the gas stream leaving the dissolver is given by:

$$
C_{I} \text { (dissolver to condenser) } ' \frac{C_{I} \text { (dissolver) }}{f_{I} \text { (dissolver) }}
$$

where:

$$
\begin{array}{lll}
\mathrm{C}_{\mathrm{I}} \text { (dissolver to condenser) } & = & \begin{array}{l}
\text { concentration of iodine in gas flow from dissolver to } \\
\text { condenser }
\end{array} \\
\mathrm{C}_{\mathrm{I}} \text { (dissolver) } & = & \begin{array}{l}
\mathrm{kg} \text { of iodine per } \mathrm{kg} \text { of dissolver solution } \\
\mathrm{fg} \text { of iodine in gas stream per } \mathrm{kg} \text { of iodine in dissolver } \\
\text { folution }
\end{array}
\end{array}
$$

The mass of iodine in the gas flow from dissolver to condenser can then be evaluated from the above two equations to give:

$$
C_{I} \text { (dissolver to condenser) }{ }^{\prime} \frac{\left.M_{I} \text { (dissolver }\right)}{\left.M_{T} \text { (dissolver }\right)} \times \frac{1}{\left.f_{I} \text { (dissolver }\right)}
$$

The iodine mass flow rate is the concentration of iodine in the gas stream from dissolver to condenser times the mass flow rate of the gas stream:

$$
\left.M_{I} \text { (dissolver to condenser) ' } C_{I} \text { (dissolver to condenser }\right) \times M_{G A S} \text { (dissolver to condenser) }
$$

where:

$$
\begin{array}{lll}
\mathrm{M}_{\mathrm{I}} \text { (dissolver to condenser) } & = & \text { mass flow of iodine from dissolver to condenser } \\
\mathrm{C}_{\mathrm{I}} \text { (dissolver to condenser) } & = & \begin{array}{l}
\text { concentration of iodine in gas flow from dissolver to } \\
\text { condenser }
\end{array} \\
\mathrm{M}_{\mathrm{GAS}} \text { (dissolver to condenser) } & = & \text { flow rate of total gas from dissolver to condenser }
\end{array}
$$


The total gas flow rate $\left(\mathrm{M}_{\mathrm{GAS}}\right)$ is calculated from the sum of the mass flow rates from dissolver to condenser for air, $\mathrm{NO}, \mathrm{NO}_{2}$, acid and water. The resulting equation for the mass flow rate of iodine from dissolver to condenser is given as follows:

$$
\left.M_{I} \text { (dissolver to condenser }\right)=\frac{\left.M_{I} \text { (dissolver }\right)}{M_{T}(\text { dissolver })} \times \frac{\left.M_{G A S} \text { (dissolver to condenser }\right)}{\left.f_{I} \text { (dissolver }\right)}
$$

Both the mass of iodine in dissolver solution $M_{I}$ (dissolver) and the total mass of solution in the dissolver $M_{T}$ (dissolver) are functions of time. $M_{I}$ (dissolver) is a function of the production of iodine from uranium dissolution, the removal of iodine by the flow of gases from the dissolver to the condenser, and the flow of condensed iodine from the condenser back to the dissolver. Similarly, $M_{T}($ dissolver $)$ is a function of the total mass of material being produced by uranium dissolution, removed by flow to the condenser and the return of condensate from the condenser. As the total mass and the mass of iodine in the dissolver varies with respect to time, these two variables are solved using a time-step solution which generates values for both variables at the end of each time step. A one-minute time step was employed; an assessment of the sensitivity of the flow rates with respect to the length of time step showed no improvement in the accuracy of the result when shorter time steps were used.

\section{$\underline{\text { Reactions of the RaLa Dissolver Condenser }}$}

The reflux condenser was used to reduce the release of nitric acid vapors. The reaction and conversion of NO and $\mathrm{NO}_{2}$ are modeled as part of the condenser:

$$
\begin{array}{ll}
2 \mathrm{NO}+\mathrm{O}_{2} & \multicolumn{2}{l}{2 \mathrm{NO}_{2}} \\
3 \mathrm{NO}_{2}+\mathrm{H}_{2} \mathrm{O} & 2 \mathrm{HNO}_{3}+\mathrm{NO}
\end{array}
$$

The mass of $\mathrm{NO}$ and $\mathrm{NO}_{2}$ in the condenser is a function of the rate at which both species are converted. The mass of $\mathrm{NO}$ decreases as $\mathrm{NO}$ is converted to $\mathrm{NO}_{2}$; and the mass of $\mathrm{NO}_{2}$ converted to $\mathrm{HNO}_{3}$.

\section{$\underline{\text { Reaction of the RaLa "Reactor" }}$}

The reactor was used to mix air with NO to convert nitric oxide to nitrogen dioxide.

$$
2 \mathrm{NO}+\mathrm{O}_{2} " 2 \mathrm{NO}_{2}
$$




\section{$\underline{\text { Reactions of the Caustic Scrubber }}$}

The final component is the scrubber, which was used to remove elemental iodine from the dissolver off-gas. In addition, the following reactions in the scrubber are modeled:

$$
\begin{aligned}
& 2 \mathrm{NO}+\mathrm{O}_{2} " 2 \mathrm{NO}_{2} \\
& 3 \mathrm{NO}_{2}+2 \mathrm{NaOH} " 2 \mathrm{NaNO}_{3}+\mathrm{NO}+\mathrm{H}_{2} \mathrm{O} \\
& \mathrm{HNO}_{3}+\mathrm{NaOH} " \mathrm{NaNO}_{3}+\mathrm{H}_{2} \mathrm{O}
\end{aligned}
$$

Based on the reactions outline above, a RaLa process model was developed that uses a combination of input data specific to each run and information based on literature values and professional opinions. The model produces values of flow rates for each process component for all the species of gases discussed above, with values generated at the end of each time-step. As discussed in the beginning of this section, the RaLa plant model was used in this assessment to estimate flow rates of elemental iodine from the dissolver condenser for a number of cases that span the range of dissolving rates that were experienced in processing of X-10 and Hanford fuel slugs. Two correlation equations were developed that allow one to estimate the elemental iodine release rate from the condenser for each RaLa dissolving batch based on the rate at which uranium was dissolved and the iodine content of the dissolved uranium slugs. The calculated release rates for each batch are then used to estimate releases of elemental, organic, and particulate iodine to Building 706-D and through the X-10 stacks, taking into account estimated occurrences of direct releases, removal in the caustic scrubber (handled separately from the plant model in this assessment), and removal in the exhaust path between the scrubber and the stack in use at the time.

Table 3.12 presents the plant model input parameters for the RaLa dissolver and dissolver condenser, the parameters that are relevant to the model as used in this assessment.

Nine cases were selected to represent the combinations of dissolution rate and iodine fraction for RaLa runs that used X-10 slugs. Similarly, eight cases were used to evaluate a correlation for the Hanford 8" slugs. For each case, the RaLa plant model was executed with the specified values of dissolution rate and iodine fraction; dissolution times were based on reported or estimated batch durations. Flow rates for each species in the plant model, including iodine, were estimated at the end of each dissolution run. The flow rates for most species were constant with respect to time. The major exceptions were flow rates for total iodine, which are a function of the reflux flow in the condenser. The iodine flow rate approaches an equilibrium value after the first hour of dissolving, and changes to the flow rate after that are minimal. For this assessment, the maximum flow rate was determined to be at the end of the dissolution.

Values for uranium dissolution rate range from 0.15 to $0.35 \mathrm{~kg} / \mathrm{min}$ and the iodine-to-uranium ratio (the iodine fraction) ranged from $1.5 \times 10^{-7}$ to $3.0 \times 10^{-7} \mathrm{~kg} \mathrm{I} / \mathrm{kg} \mathrm{U}$ for the X-10 runs. For eight-inch Hanford slugs, the dissolution rate ranged from $0.08 \mathrm{~kg} / \mathrm{min}$ to $0.23 \mathrm{~kg} / \mathrm{min}$; the lower dissolution rates are reflective of the availability of lower surface areas per unit mass of uranium. The iodine fraction was higher for the Hanford slugs, with a range from $1.8 \times 10^{-6}$ to $5.5 \times 10^{-6} \mathrm{~kg} \mathrm{I} / \mathrm{kg} \mathrm{U}$. 
Table 3.12: RaLa Plant Model Input Parameters for the Dissolver and Dissolver Condenser

\begin{tabular}{|c|c|c|c|}
\hline Parameter & Description & Value & Basis \\
\hline NSLUGS & Number of slugs dissolved. & Varies by batch. & From analytical reports or other operational records; see Section 3.4.2 \\
\hline DTIME & Length of time step (min) & $1 \mathrm{~min}$. & Model performs well at this setting; results are not sensitive to this setting. \\
\hline TTIME & Total dissolution run time (min) & Varies by batch. & From operations log books or other records; see Section 3.4.3. \\
\hline FITA1 & Iodine Fraction (kg total I / kg U) & Varies by run. & From ORIGEN calculations; see Section 3.5.1 and 3.5.2. \\
\hline FICA1 & Curies of I-131 per kg uranium & Varies by run. & From ORIGEN calculations; see Section 3.5.1 and 3.5.2. \\
\hline RUSA1 & Uranium dissolution rate & Varies by batch. & Calculated as mass of slugs dissolved divided by duration of dissolving. \\
\hline FISA1 & Fraction nonvolatile I in dissolver & 0.02 & Based on expert opinion (Yarbro, 1996). \\
\hline FIMA1 & Fraction organic iodine in dissolver & 0.01 & Based on expert opinion (Yarbro, 1996). \\
\hline MAA102 & Inleakage air mass flow $(\mathrm{kg} / \mathrm{min})$ & 0.35 & Per O. Yarbro, professional judgement. \\
\hline MAA103 & Sparger air mass flow (kg/min) & 0.02 & Per O. Yarbro, typical sparge rates are $0.25 \mathrm{CFM}$ per sq $\mathrm{ft}$ tank cross-section. \\
\hline MTA104 & Solution added to dissolver $(\mathrm{kg})$ & 180.5 & From descriptions of the RaLa chemical separation procedure. \\
\hline MUA105 & Mass of slugs dissolved (kg) & Varies by batch. & Based on number of slugs times $1.175,1.782$, or $3.564 \mathrm{~kg}(\mathrm{X}, 4 " \mathrm{~W}, 8$ "W) \\
\hline FHA101 & $\begin{array}{l}\text { ( } \mathrm{kg} \text { acid }) /(\mathrm{kg} \text { vapor) in vapor leaving } \\
\text { dissolver. }\end{array}$ & 0.01 & Per O. Yarbro, professional judgement (Yarbro, 1996). \\
\hline FWA101 & (kg water)/(kg gas) leaving dissolver & 0.2 & Per O. Yarbro, professional judgement (Yarbro, 1996). \\
\hline FIA101 & ( $\mathrm{kg}$ iodine in liquid $) /(\mathrm{kg}$ iodine in vapor) & 0.16 & Per O. Yarbro (Yarbro, 1996; Unger et al.,1970; Morgan and Holland, 1979). \\
\hline STIME & Sparger on time (min) & 15 (after dissolution) & From descriptions of the RaLa chemical separation procedure. \\
\hline FNOA2R & Fraction of $\mathrm{NO}$ reacting to $\mathrm{NO} 2$ & 0.2 & Per O. Yarbro, professional judgement (Yarbro, 1996). \\
\hline FNO2A2R & Fraction of $\mathrm{NO} 2$ reacting to $\mathrm{HNO} 3$ & 0.5 & Per O. Yarbro, professional judgement (Yarbro, 1996). \\
\hline FHA202 & $\begin{array}{l}(\mathrm{kg} \text { acid }) /(\mathrm{kg} \text { water }) \text { in condensate leaving } \\
\text { condenser }\end{array}$ & 0.006 & Per O. Yarbro, professional judgement (Yarbro, 1996). \\
\hline FWA201 & $\begin{array}{l}\text { ( } \mathrm{kg} \text { water }) /(\mathrm{kg} \text { gas) in vapor leaving } \\
\text { condenser }\end{array}$ & 0.015 & Per O. Yarbro, professional judgement (Yarbro, 1996). \\
\hline FIA2 & $\begin{array}{l}\text { Ratio of fraction of iodine leaving the } \\
\text { condenser in condensate to the that in off- } \\
\text { gas. }\end{array}$ & 0.9 & Per O. Yarbro, professional judgement (Yarbro, 1996). \\
\hline
\end{tabular}


Using results from the Plant Model runs for X-10 slugs, there was not a good fit between the calculated elemental iodine flow rate and either the estimated uranium dissolution rate or the iodine fraction. However, by plotting the elemental iodine flow rate from the condenser (calculated using the plant model) as a function of the product of dissolution rate and iodine fraction (determined from available RaLa run data and ORIGEN calculations), a very good linear approximation could be made. Figure 3.5 shows a plot of the data and the linear approximation. The correlation is well established, with no visible bias, and therefore it was possible to estimate elemental iodine flow rates from the condenser for each dissolving batch based on the following relationship:

$$
\text { Elem. I Flow Rate }(\mathrm{kg} / \mathrm{min})=0.9717 \times(\text { Diss. Rate } \times \text { I Fract. })-7.0 \times 10^{-11}
$$

Where: $\quad$ Diss. Rate $=\quad$ Uranium dissolution rate $(\mathrm{kg} / \mathrm{min})$, and I Fract. = Iodine-to-Uranium Mass Ratio $(\mathrm{kg} / \mathrm{kg})$.

This same relationship was used to estimate evolution of radioiodine from the dissolution of four-inch Hanford slugs, which were quite similar to X-10 slugs. Essentially the same process and methodology was used to determine a relationship for iodine flow from the condenser for the eight-inch Hanford slugs. Complete data were available for Runs 52, 53, 54, 55, 56, 57, 59, 60, and 62; the data set includes numbers of effective slugs dissolved per run as well as a complete dissolution chronology. By executing the RaLa plant model with these data, a similar correlation between calculated elemental iodine flow rates and (dissolution rate $\times$ iodine fraction) was determined. Using this correlation, which is represented in Figure 3.6 along with the plant model results, it was possible to determine the elemental iodine flow rate from the RaLa condenser for each dissolving batch that involved the eight-inch Hanford slugs.

The correlation between (dissolution rate $\times$ iodine fraction) and flow rates of elemental iodine from the condenser for eight-inch Hanford slugs is:

Elem. I Flow Rate $(\mathrm{kg} / \mathrm{min})=0.9657 \times($ Diss. Rate $\times$ I Fract. $)+1 \times 10^{-9}$

Four-inch Hanford slugs were quite similar to the 4-inch X-10 slugs in their construction, other than for differences in methods used to bond the aluminum coats to the slugs, which should not have affected iodine production in the slugs. Because the 4-inch Hanford slugs likely behaved more like the 4-inch X-10 slugs in the dissolver than like 8-inch Hanford slugs (e.g., from surface area considerations), the correlation equation for 4-inch X-10 slugs is used (with iodine fractions and dissolution rates determined for the appropriate Hanford slugs) to estimate iodine release rates from the RaLa condenser for runs that used 4-inch Hanford slugs. These were Runs 28-42, 44-51, and 54. For Run 54, the only run that used both 4-inch and 8-inch Hanford slugs, the correlation equation for the 4-inch X-10 slugs is used. The correlation coefficients (0.9717 and 0.9657) are close enough that the impact in using one correlation equation over the other are minimal; for Batches 54-A through 54-I, iodine release rates calculated using the correlation equation for the 4-inch X-10 slugs range from $0.24 \%$ to $0.47 \%$ higher than values calculated using the equation for the 8-inch Hanford slugs. 


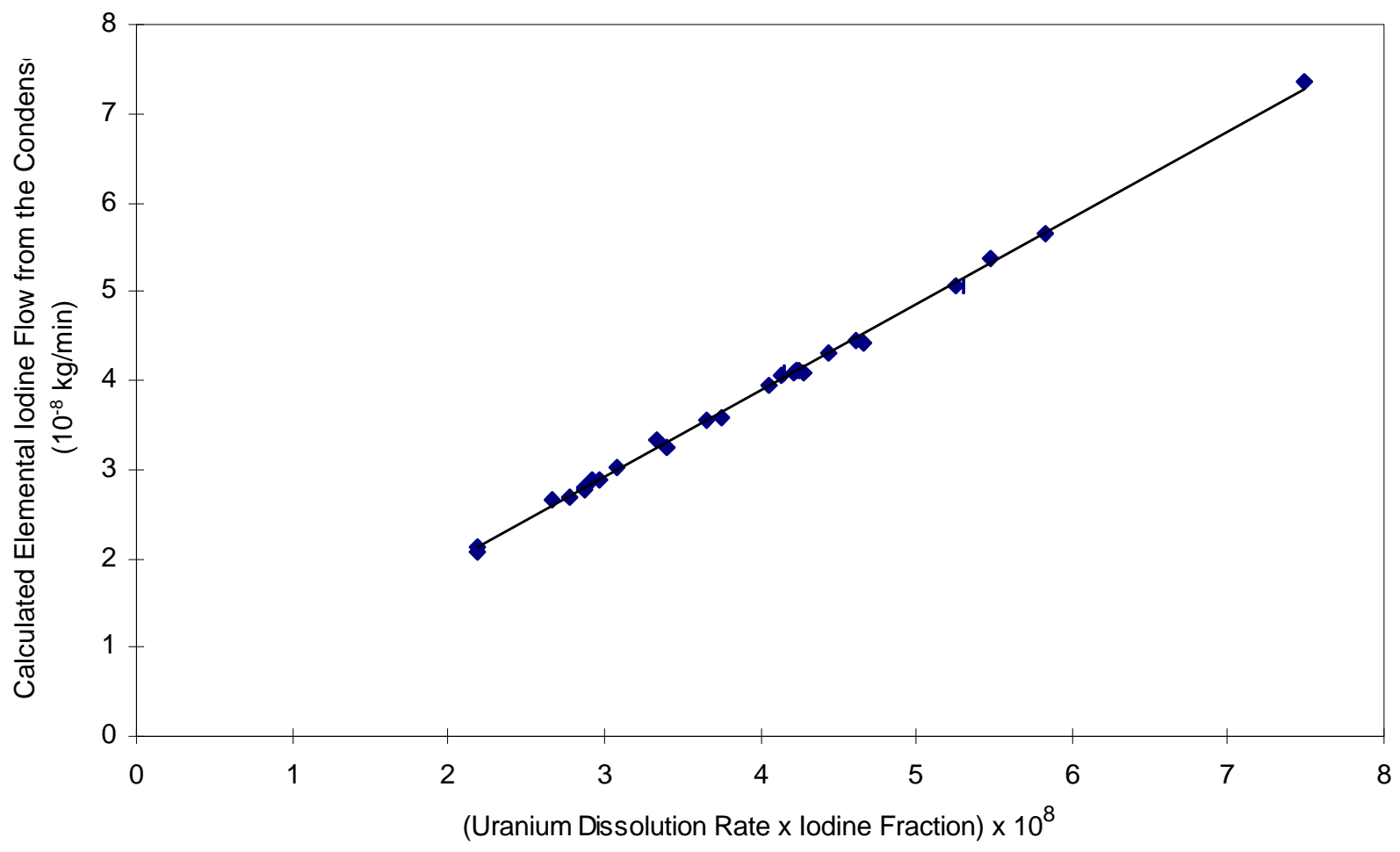

Figure 3.5: Elemental Iodine Flow Rate from the RaLa Condenser as a Function of the Product of Uranium Dissolution Rate $\times$ Iodine Fraction (4-inch X-10 Slugs) 
TASK 1 REPORT

July 1999

${ }^{131}$ I Releases from X-10 Radioactive Lanthanum Processing-

Page 3-48

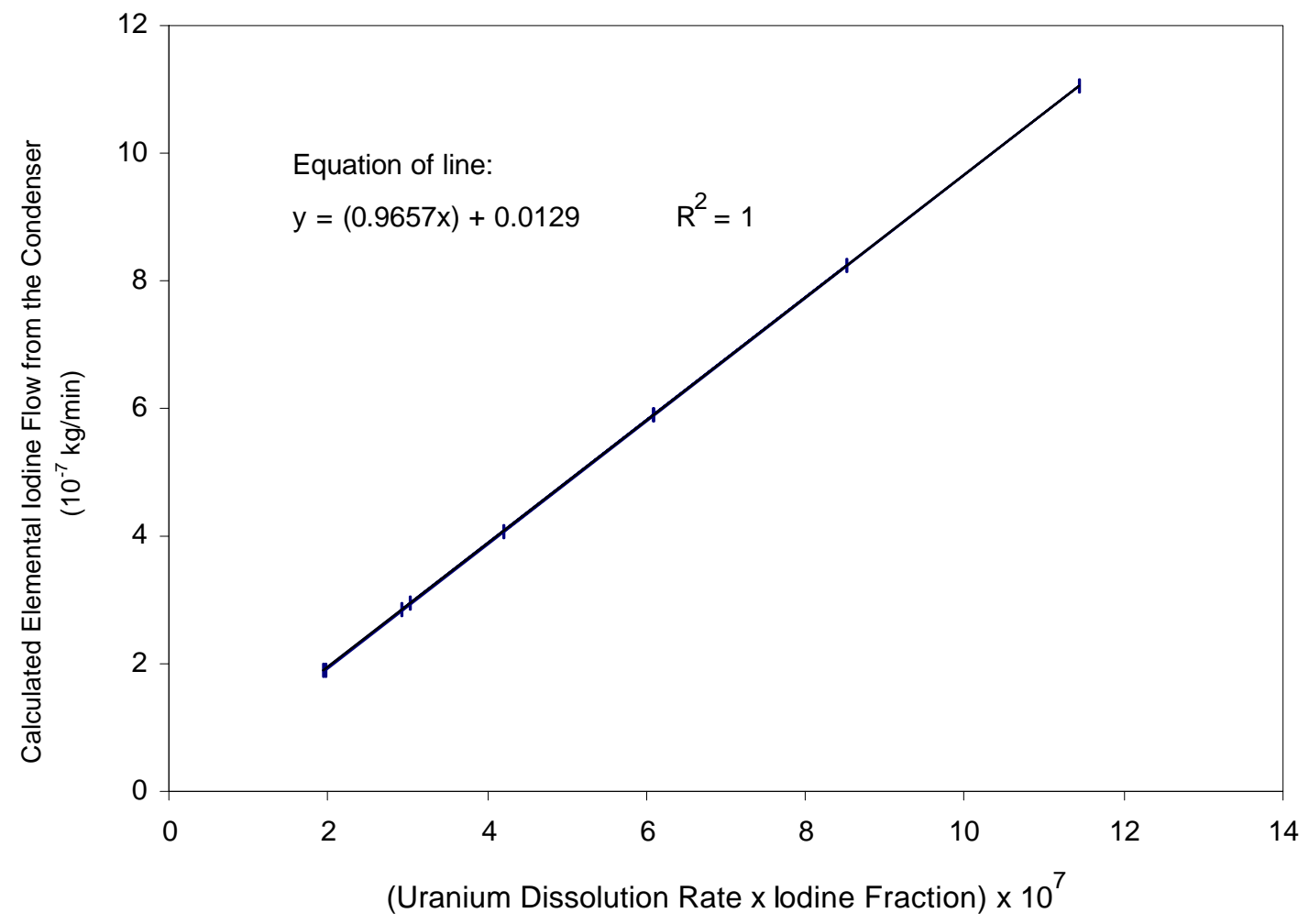

Figure 3.6: Elemental Iodine Flow Rate from the RaLa Condenser as a Function of the Product of Uranium Dissolution Rate $\times$ Iodine Fraction (8-inch Hanford Slugs) 


\subsection{Estimation of the Magnitude of Direct Releases}

As described in Section 3.4.3, each RaLa dissolving batch was classified by the project team as to its relative potential for releases of iodine to the environment from pathways that bypassed the caustic scrubber and the normal path to an elevated stack. This classification was based largely on review of Chemical Separations Operations log books. For each "direct release class," a portion of the radioiodine estimated to have left the dissolver condenser is assumed to have bypassed the caustic scrubber and the normal release path to the stack. These portions, specified as ranges of percentages entered as loguniform distributions, are based on professional judgement of the project team and the experts who were consulted after extensive review of RaLa process documentation. The percentages of direct release that are assumed, with statements of their basis, are shown in Table 3.13.

For each of the 400 realizations of the Monte Carlo simulation performed to estimate iodine releases from each RaLa batch, a value is sampled from the "Percentage Direct Release" distribution for the class that the batch was assigned. For that realization of the simulation, the sampled percentage of the calculated iodine flow from the dissolver condenser is considered to be released without being diminished by the caustic scrubber or by capture along the exhaust path to an elevated stack. The remainder of the iodine flow from the condenser has portions of contained iodine removed in the scrubber and in the exhaust path, as described in Sections 3.10 and 3.11 .

Releases by each pathway (from the building and from the stack) are calculated separately and are in turn totaled separately for each calendar year and for each hour of the accident during Run 56 in 1954. When airborne contamination was encountered in Building 706-D, it was common practice to open the building's windows until levels went back down again; in some cases, portable fans were placed in the windows to speed up the purging of contaminated air. In contrast to releases that occurred via the normal path from the dissolver off-gas system to an elevated stack, the direct releases to Building 706 are treated as ground-level releases, and no account is taken for capture in the building itself.

For dissolving batches in Class 4, the records that have been located by the project team provide insufficient information upon which to judge the extent to which direct releases were experienced. For each Class 4 batch, the percent direct release is sampled from a distribution prepared as described below, based on the prevalences of Classes 1 through 3 in those batches that had operations logs available. This method affects calendar years 1946, 1948, and 1954, the year that contained Class 4 batches. Steps used to specify a weighted distribution of percentages of direct release for Class 4 batches are as follows:

a. $\quad$ Subjective weighted probability is assigned to each possible direct release class other than Class 4 (i.e., 1,2,3). The probability of each option is based on the batches for which log books are available. For 1946 and 1948, Class 1 has a probability of $326 \div 364=0.8956$, Class 2 has a probability of $32 \div 363$ $=0.0879$, and Class 3 has a probability of $6 \div 364=0.0165$. These probabilities were based on $\log$ book data from the 364 Building 706-D RaLa batches from 1945 through 1949. 
Table 3.13: Direct Release Percentages Assumed for each Batch Class

\begin{tabular}{|c|c|c|}
\hline $\begin{array}{l}\text { Direct } \\
\text { Release } \\
\text { Class } \\
\end{array}$ & Criteria for Selection & $\begin{array}{c}\text { Assumed Percent Direct Release } \\
\text { (loguniform distributions between } \\
\text { stated values) } \\
\end{array}$ \\
\hline 1 & $\begin{array}{l}\text { Operations and/or Health Physics log } \\
\text { books are available, and provide no } \\
\text { evidence of significant off-gas leakage } \\
\text { beyond that of normal operations. }\end{array}$ & $\begin{array}{l}\qquad 0.001 \text { to } 0.1 \% \\
\text { Rationale: This level of occurrence of direct releases is } \\
\text { believed to be representative of the leakage that would } \\
\text { have resulted from normal operations. For example, } \\
\text { leakage occurred during routine collection of samples of } \\
\text { process fluids, products, and wastes, and when process } \\
\text { fluids and gases backed up instrument lines to } \\
\text { operating areas. Larson (1954) stated that "back-ups } \\
\text { into exterior lines and contamination from sampling and } \\
\text { product removal are frequent." }\end{array}$ \\
\hline 2 & $\begin{array}{l}\text { Available records provide evidence of } \\
\text { conditions conducive to leakage of } \\
\text { process off-gas (such as } \\
\text { documentation of the inability to } \\
\text { maintain sufficient negative pressure } \\
\text { on the dissolver or airborne } \\
\text { contamination in the building). }\end{array}$ & $\begin{array}{l}\qquad 0.01 \text { to } 1 \% \\
\text { Rationale: This class represents an intermediate level of } \\
\text { direct release, between that of Class } 1 \text { and Class } 3 \text {. }\end{array}$ \\
\hline 3 & $\begin{array}{l}\text { Available records provide evidence of } \\
\text { significant leakage of radioactive } \\
\text { materials from the RaLa processing } \\
\text { equipment (such as the need for } \\
\text { operators to evacuate the building or } \\
\text { wear respirators in the course of their } \\
\text { normal duties). }\end{array}$ & $\begin{array}{l}\qquad 1 \text { to } 10 \% \\
\text { Rationale: The criteria for this class are clearly } \\
\text { indicative of process leakage or malfunction. } \\
\text { Regarding the upper bound, it is believed that direct } \\
\text { release of more than 10\% of dissolver off-gas contents } \\
\text { would have resulted in major consequences within the } \\
\text { building and across the X-10 site that would have likely } \\
\text { been widely documented (as was the Run } 56 \text { accident in } \\
\text { 1954). }\end{array}$ \\
\hline
\end{tabular}

b. If option 1 in selected in an iteration, the percent direct release distribution for Class 1 is sampled (0.001 to $0.1 \%$ loguniform). If option 2 is selected, the percent direct release distribution for Class 2 is sampled (0.01 to $1 \%$ ). If option 3 in selected, the percent direct release distribution for Class 3 is sampled (1 to 10\%). In each iteration, the options that are not selected are assigned a value of zero, and a forecast cell is assigned the sum of options 1 through 3. Across 400 realizations of Monte Carlo simulation, the contributions of the three options are summed, resulting in weighted distributions of possible percentages of direct release for Class 4 batches, based on the prevalences of Classes 1-3 in those batches with logs. 
c. The same process was used for 1954 , with the probability of option 1 being $143 \div 151$, the probability of option 2 being $8 \div 151$. There was no third option in this case, because over 1950-1956 there were no Class 3 batches among the 151 batches with log books.

\subsection{Iodine Removal in the Caustic Scrubber}

Because the performance of the caustic scrubber is a critical consideration in this assessment of releases of iodine from RaLa dissolving batches, a number of different approaches and information sources were utilized. They included elicitation of expert opinion, review of the limited amount of monitoring data that were collected during RaLa runs, and consideration of a mathematical model of the performance of the scrubber.

\subsubsection{Initial Expert Opinion}

As discussed in Section 3.7.1, a number of experts in the fields of nuclear material processing, iodine behavior, and chemical engineering were consulted for their professional opinions regarding behavior of radioiodine in the RaLa process and the caustic scrubber. The summary of the meeting stated that, in the caustic scrubber, iodine capture efficiencies would be expected to be approximately $99 \%$ for elemental, 1 to $10 \%$ for volatile organic, and 1 to $10 \%$ for particulate. These efficiencies were used in the preliminary Task 1 assessment of

${ }^{131}$ I releases from X-10 radioactive lanthanum processing (Hoffman et al., 1996).

\subsubsection{Mathematical Modeling of the Caustic Scrubber}

Subsequent to completion of the preliminary Task 1 assessment, and as recommended in the preliminary Task 1 report, a mathematical model was developed and applied to describe the performance of the caustic scrubber in collecting elemental iodine in the presence of oxides of nitrogen (NOx). Since no $\mathrm{NO}_{\mathrm{x}}-\mathrm{HNO}_{\mathrm{x}}$ -iodine interactions occur, the overall model was split into two sequential parts to simplify model development. First, the $\mathrm{NO}_{\mathrm{x}}$ scrubbing portion was modeled to find the $\mathrm{H}^{+}$distribution in the column. Then this concentration profile was used in modeling the iodine distribution in the column. Since the liquid $\mathrm{I}_{2}$ is dilute relative to the $\mathrm{H}^{+}$ concentration, the reactions between $\mathrm{H}^{+}$and $\mathrm{I}_{2}$ did not appreciably affect the $\mathrm{NO}_{\mathrm{x}}$ scrubbing model.

This model for elemental iodine removal in the presence of oxides of nitrogen used a rate based approach, based on the following known information:

- $\quad$ The composition and flow rate of the gas into the bottom of the scrubber (from the RaLa plant model described in Section 3.8);

- The composition and flow rate of the liquid into the top of the scrubber (from descriptions of the RaLa chemical separation process and procedures);

- $\quad$ The hydrogen concentration $\left(\mathrm{H}^{+}\right)$throughout the scrubber (calculated from NOx scrubber model); 
- Equilibrium constants that describe partitioning of elemental iodine between liquid and vapor phases, and between dissolved $\mathrm{I}_{2}$ and the following dissolved species:

$\mathrm{I}_{3}^{-}, \mathrm{H}^{+}+\mathrm{I}^{-}+\mathrm{HIO}$, and $\mathrm{H}_{2} \mathrm{OI}^{+}+\mathrm{I}^{-}$; and

- $\quad$ All gas and liquid flow rates throughout the column (from the NOx scrubber model).

The organic iodine species were assumed to pass through the scrubber unchanged. The general procedure of the model was as follows:

1. The 9 -foot scrubber was divided into $1 / 2$-foot long equilibrium stages.

2. Calculations were started at the bottom stage of the scrubber. The composition and flow rate of the gas into this stage were known from RaLa plant model calculations.

3. A concentration of total soluble $\mathrm{I}_{2}$ in the liquid out of the first stage was guessed. Assuming the stage was well mixed, this was the concentration in the stage also.

4. The following three liquid phase equilibrium reactions were used to solve for the dissolved $\mathrm{I}_{2}$ concentration in the stage.

$$
\begin{array}{lll}
\mathrm{I}_{2}(l)+\mathrm{I}^{-} & <\longmapsto & \mathrm{I}_{3}^{-} \\
\mathrm{I}_{2}(l)+\mathrm{H}_{2} \mathrm{O} & \longleftrightarrow \longleftrightarrow-\mathrm{H}^{+}+\mathrm{I}^{-}+\mathrm{HIO} \\
\mathrm{I}_{2}(l)+\mathrm{H}_{2} \mathrm{O} & \longleftrightarrow \longrightarrow \longrightarrow & \mathrm{H}_{2} \mathrm{OI}^{+}+\mathrm{I}^{-}
\end{array}
$$

5. The vapor liquid equilibrium constant was used to find the gas phase $\mathrm{I}_{2}$ concentration leaving the stage.

$$
\mathrm{I}_{2}(l) \quad<<->\quad \mathrm{I}_{2}(g)
$$

6. An overall material balance was done on the stage to find the liquid phase $\mathrm{I}_{2}$ concentration coming into the stage.

7. Steps 3 through 6 were repeated, working up the column to the top.

8. Once at the top, a check was made to see if the liquid $\mathrm{I}_{2}$ concentration into the top of the scrubber was zero (no $\mathrm{I}_{2}$ was fed to the top of the scrubber). If not, the guess in step 3 was adjusted and the process continued until the $\mathrm{I}_{2}$ in at the top was essentially zero. Then the model was solved, and the $\mathrm{I}_{2}$ leaving the scrubber in the gas phase was the correct value.

Results of application of the scrubber model to nine dissolving batch cases are shown in Table 3.14. 


\subsubsection{Monitoring Study During Two RaLa Runs}

In a study documented by Bradshaw and Cottrell (1954) the RaLa process was monitored during two complete cycles of operation, Runs 52 and 54. During Run 52, Millipore filters and cascade impactors were used to sample in the off-gas duct, the cell ventilation duct, the 50-ft level of the stack, and inside the process building. During Run 54, a bubbler sampler was added to collect iodine at the same locations. Bubbler solution was prepared by dissolving $50 \mathrm{~g}$ of sodium thiosulfate and $5 \mathrm{~g}$ of sodium hydroxide in one liter of water. Each bubbler held 20-30 mL, and sampling periods ranged from 6 to 25 hours with a flow rates of about 1 liter per minute.

Table 3.14: Scrubber Model Inputs and Results for Nine Cases

\begin{tabular}{|c|c|l|c|c|c|}
\hline $\begin{array}{c}\text { Case } \\
\text { Number }\end{array}$ & $\begin{array}{l}\text { Uranium } \\
\text { Dissoluti } \\
\text { on Rate } \\
\text { (kg/min) }\end{array}$ & $\begin{array}{l}\text { Ratio of } \\
\text { Total } \\
\text { Iodine to } \\
\text { Uranium in } \\
\text { Fuel Slugs }\end{array}$ & $\begin{array}{c}\text { Elemental } \\
\text { Iodine Flow } \\
\text { INTO } \\
\text { Scrubber } \\
\text { (kg/min) }\end{array}$ & $\begin{array}{c}\text { Elemental } \\
\text { Iodine Flow } \\
\text { OUT OF } \\
\text { Scrubber } \\
\text { (kg/min) }\end{array}$ & $\begin{array}{c}\text { Calculated } \\
\text { Scrubber } \\
\text { Removal } \\
\text { Efficiency for } \\
\text { Elemental } \\
\text { Iodine }\end{array}$ \\
\hline \hline 1 & 0.25 & $1.50 \times 10^{-7}$ & $3.63 \times 10^{-8}$ & $2.78 \times 10^{-13}$ & $99.9992 \%$ \\
\hline 2 & 0.25 & $2.00 \times 10^{-7}$ & $4.84 \times 10^{-8}$ & $5.32 \times 10^{-13}$ & $99.9989 \%$ \\
\hline 3 & 0.25 & $2.00 \times 10^{-7}$ & $4.84 \times 10^{-8}$ & $4.58 \times 10^{-12}$ & $99.9905 \%$ \\
\hline $\begin{array}{c}\text { water through } \\
\text { scrubber) }\end{array}$ & 0.3 & $2.00 \times 10^{-7}$ & $5.81 \times 10^{-8}$ & $5.27 \times 10^{-13}$ & $99.9991 \%$ \\
\hline 4 & 0.35 & $2.00 \times 10^{-7}$ & $6.78 \times 10^{-8}$ & $5.21 \times 10^{-13}$ & $99.9992 \%$ \\
\hline 5 & 0.2 & $2.00 \times 10^{-7}$ & $3.87 \times 10^{-8}$ & $2.65 \times 10^{-13}$ & $99.9993 \%$ \\
\hline 6 & 0.15 & $2.50 \times 10^{-7}$ & $3.62 \times 10^{-8}$ & $2.58 \times 10^{-13}$ & $99.9993 \%$ \\
\hline 7 & 0.35 & $1.50 \times 10^{-7}$ & $5.09 \times 10^{-8}$ & $5.66 \times 10^{-13}$ & $99.9989 \%$ \\
\hline 8 & 0.30 & $3.00 \times 10^{-7}$ & $8.72 \times 10^{-8}$ & $9.14 \times 10^{-13}$ & $99.9990 \%$ \\
\hline 9 & & & & & \\
\hline
\end{tabular}

At a flow of about 120,000 $\mathrm{ft}^{3} \mathrm{~min}^{-3}$, results of samples from the stack during Run 54 were taken to indicate that about $33 \mathrm{Ci}$ of gaseous ${ }^{131} \mathrm{I}$ was released from the stack during the period of the run. With a calculated radioiodine inventory of Run 54 slugs of about $40,000 \mathrm{Ci}^{1}$, the stack release of $33 \mathrm{Ci}$ corresponds to a release fraction of about 0.1 percent. One factor to keep in mind is that most of the iodine thought to survive passage through the scrubber was likely in the form of organic iodides. If this form was largely unaffected by the caustic

${ }^{1}$ Approximately $650 \mathrm{~kg}$ of uranium was dissolved with an estimated average I-131 content of about $124 \mathrm{Ci} / \mathrm{kg}$ at time of discharge. With about $50 \%$ remaining at the time of dissolving, that would have left about $40,000 \mathrm{Ci}$ available in the dissolver. 
scrubber, it was likely also inefficiently captured by the caustic solution of the bubbler sampler. On reviewing the methods and results of the Bradshaw and Cottrell study, Dr. Hermon Rao made the following points: ${ }^{1}$

- $\quad$ The sodium thiosulfate in the bubbler solution served to reduce the $\mathrm{I}_{2}$ and form NaI, thus he concurred that any iodine species that passed through the caustic scrubber likely passed through the samplers as well.

- The surface area in the scrubber available for removal of iodine was much greater than in the samplers, and so the bubbler samplers would likely have been less efficient than the scrubber for the same mix of species. $^{2}$

- The measurement performed to assess the trapping efficiency of the bubbler samplers using inert iodine vapor was likely invalid given the large difference between iodine vapor and iodine species that would have been present in the dissolver stream.

- $\quad$ The trapping efficiency of the samplers was likely impacted substantially because the flow rate used $(1 \mathrm{~L} / \mathrm{min})$ was so large given the small volume of solution present $(20-30 \mathrm{~mL})$. There is also the possibility that the samplers could have saturated, given that they were collecting stable as well as active fission iodine.

- $\quad$ There were potential problems with some of the analytical methods cited in the Bradshaw and Cottrell study. If aliquots from the samplers were evaporated to dryness for counting, the NaI trapped in solution could have reacted with moisture and converted to volatile HI, which would have been lost.

- In summary, the gaseous iodine samples collected during Run 54 are not indicative of the actual iodine releases that occurred. As further evidence of this, the results from the bubbler samplers, when plotted against time, do not correlate with RaLa processing events such as dissolving or sparging.

The results of particle sampling done during RaLa Run 54 were used to estimate releases of iodine associated with particles during the other RaLa runs. For dissolving batches 54-A through 54-J, elemental iodine release rates from the RaLa condenser $(\mathrm{kg} / \mathrm{min})$ were calculated using the RaLa Plant Model equation for Hanford slugs described in Section 3.8. The distribution of the mean calculated release rates from the 10 batches were used to specify a distribution of release rates for Run 54 as a whole. A second distribution was specified based on the 13 results of gross beta radioactivity analyses of Millipore filter paper samples collected at the 50-foot elevation of the X-10 central stack during Run 54 (Bradshaw and Cottrell, 1954). A ratio to relate release rates of particle-associated iodine-131 to elemental iodine release rates from the RaLa condenser was calculated as follows:

\footnotetext{
${ }^{1}$ Personal communication between Bob Burns of the project team and Dr. Herman Rao, radiochemist, currently with Nuclear Technology Services of Roswell, Georgia. August 1996.

${ }^{2}$ Sodium thiosulfate scrubbers used for sampling of iodine from RaLa operations at the Idaho Chemical Processing plant were calculated to have been $30 \%$ efficient in one study and $50 \%$ efficient in another (Cowser, 1964).
} 


$$
\text { Run } 54 \text { Ratio }=\frac{\text { Conc }_{\text {stack }} \times f_{I-131} \times 120,000 \mathrm{ft}^{3} / \mathrm{min} \times 28,320 \mathrm{~cm}^{3} / \mathrm{ft}^{3} \times 60 \mathrm{~min} / \mathrm{h} \times 1 \times 10^{-15} \mathrm{Ci} / \mathrm{fCi}}{Q_{\text {cond. }} \times\left(1-\frac{\% D R}{100}\right)}
$$

\begin{tabular}{|c|c|c|c|}
\hline where: & Conc $c_{\text {stack }}$ & $=$ & $\begin{array}{l}\text { the gross beta concentration from Millipore filter samples } \\
\text { taken during Run } 54\left(\mathrm{fCi} \mathrm{cm}^{-3}\right) \text { at the } 50 \text { - } \mathrm{ft} \text { elevation of the X- } \\
10 \text { central stack, }\end{array}$ \\
\hline & $f_{I-131}$ & $=$ & $\begin{array}{l}\text { the estimated fraction of gross beta activity collected on the } \\
\text { filters that was from iodine- } 131 \text { [based on Bradshaw and } \\
\text { Cottrell (1954), represented by a triangular distribution } \\
\text { between } 0.79 \text { and } 0.95 \text {, with } 0.86 \text { most likely], }\end{array}$ \\
\hline & $120,000 \mathrm{~cm}^{3} / \mathrm{min}$ & $=$ & the reported flow rate in the stack, \\
\hline & $28,320 \mathrm{~cm}^{3 /} \mathrm{ft}^{3}$ & $=$ & conversion factor for units of volume, \\
\hline & $60 \mathrm{~min} / \mathrm{h}$ & $=$ & conversion factor for units of time, \\
\hline & $1 \times 10^{-15} \mathrm{Ci} / \mathrm{fCi}$ & $=$ & conversion factor for units of radioactivity, \\
\hline & $Q_{\text {stack }}$ & $=$ & $\begin{array}{l}\text { calculated elemental iodine release rate from the RaLa } \\
\text { condenser }(\mathrm{kg} / \mathrm{min}) \text {, and }\end{array}$ \\
\hline & $\% D R$ & $=$ & $\begin{array}{l}\text { The percent direct release sampled from the range for } \\
\text { applicable class of dissolving batch (Class } 1 \text { for all Run } 54 \\
\text { batches). }\end{array}$ \\
\hline
\end{tabular}

For each RaLa dissolving batch, the calculated elemental iodine release rate from the dissolver $\left(\mathrm{kg} \mathrm{min}^{-1}\right)$ is multiplied times the Run 54 Ratio $\left(\mathrm{Ci} \mathrm{h}^{-1} / \mathrm{kg} \mathrm{min}^{-1}\right.$, calculated as described above) to estimate the release rate of particle-associated iodine-131 from the central stack $\left(\mathrm{Ci} \mathrm{h}^{-1}\right)$. For dissolving batches prior to Run 29, it was necessary to correct for the fact that filters were added to the exhaust path after that run, and would have affected particle transmission through the stack during the Run 54 sampling. To account for this change in effluent treatment, the Run 54 ratio used for dissolving batches through Run 28 also included a multiplicative factor that was the ratio of estimated transmission through the exhaust path before the filters were added to transmission after the filters were added. In other words, the correction factor was determined as follows:

$$
C F_{\text {filters }}=\frac{1-\% R_{\text {part, path, 1-28 }}}{1-\% R_{\text {part, path, } 29 \text { on }}}=\frac{\text { transmission before filters added }}{\text { transmission after filters added }}
$$

where: $\quad \% R_{\text {part, path, } 1-28}=\quad$ percent removal of particulate iodine in the exhaust path for RaLa runs through Run 28

(1-10\%, loguniformly distributed). 


$$
\begin{aligned}
\% R_{\text {part, path, } 29 \text { on }}= & \text { percent removal of particulate iodine in the exhaust } \\
& \text { path for RaLa runs after Run } 28(70-99 \%, \\
& \text { uniformly distributed). }
\end{aligned}
$$

\subsubsection{Building 205 Stack Monitoring}

A number of 206 Area Operations weekly reports for 1945 have been found that discuss operation of a Building 205 stack gas monitoring system during RaLa runs. This system was apparently the monitoring system that was installed to monitor off-gas from the Chemical Processing Pilot Plant (Building 205) during the early plutonium production runs (Beall and Adams, 1944; Kanne and Branch, 1944). This system pulled an 0.1 to $0.2 \mathrm{ft}^{3} \mathrm{~min}^{-1}$ off-gas sample through a Raschig ring-filled stripping column, through which sodium hydroxide solution was flowing at a rate of $15-17 \mathrm{~cm}^{3} \mathrm{~min}^{-1}$. The stripper solution flowed through an ionization chamber, from which radiation-induced current was measured and used to calculate the concentration of radioiodine present. Measurement results that were located are as follows (Harris, 1945a-c):

a. Week ending 5/12/45 (Run 8 in Building 706-C) - Reported maximum monitored ${ }^{131}$ I activity leaving the stack was between $8.7 \times 10^{-10}$ and $8.7 \times 10^{-9} \mathrm{Ci} \mathrm{ft}^{-3}$.

b. Week ending 5/19/45 (still Run 8 in Building 706-C) - Reported maximum monitored ${ }^{131}$ I activity leaving the stack was between $1.5 \times 10^{-9}$ and $1.5 \times 10^{-8} \mathrm{Ci} \mathrm{ft}^{-3}$.

c. Week ending 5/26/45 (Run 9 in Building 706-C) - Reported maximum monitored ${ }^{131}$ I activity leaving the stack was between $2.75 \times 10^{-9}$ and $2.75 \times 10^{-8} \mathrm{Ci} \mathrm{ft}^{-3}$.

At stack flow rates reported to be between 40,000 and 50,000 $\mathrm{ft}^{3} \mathrm{~min}^{-1}$ (Kanne and Branch, 1944), the maximum reported ${ }^{131} \mathrm{I}$ concentration corresponds to a release rate of between 0.066 and $0.083 \mathrm{Ci}$ per hour. Using the methodology presented in this report, with the following assumptions, peak release rates were calculated for comparison with these measured values:

- $\quad$ Assumed 208 slugs dissolved in Run 9 (same as in Run 1 in Building 706-D), in batches of 14 slugs at a time.

- $\quad$ Assumed dissolvings were completed in the same shift that they started (i.e., possible durations ranged from 4 to 8 hours) and used the iodine-131 and total iodine inventory distributions calculated for X-10 slugs from Runs 1 through 22.

- $\quad$ Assumed reduced scrubber collection efficiency for elemental iodine due to use of water in scrubber instead of caustic (see Section 3.10.6).

- $\quad$ Results indicate a mean release rate of $11 \mathrm{Ci} / \mathrm{h}$ from the dissolver condenser and $1.3 \mathrm{Ci} / \mathrm{h}$ from the Building 205 stack.

If one accepts that the peak stack monitor reading from the week ending 5/26/45 was a valid indication of the peak release rate of elemental iodine from Run 9 in Building 706-C, and the $11 \mathrm{Ci} / \mathrm{h}$ value calculated in this 
study is an accurate representation of the peak release rate of elemental iodine from the dissolver condenser in Run 9, the ratio of $0.083 \mathrm{Ci} / \mathrm{h}$ (monitored) to $11 \mathrm{Ci} / \mathrm{h}$ (predicted) indicates that $0.75 \%$ of the elemental iodine that entered the dissolver scrubber was measured in the stack. This would represent elemental iodine capture in the scrubber and the exhaust path totaling $99.25 \%$.

If one accepts that the $1.3 \mathrm{Ci} / \mathrm{h}$ value calculated in this study is an accurate representation of the maximum release rate of elemental iodine from the Building $205 \mathrm{stack}$, then the ratio of $0.083 \mathrm{Ci} / \mathrm{h}$ to $1.3 \mathrm{Ci} / \mathrm{h}$ would indicate that the monitoring system measured about $6 \%$ of the elemental iodine that exited the stack.

As in the study by Bradshaw and Cottrell, if organic iodides were largely unaffected by the scrubber, they were likely also inefficiently captured by the scrubbing solution of the monitoring system. As a result, monitoring results likely under-represent actual total radioiodine releases from the Building 205 stack.

\subsubsection{Expert Opinion Revisited}

After completion of the mathematical modeling of the RaLa caustic scrubber, a number of the iodine experts named in Section 3.7 were again consulted in preparation for establishing the approach for reflecting scrubber performance this assessment. Several of the experts consulted view the scrubber modeling results as an affirmation of the fact that caustic scrubbers in theory can be very efficient devices for removal of elemental iodine from contaminated air, as was stated in the initial summary of elicited expert opinion on the subject. But there is widespread belief among the experts that the scrubber model, which indicated $99.999 \%$ removal of elemental iodine by caustic scrubbing and $99.99 \%$ by water scrubbing, predicted unrealistically high capture efficiencies. While the scrubber model likely proper performed its calculations in accordance with the equations on which it was based, the widespread belief is that the model did not fully represent the real-world environment in which the A-4 caustic scrubber was operated, where conditions often varied significantly from design specifications and operations often varied from procedure.

One situation that is mentioned by the experts as evidence that the model over predicted iodine capture is the fact that the scrubber model predicted that essentially all of the elemental iodine entering the scrubber column was removed from the vapor in the first stage of the column. If the scrubber had that much excess capacity, it would not likely have experienced noticeably reduced efficiency when caustic solution flowed at a reduced rate; however, during Batch 59-C, high airborne radioactivity was detected across the central X-10 site, and it was reported that "the air activity was the result of changing the flow of water through the caustic scrubber air filtration." According to Fred Weber, this should only have occurred if the flow rate dropped so low that the packing of the column was no longer being effectively wetted.

Orlan Yarbro and Bill Burch state their belief that one should not assume any values for collection efficiency above $99 \%$, and recommend that the lower bound of the range of assumed elemental iodine collection efficiencies be raised above $95 \%$, based on the monitoring data described above and the results of the scrubber modeling. 
Cal Heeb agrees that capture efficiencies above $99 \%$ should not be assumed. Leland Burger states his belief that a range of about 97 to $99.5 \%$ (or just 99\%) for caustic scrubbing and 50 to $70 \%$ for water scrubbing are more realistic than the higher values obtained from the scrubber modeling. Burger bases his opinions on experience with caustic scrubbing from Windscale, La Hague, Tokai Mura, and Hanford. While Yarbro and Burch believe that efficiency with water in scrubber would be greater than $90 \%$, Hermon Rao expects that the elemental iodine collection efficiency would drop from 98 to $99 \%$ with caustic scrubbing to approximately $95 \%$ with water scrubbing.

\subsubsection{Experience with Scrubbers at Other Sites}

Because of the paucity of measurements at X-10 relevant to the efficiency of the RaLa caustic scrubber, an effort was undertaken to identify relevant experiences and measurements at other sites of comparable nuclear material processing campaigns. Relevant findings are summarized below.

- $\quad$ Caustic scrubbers had been used at the Idaho Chemical Processing Plant (ICPP) for several years by 1960, with estimated efficiency of $95 \%$ for iodine removal (Browning, 1960).

- $\quad$ Tests of a caustic scrubber designed for removing iodine from the containment vessel of the Oak Ridge Research Reactor showed the 5\% NaOH solution used to be $99 \%$ efficient (Browning, 1960).

- The ICPP RaLa caustic scrubber initially used one molar $\mathrm{NaOH}$ solution, which was recirculated at $8 \mathrm{gpm}$. After iodine releases were found to be unacceptably high, the scrubber solution was modified by adding 0.1 molar sodium thiosulfate. This change increased the iodine removal efficiency of the scrubber from 90 percent to 97 percent (Cederberg and MacQueen, date unknown).

- $\quad$ Tests of British BEPO reactor caustic scrubbers showed decontamination factors for iodine to be 29 and 32. These would convert to removal efficiencies of 97\% (May and Morris, 1958 as cited in Holmes, 1961).

- Water scrubbers installed at Hanford in 1948 removed an estimated $85 \%$ of iodine in vessel vent gases (Roberts, 1958 as cited in Burger, 1991).

\subsubsection{Representation of Scrubber Collection Efficiency}

Based on information from other sites presented above (particularly the most directly applicable results of measurements at ICPP and Hanford), and to reflect the range of expert opinion received on the subject, the fraction of elemental iodine removed by the caustic scrubber is assumed to be between $90 \%$ and $99 \%$, with 97\% most likely (log-triangular distribution). Removal of organic iodides is estimated to have been between 1 and 10 percent (loguniform distribution). A removal factor is not applied for particle-associated iodine, releases of which are estimated based on Run 54 stack sampling as described in Section 3.10.3. 
There are indications in Chemical Separations Log Books that water was used instead of caustic solution in the RaLa dissolver off-gas scrubber during the following RaLa batches:

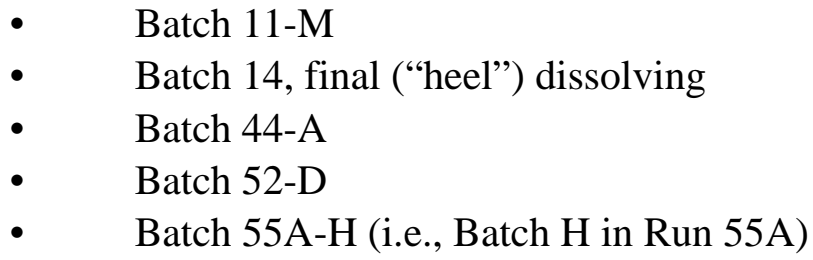

In this analysis, the fraction of iodine removal by water scrubbing is represented by a triangular distribution between 50 and 95 percent, with 85 percent most likely, for elemental iodine and between 1 and 10 percent for volatile organic iodides (loguniform distributions). Again, a removal factor is not applied for particleassociated iodine, releases of which are estimated based on Run 54 stack sampling as described in Section 3.10.3.

During Batch 17-A in 1947, the Operations log book indicates that the scrubber pit was found to be dry during the dissolving. This indicated that no fluid had been flowing through the scrubber for an unspecified portion of the first dissolving of Run 17; in effect, the scrubber was out of service for a portion of the dissolving. Per 706-D Operations shift log book \#9, “The pit is dry. Doesn't seem to help the system any (A4). High air count experienced on $3^{\text {rd }}$ level due to A4 off-gas system being on fritz." To reflect the fact that the scrubber was out of service for a portion of the dissolving of Batch 17-A, the elemental iodine removal efficiency is estimated as follows:

- The fraction of the time that the scrubber was dry was specified as an uncertain parameter, ranging between .25 and .75 (uniform distribution).

- $\quad$ Based on the sampled fraction of time that the scrubber was out of service, an overall removal efficiency for the scrubber was calculated as a weighted average of $0 \%$ efficiency and the value sampled from the usual distribution of efficiency of the caustic scrubber (90\% to 99\%, with $97 \%$ most likely; log-triangular distribution).

- Over 400 realizations of Monte Carlo simulation, a distribution of overall elemental iodine removal efficiency for Batch 17-A was developed and applied in the release calculations for that batch.

On two occasions, there are indications in the Operations log books that the flow rate of caustic solution through the RaLa dissolver off-gas scrubber was "cut back." In one case, flow rates were reportedly cut back in order to reduce the number of samples that had to be taken of scrubber solution. The scrubber flow rate was reduced during the dissolving of Batches 32-A and 32-B in April of 1949 and Batch 59-C in March of 1955. Based on the records of increased air contamination when caustic flow rates were reduced, the fraction of iodine removal by caustic scrubbing for Batches 32-A, 32-B, and 59-C is assumed to be between 75 and 99 percent for elemental iodine (uniformly distributed) and between 1 and 10 percent for volatile organic iodides. 


\subsection{Iodine Removal in the Exhaust Path}

Elemental iodine is known to deposit or "plate out" in varying extents on surfaces with which it comes in contact, such as sample tubing or exhaust path piping or ducts. Some fraction of the elemental iodine that escaped the RaLa scrubber was likely deposited in the line between the scrubber and the Building 205 stack or the later central off-gas stack. Calculations by Voillequé (1996) indicate a median transmission of elemental iodine through the line between Building 706-D and the Building 205 stack of 60 percent, with 95 percent confidence bounds ranging from 30 to 80 percent. These estimates are for a release rate that was approximately uniform over a 20-hour period, with 2-inch line diameter, 800 to 1200-foot line length, and a flow rate ranging from 24 to $36 \mathrm{ft}^{3} \mathrm{~min}^{-1}$. Voillequé states that condensation in the line would be another significant removal mechanism for elemental iodine that was not modeled.

There is extensive documentation that condensation did occur in the 706-D off-gas line, which traveled outdoors above ground and underground for portions of its path. For example, during Run 33, the dissolver scrubber-Building 205 off-gas line condensate pot was a source of air contamination (Ramsey et al., 1949). A small blower was added to vent air from the condensate collection pot to above 706-D roof level. For this source term analysis, 10 percent was added to the median and upper-bound line-loss values corresponding to Voillequé's transmission estimates to account for effects of condensation. The lower-bound value was maintained at 20 percent because it is not known that condensation was a continuous phenomenon throughout the year. The line-loss correction factor, sampled from a triangular distribution between $20 \%$ and $80 \%$ with $50 \%$ most likely, is only applied to the elemental component of the iodine release.

Removal of particulates in the Building 706-D exhaust path apparently significantly increased in late 1948 (after Run 28), when filter houses were installed on the cell ventilation, dissolver off-gas, and secondary equipment off-gas lines. The "temporary" filter building contained fiberglass filters backed by U.S. Army Chemical Warfare Service asbestos-base paper filters; Run 29 sampling indicated a removal efficiency of $97 \%$ (Thompson, 1949; Miller, 1949). And beginning in 1950, RaLa exhausts were routed through a central offgas treatment facility that included an electrostatic precipitator and filters.

Reflecting the iodine capture mechanisms described above, the fraction of particulate iodine removed from the exhaust stream along the exhaust path is estimated to be between 1 and 10 percent through Run 28 in late 1948 (loguniformly distributed), and between 70 and 99 percent (uniformly distributed) from 1949 through 1956. A removal factor is not applied for particle-associated iodine, releases of which are estimated based on Run 54 stack sampling as described in Section 3.10.3; removal of particle-associated iodine would have been directly reflected in the Run 54 stack sampling. These assumed particle loss factors were, however, used in the correction of particle-associated iodine releases through Run 58 for the absence of the filters that were added in the exhaust path afer Run 28 (see Section 3.10.3). 


\subsection{The Source Term Calculations}

The following steps are taken to calculate the release rates for each iodine species for each of the $733 \mathrm{RaLa}$ dissolving batches. For this analysis, the releases from the individual batches are summed to yield annual totals both of releases of each iodine species from the RaLa processing building and of releases from the elevated stack in use at the time.

1. The number of effective slugs dissolved is converted to a uranium mass dissolved based on the following masses of uranium fuel slugs used in RaLa processing:

$$
\begin{array}{ll}
\text { X-10 slugs } & 1.175 \mathrm{~kg} \\
\text { 4-inch Hanford slugs } & 1.782 \mathrm{~kg} \\
\text { 8-inch Hanford slugs } & 3.564 \mathrm{~kg}
\end{array}
$$

2. The fraction of total iodine that remained at the time of dissolution is calculated using the decay time sampled from the appropriate decay time distribution (X-10 or Hanford) and the equation for correction for decay presented in Section 3.6.

3. The fraction of I-131 remaining at the time of dissolution is calculated using the sampled decay time and the equation for simple radioactive decay (See Section 3.6).

4. The fraction of total iodine mass that ${ }^{131}$ I comprised is calculated by multiplying:

a) the ${ }^{131} \mathrm{I}$ inventory from ORIGEN for the batch in question $(\mathrm{Ci} / \mathrm{kg})$; times

b) a $8.06 \times 10^{-6} \mathrm{~g} / \mathrm{Ci}$ factor for ${ }^{131} \mathrm{I}$; times

c) the fraction of ${ }^{131}$ I remaining (from step 3); and dividing by:

d) (the iodine fraction [ $\mathrm{g} \mathrm{I} / \mathrm{kg} \mathrm{U}$ ] from ORIGEN) $\times($ fraction of total I remaining)

5. The release rate of elemental iodine from the dissolver condenser $(\mathrm{kg} / \mathrm{min})$ is calculated using one of the RaLa plant model correlation equations described in Section 3.8. The inputs to the applicable equation are:

a) mass of uranium dissolved $\div$ duration of dissolving (the uranium dissolution rate),

b) the iodine fraction ( $\mathrm{g}$ total $\mathrm{I} / \mathrm{kg} \mathrm{U}$ ) calculated using ORIGEN,

c) the fraction of total iodine remaining [from Step 2], and

d) a factor of 0.001 to convert the $\mathrm{g} / \mathrm{kg}$ of the iodine fraction to $\mathrm{kg} / \mathrm{kg}$. 
6. The results of applying the plant model correlation equation are then multiplied by the inventory uncertainty factors discussed in Tables 3.7 and 3.8 to yield distributions of elemental iodine release from the condenser $(\mathrm{kg} / \mathrm{min})$, with additional uncertainties reflected.

7. The percentages of the iodine in the dissolver that were elemental, volatile organic, nonvolatile organic, and particulate are then calculated using the method described in Section 3.7.

8. The percentages of iodine in the dissolver off-gas that were elemental, organic, and particulate are calculated by dividing the fraction that each constituted in the dissolver (from Step 7) by the sum of the fractions that the three constituted in the dissolver (excluding the nonvolatile organic fraction), and multiplying the result by 100 .

9. The total ${ }^{131} \mathrm{I}$ flow rate from the condenser $(\mathrm{Ci} / \mathrm{hr})$ is calculated by dividing:

a) the elemental iodine flow rate from the condenser $(\mathrm{kg} / \mathrm{min}$ from Step 6) by

b) (the percentage of elemental I in the off-gas $\div 100$ ) and multiplying by

c) $\left(\right.$ the ${ }^{131} \mathrm{I}$ fraction of total iodine $) \times(1000 \mathrm{~g} / \mathrm{kg}) \times(60 \mathrm{~min} / \mathrm{hr}) \times(124,000 \mathrm{Ci} / \mathrm{g})$.

10. The elemental ${ }^{131} \mathrm{I}$ flow rate from the condenser $(\mathrm{Ci} / \mathrm{hr})$ is calculated by multiplying:

a) the elemental iodine flow rate from the condenser $(\mathrm{kg} / \mathrm{min}$ from Step 6) by

b) $\left(\right.$ the ${ }^{131} \mathrm{I}$ fraction of total iodine $) \times(1000 \mathrm{~g} / \mathrm{kg}) \times(60 \mathrm{~min} / \mathrm{hr}) \times(124,000 \mathrm{Ci} / \mathrm{g})$.

11. The volatile organic ${ }^{131} \mathrm{I}$ flow rate from the condenser $(\mathrm{Ci} / \mathrm{hr})$ is calculated by multiplying (the total

${ }^{131}$ I flow rate from the condenser) $\times$ (the percentage of iodine in the off-gas that was volatile organic $\div 100$ ).

12. The particulate ${ }^{131}$ Iflow rate from the condenser $(\mathrm{Ci} / \mathrm{hr})$ is calculated by multiplying (the total ${ }^{131} \mathrm{I}$ flow rate from the condenser) $\times$ (the percentage of iodine in the off-gas that was particulate $\div 100$ ).

13. The following equation is then used in an Excel spreadsheet with the Crystal Ball extension for Monte Carlo simulation (Decisioneering, 1996) to estimate release rates of each species of ${ }^{131} \mathrm{I}$ (other than particle-associated iodine) from the X-10 stack for each dissolving batch:

$$
R R S_{\text {species }} \text { ' }\left[I F C \times\left(1 \& \frac{\% D R}{100}\right)\right] \times\left[1-\frac{\% R_{s c r}}{100}-\left(1 \& \frac{\% R_{s c r}}{100}\right)\left(\frac{\% R_{p a t h}}{100}\right)\right]
$$


where:

$\begin{array}{lll}R R S_{\text {species }} & = & \text { release rate of a given iodine species from the stack, } \mathrm{Ci} / \mathrm{h} \\ I F C & = & \text { flow rate of that species from the dissolver condenser, } \mathrm{Ci} / \mathrm{h} \\ \% D R & = & \text { percent direct release for the batch in question } \\ \% R_{\text {scr }} & = & \text { percent removal of the iodine species in the scrubber } \\ \% R_{\text {path }} & = & \text { percent removal of the iodine species in the exhaust path }\end{array}$

14. Release rates of particle-associated iodine from the RaLa stack are calculated by multiplying the elemental iodine release rate from the RaLa condenser ( $\mathrm{kg} / \mathrm{min}$, from Step 5) times the Run 54 Ratio (Ci/hr per $\mathrm{Kg} / \mathrm{min}$, calculated as described in Section 3.10.3).

15. The following equation is then used in Monte Carlo simulation to estimate release rates of each species of ${ }^{131}$ I from the RaLa processing building for each dissolving batch:

$$
R R B_{\text {species }}=I F C \times\left(\frac{\% D R}{100}\right)
$$

where:
$R R B_{\text {species }}=$ release rate of a given iodine species from the building, $\mathrm{Ci} / \mathrm{h}$
$I F C=$ flow rate of that species from the dissolver condenser, $\mathrm{Ci} / \mathrm{h}$
$\% D R=$ percent direct release for the batch in question

16. Each calculated release rate $(\mathrm{Ci} / \mathrm{h})$ from Steps 13,14 , and 15 above is multiplied times the documented or estimated duration of the dissolving period (h) to calculate the release totals for the batch $(\mathrm{Ci})$.

17. To calculate annual release totals, release totals for all batches are summed within each year from 1944 through 1956. Grand totals across all 13 years of RaLa processing are also calculated by summing the contributions of all 732 "routine" batches and the Batch 56-D accident in 1954. The calculation of these annual totals and grand totals is done by simultaneously calculating the releases from each batch and summing their contributions via Monte Carlo simulation. In order to ensure that the uncertainty bounds of the annual totals and the grand totals are not inappropriately narrowed in this summation process, assumptions that are specified as distributions are sampled once for each batch, once for each run, or once across all batches depending on the nature of the parameter.

Some assumptions are sampled once across all batches within the summation period (i.e., a year or the 13-year period). This is because the distributions for these assumptions represent systematic uncertainty that applies to the RaLa processing operation as a whole. The value sampled from each distribution is applied equally for each batch. Assumption that fall in this category include: 
a. Inventory calculation uncertainty factors: a distribution is sampled once for each factor across all batches that used X-10 slugs and a second distribution is sampled once across all batches that used Hanford slugs.

b. $\quad$ Percentages of particulate, volatile organic, and nonvolatile organic present in the RaLa dissolver: a distribution for each of these parameters is sampled once across all batches in the summation period.

c. $\quad$ Percent removal of elemental, organic, and particulate iodine in the caustic scrubber: a distribution for each of these parameters is sampled once across all batches in the summation period. Separate distributions are sampled once and applied to all batches that involved water instead of caustic solution in the scrubber, decreased scrubber flow, or scrubber malfunction (Batch 17-A).

d. $\quad$ Percent removal of elemental and particulate iodine in the exhaust path: a distribution for each of these parameters is sampled once across all batches in the summation period. Separate distributions for particulate iodine removal are sampled once and applied to all batches before and after filters were added to the exhaust path. This occurred between Runs 28 and 29.

e. The uncertainty factor for 706-C runs: A single distribution is sampled once and applied across all batches that were conducted in Building 706-C.

Distributions for the following assumptions are sampled once for each run and applied to all batches in that run.

a. Hanford slug ${ }^{131} \mathrm{I}$ and total iodine inventory distributions: Because distributions were of $\mathrm{Ci} / \mathrm{kg}$ ${ }^{131} \mathrm{I}$ and $\mathrm{g}$ total iodine/kg calculated specifically for each run, a distribution for each parameter is sampled once for each run and applied across all batches in the run.

b. Hanford slug decay times: Decay times for Hanford slugs were dominated by the time spent in transit from Richland, Washington, and all slugs for a given run traveled together. Because of this, a Hanford decay time distribution is sampled once for each run and applied cross all batches in the run.

c. The Run 54 Ratio used to estimate particulate iodine emissions from the X-10 stack based on Run 54 monitoring and estimated release rates from the RaLa condenser: Because the distributions for this parameter were determined based on measurements made across all of the batches in a given run (Run 54), a distribution is sampled once for each run and applied to all batches in the run. One distribution is applied for batches through Run 28, and a second for batches after Run 28.

Distributions for the following assumptions are sampled for each individual dissolving batch:

a. Duration of dissolving

b. $\quad$ Percent direct release of iodine evolved from the dissolver. 
c. $\quad \underline{X}-10$ slug ${ }^{131}$ I and total iodine inventory distributions: Because distributions were of Ci/ $/ \mathrm{kg}^{131} \mathrm{I}$ and $g$ total iodine $/ \mathrm{kg}$ were specified based on the variation of these parameters across the 21 batches that used X-10 slugs, and were not specified for individual runs or batches, the same distribution for each parameter is sampled individually for each batch.

d. $\quad$ X-10 slug decay time: $\mathrm{X}-10$ slugs for RaLa runs were pushed in numerous groups for each run. Subsets of slugs within a given run may have had different exposure conditions and chronologies of discharge from the reactor and charging to the dissolver. The same distribution for this parameter is sampled individually for each dissolving batch.

The nine early RaLa runs that were performed in Building 706-C, the source term calculations are performed slightly differently. This is because there is less operational information available on these early runs, and because some aspects of this assessment are specifically tailored to the facilities in Building 706-D. The primary focus of this assessment was Building 706-D, in which over $98 \%$ of the ${ }^{131}$ I handled in RaLa slugs at $\mathrm{X}-10$ was processed. A less rigorous evaluation of operational details was possible for Building 706-C runs within the scope of this study. For the three RaLa runs in 1944 and the six runs in 1945, the calculations are performed as follows:

- $\quad$ The X-10 slugs used in these runs are assumed to have the same range of ${ }^{131} \mathrm{I}$ and total iodine contents as the slugs used in Runs 1 through 22 (see Section 3.5.1).

- The numbers of dissolving batches per run and the numbers of slugs dissolved in each batch are estimated to be as follows, based on the available information from Operations log books and a historical summary (Thompson, 1949).

$\begin{array}{lll}- & \text { Run 1: } & 15 \text { batches } \times 12 \text { slugs } \\ \text { S } & \text { Run 2: } & 16 \text { batches } \times 11 \text { slugs, } 1 \text { batch } \times 4 \text { slugs } \\ \text { S } & \text { Run 3: } & 10 \text { batches } \times 18 \text { slugs } \\ \text { S } & \text { Run 4: } & 15 \text { batches } \times 12 \text { slugs } \\ \text { S } & \text { Run 5: } & 15 \text { Batches } \times 14 \text { slugs } \\ \text { S } & \text { Run 6: } & 15 \text { Batches } \times 14 \text { slugs } \\ \text { S } & \text { Run 7: } & 15 \text { Batches } \times 14 \text { slugs } \\ \text { S } & \text { Run 8: } & 15 \text { Batches } \times 14 \text { slugs } \\ \text { S } & \text { Run 9: } & 15 \text { Batches } \times 14 \text { slugs }\end{array}$

$=180$ slugs total

$=180$ slugs total

$=180$ slugs total

$=180$ slugs total

$=210$ slugs total

$=210$ slugs total

$=210$ slugs total

$=210$ slugs total

$=210$ slugs total

- All dissolvings are assumed to be finished within one shift, that is durations are sampled from a uniform distribution between 4 and 8 hours.

- $\quad$ All batches were assigned direct release class 1, as there are no clear indications of leakage of process off-gas beyond that of normal operations.

- Scrubber removal efficiency for elemental iodine is represented as a triangular distribution between 50 and $95 \%$, with $85 \%$ most likely, as it is documented that water was used in the Building 706-C scrubber rather than caustic solution (Webster, 1944). 
- To account for the uncertainty introduced by the transfer of the methods of the source term assessment to the facilities in 706-C, when most information available pertains to the permanent facilities in 706-D, a subjective, multiplicative uncertainty factor is applied in the calculations for the Building 706-C runs. The uncertainty associated with use of the inventory results from later runs for the 706-C runs is not thought to be significant, as slug composition and conditions of irradiation in the Clinton Pile were quite constant. There is some uncertainty in applying the plant model correlation equations to the facilities in 706-C, as the operational details of the early facilities have not been examined to the level of detail as those in 706-D.

The uncertainty factor for Building 706-C runs, which is represented as a triangular distribution between 0.5 and 1.5, with 1.0 most likely, is applied to the results of the plant model correlation equation (see Steps 5 and 6 above).

\subsection{Source Term Results for Annual Releases}

The results of the Task 1 assessment of annual releases from radioactive lanthanum processing are given in Tables 3.15 (releases from the X-10 stack) and 3.16 (releases directly from the process building) and in Figures 3.7 through 3.12. The annual values are stated as central estimates and $95 \%$ subjective confidence intervals for ${ }^{131}$ I in each of the identified forms, based on 400 Monte Carlo simulations using Latin Hypercube sampling and the methods described above. The results for the $1954 \mathrm{RaLa}$ processing accident are not included in the totals for 1954 in these tables or figures, but are reflected in the grand totals presented in Tables 3.15 and 3.16.

Figure 3.13 presents a plot of annual elemental iodine releases from the X-10 stack along with a summary table of key operational parameters and events that were important factors in determining the magnitudes of iodine releases. 
Table 3.15: Annual Iodine Releases from RaLa Processing via the X-10 Stack

\begin{tabular}{|c|c|c|c|c|c|c|c|c|c|}
\hline & \multicolumn{3}{|c|}{ Elemental I-131 (Ci) } & \multicolumn{3}{|c|}{ Organic I-131 (Ci) } & \multicolumn{3}{|c|}{ Partculate I-131 (Ci) } \\
\hline & $2.5 \%$-ile & Central Value & $97.5 \%$-ile & $2.5 \%$-ile & Central Value & $97.5 \%$-ile & $2.5 \%$-ile & Central Value & $97.5 \%$-ile \\
\hline 1944 & 75 & 250 & 6600 & 2.9 & 12 & 60 & 0.015 & 0.082 & 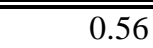 \\
\hline 1945 & 510 & 1,200 & 2,400 & 43 & 170 & 810 & 0.44 & 1.4 & 5.6 \\
\hline 1946 & 260 & 670 & 1,600 & 41 & 160 & 760 & 0.0076 & 0.13 & 2.0 \\
\hline 1947 & 330 & 870 & 2,200 & 56 & 220 & 990 & 0.0032 & 0.066 & 1.0 \\
\hline 1948 & 150 & 510 & 1,200 & 34 & 130 & 610 & 0.22 & 0.89 & 4.3 \\
\hline 1949 & 270 & 790 & 1,700 & 41 & 160 & 780 & 0.40 & 3.1 & 27 \\
\hline 1950 & 140 & 400 & 890 & 26 & 100 & 410 & 0.025 & 0.081 & 0.28 \\
\hline 1951 & 200 & 500 & 890 & 18 & 71 & 290 & 0.0070 & 0.037 & 0.18 \\
\hline 1952 & 550 & 1,600 & 4,100 & 110 & 410 & 1,700 & 0.11 & 0.32 & 0.83 \\
\hline 1953 & 910 & 2,700 & 5,500 & 150 & 590 & 2,400 & 0.10 & 0.37 & 1.6 \\
\hline $1954 *$ & 1,200 & 2,900 & 6,500 & 160 & 630 & 2,400 & 0.082 & 0.32 & 1.3 \\
\hline 1955 & 690 & 1,900 & 4,400 & 110 & 430 & 1,600 & 0.055 & 0.19 & 0.61 \\
\hline 1956 & 840 & 2,700 & 5,700 & 160 & 670 & 2,400 & 0.10 & 0.33 & 1.2 \\
\hline Totals & 5,600 & $\bar{~} 15,000$ & 36,000 & 940 & 3,600 & 17,000 & 3.6 & "6.5 & $\overline{14}$ \\
\hline
\end{tabular}

Table 3.16: Annual Iodine Releases from the RaLa Processing Building

\begin{tabular}{|c|c|c|c|c|c|c|c|c|c|}
\hline & \multicolumn{3}{|c|}{ Elemental I-131 (Ci) } & \multicolumn{3}{|c|}{ Organic I-131 (Ci) } & \multicolumn{3}{|c|}{ Partculate I-131 (Ci) } \\
\hline & $2.5 \%$-ile & Central Value & $97.5 \%$-ile & $2.5 \%$-ile & Central Value & $97.5 \%$-ile & $2.5 \%$-ile & Central Value & $97.5 \%$-ile \\
\hline 1944 & 0.27 & 0.52 & 0.94 & 0.00064 & 0.0027 & 0.015 & 0.000044 & 0.00016 & 0.00061 \\
\hline 1945 & 34 & 62 & 100 & 0.084 & 0.36 & 1.8 & 0.006 & 0.020 & 0.070 \\
\hline 1946 & 9.2 & 16 & 40 & 0.018 & 0.10 & 0.58 & 0.0015 & 0.005 & 0.024 \\
\hline 1947 & 11 & 19 & 38 & 0.028 & 0.11 & 0.57 & 0.0017 & 0.006 & 0.026 \\
\hline 1948 & 4.8 & 16 & 57 & 0.015 & 0.091 & 0.82 & 0.00085 & 0.0048 & 0.036 \\
\hline 1949 & 5.5 & 12 & 27 & 0.014 & 0.071 & 0.43 & 0.00085 & 0.0039 & 0.019 \\
\hline 1950 & 2.2 & 5.3 & 13 & 0.006 & 0.030 & 0.15 & 0.00033 & 0.0017 & 0.006 \\
\hline 1951 & 0.75 & 2.6 & 5.8 & 0.0024 & 0.015 & 0.066 & 0.00013 & 0.00088 & 0.0032 \\
\hline 1952 & 10 & 17 & 25 & 0.023 & 0.092 & 0.37 & 0.0014 & 0.0053 & 0.018 \\
\hline 1953 & 17 & 40 & 98 & 0.051 & 0.23 & 1.2 & 0.0026 & 0.013 & 0.057 \\
\hline $1954 *$ & 22 & 52 & 99 & 0.059 & 0.28 & 1.3 & 0.0036 & 0.016 & 0.063 \\
\hline 1955 & 12 & 28 & 71 & 0.034 & 0.18 & 0.71 & 0.0020 & 0.0094 & 0.038 \\
\hline 1956 & 14 & 26 & 43 & 0.030 & 0.14 & 0.56 & 0.0022 & 0.0081 & 0.027 \\
\hline Totals* & 320 & 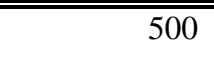 & 780 & 0.70 & 2.8 & 14 & 0.046 & 0.15 & 0.54 \\
\hline urces: & 6,300 & 16,000 & 36,000 & 940 & 3,600 & 17,000 & 3.8 & 6.7 & 14 \\
\hline
\end{tabular}

Total* Iodine-131 Released, all Sources and all Forms: 95\% confidence interval is 8,800 to $42,000 \mathrm{Ci}$, with central value of $21,000 \mathrm{Ci}$.

* Note: The 1954 totals do not include contributions from the Run 56 accident, but the grand totals do. See Section 3.15 for details concerning that accident. 
Figure 3.7: Annual Elemental I-131 Releases from the X-10 Stack (Ci)

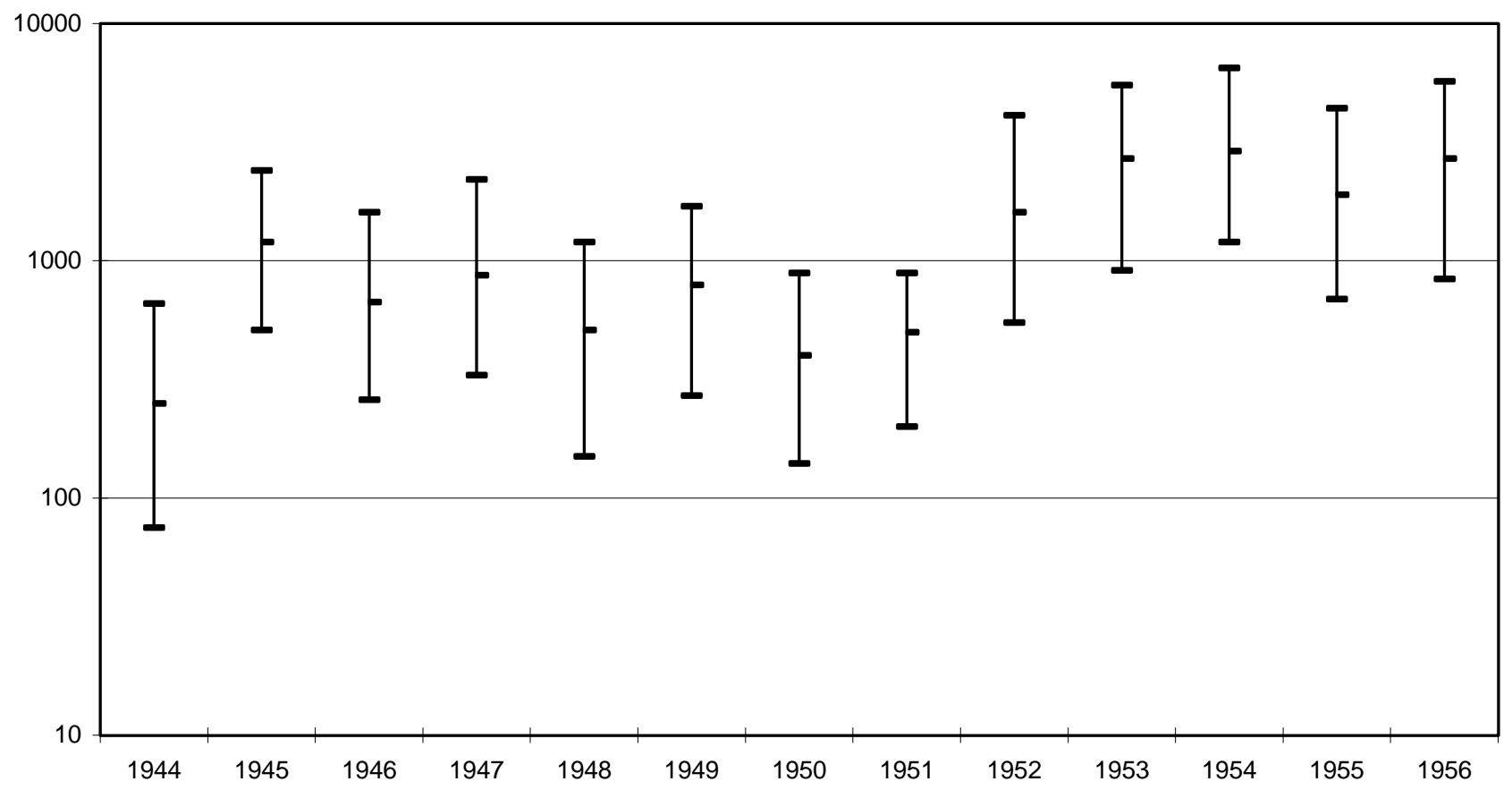

Figure 3.8: Annual Elemental I-131 Releases from the RaLa Process Building (Ci)

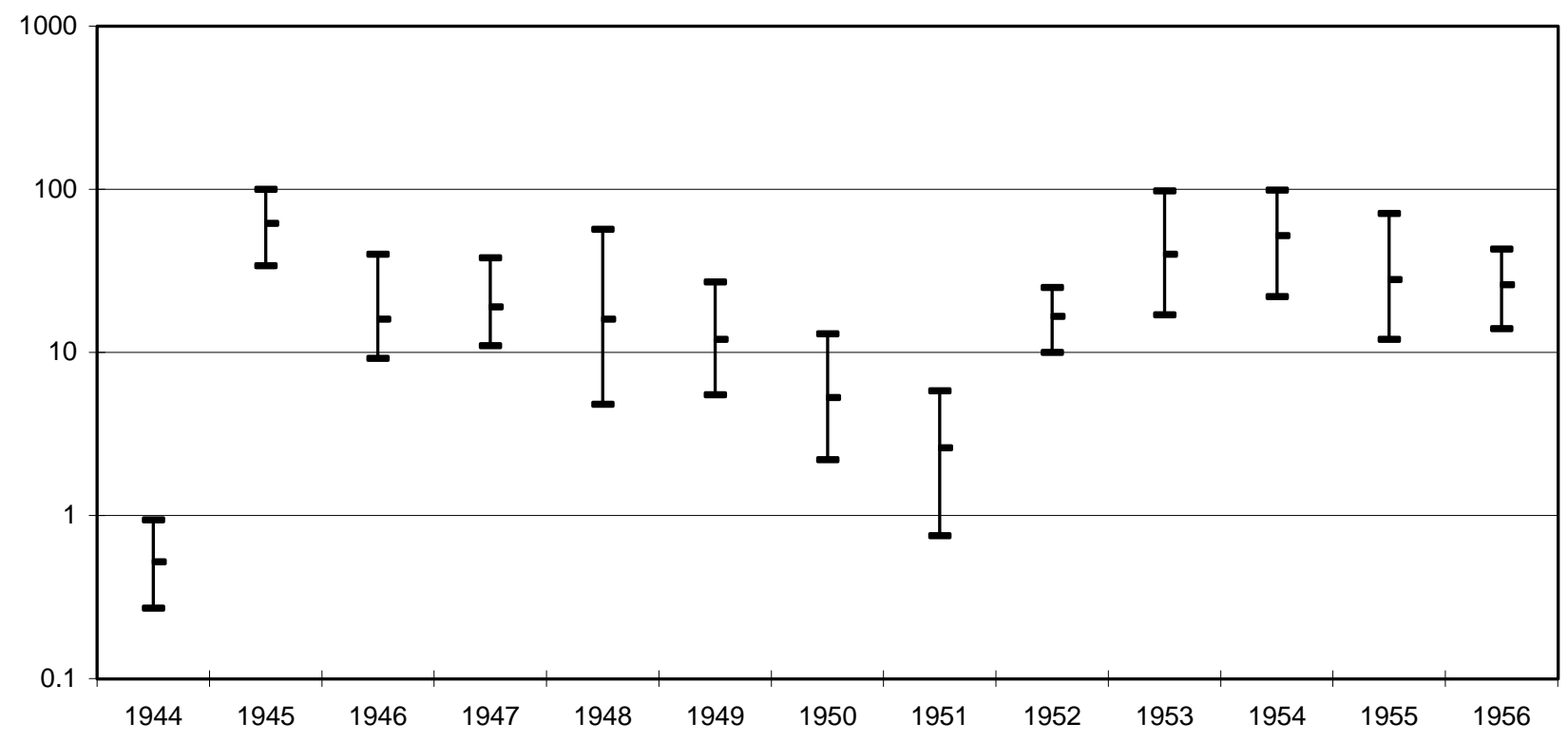


Figure 3.9: Annual Organic I-131 Releases from the X-10 Stack (Ci)

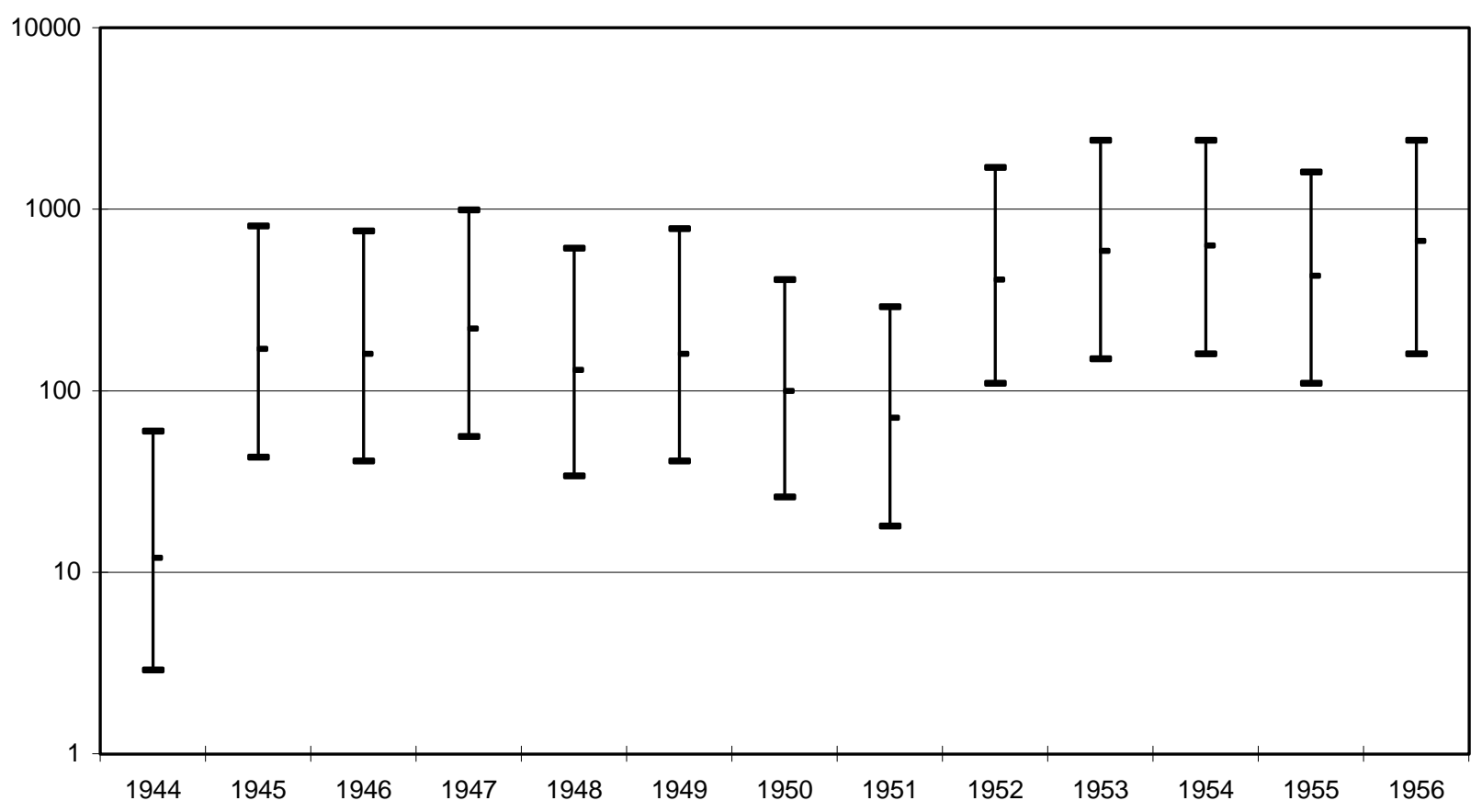

Figure 3.10: Annual Organic I-131 Releases

from the RaLa Process Building (Ci)

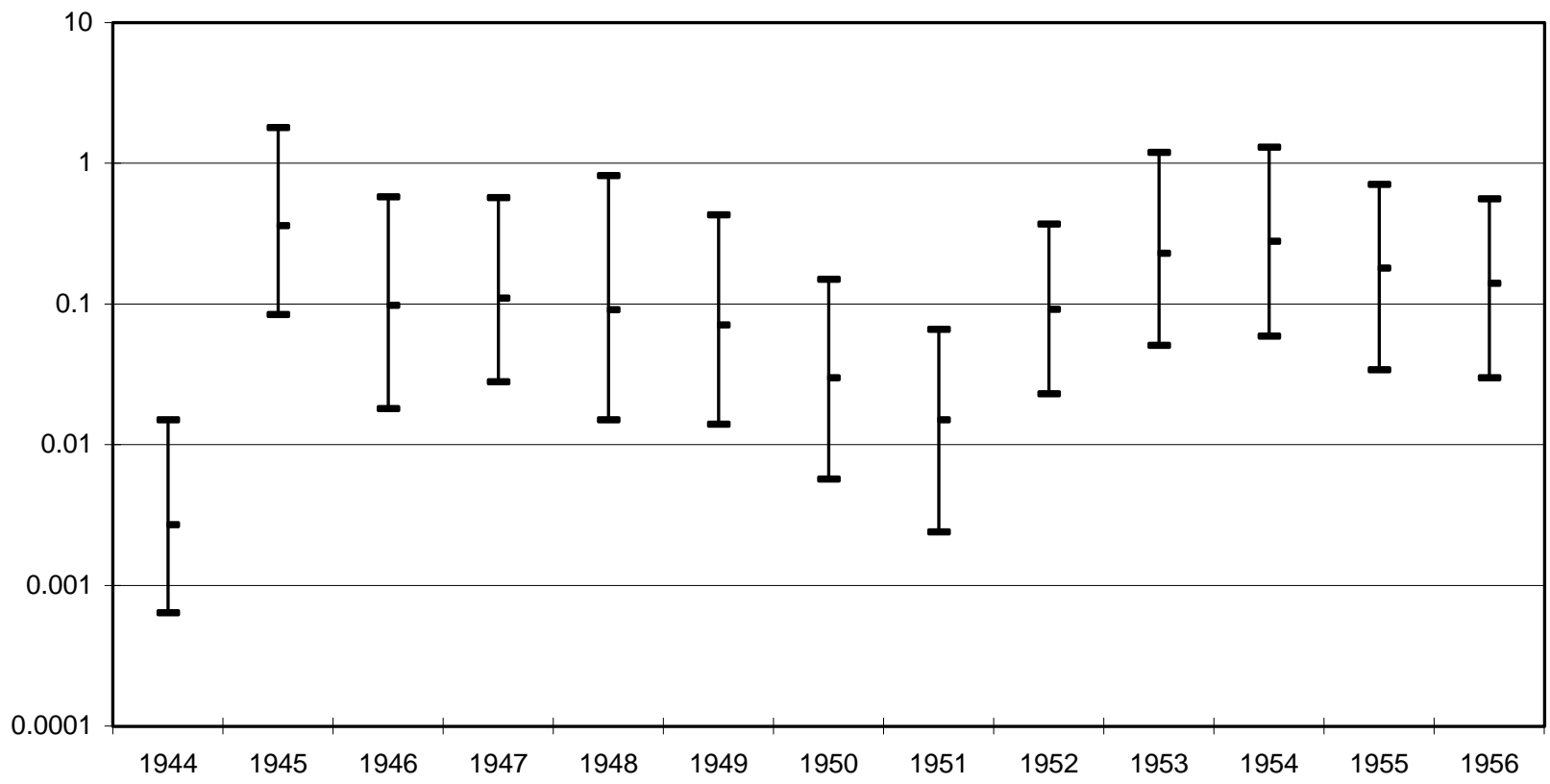


Figure 3.11: Annual Particulate I-131 Releases

from the X-10 Stack (Ci)

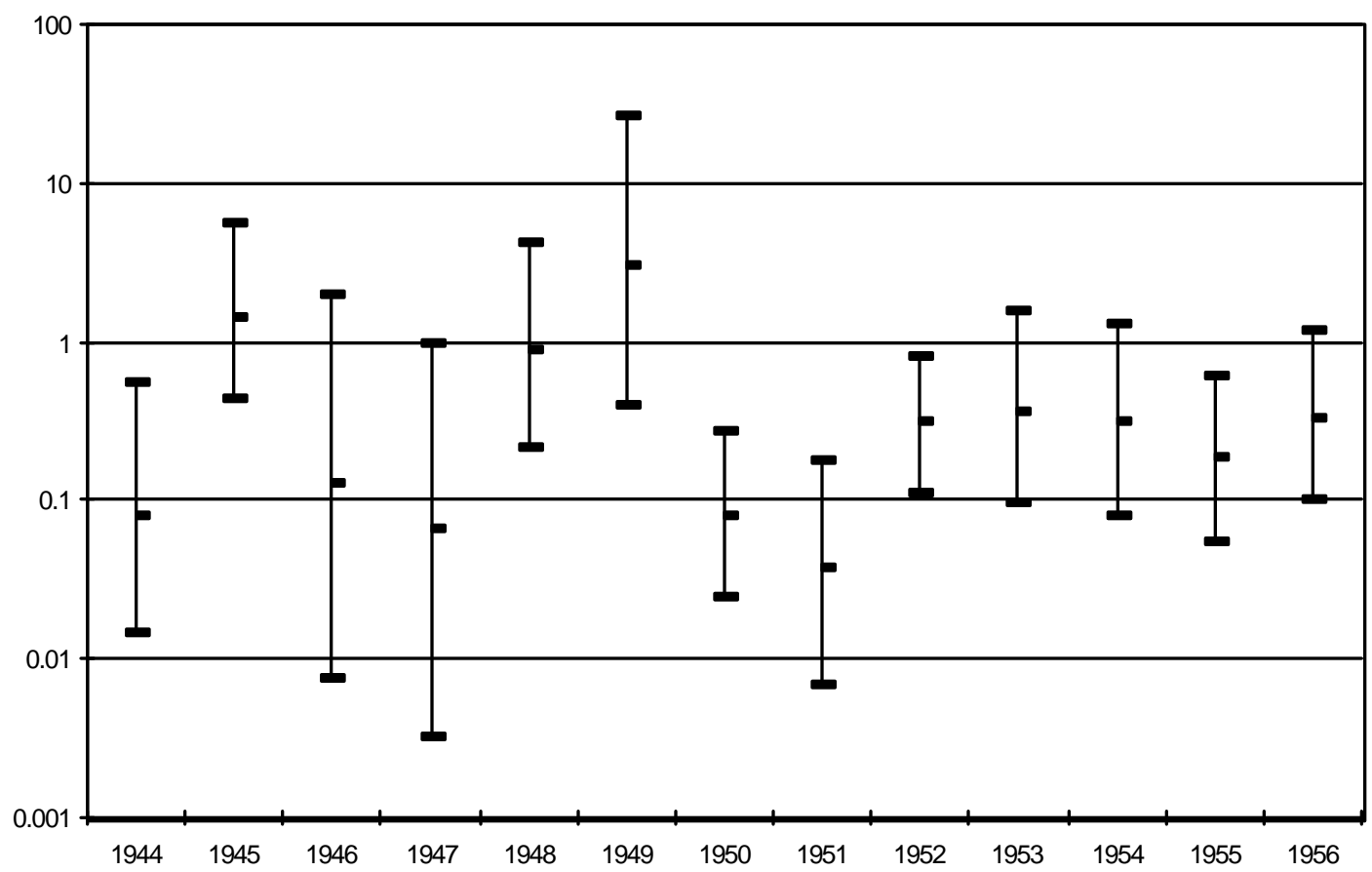

Figure 3.12: Annual Particulate I-131 Releases

from the RaLa Process Building (Ci)

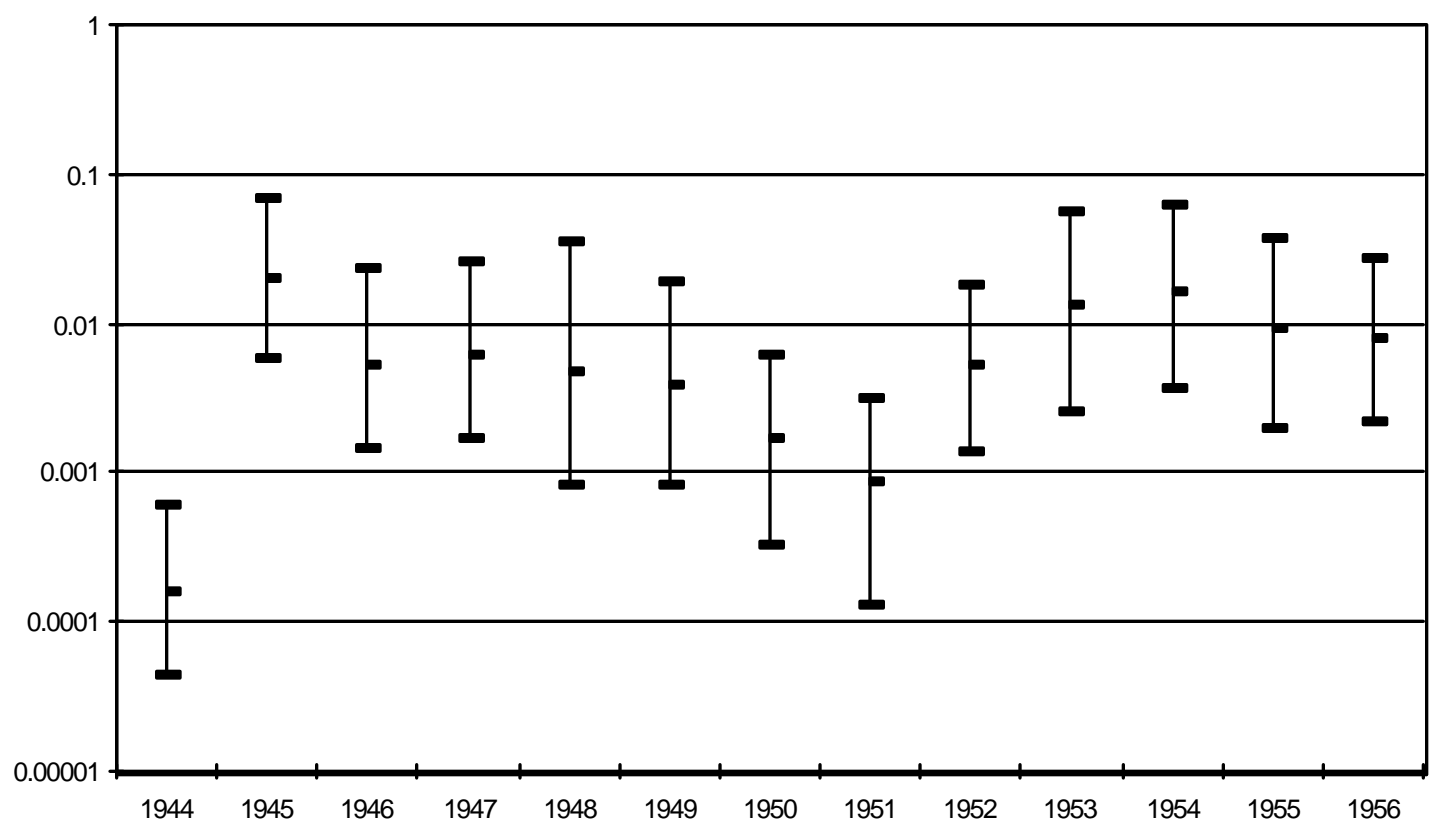


Figure 3.13: Annual Elemental I-131 Releases from the X-10 Stack (Ci) with Operational Data

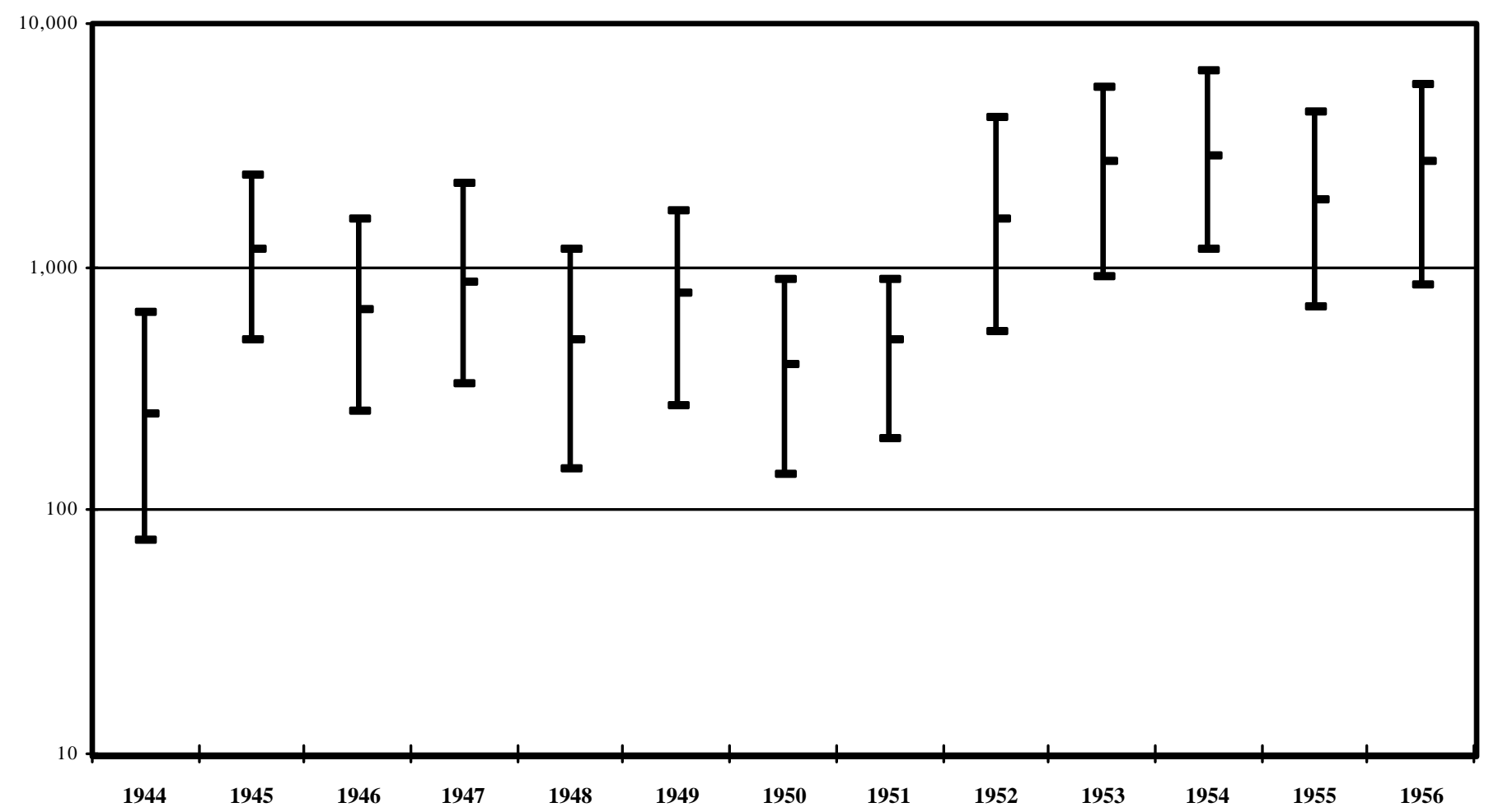

\begin{tabular}{|c|c|c|c|c|c|c|c|c|c|c|c|c|c|}
\hline Number of Dissolving Batches: & 41 & 185 & 97 & 136 & 77 & 36 & 17 & 9 & 38 & 22 & 28 & 21 & 24 \\
\hline Approx. kCi I-131 in Dissolved Slugs ${ }^{1}$ : & 2.5 & 32 & 30 & 39 & 24 & 34 & 22 & 15 & 83 & 100 & 140 & 93 & 140 \\
\hline \multicolumn{14}{|l|}{ Number of Batches with Direct Release... } \\
\hline Class 2 (evidence of leakage): & 0 & 10 & 14 & 5 & 0 & 3 & 1 & 0 & 0 & 2 & 3 & 2 & 0 \\
\hline Class 3 (evidence of severe leakage): & 0 & 5 & 0 & 1 & 0 & 0 & 0 & 0 & 0 & 0 & 0 & 0 & 0 \\
\hline Class 4 (no basis to judge leakage): & 0 & 0 & 17 & 0 & 60 & 0 & 0 & 0 & 0 & 0 & 8 & 0 & 0 \\
\hline No. of Batches with Water in Scrubber: & 41 & 90 & 2 & (a) & 0 & (b) & 0 & 1 & 0 & 1 & 1 & (c) & 0 \\
\hline
\end{tabular}

${ }^{1}$ Based on the number of slugs from each source, the average ${ }^{131}$ I content over the year, and a typical decay time ( $8 \mathrm{~h}$ for X-10 and $6 \mathrm{~d}$ for Hanford slugs)

(a) Scrubber malfunctioned for portion of Batch 17-A.

(b) Flow rate of caustic solution in scrubber was reduced during Batches 32-A and 32-B

(c) Flow rate of caustic solution in scrubber was reduced during Batch 59-C. 


\subsection{Sensitivity Analysis for the Source Terms for Annual Releases}

This source term analysis is structured as a set of calculations of releases from individual RaLa dissolving batches. In the end, the results of the individual batches are summed to yield annual release totals. To indicate the sensitivity of the calculations to various input parameters and assumptions, a set of RaLa batches was selected for performance of sensitivity analyses. Each selected batch is unique from an operational standpoint or was within a year of peak releases. The results of the sensitivity analyses are as follows:

a. Batch 55-D: from the year with the highest elemental iodine releases (1954).

The top five contributors to the overall uncertainty in the elemental ${ }^{131}$ I releases from RaLa processing during Batch 55-D, with their contribution to total uncertainty, were as follows:

1. Percent removal of elemental ${ }^{131} \mathrm{I}$ in the caustic scrubber $\ldots \ldots \ldots \ldots \ldots \ldots$

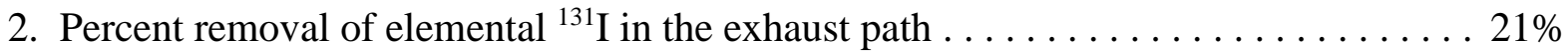

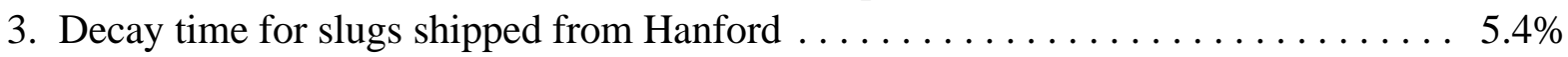

4. Percent direct release of dissolver off-gas $\ldots \ldots \ldots \ldots \ldots \ldots \ldots \ldots \ldots \ldots \ldots$

5. Uncertainty in inventory calculations due to uncertainty in recorded

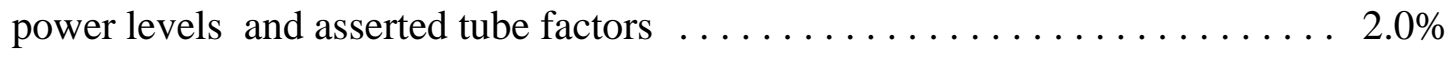

b. Batch 68-A: from the year with the highest volatile organic iodine releases (1956).

The top five contributors to the overall uncertainty in the organic ${ }^{131}$ I releases from RaLa processing during Batch 68-A, with their contribution to total uncertainty, were as follows:

1. Percent of iodine in dissolver present in volatile organic form $\ldots \ldots \ldots \ldots \ldots . \ldots 90$

2. Decay time for slugs shipped from Hanford $\ldots \ldots \ldots \ldots \ldots \ldots \ldots \ldots \ldots \ldots \ldots \ldots \ldots$

3. The ${ }^{131}$ I content of Hanford fuel slugs ( $\mathrm{Ci} / \mathrm{kg} \mathrm{U}$; from ORIGEN) $\ldots \ldots \ldots \ldots \ldots 1.3 \%$

4. The percentage of iodine in dissolver present in nonvolatile organic form $\ldots \ldots 1.2 \%$

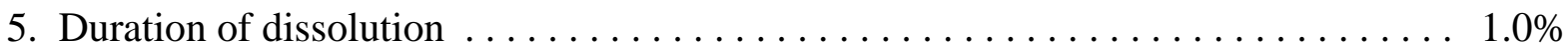

c. Batch 18-F: from the year with the highest particulate iodine release (1947).

The top five contributors to the overall uncertainty in the particulate ${ }^{131}$ I releases from the $\mathrm{X}-10$ stack during Batch 18-F, with their contribution to total uncertainty, were as follows:

1. The total iodine content of $\mathrm{X}-10$ slugs (g I / kg U; from ORIGEN) . . . . . . . 70\%

2. The ratio of measured particulate iodine release from the stack to calculated elemental iodine release from the dissolver (from Run 54 monitoring) . . . . . . . 11\%

3. Uncertainty in inventory calculations due to uncertainty in power-to-peak

flux conversion factors for the Clinton Pile $\ldots \ldots \ldots \ldots \ldots \ldots \ldots \ldots \ldots \ldots \ldots . \ldots \ldots$

4. Uncertainty in inventory calculations due to uncertainty in physical constants

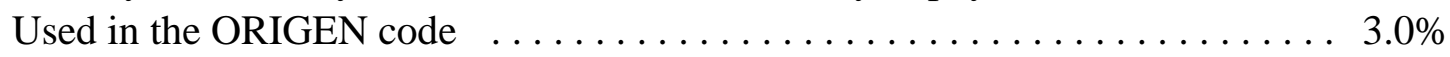


5. Uncertainty in inventory calculations due to uncertainty in relative neutron flux values for different positions in the Clinton Pile $\ldots \ldots \ldots \ldots \ldots . . .2 .8 \%$

d. $\quad$ Batch 26-E from 1948: with large uncertainty in degree of direct release (Class 4).

The top five contributors to the overall uncertainty in the total elemental ${ }^{131}$ I release from all sources during Batch 26-E, with their contribution to total uncertainty, were as follows:

1. Percent removal of elemental ${ }^{131} \mathrm{I}$ in the caustic scrubber $\ldots \ldots \ldots \ldots \ldots \ldots \ldots$

2. Percent removal of elemental ${ }^{131} \mathrm{I}$ in the exhaust path $\ldots \ldots \ldots \ldots \ldots \ldots \ldots \ldots$

3. The ${ }^{131} \mathrm{I}$ content of $\mathrm{X}-10$ fuel slugs ( $\mathrm{Ci} / \mathrm{kg} \mathrm{U}$; from ORIGEN) $\ldots \ldots \ldots \ldots \ldots .6 \%$

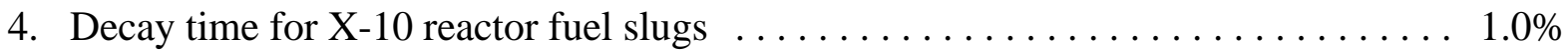

5. Uncertainty in inventory calculations due to uncertainty in power-to-peak

flux conversion factors for the Clinton Pile $\ldots \ldots \ldots \ldots \ldots \ldots \ldots . . . \ldots \ldots$

e. Batch 55-A-H from 1954: a batch in which there was water in the dissolver scrubber.

The top five contributors to the overall uncertainty in the total elemental ${ }^{131}$ I release from the X-10 stack during Batch 55-A-H, with their contribution to total uncertainty, were as follows:

1. Percent removal of elemental ${ }^{131} \mathrm{I}$ by water in the scrubber $\ldots \ldots \ldots \ldots \ldots \ldots$

2. Percent removal of elemental ${ }^{131} \mathrm{I}$ in the exhaust path $\ldots \ldots \ldots \ldots \ldots \ldots \ldots$

3. Decay time for slugs shipped from Hanford $\ldots \ldots \ldots \ldots \ldots \ldots \ldots \ldots \ldots \ldots \ldots \ldots \ldots \ldots \ldots$

4. Uncertainty in inventory calculations due to uncertainty in physical constants used in ORIGEN and actual reactor power variation $\ldots \ldots \ldots \ldots . .7 \%$

5. Uncertainty in inventory calculations due to uncertainty in recorded reactor power levels and asserted tube factors $\ldots \ldots \ldots \ldots \ldots \ldots \ldots \ldots \ldots \ldots \ldots$

f. Batch 66-A from 1956: a batch in which the Hanford reactor of slug origin was unknown, and weighted average iodine inventories were calculated.

The top five contributors to the overall uncertainty in the total elemental ${ }^{131}$ I release from the X-10 stack during Batch 66-A, with their contribution to total uncertainty, were as follows:

1. Calculated g iodine / $\mathrm{kg} \mathrm{U}$ for Run 66 slugs from the $\mathrm{H}$ Reactor . . . . . . . . . 79\%

2. Calculated g iodine / $\mathrm{kg} U$ for Run 66 slugs from the $\mathrm{C}$ Reactor . . . . . . . . . 7.0\%

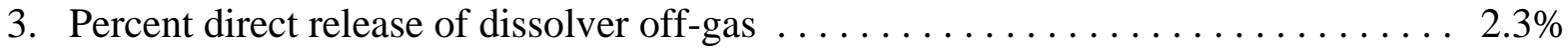

4. Decay time for slugs shipped from Hanford $\ldots \ldots \ldots \ldots \ldots \ldots \ldots \ldots \ldots \ldots$

5. Uncertainty in inventory calculations due to uncertainty in physical constants used in ORIGEN and actual reactor power variation $\ldots \ldots \ldots \ldots . . .6 \%$ 
g. Batch 706-C-5: a batch during operations in Building 706-C.

The top five contributors to the overall uncertainty in the total elemental ${ }^{131}$ I release from the X-10 stack during 706-C Run 5's first dissolving batch, with their contribution to total uncertainty, were:

1. Percent removal of elemental ${ }^{131} \mathrm{I}$ in the caustic scrubber . . . . . . . . . . . $62 \%$

2. Percent removal of elemental ${ }^{131} \mathrm{I}$ in the exhaust path ............... $20 \%$

3. Uncertainty Factor for transfer of methods to Building $706-\mathrm{C} \ldots \ldots \ldots \ldots \ldots$

4. Uncertainty in inventory calculations due to uncertainty in Clinton Pile

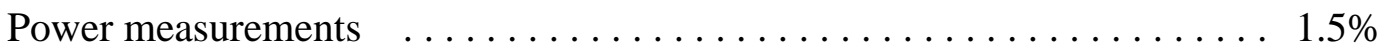

5. The ${ }^{131} \mathrm{I}$ content of $\mathrm{X}-10$ slugs $(\mathrm{Ci} / \mathrm{kg} \mathrm{U}$; from ORIGEN) $\ldots \ldots \ldots \ldots \ldots \ldots$

h. Batch 17-A in 1947, during which the caustic scrubber malfunctioned.

The top five contributors to the overall uncertainty in the total elemental ${ }^{131}$ I release from the X-10 stack during Batch 17-A, with their contribution to total uncertainty, were as follows:

1. Percent removal of elemental ${ }^{131} \mathrm{I}$ in the caustic scrubber ............. $47 \%$

2. Percent removal of elemental ${ }^{131} \mathrm{I}$ in the exhaust path ............... $36 \%$

3. Uncertainty in inventory calculations due to uncertainty in physical constants

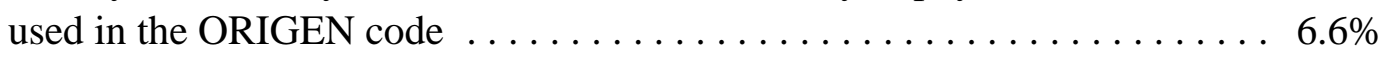

4. Uncertainty in inventory calculations due to uncertainty in power-to-peak

flux conversion factors for the Clinton Pile $\ldots \ldots \ldots \ldots \ldots \ldots \ldots . \ldots \ldots$

5. The ${ }^{131} \mathrm{I}$ content of $\mathrm{X}-10$ slugs ( $\mathrm{Ci} / \mathrm{kg} \mathrm{U}$; from ORIGEN) $\ldots \ldots \ldots \ldots \ldots 1.0 \%$

i. $\quad$ Grand total elemental iodine-131 release.

The top five contributors to the overall uncertainty in the grand total elemental ${ }^{131}$ I release from the X-10 site (over all 733 batches, including the 1954 accident), with their contribution to total uncertainty, were as follows:

1. Percent removal of elemental ${ }^{131} \mathrm{I}$ in the caustic scrubber $\ldots \ldots \ldots \ldots \ldots . \ldots .2 \%$

2. Percent removal of elemental ${ }^{131} \mathrm{I}$ in the exhaust path $\ldots \ldots \ldots \ldots \ldots \ldots . .2 .8 \%$

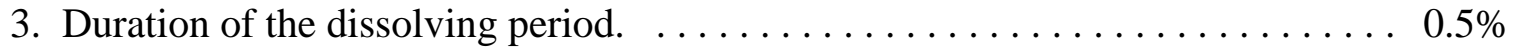

4. Uncertainty in inventory calculations due to uncertainty in power-to-peak

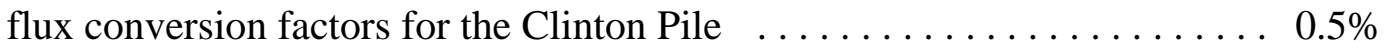

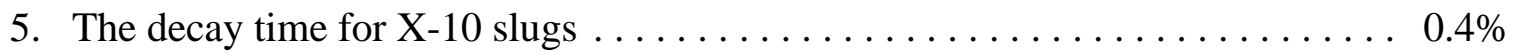

j. Grand total organic iodine-131 release.

The top five contributors to the overall uncertainty in the grand total volatile organic ${ }^{131}$ I release from the X-10 site (over all 733 batches, including the 1954 accident), with their contribution to total uncertainty, were as follows: 
1. Percent of iodine in dissolver present in volatile organic form $\ldots \ldots \ldots \ldots \ldots 11 \%$

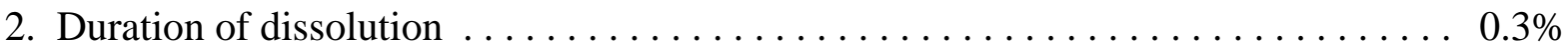

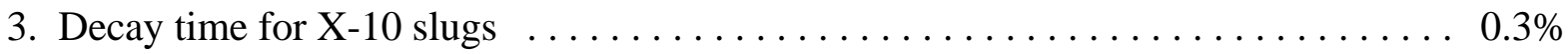

4. The ${ }^{131} \mathrm{I}$ content of Hanford fuel slugs ( $\mathrm{Ci} / \mathrm{kg} \mathrm{U}$; from ORIGEN) $\ldots \ldots \ldots \ldots . .3 \%$

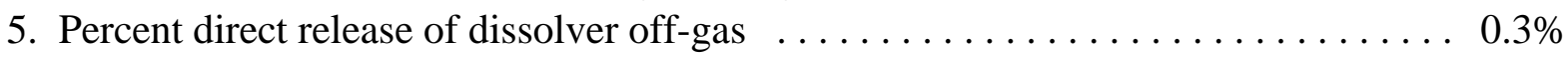

k. $\quad$ Grand total particulate iodine-131 release.

The top five contributors to the overall uncertainty in the grand total particulate ${ }^{131} \mathrm{I}$ release from the X-10 site (over all 733 batches, including the 1954 accident), with their contribution to total uncertainty, were as follows:

1. The ratio of measured particulate iodine release from the stack to calculated elemental iodine release from the dissolver (from Run 54 monitoring) . . . . . . $0.4 \%$

2. The total iodine content of $\mathrm{X}-10$ slugs ( $\mathrm{g}$ / / kg U; from ORIGEN) .......... $0.3 \%$

3. Uncertainty in inventory calculations due to uncertainty in physical constants

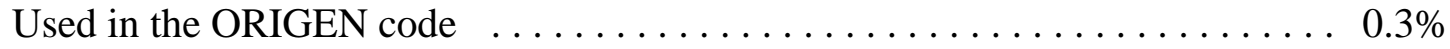

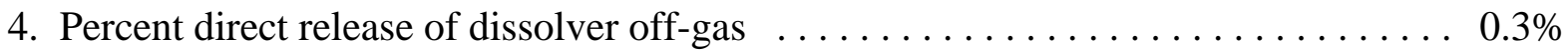

5. Uncertainty in inventory calculations due to uncertainty in power-to-peak

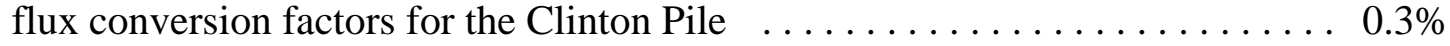

\subsection{The Run 56 Accident of April 29, 1954}

On April 29, 1954, an accident occurred during processing of RaLa Run 56 that was called "the most serious accidental release of activity ever experienced in the history of the process" (Rupp and Witkowski, 1955). Because of the apparent association of the event with a significant episodic release, it was analyzed separately from annual releases in this assessment.

\subsubsection{Information about the Accident}

It is documented in Building 706-D log books that 161 8-inch Hanford slugs were loaded to the dissolver to start Run 56 ${ }^{1}$. Three successful dissolvings of about 18 effective slugs each had occurred (Batches 56-A through 56-C). Batch 56-A dissolving lasted about 4 hours. The remaining, partially dissolved slugs sat dry in the dissolver for about 28 hours after Batch 56-C, and they became thermally hot due to radioactive decay. When acid was poured in to the dissolver to start Batch 56-D, a violent reaction occurred, forcing dissolver solution up the slug loading chute and solution addition lines. The peak release lasted some length of time

\footnotetext{
${ }^{1}$ Several memos report the number of slugs loaded was 101. While the Building 706-D log books are thought to be a more reliable source of information, the number of slugs loaded prior to Run 56 does not directly enter into the calculations of releases from the accident. The number of slugs dissolved is estimated based on the dissolving rate reported for Batch 56-A and the estimated duration of the reaction in Run 56 before the cooling water was turned on.
} 
(reported by different references to have been as short as 10 minutes and as long as about two hours) before the off-gas treatment system could "recover" (Stanley, 1954; Rupp and Witkowski, 1955; Larson, 1954).

Copies of several documents that describe the Run 56 accident are presented in Appendix 30.

\subsubsection{Assessment of Iodine Releases from the Event}

In order for releases from the Run 56 accident to be estimated, the key assumptions that have to be made deal with the following areas:

- $\quad$ The time that the release started,

- The rate of dissolution of uranium during the "violent reaction,"

- The length of time the dissolution reaction continued,

- The fraction of evolved gases that bypassed the caustic scrubber, and

- The efficiency of the caustic scrubber under the conditions at hand.

As stated above, there were reports of an exceptionally violent reaction after the acid was added for Batch 56D, likely caused by the fact that the fuel slugs had become thermally hotter than normal.

\section{$\underline{\text { Start of Release - }}$}

A letter written over a week after the accident (Stanley, 1954c) and health physics log book A-569 (ORNL, 1954) indicate that the elevated release started at 4:58 p.m. Earlier letters (i.e., written sooner after the accident) and the original log book entry say the release started as early as 4:48 p.m. and as late as 5:15 p.m.(Stanley 1954a,b).

ORHASP member Bob Peelle, who was working at X-10 the day of the acccident, estimates that the release started around 4:45 p.m. based on his observation of the group of people who clocked out at 4:30 p.m. and reached the gate house as he observed it after evacuating his building.

\section{$\underline{\text { Rate of Reaction - }}$}

Since the rate of the uranium dissolution reaction increases with increased temperature, a higher than normal dissolution rate may have occurred during the initial portion of the accident. At the same time, many reviewers of the draft Task 1 report indicated that they believe that the reaction rate was not likely substantially higher than normal during the event. Factors affecting their opinions include:

- The RaLa dissolvings were normally conducted just below the boiling point of the dissolver solution. Once the solution began to boil, it could not have gotten any hotter.

- Boiling would have caused nucleation, voids, and blanketing of metal. These effects would have limited the contact of the acid in the dissolver with the uranium metal surfaces and thereby reduced dissolution rates to some extent. 
- The reported loss of acid through the slug loading chute and solution addition lines would have likely limited the dissolving reaction somewhat.

In this assessment, the documented dissolution rate of Batch 56-A was used as a point of reference for specifying the dissolution rate for Batch 56-D; it was 18 slugs over 4 hours, or about 4.5 slugs per hour. Cal Heeb indicates that the dissolution rate from batches after 56-A were likely somewhat higher than that of 56-A if there was no heel in the dissolver when 56-A dissolving began. Subsequent dissolving batches, which would have had a heel in place, likely started more readily due to the increased surface area of the metal heel.

Based upon the above information, the dissolution rate for the Run 56 accident was assumed to have been between 0.70 and 1.25 the "normal" rate indicated by the log book data for bath 56-A.

\section{Duration of the Release -}

An April 30, 1954 memo states that the operators evacuated the building immediately on hearing the radiation monitors alarms (Stanley, 1954a). Log books and letters indicate than workers entered the building to turn on cooling water and stop the dissolution reaction. Letters indicate that the workers were sent into the building at "approximately 5:02" or immediately after the health physicist/lab shift supervisor discussion that started at 5:01. The original health physics log book entry said that heavy fumes were still seen at 5:20; this was crossed out and changed to 5:00. While the first letter after the event said the release went on for 2 hours before the off-gas system could "take care of it," this statement was apparently discredited upon further examination; subsequent reports say that the system recovered within about 10 minutes of the initial burst. When the workers who entered the building to turn on the cooling water exited (around 5:10 or 5:15 p.m.), fumes escaping the dissolver had reportedly ceased.

For this assessment, the direct release from the RaLa dissolver was assumed to have lasted from 4:40 p.m. on April 29, 1954, to 5:10 p.m. that same day. But the duration of the release from the dissolver did not equal the duration of the release from the building. It would have taken a while for the building to clear out what had escaped from the dissolver.

\section{Fraction of Evolved Gases that Bypassed the Caustic Scrubber -}

It appears that the RaLa dissolver was pressurized for some time after the nitric acid was added, that is the offgas system was not maintaining a vacuum on the dissolver like it was supposed to. Larson (1954) states that "gases were given off in too great a volume for the off-gas system to handle."

Yarbro and Burch believe that the off-gas system was still the path of least resistance for evolved gases during the period of excessive evolution of gases, and that less than $50 \%$ of evolved gases would have bypassed the scrubber. They state that, if more than $50 \%$ of evolved gases had bypassed the scrubber, the amount of ruthenium-103 than would also have escaped and would have quickly attached to "cold" surfaces would have 
led to dose rates in the building that dropped off slower than the 20-hour half-life that was indicated (Stanley, 1954a).

Leland Burger indicates that the rapid temperature rise was a condition that often leads to fast iodine release. Burger believes that the reports of a very rapid reaction over a short time period supports assumption of a direct release fraction approaching 100\% during the initial violent reaction. He believes that a fast reaction could have easily caused direct release of gases and fluids into the building due to the high differential pressure across the scrubber. Heeb believes a $20-50 \%$ direct release fraction is reasonable.

Wichner believes that it would be preferable to back up the estimate of the direct release fraction based on some model or based on building and exterior measurements directly. An approach of this type would indeed be preferred, but options are limited at this time by the lack of suitable measurements. We have only the following information regarding the radiological consequences of the accident (Stanley, 1954a):

1) Indications of which buildings on site experienced high air activity (no values are given).

2) Radiation levels at the windows on the roof about 20 feet above the RaLa processing cells were greater than $10 \mathrm{R} / \mathrm{hr}$ during the night after the accident. This is an indication of residual radiation levels, not a measure of airborne radioactivity.

3) Radiation levels in the RaLa building exceeded $100 \mathrm{R} / \mathrm{h}$ on the third level during the incident (measured around 5 p.m. with a "paper chamber cutie pie"). By 7:00 a.m., the first floor was $100 \mathrm{mr} / \mathrm{h}$ and air activity was below tolerance levels.

4) Dose rates from surface contamination in/on other on-site buildings was about 1 to $5 \mathrm{mr} / \mathrm{h}$.

5) Counts of samples from the Settling Basin inlet showed 651 counts at 7 p.m., 10335 counts at 11 p.m., and 1117 counts at 3 a.m. White Oak Dam samples yielded 24 counts at 2:30 a.m. and 16 counts at 5:30 a.m. ("it will be several hours before this activity reaches the dam.")

6) The air monitor at the rock quarry on Bethel Valley Road reportedly showed "a very slight rise" after the incident.

7) We have preliminary results of readings of the film badges worn by 8 people who were involved in the incident or high-level clean up activities.

Based on the available information and the expert interpretation provided, the percentage of direct release is assumed to have been between 25 and $80 \%$ (uniform distribution) during the period of direct release.

\section{Scrubber Elemental Iodine Removal Efficiency -}

Yarbro and Burch believe that the condenser and scrubber were "flooded" by the excessive quantities of gases and vapors leaving the dissolver. In the condenser, the large quantities of vapor likely caused excessive condensation of water that could have impeded air flow through the condenser. And the excessive flow of air and vapor up the scrubber likely impeded the gravity-fed flow of caustic solution down over the rings in the scrubber. Yarbro and Burch believe that the efficiency of the scrubber while flooded would still have been 
$90 \%$ or greater. However, there is little information with which to judge the possible impacts of flooding and channeling in the scrubber during the period of abnormally high evolution of gases and vapors.

The range of percent elemental iodine removal in the caustic scrubber is assumed to be between $65 \%$ and $95 \%$, with $90 \%$ most likely (triangular distribution) over the course of the accident.

\section{Fuel Slug Inventories -}

The I-131 content of the Run 56 slugs is represented as a weighted average of results of ORIGEN calculations for Hanford's C and H reactors and a subjective representation of the probability that Run 56 slugs came from the $\mathrm{H}$ reactor, the $\mathrm{C}$ reactor, or other reactors (see Section 3.5.2).

\section{Calculation of Release Rates -}

The release rates of I-131 from the dissolver are estimated using the correlation equations from the RaLa plant model. Other than as discussed above for elemental iodine, removal of iodine species in the scrubber and exhaust path are assessed as described in Sections 3.10 and 3.11. Except as modified above for specification of dissolution rate, the release rate calculations proceed as summarized in Section 3.12.

Release rates were calculated for five 30-minute periods. The period of direct release from the dissolver occurred partially (10 minutes) within the 4:30 p.m. to 5:00 p.m. period and partially (20 minutes) within the 5:00 p.m. to 5:30 p.m. period. Releases from the RaLa processing building were estimated for each of the five 30-minute periods. Releases from the X-10 central stack were only estimated for the initial two 30-minute periods, as releases via this pathway would have ceased for all practical purposes when the dissolution reaction stopped.

Release rates of iodine-131 from the RaLa processing building after the direct release from the dissolver ended were calculated using the following equation:

$$
P_{2}=P_{1}\left(1-e^{\frac{-F \times t_{r e l}}{V_{b l d g}}}\right)\left(e^{\frac{-F \times t_{\text {post-rel }}}{V_{\text {bldg }}}}\right)
$$

where: $\quad P_{2}=\quad$ release rate from the building $(\mathrm{Ci} / \mathrm{min})$,

$P_{1}=$ release rate into the building from the dissolver $(\mathrm{Ci} / \mathrm{min})$, 


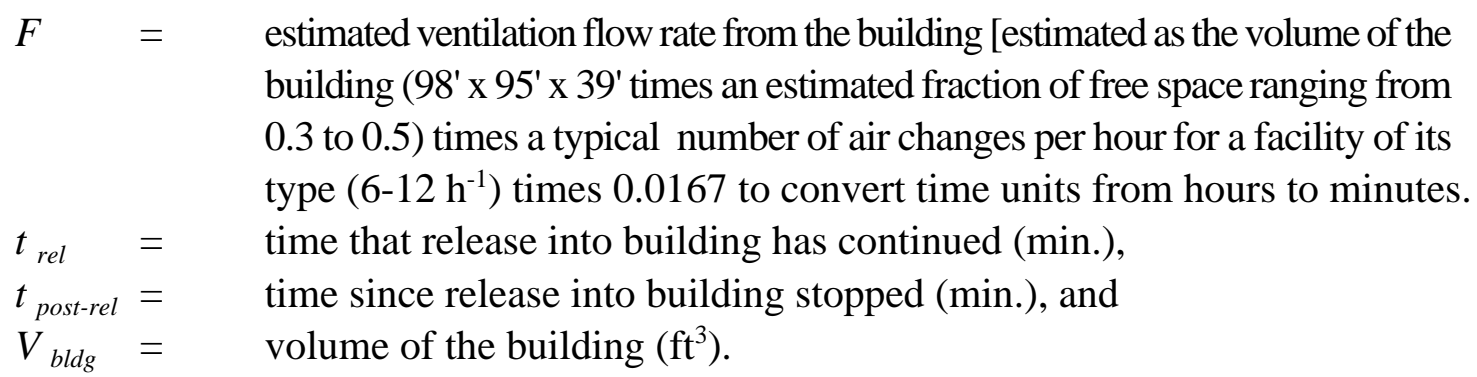

The results of the source term assessment for the Run 56 accident are shown in Table 3.17. The values are stated as central estimates and $95 \%$ subjective confidence intervals for ${ }^{131} \mathrm{I}$ in each of the identified forms, based on 400 Monte Carlo simulations using Latin Hypercube sampling and the methods described above.

\subsection{Sensitivity Analysis for the 1954 Accident Source Term}

The top five contributors to the overall uncertainty in the total elemental ${ }^{131}$ I release from the RaLa processing building during the first half hour of the Run 56 accident, with their contribution to total uncertainty, were as follows:

1. Percent of off-gas experiencing direct release (bypassing scrubber) $\ldots \ldots \ldots \ldots 58 \%$

2. Dissolution rate of uranium when dissolving was active $\ldots \ldots \ldots \ldots \ldots \ldots \ldots$

3. Decay time for slugs shipped from Hanford $\ldots \ldots \ldots \ldots \ldots \ldots \ldots \ldots .6 .6 \%$

4. ${ }^{131} \mathrm{I}$ content of Run $56 \mathrm{H}$-reactor slugs (Ci / kg U; from ORIGEN) . ......... $6.2 \%$

5. Uncertainty in inventory calculations due to uncertainty in recorded reactor power levels and asserted tube factors $\ldots \ldots \ldots \ldots \ldots \ldots \ldots \ldots \ldots \ldots$

The top five contributors to the overall uncertainty in the total elemental ${ }^{131} \mathrm{I}$ release from the X-10 central stack during the first half hour of the Run 56 accident, with their contribution to total uncertainty, were as follows:

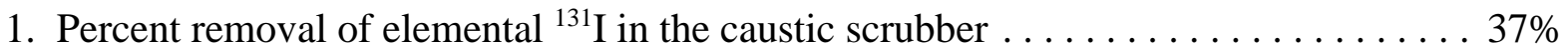

2. Percent of off-gas experiencing direct release (bypassing scrubber) $\ldots \ldots \ldots \ldots 32 \%$

3. Percent removal of elemental ${ }^{131} \mathrm{I}$ in the exhaust path ................... $15 \%$

4. Dissolution rate of uranium when dissolving was active $\ldots \ldots \ldots \ldots \ldots \ldots .1 \%$

5. Decay time for slugs shipped from Hanford $\ldots \ldots \ldots \ldots \ldots \ldots \ldots \ldots \ldots \ldots$ 
Table 3.17: Iodine-131 Releases from the April 29, 1954 Accident During RaLa Processing

\begin{tabular}{cccc} 
Time & \multicolumn{3}{c}{ Elemental I-131 from the RaLa Building } \\
Period & 2.5\%-ile & Central Value & 97.5\%-ile \\
\hline \hline 4:30 to 5:00 & 57 & 140 & 300 \\
5:00 to 5:30 & 43 & 110 & 220 \\
5:30 to 6:00 & 0.16 & 1.2 & 5.4 \\
6:00 to 6:30 & 0.00046 & 0.013 & 0.22 \\
6:30 to 7:00 & 0.0000013 & 0.00014 & 0.0080
\end{tabular}

\begin{tabular}{ccc}
\multicolumn{3}{c}{ Organic I-131 from the RaLa Building } \\
$2.5 \%$-ile & Central Value & $97.5 \%$-ile \\
\hline 0.15 & 0.83 & 3.4 \\
0.12 & 0.61 & 2.6 \\
0.00068 & 0.0063 & 0.052 \\
0.0000021 & 0.000065 & 0.0018 \\
0.0000000054 & 0.00000077 & 0.000061
\end{tabular}

\begin{tabular}{ccc}
\multicolumn{3}{c}{ Particulate I-131 from the RaLa Building } \\
$2.5 \%$-ile & Central Value & $97.5 \%$-ile \\
\hline \hline 0.010 & 0.046 & 0.17 \\
0.0083 & 0.034 & 0.12 \\
0.000039 & 0.00036 & 0.0023 \\
0.00000012 & 0.0000041 & 0.000080 \\
0.00000000038 & 0.000000042 & 0.0000032
\end{tabular}

\begin{tabular}{cccc} 
Time & \multicolumn{3}{c}{ Elemental I-131 from the Central Stack } \\
Period & $2.5 \%$-ile & Central Value & 97.5\%-ile \\
\hline \hline 4:30 to 5:00 & 2.9 & 10 & 33 \\
5:00 to 5:30 & 1.4 & 5.2 & 17
\end{tabular}

\begin{tabular}{|c|c|c|}
\hline \multicolumn{3}{|c|}{ Organic I-131 from the Central Stack } \\
\hline $2.5 \%$-ile & Central Value & $97.5 \%$-ile \\
\hline 0.12 & 0.85 & 3.8 \\
\hline 0.061 & 0.43 & 1.9 \\
\hline
\end{tabular}

\begin{tabular}{ccc}
\multicolumn{3}{c}{ Particulate I-131 from the Central Stack } \\
$2.5 \%$-ile & Central Value & $97.5 \%$-ile \\
\hline \hline 0.000017 & 0.00029 & 0.0022 \\
0.0000085 & 0.00015 & 0.0011
\end{tabular}

\section{Grand Totals for the Accident}

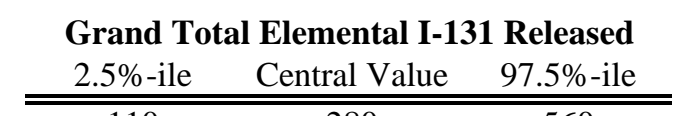

110

560

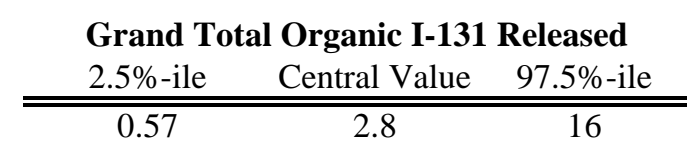

\begin{tabular}{|c|c|c|}
\hline \multicolumn{3}{|c|}{ Grand Total Particulate I-131 Released } \\
\hline $2.5 \%$-ile & Central Value & $97.5 \%$-ile \\
\hline 0.017 & 0.080 & 0.32 \\
\hline
\end{tabular}

Grand Total Iodine-131 Released During the Accident: The 95\% confidence interval is from 110 to $560 \mathrm{Ci}$, with a central value of $280 \mathrm{Ci}$. 


\subsection{References}

Barnard, M. G., 1949. 100 Areas Monthly Reports- 1949. Hanford Report 45828, Hanford Operations Division.

Beall, S. E. and J. W. Adams, 1944. Progress Report, Problem Assignment No. 242-X17E, 205 Stack Gas Monitoring. Clinton Laboratories, Oak Ridge, TN. Report CE-2205. December.

Bradshaw, R. L. and W. D. Cottrell, 1954. A Study of the Contribution of the RaLa Process to Atmospheric Contamination at ORNL. Health Physics Division. ORNL Central Files No. 54-11-86. November 1.

Browning, W. E., Jr., 1960. Removal of Fission-product Activity from Gases. Nucl.Safety 1:3. March.

Burger, L. L., 1991. Fission Product Iodine During Early Hanford-site Operations: Its Production and Behavior During Fuel Reprocessing, Off-gas Treatment and Release to the Atmosphere. Pacific Northwest Laboratory, Richland, WA. Hanford Environmental Dose Reconstruction Project Report PNL-7210 HEDR. May.

Cederberg, G. K., and D. K. MacQueen, date unknown. Containment of Iodine-131 Released by the RaLa Process. Idaho Chemical Processing Plant. AEC Research and Development Report IDO-14566.

ChemRisk, 1993a. Identification of Important Environmental Pathways for Materials Released from the Oak Ridge Reservation. Report of Tasks 3 \& 4 of the Oak Ridge Dose Reconstruction Feasibility Study. Volume II, Part B of the Oak Ridge Health Studies Phase I Report. ChemRisk, a Division of McLaren/Hart Environmental Engineering Corporation, Alameda, CA. September.

ChemRisk, 1993b. A Summary of Historical Activities on the Oak Ridge Reservation with Emphasis on Information Concerning Off-site Emissions of Hazardous Materials. Report of Tasks $1 \& 2$ of the Oak Ridge Dose Reconstruction Feasibility Study. Volume II, Part A of the Oak Ridge Health Studies Phase I Report. ChemRisk, a Division of McLaren/Hart Environmental Engineering Corporation, Alameda, CA. September.

Coughlen, C. P., 1950. Determination of Potential Sources of Area Atmospheric Radio-Active Contamination. Reactor Technology Division. Report ORNL-677. June 8.

Cowser, K. E., 1964. Current Practices in the Release and Monitoring of 131I at NRTS, Hanford, Savannah River, and ORNL. Oak Ridge National Laboratory, Nuclear Safety Information Center, Oak Ridge, TN. Report ORNL-NSIC-3. August 11.

Decisioneering, Inc., 1996. Crystal Ball ${ }^{\circledR}$ Forecasting \& Risk Analysis for Spreadsheet Users, Version 4.0. Denver, Colorado. 
Glasstone, S. and L. Redman, 1972. An Introduction to Nuclear Weapons. WASH-1037.

Harris, R. K., 1945a. Report on 206 Area Operations for Week Ending 5-12-45. Clinton Laboratories, Oak Ridge, TN. Central Files Memorandum No. 45-5-222. May 16.

Harris, R. K., 1945b. 206 Area Operation Report for Week Ending 5/19/45. Clinton Laboratories, Oak Ridge, TN. Central Files Memorandum No. 45-5-262. May 23.

Harris, R. K., 1945c. 206 Area Operations Report for Week Ending 5/26/45. Clinton Laboratories, Oak Ridge, TN. Central Files Memorandum No. 45-5-332. May 31.

Heeb, C. M., 1991. Uncertainties in Source Term Calculations Generated by the ORIGEN2 Computer Code for Hanford Production Reactors. PNL-7223 HEDR. Battelle, Pacific Northwest Laboratories, March.

Heeb, C. M. and D. J. Bates, 1994. Radionuclide Releases to the Columbia River from Hanford Operations, 1944-1971. Battelle, Pacific Northwest Laboratories, Richland, Washington. Report PNWD-2222 HEDR. January.

Hoffman, F. O., A. I. Apostoaei, S. K. Nair, T. E. Widner, and R. E. Burns, 1996. First Iteration Dose and Health Risk Assessment for Iodine-131 Emissions from X-10 Radioactive Lanthanum Processing. Oak Ridge Health Studies, Oak Ridge Dose Reconstruction, Preliminary Task 1 Report. ChemRisk Division, McLaren/Hart Environmental Services. April.

Holmes, J. M., 1961. Removal of Contaminants from Gas Streams. Nuclear Safety 2:4. 1961.

Jaech, J. L., undated. Monthly Summary of Dissolver Data. Hanford Report FTS-CLVI-73.

Jones, H., L. B. Watson, T. Arnette, and I. Conveyou, 1945. Neutron Distribution in the Clinton Pile. Clinton Labs, Oak Ridge, TN. Report CP 2602. February.

Kanne, W. R., and G. M. Branch, 1944. Final Report-Activities in the Off Gas from the Metal Solution Step, Problem Assignment No. 242-X8P. Clinton Laboratories, Oak Ridge, TM. Report CP-1615. May.

LANL, 1956. Memos Regarding Operation Quick and Dirty. Los Alamos National Laboratory, Central Records Center, TR-6797, Group 1 of 7, Envelope 16.

Larson, C. E. 1954. RaLa Production. Oak Ridge National Laboratory Central Files Memorandum 54-645 to K. A. Kasschau of the USAEC, Oak Ridge. June 2.

May, F. G., and J. B. Morris, 1958. Performance of the BEPO Scrubber for Iodine Removal. Commercial Brittish Report AERE-CE/M-226. May. 
Miller, E. C., Ed., 1949. Reactor Technology Progress Report for Quarter Ending May 31, 1949. Technical Division. Report ORNL-267. July 18.

Morgan, J. G., and W. D. Holland, 1979. Iodine Evolution from Nitric Acid Solutions. Report ORNL/TM7032. November.

ORNL, 1950. Operations Notebook No. 82. Assigned to H. Blauer on February 24, 1950. Oak Ridge National Laboratory, Oak Ridge, TN.

Ramsey, M. E., E. J. Witkowski, A. F. Rupp, J. A. Cox, and L. B. Emlet, 1949. Operations Division Monthly Report for Month Ending June 30, 1949. ORNL Report ORNL-288. June 27.

Roberson, J. K. Radiological Warfare Program. Oak Ridge National Laboratory Central Files Memorandum 48-6-375 to K. Z. Morgan. June 29.

Roberts, R. E., 1958. History of Airborne Contamination and Control-200 Areas. Hanford Atomic Products Operation, Richland, WA. Report HW-55569.

RSIC, 1991. Documentation for CCC-371/ORIGEN 2.1 Code package. Radiation Shielding Information Center, Oak Ridge National Laboratory, Oak Ridge, TN, December.

Rupp, A. F. and E. J. Witkowski, 1955. RaLa Production - 1954. Oak Ridge National Laboratory, Oak Ridge, TN. Central Files Number 55-1-211.

Stanley, W. M., Jr., 1954. Area Contamination on April 29, 1954. Oak Ridge National Laboratory, Oak Ridge, TN. Inter-Company Correspondence to C.E. Larson. April 30.

Thompson, W. E., Jr., 1949. History of the Barium-Lanthanum Process and Production. Report ORNL246 Special (with deletions). June 22.

Unger, W. E. et al., 1970. LMFBR Fuel Cycle Studies Progress Report for September 1970, No. 19. Report ORNL/TM-3180. October.

USEPA, 1988. Limiting Values of Radionuclide Intake and Air Concentration and Dose Conversion Factors for Inhalation, Submersion, and Ingestion. Federal Guidance Report 11. U. S. Environmental Protection Agency, Office of Radiation Programs. Washington, D.C. September.

USGAO, 1993. Examples of Post World War II Radiation Releases at U.S. Nuclear Sites. Fact Sheet for the Chairman, Committee on Governmental Affairs, U.S. Senate. GAO/RCED-94-51FS.United States General Accounting Office, Washington, D.C. November.

Voillequé, P. G., 1996. Transmission of $\mathrm{I}_{2}$ Through the Line from Dissolver to Stack at X-10. MJP Risk Assessment, Inc., Idaho Falls, ID. Memo to Shyam Nair of the project team. January 30. 
Webster, D. S., 1944. Preparation of Radioactive Barium-Lanthanum, Problem Assignment No. 263X60G. Clinton Laboratories, Oak Ridge, TN. Report CN-2196. November.

Winters, C.E., 1949a. Seventeenth Progress Report on Oak Ridge National Laboratory Waste Disposal. Oak Ridge National Laboratory, Oak Ridge, TN. Central Files Memorandum 49-3-195. March 15.

Winters, C.E., 1949b. Nineteenth Progress Report on Oak Ridge National Laboratory Waste Disposal. Oak Ridge National Laboratory, Oak Ridge, TN. Central Files Memorandum 49-5-222. May 15.

Yarbro, O., 1996. Information for Modeling the RaLa Process. Memorandum from Orlan Yarbro to Talaat Ijaz of the project team. July 24. 
TASK 1 REPORT

July 1999

${ }^{131}$ I Releases from X-10 Radioactive Lanthanum Processing-

Page 3-86

Source Term Assessment

This page intentionally left blank. 


\subsection{ATMOSPHERIC DISPERSION AND RADIOIODINE CHEMISTRY}

\subsection{Introduction}

Most releases of ${ }^{131}$ I from the radioactive lanthanum processing facility occurred from two X-10 stacks. The Chemical Processing Pilot Plant Stack (called the Building 205 Stack and later the 3020 Stack) was used between 1944 and 1950. The central off-gas-processing stack (called the 900 Area, Isotope Area, or 3039 Stack) was used between 1950 and 1956. Some releases also occurred directly from the building housing the lanthanum processing facility. Once released into the atmosphere, ${ }^{131}$ I was dispersed and transported downwind by prevailing winds. During transport, it underwent a number of chemical transformations because of the reactions of radioiodine in different chemical forms with other atmospheric chemicals and moisture. The different chemical forms of radioiodine were also removed from the atmosphere in differing amounts by the processes of wet and dry deposition.

The primary pathways by which an individual member of the public could have been exposed to ${ }^{131}$ I released from the radioactive lanthanum operation have been presented in Section 2 . In order to calculate the exposures to individual members of the public, it is necessary to calculate the ground-level concentrations of ${ }^{131} \mathrm{I}$ in air and its concentrations on pasture vegetation grazed by cows and goats. Estimation of ground-level ${ }^{131} \mathrm{I}$ concentrations in air at specific locations around the X-10 facility is the primary focus of this section.

For this analysis, a new dispersion model ( $\underline{S} E N E S \underline{O}$ ak $\underline{R}$ idge $\underline{A}$ tmospheric Dispersion $\underline{M}$ odel for Iodine-131; SORAMI), based on a Gaussian Plume modeling approach (Hanna et al., 1982), was developed. The primary reasons for developing a new code were to address the calculation of the wet deposition velocities in the manner presented in Section 5 of this report, to address the depletion of radioiodine from the plume as a result of removal by the processes of wet and dry deposition, and to simulate the chemical transformations of iodine from one chemical form to another as a function of downwind distance. The transformation chemistry of iodine in the atmosphere is discussed first in Section 4.2. Wet and dry deposition processes are directly dependent on the chemical forms of radioiodine; therefore, they also become functions of downwind distance from the source. The governing mathematical equations of SORAMI are presented in Section 4.3.1.

SORAMI uses meteorological data from a single station to model the transport of radioiodine in the valley-ridge region of the Oak Ridge Reservation. This implies that the model does not account for modification of airflow patterns because of changes in topographic elevations (ridges and valleys) at distances of a kilometer and more from the meteorological station. The airflow patterns in the Oak Ridge Reservation are presented in Appendix 4A. SORAMI was validated both with and without the inclusion of the processes of deposition, plume depletion, and iodine chemistry, as discussed in Sections 4.3.3 and 4.3.4. Final simulations were then conducted separately for the routine releases and the 1954 accident. For both routine releases and the accident, separate simulations were conducted for releases from the stack and from the building vents and openings. Results of the simulations are presented in Section 4.4. SORAMI was also benchmarked with a public-domain model ISC3-ST (USEPA, 1995), as described in Section 4.3.3.1. 
An area covered by a radius of $38 \mathrm{~km}$ from Stack 3039 was selected as the region of interest within which the model predictions would be used for the estimation of ground-level concentrations of ${ }^{131} \mathrm{I}$ in air. Within this domain, 41 specific locations of interest were identified on the basis of the presence of backyard cows, dairy- and beef-cattle farms, pastures, or population clusters susceptible to ${ }^{131}$ I exposure from pathways of secondary importance such as inhalation. These locations of concern are presented in Appendix 11A, and the predicted concentrations of ${ }^{131} \mathrm{I}$ in air at these locations are presented in Appendix 11B. The modeling domain was discretized using a polar grid system, and the concentrations of ${ }^{131}$ I were first estimated using SORAMI at the nodes of this grid system. Concentrations at the 41 locations were then obtained using an interpolation scheme. Description of the interpolation scheme and its accuracy are presented in Section 4.3.3.2.

\subsection{Atmospheric Chemistry}

\subsubsection{Background}

During its transport in the atmosphere, ${ }^{131} \mathrm{I}$ undergoes several chemical transformations depending on the chemical forms in which it is released from the stack. In addition, all forms of ${ }^{131}$ I undergo radioactive decay with a half-life of $8.04 \mathrm{~d}$. For travel times of less than an hour, within which most of the chemical transformations occur, as shown below, removal of ${ }^{131}$ I by radioactive decay would be insignificant.

As discussed in Section 3, ${ }^{131}$ I was released from the RaLa processing facility in three different chemical forms: a reactive form (consisting primarily of ${ }^{131} \mathrm{I}$ in elemental form), a nonreactive form (consisting of ${ }^{131} \mathrm{I}$ in organic forms), and a particulate form. A detailed literature survey of the reactions of iodine in the atmosphere was conducted. Some of the conclusions that have direct significance to this study are presented below.

- Elemental iodine $\left(\mathrm{I}_{2}\right)$ can photodissociate rapidly in the presence of sunlight with a halflife of $20 \mathrm{~s}$.

- Methyl iodide can photodissociate in the atmosphere in the presence of sunlight with a half-life of $64 \mathrm{~h}$, which indicates that only the iodine released in elemental form undergoes significant photodissociation.

- Models of iodine chemistry did not contain reactions for the formation of methyl iodide in the atmosphere. Methyl iodide is introduced into the atmosphere primarily from the degradation of ocean biomass. Methyl iodide may also be produced (theoretically) by a few free-radical reactions, but the amount is probably small relative to the oceanic sources. Urban and industrial air samples show high quantities of methyl iodide, indicating possible pathways of formation involving industrial effluents. Knowledge about the formation of methyl iodide from elemental iodine is important for this study because, as discussed below, measurements have indicated that this transformation does occur in the atmosphere.

An experimental study was conducted at Hanford (Ludwick, 1964) in which iodine was released into the atmosphere in elemental form. Measurements of different forms of iodine in the atmosphere indicated that beyond a distance of roughly $3 \mathrm{~km}, 30 \%$ of iodine was in particulate 
form, 36\% was in organic form, and the remaining $34 \%$ was in the elemental form. Ludwick (1967) used stack gas measurements and measurements 5 miles downwind of the stack to estimate that the original iodine in elemental form partitioned into $15 \%, 43 \%$, and $42 \%$ particulate, organic, and elemental forms, respectively. Ramsdell et al. (1994), after a review of several papers, concluded that the partitioning of iodine into different forms at $3200 \mathrm{~m}$ in Ludwick's experiments (Ludwick, 1964) was consistent with the results of other measurements of iodine in the plumes from other stacks at the Hanford site (Ludwick, 1967; Perkins, 1963; 1964), with the partitioning of iodine in the plume following the Chernobyl accident (Aoyama et al., 1986; Bondietti and Brantley, 1986; Cambray et al., 1987; Mueck, 1988), and with the partitioning of natural iodine in the atmosphere (VoillequL, 1979).

A number of references (Garland, 1967; Clough et al., 1965; Chamberlain and Wiffen, 1959) indicate that the majority of iodine is sorbed onto submicron-range aerosol particles. If the relative humidities at Hanford and Oak Ridge were comparable, the partitioning of elemental iodine into particulate form would occur in similar proportions at both places. Measurements of particle concentrations in the submicron range are not directly available from either Oak Ridge or Hanford for the period of interest (1944-1956). Recent measurements of PM10 (particles less than $10 \mu \mathrm{m}$ in size) at Oak Ridge (18-24 $\mathrm{g} \mathrm{m}^{-3}$ between 1992 and 1996; McElhoe, 1998) and Hanford (32 $\mu \mathrm{g} \mathrm{m}^{-3}$; Neitzel et al., 1993) indicate comparable numbers.

A comparison of relative humidities at the two sites (Table 4.1) indicates that the relative humidity is consistently higher at Oak Ridge than at Hanford. Since conversion of condensation nuclei (aerosol particles on which condensation of water can occur under subsaturated conditions) to submicron size aqueous aerosols is enhanced by increased relative humidity in the atmosphere (Winkler, 1973; Fitzgerald, 1975), it is reasonable to assume that there would be increased sorption of iodine onto aerosols (both dry and aqueous) at Oak Ridge, resulting in a higher rate of transformation of the elemental form of iodine into the particulate form.

Moyers and Duce (1972) provide sorption mechanisms for the dissolution of gaseous $\mathrm{I}_{2}$ and HI species into aqueous, submicron aerosols formed on sea-salt condensation nuclei in a marine atmosphere. They also provide speciation reactions for iodine in the aqueous phase, which lead to the formation of $\mathrm{I}^{-} \mathrm{IO}_{3}{ }^{-}$, and $\mathrm{I}_{2}$. Perkins (1964) had also observed the same three forms of iodine in the particulate matter. Presence of these forms of iodine in the atmosphere suggests that the mechanisms suggested by Moyers and Duce (1972) are also applicable to the nonmarine atmosphere. However, it must be noted that the occurrence of these mechanisms in a nonmarine environment has not been directly established by measurements. 
Table 4.1 Relative humidity for Oak Ridge ${ }^{\text {a }}$ (Fitzpatrick, 1982) and Hanford (Stone et al., 1983).

\begin{tabular}{ccc}
\hline & \multicolumn{2}{c}{ Relative humidity (\%) } \\
Month & Oak Ridge & Hanford \\
\hline January & 72.3 & 76.4 \\
February & 68.1 & 70.7 \\
March & 62.0 & 55.9 \\
April & 59.3 & 46.9 \\
May & 68.1 & 43.0 \\
June & 70.5 & 39.7 \\
July & 73.6 & 32.2 \\
August & 74.5 & 35.6 \\
September & 72.6 & 41.6 \\
October & 72.7 & 56.8 \\
November & 72.1 & 73.6 \\
December & 73.6 & 80.0 \\
Annual Average & 70.0 & 54.3 \\
\hline
\end{tabular}

${ }^{a}$ The Oak Ridge data represent hourly records between January 01, 1955, and February 28, 1965, and between December 1 , 1965, and December 31, 1972.

\subsubsection{Approach and Input Parameters}

For estimating the concentrations of radioiodine in different chemical forms at ground-level at the various locations (all of which are at or beyond $3 \mathrm{~km}$ from the stack), the following approach is used, based on the background presented in Section 4.2.1.

- If all of the ${ }^{131}$ I were to be released in elemental form and if the Oak Ridge atmospheric conditions were identical to those at Hanford, it can be assumed that the proportion of ${ }^{131} \mathrm{I}$ in each of the three chemical forms identified earlier would be equal at all locations of concern. Because Oak Ridge has a significantly more humid climate than Hanford, the amount of particulate formed from the release of ${ }^{131} \mathrm{I}$ in elemental form would be higher at Oak Ridge. Thus, as the proportion of particulate form increases downwind of the source, the proportion of the elemental form decreases; there would be no change in the proportion of organic form.

- It is assumed that ${ }^{131}$ I released in an organic form would not undergo any change because of its slow rate of photodissociation, and all ${ }^{131} \mathrm{I}$ in organic form would reach the locations of interest with appropriate dilution from dispersion.

- $\quad$ Similarly, all the ${ }^{131}$ I released in particulate form would reach the locations of interest with appropriate dilution from dispersion and without undergoing any chemical changes.

- Iodine chemistry is assumed to remain the same throughout the year with no seasonal patterns.

The term $g_{k j}$ is used to represent the fraction of iodine that is transformed from form $\mathrm{j}$ to form $\mathrm{k}$ (or remains in form $\mathrm{j}$, if $\mathrm{k}=\mathrm{j}$ ). Based on the discussions provided earlier, if $\mathrm{k}$ and $\mathrm{j}$ take on the values 1, 2, and 3 for elemental, organic, and particulate forms, respectively, then $g_{12}=g_{13}=g_{23}$ $=g_{32}=0$ and $g_{22}=g_{33}=1$. In other words, none of the ${ }^{131}$ I released in particulate and organic forms ends up as elemental ${ }^{131} \mathrm{I}\left(g_{12}=g_{13}=0\right)$; none of the ${ }^{131} \mathrm{I}$ released as organic ends up as particulate ${ }^{131} \mathrm{I}\left(g_{32}=0\right)$; none of the ${ }^{131} \mathrm{I}$ released in particulate form ends up as organic ${ }^{131} \mathrm{I}\left(g_{23}\right.$ 
$=0)$; all of the ${ }^{131}$ I released as organic ${ }^{131}$ I remains in organic form $\left(g_{22}=1\right)$; and all of the ${ }^{131} \mathrm{I}$ released as particulate ${ }^{131} \mathrm{I}$ remains in particulate form $\left(g_{33}=1\right)$. The only parameters which then must be defined with specific numerical values (based on the earlier discussion) are $g_{11}, g_{21}$, and $g_{31}$ (the fractions of iodine released in elemental form that end up in elemental, particulate, and organic forms, respectively). Therefore, starting with three forms at release and three forms at the receptor location, five non-zero combinations of $g_{k j}\left(g_{11}, g_{22}, g_{33}, g_{21}\right.$, and $\left.g_{31}\right)$ exist that link the chemical forms of ${ }^{131} \mathrm{I}$ at the source to those at the receptor.

Uniform distributions are chosen for three parameters: (a) the fraction of elemental iodine released that is converted to organic form $\left(g_{21}\right)$; (b) the fraction of elemental iodine released that is converted to particulate form $\left(g_{31}\right)$; and (c) the fraction that remains unconverted $\left(g_{11}\right)$.

For $g_{21}$, a uniform distribution was assumed with a central value of 0.33 and a large range $(0.2$ 0.45). This assumption results directly from the literature data and the conclusions from Ramsdell et al. (1994), accounting for uncertainties. For $g_{31}$, a larger range of probable values was selected (0.2-0.8) to account for the increased humidity at Oak Ridge compared to that at Hanford, to account for the lack of confirmatory knowledge regarding the applicability of the mechanisms of Moyers and Duce (1972) to the nonmarine atmosphere at Oak Ridge, and to reflect the range of measured values from the literature surveys discussed earlier. The upperbound value was increased to 0.8 to account for generally high relative humidity at Oak Ridge (Table 4.1) and also to account for the very high relative humidity during summer nights at Oak Ridge (Holland, 1953). The lower value was set at a level (0.2) above the measured values, to reflect the generally higher relative humidity at Oak Ridge than at Hanford. Finally, $g_{11}$, the fraction that remains in elemental form, is calculated as $\left(1-g_{21}-g_{31}\right)$. The sampling steps for each set of values of $g_{11}, g_{21}$, and $g_{31}$ corresponding to one simulation of the uncertainty analysis are shown below. These steps ensure that the distributions of $g_{11}, g_{21}$, and $g_{31}$ are sampled appropriately.

Step 1. Sample the distribution of $g_{21}$ (uniform between 0.2 and 0.45) using a Latin Hypercube sampling (NCRP, 1996). Let $\Phi$ be the sampled value. This is the value of $g_{21}$.

Step 2. Sample a uniform distribution between 0 and 1 using a Latin Hypercube sampling (NCRP, 1996). Let $\eta$ be the sampled value.

Step 3. Obtain K using

$$
\mathrm{K}=0.2+\eta \frac{(0.45-\Phi)}{(0.45-0.2)}(0.8-0.2)
$$

Value obtained for $\mathrm{K}$ represents the value for $g_{31}$. It should be noted that if the interval of $(0.2,0.8)$ is sampled directly as $\mathrm{K}$, it can result in $(\Phi+\mathrm{K})$ being greater than 1 for some combinations of $\Phi$ and $\mathrm{K}$. 
Step 4. Obtain $g_{11}$ as $1-g_{21}-g_{31}$.

These steps ensure that for each iteration, a nonnegative value between 0 and 1 is obtained for $g_{11}, g_{21}$, and $g_{31}$ and the sum of the three terms adds up to 1 . The $95 \%$ subjective confidence intervals for $\mathrm{g}_{11}, \mathrm{~g}_{21}$, and $\mathrm{g}_{31}$ were obtained as $(0.11,0.52),(0.21,0.44)$, and $(0.2,0.66)$.

\subsubsection{Distance to Complete Transformation, $x_{c}$}

Literature data indicate that the conversion of iodine from elemental form to the other forms is complete well within $3.2 \mathrm{~km}$ of the source (Ramsdell et al., 1994). This distance, denoted by $x_{c}$, was treated as an uncertain parameter and was allowed to take a uniform distribution between 2 and $3.2 \mathrm{~km}$ to indicate the large uncertainty in the exact value of the distance at which the chemical transformation process is complete.

\subsection{Atmospheric Dispersion Simulations}

\subsubsection{The SORAMI Model}

As shown in Section 3, part of the routine releases occurred directly from building vents and openings. A major portion of the release from the 1954 accident on April 29 also occurred directly from the building. Wet and dry deposition can remove significant portions of contaminants from the plumes released at or near ground-levels (Horst, 1984; 1977). Therefore, if the depletion from wet and dry deposition processes is not accounted for, the predicted concentrations at downwind distances would be significantly biased toward overprediction. To account for the depletion from wet and dry deposition processes and from the atmospheric chemistry of iodine described in Section 4.2, a modified form of the Gaussian Plume Model was developed. The downwind concentration of ${ }^{131} \mathrm{I}$ in air in chemical form $k$ is calculated as follows:

$$
\begin{aligned}
C_{a, k}=\left[\sum_{j=1}^{3} Q_{0, j} g_{k j}\left\{\left(\frac{Q_{j}}{Q_{0, j}}\right)_{0, x_{c}} \cdot\left(\frac{Q_{k}}{Q_{0, k}}\right)_{x_{c}, x}\right\}\right] & \frac{1}{2 \pi \sigma_{y} \sigma_{z} u} \cdot e^{-\frac{y^{2}}{2 \sigma_{y}^{2}}} \cdot\left\{e^{-\frac{(z-h)^{2}}{2 \sigma_{z}^{2}}}+e^{-\frac{(z+h)^{2}}{2 \sigma_{z}^{2}}}\right\}
\end{aligned}
$$

where,

$$
\begin{aligned}
& C_{a, k}=\text { the concentration of }{ }^{131} \mathrm{I} \text { in chemical form } k \text { at location }(x, y, z)\left[\mathrm{Bq} \mathrm{m}^{-3}\right] \text {; } \\
& Q_{0, j} \quad=\text { the release rate of }{ }^{131} \mathrm{I} \text { in form } j \text { from a source located at }(0,0, h)\left[\mathrm{Bq} \mathrm{s}^{-1}\right] \text {; } \\
& \left(Q_{j} / Q_{0, j}\right)_{x 1-x 2}=\text { source depletion correction for the } j^{\text {th }} \text { chemical form between downwind } \\
& \text { distances } x 1 \text { and } x 2 \text { [dimensionless]; } \\
& g_{k j} \quad=\text { fraction of }{ }^{131} \mathrm{I} \text { released in chemical form } j \text { that is transformed to } \\
& \text { chemical form } k \text { within the distance } x_{c} \text {; } \\
& x_{c} \quad=\text { distance from source at which all chemical transformations are assumed } \\
& \text { to be complete }[\mathrm{m}] \text {; }
\end{aligned}
$$


$h \quad=$ the effective release height $(=$ height of physical stack + plume rise $)[\mathrm{m}]$;

$\sigma_{y} \quad=$ crosswind dispersion coefficient of a contaminant in air [m];

$\sigma_{z} \quad=$ vertical dispersion coefficient of a contaminant in air $[\mathrm{m}]$; and

$u \quad=$ average wind speed at the source elevation during the hour being simulated $\left[\mathrm{m} \mathrm{s}^{-1}\right]$.

The source depletion correction terms were obtained from the formulation presented in Hanna et al. (1982) as shown below.

$$
\frac{Q_{j}(x)}{Q_{0, j}}=\left[\exp \int_{0}^{x} \frac{d \varepsilon}{\sigma_{z} \exp \left(h^{2} / 2 \sigma_{z}^{2}\right)}\right]^{-\left(\frac{2}{\pi}\right)^{\frac{1}{2}}\left(\frac{V_{d e p, j}}{u}\right)}
$$

where

$$
\begin{aligned}
\varepsilon & =\text { integration variable that defines the downwind distance from source }[\mathrm{m}] ; \\
& \text { and } \\
V_{d e p, j} & \text { total deposition velocity of the } j^{\text {th }} \text { chemical form of }{ }^{131} \mathrm{I}\left[\mathrm{m} \mathrm{s}^{-1}\right]
\end{aligned}
$$

Other quantities in Equation 4.2 have been defined earlier. It should be noted that $\sigma_{z}$ is a function of downwind distance in Equation 4.2. To derive the quantity $\left(\mathrm{Q}_{\mathrm{j}} / \mathrm{Q}_{0, \mathrm{j}}\right)_{\mathrm{xc}, \mathrm{x}}$ in Equation 4.1, the following relationship is used.

$$
\left(\frac{Q_{j}}{Q_{0, j}}\right)_{x_{c}, x}=\frac{\left(\frac{Q}{Q_{0, j}}\right)_{0, x}}{\left(\frac{Q}{Q_{0, j}}\right)_{0, x_{c}}}
$$

Equation 4.3 can be derived directly from Equation 4.2.

Other processes not explicitly shown in the above equations but included in the new code are the plume rise (based on Briggs, 1969; 1975), vertical wind-speed profile for the estimation of wind speed at stack height, and distances to virtual point sources for ground-level or near-ground area sources (based on Turner, 1970). The total deposition velocity in Equation 4.2 is estimated using the following equation.

$$
V_{d e p, j}=V_{d, j}+V_{w, j}
$$

where

$V_{d, j}=$ dry deposition velocity of the $j^{\text {th }}$ chemical form of ${ }^{131} \mathrm{I}\left[\mathrm{m} \mathrm{s}^{-1}\right]$ and

$V_{w, j}=$ wet deposition velocity of the $j^{\text {th }}$ chemical form of ${ }^{131} \mathrm{I}\left[\mathrm{m} \mathrm{s}^{-1}\right]$.

Estimation of dry and wet deposition velocities for each chemical form of ${ }^{131} \mathrm{I}$ is discussed in detail in Section 4.3.2 and Section 5. 


\subsubsection{Input Parameters}

Two separate analyses were conducted for the releases of ${ }^{131}$ I between 1944 and 1956 . The first one was conducted for the routine releases. These releases were represented by annual average estimates of source terms from the stacks and the building housing the radioactive lanthanum facility. For the analysis of routine releases, the SORAMI model was first validated using field data on concentrations of ${ }^{131} \mathrm{I}$ in air from 1967 to 1969 without including the processes of plume depletion, dry and wet deposition, and iodine chemistry. For benchmarking the SORAMI code, these predictions were compared with those obtained from a public-domain model, ISC3-ST (USEPA, 1995), as described in Section 4.3.3.1. The SORAMI model was then validated with the same field data after including the processes of plume depletion, dry and wet deposition, and iodine chemistry, as described in Section 4.3.3.2. Results of the model simulations for routine releases are presented in Section 4.4.1.

The second analysis was conducted for the accidental release of April 29, 1954. As presented in Section 3, the 1954 release lasted for just under an hour for releases from the stack and for 2.5 hours for releases from the building housing the RaLa processing facility. For the analysis of the accidental release, the SORAMI model was first validated using a limited number of short-term monitoring and release data, as discussed in Section 4.3.4.

Stack parameters from Stack 3020 were used for the analysis of releases from both Stack 3020 and Stack 3039. The rationale for adopting this approach is presented in Section 4.3.2.1. The error associated with using Stack 3020 for the entire analysis for all years was estimated explicitly and is presented in Section 4.3.3.3.

\subsubsection{1 $\quad$ Routine Releases}

The primary input parameters for the simulation of the SORAMI model on an hourly basis are described below. Rationales for the development of all parameter values were discussed with Dr. Frank Gifford as part of an expert elicitation process to account for the uncertainties in the input parameters. Input parameters for the chemistry characterization are presented in Section 4.2.3.

\section{Hourly Wind Speed, Wind Direction, and Atmospheric Stability}

The SORAMI model requires, for routine releases, the input of wind speed, wind direction, and atmospheric stability for each hour of simulation. Directly usable data in electronic form for the years 1944 to 1956 were not available even though summaries for some of these years are available (Holland, 1953). Some data were available from strip-chart recordings for 1950 and later years. Electronic data from the X-10 meteorological station MT2 (Oak Ridge Reservation Environmental Report, 1992) were available for ten years from 1987 to 1996. The data represented hourly measurements of the wind speed, wind-direction, and stability class at an elevation of $10 \mathrm{~m}$ above the ground. The data were also available at elevations of 30 and $100 \mathrm{~m}$. The data from 10-m elevation were used in accordance with standard industry practice. It should be noted that the use of either $30-\mathrm{m}$ or $100-\mathrm{m}$ data also requires an internal calculation by the 
model of wind speeds at the stack-tip elevation. These data were statistically analyzed to generate the wind speed, wind direction, and atmospheric stability data.

The objective of the statistical analysis was to generate the probabilities of obtaining specific values of wind speed $(u)$, wind direction $(d)$, and atmospheric stability $(s)$ for a given hour of the day for a given month. The following assumptions were made for the statistical analysis:

- Significant variations of $u, d$, and $s$ occur on an hourly scale. Once the hourly variations in $u, d$, and $s$ are accounted for, seasonal variations in the values of $u, d$, and $s$ are handled on a monthly scale. In other words, each month has a statistically different pattern of diurnal distributions of $u, d$, and $s$ from another month. Furthermore, within any month, each day is statistically the same as any other day in that month.

The rationale for the use of one month as the time scale for accounting for seasonal variations of $u, d$, and $s$ is driven primarily by the amount of available data. For example, for the month of January, the choice of the monthly time scale makes available 31 data points for a given hour of the day (e.g., 8 a.m.) from one year's meteorological data set. For the ten-year period between 1987 and 1996, it makes available $310(=31 \times 10)$ data points for that hour. Thus, 310 alternate values of $u, d$, and $s$ are available for the development of their probabilities of occurrence for a given hour in January.

If the time scale is made finer than 1 month, the number of data points is reduced and the uncertainties on the probabilities increase. For example, for a time scale of one week, only 7 data points are available from one year's data for a given hour, and from 10 years' data, only 70 points are available. Furthermore, there is no overwhelmingly compelling rationale for the meteorology of one week in January to be statistically different from that of another week. To test the hypothesis that the time-scale of one month to define meteorological parameters is adequate, field data on three weekly releases in the 1960s were used to validate SORAMI on a weekly basis. The results of this validation are presented in Section 4.3.3.4.

- The probabilities of the occurrences of $u, d$, and $s$ during a given hour of the day for a given month are assumed to be mutually dependent. In other words $u, d$, and $s$ are treated as correlated parameters. For any given hour, the meteorological data provide simultaneous measurements of these three parameters. This allows the generation of a joint probability distribution for the three parameters for a given hour of the day in a specific month using the definition of conditional probability:

$$
\mathrm{p}\left(u_{i, m}, s_{i, m}, d_{i, m}\right)=\mathrm{p}\left(d_{i, m}\right) \times \mathrm{p}\left(u_{i,\left.m\right|_{d_{i, m}}}\right) \times \mathrm{p}\left(s_{i, m \mid u_{i, m}, d_{i, m}}\right)
$$

where

$$
\begin{array}{ll}
i & =\text { subscript representing an hour of the day; } \\
m & =\text { subscript representing the month; } \\
p(u, s, d)= & \text { the joint probability of the simultaneous occurrence of events } u, s, \\
& \text { and } d ; \\
p(d) \quad= & \text { probability of event } d ;
\end{array}
$$




$$
\begin{array}{ll}
p\left(\left.u\right|_{d}\right) & =\text { probability of event } u \text { given that } d \text { has occurred; } \\
p\left(\left.s\right|_{u, d}\right) & =\text { probability of event } s \text { given that } u \text { and } d \text { have occurred; } \\
u & =\text { wind speed; } \\
d & =\text { wind direction; and } \\
s & =\text { atmospheric stability class. }
\end{array}
$$

Sampling from the joint-probability domain then permits the generation of implicitly correlated sets of $u, d$, and $s$ for a given hour of the day in a specific month.

The following approach was used to estimate $u, d$, and $s$ values for a given hour of the day in a specific month:

- Wind speed was divided into six classes, 0-1.5 $\mathrm{m} \mathrm{s}^{-1}, 1.5-3 \mathrm{~m} \mathrm{~s}^{-1}, 3-4.5 \mathrm{~m} \mathrm{~s}^{-1}, 4.5-6 \mathrm{~m} \mathrm{~s}^{-1}$, $6-7.5 \mathrm{~m} \mathrm{~s}^{-1}$, and $>7.5 \mathrm{~m} \mathrm{~s}^{-1}$; wind direction was divided into 36 sectors of $10^{\circ}$ each; and the atmospheric stability is divided into six discrete classes, A to F, according to the Pasquill-Gifford classification scheme.

Using the meteorological data from 1987 through 1996 (for each hour of the day for a given month) the cumulative joint probability distributions were developed for the simultaneous occurrences of a given wind speed class, wind direction class, and the atmospheric stability class using Equation 4-5. Specifically, the probability of the occurrence of each of the 36 wind directions was first developed for each hour of the day for each month of the year. This represents the first term in Equation 4.5. Next, the probability of the occurrence of each of the six wind speed classes for each wind direction class was developed. This represents the second term in Equation 4.5. Finally, the probability of the occurrence of each atmospheric stability class for each wind speed class and for each wind direction class was developed. This represents the last term in Equation 4.5.

- The probability density functions (PDFs) representing the three terms on the right hand side of Equation 4.5, developed in the previous step, were then sampled using a Latin Hypercube Monte Carlo sampling scheme (NCRP, 1996). First the PDF for wind direction $(d)$ is sampled for a given hour of day for a given month. Once the wind direction is known, the PDF for the wind speed $(u)$ for the just-sampled wind direction is sampled for the same hour of the day for the same month. Finally, the PDF for the stability class $(s)$ is sampled for the just-sampled wind speed and wind direction. This process is repeated 400 times for each hour of the day for the 12 calendar months.

- The 12 sets of 400 values of implicitly correlated $d, s$, and $u$ for each hour of the day for each month were then used to generate the meteorological input files in the format required by SORAMI.

\section{$\underline{\text { Lateral and Vertical Dispersion Coefficients }}$}

For the rural simulation mode, SORAMI uses Briggs' approximations (Hanna et al., 1982) to the Pasquill-Gifford curves (Turner, 1970) for the estimation of $\sigma_{\mathrm{y}}$ and $\sigma_{\mathrm{z}}$, the lateral and vertical 
dispersion coefficients, respectively. An uncertainty factor was assigned to each of these estimates. The uncertainty factor was sampled using a uniform distribution between 0.67 and 1.5 for each stability category. The uncertainty factor is used to multiply the values of $\sigma_{\mathrm{y}}$ and $\sigma_{\mathrm{z}}$ estimated by the Briggs' approximation for each stability class. The upper and lower bounds of the uncertainty factor were developed such that the lower and upper bounds of one class are very close to the expected value of the adjacent classes. In other words, on a plot of $\sigma_{\mathrm{y}} \mathrm{vs}$. downwind distance (or $\sigma_{\mathrm{z}}$ vs. distance), a band of probable values of $\sigma_{\mathrm{y}}\left(\right.$ or $\sigma_{\mathrm{z}}$ ) was developed around the expected curve.

\section{Mixing Heights}

When the vertical extent of the plume becomes equal to the mixing height at a given downwind distance from the source, the plume extent in the vertical is assumed to become equal to the mixing height beyond that distance, and the contaminant is assumed to be uniformly distributed in the vertical dimension below the mixing height. Mixing heights representative of different stability classes were obtained from Holzworth (1972) for the Oak Ridge region. Holzworth (1972) provides contours and tables of the morning and afternoon annual average mixing heights for the contiguous United States. Uniform distributions of mixing heights were developed from the contours and tables. Afternoon conditions were assumed to represent stability classes A and $\mathrm{B}$ (unstable categories), morning conditions were assumed to represent stability classes $\mathrm{E}$ and $\mathrm{F}$ (stable categories), and the information for stability classes C and D (neutral category) was derived from the values for stable and unstable conditions. Sufficiently large ranges of parameter values were used to represent the uncertainty in the knowledge of mixing heights. Table 4.2 presents the mixing height data used in the analysis. For each of the 400 alternate realizations of $u, d$, and $s$, the mixing height is sampled from the appropriate distribution in Table 4.2 depending on the sampled stability category (values of $s$ ).

Table 4.2 Mixing height data used for analysis. A uniform distribution was chosen between the minimum and maximum values.

\begin{tabular}{lcc}
\hline Stability Category & Minimum mixing height $(\mathbf{m})$ & Maximum mixing height $(\mathbf{m})$ \\
\hline A, B (unstable) & 1000 & 2000 \\
C, D (neutral) & 500 & 1500 \\
E, F (stable) & 300 & 650 \\
\hline
\end{tabular}

Release information such as emission rate, height and diameter of the stack, and temperature and velocity of the effluent releases.

A unit emission rate of ${ }^{131} \mathrm{I}$ was prescribed for each hour to obtain the ground-level air concentration per unit release $(\chi / Q)$, which can then be multiplied by the actual emission rate to estimate the hourly air concentration for each given hour at the desired location of concern. Table 4.3 presents the values of other parameters used in the analysis. As mentioned earlier, Stack 3020 was used for ${ }^{131}$ I releases between 1944 and 1950, and Stack 3039 was used between 
1950 and 1956. However, because of the close proximity and elevations of the two stacks, it was decided to treat all releases as having occurred from Stack 3020.

Table 4.3 Stack Release Parameters

\begin{tabular}{ccccc}
\hline Stack & $\begin{array}{c}\text { Release } \\
\text { Elevation }(\mathbf{m})\end{array}$ & $\begin{array}{c}\text { Release } \\
\text { Temperature }\left({ }^{\circ} \mathbf{K}\right)\end{array}$ & $\begin{array}{c}\text { Release } \\
\text { Velocity }\left(\mathbf{m ~ s}^{-\mathbf{1}}\right)\end{array}$ & $\begin{array}{c}\text { Stack } \\
\text { Diameter (m) }\end{array}$ \\
\hline 3020 & 80 & 293 & 26 & 0.9 \\
3039 & 81 & 293 & 12 & 2.4 \\
\hline
\end{tabular}

The plume rise for the two stacks would be different because of differences in the release velocities of the stack gases and the stack diameters. Differences in plume rise can lead to different effective release heights for the two stacks and hence differences in predicted groundlevel concentrations in air. Since releases from both stacks occurred at ambient temperatures, the plume rise because of the buoyancy of the exit gases can be neglected. Momentum plume rise is a function of the stack exit velocity, stack diameter, prevailing wind speeds, downwind distance, and atmospheric stability.

Plume rises were estimated using the same mathematical formulations as used by SORAMI for the two stacks under various combinations of wind speed, downwind distance, and atmospheric stability. The effect of the plume rise on the predicted ground-level air concentrations, according to Equation 4.5, was then evaluated as a ratio of the concentration predicted by Stack 3020 to the concentration predicted by Stack 3039. The ratio was found to decrease and approach 1 as a function of increasing downwind distance, increasing velocity, and decreasing atmospheric stability (as stability class decreases from $\mathrm{F}$ to $\mathrm{A}$ ). For a downwind distance of $5 \mathrm{~km}$ and wind speed of $1 \mathrm{~m} \mathrm{~s}^{-1}$, the ratios were estimated as $1,1,1,1.2,2$, and 3.4 for stability classes $\mathrm{A}, \mathrm{B}, \mathrm{C}$, $\mathrm{D}, \mathrm{E}$, and F, respectively. For a downwind distance of $30 \mathrm{~km}$ and wind speed of $1 \mathrm{~m} \mathrm{~s}^{-1}$, the ratios were estimated as $1,1,1,1,1.4$, and 1.7 for stability classes $\mathrm{A}, \mathrm{B}, \mathrm{C}, \mathrm{D}$, E, and F, respectively. The median and mean wind speeds at the X-10 site (using hourly meteorological data between 1987 and 1996) were estimated as $1.2 \mathrm{~m} \mathrm{~s}^{-1}$ and $1.4 \mathrm{~m} \mathrm{~s}^{-1}$ respectively, and stability class D was observed as the most frequent atmospheric stability ( $40 \%$ of all occurrences).

On an annual average basis, therefore, the net effect of these differences in plume rise on the predicted ground-level concentrations is not expected to be significant. This was verified by the separate estimations of the predicted downwind concentrations at 4 and $20 \mathrm{~km}$ distances from the two stacks using a public-domain model ISC2-LT (USEPA, 1992) and annual average meteorological data obtained from the measurements between 1987 and 1994 at the MT2 Station. The results of this analysis showed that the maximum value of the ratio of the median annual average concentration predicted by using Stack 3020 to that predicted by using Stack 3039 was 1.1 at a 4-km distance. Therefore, it was decided to use Stack 3020 for estimating the ground-level air concentrations for all years between 1944 and 1956. Additional validation of SORAMI was conducted, as discussed in Section 4.3.3.3, to verify that Stack 3020 can be used to model releases for all years between 1944 and 1956. 


\section{$\underline{\text { Ambient Temperature }}$}

Ambient temperature was assumed to remain at $293^{\circ} \mathrm{K}$. The temperature data are used in the model to calculate the buoyancy plume rise from the stack release as a function of the temperature difference between the ambient air and stack effluents. Because effluents were released to the stack at ambient conditions, the contribution of buoyancy to the plume rise would be small compared to the momentum contribution.

\section{Dry and Wet Deposition Velocities}

Dry and wet deposition processes contribute to the depletion of radioiodine from the plume. They also contribute to the accumulation of radioiodine on the pasture grass. Depletion of radioiodine from the plume is controlled by deposition processes that must account for the presence of all major landforms and features in the plume path. Since SORAMI uses a Gaussian plume approach, an average value of the deposition velocity representative of the study region is required. At a given downwind location, the air concentration will be controlled by the depletion that occurred between the source and that location. Therefore, the deposition velocities to account for the depletion must be representative of all features between the source and that location. However, the amount deposited on the pasture grass or other vegetation at that location will be controlled only by the deposition rates on the pasture grass or other vegetation that is present. Therefore, deposition velocities for estimating concentrations of ${ }^{131} \mathrm{I}$ in air and on pasture grass or other vegetation are treated differently.

The wet deposition component of the total deposition velocity (Equation 4.4) is independent of the landform features. It is dependent only on the amount of rainfall and rainfall intensities. Therefore, the wet deposition component should be treated in the same manner to account for depletion as well as for deposition on pasture grass. However, the dispersion analysis, which includes the depletion process, is conducted using an hourly time step; whereas the analysis of deposition to pasture grass and other vegetation is conducted on an annual average basis. The differences in the parameterization for the two time scales of analysis, the approaches for the evaluation of wet deposition velocities, and the choice of input parameters are presented in Section 5. Description of the development of hourly rainfall data for the Oak Ridge region for any day of a given month is presented in Section 5. Since direct measurements for all years of concern (1944-1956) were not available, the rainfall data were statistically prepared in a manner similar to that used for the preparation of the meteorological data from a number of years of available data.

Information on the values of dry deposition velocities for the Oak Ridge region were obtained from Dr. Tilden Meyers of the National Oceanic and Atmospheric Association's Atmospheric Turbulence and Diffusion Division (ATDD) at Oak Ridge (Meyers, 1998) and from Dr. Stephen Lindberg of the Oak Ridge National Laboratory (1998). For the dry deposition of iodine in elemental form, a uniform distribution between 1 and $6 \mathrm{~cm} \mathrm{~s}^{-1}$ was selected as representative of a study region consisting of forest canopies and pastureland. This range of values is consistent with measurements of other highly reactive chemical species, such as gaseous $\mathrm{HNO}_{3}$, in the Oak Ridge region. For the deposition of iodine in the particulate form, a log-uniform distribution between 0.05 and $0.5 \mathrm{~cm} \mathrm{~s}^{-1}$ was selected for the study region. This range of values is consistent 
with measurements on submicron size particles, such as sulfate aerosols, in the Oak Ridge region. For the organic form, a log-uniform distribution between 0.001 and $0.05 \mathrm{~cm} \mathrm{~s}^{-1}$ was selected, consistent with the deposition velocities presented in Section 5. The organic form of iodine is extremely nonreactive. Hence, the range of values used is applicable to deposition on both forest canopy and pasture grass.

\section{Release Geometry for Releases from Buildings}

The building that housed the radioactive lanthanum processing facility was approximately $29 \mathrm{~m}$ $\times 30 \mathrm{~m}$ in area (Clinton Laboratory Drawing No. CL-706D-9 dated November 18, 1944). The building was $12 \mathrm{~m}$ tall at its highest point (Clinton Laboratory Drawing No. CL-706-D-54 dated January 8, 1945). Since releases from the building occurred from vents and openings at various levels within the building, an average, constant release height of $10 \mathrm{~m}$ was chosen. The entire release is treated as a single area source with a diameter that marginally exceeds the longest diagonal of the building. The diameter was treated as an uncertain parameter with a uniform distribution varying from $29 \mathrm{~m}$ to $44 \mathrm{~m}$. As mentioned earlier, the area source is converted into a virtual point source at an elevation of $10 \mathrm{~m}$ and upwind of the building. The distance from the building to the virtual point source is estimated on the basis of the source diameter at the building and the atmospheric stability, both being expressed as uncertain variables. The distance from the virtual source as well as the vertical extent of the plume at the location of the building (downwind from the virtual point source) then automatically emerge as uncertain variables because of the treatment of the prescribed release height of $10 \mathrm{~m}$ as the height of the virtual source. Therefore, it is appropriate to describe the release height as a constant.

Ramsdell and Fosmire (1998) show that the building wake effects are important for predictions in the first few hundred meters from the source (typically 200-300 m). They also show that most building wake models in use, such as Fuquay model (Gifford, 1968), NRC Regulatory Guide 1.145 (USNRC, 1982), Ramsdell's model (1990), and Huber's model (1984), converge to openterrain solutions obtained from Gaussian plume models beyond 300-400 $\mathrm{m}$ from the source. Since the receptor locations of interest for this study are beyond $3 \mathrm{~km}$ from the RaLa processing facility, it was decided that it was not necessary to model the building wake effects from the Building 706-D (housing the RaLa processing facility) or those from the presence of other buildings in the vicinity. This assumption may still have serious implications on predictions within the first 300-400 m as shown by Ramsdell and Fosmire (1998), but very little impact on predictions at distances beyond $3 \mathrm{~km}$.

\subsubsection{1954 Accident}

As discussed in Section 3, the accident on April 29, 1954, started sometime before 5:00 p.m. and resulted in releases of ${ }^{131} \mathrm{I}$ until 7:00 p.m. Releases from the stack ceased at about 5:30 p.m., while direct releases from the building continued to occur until 7:00 p.m. Meteorological parameters were obtained from an inter-company (Oak Ridge National Laboratory) correspondence from P. R. Guinn to J. C. Hart (Guinn, 1954). These parameters represent data at half-hour intervals from the X-10 Health Physics Group Meteorological Station 012, a midvalley location within Bethel Valley (Holland, 1953; Hillsmeier, 1963). Table 4.4 presents the data from Guinn (1954). 
Meteorological parameters were also available from the same station in the form of strip charts, which were converted to hourly data records at the National Oceanic and Atmospheric Association's ATDD facility at Oak Ridge. These data were interpreted using Holland (1953) and Hillsmeier (1963) and after consultations with Dr. R. P. Hosker and Dr. W. Pendergrass of the National Oceanic and Atmospheric Association's ATDD facility at Oak Ridge and with Dr. F. A. Gifford. These data, available at hourly intervals, are presented in Table 4.5.

Table 4.4 Weather conditions between 4 and 8 p.m. on April 29, 1954 (Guinn, 1954), from the X-10 Health Physics Group Meteorological Station 012.

\begin{tabular}{cccccc}
\hline Time (p.m.) & $\begin{array}{c}\text { Temperature } \\
\left({ }^{\circ} \mathbf{F}\right)\end{array}$ & $\begin{array}{c}\text { Relative } \\
\text { Humidity }(\%)\end{array}$ & $\begin{array}{c}\text { Temperature } \\
\text { Difference }\left({ }^{\circ} \mathbf{F}\right)\end{array}$ & $\begin{array}{c}\text { Direction } \\
\text { (from) }\end{array}$ & $\begin{array}{c}\text { Speed } \\
\text { (miles/hour) }\end{array}$ \\
\hline $4: 00$ & 78 & 57 & -2 & $\mathrm{~W}$ & 9 \\
$4: 30$ & 73 & 95 & -2 & $\mathrm{~S}$ & 12 \\
$5: 00$ & 74 & 70 & -1 & $\mathrm{SE}$ & 8 \\
$5: 30$ & 75 & 66 & -1 & $\mathrm{~S}$ & 8 \\
$6: 00$ & 73 & 69 & -1 & $\mathrm{~S}$ & 6 \\
$6: 30$ & 65 & 90 & -1 & $\mathrm{~S}$ & 15 \\
$7: 00$ & 65 & 95 & -1 & $\mathrm{DI}$ & 12 \\
$7: 30$ & 64 & 100 & -1 & $\mathrm{DI}$ & 3 \\
8:00 & 65 & 100 & -1 & $\mathrm{DI}$ & 1 \\
\hline
\end{tabular}

Table 4.5 Meteorological data from strip-chart records for April 29, 1954, from X-10 Health Physics Group Meteorological Station 012 within Bethel Valley.

\begin{tabular}{cccccc}
\hline Time (pm) & $\begin{array}{c}\text { Wind Direction } \\
\text { (from) }\end{array}$ & $\begin{array}{c}\text { Wind Speed } \\
(\text { miles/hour) }\end{array}$ & $\begin{array}{c}\text { Rainfall } \\
\text { (inches) }\end{array}$ & $\begin{array}{c}\text { Temperature } \\
\left({ }^{\circ} \mathbf{F}\right)\end{array}$ & $\begin{array}{c}\text { Temperature } \\
\text { Difference }\left({ }^{\circ} \mathbf{F}\right)\end{array}$ \\
\hline $4: 00$ & SSE & 8 & 0 & 74 & -2 \\
5:00 & S & 5 & 0 & 73 & -1 \\
6:00 & SSE & 9 & 0.67 & 65 & -1 \\
7:00 & E & 2 & 0 & 65 & -1 \\
$8: 00$ & N & 0 & 0 & 65 & 0 \\
\hline
\end{tabular}


Comparison of the two data sets indicates differences in the half-hour-averaged and hourlyaveraged values. Measurements of contamination following the accident in the immediate vicinity of the site indicated presence of radioactivity along a northwest direction, as shown in Figure 4.1. The half-hour-averaged data in Table 4.4 indicates winds to the northwest at 5:00 p.m. It was, therefore, decided to use the half-hour-averaged meteorology for the analysis of the accident. Uncertainties in the measurement of parameter values were addressed using the approach presented below.

\section{$\underline{\text { Wind Direction }}$}

Each wind direction from Table 4.4 was interpreted as representing a $22.5^{\circ}$ sector from which the wind blew towards the monitoring station. Each reading would, therefore, represent a full sector of $22.5^{\circ}$, and the maximum uncertainty associated with each reading would be $\pm 11.25^{\circ}$ on either side of the designated direction. For each of the five half-hour periods of the accident, a maximum uncertainty of $\pm 11.25^{\circ}$ was accordingly assigned about the readings recorded in Table 4.4, and the direction was sampled using a uniform distribution. For example, if the direction from which the wind is blowing is indicated as SE (135 clockwise from north), the actual wind direction was specified as a random value from a uniform distribution from $125.75^{\circ}$ to $146.25^{\circ}$ representing a $\pm 11.25^{\circ}$ region about $\mathrm{SE}$.

\section{Wind Speed}

To address the uncertainty associated with a given integer value of the wind speed in Table 4.4, the lower limit of wind speed was chosen as 0.5 subtracted from the integer value, and the upper limit of wind speed was chosen as 0.5 added to the integer value. Thus, a value of $8 \mathrm{mph}$ was treated as a uniform distribution from 7.5 to $8.5 \mathrm{mph}$, and a value of $3 \mathrm{mph}$ was treated as a uniform distribution between 2.5 and $3.5 \mathrm{mph}$.

\section{Atmospheric Stability and Dispersion Coefficients}

The temperature differences presented in Table 4.4, which are consistent with those in Table 4.5, were used to evaluate the stability during a given hour. The temperature difference was interpreted as representing the atmospheric temperature difference between two measuring heights. The reading was converted into temperature difference per unit rise in elevation. The two elevations were presented in Holland (1953) as $4 \mathrm{ft}$ and $183 \mathrm{ft}$, whereas in Hillsmeier (1963), they were presented as $5 \mathrm{ft}$ and $145 \mathrm{ft}$. However, for each of the five half-hour periods, the same stability class was obtained using either of the two sets of values for elevation. Atmospheric stabilities were estimated from the stability classification procedure promulgated by the Nuclear Regulatory Commission and presented in Brenk et al. (1983). Vertical and horizontal dispersion coefficients $\left(\sigma_{z}\right.$ and $\sigma_{y}$, respectively) are directly affected by the stability category. Uncertainty in $\sigma_{\mathrm{z}}$ and $\sigma_{\mathrm{y}}$ were assigned in the same manner as presented in Section 4.3.2.1 for routine releases. 


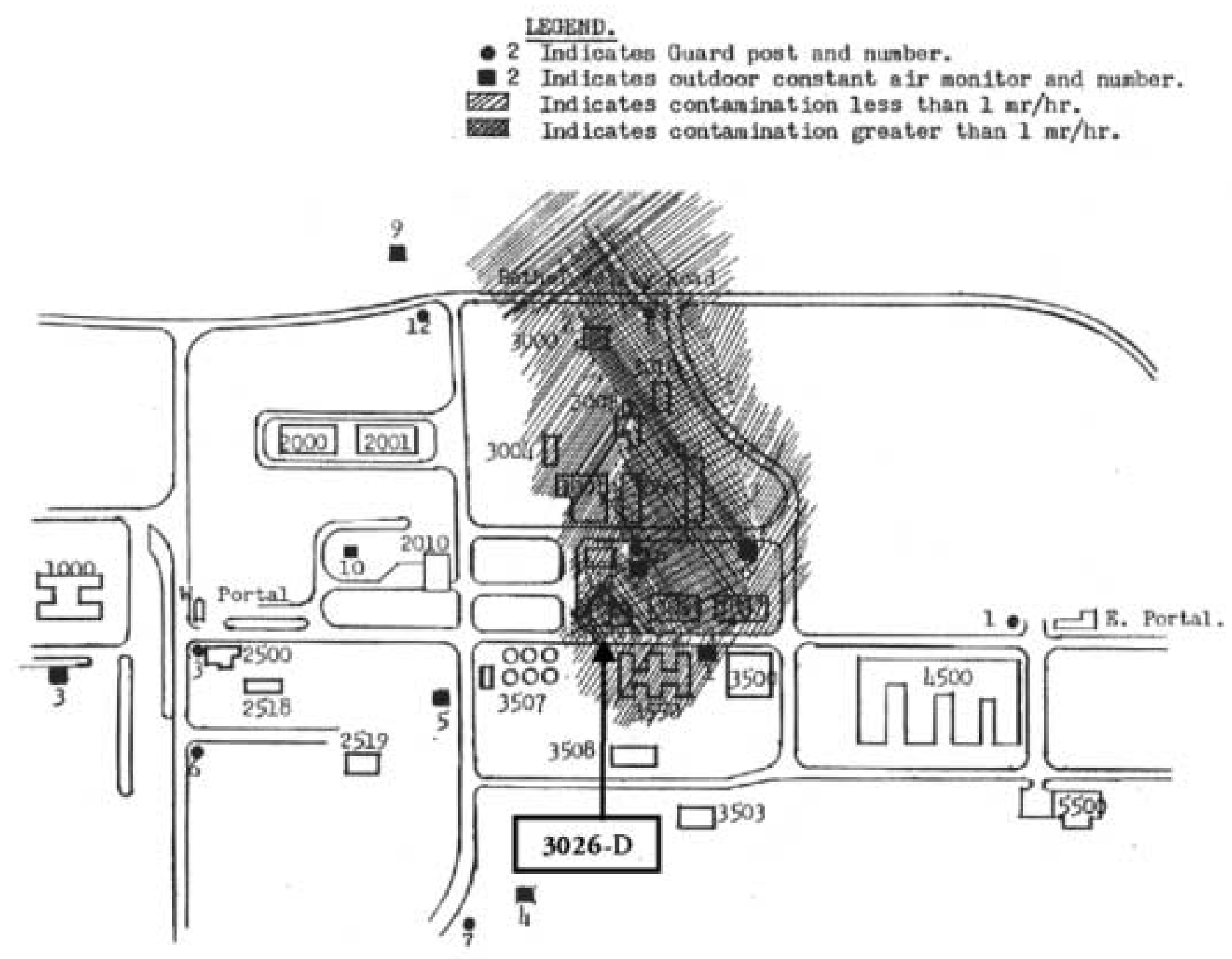

Figure 4.1: Map indieating the extent of contamination from the April 29, 1954 aceident (adapted from Guinn, 1954). 


\section{Other Parameters}

Other parameters are treated in the same manner as described in Section 4.3.2.1 for routine releases. Input parameters for the chemistry characterization are obtained according to the methods presented in Section 4.2.2. Input parameters for dry deposition velocity were obtained in the same manner as was done for the simulation of routine releases for each chemical form of

${ }^{131}$ I. Wet deposition rates are primarily dependent on the occurrence of rainfall during the hours of the accident. The amount of rainfall is obtained directly from the hourly records as presented in Table 4.5. Table 4.5 indicates that there was only one period, between 6:00 and 7:00 p.m., during which any precipitation occurred on April 29, 1954. The amounts of rainfall in the two half-hour periods between 6:00 and 7:00 p.m., however, are unknown. However, Guinn (1954) states that the rain started at 6:15 p.m. This means that there was a 15-min. period in the first half-hour period and the full 30-min. period during the second half-hour period during which precipitation occurred. Assuming that the rainfall intensity was fairly uniform during the 45minute period, the first fifteen minutes, between 6:15 and 6:30 p.m. would account for one-third ( 0.22 inches) of the entire rainfall ( 0.67 inches). It was decided to use this value as the most likely value of the rainfall in the period between 6:00 and 6:30 p.m. A triangular distribution with a large range spanning 0.11 to 0.44 inches was assigned to account for the uncertainty in the knowledge of the rainfall intensity during this period. Rainfall for the second period between 6:30 and 7:00 p.m., was then obtained as the difference between the total rainfall and the rainfall between 6:00 and 6:30 p.m. An uncertainty of $\pm 10 \%$ was assigned, along with a triangular distribution with a most likely value of 1 , to the total rainfall for the entire period to account for any errors in measurements. Estimation of the wet deposition velocities of individual chemical forms for the accidental release using a half-hourly time step is conducted in the manner presented in Section 5.

\subsubsection{Model Validation for Routine Releases}

Annual average release rates from Bethel Valley Stacks 3039, 3020, and 2026 and Melton Valley Stacks 7911 and 7512, and measured ground-level air concentrations from 9 monitoring locations (Figure 4.2) surrounding the X-10 facility were available for the 1967-1969 period. Further monitoring data after 1974 are also available. However, it is not clear whether the source of ${ }^{131} \mathrm{I}$ during these years was the X-10 stack in Bethel Valley. Data beyond 1987 clearly indicates that more than $65 \%$ of the releases occurred from Melton Valley Stack 7911.

Using the hourly meteorological data, generated according to the discussions presented in Section 3.2.1 and constant release rates matching the reported releases in 1967, 1968, and 1969 (Table 4.6), the annual average concentrations were estimated for the 9 monitoring locations using the SORAMI model with and without including the processes of plume depletion, wet and dry deposition, and iodine chemistry. The purpose of the simulation without the inclusion of these processes was to benchmark SORAMI with a public domain model, ISC3-ST (USEPA, 1995). For this simulation all of the releases were assumed to have occurred from Stack 3020. 


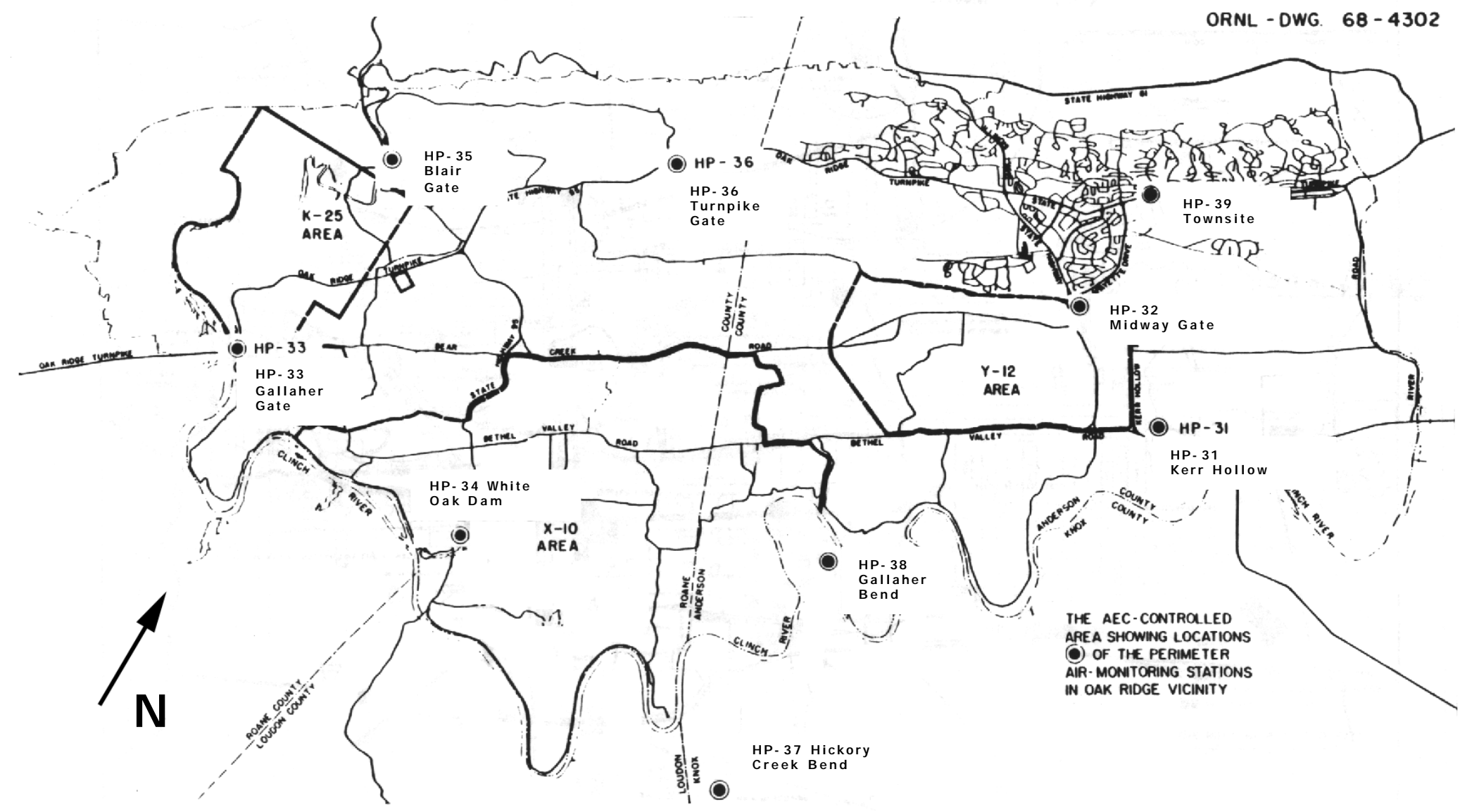

Figure 4.2: $\quad$ Locations of the Health Physics Group monitoring stations. 
Table 4.6 Amounts of ${ }^{131}$ I released from Bethel and Melton Valley Stacks between 1967 and 1969 (Binford et al., 1970).

\begin{tabular}{|c|c|c|c|c|c|c|}
\hline \multirow[b]{2}{*}{ Year } & \multicolumn{3}{|c|}{$\begin{array}{c}\text { Releases from } \\
\text { Bethel Valley Stacks }\left(10^{10} \mathrm{~Bq}\right) \\
\end{array}$} & \multicolumn{2}{|c|}{$\begin{array}{c}\text { Releases from } \\
\text { Melton Valley Stacks }\left(10^{10} \mathrm{~Bq}\right)\end{array}$} & \multirow{2}{*}{$\begin{array}{c}\begin{array}{c}\text { Total } \\
\text { Releases }\end{array} \\
\left(1^{10} \mathbf{B q}\right)\end{array}$} \\
\hline & Stack 2026 & Stack 3039 & Stack 3020 & Stack 7512 & Stack 7911 & \\
\hline 1967 & $\begin{array}{c}11 \\
(13 \%)\end{array}$ & $\begin{array}{c}64 \\
(78 \%) \\
\end{array}$ & $\begin{array}{c}0.6 \\
(1 \%)\end{array}$ & $\begin{array}{c}0 \\
(0 \%)\end{array}$ & $\begin{array}{c}7 \\
(8 \%)\end{array}$ & 83 \\
\hline 1968 & $\begin{array}{c}2.2 \\
(6 \%) \\
\end{array}$ & $\begin{array}{c}27 \\
(72 \%)\end{array}$ & $\begin{array}{c}0.2 \\
(1 \%)\end{array}$ & $\begin{array}{c}1.2 \\
(3 \%)\end{array}$ & $\begin{array}{c}6.8 \\
(18 \%)\end{array}$ & 38 \\
\hline 1969 & $\begin{array}{c}23 \\
(39 \%)\end{array}$ & $\begin{array}{c}30 \\
(51 \%)\end{array}$ & $\begin{array}{l}0.1 \\
(0 \%)\end{array}$ & $\begin{array}{c}3.0 \\
(5 \%)\end{array}$ & $\begin{array}{c}2.6 \\
(4 \%)\end{array}$ & 59 \\
\hline
\end{tabular}

Two separate simulations were made for the case that included the processes of plume depletion, deposition, and iodine chemistry: (1) all releases were treated as having occurred from Stack 3020 and (2) releases were treated as having occurred from Stack 3020 and Stack 7911. The purpose of conducting the first of these simulations was to study the effect of including the processes of plume depletion, deposition, and iodine chemistry by comparing the result of this simulation with the results from the benchmarking exercise. The purpose of the second simulation, where two sources were used, was to conduct a more realistic simulation of the releases. For all Bethel Valley releases, this simulation used Stack 3020 as the point of release, and for all Melton Valley releases, it used Stack 7911, which served the HFIR, TRU, and TUFCDF operations at ORNL, as the point of release.

Measurements at the monitoring stations involved the adsorption of gaseous iodine in an activated charcoal canister located downstream from a Vose LB-5211 particulate filter (Oakes et al., 1981). The collection efficiency of particulate iodine was reported to be greater than $99 \%$, and the collection efficiency of methyl iodide was reported to be greater than $60 \%$ depending on factors such as relative humidity (Oakes et al., 1981). The measured concentrations were converted to annual average ground-level concentrations of ${ }^{131} \mathrm{I}$ in air and were reported by Binford et al. (1970). Manneschmidt (1961) presents information on stack monitors that were placed on all stack samplers in 1959. The stack samplers were described as inventory type samplers, consisting of a filter and a charcoal cartridge. Data presented in Table 4.6 were developed from a series of monthly reports authored by L. C. Lasher (Lasher, 1967a; 1967b; 1967c; 1967d; 1967e; 1967f; 1967g; 1967h; 1967i; 1967j; 1968a; 1968b; 1968c; 1968d; 1968e; 1968f; 1968g; 1968h; 1968i; 1968j; 1968k; 1969a; 1969b; 1969c; 1969d; 1969e; 1969f; 1969g; 1969h; 1969i; 1969j; 1969k; 1969l; 1969m; 1970a; 1970b). It should be noted that individual quantities of iodine in the three chemical forms or the quantities released or measured at the monitoring stations in gaseous or particulate forms were not separately reported and are, therefore, unavailable. Table 4.7 presents the annual average concentrations of ${ }^{131} \mathrm{I}$ at the nine monitoring stations. Table 4.7 also presents the distance and direction of each monitoring station from the RaLa processing facility. 
Table 4.7 Measured annual average ground-level concentrations of ${ }^{131} \mathrm{I}$.

\begin{tabular}{|c|c|c|c|}
\hline \multirow[t]{2}{*}{$\begin{array}{l}\text { Ambient }{ }^{131} \text { I monitoring station; distance and } \\
\text { Direction }^{\text {a }} \text { of the station from the RaLa Stack }\end{array}$} & \multicolumn{3}{|c|}{$\begin{array}{c}\text { Measured Concentrations } \\
\left(\mathbf{1 0}^{-4} \mathrm{~Bq} \mathrm{~m}^{-3}\right)\end{array}$} \\
\hline & 1967 & 1968 & 1969 \\
\hline Kerr Hollow (HP-31); 11.4 km, 49 & 10.4 & 5.18 & 8.51 \\
\hline Midway Gate (HP-32); $10.6 \mathrm{~km}, 37^{\circ}$ & 7.03 & 3.33 & 7.03 \\
\hline Gallaher Gate (HP-33); $7.4 \mathrm{~km}, 274^{\circ}$ & 8.51 & 4.44 & 8.88 \\
\hline White Oak Dam (HP-34); $3.1 \mathrm{~km}, 189^{\circ}$ & 9.62 & 8.88 & 7.40 \\
\hline Blair Gate (HP-35); $7.0 \mathrm{~km}, 292^{\circ}$ & 3.70 & 3.70 & 5.18 \\
\hline Turnpike Gate (HP-36); $8.8 \mathrm{~km}, 14^{\circ}$ & 3.33 & 3.33 & 3.70 \\
\hline Hickory Creek Bend (HP-37); $7.6 \mathrm{~km}, 119^{\circ}$ & 2.96 & 3.70 & 4.07 \\
\hline Gallaher Bend (HP-38); $13.8 \mathrm{~km}, 31^{\circ}$ & 8.51 & 6.66 & 9.25 \\
\hline Townsite (HP-39); $5.6 \mathrm{~km}, 78^{\circ}$ & 6.29 & 5.18 & 4.44 \\
\hline
\end{tabular}

It should be noted that all releases from the Bethel Valley Stacks were treated as having occurred from Stack 3020 and all releases from the Melton Valley were treated as having occurred from Stack 7911. Elevations of these stacks are presented in Figure 4.3. As seen from Table 4.6, most of the releases occurred from the Bethel Valley stacks for each of the three years. Release from Bethel Valley in turn was dominated by Stack 3039, while that from the Melton Valley was dominated by Stack 7911. Stack 7911 is located 1,300 m east and 1,070 m south of Stack 3039. It was assumed that the meteorological data from the Bethel Valley Station MT2 are applicable to the meteorology of the Melton Valley.

As discussed in Section 4.3.2.1, the hourly meteorological input for a given month is representative of any day in the month. Therefore, 400 alternate realizations of the ratio of air concentration at a receptor point to the source term $\left(\chi / Q\right.$ in $\left.\mathrm{s} \mathrm{m}^{-3}\right)$ for each hour of the day for each of the twelve months were developed using Equations 4.1 through 4.4. From these, 400 alternate one-day average values of the $\chi / Q$ ratios were developed for each month by averaging over the 24 hours. These 400 values were then randomly sampled with replacement, 31 times, and the 31 values were summed to represent one alternate realization of the 31-day timeintegrated $\chi / Q$ ratio for the month of January. The same process was repeated 400 times to represent 400 alternate realizations of the 31-day time-integrated concentrations for January. The process was repeated for each calendar month. For each month, the lower bound, central estimate, and upper bound of the $95 \%$ subjective confidence interval and arithmetic mean values were developed from the 400 alternate realizations.

The largest scale of meteorological data analysis for the development of the meteorological input data was one month. Because the fluctuations from month to month in the 400 alternate realizations would cancel each other out during the process of addition, the 400 individual values of the monthly time-integrated $\chi / Q$ ratios from one month cannot be directly added to those of the next month in order to obtain 400 values of the annual average $\chi / Q$ ratios. For this reason, the lower bound, central estimate, and upper bound values of the $95 \%$ subjective confidence 


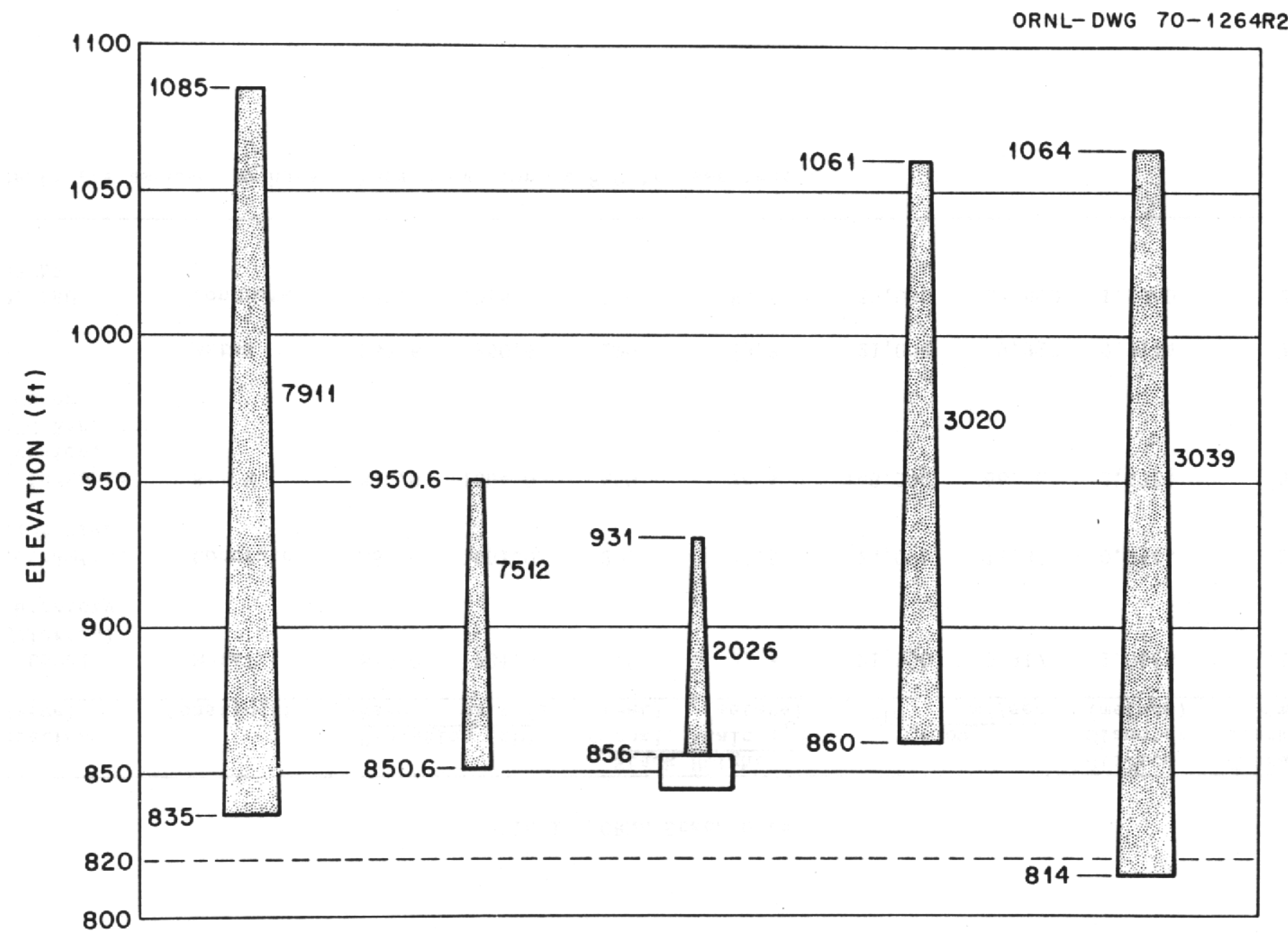

Figure 4.3: Elevations of Bethel and Melton Valley stacks (Binford et al., 1970). 
interval and the arithmetic means of the $\chi / Q$ ratios for all the 12 months were summed up and divided by 365 days to obtain, respectively, the lower bound, central estimate, and upper bound values of the $95 \%$ subjective confidence interval and the arithmetic means of annual average $\chi / Q$ ratios. Concentrations at each location of interest were then estimated using Equation 4.1.

\subsubsection{Model validation without plume depletion, deposition, and iodine chemistry}

The first analysis was conducted without including the processes of plume depletion, deposition, and chemistry components to ensure that SORAMI, developed using Equations 4.1 through 4.4, provides similar results to those obtained from field measurements and from a public-domain model, ISC3-ST (USEPA, 1995). It should be noted that the ISC3-ST model does not handle chemical transformations, and its wet deposition formulation is different from the formulation used in this study (Section 5). Therefore, for benchmarking purposes, it was decided to use both SORAMI and ISC3-ST without including these processes. As stated earlier, all of the releases were assumed to have occurred from Stack 3020 for these simulations. Ratios of predicted estimates of the central value (median) of the annual average ground-level concentrations of ${ }^{131} \mathrm{I}$ in air at each of the nine monitoring stations were divided by the measured annual average concentrations presented in Table 4.7 to obtain the $\mathrm{P}$ (predicted)/O (observed) ratio for each of the nine monitoring stations as shown in Table 4.8. Predictions from the SORAMI model are seen to be consistently higher than the ISC3-ST predictions. The reasons for this are not clear. Predictions from the ISC3-ST are seen to be within a factor of 3 of the observations, while those from SORAMI are within a factor of 2.

Table 4.8 Comparison of the $\mathrm{P} / \mathrm{O}$ ratios of the annual average concentrations of ${ }^{131} \mathrm{I}$ from the SORAMI model and the public-domain model, ISC3-ST (USEPA, 1995).

\begin{tabular}{lcc}
\hline \multicolumn{1}{c}{$\begin{array}{c}\text { Ambient } \\
\text { monitoring station }\end{array}$} & $\begin{array}{c}\text { Geometric mean of P/O Ratios } \\
\text { Using the ISC3-ST } \\
\text { model }\end{array}$ & $\begin{array}{c}\text { Using the SORAMI } \\
\text { model }\end{array}$ \\
\hline Kerr Hollow (HP-31) & 0.80 & 0.99 \\
Midway Gate (HP-32) & 0.84 & 1.05 \\
Gallaher Gate (HP-33) & 0.34 & 0.45 \\
White Oak Dam (HP-34) & 0.85 & 1.19 \\
Blair Gate (HP-35) & 0.37 & 0.51 \\
Turnpike Gate (HP-36) & 0.99 & 1.26 \\
Hickory Creek Bend (HP-37) & 1.72 & 2.05 \\
Gallaher Bend (HP-38) & 1.74 & 2.00 \\
Townsite (HP-39) & 0.51 & 0.69 \\
\hline Average of the Geometric Mean P/O ratios & 0.91 & 1.13 \\
\hline
\end{tabular}




\subsubsection{Model validation with plume depletion, deposition, and iodine chemistry}

Implementation of the source depletion formulation within the SORAMI model, based on Equations 4.1 through 4.4, was first independently verified using the example problems provided in Horst $(1984 ; 1977)$. Using the input parameters presented in Section 4.3.2.1, the model was then tested with the plume depletion, dry and wet deposition, and chemistry components. Since amounts of iodine released in individual chemical forms during the 1967-1969 period are not known, it was decided to estimate the concentrations of iodine at the 9 receptor locations on the basis of three different assumptions: (1) all of the iodine was released in elemental form; (2) all of the iodine was released in the organic form; and (3) all of the iodine was released in the particulate form. As explained in Section 4.2, $1 \mathrm{~Bq}$ of iodine released in elemental form would result in different fractions of elemental, organic, and particulate forms of iodine at a receptor location. Therefore, for the case when all iodine was assumed to have been released in the elemental form, the concentrations of all three forms were added for each location to represent the total iodine concentration at each location.

Concentrations were predicted at the nodes of a polar grid along each of the equally spaced 16 compass directions starting with $\mathrm{N}$ and going clockwise $22.5^{\circ}$ to $\mathrm{NNE}, \mathrm{NE}$, and so on until NNW. Along each direction, 25 equally spaced nodes, $1.5 \mathrm{~km}$ apart, were selected. Thus, ground-level concentrations of ${ }^{131} \mathrm{I}$ in air were predicted using the SORAMI model at each of the $400(25 \times 16)$ nodes of the polar grid. Concentrations at each of the 9 monitoring stations were then estimated from the predicted concentrations at the four nodes surrounding the location of the monitoring station using an interpolation scheme. The interpolation scheme used a weighted approach, with weights calculated as the inverse of the square of distance from the monitoring station (where the prediction was needed) to each of the four surrounding nodes. The concentration at the monitoring station was then estimated as the weighted sum of the product of the normalized weight and predicted concentration at each of the four surrounding nodes.

Additionally, to compare the accuracy of the interpolation scheme, the ground-level air concentrations were also predicted by directly specifying the coordinates of the nine monitoring locations with respect to the RaLa Stack (from Table 4.7) in the SORAMI model.

Ratios of predicted estimates of the central value (median) of the annual average ground-level concentrations of ${ }^{131} \mathrm{I}$ in air at each of the nine monitoring stations were then divided by the measured annual average concentrations presented in Table 4.7 to obtain the $\mathrm{P}$ (predicted)/O (observed) ratio for each of the nine monitoring stations. The geometric means of the $\mathrm{P} / \mathrm{O}$ ratios for each of the monitoring stations using estimates for all three years are presented in Tables 4.9 and 4.10. Table 4.9 presents the geometric mean $\mathrm{P} / \mathrm{O}$ ratios for the case in which all of the releases were treated as having occurred from Stack 3020. Table 4.10 presents the geometric mean $\mathrm{P} / \mathrm{O}$ ratios for the case in which all of the Bethel Valley releases were treated as having occurred from Stack 3020 and all of the Melton Valley releases were treated as having occurred from Stack 7911. 
Table 4.9 The geometric mean $\mathrm{P} / \mathrm{O}$ ratios of the predicted annual average concentrations of ${ }^{131}$ I from SORAMI with the inclusion of dry and wet deposition, plume depletion, and chemistry, and assuming that all of the releases between 1967 and 1969 occurred from Stack 3020, ${ }^{\text {a }}$ to the measured concentrations.

\begin{tabular}{|c|c|c|c|c|c|c|}
\hline \multirow{3}{*}{$\begin{array}{c}\text { Ambient }{ }^{131} \mathbf{I} \\
\text { monitoring station }\end{array}$} & \multicolumn{6}{|c|}{ Geometric mean of P/O Ratios } \\
\hline & \multicolumn{2}{|c|}{$\begin{array}{l}\text { Assuming all }{ }^{131} \mathbf{I} \\
\text { was released in } \\
\text { elemental form }\end{array}$} & \multicolumn{2}{|c|}{$\begin{array}{l}\text { Assuming all }{ }^{131} \mathbf{I} \\
\text { was released in } \\
\text { organic form }\end{array}$} & \multicolumn{2}{|c|}{$\begin{array}{c}\text { Assuming all }{ }^{131} \mathbf{I} \text { was } \\
\text { released as } \\
\text { particulate }\end{array}$} \\
\hline & $\begin{array}{l}\text { Inter- } \\
\text { polated }\end{array}$ & $\begin{array}{c}\text { Directly } \\
\text { calculated }\end{array}$ & $\begin{array}{l}\text { Inter- } \\
\text { polated }\end{array}$ & $\begin{array}{c}\text { Directly } \\
\text { calculated }\end{array}$ & $\begin{array}{c}\text { Inter- } \\
\text { polated }\end{array}$ & $\begin{array}{c}\text { Directly } \\
\text { calculated }\end{array}$ \\
\hline Kerr Hollow (HP-31) & 0.73 & 0.77 & 0.90 & 0.96 & 0.87 & 0.92 \\
\hline Midway Gate (HP-32) & 0.96 & 0.84 & 1.18 & 1.03 & 1.14 & 0.99 \\
\hline Gallaher Gate (HP-33) & 0.47 & 0.38 & 0.55 & 0.45 & 0.55 & 0.44 \\
\hline White Oak Dam (HP-34) & 1.15 & 1.10 & 1.26 & 1.21 & 1.25 & 1.21 \\
\hline Blair Gate (HP-35) & 0.45 & 0.42 & 0.54 & 0.51 & 0.53 & 0.49 \\
\hline Turnpike Gate (HP-36) & 0.84 & 1.01 & 1.03 & 1.23 & 0.99 & 1.20 \\
\hline Hickory Creek Bend (HP-37) & 1.86 & 1.73 & 2.17 & 2.03 & 2.12 & 1.98 \\
\hline Gallaher Bend (HP-38) & 1.63 & 1.75 & 1.83 & 1.97 & 1.81 & 1.94 \\
\hline Townsite (HP-39) & 0.50 & 0.53 & 0.65 & 0.67 & 0.62 & 0.64 \\
\hline $\begin{array}{l}\text { Average of the Geometric } \\
\text { Mean } \mathrm{P} / \mathrm{O} \text { ratios }\end{array}$ & 0.95 & 0.95 & 1.12 & 1.12 & 1.10 & 1.09 \\
\hline
\end{tabular}

a Interpolated concentrations represent concentrations estimated from predicted values for four surrounding nodes using an inverse distance square weighting method. Directly calculated concentrations represent concentrations predicted directly for the monitoring stations.

b The predicted concentrations $(\mathrm{P})$ represent the concentrations of ${ }^{131} \mathrm{I}$ in all three chemical forms at the receptor location from a release in elemental form. 
Table 4.10 The geometric mean $\mathrm{P} / \mathrm{O}$ ratios of the predicted annual average concentrations of ${ }^{131}$ I from SORAMI with the inclusion of dry and wet deposition, plume depletion, and chemistry, and assuming that all of the Bethel Valley releases between 1967 and 1969 occurred from Stack 3020 and all of the Melton Valley releases between 1967 and 1969 occurred from Stack $7911,{ }^{a}$ to the measured concentrations.

\begin{tabular}{|c|c|c|c|c|c|c|}
\hline \multirow{3}{*}{$\begin{array}{c}\text { Ambient }{ }^{131} \mathbf{I} \\
\text { monitoring station }\end{array}$} & \multicolumn{6}{|c|}{ Geometric mean of P/O Ratios } \\
\hline & \multicolumn{2}{|c|}{$\begin{array}{l}\text { Assuming all }{ }^{131} \mathbf{I} \\
\text { was released in } \\
\text { elemental form }{ }^{\mathrm{b}}\end{array}$} & \multicolumn{2}{|c|}{$\begin{array}{c}\text { Assuming all }{ }^{131} \mathbf{I} \\
\text { was released in } \\
\text { organic form }\end{array}$} & \multicolumn{2}{|c|}{$\begin{array}{c}\text { Assuming all }{ }^{131} \mathbf{I} \\
\text { was released as } \\
\text { particulate } \\
\end{array}$} \\
\hline & $\begin{array}{l}\text { Inter- } \\
\text { polated }\end{array}$ & $\begin{array}{l}\text { Directly } \\
\text { calculated }\end{array}$ & $\begin{array}{l}\text { Inter- } \\
\text { polated }\end{array}$ & $\begin{array}{l}\text { Directly } \\
\text { calculated }\end{array}$ & $\begin{array}{l}\text { Inter- } \\
\text { polated }\end{array}$ & $\begin{array}{l}\text { Directly } \\
\text { calculated }\end{array}$ \\
\hline Kerr Hollow (HP-31) & 0.72 & 0.75 & 0.89 & 0.94 & 0.86 & 0.90 \\
\hline Midway Gate (HP-32) & 0.92 & 0.80 & 1.12 & 0.98 & 1.08 & 0.95 \\
\hline Gallaher Gate (HP-33) & 0.45 & 0.36 & 0.53 & 0.43 & 0.53 & 0.42 \\
\hline White Oak Dam (HP-34) & 1.18 & 1.14 & 1.30 & 1.26 & 1.29 & 1.25 \\
\hline Blair Gate (HP-35) & 0.43 & 0.41 & 0.53 & 0.50 & 0.52 & 0.48 \\
\hline Turnpike Gate (HP-36) & 0.79 & 0.94 & 0.97 & 1.15 & 0.94 & 1.12 \\
\hline Hickory Creek Bend (HP-37) & 1.91 & 1.77 & 2.22 & 2.07 & 2.17 & 2.02 \\
\hline Gallaher Bend (HP-38) & 1.66 & 1.75 & 1.87 & 1.97 & 1.84 & 1.94 \\
\hline Townsite (HP-39) & 0.49 & 0.51 & 0.64 & 0.65 & 0.60 & 0.62 \\
\hline $\begin{array}{l}\text { Average of the Geometric } \\
\text { Mean } \mathrm{P} / \mathrm{O} \text { ratios }\end{array}$ & 0.95 & 0.94 & 1.12 & 1.11 & 1.09 & 1.08 \\
\hline
\end{tabular}

a Interpolated concentrations represent concentrations estimated from predicted values for four surrounding nodes using an inverse distance square weighting method. Directly calculated concentrations represent concentrations predicted directly for the monitoring stations.

b The predicted concentrations $(\mathrm{P})$ represent the concentrations of ${ }^{131} \mathrm{I}$ in all three chemical forms at the receptor location from a release in elemental form. 
It should be noted that the directly calculated values of geometric mean $\mathrm{P} / \mathrm{O}$ ratios in Table 4.10 were estimated using stack parameters listed in Table 4.3 for Stack 3020 and using relevant values of the stack height (87.3), the stack diameter $(1.5 \mathrm{~m})$ and the stack exit velocity $\left(13 \mathrm{~m} \mathrm{~s}^{-1}\right)$ for Stack 7911 (Binford et al., 1970). The interpolated values of geometric mean P/O ratios in Table 4.10 were estimated using stack parameters listed in Table 4.3 for Stack 3020 for both Stacks 3020 and stack 7911, primarily because of the large computational time required for the simulation for 400 nodes of the grid.

The geometric mean $\mathrm{P} / \mathrm{O}$ ratios in Tables 4.9 and 4.10 do not show significant differences between interpolated and directly calculated estimates of concentrations at the nine monitoring stations. The accuracy of the interpolation was further tested, as described in Section 4.3.3.4, using the 41 locations of interest.

Comparison of directly calculated geometric mean P/O ratios from Stack 3020 alone (Table 4.9) and from the combination of Stacks 3020 and 7911 (Table 4.10) also shows good agreement between the two sets of predictions. It must be noted, however, that the majority of releases for all three years occurred from the Bethel Valley Stack 3039. The verification study presented in Section 4.3.3.3 addresses the effect on the magnitude of the error arising from the use of Stack 3020 as a surrogate for Stack 3039.

The wet and dry deposition rates are highest for the elemental form of iodine followed by those for the particulate and organic forms. Therefore, removal of elemental ${ }^{131} \mathrm{I}$ from the plume by dry and wet deposition would result in the lowest concentrations of ${ }^{131} \mathrm{I}$ at a receptor location if all of the ${ }^{131} \mathrm{I}$ is released in elemental form. The highest concentrations of ${ }^{131} \mathrm{I}$ at a receptor location would result if all of the ${ }^{131} \mathrm{I}$ are released in organic form, because of its extremely low deposition velocity. The geometric means of the $\mathrm{P} / \mathrm{O}$ ratios presented in Tables 4.9 and 4.10 clearly reflect these observations. Furthermore, the predicted concentrations with the inclusion of plume depletion, deposition, and chemistry should be lower than the predicted concentrations without the inclusion of these processes. Comparison of the results presented in Tables 4.9 and Table 4.10 (columns containing results of directly calculated values) with those in the third column of Table 4.8 clearly bears this out. The P/O ratios corresponding to column 4 of Table 4.10 are also shown in Figure 4.4 for the case for which all of the iodine was released in the organic form. It is clearly seen that most predictions fall within a factor of two of the observations. Figures 4.5 through 4.7 present the $95 \%$ subjective confidence intervals of the predicted ground-level concentrations of ${ }^{131} \mathrm{I}$ in air at the monitoring stations along with the measurements. These figures represent the directly calculated values for release as elemental iodine for 1967, 1968, and 1969, respectively. Most measured concentrations are seen to lie within the $95 \%$ subjective confidence intervals of the predicted concentrations. 


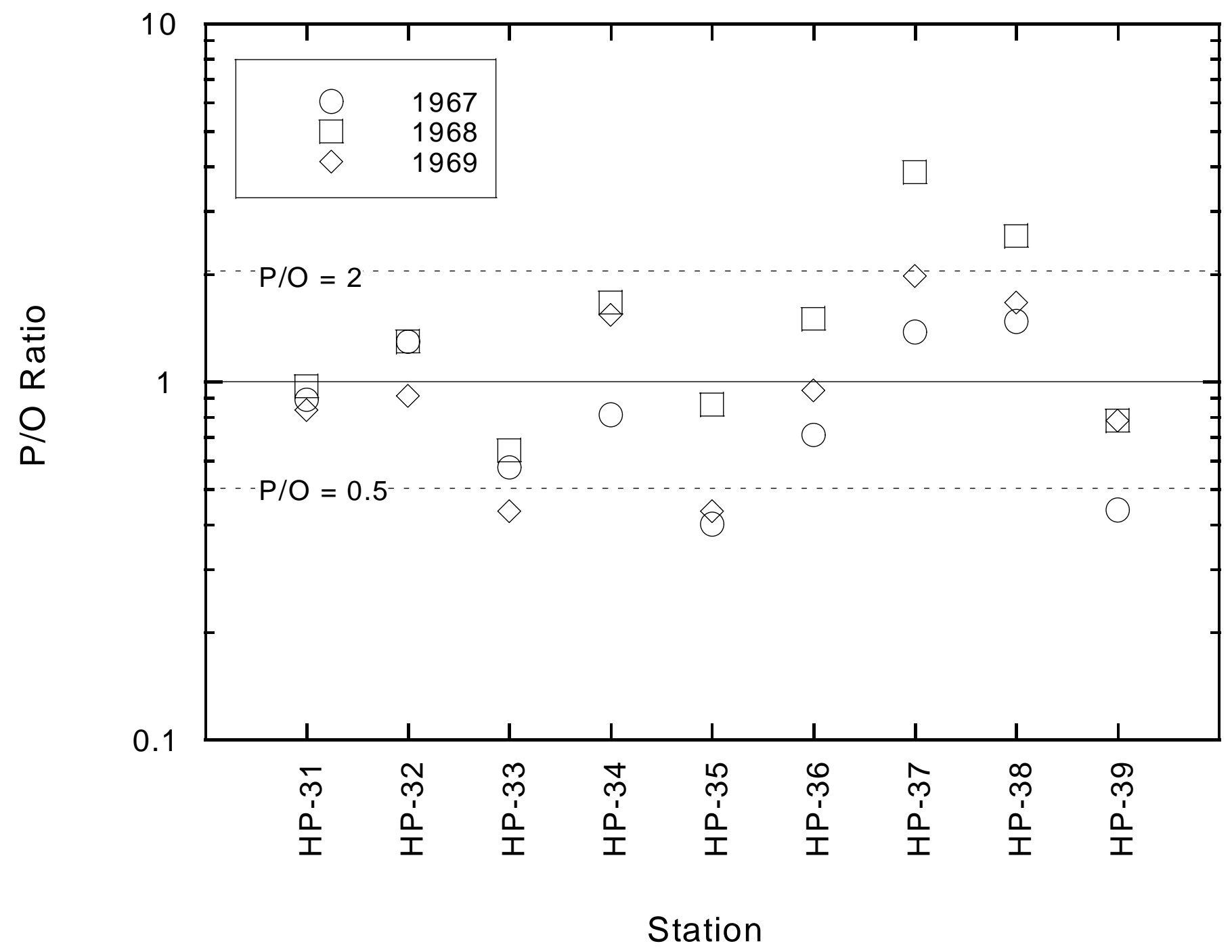

Figure 4.4 The P/O ratios from the SORAMI model for the case in which all the ${ }^{131} \mathrm{I}$ was assumed to be released in the organic form. Releases from Bethel Valley were treated as having occurred from Stack 3020 and releases from Melton Valley were treated as having occurred from Stack 7911. The processes of plume depletion, wet and dry deposition, and iodine chemistry were included, and concentrations were directly estimated at the monitoring stations. 


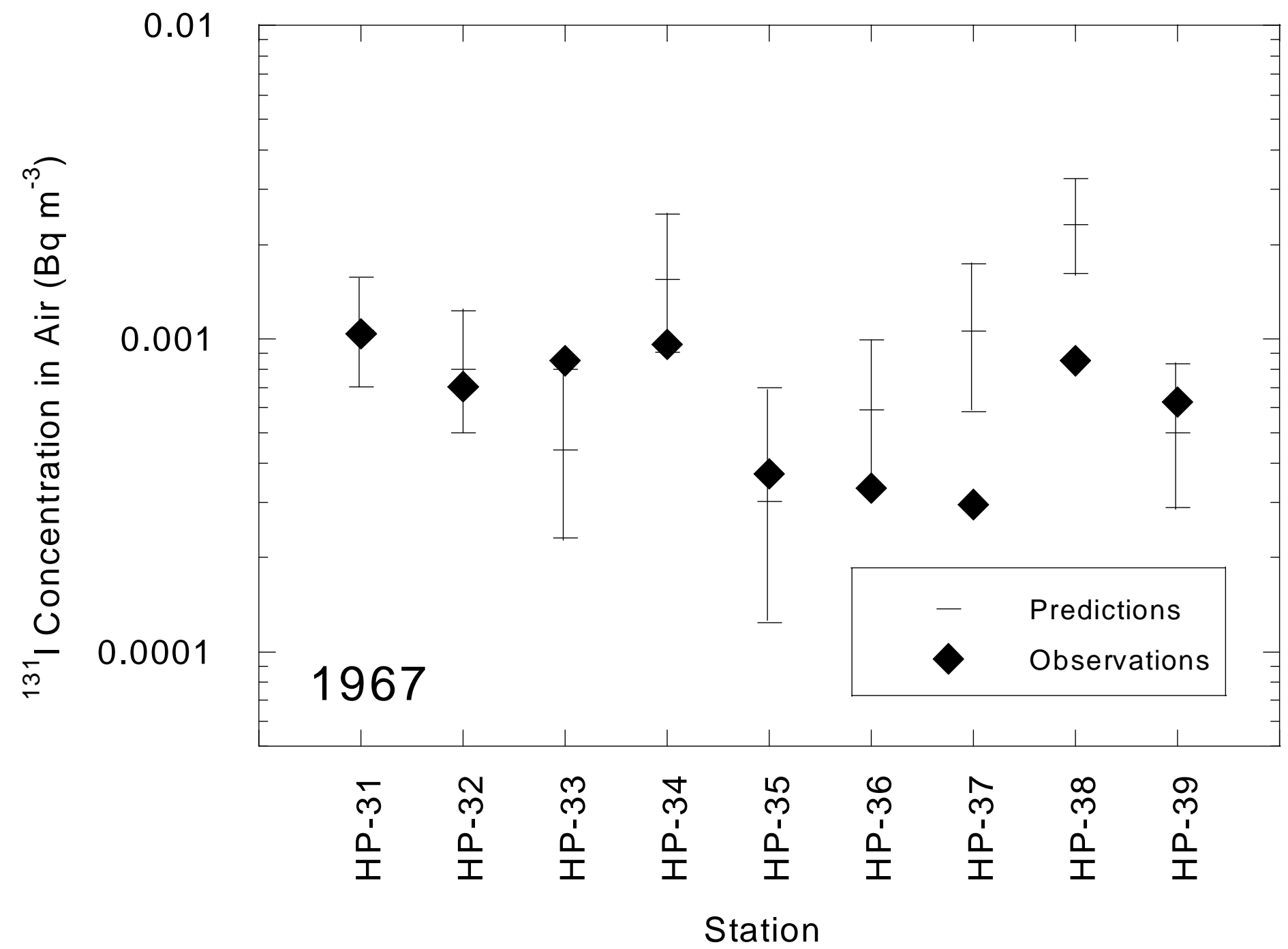

Figure 4.5 Measured concentrations and 95\% confidence intervals of the predicted concentrations from the SORAMI model for 1967 for the case in which all the ${ }^{131} \mathrm{I}$ was assumed to be released in the organic form. Releases from Bethel Valley were treated as having occurred from Stack 3020 and releases from Melton Valley were treated as having occurred from Stack 7911. The processes of plume depletion, wet and dry deposition, and iodine chemistry were included, and concentrations were directly estimated at the monitoring stations. 


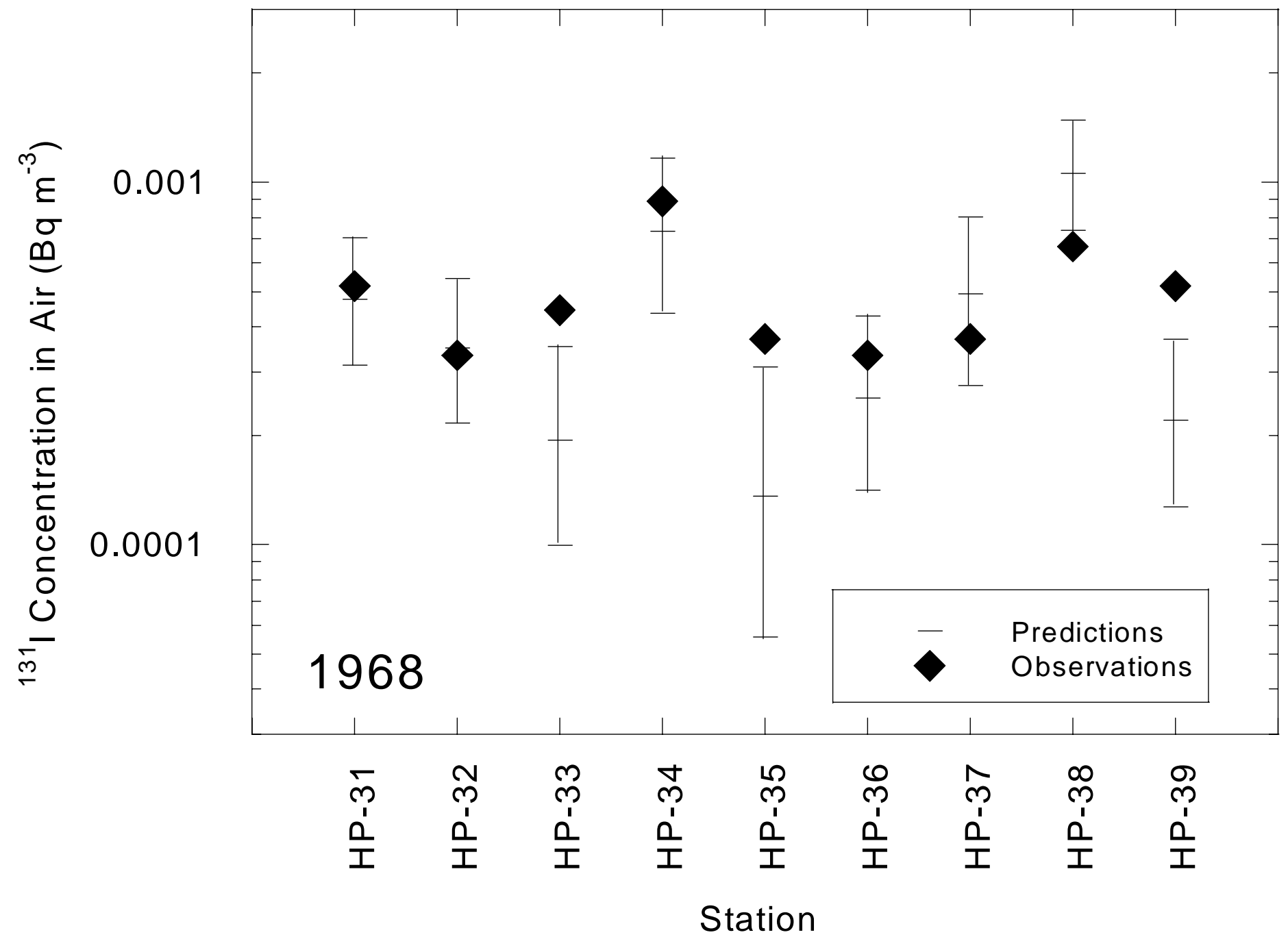

Figure 4.6

Measured concentrations and 95\% confidence intervals of the predicted concentrations from the SORAMI model for 1968 for the case in which all the ${ }^{131}$ I was assumed to be released in the organic form. Releases from Bethel Valley were treated as having occurred from Stack 3020 and releases from Melton Valley were treated as having occurred from Stack 7911. The processes of plume depletion, wet and dry deposition, and iodine chemistry were included, and concentrations were directly estimated at the monitoring stations. 


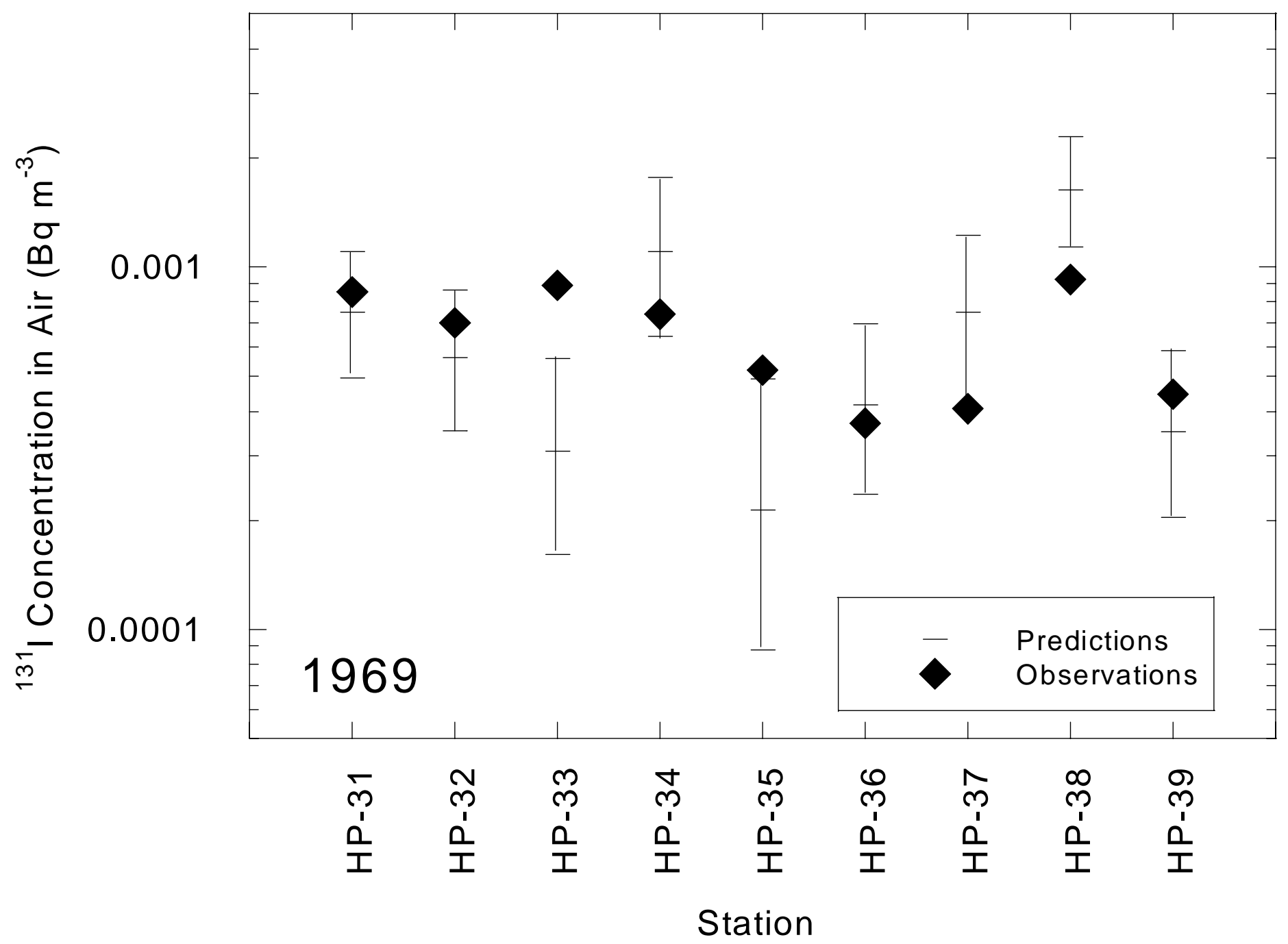

Figure 4.7 Measured concentrations and 95\% confidence intervals of the predicted concentrations from the SORAMI model for 1969 for the case in which all the ${ }^{131}$ I was assumed to be released in the organic form. Releases from Bethel Valley were treated as having occurred from Stack 3020 and releases from Melton Valley were treated as having occurred from Stack 7911. The processes of plume depletion, wet and dry deposition, and iodine chemistry were included, and concentrations were directly estimated at the monitoring stations. 
Validation studies presented in this section and in Section 4.3.3.1 clearly show that the SORAMI model provides reasonable predictions (within a factor of 2) for most locations, and therefore, its use for the reconstruction of doses and risks is justified. There are a few locations where the model consistently provides under- or overestimates of the measured concentrations. However, considering that the predictions are still within a factor of two, it was not found necessary to develop bias correction factors for specific locations or specific directions from the RaLa Stack.

The validation study conducted here could have been improved further by estimating concentrations from each stack separately using individual source terms and stack parameters. However, because most of the release occurred from Stack 3039 for Bethel Valley releases and from Stack 7911 for Melton Valley releases, the closeness of the stacks in each valley, and the fact that the geometric mean $\mathrm{P} / \mathrm{O}$ ratios are within a factor of 2 , it was decided that further refinements of these calculations are not necessary.

\subsubsection{Verification for the use of Stack 3020 for all years of simulation}

As the stack diameter, stack exit velocity, and stack elevation are different for Stacks 3020 and 3039 , the plume rises would be expected to be slightly different for the two stacks, depending on the distance to the ground-level receptor, the atmospheric stability, and the wind speed, as discussed in Section 4.3.2.1. To evaluate the effect on the predicted ground-level concentration of ${ }^{131} \mathrm{I}$ in air, two separate simulations were made, one with Stack 3020 as the point of release, and the other with Stack 3039 as the point of release using their individual release parameters. The $\chi / Q$ ratios were then estimated for each of the 400 nodes between 2 and $38 \mathrm{~km}$. Depletion, deposition, and chemistry were included in the analysis. The $\chi / Q$ ratios were estimated for each of the 5 categories at each node: for release in elemental form and arrival at the node in organic form (after chemical transformation); for release in elemental form and arrival at the node in particulate form (after chemical transformation); for release in elemental form and arrival at the node in elemental form (remainder after chemical transformation into organic and particulate forms); for release in organic form and arrival at the node in organic form; and for release in particulate form and arrival at the grid-node in particulate form. The average estimates of the differences in the lower and upper bounds of the $95 \%$ subjective confidence interval, the median, and the arithmetic mean of the predicted $\chi / \mathrm{Q}$ ratios at the 400 grid-nodes were then estimated using the following equation:

$$
\varepsilon_{i}=\left(\frac{1}{4}\right) \cdot \sum_{j=1}^{400} \sum_{k=1}^{5} \frac{\left.\mid(\chi / Q)_{\text {Stack } 3020, i, j, k}-(\chi / Q)_{\text {Stack } 3039, i, j, k}\right) \mid}{(\chi / Q)_{\text {Stack } 3020, i, j, k}}
$$

where

$\varepsilon_{i}=$ the average absolute $\%$ difference in the estimate of the $\chi / \mathrm{Q}$ ratios predicted from Stack 3020 and Stack 3039 for the statistic $i$;

$i=$ the particular statistic being evaluated; e.g., the median, the arithmetic mean, or the lower or upper bound of the $95 \%$ subjective confidence interval; 
$\begin{array}{ll}j & =\text { the } j^{\text {th }} \text { node } \text {, and } \\ k & =\text { the } k^{\text {th }} \text { category. }\end{array}$

The average absolute percent differences for the lower bound of the $95 \%$ subjective confidence interval, the median, and the upper bound of the $95 \%$ subjective confidence interval and for the arithmetic mean were estimated as $4.8 \%, 4.8 \%, 5.1 \%$, and $4.8 \%$, respectively, using all of the 400 nodes and all five categories of $\chi / Q$ ratios. The error in using one stack in preference to the other will not, therefore, result in major differences in the estimates of doses and risks to the exposed individual. This is also borne out by the validation study presented in Section 4.3.3.2. Thus, the use of Stack 3020 for all years for the dispersion analysis is justified.

\subsubsection{Verification of the interpolation scheme}

In order to verify the interpolation scheme (Section 4.3.2.2) that was used for the estimation of concentrations at locations other than the nodes of the grid within the study domain of 38-km radius around the RaLa processing facility, the $\chi / \mathrm{Q}$ ratios were predicted using both the interpolation scheme (using predictions from SORAMI at grid nodes) and using SORAMI directly for the 41 locations of interest (see Appendix 11A for the locations of interest). As in Section 4.3.3.3, the $\chi / Q$ ratios were estimated for each of the 5 categories at each location of interest: for release in elemental form and arrival at the node/receptor location in organic form (after chemical transformation); for release in elemental form and arrival at the node/receptor location in particulate form (after chemical transformation); for release in elemental form and arrival at the node/receptor location in elemental form (remainder after chemical transformation into organic and particulate forms); for release in organic form and arrival at the node/receptor location in organic form; and for release in particulate form and arrival at the node/receptor location in particulate form. The average estimates of the error in the lower and upper bounds of the $95 \%$ subjective confidence interval, the median, and the arithmetic mean of the predicted $\chi / Q$ ratios at the 41 locations of interest were then estimated using the following equation:

$$
\varepsilon_{i}=\left(\frac{100}{41}\right) \cdot \sum_{j=1}^{41} \sum_{k=1}^{5} \frac{\left.\mid(\chi / Q)_{\text {Interpolated }, i, j, k}-(\chi / Q)_{\text {Direct }, i, j, k}\right) \mid}{(\chi / Q)_{\text {Direct }, i, j, k}}
$$

where

$\varepsilon_{i}=$ the average absolute $\%$ error in the estimate of the $\chi / \mathrm{Q}$ ratios predicted from interpolation and from direct estimation using the SORAMI model for the statistic $i$;

$i=$ the particular statistic being evaluated; e.g., the median, the arithmetic mean, or the lower or upper bound of the $95 \%$ subjective confidence interval;

$j \quad=\quad$ the $j^{\text {th }}$ location of interest; and

$k=$ the $k^{\text {th }}$ category. 
The average absolute percent differences for the lower bound of the $95 \%$ subjective confidence interval, the median, and the upper bound of the $95 \%$ subjective confidence interval and for the arithmetic mean were estimated as $8.5 \%, 6.5 \%, 7.1 \%$, and $6.4 \%$, respectively, using the 41 locations of interest and all five categories of $\chi / Q$ ratios for releases from the building housing the RaLa processing facility; the corresponding values for releases from the stack were $8.1 \%$, $6.3 \%, 6.2 \%$ and $6.2 \%$, respectively. The error in using the interpolation scheme instead of direct estimation will not, therefore, result in major errors in the estimates of doses and risks to an exposed individual. Furthermore, the interpolation scheme allows for easy estimation of concentrations at other locations of interest not addressed directly in this report within the 38-km radius of the RaLa processing facility.

\subsubsection{Model Validation for Short-Term Releases}

Release data on ${ }^{131}$ I were available for three weekly periods in the 1960s along with reported measured weekly average concentrations of ${ }^{131} \mathrm{I}$ in air at one location for each of the releases. During the week ending 17 January 1965, $1 \times 10^{11} \mathrm{~Bq}$ of ${ }^{131} \mathrm{I}$ was released (Lasher, 1965), and the maximum weekly average concentration of ${ }^{131}$ I was measured at Station HP-34 (Figure 4.3) as $0.0074 \mathrm{~Bq} \mathrm{~m}^{-3}$ (ORNL, 1966). About $90 \%$ of the release was from Stack 3039.

During the week ending 15 May 1966, $3.7 \times 10^{10} \mathrm{~Bq}$ of ${ }^{131} \mathrm{I}$ was released (Lasher, 1966), and the maximum weekly average concentration of ${ }^{131}$ I was measured at Station HP-38 (Figure 4.3) as $0.0044 \mathrm{~Bq} \mathrm{~m}^{-3}$ (ORNL, 1967). About $94 \%$ of the release was from Stack 3039, 3.5\% was from Stack 2026, and 2\% was from Stack 3020.

Finally, during the week ending 9 July $1967,1.5 \times 10^{10} \mathrm{~Bq}$ of ${ }^{131} \mathrm{I}$ was released (Lasher, 1967d), and the maximum weekly average concentration of ${ }^{131} \mathrm{I}$ was measured at Station HP-33 (Figure 4.3) as $0.0081 \mathrm{~Bq} \mathrm{~m}^{-3}$ (ORNL, 1968). About $74 \%$ of the release was from Stack 3039, $21 \%$ was from Stack 2026, and 5\% was from Stack 3020.

Since the meteorological parameters were developed for each of the specified months (as discussed in Section 4.3.2.1), it was possible to estimate the ground-level concentrations of ${ }^{131} \mathrm{I}$ using the meteorological data for each month separately. The results of this validation analysis are presented in Table 4.11. Again, the chemical form(s) in which iodine was released at the source was not known. Therefore, three separate analyses were conducted, as in the case of the validation for routine releases, with the assumption that all of the iodine was released (a) in elemental form, (b) in organic form, or (c) in particulate form. For this exercise also, all of the releases were assumed to have occurred from Stack 3020. The 400 alternate values of 7-day, time-integrated $\chi / Q$ ratios were developed from the hourly meteorological data in a similar manner to that used to develop the 31-day time-integrated ratios (as presented in Section 4.3.3); from these, the weekly average $\chi / \mathrm{Q}$ ratios were estimated. These ratios were then multiplied by the constant release rate for release in each chemical form, using Equation 4.1 to obtain the 400 alternate realizations of the weekly average concentration of ${ }^{131} \mathrm{I}$ in air. 
Table 4.11 Results from the validation of SORAMI model for short-term (weekly) releases.

\begin{tabular}{|c|c|c|c|c|c|}
\hline \multirow[b]{2}{*}{$\begin{array}{l}\text { Period (week } \\
\text { ending) }\end{array}$} & \multirow[b]{2}{*}{$\begin{array}{l}\text { Monitoring } \\
\text { station }\end{array}$} & \multirow{2}{*}{$\begin{array}{c}\text { Measured } \\
\text { weekly }{ }^{131} \mathrm{I} \\
\text { concentration } \\
\left(10^{-3} \mathrm{~Bq} \mathrm{~m}^{-3}\right)\end{array}$} & \multicolumn{3}{|c|}{$\begin{array}{l}95 \% \text { Subjective Confidence Interval of the } \\
\text { predicted ground-level concentration of }{ }^{131} \mathrm{I} \\
\text { in air }\left(\mathbf{1 0}^{-3} \mathrm{~Bq} \mathrm{~m}^{-3}\right)\end{array}$} \\
\hline & & & $\begin{array}{c}\text { Release as } \\
\text { elemental }{ }^{131} \mathbf{I}\end{array}$ & $\begin{array}{c}\text { Release as } \\
\text { organic }{ }^{131} \mathbf{I}\end{array}$ & $\begin{array}{c}\text { Release as } \\
\text { particulate }^{131} \mathbf{I}\end{array}$ \\
\hline 17 January 1965 & HP-34 & 7.4 & $(1.8-26.4)$ & $(2.9-27.7)$ & $(2.1-31.4)$ \\
\hline & & 4. & & & \\
\hline 9 July 1967 & HP-33 & 8.1 & $(0.04-0.6)$ & $(0.04-0.8)$ & $(0.03-0.7)$ \\
\hline
\end{tabular}

Results for January 1965 and May 1966 clearly show that the measured concentrations are contained within the lower and upper bounds of the $95 \%$ subjective confidence intervals of the predicted concentrations. However, the predicted concentrations grossly underestimate the measured concentrations for July 1967. The reasons for this are not clear. Results of routine releases, presented in Tables 4.8, 4.9, and 4.10, also indicate that the SORAMI model typically underpredicts at the Gallaher Gate Station (HP-33). Because more short-term monitoring and release data were not available, further short-term validation could not be performed. However, it is clear that the model can be used for predicting downwind ground-level concentrations of ${ }^{131} \mathrm{I}$ from the accident on April 29, 1954.

\subsection{Results}

\subsubsection{Routine Releases}

Distributions of ground-level $\chi / Q$ ratios were developed in the same manner as described in Section 4.3.3 for each of the five categories of release: for release in elemental form and arrival at the node/receptor location in organic form (after chemical transformation); for release in elemental form and arrival at the node/receptor location in particulate form (after chemical transformation); for release in elemental form and arrival at the node/receptor location in elemental form (remainder after chemical transformation into organic and particulate forms); for release in organic form and arrival at the node/receptor location in organic form; and for release in particulate form and arrival at the node/receptor location in particulate form. Using these $\chi / Q$ ratios, the source terms presented in Section 3, and Equation 4.1, concentrations of ${ }^{131} \mathrm{I}$ in the three forms were estimated for each of the 400 nodes. Contours of the lower- and upper-bound estimates of the $95 \%$ subjective confidence interval of the annual average ground-level concentrations of total ${ }^{131} \mathrm{I}\left({ }^{131} \mathrm{I}\right.$ in all 3 forms) are presented in Figures 4.8 and 4.9, respectively. These contours represent the 13-year average (1944-1956) of the predicted annual average ground-level concentrations of total ${ }^{131} \mathrm{I}$ in air. These concentrations along with central estimates are reported in Appendix 11B for the 41 locations of interest. 


\subsubsection{Accident}

Transport simulations for each of the five half-hour periods of the accident that occurred between 4:30 and 7:00 p.m. on April 29, 1954, were conducted using the input parameters presented in Section 4.3.2.2. Results were first developed as $\chi / Q$ ratios for the five categories of release as described in Section 4.4.1 for each half-hour period, for each of the nodes. These half-hourly ratios were multiplied with half-hourly release rates from appropriate sources (stack or building) and for appropriate chemical forms, using Equation 4.1, to obtain the concentrations of ${ }^{131} \mathrm{I}$ in air in each of the three chemical forms at each of the 41 locations of interest and the monitoring stations. From the hourly concentrations, the time-integrated concentrations of ${ }^{131} \mathrm{I}$ in all three chemical forms were developed. Time-integrated concentrations were estimated for 464 nodes on a polar grid. The 400 nodes on the polar grid are identical to the ones used in the analysis for routine releases. Sixty-four additional nodes were added to include more locations within the first $2 \mathrm{~km}$ to compare the results of the predictions with the onsite radiation measurements made immediately after the accident, as shown in Figure 4.1. The lower-bound, central, and upperbound estimates of the $95 \%$ subjective confidence intervals of the total time-integrated concentrations of ${ }^{131} \mathrm{I}$ are presented in Figures 4.10, 4.11, and 4.12 respectively. It should be noted that the concentrations in these figures are representative of emissions from both sources, the Stack 3039 and the building housing the radioactive lanthanum processing facility.

Two distinct plumes developed in response to the prevailing wind directions during the five halfhour periods of the accident. The northwest direction of contaminant transport is corroborated by the radiation measurements shown in Figure 4.1. However, the transport towards the north cannot be corroborated by the measurements shown in Figure 4.1, because there were no monitoring stations towards the north. Therefore, the movement of the plume towards the north, as predicted by SORAMI, is quite plausible.

It was noted that the $95 \%$ subjective confidence intervals span several orders of magnitude for some locations. The primary reason for this was found to be the uncertainty assigned to the wind direction. An uncertainty range of $\pm 11.25^{\circ}$ was assigned to each measured value shown in Table 4.4. This results in a wide range of cross-wind distances ( $y$ in Equation 4.1) from the plume centerline (along the wind direction) for a given location. The exponential term containing the $y$ and $\sigma_{z}$ in Equation 4.1 accordingly takes a wide range of values from 0 (when the plume centerline is far from the location of interest) to some large finite value (when the location of concern is well within the plume). As seen from Figures 4.10, 4.11 and 4.12, the directions of plume travel during the five half-hour periods are such that most of the locations of concern do not fall within the plume and some are exposed only to the edge of the plume. Particularly noteworthy in these figures is the fact that the $95 \%$ subjective confidence interval is quite large towards the northwest direction to which the wind was flowing for one half-hour period between 5:00 and 5:30 p.m. The plume along the north shows a much smaller 95\% subjective confidence interval for the predicted time integrated concentrations. The winds were towards the north for 4 of the 5 half-hour periods of the accident. 
TASK 1 REPORT

${ }^{131}$ I Releases from X-10 Radioactive Lanthanum Processing July 1999 Atmospheric Dispersion/Radioiodine Chemistry

Page 4-37
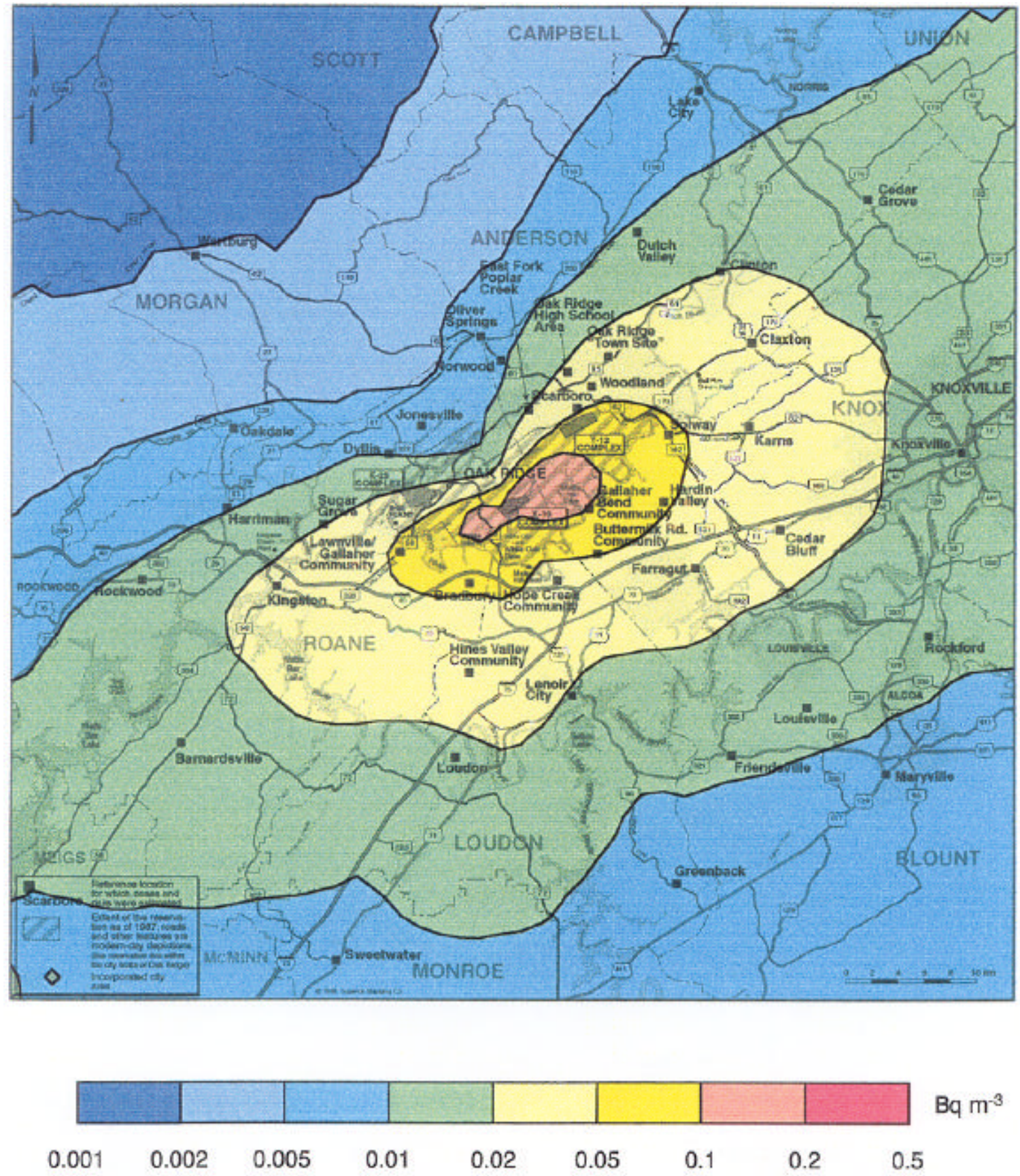

Figure 4.8 Contours for the lower bound estimates (2.5 percentile) of the 95\% subjective confidence interval for the annual average, ground-level concentrations of total ${ }^{131} \mathrm{I}\left({ }^{131} \mathrm{I}\right.$ in all three chemical forms) in air. The actual concentration at a given location is highly likely to be greater than the value presented in this figure. 

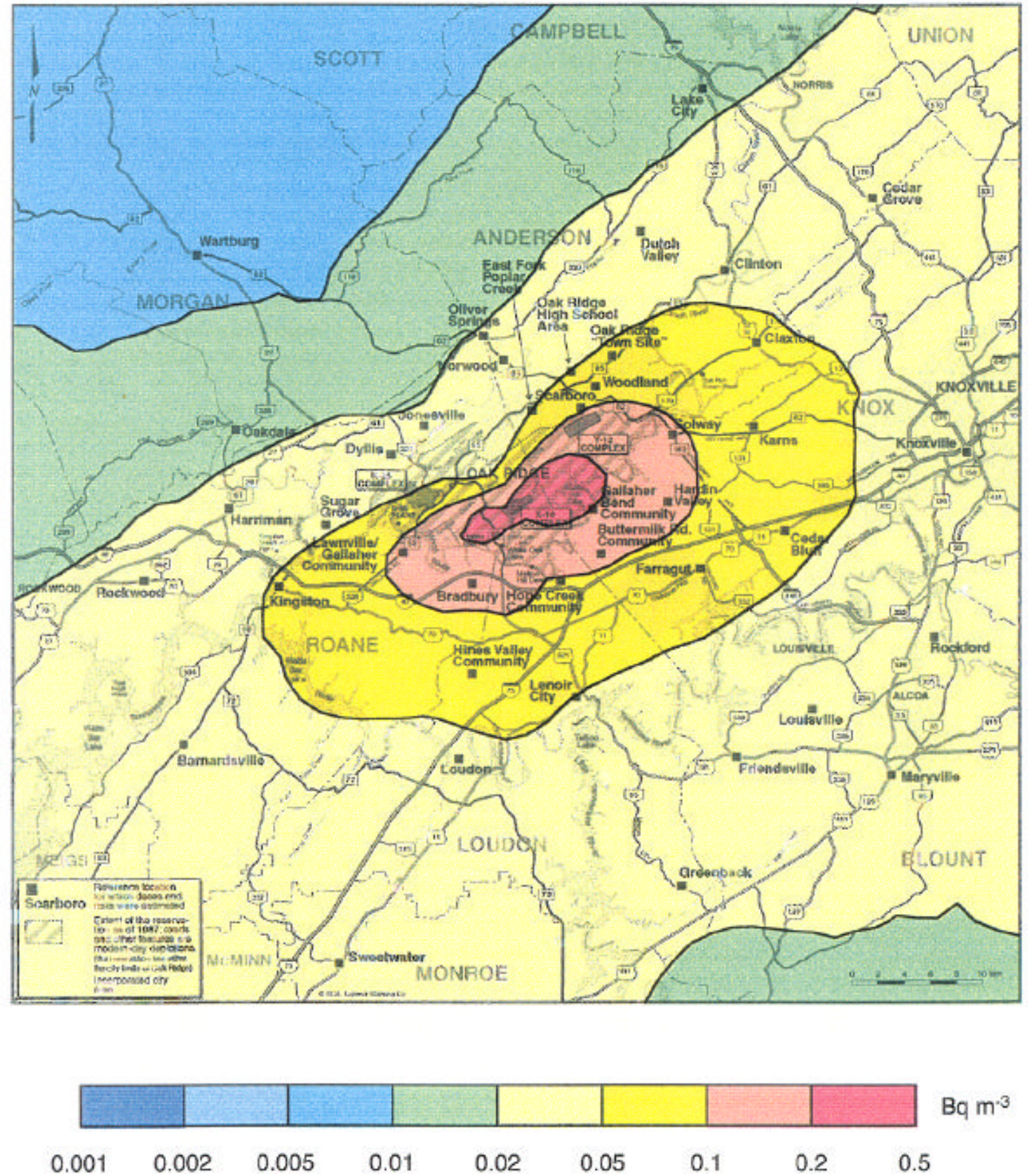

Figure 4.9 Contours for the upper bound estimates (97.5 percentile) of the 95\% subjective confidence interval for the annual average, ground-level concentrations of total ${ }^{131} \mathrm{I}\left({ }^{131} \mathrm{I}\right.$ in all three chemical forms) in air. The actual concentration at a given location is highly likely to be less than the value presented in this figure. 
TASK 1 REPORT

${ }^{131}$ I Releases from X-10 Radioactive Lanthanum Processing July 1999

Page 4-39
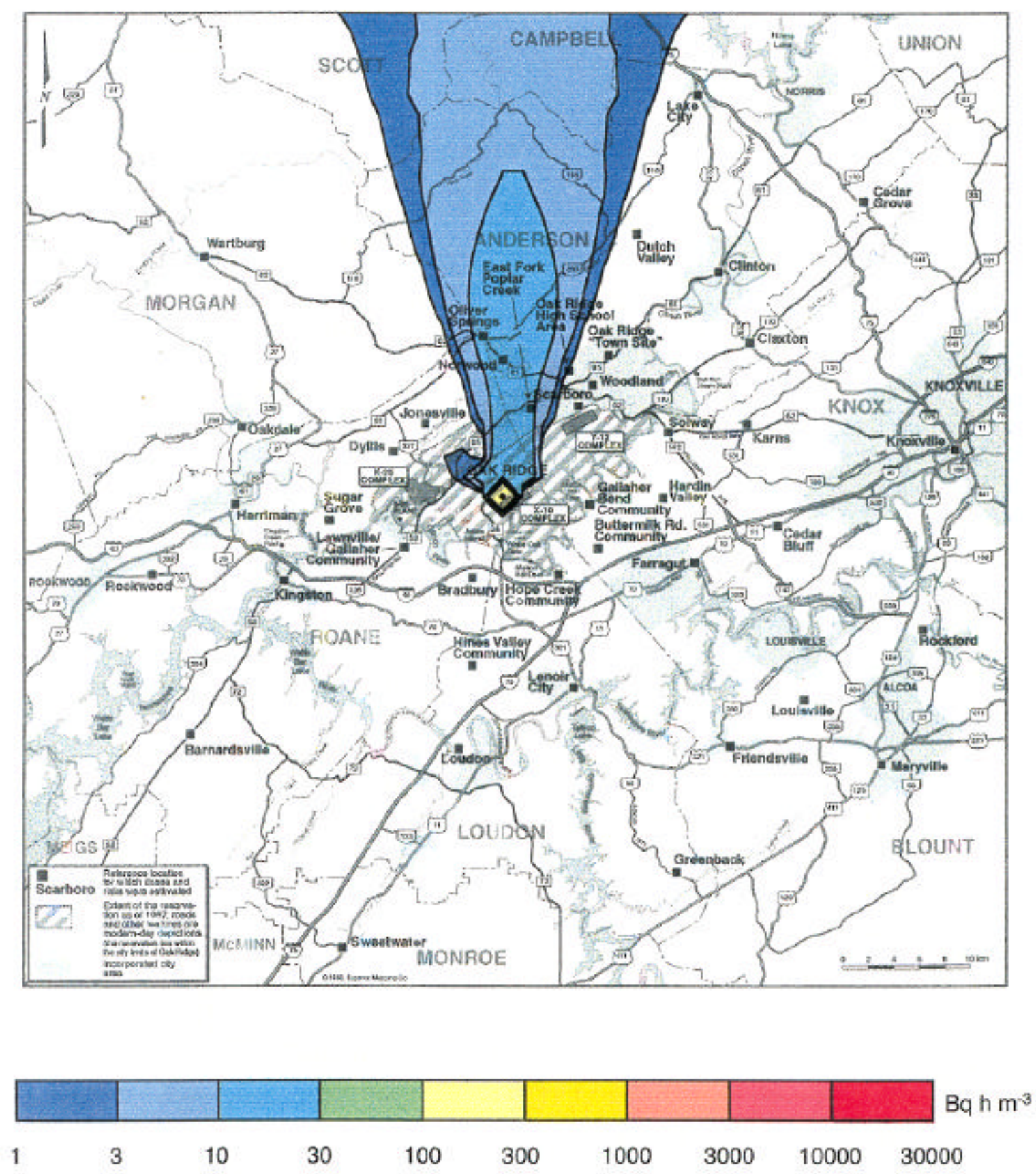

Figure 4.10 Contours for the lower bound estimates ( 2.5 percentile) of the $95 \%$ subjective confidence interval for the time-integrated, ground-level concentrations of total ${ }^{131} \mathrm{I}\left({ }^{131} \mathrm{I}\right.$ in all three chemical forms) in air following the accident on April 29, 1954. The actual concentration at a given location is highly likely to be greater than the value presented in this figure. 
TASK 1 REPORT

${ }^{131}$ I Releases from X-10 Radioactive Lanthanum Processing July 1999 Atmospheric Dispersion/Radioiodine Chemistry

Page 4-40
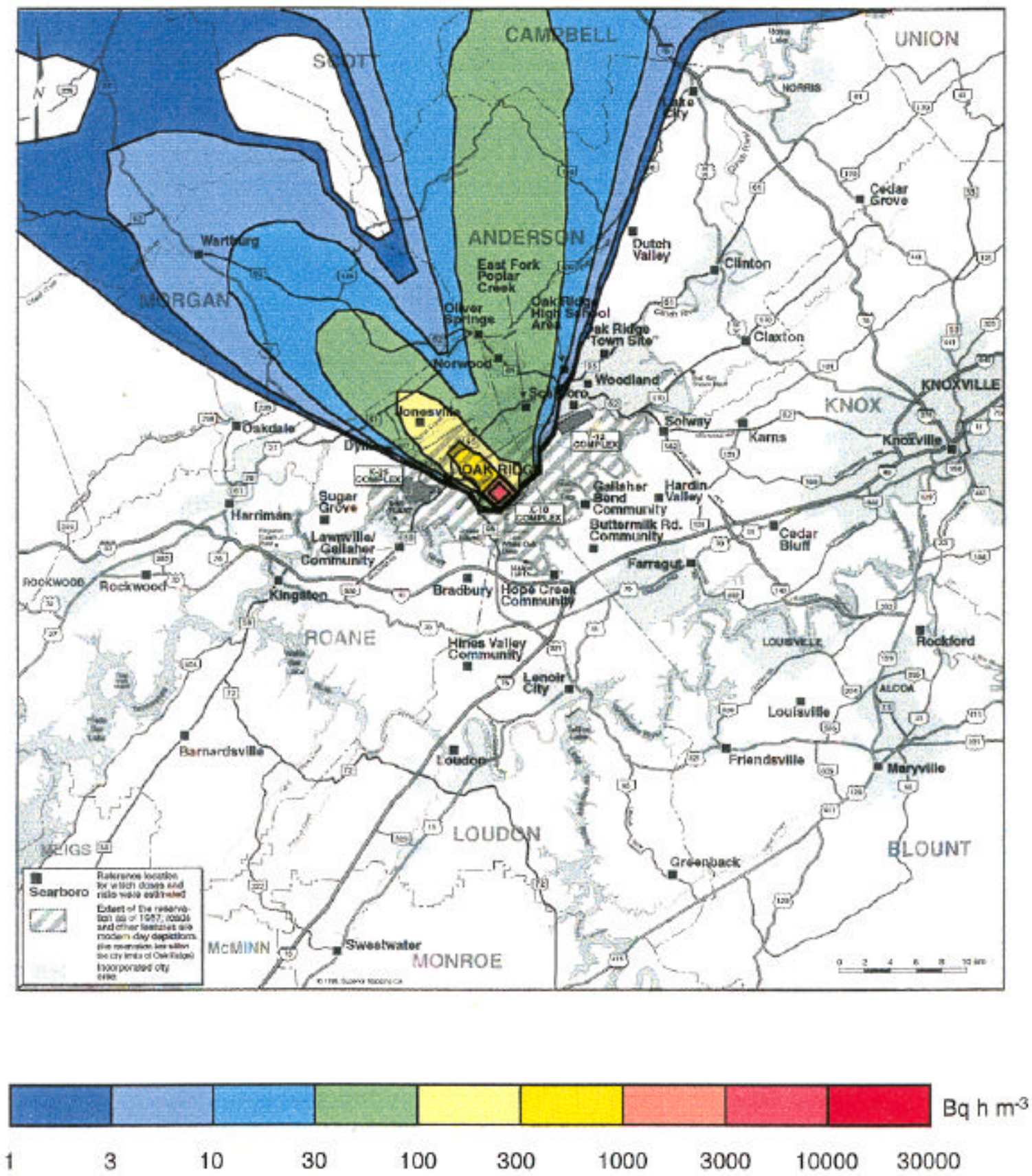

Figure 4.11 Contours of the central estimate $\left(50^{\text {th }}\right.$ percentile) of the $95 \%$ subjective confidence interval for the time-integrated, ground-level concentrations of ${ }^{131} \mathrm{I}\left({ }^{131} \mathrm{I}\right.$ in all three chemical forms) in air following the accident on April 29, 1954. 

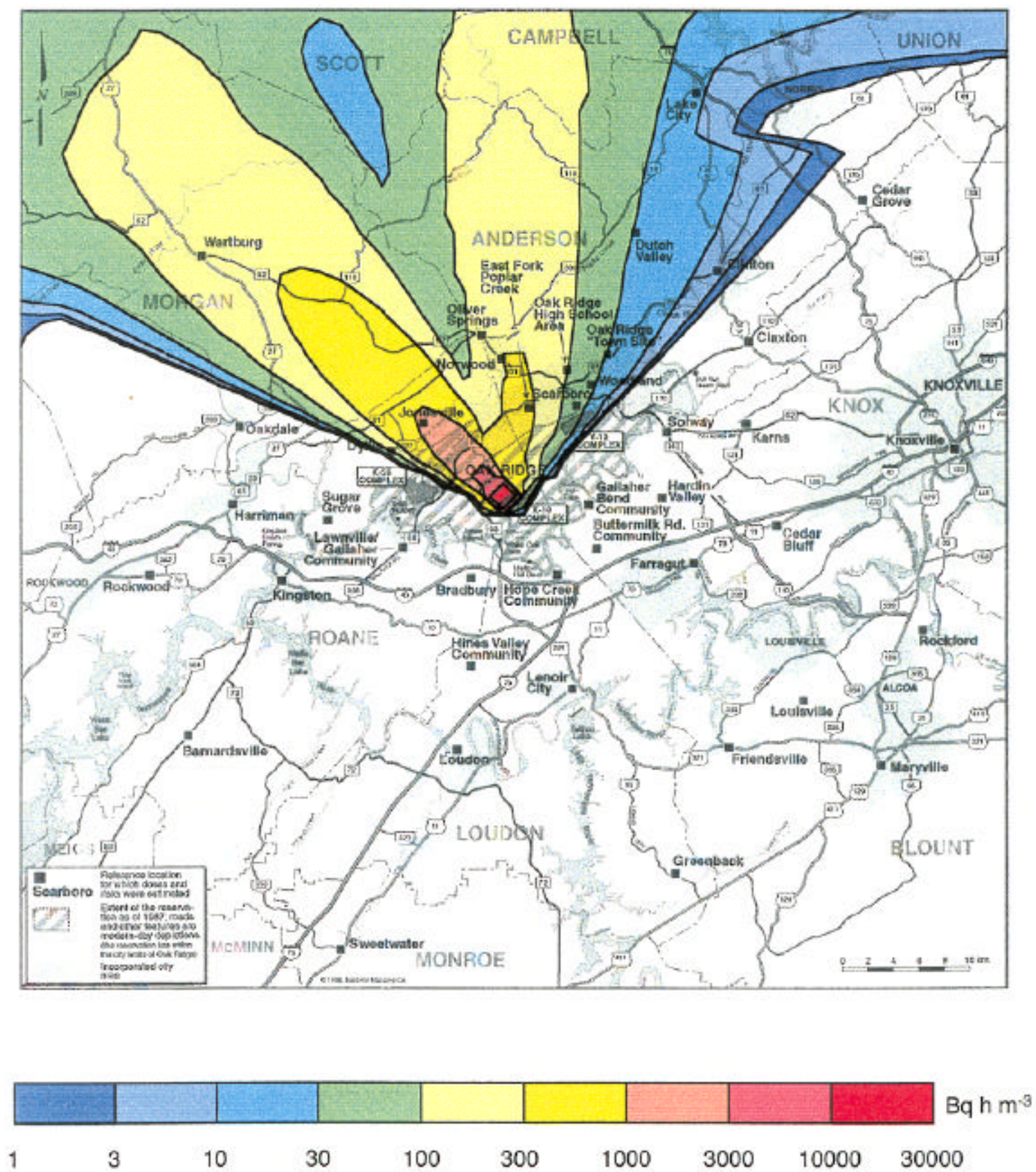

Figure 4.12 Contours for the upper bound estimates (97.5 percentile) of the $95 \%$ subjective confidence interval for the time-integrated, ground-level concentrations of total ${ }^{131} \mathrm{I}\left({ }^{131} \mathrm{I}\right.$ in all three chemical forms) in air following the accident on April 29, 1954. The actual concentration at a given location is highly likely to be less than the value presented in this figure. 
TASK 1 REPORT

July 1999

${ }^{131}$ I Releases from X-10 Radioactive Lanthanum Processing -

Page 4-42

This page intentionally left blank. 


\subsection{Future Refinements}

For the routine releases, the atmospheric dispersion modeling in this analysis used hourly specifications of all data except the source term. In the next phase of the analysis, it is recommended that the entire analysis from release to health risk be conducted at the scale of one hour. Even with the availability of source term information at the fine resolution of one hour, the recommended analysis would be a significantly major undertaking because of the enormous computer memory and storage requirements for the completion of the analysis from source to lifetime risk for the thirteen years of release.

Uncertainty in the characterizations of wind direction in the Oak Ridge Reservation should be reduced to reduce the uncertainty in the predicted range of time-integrated concentrations of ${ }^{131} \mathrm{I}$ in air from the accidental release of April 29, 1954. Eckman et al. (1992) used a network of meteorological monitoring stations to analyze the wind data in the Oak Ridge region. In that study the meteorological data were recorded at 15-min. intervals. Correlations among same parameters measured at different stations and among different parameters measured at the same stations can be developed and used along with the meteorological data available from strip charts from various stations in Oak Ridge during the time of the accident to establish an interpolated wind-field structure for the Oak Ridge region during the accident. This methodology would allow the determination of the distribution of probable directions of the travel of a single puff of

${ }^{131}$ I released to the atmosphere at a specific time during the accident. A combined modeling approach that addresses transport of individual puffs while modeling the growth of the puff in a Gaussian sense would allow tracking of several such puffs, each with an alternate path of plume travel. This would be a significant improvement over the current analysis. However, it must be noted that the applicability of this approach is entirely dependent on the development of a defensible, probability-based wind field structure. In addition, this exercise was clearly out of the scope and budget of the current project. However, the analysis performed in this study provides the justification for the conduct of the more detailed analysis suggested here. The proposed analysis is also crucial to the determination of defensible health impacts to real members of the public. 


\subsection{References}

Aoyama, M., Hirose, K., Suzuki, Y, Inoue, H, and Sugimura, Y. "High Level radioactive Nuclides in Japan in May." Nature 321:819-820, 1986.

Binford, F. T., Hamrick, T. P., and Cope, B. H. "Some Techniques for Estimating the Results of the Emission of Radioactive Effluent from ORNL Stacks.” ORNL-TM-3187, 1970.

Bondietti, E. A. and Brantley, J. N. "Characteristics of Chernobyl Radioactivity in Tennessee." Nature 322:313-314, 1986.

Brenk, H. D., Fairobent, J. E., and Markee, E. H., Jr. "Transport of Radionuclides in the Atmosphere." In Radiological Assessment. A Textbook on Environmental Dose Assessment. Editors: J. E. Till and H. R. Meyer, NURE/CR-3332, ORNL-5968. 1983.

Briggs, G. A. "Plume Rise Predictions," in Lectures on Air Pollution and Environmental Impact Analysis, Work Proceedings, Boston, Massachusetts, September 29 - October 3, 1975, pp 59111, American Meteorological Society, Boston, Massachusetts.

Briggs, G. A. Plume Rise, USACE Critical review Series, TLD-25075, National Technical Information Service, Springfield, Virginia 22161. 1969.

Cambray, R. S., Cawse, P. A., Garland, J. A., Gibson, A. B., Johnson, P., Lewis, G. N. J., Newton, D., Salmon, L., and Wade, B. O. "Observations on Radioactivity from the Chernobyl Accident." Nuclear Energy 26:77-101, 1987.

Chamberlain, A. C. and Wiffen, R. D. "Some observations on the Behavior of Radioiodine Vapour in the Atmosphere." Gefis. Para e appl. (journal) 42:42-48, 1959.

Clough, W. S., Cousins, L. B., and Eggleton, E. J. "Radio-Iodine Adsorption on Particulate Matter." J. Air and Water Pollution 9:769:789, 1965.

Eckman, R. M., Dobosy, R. J., Pendergrass, W. R. "Preliminary Analysis of Wind Data for the Oak Ridge Site.” NOAA/ATDD TM/ERL/ARI-193, 1992.

Fitzgerald, J. W. "Approximate Formulas for the Equilibrium Size of An Aerosol Particle as a Function of Its Dry Size and Composition and the Ambient Humidity." J. Applied Meteorology 14:1044-1049, 1975.

Fitzpatrick, F. C. "Oak Ridge National Laboratory Site Data for Safety Analysis Reports." ORNL/ENG/TM-19, 1982.

Garland, J. A. “The Absorption of Iodine by Atmospheric Particles.” J. Nuclear Energy 21:687. 1967. 
Gifford, F. A. "An Outline of Theories of Diffusion in the Lowest Layers of the Atmosphere." In Meteorology and Atomic Energy - 1968. TID-24190, U.S. Atomic Energy Commission, pp. 65-116. National Technical Information Service, Springfield, VA, 1968.

Guinn, P. R. "Incident in Bldg. 3026-D." Inter-company Correspondence to J. C. Hart, Oak Ridge National Laboratory. May 17, 1954.

Hanna S. R., Briggs, G. A., and Hosker, R. P., Jr. "Handbook on Atmospheric Diffusion.” Office of Health and Environmental Research, Office of Energy Research, U. S. Department of Energy. DOE/TIC-11223 (DE82002045). 1982.

Hillsmeier, W. F. "Supplementary Meteorological Data for oak Ridge." NOAA/ATDD Report ORO-199. Oak Ridge, TN. 1963.

Holland, J.Z. "A Meteorological Survey of the Oak Ridge Area: Final Report Covering the Period 1948-1952." NOAA/ATDD Report ORO-99. Oak Ridge, TN. 1953. (ChemRisk Repository No. 1211)

Holzworth, G. C. "Mixing Heights, Wind Speeds, and Potential for Urban air Pollution Throughout the Contiguous United States." Office of Air Programs Publication No. 101. U.S. Environmental Protection Agency, Research Triangle Park, NC, 1972.

Horst, T. W. "The Modification of Plume Models to Account for Dry Deposition." BoundaryLayer Meteorology. Vol. 30, pp. 413-430, 1984.

Horst, T. W. "A Surface Depletion Model for Deposition from A Gaussian Plume." Atmospheric Environment. Vol. 11, pp 41-46, 1977.

Huber, A. H. "Evaluation of A Method for Estimating Pollution Concentrations Downwind of Influencing Buildings.” Atmospheric Environment 18(11):2311-2338, 1984).

Lasher, L. C. ORNL Central Files Memorandum 70-2-43, 1970a.

Lasher, L. C. ORNL Central Files Memorandum 70-2-8, 1970 b.

Lasher, L. C. ORNL Central Files Memorandum 69-12-37, 1969a.

Lasher, L. C. ORNL Central Files Memorandum 69-10-76, 1969 b.

Lasher, L. C. ORNL Central Files Memorandum 69-10-72, 1969 c.

Lasher, L. C. ORNL Central Files Memorandum 69-9-58, 1969d.

Lasher, L. C. ORNL Central Files Memorandum 69-8-22, 1969e.

Lasher, L. C. ORNL Central Files Memorandum 69-7-18, $1969 f$. 
Lasher, L. C. ORNL Central Files Memorandum 69-6-51, 1969g.

Lasher, L. C. ORNL Central Files Memorandum 69-6-4, 1969 h.

Lasher, L. C. ORNL Central Files Memorandum 69-4-36, 1969 i.

Lasher, L. C. ORNL Central Files Memorandum 69-3-55, 1969j.

Lasher, L. C. ORNL Central Files Memorandum 69-2-38, 1969k.

Lasher, L. C. ORNL Central Files Memorandum 69-1-24, 19691.

Lasher, L. C. ORNL Central Files Memorandum 69-1-18, 1969m.

Lasher, L. C. ORNL Central Files Memorandum 68-11-48, $1968 \mathrm{a}$.

Lasher, L. C. ORNL Central Files Memorandum 68-9-42, 1968 b.

Lasher, L. C. ORNL Central Files Memorandum 68-8-51, $1968 \mathrm{c}$.

Lasher, L. C. ORNL Central Files Memorandum 68-8-34, 1968 d.

Lasher, L. C. ORNL Central Files Memorandum 68-7-2, 1968 e.

Lasher, L. C. ORNL Central Files Memorandum 68-5-49, $1968 f$.

Lasher, L. C. ORNL Central Files Memorandum 68-4-50, 1968g.

Lasher, L. C. ORNL Central Files Memorandum 68-4-11, 1968 h.

Lasher, L. C. ORNL Central Files Memorandum 68-3-6, 1968 i.

Lasher, L. C. ORNL Central Files Memorandum 68-2-8, 1968j.

Lasher, L. C. ORNL Central Files Memorandum 68-1-30, 1968k.

Lasher, L. C. ORNL Central Files Memorandum 67-12-26, 1967a.

Lasher, L. C. ORNL Central Files Memorandum 67-11-29, 1967 b.

Lasher, L. C. ORNL Central Files Memorandum 67-10-36, 1967 c.

Lasher, L. C. ORNL Central Files Memorandum 67-9-10, 1967d.

Lasher, L. C. ORNL Central Files Memorandum 67-8-17, 1967e. 
${ }^{131}$ I Releases from X-10 Radioactive Lanthanum Processing -

Lasher, L. C. ORNL Central Files Memorandum 67-7-9, $1967 f$.

Lasher, L. C. ORNL Central Files Memorandum 67-6-11, 1967g.

Lasher, L. C. ORNL Central Files Memorandum 67-5-21, $1967 \mathrm{~h}$.

Lasher, L. C. ORNL Central Files Memorandum 67-4-8, 1967 i.

Lasher, L. C. ORNL Central Files Memorandum 67-3-39, 1967j.

Lasher, L. C. "Radioactive Waste Disposal Operations Report for the Month of May, 1966."

ORNL Central Files Memorandum 66-7-9, July 6, 1966.

Lasher, L. C. "Radioactive Waste Disposal Operations Report for January, 1965." ORNL Central Files Memorandum 65-3-11, March 5, 1965.

Lindberg, S. Personal communication with S. K. Nair, 1998.

Ludwick, J. D. “A Portable Boom-type Air Sampler.” Pacific Northwest laboratory Annual Report for 1966 to the USAEC Division of Biology and Medicine. Vol. II: Physical Sciences, Part 1, Atmospheric Sciences. BNWL-481 1. D. W. Pearce and M. R. Compton, eds. pp 87-92, 1967.

Ludwick, J. D. "Investigation of the Nature of ${ }^{131}$ I in the Atmosphere." In: Hanford Radiological Sciences Research and Development Annual Report for 1963. (C.C. Gamertsfelder and J.K. Green, Eds.). HW-81746. Hanford Atomic Products Operation. Richland, Washington. 1964. (ChemRisk Repository No. 2189)

Manneschmidt, J. F. "Equipment and Procedures for Stack gas Monitoring at ORNL." Presented at the Seventh AEC Air Cleaning Conference at Brookhaven National Laboratory, October 10-12, 1961.

McElhoe, H. B. E-mail transmittal of ETTP TSP/PM10 Annual Data to S. K. Nair. 1998.

Meyers, T. Personal communication with S. K. Nair, 1998.

Moyers, J. L. and Duce, R. A. "Gaseous and Particulate Iodine in the Marine Atmosphere." J. of Geophysical Research 77(27):5229-5238, 1972.

Mueck, K. "Variations in Activity Concentration and Radionuclide Ratio in Air After the Chernobyl Accident and Its Relevance to Inhalation Dose Estimates." Radiation Protection Dosimetry 22:219-229, 1988.

NCRP. "A Guide for the Uncertainty Analysis in Dose and Risk Assessments Related to Environmental Contamination." NCRP Commentary No. 14. National Council on Radiation Protection and Measurements, Bethesda, MD, 1996. 
Nietzel et al. "Hanford Site Environmental Policy Act (NEPA) Characterization." PNNL-6415, Rev. 9, 1993.

Oak Ridge Reservation Environmental Report for 1991. Volume I: Narrative, Summary, and Conclusions. ES/ESH-22/V1, Oak Ridge, TN, 1992.

Oakes, T. W., Montford, M A., Shank, K. E., Wagner, E. B., Scott, T. G., and Eldridge, J. S. "Methods and Procedures Utilized in Environmental Management Activities at Oak Ridge National Laboratory.” ORNL/TM-7212, 1981.

ORNL. Oak Ridge National Laboratory. "Health Physics and Safety Annual Report for 1967." Report ORNL-4286. August, 1968.

ORNL. Oak Ridge National Laboratory. "Health Physics and Safety Annual Report for 1966." Report ORNL-4146. August, 1967.

ORNL. Oak Ridge National Laboratory. "Health Physics and Safety Annual Report for 1965." Report ORNL-3969. July, 1966.

Perkins, R. W. "Physical and Chemical Forms of I-131 from Fallout and Chemical Processing Plants." In Hanford Radiological Sciences Research and Development Annual Report for 1963. HW-81746. C. C. Gamertsfelder and J. K. Green, eds. pp 3.55-3.58, 1964.

Perkins, R. W. "Studies of Radoiodine and Other Fallout Radionuclides in Air." In Hanford Radiological Sciences research and Development Annual Report for 1962. HW-77609. . C. Gamertsfelder and J. K. Green, eds. pp 3.36-3.48, 1963.

Ramsdell, J. V., Jr., and Fosmire, C. J. "Estimating Concentrations in Plumes Released in the Vicinity of Buildings: Model Development." Atmospheric Environment. 32(10):1663-1677, 1998.

Ramsdell, J. V., Jr., Simonen, C. A., and Burk, K. W. "Regional Atmospheric Transport Code for Hanford Emission Tracking (RATCHET)." Hanford Environmental Dose Reconstruction Project. PNWD-2224 HEDR, UC-000, Battelle Pacific Northwest Laboratories, Richland, WA, 1994.

Ramsdell, J. V., Jr. "Diffusion in Building Wakes for Ground-Level Releases." Atmospheric Environment 24B(3):377-388, 1990.

Stone, W. A., Thorp, J. M., Gifford, O. P., and Hoitink, D. J. "Climatological Summary for the Hanford Area." PNL-4622, 1983.

Turner, D. B. "Workbook of Atmospheric Dispersion Estimates." PHS Publication No. 999-AP26. U.S. Department of Health, Education, and Welfare, National Air Pollution Control Administration. Cincinnati. OH. 1970. 
USEPA. U.S. Environmental Protection Agency. "User's Guide for the Industrial Source Complex (ISC3) Dispersion Models. Vols. I and II." EPA-454/B-95-003. Research Triangle Park. NC. 1995.

USEPA. U.S. Environmental Protection Agency. "User's Guide for the Industrial Source Complex (ISC2) Dispersion Models. Vols. I and II." EPA-450/4-92-008. Research Triangle Park. NC. 1992.

UNRC. U.S. Nuclear Regulatory Commission. "Atmospheric Dispersion Models for Potential Accident Consequence Assessments at Nuclear Power Plants." U. S. NRC Regulatory Guide 1.145 Revision 1. U.S. NRC, Washington, D.C., 1982.

Voillequé, P. G. "Iodine Species in Reactor Effluents and in the Environment." EPRI-NP-1269. Electric Power Research Institute, Palo Alto, CA. 1979.

Winkler, P. "The Growth of Atmospheric Aerosol Particles as a Function of the Relative Humidity - II. An Improved Concept of Mixed Nuclei.” Aerosol Science 4:373-387, 1973. 
TASK 1 REPORT

July 1999

${ }^{131}$ I Releases from X-10 Radioactive Lanthanum Processing -

Page 4-50 Atmospheric Dispersion/Radioiodine Chemistry

This page intentionally left blank. 


\subsection{TRANSFER OF ${ }^{131}$ I FROM AIR TO VEGETATION}

Iodine from the atmosphere is deposited on both the vegetation canopy and the ground. Deposition can occur under dry conditions, or it can be associated with precipitation. Of the total iodine intercepted by vegetation surfaces, some may be removed by environmental weathering processes such as wind, rain, and plant senescence, while the rest is retained. In addition, iodine deposited on the ground can be taken up by the roots of a plant.

Two categories of vegetation are important for the bioaccumulation of ${ }^{131} \mathrm{I}$. The first is "pasture grass," which is consumed by grazing animals and contributes to the contamination of milk and meat. The second category, "leafy vegetables," is composed of vegetables such as lettuce, cabbage, and spinach. These vegetables are usually consumed a short time after harvest and thus may carry significant levels of ${ }^{131} \mathrm{I}$. Other types of crops that accumulate ${ }^{131} \mathrm{I}$ deposited from the air are normally stored for a sufficiently long time that their contribution to the total intake of ${ }^{131}$ I by humans is negligible due to radioactive decay of ${ }^{131} \mathrm{I}$.

\subsection{Description of Processes and Model Assumptions}

To develop the governing equations of transfer of ${ }^{131} \mathrm{I}$ from air to vegetation and ground, the following assumptions were made, based on our current state of knowledge.

- Three physico-chemical forms of iodine are considered to be present in air:

a. a highly reactive form, assumed to be molecular or elemental iodine $\left(\mathrm{I}_{2}\right)$;

b. a particulate form, which accounts for iodine attached to atmospheric aerosols; and

c. a nonreactive form, assumed to be organic iodine (e.g., $\left.\mathrm{CH}_{3} \mathrm{I}\right)$.

- Iodine is deposited on vegetation by both dry and wet deposition processes. Dry deposition refers to the process of direct removal of iodine from air by collection on soil, vegetation, or water surfaces. Wet deposition refers to the removal of iodine from air to land or water surfaces by various types of precipitation, including rain and snow.

- Dry deposition occurs even during periods of precipitation.

- Uptake of ${ }^{131}$ I from soil by plants is negligible when compared with direct deposition of ${ }^{131}$ I from the atmosphere.

- For the April 29, 1954, accident, the source term varied significantly with time. The deposition rates of the three chemical forms of ${ }^{131} \mathrm{I}$ on vegetation vary on a half-hourly basis, reflecting the half-hourly variations of ${ }^{131}$ I release rates, ${ }^{131}$ I concentrations in air, and precipitation rates. A transient approach is used to estimate the time-integrated concentration of ${ }^{131}$ I on the vegetation. 
- For routine releases, the concentrations in air did not vary significantly over time; therefore, an annual average approach is used. In this case, the iodine deposition rates can be considered constant during a given year and equal to the annual averages.

\subsection{Modeling Approach}

\section{Accidental Release of 1954}

A governing equation to estimate transient concentrations of ${ }^{131} \mathrm{I}$ on pasture grass and on leafy vegetation from half-hourly changes in ground-level air concentrations was developed based on the assumptions stated above. This model uses a time-dependent approach involving the input and removal of ${ }^{131}$ I to and from pasture grass or leafy vegetables.

The transfer of ${ }^{131} \mathrm{I}$ from air to vegetation is given by Equation 5.1 for pasture grass and by Equation 5.2 for leafy vegetables. The resulting concentrations of ${ }^{131} \mathrm{I}$ in pasture grass and leafy vegetables are given by Equations 5.3 and 5.4, respectively.

$$
\begin{aligned}
& \frac{d C_{p, k}}{d t}=\left[V_{d, k} \cdot\left(\frac{r}{Y}\right)_{d r y, k}+V_{w, k} \cdot\left(\frac{r}{Y}\right)_{w e t, k}\right] \cdot C_{a, k}-\lambda_{e f f} \cdot C_{p, k} \\
& \frac{d C_{v, k}}{d t}=\left[V_{d, k} \cdot\left(\frac{r}{Y}\right)_{d r y, k}+V_{w, k} \cdot\left(\frac{r}{Y}\right)_{w e t, k}\right] \cdot \gamma_{\text {drymass:freshmass }} \cdot C_{a, k}-\lambda_{e f f} \cdot C_{v, k} \\
& C_{p}=\sum_{k=1}^{3} C_{p, k} \\
& C_{p}=\sum_{k=1}^{3} C_{p, k}
\end{aligned}
$$

where

$k=$ the index for the physico-chemical form of iodine: $k=1$ for the highly reactive form, $k=2$ for the organic form, and $k=3$ for the particulate form;

$C_{a, k}=$ the concentration of physico-chemical form $k$ of ${ }^{131} \mathrm{I}$ in air $\left[\mathrm{Bq} \mathrm{m}^{-3}{ }_{\text {air }}\right]$;

$C_{p, k}=$ the concentration of physico-chemical form $k$ of ${ }^{131} \mathrm{I}$ in pasture grass [Bq $\mathrm{kg}^{-1}$ dry mass];

$C_{p} \quad=$ the total concentration of ${ }^{131} \mathrm{I}$ in pasture grass $\left[\mathrm{Bq} \mathrm{kg}^{-1}\right.$ dry mass $]$;

$C_{v, k}=$ the concentration of physico-chemical form $k$ of ${ }^{131} \mathrm{I}$ in leafy vegetables $\left[\mathrm{Bq} \mathrm{kg}{ }^{-1}\right.$ fresh mass]; 


$$
\begin{aligned}
& C_{v} \quad=\quad \text { the total concentration of }{ }^{131} \mathrm{I} \text { in leafy vegetables }\left[\mathrm{Bq} \mathrm{kg}^{-1} \text { fresh mass }\right] \text {; } \\
& V_{d, k} \quad=\quad \text { the total dry deposition velocity }\left[\mathrm{m} \mathrm{h}^{-1}\right] \text { for form } k \text {; } \\
& V_{w, k} \quad=\quad \text { the total wet deposition velocity }\left[\mathrm{m} \mathrm{h}^{-1}\right] \text { for form } k \text {; } \\
& (r / Y)_{d r y, k} \quad=\quad \text { the mass interception factor (dry deposition) }\left[\mathrm{m}^{2} \mathrm{~kg}^{-1} \text { dry mass }\right] \text {; } \\
& (r / Y)_{\text {wet }, k} \quad=\quad \text { the mass interception factor (wet deposition) }\left[\mathrm{m}^{2} \mathrm{~kg}^{-1} \text { dry mass }\right] \text {; } \\
& \gamma_{\text {dry mass: fresh mass }}=\quad \text { the ratio of the dehydrated vegetation sample and its } \\
& \text { fresh mass }\left[\mathrm{kg}_{\mathrm{dry}} \mathrm{kg}_{\mathrm{wt}}{ }^{-1}\right. \text {; } \\
& t \quad=\quad \text { time }(\mathrm{h}) \text {; and } \\
& \lambda_{\text {eff }}=\text { the effective removal rate constant }\left(\mathrm{h}^{-1}\right) \text { that accounts for the } \\
& \text { processes of removal by weathering and radioactive decay } \\
& \text { (estimated as } 0.693 / T_{\text {eff }} \text {. } \\
& T_{\text {eff }} \quad=\quad \text { the effective half-life of iodine on vegetation }[\mathrm{h}]=\frac{T_{w} \cdot T_{r}}{T_{w}+T_{r}}
\end{aligned}
$$

where

$$
\begin{array}{ll}
T_{w}= & \text { the removal half-life from weathering processes, and } \\
T_{r}= & \text { the radiological half-life of }{ }^{131} \mathrm{I}(8.04 \mathrm{~d}) .
\end{array}
$$

The wet deposition velocity is defined as follows:

$$
V_{w}=W R \cdot P
$$

$$
\begin{aligned}
& W R=\text { the washout ratio }\left[\mathrm{m}^{3} \text { air }\left(\mathrm{L}^{-1} \text { rain }\right)\right] \text {, and } \\
& P \quad=\text { the half-hourly precipitation rate }\left[\mathrm{mm} \mathrm{h}^{-1}\right] .
\end{aligned}
$$

The washout ratio is defined in Section 5.3.2.1. Equations 5.1 and 5.2 are solved on a halfhourly basis. For each half-hour period, the input of a specific chemical form of ${ }^{131}$ I to the vegetation (given by the first terms on the right hand sides of Equations 5.1 and 5.2) takes a constant value defined by (1) the constant concentration of the chemical form in air $\left(C_{a, k}\right)$ during that half hour, (2) the constant dry deposition velocity and mass interception factor for the chemical form, and (3) the constant wet deposition velocity during the half-hour period. Closedform analytical solutions of the two equations are then used for each half-hour period to obtain the time-dependent concentrations of the different forms of ${ }^{131} \mathrm{I}$ on the pasture grass and vegetation. The generic analytical solution for a first-order ordinary differential equation described by

$$
\frac{d C}{d t}=s-\lambda_{e f f} C
$$


is obtained using the following equation:

$$
C=\left[s-\left(s-\lambda_{e f f} C_{o}\right) \cdot e^{-\lambda_{e f f} t}\right] \cdot\left(\frac{1}{\lambda_{e f f}}\right)
$$

where

$$
\begin{aligned}
& C=\quad \begin{array}{l}
\text { the concentration of }{ }^{131} \mathrm{I} \text { in pasture grass or leafy vegetables }\left[\mathrm{Bq} \mathrm{kg}^{-1}\right] \text { at a } \\
\text { given time } t
\end{array} \\
& \lambda_{\varepsilon f f}=\text { the constant source of }{ }^{131} \mathrm{I} \text { to the vegetation }\left[\mathrm{Bq} \mathrm{kg}^{-1} \mathrm{~h}^{-1}\right] \\
& C_{o}=\text { the initial concentration of }{ }^{131} \mathrm{I} \text { on the vegetation }\left[\mathrm{Bq} \mathrm{kg}^{-1}\right] .
\end{aligned}
$$

This solution is used with $t=0.5 \mathrm{hr}$ for each half-hour period. The initial concentration of ${ }^{131} \mathrm{I}$ for each half-hour is obtained from the solution for the previous half-hour period. The pasture grass and vegetation are assumed to be in pristine condition at the start of the simulation before the accident started $\left(\mathrm{C}_{\mathrm{o}}=0 \mathrm{~Bq} \mathrm{~kg}^{-1}\right)$. The necessary parameter values are described in detail in the next sections.

\section{$\underline{\text { Routine Releases }}$}

For routine releases, estimates of ${ }^{131}$ I concentrations on pasture grass and on vegetation are derived from the ground-level air concentrations using a constant deposition rate of ${ }^{131}$ I from air equal to the annual average deposition rate. This model uses a steady-state approach that is applicable to a continuous release described by the annual average concentration of iodine in air.

Transfer of ${ }^{131}$ I from air to vegetation is given by Equation 5.6 for pasture grass and by Equation 5.7 for leafy vegetables.

$$
\begin{aligned}
C_{p, k} & =A P_{k} \cdot C_{a, k} \\
& =\left[V_{d, k} \cdot\left(\frac{r}{Y}\right)_{d r y, k}+V_{w, k} \cdot\left(\frac{r}{Y}\right)_{w e t, k}\right] \cdot \frac{1-\exp \left(-\lambda_{e f f} \cdot t_{a g}\right)}{\lambda_{e f f}} \cdot C_{a, k} \\
C_{v, k} & =A V_{k} \cdot C_{a, k} \\
& =\left[V_{d, k} \cdot\left(\frac{r}{Y}\right)_{d r y, k}+V_{w, k} \cdot\left(\frac{r}{Y}\right)_{w e t, k}\right] \cdot \gamma_{\text {drymass freshmass }} \cdot \frac{1-\exp \left(\lambda_{e f f} \cdot t_{a g}\right)}{\lambda_{\text {eff }}} \cdot C_{a, k}
\end{aligned}
$$


where

$$
\begin{aligned}
& C_{p, k} \quad=\text { the concentration of }{ }^{131} \mathrm{I} \text { in pasture grass }\left[\mathrm{Bq} \mathrm{kg}^{-1} \text { dry mass }\right] \text {; } \\
& A P_{k} \quad=\text { the transfer factor of }{ }^{131} \mathrm{I} \text { from air to pasture grass for the physico- } \\
& \text { chemical form } k\left[\mathrm{~Bq} \mathrm{~kg}^{-1} \text { dry mass per } \mathrm{Bq} \mathrm{m}^{-3}{ }_{\text {air }}\right. \text {; } \\
& k \quad=\text { the index for the physico-chemical form of iodine: } k=1 \text { for the } \\
& \text { highly reactive form, } k=2 \text { for the particulate form, and } k=3 \text { for the } \\
& \text { organic form; } \\
& C_{a, k} \quad=\text { the concentration of physico-chemical form } k \text { of }{ }^{131} \mathrm{I} \text { in air }\left[\mathrm{Bq} \mathrm{m}{ }^{-3} \text { air }\right] \text {; } \\
& C_{v, k} \quad=\text { the concentration of }{ }^{131} \mathrm{I} \text { in leafy vegetables }\left[\mathrm{Bq} \mathrm{kg}{ }^{-1} \text { fresh mass }\right] \text {; } \\
& A V_{k} \quad=\text { the transfer factor of }{ }^{131} \mathrm{I} \text { from air to leafy vegetables for the physico- } \\
& \text { chemical form } k\left[\mathrm{~Bq} \mathrm{~kg}{ }^{-1} \text { fresh mass per } \mathrm{Bq} \mathrm{m}^{-3}\right] \text {; } \\
& V_{d, k} \quad=\text { the total dry deposition velocity }\left[\mathrm{m} \mathrm{d}^{-1}\right] \text { for form } k \text {; } \\
& V_{w, k} \quad=\text { the total wet deposition velocity }\left[\mathrm{m} \mathrm{d}^{-1}\right] \text { for form } k \text {; } \\
& (r / Y)_{d r y, k}=\text { the mass interception factor (dry deposition) }\left[\mathrm{m}^{2}\left(\mathrm{~kg}^{-1} \text { dry mass }\right)\right] \text {; } \\
& (r / Y)_{\text {wet }, k}=\text { the mass interception factor (wet deposition) }\left[\mathrm{m}^{2}\left(\mathrm{~kg}^{-1} \text { dry mass }\right)\right] \text {. } \\
& \gamma_{\text {dry mass: fresh mass }}=\text { the ratio of the dehydrated vegetation sample and its fresh mass } \\
& {\left[\operatorname{kg}_{\text {dry }}\left(\mathrm{kg}^{-1} \text { wet }\right)\right] \text {; }} \\
& t_{a g} \quad=\text { the time period of exposure of the standing crop biomass [d]; and } \\
& \lambda_{\text {eff }}=\text { the effective removal rate constant }\left[\mathrm{d}^{-1}\right] 0.693 / T_{\text {eff. }} \\
& T_{\text {eff }} \quad=\text { the effective half-life of iodine on vegetation }[\mathrm{d}]=\frac{T_{w} \cdot T_{r}}{T_{w}+T_{r}}
\end{aligned}
$$

For routine releases, the wet deposition velocity is given by the following:

$$
V_{w}=W R \cdot R
$$

$W R=$ the washout ratio $\left[\mathrm{m}^{3}\right.$ air $\left(\mathrm{L}^{-1}\right.$ rain $\left.)\right]$, and

$R \quad=$ the annual average precipitation rate, expressed on a daily basis $\left[\mathrm{mm} \mathrm{d}^{-1}\right]$. 
The necessary parameter values are discussed in detail in the next sections. Equations 5.6 and 5.7 produce estimates of the annual average concentrations of ${ }^{131} \mathrm{I}$ in vegetation. The concentrations in air and in vegetation vary from one year to the next. However, the deposition velocities and the mass interception factors are annual averages, and they apply for all years of release.

\subsection{Input Parameters}

\subsubsection{Dry Deposition}

The combination of processes by which an airborne contaminant is transferred to vegetation without being carried by rain is referred to as dry deposition. In Equations 5.1, 5.2, 5.6 and 5.7, dry deposition is described by the total deposition velocity $\left(V_{d}\right)$ and by the mass interception factor $(r / Y)_{d r y}$. The total dry deposition velocity relates the deposition flux $\left[\mathrm{Bq} \mathrm{m} \mathrm{s}^{-1}\right]$ to the concentration in air $\left[\mathrm{Bq} \mathrm{m}^{-3}\right]$, and accounts for the total deposition per unit time per unit area. The area includes vegetation, detritus, the root mat, and soil. However, many measurements (Chamberlain and Chadwick, 1953; 1966; Chamberlain, 1960; Heinemann and Vogt, 1980) were performed for the vegetation dry deposition velocity $\left(V_{v}\right)$ which accounts for the deposition just to the vegetation. The vegetation dry deposition velocity $\left(V_{v}\right)$ is mathematically given by the product between the total dry deposition velocity $\left(V_{d}\right)$ and the interception fraction $(r)$, which expresses the amount of contaminant that is retained on the vegetation from the net flux to the ground (Equation 5.9).

$$
V_{v}=V_{d} \cdot r
$$

Furthermore, the vegetation dry deposition velocity $\left(V_{v}\right)$ can be normalized to the biomass of the vegetation $(Y)$. The normalized dry deposition velocity $\left(V_{D}\right)$ is mathematically given by the product of the total dry deposition velocity $\left(V_{d}\right)$ and the mass interception factor $(r / Y)_{d r y}$ (Equation 5.10).

$$
V_{D}=V_{d} \cdot\left(\frac{r}{Y}\right)_{d r y}
$$

Dry deposition of elemental iodine is enhanced if the vegetation is moist, as, for instance, after rain or because of morning dew. During the 1954 accident, iodine was released for 2.5 hours, starting after about 4:30 p.m. on April 29, which was a warm day (maximum temperature $78^{\circ} \mathrm{F}$ ) with no precipitation during the afternoon hours. Thus, it can be assumed that the vegetation was dry during the first hours of the accident. However, rain was recorded between 6:15 and 7 p.m. on April 29, 1954. Therefore, wet deposition as well as dry deposition on wet vegetation occurred during the accident. Wet deposition is discussed in the next section.

The normalized dry deposition velocity $\left(V_{D}\right)$ can be determined from field experiments. An extensive set of experimental measurements of $V_{D}$ has been reported by Vogt et al. (1976) and Heinemann and Vogt (1980). These measurements were used for calibration purposes. In other words, after selection of the appropriate parameter values for $V_{d}$ and $(r / Y)_{d r y}, V_{D}$ for each form of

${ }^{131}$ I was estimated using Equation 5.10, and these values of $V_{D}$ were compared with the measured 
values. Then the values of $V_{d}$ and $(r / Y)_{d r y}$ were adjusted, and the entire process was repeated until the calculated values for $V_{D}$ matched the experimentally measured values. Distinctions were made between dry deposition on moist vegetation and dry deposition on dry vegetation for the April 29, 1954, accident situation. The selected parameter ranges are listed in Table 5.1 for $V_{d}$ and in Table 5.2 for $(r / Y)_{d r y}$. The comparison between the calculated and measured values for the normalized deposition velocity $\left(V_{D}\right)$ is shown in Table 5.3.

For routine releases, annual averages for the deposition velocity were selected. In the course of a year, all deposition mechanisms will eventually occur (dry deposition on moist vegetation, dry deposition on dry vegetation, and wet deposition). The subjective probability distributions for the deposition velocities have been selected to include all these possible situations. Also, the subjective probability distributions for the annual average deposition velocities were chosen to be tighter (more sampling towards the central values) than the distributions for the accident situation, because an annual average should generally have a smaller variation than samples taken at given moments in time.

It should be noted that the parameter values for $V_{d}$ presented in this section are used only for the estimation of concentrations of ${ }^{131} \mathrm{I}$ on pasture grass and vegetation, and not for the estimation of the depletion of ${ }^{131} \mathrm{I}$ from the plume. The parameter values for $V_{d}$ presented in Section 4.3.2 are applicable for the overall regional dry deposition and are used to estimate the depletion as described in Section 4.3. Total dry deposition from the plume accounts for the deposition on trees in forested areas and or lakes and other nonvegetated areas that are not grazed by the cows or goats. In this section, the focus is primarily on the dry deposition that occurs on pasture grass and leafy vegetation.

Table 5.1 Selected values and distribution shapes for the total dry deposition velocity $V_{d}$.

\begin{tabular}{|c|c|c|c|c|c|}
\hline \multirow{2}{*}{$\begin{array}{l}\text { Chemical form } \\
\qquad \text { of }^{131} \mathbf{I}\end{array}$} & \multicolumn{4}{|c|}{$\mathrm{V}_{\mathrm{d}}\left[\mathrm{cm} \mathrm{s}^{-1}\right]$} & \multirow[t]{2}{*}{ Type of Release } \\
\hline & lower $^{a}$ & upper $^{\mathrm{a}}$ & mode & Distribution & \\
\hline Elemental & 0.3 & 4.0 & & log-uniform & Routine \\
\hline Particulate & 0.05 & 0.5 & 0.15 & log-triangular & Routine \\
\hline Organic & 0.001 & 0.05 & 0.0071 & log-triangular & Routine \\
\hline Elemental & 0.3 & 3.0 & & log-uniform & $\begin{array}{c}\text { Accident } \\
\text { (dry vegetation) }\end{array}$ \\
\hline Elemental & 1.0 & 6.0 & & log-uniform & $\begin{array}{c}\text { Accident } \\
\text { (moist vegetation) }\end{array}$ \\
\hline Particulate & 0.05 & 0.5 & & log-uniform & Accident \\
\hline Organic & 0.001 & 0.05 & & log-uniform & Accident \\
\hline
\end{tabular}

${ }^{\text {a }}$ Lower and upper values represent the extreme limits of the log-uniform distribution. 
Table 5.2 Selected values and distribution shapes for the mass interception factor $(r / Y)_{d r y}$ for dry deposition.

\begin{tabular}{|c|c|c|c|c|c|}
\hline \multirow{2}{*}{$\begin{array}{c}\text { Chemical form } \\
\text { of }{ }^{131} I\end{array}$} & \multicolumn{4}{|c|}{$(r / Y)_{d r y}\left[\mathrm{~m}^{2} \mathrm{~kg}^{-1}(\mathrm{dry}\right.$ mass $\left.)\right]$} & \multirow[t]{2}{*}{ Type of Release } \\
\hline & Lower $^{\text {a }}$ & Upper $^{\mathrm{a}}$ & mode & Distribution & \\
\hline Elemental & 0.8 & 4.0 & 2.4 & Triangular & routine \\
\hline Particulate & 0.5 & 4.0 & 2.3 & Triangular & routine \\
\hline Organic & 0.8 & 4.0 & 2.4 & Triangular & routine \\
\hline Elemental & 0.8 & 4.0 & & Uniform & accident \\
\hline Particulate & 0.5 & 4.0 & & Uniform & accident \\
\hline Organic & 0.8 & 4.0 & & Uniform & accident \\
\hline
\end{tabular}

${ }^{a}$ Lower and upper values represent the extreme limits of the uniform distribution.

Table 5.3 Comparison between calculated and measured values for normalized deposition velocities $\left(V_{D}\right)\left[\mathrm{cm}^{3} \mathrm{~g}^{-1} \mathrm{~s}^{-1}\right]$.

\begin{tabular}{|c|c|c|c|c|c|c|}
\hline \multirow{3}{*}{$\begin{array}{c}\text { Chemical form } \\
\text { Elemental } \\
\text { (moist vegetation) }\end{array}$} & \multicolumn{4}{|c|}{ Calculated } & \multicolumn{2}{|c|}{ Measured } \\
\hline & \multicolumn{2}{|c|}{$\begin{array}{c}\text { Accident } \\
\text { 95\% subjective } \\
\text { confidence interval }\end{array}$} & \multicolumn{2}{|c|}{$\begin{array}{c}\text { Routine } \\
95 \% \text { subjective } \\
\text { confidence interval }\end{array}$} & \multirow{2}{*}{$\frac{\text { lower }^{\mathrm{a}}}{51^{\mathrm{b}}}$} & \multirow{2}{*}{$\frac{\text { upper }^{\mathrm{a}}}{153^{\mathrm{b}}}$} \\
\hline & 22.4 & 136 & & & & \\
\hline $\begin{array}{c}\text { Elemental } \\
\text { (dry vegetation) }\end{array}$ & 7.7 & 66 & & & $9^{c}$ & $132^{\mathrm{c}}$ \\
\hline $\begin{array}{c}\text { Elemental } \\
\text { (annual average) }\end{array}$ & & & 6.4 & 83 & & \\
\hline Particulate & 0.7 & 13.4 & 0.7 & 10.9 & $2.9^{\mathrm{d}}$ & $12.0^{\mathrm{d}}$ \\
\hline Organic & 0.021 & 1.2 & 0.028 & 0.9 & $0.04^{\mathrm{d}}$ & $0.8^{\mathrm{d}}$ \\
\hline
\end{tabular}

\footnotetext{
${ }^{\mathrm{a}}$ Lower and upper values represent the extreme observed values.

${ }^{\mathrm{b}}$ Heinemann and Vogt (1980) (an extreme value of $249 \mathrm{~cm}^{3} \mathrm{~g}^{-1} \mathrm{~s}^{-1}$ was eliminated).

${ }^{c}$ Heinemann and Vogt (1980) (an extreme value of $226 \mathrm{~cm}^{3} \mathrm{~g}^{-1} \mathrm{~s}^{-1}$ was eliminated).

${ }^{\mathrm{d}}$ Heinemann and Vogt (1980).
}

\subsubsection{Wet Deposition}

The combination of processes by which a contaminant present in air is transferred to vegetation by rainfall is referred to as wet deposition. The mechanisms which contribute to wet deposition are in-cloud and below-cloud transfer of contaminants to rain drops. The contaminant is first transferred to a rain droplet, then mixed within the rain droplet, and finally deposited on the plant surfaces. 


\subsubsection{Transfer to rain water}

Transfer of iodine to rain water is described by a washout ratio (WR, $\mathrm{m}^{3} \mathrm{~L}^{-1}$ ), which is defined as the ratio of the concentration of iodine in the rainwater $\left(\mathrm{Bq} \mathrm{L}^{-1}\right.$ rain $)$ to the concentration in air $(\mathrm{Bq}$ $\mathrm{m}^{-3}$ air $)$ at ground level $\left(\mathrm{Bq} \mathrm{m}^{-3}\right)$. The deposition rate of iodine is obtained when the wet deposition velocity defined by Equation 5.5 is multiplied by the concentration of iodine in the air.

\section{Approach for the Accidental Release}

The washout ratio was assumed to depend on the amount of rain during each rain event, and each rain event is assumed to last for a half-hour period. Thus, a rainfall lasting several hours would be treated as several events each lasting for a half hour. The greater the amount of rain per rain event, the smaller the washout ratio as defined by the following empirical equation (Slinn, 1978; NRC/CEC, 1994):

$$
W R=W R_{1}^{h} \cdot P^{-s}
$$

where

$$
\begin{aligned}
& W R_{l}{ }^{h} \quad=\text { the washout ratio for a 1-mm half-hourly rain; } \\
& P \quad=\text { the precipitation rate during a half-hour rain event }\left[\mathrm{mm} \mathrm{h}^{-1}\right] \text {; and } \\
& s=\text { the rain exponent, an empirical parameter [unitless] that accounts for the } \\
& \text { observed decrease in the washout ratio with increasing rates of precipitation. }
\end{aligned}
$$

A similar approach was used by NCI (1997) using washout ratios and precipitation rates corresponding to daily values:

$$
W R_{d}=W R_{1}^{N C I} \cdot E^{-\alpha}
$$

where

$$
\begin{aligned}
& W R_{l}^{N C I}=\text { the washout ratio for a 1-mm daily rain; } \\
& \begin{aligned}
E= & \text { the precipitation rate per rain event (per day) }\left[\mathrm{mm} \mathrm{d}^{-1}\right] ; \text { and }
\end{aligned} \\
& \alpha=0.7=\begin{array}{l}
\text { the rain exponent, an empirical parameter [unitless] that accounts for the } \\
\text { observed decrease in the washout ratio with increasing rates of precipitation. }
\end{array}
\end{aligned}
$$


Since parameter values for $W R_{l}^{N C I}$ and $\alpha$ were readily available (NCI, 1997), the washout ratio, $W R_{l}{ }^{h}$, was derived by equating the net daily deposition rate from Equation 5.12 with that derived from Equation 5.11 as follows:

$$
\sum_{i=1}^{48} W R \cdot P_{i} \cdot C_{a, k, i}=W R_{d} \cdot E \cdot C_{a, k, d}
$$

where

$$
\begin{aligned}
P_{i}= & \text { the rainfall in the half-hour period } i \\
C_{a, k, i}= & \text { the concentration of }{ }^{131} \mathrm{I} \text { in chemical form } k \text { in air during the half-hour period } \\
& i \text { and }
\end{aligned}
$$

Thus, $C_{a, k, d}=(1 / 48) \Sigma C_{a, k, i}$ and $E=\Sigma P_{i}$, the total rainfall for the day. Substituting these relationships in Equation 5.13, remembering that rainfall in a given half-hour period $\left(P_{i}\right)$ can remove only contaminant present in that half-hour (at concentration $C_{a, k, i}$ ), and treating $W R$ as a constant during a given day yields the following equality.

$$
W R=W R_{d} / 48
$$

Using Equations 5.11, 5.12, and 5.13, the following equation relating $W R_{I}^{h}$ and $W R_{l}^{N C I}$ is obtained:

$$
W R_{1}^{h}=\frac{1}{48} \cdot \frac{\left(W R_{1}^{N C I} \cdot E^{-\alpha}\right)}{P_{a}^{-s}}
$$

where $P_{a}$ is the average rainfall for the day $(=E / 48)$. Thus, the washout ratio for a rain of $1 \mathrm{~mm}$ $\mathrm{hr}^{-1}$ is estimated on a daily basis from the known half-hourly data on $P$. Selection of parameter values for the amount of rainfall during each half-hour period of nonzero precipitation is described in Section 4.3.2.2.

\section{Approach for Routine Releases}

Two separate analyses were conducted for the estimation of wet deposition velocities for routine releases. The first analysis was conducted to account for depletion of ${ }^{131} \mathrm{I}$ from the contaminated plume according to Equations 4.1 through 4.5 (Section 4.3). The second analysis was conducted to estimate the amount of ${ }^{131}$ I that was deposited on the pasture grass and vegetation. The separate analysis was necessitated because of the different temporal scales of analysis used to address the atmospheric transport and the deposition processes. Details of both analyses are presented below. 


\section{A. Estimation of wet deposition velocity for the plume depletion calculations.}

As described in Section 4.3, the atmospheric transport analysis is conducted on an hourly scale for the routine releases. This requires that the plume depletion according to Equations 4.1 through 4.5 should also be handled at the same scale. The contribution of wet deposition on an hourly scale to plume depletion is addressed by evaluating the wet deposition velocity for each hour that has a nonzero precipitation. The approach is similar to that described in Equations 5.11 through 5.15, except that the time-step of the analysis is changed from a half-hour to one hour. Accordingly, in Equation 5.11, $W R_{1}{ }^{h}$ represents the washout ratio for a 1-mm hourly rainfall, and $\mathrm{P}$ represents the precipitation rate per hourly event. Equation 5.11 and 5.12, therefore, remain the same except for the modified interpretation of the two parameters as described in the previous sentence. Equation 5.13 can be rewritten as follows for a one-hour analysis:

$$
\sum_{i=1}^{24} W R \cdot P_{i} \cdot C_{a, k, i}=W R_{d} \cdot E \cdot C_{a, k, d}
$$

where

$$
\begin{aligned}
& P_{i}=\text { the rainfall in hour } i \\
& C_{a, k, i}=\text { the concentration of }{ }^{131} \mathrm{I} \text { in chemical form } k \text { in air during the hour } i \text {; and } \\
& C_{a, k, d}=\text { the average concentration of }{ }^{131} \mathrm{I} \text { in chemical form } k \text { in air during the whole }
\end{aligned}
$$

Thus, $C_{a, k, d}=(1 / 24) \Sigma C_{a, k, i}$ and $E=\Sigma P_{i}$, the total rainfall for the day. Substituting these relationships in Equation 5.16, remembering that rainfall in a given hour $\left(P_{i}\right)$ can remove only contaminant present in that hour (at concentration $C_{a, k, i}$ ), and treating $W R$ as a constant during a given day yields the following equality:

$$
W R=W R_{d} / 24
$$

Using Equations 5.12, 5.16, and 5.17, the following equation relating $W R_{l}{ }^{h}$ and $W R_{l}^{N C I}$ is obtained:

$$
W R_{1}^{h}=\frac{1}{24} \cdot \frac{\left(W R_{1}^{N C I} \cdot E^{-\alpha}\right)}{P_{a}^{-s}}
$$

where $P_{a}$ is the average rainfall for the day $(=E / 24)$. Thus, the washout ratio for a rain of $1 \mathrm{~mm}$ $\mathrm{hr}^{-1}$ is estimated on a daily basis from the hourly data on $P$. The hourly rainfall data are generated for the 1944-1956 period using the approach described later in this section. 


\section{B. Estimation of wet deposition velocity for the deposition calculations.}

In the case of routine releases, the contaminant concentration in air is assumed to be constant over a long period of time (e.g., one year.) A specific approach for wet deposition was used to accommodate such a large time step.

A day when precipitation is recorded is defined as a "rain event." During a rain event, the washout ratio depends on the amount of rain $\left(\mathrm{mm} \mathrm{day}^{-1}\right)$; Equation 5.11. The larger the amount of rain per rain event, the smaller the washout ratio as defined by the following empirical equation (Slinn, 1978; NRC/CEC, 1994). For a daily rain event, the washout ratio can be expressed as follows.

$$
W R=W R_{1}^{d} \cdot I^{-s}
$$

where

$W R^{d}{ }_{1} \quad=$ the washout ratio for a $1-\mathrm{mm}$ daily rain;

$I=$ the precipitation rate per rain event (per day) defined as the amount of rain divided by the total duration of rain in a year, and expressed in $\left[\mathrm{mm} \mathrm{d}^{-1}\right]$; and

$s=\quad=$ "the rain exponent," an empirical parameter [unitless] that accounts for the observed decrease in the washout ratio with increasing rates of precipitation.

Rain does not occur every day during a year. The total amount of precipitation in one year should be the same if calculated using an annual average approach, or if calculated by summing the amount of rain from each individual rain event.

To estimate the washout ratio to be used for routine releases, the approach and the data reported by NCI (1997; Eq. 5.12) were used in this study. The rain exponent used by NCI ( $\alpha=0.7$; NCI, $1997)$ is different from the values $(s \cong 0.4)$ reported by Slinn, (1978) and NRC/CEC (1994). Imposing the condition that, on average, the washout ratio should be the same regardless of the approach at a given precipitation rate $(I=E)$, the following relationship can be written:

$$
W R=W R_{1}^{d} \cdot I^{-s}=W R_{1}^{N C I} \cdot E^{-\alpha}
$$

Using $s=0.4$ (Slinn, 1978; NRC/CEC, 1994), $\alpha=0.7$ (NCI, 1997), and the average rain per rain event (rainy day) for Oak Ridge $I=E=10 \mathrm{~mm} \mathrm{~d}^{-1}$, one obtains the following equation:

$$
W R_{1}^{d} \cong 0.5 \cdot W R_{1}^{N C I}
$$

The values of $\mathrm{WR}_{1}{ }^{\mathrm{NCI}}$ for each chemical form of iodine are discussed below. 


\section{Washout Ratio for Elemental Iodine (I 2 )}

The washout ratio for a gas is a measure of the solubility of the gas; as stated by Slinn (1978), essentially all raindrops attain their equilibrium concentration of a gas in less than $10 \mathrm{~m}$ of fall.

The distribution of $W R_{l}{ }^{N C I}$ (for the elemental form of ${ }^{131} \mathrm{I}$ ) was assumed to be log-triangular with a minimum value of $2100 \mathrm{~m}^{3}$ air $\mathrm{L}^{-1}$ rain, a mode of $5000 \mathrm{~m}^{3}$ air $\mathrm{L}^{-1}$ rain, and a maximum value of $10,700 \mathrm{~m}_{\text {air }}^{3} \mathrm{~L}_{\text {rain }}^{-1}$ (Table 5.8). This range is based on a review of NCRP recommendations (NCRP, 1993), on an expert elicitation performed for the U.S. Nuclear Regulatory Commission and the European Community (NRC/CEC, 1994), on NCI (1997), and on Slinn (1978).

\section{Washout Ratio for Particulate Iodine}

The diameter of the particles is assumed to be very small $(\mathrm{d}<3 \mu \mathrm{m})$. Particles may be removed by rain both during the formation of rain in the cloud and by scavenging below the cloud; the process is highly dependent on the rain event (type, rate, drop size, etc.).

The distribution of $W R_{1}^{N C I}$ for particulate ${ }^{131} \mathrm{I}$ is assumed to be log-triangular between 1000 and $5400 \mathrm{~m}^{3}$ air $\mathrm{L}^{-1}$ rain with a mode of $2500 \mathrm{~m}^{3}$ air $\mathrm{L}_{\text {rain }}^{-1}$ (Table 5.8). This range is based on measurements made in the USA in the aftermath of the Chernobyl accident and summarized by Richmond et al. (1988) and on an expert elicitation performed for the U.S. Nuclear Regulatory Commission and the European Community (NRC/CEC, 1994).

\section{Washout Ratio for Organic Iodine}

The distribution of $\mathrm{WR}_{1}{ }^{\mathrm{NCI}}$ for organic forms of ${ }^{131} \mathrm{I}$ is assumed to be log-triangular between 4.0 and $18.0 \mathrm{~m}^{3}$ air $\mathrm{L}^{-1}$ rain with a mode of $8 \mathrm{~m}^{3}$ air $\mathrm{L}^{-1}$ rain (Table 5.8; Slinn, 1978; NRC/CEC, 1994; NCI, 1997).

\section{$\underline{\text { Hourly Precipitation Rate }}$}

The hourly precipitation rate was developed from ten years of hourly rainfall data available from measurements conducted at the ATDD (NOAA) station on Illinois Avenue in Oak Ridge between 1986 and 1996. (The 1995 dataset was not complete and therefore was not used.) Since direct rainfall data for the years 1944 to 1956 were not available in electronic form, it was decided to generate statistical descriptions of hourly rainfall along with estimates of underlying uncertainty. Using the ATDD data from 1986 to 1996, hourly rainfall estimates were generated for each hour of the day for a given month along with estimates of uncertainty for each hour. It was not possible to generate hourly estimates for every single day of the month because ten years' data would provide only ten measured values for each hour and day of a month. For example, for 3 p.m. on 15 January, the ten years of data include exactly ten measurements. But for 3 p.m. on any given day in January, there are 31 representative measurements for each year. Therefore, focusing on a "representative day of a month" allows the use of $310(31$ days $\times 10$ years) measured values for every hour of a day in January. The next step in the process was to develop a strategy to address the uncertainty associated with the measurements. Previous methods to generate rainfall estimates have relied on a Markov Chain approach (Richardson and Wright, 1984; Richardson, 1981; Schroeder et al., 1985). The Markov Chain approach models a 
stochastic process using the assumption that the event at a given time step $(t+1)$ is dependent only on the previous time step $(t)$. In other words, occurrence of rain at 4 p.m. is determined only on the basis of whether it did or did not rain at 3 p.m.

The 310 sets of hourly information available for the month of January were analyzed to determine the frequency with which it rained a certain amount in any given hour, given (a) that it did not rain during the previous hour, or (b) that it rained during the previous hour. These frequencies represent the probabilities of the occurrence of rainfall of a given magnitude during a given hour assuming that it did or did not rain during the previous hour. Similarly 280 (28 days $\times 10$ years $)$ and $310(31 \times 10$ years $)$ sets of hourly information can be used to generate the conditional probabilities for February and March, respectively, and so on.

Statistical analysis of the 10 years of data provided the probabilities for each month of the occurrence of a given amount of rain given information on whether or not rain occurred in the previous hour. Using an arbitrary initial condition of no rainfall during the last hour of December, 400 alternate sets of hourly rainfall data were then generated sequentially for each hour of the "representative day" for each of the 12 months from January through December. Sampling of the conditional probabilities for each hour was conducted using Latin Hypercube based Monte Carlo methodology described in IAEA (1989). In order to make the predicted 400 sets of hourly rainfall data independent of the initial condition, the first 50 sets were not included in the 400 sets used. Thus, each of the 400 data sets for a given month contains 24 hours of simulated rainfall data for a given day.

The next step was to simulate complete rainfall data for all days of one month. For January, the 400 alternate sets of 24 hours of rainfall data generated by the above method were sampled 31 times at random. This resulted in one full set of 24-hour rainfall estimates for January. This process was repeated 400 times to obtain 400 sets of complete hourly data for the 31 days of January; the whole process was repeated for each month of the year.

Statistics for the final simulated rainfall data sets are presented in Table 5.4. Comparisons of the statistics of the predicted rainfall with those of the observed rainfall for 30 years presented in Holland (1953) and those of the 10 years of data between 1986 and 1996 are provided in Tables 5.4 and 5.5. Table 5.5 presents the estimates of the rainfall rate and the annual average rainfall rate per rain event $(\mathrm{E})$. It is not entirely clear why the ranges of modeled monthly rainfall are consistently larger than the measured ranges; however, including more years of hourly measurements of rainfall and establishing correlations among hourly rainfall and other meteorological parameters (e.g., atmospheric stability and temperature) can help reduce the large uncertainty in the prediction of monthly rainfall. 
Table 5.4 Annual statistics for the $\mathbf{4 0 0}$ sets of a full year's estimated hourly rainfall using the Monte Carlo Markov Chain model, annual statistics from the monthly average data provided in Holland (1953), and annual statistics of the measurements from ATDD between 1986 and 1996.

\begin{tabular}{|c|c|c|c|c|}
\hline Annual average rainfall rate $(R)$ & $\begin{array}{c}2.5 \\
\text { percentile } \\
{\left[\mathrm{mm} \mathrm{d}^{-1}\right]}\end{array}$ & $\begin{array}{c}\text { Median } \\
\text { Rainfall } \\
{\left[\mathbf{m m ~ d}^{-1}\right]}\end{array}$ & $\begin{array}{c}97.5 \\
\text { percentile } \\
{\left[\mathrm{mm} \mathrm{d}^{-1}\right]} \\
\end{array}$ & $\begin{array}{l}\text { Average } \\
{\left[\mathrm{mm} \mathrm{d}^{-1}\right]}\end{array}$ \\
\hline Model results & 2.36 & 3.70 & 4.91 & 3.69 \\
\hline 1921-1950 data for $\mathrm{X}-10^{\mathrm{a}}$ (Holland, 1953) & & & & 3.42 \\
\hline ATDD (1986-1996) Data & 2.75 & 3.75 & 4.65 & 3.77 \\
\hline
\end{tabular}

${ }^{a}$ Only the mean value was reported.

Table 5.5 Monthly statistics for the 400 sets of a full year's estimated hourly rainfall using the Monte Carlo Markov Chain model, monthly average measurements from Holland (1953), and monthly statistics of the measurements from ATDD between 1986 and 1996.

\begin{tabular}{|c|c|c|c|}
\hline Month & $\begin{array}{c}\text { ATDD Total rainfall } \\
(\mathrm{mm})(2.5 \%, 50 \%, 97.5 \%) \\
\text { average }\end{array}$ & $\begin{array}{c}\text { Holland (1953) } \\
(\mathrm{mm}) \text { average }\end{array}$ & $\begin{array}{c}\text { Modeled }(\mathbf{m m}) \\
(2.5 \%, 50 \%, 97.5 \%), \\
\text { average } \\
\end{array}$ \\
\hline January & $(41,130,192), 122$ & 125 & $(0,110,263), 118$ \\
\hline February & $(84,131,239), 143$ & 125 & $(0,122,290), 130$ \\
\hline March & $(70,120,229), 132$ & 129 & $(0,119,303), 132$ \\
\hline April & $(43,82,217), 94$ & 101 & $(0,107,314), 118$ \\
\hline May & $(54,74,165), 96$ & 100 & $(2,87,253), 98$ \\
\hline June & $(19,106,261), 115$ & 99 & $(13,104,294), 119$ \\
\hline July & $(56,116,286), 133$ & 119 & $(14,96,241), 103$ \\
\hline August & $(52,86,127), 88$ & 97 & $(3,63,191), 70$ \\
\hline September & $(40,107,212), 111$ & 74 & $(1,111,349), 123$ \\
\hline October & $(23,59,113), 62$ & 65 & $(1,71,192), 80$ \\
\hline November & $(57,105,199), 118$ & 96 & $(3,104,295), 116$ \\
\hline December & $(73,139,313), 161$ & 120 & $(1,125,348), 138$ \\
\hline
\end{tabular}

\footnotetext{
${ }^{a}$ Only mean values were reported.
} 
The comparisons of measurements to modeling results show good agreement between the modeled data set and the actual measurements of precipitation at Oak Ridge during recent years. The 95\% subjective confidence interval of modeled rainfall is larger than that of the measurements (Table 5.5). Analysis of the rainfall data measured at ATDD between 1986 and 1996 are provided in Table 5.6. The last column in Table 5.6 was used to generate the distribution of annual average rainfall rate per rain event $(E)$.

Table 5.6 Analysis of the rainfall data measured at ATDD between 1986 and 1992 .

\begin{tabular}{|c|c|c|c|}
\hline Year & $\begin{array}{c}\text { Total Rainfall } \\
{[\mathrm{mm}]}\end{array}$ & $\begin{array}{c}\text { Annual average } \\
\text { rainfall rate }\left[\mathrm{mm} \mathrm{d}^{-1}\right]\end{array}$ & $\begin{array}{c}\text { Annual average } \\
\text { rainfall rate } \\
\text { per rain event } \\
{\left[\mathrm{mm} \mathrm{d}^{-1}\right]}\end{array}$ \\
\hline 1986 & 990 & 2.71 & 8.38 \\
\hline 1987 & 1027 & 2.81 & 8.35 \\
\hline 1988 & 1244 & 3.41 & 11.52 \\
\hline 1989 & 1683 & 4.61 & 11.37 \\
\hline 1990 & 1479 & 4.05 & 11.64 \\
\hline 1991 & 1537 & 4.21 & 12.10 \\
\hline 1992 & 1113 & 3.05 & 8.12 \\
\hline 1993 & 1244 & 3.41 & 10.03 \\
\hline 1994 & 1665 & 4.56 & 12.24 \\
\hline 1996 & 1679 & 4.60 & 11.66 \\
\hline $\begin{array}{c}1986-1996 \\
\text { average }\end{array}$ & 1366 & 3.74 & 10.54 \\
\hline
\end{tabular}

${ }^{\mathrm{a}}$ Normalized by the number of days of nonzero rainfall in the year ( 1 rainy day $=1$ rain event).

A comprehensive summary of the state of knowledge about the rain exponent ( $\alpha$ or $s$ ) is found NRC/CEC's (NRC/CEC, 1994) report of a formal expert elicitation on radionuclide deposition issues. The results of the elicitation, as well as other sources of information, are presented in Table 5.7 
Table 5.7 Summary of literature review on the rain exponent ( $\alpha$ or $s$ ).

\begin{tabular}{lcl}
\hline & $s$ & \multicolumn{1}{c}{ Reference } \\
\hline Elemental iodine & 0.4 & Brenk and Vogt (1981) \\
Aerosols & 0.5 & Brenk and Vogt (1981) \\
Elemental iodine & 0.4 & Schwartz (1985) cited by NRC/CEC (1994) \\
Methyl iodide & 0.4 & Schwartz (1985) cited by NRC/CEC (1994) \\
Particulate iodine & $0.3 \pm 0.12$ & NRC/CEC (1994); Expert B \\
Gaseous iodine & 0.3 & NRC/CEC (1994); Expert C \\
Particulate iodine & 0.25 & NRC/CEC (1994); Expert G \\
Particulate iodine & 0.25 & NRC/CEC (1994); Expert H \\
\hline
\end{tabular}

The rain exponent $(s)$ was also considered an uncertain variable. Based on the available information (Table 5.7), a relatively large range $(0.2-0.6$, central value 0.4 ; uniform distribution) was chosen for this parameter.

\subsubsection{Interception and initial retention by pasture vegetation.}

The transfer of iodine carried by water droplets from a deposit in rain to vegetation surfaces is described in this work by the mass interception factor $(r / Y)_{\text {wet }}\left[\mathrm{m}^{2} \mathrm{~kg}^{-1}\right.$ dry mass $]$, defined as the fraction of material in rain deposited per square meter of the ground surface intercepted and retained on the plant, normalized to the dry mass of the vegetation per unit area of soil. For ${ }^{131} \mathrm{I}$, such factors have been experimentally determined by Hoffman et al. (1992).

Iodine is present in rainwater in ionic form, and also as particles or organic and inorganic forms dissolved in water. The mass interception factor for wet deposition is a function of the biomass $Y$, of the rainfall rate $R$, of the amount of rain during the deposition process, and of the vegetation type (Hoffman et al., 1992). Negative ionic forms of iodine are not readily retained by the leaves of the plant, because the plant surface is usually negatively charged. On the other hand, positive ions, particles, and neutral molecules are more readily retained on the plant.

In the case of wet deposition of iodide, biomass and vegetation type are of minor importance in comparison to the amounts of rain and the intensity of rain (Hoffman et al., 1992).

In the case of wet deposition for insoluble particles in rainwater, retention is higher than for soluble compounds, and rain amount, rain intensity, and vegetation biomass are all of about the same importance. 


\section{Elemental Iodine}

Atmospheric elemental iodine is probably found in rainwater in either anionic or molecular form. The concentration of the molecular dissolved form is assumed to be negligible in comparison with the ionic forms; that is, elemental iodine from the atmosphere is transferred in rainwater as iodide. Therefore, the effective mass interception factor for atmospheric elemental iodine is equal to the mass interception factor measured for iodide by Hoffman et al. (1992).

$$
\left(\frac{r}{Y}\right)_{\text {wet }}^{\text {elemental }}=\left(\frac{r}{Y}\right)_{\text {iodide }}
$$

\section{$\underline{\text { Particulate Iodine }}$}

Atmospheric iodine attached to particles is transferred in rainwater as particles. However, an important fraction of iodine trapped to the particles in a water droplet will be released into solution as ions. The fraction of iodine that is still trapped on the particles is denoted as " $d$," and an effective mass interception factor is calculated as follows.

$$
\left(\frac{r}{Y}\right)_{\text {wet }}^{\text {particulate }}=d \cdot\left(\frac{r}{Y}\right)_{\text {particles }}+(1-d) \cdot\left(\frac{r}{Y}\right)_{\text {iodide }}
$$

The fraction " $d$ " of iodine still trapped on the particles was also treated as an uncertain variable in order to account for the lack of knowledge about the value of this parameter. The selected range for $d$ is $0.1-0.5$, and the selected distribution is uniform.

\section{$\underline{\text { Organic Iodine }}$}

Dissolution is assumed to be the only process by which atmospheric organic iodine is transferred into rain water. Dissolved organic iodine is assumed to be retained on the plant surfaces as efficiently as iodide.

$$
\left(\frac{r}{Y}\right)_{\text {wet }}^{\text {organic }}=\left(\frac{r}{Y}\right)_{\text {iodide }}
$$

The mass interception fraction was measured in Oak Ridge, Tennessee, by Hoffman et al. (1992) in an experiment with simulated rain containing both soluble radionuclides and insoluble particles labeled with radionuclides. The experiment determined empirical relationships between the mass interception fraction for iodide and for insoluble particles, and the total rain amount, the rain intensity and the biomass of pasture grass. The mass interception factor was found to depend strongly on the total rain amount.

$$
\left(\frac{r}{Y}\right)=\left(\frac{r}{Y}\right)_{1 m m} \cdot P_{0}^{K}
$$


where

$$
\begin{aligned}
& P_{0}=\text { the precipitation amount per rain event }[\mathrm{mm}] \\
& (\mathrm{r} / \mathrm{Y})_{1 \mathrm{~mm}}=\quad \begin{array}{l}
\text { the mass interception fraction for a unit precipitation }\left[\mathrm{m}^{2} \mathrm{~kg}^{-1}\right. \text { dry mass per } \\
K
\end{array} \quad=\quad \text { the coefficient of the functional fit [unitless]. }
\end{aligned}
$$

However, in the present report, one hour containing rain is considered one rain event. Therefore, the total amount of rain in a rain event is numerically equal to the rain per precipitation rate $\left(P_{0}\right.$ $=P)$.

For iodide, the measured value for the mass interception fraction $(\mathrm{r} / \mathrm{Y})_{1 \mathrm{~mm}}$ for a unit rain amount is found to range between 1 and $4 \mathrm{~m}^{2} \mathrm{~kg}^{-2}$ dry mass (Hoffman et al., 1992). A uniform distribution was assigned to represent the uncertainty in this parameter. The exponent describing the dependence on the rain amount has a value of $K_{\text {iodide }}=-0.909$ (Hoffman et al., 1992). No uncertainty was associated with this parameter.

Iodine released from X-10 was attached to small aerosol particles. Thus, out of the entire set of experiments performed by Hoffman et al. (1992), measurements using $3-\mu \mathrm{m}$ insoluble microspheres are considered the most appropriate to describe wet interception of iodine attached to particles. The mass interception factor $(\mathrm{r} / \mathrm{Y})_{1 \mathrm{~mm}}$ for a unit rain amount for particulates is found to range between 2 and $6 \mathrm{~m}^{2} \mathrm{~kg}^{-1}$ dry mass (Hoffman et al., 1992). Uncertainty in this parameter is expressed using a uniform distribution. The exponent relating the mass interception factor to the rain amount was found by Hoffman et al. (1992) to be $K_{\text {particulate }}=-0.207$.

\subsubsection{Weathering from Vegetation Surfaces}

After radionuclides are deposited on vegetation, removal processes combine with radioactive decay to reduce the initial retained quantity. Reduction in the initial concentration by cuticle sloughing, by growth dilution, by wind and water, or by grazing by insects and larger herbivores are some of the processes that produce the effect often referred to as "weathering." These factors are combined in the following expression for the effective decay factor, presented as a function of the effective half-life $\left(T_{\text {eff }}\right)$.

$$
\lambda_{\text {eff }}=0.693 / T_{\text {eff }}
$$

where

$$
\begin{array}{r}
T_{\text {eff }}=\frac{T_{w} \cdot T_{r}}{T_{w}+T_{r}} \\
T_{w}=\text { the weathering half-life, and } \\
T_{r} \quad=\text { the radioactive half-life. }
\end{array}
$$


The 1954 accident occurred in late April. Contaminated grass was available for grazing cows for at most two months following the accident. During spring, pasture grass grows rapidly, so that a "growth dilution" phenomenon can be observed. Growth dilution represents the decrease of the concentration in grass after an initial deposition event because of a rapid increase in the mass of the plant. Therefore, smaller values for the weathering half-life are likely during springtime as compared to other seasons.

The selection of the range for this parameter is based on professional judgment, on the work of Miller and Hoffman (1983), on more recent work of Mück et al. (1994), and on an IAEA review document (IAEA, 1996).

For routine releases, the range selected for the weathering half-life of ${ }^{131}$ I on pasture grass is 6 to 17 days. This choice gives an effective half-life $\left(T_{\text {eff }}\right)$ of about 3-5 days. A uniform distribution was selected. On the other hand, for the 1954 accident, to account for a higher growth dilution during spring time, a weathering half-life of 3 to 8 days was chosen. The corresponding effective half-life is 2 to 4 days. A uniform distribution was assigned.

\subsubsection{The Ratio between the Mass of a Dehydrated Vegetation Sample and its Fresh Mass}

The dry-weight-to-wet-weight conversion factor for leafy vegetables is based on the values obtained from a literature review reported by Snyder et al. (1994). A uniform distribution between 0.05 and $0.09\left[\mathrm{~kg}_{\mathrm{dry}} \mathrm{kg}^{-1}\right.$ wet $]$ was chosen for this parameter.

\subsubsection{Summary of Input Parameters}

A summary of the input parameters used for the estimation of the transfer of ${ }^{131}$ I from air to vegetation is presented in Table 5.8. 
Table 5.8 Parameter distributions used for estimation of the transfer of ${ }^{131}$ I from air to vegetation.

\begin{tabular}{|c|c|c|c|c|c|c|c|c|}
\hline \multicolumn{2}{|c|}{ Parameter } & \multirow{2}{*}{$\begin{array}{l}\text { Physico- } \\
\text { chemical } \\
\text { form of } \\
\text { iodine }\end{array}$} & \multirow[t]{2}{*}{ Units } & \multicolumn{4}{|c|}{ Distribution } & \multirow[t]{2}{*}{ Type of release } \\
\hline Name & Symbol & & & $\min$. & $\max$. & mode & Shape & \\
\hline \multirow[t]{7}{*}{$\begin{array}{l}\text { Total dry deposition } \\
\text { velocity }\end{array}$} & $V_{d}$ & elemental $^{\mathrm{a}}$ & {$\left[\mathrm{cm} \mathrm{s}^{-1}\right]$} & 0.3 & 4 & & Log-uniform & routine \\
\hline & & particulate $^{\mathrm{b}}$ & {$\left[\mathrm{cm} \mathrm{s}^{-1}\right]$} & 0.05 & 0.5 & 0.15 & Log-triangular & routine \\
\hline & & organic $^{c}$ & {$\left[\mathrm{~cm} \mathrm{~s}^{-1}\right]$} & 0.001 & 0.05 & 0.0071 & Log-triangular & routine \\
\hline & $V_{d}$ & elemental & {$\left[\mathrm{cm} \mathrm{s}^{-1}\right]$} & 0.3 & 3 & & Log-uniform & Accidental (dry vegetation) \\
\hline & & elemental & {$\left[\mathrm{cm} \mathrm{s}^{-1}\right]$} & 1 & 6 & & Log-uniform & accidental (moist vegetation) \\
\hline & & particulate & {$\left[\mathrm{cm} \mathrm{s}^{-1}\right]$} & 0.05 & 0.5 & & Log-uniform & Accidental \\
\hline & & organic & {$\left[\mathrm{cm} \mathrm{s}^{-1}\right]$} & 0.001 & 0.05 & & Log-uniform & Accidental \\
\hline \multirow[t]{6}{*}{$\begin{array}{l}\text { Mass Interception } \\
\text { factor for dry } \\
\text { deposition }\end{array}$} & $(r / Y)_{d r y}$ & elemental & {$\left[\mathrm{m}^{2} \mathrm{~kg}^{-1}\right.$ dry] } & 0.8 & 4 & 2.4 & Triangular & routine \\
\hline & & particulate & {$\left[\mathrm{m}^{2} \mathrm{~kg}^{-1} \mathrm{dry}\right]$} & 0.5 & 4 & 2.3 & Triangular & routine \\
\hline & & organic & {$\left[\mathrm{m}^{2} \mathrm{~kg}^{-1}\right.$ dry] } & 0.8 & 4 & 2.4 & Triangular & routine \\
\hline & $(r / Y)_{d r y}$ & elemental & {$\left[\mathrm{m}^{2} \mathrm{~kg}^{-1} \mathrm{dry}\right]$} & 0.8 & 4 & & Uniform & Accidental \\
\hline & & particulate & {$\left[\mathrm{m}^{2} \mathrm{~kg}^{-1}\right.$ dry] } & 0.5 & 4 & & Uniform & Accidental \\
\hline & & organic & {$\left[\mathrm{m}^{2} \mathrm{~kg}^{-1} \mathrm{dry}\right]$} & 0.8 & 4 & & Uniform & Accidental \\
\hline
\end{tabular}


Table 5.8 (Continued)

\begin{tabular}{|c|c|c|c|c|c|c|c|c|}
\hline \multicolumn{2}{|l|}{ Parameter } & \multirow[t]{2}{*}{$\begin{array}{c}\text { Physico-chemical } \\
\text { form of iodine }\end{array}$} & \multirow[t]{2}{*}{ Units } & \multicolumn{4}{|c|}{ Distribution } & \multirow[t]{2}{*}{ Type of release } \\
\hline Name & Symbol & & & min. & max. & mode & shape & \\
\hline \multirow[t]{3}{*}{$\begin{array}{l}\text { Washout ratio per unit } \\
\text { precipitation rate }\end{array}$} & $W R_{I}^{N C I}$ & elemental & {$\left[\mathrm{m}_{\text {air }}^{3} \mathrm{~L}_{\text {rain }}^{-1}\right]$} & 2100 & 10,700 & 5000 & $\begin{array}{l}\text { Log- } \\
\text { triangular }\end{array}$ & accidental and routine \\
\hline & & particulate & {$\left[\mathrm{m}_{\text {air }}^{3} \mathrm{~L}_{\text {rain }}^{-1}\right]$} & 1000 & 5400 & 2500 & $\begin{array}{l}\text { Log- } \\
\text { triangular }\end{array}$ & accidental and routine \\
\hline & & organic & {$\left[\mathrm{m}_{\text {air }}^{3} \mathrm{~L}_{\text {rain }}^{-1}\right]$} & 4 & 18 & 8 & $\begin{array}{l}\text { Log- } \\
\text { triangular }\end{array}$ & accidental and routine \\
\hline Rain Exponent & $s$ & all species & [unitless ] & 0.2 & 0.6 & & uniform & accidental and routine \\
\hline $\begin{array}{l}\text { Mass interception factor } \\
\text { for wet deposition }\end{array}$ & $(r / Y)_{1 m m}$ & $\begin{array}{c}\text { as iodide } \\
\text { as particulate }\end{array}$ & $\begin{array}{l}{\left[\mathrm{m}^{2} \mathrm{~kg}^{-1} \text { dry }\right]} \\
{\left[\mathrm{m}^{2} \mathrm{~kg}^{-1} \text { dry }\right]}\end{array}$ & $\begin{array}{l}1.0 \\
2.0\end{array}$ & $\begin{array}{l}4.0 \\
6.0\end{array}$ & & $\begin{array}{l}\text { uniform } \\
\text { uniform }\end{array}$ & $\begin{array}{l}\text { accidental and routine } \\
\text { accidental and routine }\end{array}$ \\
\hline $\begin{array}{l}\text { Coefficient of the power } \\
\text { function fit }\end{array}$ & $K$ & $\begin{array}{c}\text { iodide } \\
\text { particulate }\end{array}$ & $\begin{array}{l}\text { [unitless] } \\
\text { [unitless] }\end{array}$ & & & $\begin{array}{l}-0.909 \\
-0.207\end{array}$ & $\begin{array}{l}\text { fixed value } \\
\text { fixed value }\end{array}$ & $\begin{array}{l}\text { accidental and routine } \\
\text { accidental and routine }\end{array}$ \\
\hline $\begin{array}{l}\text { Fraction of iodine trapped } \\
\text { on dissolved particles }\end{array}$ & $d$ & -- & [unitless] & 0.1 & 0.5 & & uniform & accidental and routine \\
\hline $\begin{array}{l}\text { Annual average amount of } \\
\text { rain per rain event }\end{array}$ & $I$ or $E$ & -- & {$\left[\mathrm{mm} \mathrm{d}^{-1}\right]$} & & & $\begin{array}{l}10.5^{\mathrm{d}} \\
(1.23)^{\mathrm{e}}\end{array}$ & normal & Routine \\
\hline $\begin{array}{l}\text { Annual average } \\
\text { precipitation rate }\end{array}$ & $\boldsymbol{R}$ & -- & {$\left[\mathrm{mm} \mathrm{d}^{-1}\right]$} & & & $\begin{array}{c}3.7^{\mathrm{d}} \\
(0.68)^{\mathrm{e}}\end{array}$ & normal & Routine \\
\hline
\end{tabular}


${ }^{131}$ I Releases from X-10 Radioactive Lanthanum Processing -

TASK 1 REPORT

July 1999

Transfer of ${ }^{131}$ I from Air to Vegetation

Page 5-23

Table 5.8 (Continued)

\begin{tabular}{|c|c|c|c|c|c|c|c|c|}
\hline \multicolumn{2}{|l|}{ Parameter } & \multirow[t]{2}{*}{$\begin{array}{c}\text { Physico-chemical } \\
\text { form of iodine }\end{array}$} & \multirow[t]{2}{*}{ Units } & \multicolumn{4}{|c|}{ Distribution } & \multirow[t]{2}{*}{ Type of release } \\
\hline Name & Symbol & & & min. & max. & mode & shape & \\
\hline \multirow[t]{2}{*}{$\begin{array}{c}\text { Weathering half-life on } \\
\text { vegetation }\end{array}$} & $T_{w}$ & all species & {$[\mathrm{d}]$} & 6 & 17 & & uniform & Routine \\
\hline & & all species & [d] & 3 & 8 & & uniform & Accidental \\
\hline $\begin{array}{c}\text { Time period of exposure } \\
\text { of the standing crop } \\
\text { biomass }\end{array}$ & $T_{a g}$ & all species & {$[\mathrm{d}]$} & 10 & 45 & & uniform & accidental and routine \\
\hline $\begin{array}{l}\text { Ratio of dehydrated to } \\
\text { fresh mass for grass }\end{array}$ & $\begin{array}{c}\gamma_{\text {dry mass: fresh }} \\
\text { mass }\end{array}$ & all species & [unitless ] & 0.05 & 0.09 & & uniform & accidental and routine \\
\hline
\end{tabular}

${ }^{\mathrm{a}}$ elemental $=$ reactive form of iodine

${ }^{\mathrm{b}}$ particulate $=$ iodine attached to aerosols

organic $=$ nonreactive form of iodine

d mean

e standard deviation 


\section{$5.4 \quad$ References}

Brenk, H.D., and Vogt, K.J. 1981. The calculation of wet deposition from radioactive plumes. Nuclear Safety 22(3).

Chamberlain, A.C. 1960. Aspects of the deposition of radioactive and other gases and particles. Int.J.Air.Pollut. 3:63.

Chamberlain, A.C., and Chadwick, R.C. 1986. Deposition of airborne radioiodine vapor. Nucleonics 11(8):22. (ChemRisk Repository No. 2162)

Chamberlain, A.C., and Chadwick, R.C. 1966. Transport of iodine from the atmosphere to ground. Tellus 18:226. (ChemRisk Repository No. 2160)

Chamberlain, A.C., and Chadwick, R.C. 1953. Deposition of airborne radio-iodine vapour. Nucleonics 11(22).

Heinemann, K., and Vogt, K.J. 1980. Measurements of the deposition of iodine onto vegetation and of the biological half-life of iodine on vegetation. Health Physics 39:463-474. (ChemRisk Repository No. 2159)

Hoffman, F.O., Thiessen, K.M., Frank, M.L., and Blaylock, B.G. 1992. Quantification of the interception and initial retention of the radioactive contaminants deposited on pasture grass by simulated rain. Atmos. Env. 26A(18):3313-3321. (ChemRisk Repository No.2158)

Holland, M.C. 1953. A Meteorological Survey of the Oak Ridge Area. Final Report covering the period 1948-52. ORO-99. (ChemRisk Repository No. 2169)

IAEA (International Atomic Energy Agency). 1989. Evaluating the Reliability of Predictions Made Using Environmental Transfer Models, Safety Series No. 100, Vienna.

IAEA (International Atomic Energy Agency). 1996. Modelling of radionuclide interception and loss processes in vegetation and of transfer in semi-natural ecosystems. Second report of the VAMP Terrestrial Working Group. IAEA-TECDOC-857.

Miller, C.W. and Hoffman, F.O. 1983. An examination of the environmental half-lives for radionuclides deposited on vegetation. Health Physics 45(3):731-744. (ChemRisk Repository No. 2180)

Mück, K., Roth, K., Gerzabeck, M.H., and Oberländer, H.-E. 1984. Effective Half-lives of Iand Cs-Isotopes in Grassland Shortly After Fallout. J. Environ. Radioactivity 24:127-143. (ChemRisk Repository No. 2179) 
NCI (National Cancer Institute). 1997. Estimated Exposures and Thyroid Doses Received by the American People from Iodine-131 in Fallout Following Nevada Atmospheric Nuclear Bomb Tests. A report from the National Cancer Institute. U.S. Department of Health and Human Services. National Institutes of Health. National Cancer Institute, Washington, D.C.

NCRP (National Council on Radiation Protection and Measurements). 1993. Uncertainty in NCRP Screening Models Relating to Atmospheric Transport. Deposition and Uptake by Humans. NCRP Commentary No. 8. (ChemRisk Repository No. 4018 )

NRC/CEC (U.S. Nuclear Regulatory Commission; Commission of the European Communities). 1994. Probabilistic Accident Consequence Uncertainty Analysis. Dispersion and Deposition Uncertainty Assessment. NUREG/CR-6244, EUR 15855EN, SAND94-1453, Vol. 1 \& 2. (ChemRisk Repository No. 2183)

Richardson, C.W. 1981. Stochastic Simulation of Daily Precipitation, Temperature and Solar Radiation. Water Resources Research 17 (1):182-190.

Richardson, C.W., and Wright, D.A. 1984. WGEN: A Model for Generating Daily Weather Variables. ARS-8 (USDA). 1984.

Richmond, C.R., Hoffman, F.O., Blaylock, B.G., Miller, C.W., Eckerman, K.F., Ng, Y.C., Lesslie, P.A., and Till, J.E. 1988. The Potential Use of Chernobyl Fallout Data to Test and Evaluate the Predictions of Environmental Radiological Assessment Models. ORNL-6466. (ChemRisk Repository No. 2185)

Schroeder, P.R., Peyton, R.L., McEnroe, B.M., and Sjostrom, J.W. 1985. The Hydrologic Evaluation of Landfill Performance (HELP) Model. United States Environmental Protection Agency. (ChemRisk Repository No. 2166)

Slinn, W.G.N. 1978. Parameterization for Resuspension and for Wet and Dry Deposition of Particles and Gases for Use in Radiation Dose Calculations. Nuclear safety 19(2). (ChemRisk Repository No. 2161)

Snyder, S.F., Ikenberry, T.A., Farris, W.T., Gilbert, R.O., and Napier, B.A. 1994. Parameters Used in the Environmental and Radiological Dose Modules (DESCARTES, CIDER and CRD Codes) of the Hanford Environmental Dose Reconstruction Integrated Codes (HEDRIC). Pacific Northwest Laboratory. PNWD-2023. (ChemRisk Repository No. 3076)

Schwarz, G. 1985. Deposition and post-deposition behaviour of radionuclides in urban environments. A paper presented at the Workshop on Methods for Assessing the off-site Radiological Consequences of Nuclear Accidents, Commission of the European Communities, Luxembourg, April 15-19, 1985.

Vogt, K.J., Heinemann, K., Horbert, M., Matthes, W., and Stoeppler, M. 1976. Untersuchung zur Ablagerung von Jod und Aerosolen. Abschlußbericht, Zentralabteilung Strahlenschutz, Kernforschungsanlage Julich. 
TASK 1 REPORT

July 1999

${ }^{131}$ I Releases from X-10 Radioactive Lanthanum Processing -

Page 5-26 Transfer of ${ }^{131}$ I from Air to Vegetation

This page intentionally left blank. 


\subsection{TRANSFER OF ${ }^{131}$ I FROM PASTURE TO VARIOUS FOOD PRODUCTS}

Iodine deposited onto the ground surface or on vegetation is ingested by grazing animals (i.e., cows, goats) and by chickens and is transferred to animal food products such as milk, meat, cottage cheese, and eggs. In addition, lactating women who consume ${ }^{131}$ I-contaminated foods will produce breast milk containing ${ }^{131} \mathrm{I}$.

This section discusses the approaches used in the present study for estimating the concentrations of ${ }^{131} \mathrm{I}$ in cow's and goat's milk, cottage cheese, meat, eggs, and mother's milk. Detailed measurements of the concentrations of ${ }^{131} \mathrm{I}$ in food products are not available for the time periods during which ${ }^{131} \mathrm{I}$ was released from the X-10 RaLa operation. As a result, mathematical models must be used to estimate the transfer of ${ }^{131} \mathrm{I}$ from vegetation to food products. The modeling approaches and input parameters for the transfer to cow's and goat's milk as well as to beef are presented in Sections 6.1-6.2. Transfer to cottage cheese, eggs, and mother's milk is described in Section 6.3.

\subsection{Description and Assumptions for Modeling the Transfer of ${ }^{131} \mathrm{I}$ from Pasture to Milk and Meat}

The present study examines the transfer of ${ }^{131}$ I to milk of cows and goats and to meat products. Two general categories of cows are considered in the present study. The first, a "commercial cow," is normally raised and managed in a relatively large herd, with milk (and meat) sold commercially. The second, a "backyard cow," is a cow raised by its owner primarily for the athome consumption of dairy products and meat. Differences between the two involve location of pasture and, in the case of dairy cattle, breed of cows and amount of milk produced. Commercial dairy herds are assumed to have been located on farms in different areas, both in- and outside areas contaminated by releases from RaLa processing at the X-10 facility (Sections 7 and 8), with the milk from such commercial cows pooled for retail sale locally. On the other hand, backyard cows are assumed to have grazed entirely from local pastures with supplementary feeding as described later, and the milk from these cows is consumed locally by family members and neighbors. During the years 1944 to 1956, the predominant breed of commercial cow in the region of interest was the Holstein, while Jerseys were used primarily as backyard cows. The milk production of the Holstein at 4-6 gallons per day was generally larger than that of the Jersey cow at 1-2 gallons per day (Miller, 1996).

Estimation of the transfer of ${ }^{131}$ I to milk and meat is based primarily on the ingestion of ${ }^{131} \mathrm{I}$ in contaminated pasture vegetation for the following reasons:

- The contribution of ${ }^{131} \mathrm{I}$ in the animal diet due to soil ingestion by cows or goats is negligible in comparison with the ingestion of contaminated feed.

- The contribution of ${ }^{131} \mathrm{I}$ inhaled by cows or goats to the total ${ }^{131} \mathrm{I}$ intake is negligible in comparison with the ingestion of ${ }^{131} \mathrm{I}$ in contaminated feed. 
Two types of release situations are relevant for ${ }^{131} \mathrm{I}$ emissions from X-10: an episodic release (April 1954 accident) and routine releases (all other releases). Two modeling approaches for the transfer from pasture to milk and beef are used in this study to differentiate between the two release situations.

\subsubsection{Accidental Releases}

For the April 29, 1954, accident, a transient approach is used to estimate ${ }^{131}$ I concentrations in grass and milk over a daily time scale. The following equation is used to estimate the transfer of ${ }^{131}$ I from ingested feed to milk.

$$
\frac{d C_{m m}}{d t}=\frac{f_{m}}{L} Q_{m} p_{m} C_{p}-\lambda_{e f f, m} C_{m m}
$$

where

$$
\begin{aligned}
& C_{m m}=\text { the concentration of }{ }^{131} \mathrm{I} \text { in milk }\left[\mathrm{Bq} \mathrm{L}^{-1} \text { milk }\right] \text { at the time of milking; } \\
& C_{p}=\text { the concentration of total }{ }^{131} \mathrm{I} \text { in pasture grass }\left[\mathrm{Bq} \mathrm{kg}{ }^{-1} \text { dry mass }\right] \text {; } \\
& p_{m}=\text { the fraction of feed that is contaminated [unitless]; } \\
& Q_{m}=\text { the feed ingestion rate for cow or goat }\left[\mathrm{kg}_{\mathrm{dry} \text { mass }} \mathrm{d}^{-1}\right] \text {; } \\
& f_{m}=\text { the fraction of total }{ }^{131} \mathrm{I} \text { ingested by a cow or goat that ends up in milk } \\
& \text { [unitless]; } \\
& L=\text { the volume of milk contained in the milk compartment (udder) [L]; and } \\
& \lambda_{\text {eff, } m}=\quad \text { the effective removal rate of }{ }^{131} \mathrm{I} \text { from milk }\left[\mathrm{d}^{-1}\right] .
\end{aligned}
$$

In Equation 6.1, $C_{p}$ represents the total concentration of ${ }^{131} \mathrm{I}$ in all chemical forms on pasture grass. Once ingested, all chemical forms of ${ }^{131} \mathrm{I}$ will be transferred to milk and meat in an identical fashion; therefore, there is no longer a need to maintain distinctions among the various species of ${ }^{131} \mathrm{I}$. Also, $f_{m}$ and $L$ are artifacts of the modeling approach for the development of the governing equation. The modeling approach uses a compartmental model for milk where milk is treated as one compartment within the cow's or goat's body. A fraction of total iodine intake $\left(f_{m}\right)$ is transferred to milk. However, the amount of milk, $L$, in the udder is not a constant; iodine increases proportionally to the amount of milk produced, and consequently $f_{m}$ changes as a function of time. These parameters cannot be measured directly. However, it is possible to empirically derive parameter values for the quantity $\left(f_{m} / L\right)$. Literature values for these quantities are available (Faw and Shultis, 1993).

The dynamic model for the transfer to milk (Equation 6.1) allows the same level of timedependent detail as was given for the estimation of iodine concentrations in air and in pasture grass. As with the transfer of iodine from air to pasture, the analytical solution of the equation is 
used to obtain the iodine concentrations in milk for every hour using a constant source term for that hour. The generic analytical solution is obtained as follows.

$$
\begin{aligned}
& \frac{d C_{m}}{d t}=s-\lambda C_{m} \\
& C_{m}=\left[s-\left(s-\lambda C_{m o}\right) \cdot e^{-\lambda t}\right] \cdot\left(\frac{1}{\lambda}\right)
\end{aligned}
$$

where

$C_{m}=$ the concentration of ${ }^{131} \mathrm{I}$ in milk $\left[\mathrm{Bq} \mathrm{L}^{-1}\right]$ in the hour of interest;

$s=$ a constant source term $\left[\mathrm{Bq} \mathrm{L} \mathrm{L}^{-1} \mathrm{~h}^{-1}\right]$ representing the input into the milk compartment in the hour of interest;

$\lambda=$ the removal rate of ${ }^{131} \mathrm{I}$ from the milk compartment $\left[\mathrm{h}^{-1}\right]$; and

$C_{m o}=$ concentration in milk $\left[\mathrm{Bq} \mathrm{L}^{-1}\right]$ in the previous hour.

\subsubsection{Routine Releases}

For routine releases, the concentration of iodine in pasture grass, milk, or meat will not vary significantly from one time period to another, because of continued addition of iodine at a nearly constant rate from the X-10 stack, continued atmospheric dispersion, and continued deposition from the contaminated plume. In this case, a situation of quasi-equilibrium is achieved, and an approach based on the annual average concentration of ${ }^{131} \mathrm{I}$ in pasture vegetation can be used. The following equations are used to estimate the transfer of ${ }^{131} \mathrm{I}$ from ingested feed to milk or meat of cows or goats.

$$
C_{m m a, j}=\sum_{k=1}^{3} C_{a a, j, k} \cdot A P_{k} \cdot P M=\sum_{k=1}^{3} C_{a a, j, k} \cdot A P_{k} \cdot\left(p_{m} \cdot Q_{m} \cdot F_{m}\right)
$$

where

$C_{m m a, j}=$ the concentration of iodine in milk $\left[\mathrm{Bq} \mathrm{L}^{-1}\right.$ milk $]$ at time of milking;

$C_{a a, j, k}=$ the annual average concentration in air of physico-chemical form $k$, for year $j$ of release $\left[\mathrm{Bq} \mathrm{m}{ }^{-3}\right.$ air] (Section 4$)$;

$A P_{k}=$ air-to-pasture transfer factor for physico-chemical form $k\left[\mathrm{~m}^{3}\right.$ air $\mathrm{kg}^{-1}$ dry mass $]$ (Section 5);

$P M=$ the pasture-to-milk transfer factor $\left[\mathrm{Bq} \mathrm{L}^{-1}\right.$ milk per $\mathrm{Bq} \mathrm{kg}^{-1}$ dry mass $]$; 
$p_{m}=$ the fraction of feed that is contaminated for dairy animals [unitless];

$Q_{m}=$ the feed ingestion rate for cow or goat $\left[\mathrm{kg}_{\text {dry mass }} \mathrm{d}^{-1}\right]$; and

$F_{m}=$ the feed-to-milk transfer coefficient for cow or goat $\left[\mathrm{d} \mathrm{L}^{-1}\right]$.

The concentration of ${ }^{131} \mathrm{I}$ in the muscle of beef cattle is estimated using the following equation.

$$
C_{f s a, j}=\sum_{k=1}^{3} C_{a a, j, k} \cdot A P_{k} \cdot P F=\sum_{k=1}^{3} C_{a a, j, k} \cdot A P_{k} \cdot\left(p_{f} \cdot Q_{f} \cdot F_{f}\right)
$$

where

$$
\begin{aligned}
& C_{f s a, j}=\text { the concentration of iodine in beef }\left[\mathrm{Bq} \mathrm{kg}^{-1}\right] \text { at slaughtering for year } j \text { of release; } \\
& P F=\text { the pasture-to-beef transfer factor }\left[\mathrm{Bq} \mathrm{kg}{ }^{-1} \text { per } \mathrm{Bq} \mathrm{kg}^{-1} \text { dry mass }\right] ; \\
& p_{f}=\text { the fraction of feed that is contaminated for beef cattle [unitless]; } \\
& Q_{f}=\text { the feed ingestion rate for beef cattle }\left[\mathrm{kg}_{\text {dry mass }} \mathrm{d}^{-1}\right] ; \text { and } \\
& F_{f}=\text { the feed-to-muscle or feed-to-meat transfer coefficient for beef cattle }\left[\mathrm{d} \mathrm{kg}^{-1}\right] .
\end{aligned}
$$

\subsection{Parameter Distributions}

The ranges of input parameters and the shapes of the subjective probability distributions used to estimate the transfer of ${ }^{131}$ I from pasture to milk and beef (Table 6.1) were selected from a review of the dose available to the literature, and from the recommendations by Dr. James K. Miller from the University of Tennessee, who has investigated the metabolism of iodine in cattle and dairy cows and has also worked with beef cattle management in the Tennessee area. The rationales for the choices of specific parameter- values are presented in the following sections.

\subsubsection{Feed intake rates for cows and goats}

In the United States, the management of ruminant herbivores includes various practices. Backyard cows are allowed to graze on open pastures during the entire grazing season (uncontrolled grazing). On the other hand, pasture management for commercial cows includes strip and rotational grazing, in which animals are moved between two to six pastures during the grazing season. For both backyard cows and commercial cows, stored feed is usually provided to complement their diet of fresh pasture grass. More stored feed is provided during wintertime.

The feed intake rates $\left(Q_{m}\right)$ for dairy cows, beef cattle and goats were selected based on the recommendations of Miller (1996) and on studies by Koranda (1965) and NCI (1997). Other 
significant literature was also reviewed (Hoffman and Baes, 1979; Schwartz and Hoffman 1980). The intake rates, expressed in kilograms of dry mass per day, apply for every day of the year. The same central values for intake rates can be used for both the accidental and routine releases. However, the annual average intake rates used for routine releases have a tighter uncertainty range than the uncertainty ranges for the April 29, 1954, accident.

For commercial cows in Tennessee areas, NCI (1997) found an average intake rate of $11.8 \mathrm{~kg}_{\mathrm{dry}}$ mass $\mathrm{d}^{-1}$, based on a relationship that gives the intake rate as a fraction of total body weight of the animal. Also, Koranda (1965) estimated an ingestion rate of $11.8 \mathrm{~kg}_{\text {dry mass }} \mathrm{d}^{-1}$ for dairy cows managed in strip or rotational grazing systems. For dairy cows grazing on open pastures (backyard cows), Koranda (1965) reported an average ingestion rate of $9.1 \mathrm{~kg}_{\text {dry mass }} \mathrm{d}^{-1}$.

For the April 29, 1954, accident, the selected range for the daily intake rate for backyard cows is from 6 to $18 \mathrm{~kg}_{\text {dry mass }} \mathrm{d}^{-1}$, based on the values reported by Koranda (1965). The most probable value is $9 \mathrm{~kg}_{\mathrm{dry}}$ mass $\mathrm{d}^{-1}$, and the distribution is triangular. For routine releases, a range of 7 to 14 $\mathrm{kg}_{\text {dry mass }} \mathrm{d}^{-1}$ was selected. A triangular distribution with a mode of $9 \mathrm{~kg}_{\text {dry mass }} \mathrm{d}^{-1}$ was assigned.

For the April 29, 1954, accident, the selected range for the daily intake rate for commercial cows is from 10 to $20 \mathrm{~kg}_{\text {dry mass }} \mathrm{d}^{-1}$. The most probable value is $12 \mathrm{~kg}_{\text {dry mass }} \mathrm{d}^{-1}$, and the distribution is triangular. For routine releases, possible values for the annual average ingestion rate range between 10 and $18 \mathrm{~kg}_{\text {dry mass }} \mathrm{d}^{-1}$ (Koranda, 1965). A triangular distribution with a mode of 12 $\mathrm{kg}_{\text {dry mass }} \mathrm{d}^{-1}$ was assigned.

Dairy goats consume between 0.7 and $4.0 \mathrm{~kg}_{\text {dry mass }} \mathrm{d}^{-1}$ (Hoffman and Baes, 1979). For an accidental release, any value in this range is considered possible; thus, a uniform distribution was selected. For routine releases, intake rates in the same range are possible, but the most probable value for the annual average is $2.0 \mathrm{~kg}_{\text {dry mass }} \mathrm{d}^{-1}$. The selected distribution is triangular. 
Table 6.1 Parameter distributions used for calculations of the transfer of ${ }^{131} \mathrm{I}$ from pasture to milk and beef.

\begin{tabular}{|c|c|c|c|c|c|c|}
\hline \multirow[b]{2}{*}{ Parameter } & \multirow[b]{2}{*}{ Units } & \multicolumn{4}{|c|}{ Distribution } & \multirow[b]{2}{*}{$\begin{array}{l}\text { type of } \\
\text { release }\end{array}$} \\
\hline & & $\min$. & $\max$. & $\begin{array}{c}\text { Central } \\
\text { value }\end{array}$ & Shape & \\
\hline \multicolumn{7}{|c|}{ Backyard cow } \\
\hline$Q_{m, A}$ & {$\left[\mathrm{~kg}_{\text {dry }} \mathrm{d}^{-1}\right]$} & 6 & 18 & 9 & Triangular & accidental \\
\hline$Q_{m}$ & {$\left[\mathrm{~kg}_{\text {dry }} \mathrm{d}^{-1}\right]$} & 7 & 14 & 9 & Triangular & routine \\
\hline$p_{m, A}$ & [\%] & 60 & 100 & 75 & Triangular & accidental \\
\hline$p_{m}$ & {$[\%]$} & 50 & 75 & 60 & Triangular & routine \\
\hline$\left(f_{m} / L\right)$ & {$\left[\mathrm{L}^{-1}\right]$} & $4.0 \times 10^{-3}$ & $2.2 \times 10^{-2}$ & $8.0 \times 10^{-3}$ & Triangular & accidental \\
\hline$F_{m, A}$ & {$\left[\mathrm{~d} \mathrm{~L}^{-1}\right]$} & & & $0.009^{\mathrm{a}}(1.9)^{\mathrm{b}}$ & Lognormal & accidental \\
\hline$F_{m}$ & {$\left[\mathrm{~d} \mathrm{~L}^{-1}\right]$} & & & $0.009^{\mathrm{a}}(1.8)^{\mathrm{b}}$ & Lognormal & routine \\
\hline \multicolumn{7}{|c|}{ Commercial cow } \\
\hline$Q_{m, A}$ & {$\left[\mathrm{~kg}_{\text {dry }} \mathrm{d}^{-1}\right]$} & 10 & 20 & 12 & Triangular & accidental \\
\hline$Q_{m}$ & {$\left[\mathrm{~kg}_{\text {dry }} \mathrm{d}^{-1}\right]$} & 10 & 18 & 12 & triangular & routine \\
\hline$p_{m, A}$ & {$[\%]$} & 40 & 95 & 50 & triangular & accidental \\
\hline$p_{m}$ & {$[\%]$} & 35 & 5 & 40 & triangular & routine \\
\hline$\left(f_{m} / L\right)$ & {$\left[\mathrm{L}^{-1}\right]$} & $4.0 \times 10^{-3}$ & $2.2 \times 10^{-2}$ & $8.0 \times 10^{-3}$ & triangular & accidental \\
\hline$F_{m, A}$ & {$\left[\mathrm{~d} \mathrm{~L}^{-1}\right]$} & & & $0.006^{\mathrm{a}}(1.5)^{\mathrm{b}}$ & lognormal & accidental \\
\hline$F_{m}$ & {$\left[\mathrm{~d} \mathrm{~L}^{-1}\right]$} & & & $0.006^{\mathrm{a}}(1.4)^{\mathrm{b}}$ & lognormal & routine \\
\hline \multicolumn{7}{|c|}{ Goat } \\
\hline$Q_{m, A}$ & {$\left[\mathrm{~kg}_{\text {dry }} \mathrm{d}^{-1}\right]$} & 0.7 & 4.0 & -- & uniform & accidental \\
\hline$Q_{m}$ & {$\left[\mathrm{~kg}_{\mathrm{dry}} \mathrm{d}^{-1}\right]$} & 0.7 & 4.0 & 2.0 & triangular & routine \\
\hline$p_{m, A}$ & {$[\%]$} & 30 & 100 & -- & uniform & accidental \\
\hline$p_{m}$ & {$[\%]$} & 30 & 100 & 65 & triangular & routine \\
\hline$\left(f_{m} / L\right)$ & {$\left[\mathrm{L}^{-1}\right]$} & $4.0 \times 10^{-3}$ & $2.2 \times 10^{-2}$ & $8.0 \times 10^{-3}$ & triangular & accidental \\
\hline$F_{m, A}$ & {$\left[\mathrm{~d} \mathrm{~L}^{-1}\right]$} & 0.02 & 0.9 & 0.22 & Log-triangular & accidental \\
\hline$F_{m}$ & {$\left[\mathrm{~d} \mathrm{~L}^{-1}\right]$} & 0.03 & 0.8 & 0.22 & Log-triangular & routine \\
\hline \multicolumn{7}{|c|}{ Beef cattle } \\
\hline$Q_{f}$ & {$\left[\mathrm{~kg}_{\text {dry }} \mathrm{d}^{-1}\right]$} & 7 & 14 & 9 & triangular & routine \\
\hline$p_{f}$ & [\%] & 40 & 75 & 60 & triangular & routine \\
\hline$F_{f}$ & {$\left[\mathrm{~d} \mathrm{~kg}^{-1}\right]$} & 0.0005 & 0.02 & 0.003 & Log-triangular & routine \\
\hline
\end{tabular}

${ }^{\text {a }}$ geometric mean

b geometric standard deviation 
Beef cattle are usually allowed to graze freely, more like backyard cows. Thus, the ingestion rate for beef cattle $\left(\mathrm{Q}_{\mathrm{f}}\right)$ was chosen to be the same as the ingestion rate of backyard cows.

\subsubsection{Fraction of feed that is contaminated}

Consultation with Miller (1996) indicates that the grazing period for dairy cows in the Tennessee area lasts year-round. One can identify two main seasons for grazing: (a) a winter season, from mid-October to mid-February, and (b) a summer season, from mid-February to mid-October. In the winter season, both backyard cows and commercial cows receive about $17 \%$ of the feed as fresh pasture grass. However, during the summer period, $75 \%$ of the feed for a backyard cow is fresh pasture grass. During the same period, a commercial cow receives only $50 \%$ fresh pasture grass (Figure 6.1). The rest of the feed is considered to be uncontaminated, due to a long storage time as compared to the 8.04 day half-life of ${ }^{131} \mathrm{I}$.

The National Cancer Institute (NCI, 1997) investigated dairy and beef cattle grazing patterns for the entire United States. For Tennessee, their findings are close to the values provided by Miller (1996) for a commercial dairy herd. That is, during the summer grazing season about $50 \%$ of the feed is fresh pasture grass, and during the winter grazing season $15 \%$ to $20 \%$ of the feed is pasture grass. However, for some of the winter months (December through February), NCI (1997) indicates that feed provided to cows contains no pasture grass (Figure 6.1).

The present study relies on the information provided by Miller (1996) because of his overall experience in management of dairy cows in East Tennessee area.

In the case of the April 29, 1954, accident, the fraction of contaminated feed must be determined taking into account the specific time of the year in which the accident occurred. The 1954 accident occurred in late April, and pasture grass could have been contaminated during most of the month of May. For this period, in Tennessee regions, about $75 \%$ of the feed intake for backyard cows would be fresh pasture grass (Figure 6.1). For commercial cows, 50\% of the intake would consist of fresh pasture grass. If cows had not yet been started on a summer grazing regime, this fraction can be as low as $45 \%$ for backyard cows, and $35 \%$ for commercial cows [values for the month of transition from the winter to the summer season (Figure 6.1)]. However, given the weather characteristics during April-May 1954, it is highly probable that cows were provided less stored feed than the amount normally provided during late winter. Thus, lower limits of the distributions were set to $60 \%$ for a backyard cow and to $40 \%$ for commercial cows. Individual cows may consume more pasture grass than the average given by Miller (1996). The variation in consumption of pasture grass is not well known, but it should be less than the seasonal variation (by $30 \%$ for backyard cows and by $15 \%$ for commercial cows). As an upper limit for the fraction of feed that is pasture grass, a value of $100 \%$ is selected for backyard cows and a value of $95 \%$ is selected for commercial cows. 


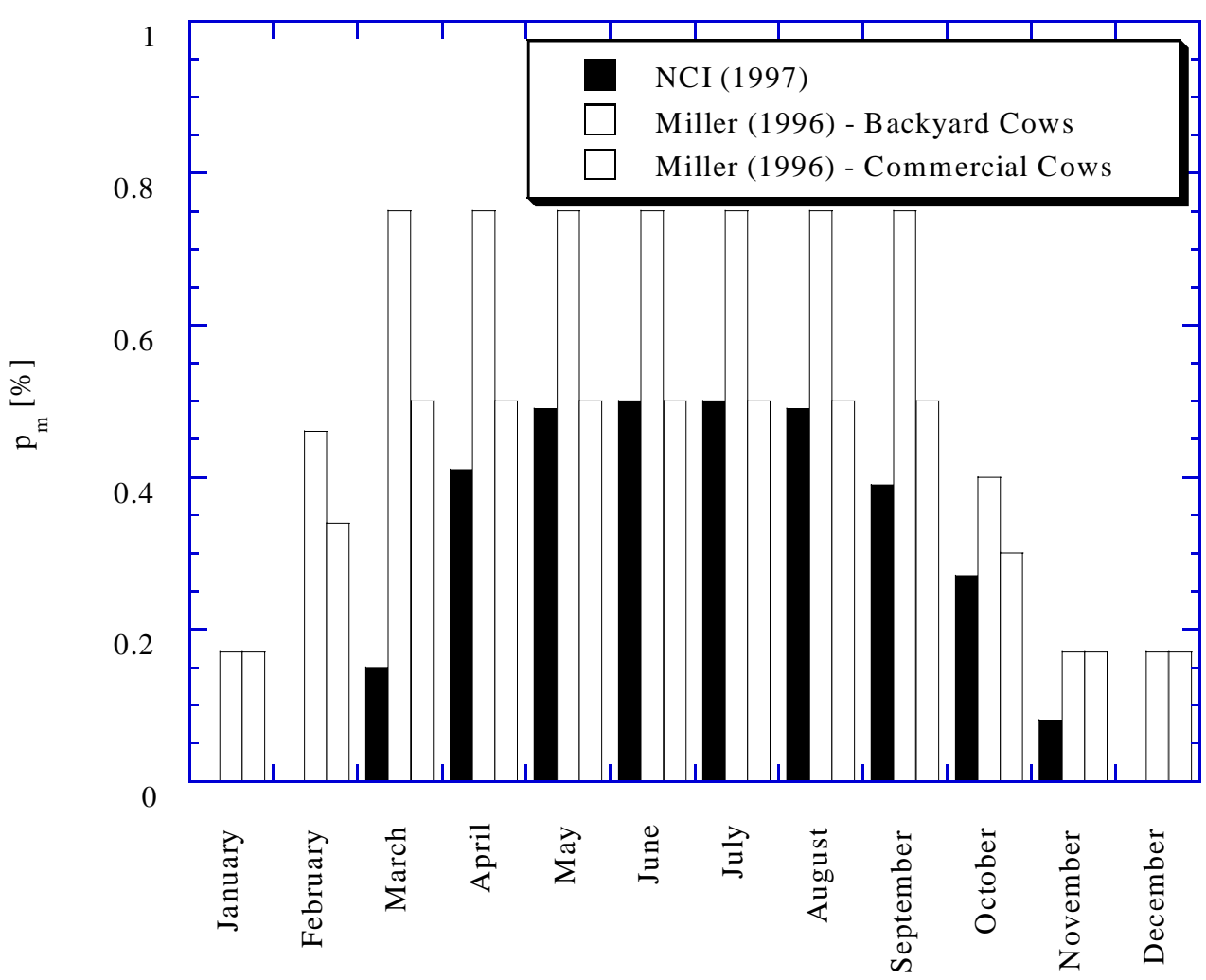

Figure 6.1 Fraction of the cow's feed that is fresh pasture $\left(p_{m}\right)$, in different grazing seasons. 
For routine releases, a subjective probability distribution was chosen to represent the state of knowledge about the annual average fraction of feed that is contaminated. Triangular distributions were selected for the fraction of feed that is contaminated $\left(p_{m}\right)$. The most probable values are $40 \%$ for commercial cows and $60 \%$ for backyard cows; these are derived as timeweighted averages of the percentages reported above. The maximum values of these triangular distributions were set to the typical values for the summer grazing season: $75 \%$ for backyard cows, and $50 \%$ for commercial cows. In addition, it is assumed that a dairy cow always receives stored feed as a supplement to fresh pasture grass. The minimum values of $p_{m}$ for an annual average must be larger than the values for late winter (February): $40 \%$ for a backyard cow, and $30 \%$ for a commercial cow. The minimum values for the triangular distribution were selected as $50 \%$ for a backyard cow and $35 \%$ for commercial cows. Thus, for routine releases, the selected distribution for the fraction of the feed of backyard cows that is contaminated is triangular from $50 \%$ to $75 \%$, with a mode of $60 \%$. The selected distribution for the fraction of the feed of commercial cows that is contaminated is triangular from $35 \%$ to $50 \%$, with a mode of $40 \%$.

According to Miller (1996) goats receive stored feed in amounts that represent up to $70 \%$ of the total intake. However, it is conceivable that no food supplements are provided, case in which $100 \%$ of the feed is contaminated pasture grass. For accidental releases, the selected distribution for the fraction of contaminated feed is uniform between $30 \%$ and $100 \%$. For routine releases, a triangular distribution was selected having a mode of $65 \%$, and the same range of $30 \%$ to $100 \%$. The range and the central tendency of the two distributions is preserved, but the distribution for routine releases will produce more samples near the center of the distribution, which is desired for the annual average case.

\subsubsection{The $f_{m} / L$ ratio and $\lambda_{e f f, m}$}

Parameter values for $f_{m} / L$, described in Equation 6.1, were directly obtained from Faw and Shultis (1993). For ${ }^{131}$ I transfer to milk, the nominal value for $f_{m} / L$ is $8.0 \times 10^{-3} \mathrm{~L}^{-1}$ with a range of 4.0 to $22 \times 10^{-3} \mathrm{~L}^{-1}$. These values were used for cows and goats.

Abundant measurements of the transfer to milk are available for the case of a single dose of iodine ingested by the cow. In this case, the transfer is described by a feed-to-milk transfer coefficient $\left(F_{m}\right)$ (Section 6.2.4).

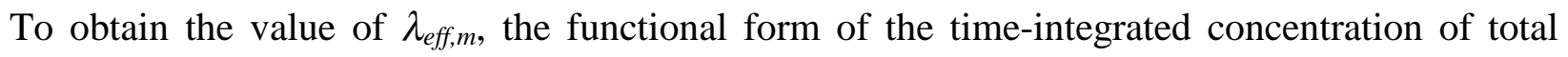
iodine was derived from the analytical solution of Equation 6.1 for an instantaneous dose of iodine administered to a cow at time $\mathrm{t}=0$. The time-integrated function can be converted to the total quantity of iodine produced in cow's milk by multiplying the concentration by the total yield. This allows the development of a theoretical, functional relationship between $f_{m} / L, \lambda_{\text {eff, } m}$, and $F_{m}$, the pasture to milk transfer coefficient for iodine, as follows:

$$
\frac{f_{m}}{L}=F_{m, m} \cdot \lambda_{e f f, m}
$$


The value of $\lambda_{\text {eff, } m}$ was obtained from Equation 6.4 using the available literature data on the other parameters in the equation. The solution of Equation 6.1 was then tested and validated for various initial conditions by calculating time-integrated concentrations of iodine in cows' milk and meat and comparing it with the solution obtained from a steady-state equilibrium-based approach.

\subsubsection{Transfer of Iodine from Feed to Milk of Cows}

\subsubsection{Review of information}

Extensive literature is available on the variability of measured values for $F_{m}$ for radioiodine. In an early literature review, Hoffman (1978) reported a range of $4.2 \times 10^{-3}$ to $3.5 \times 10^{-2} \mathrm{~d} \mathrm{~L} \mathrm{~L}^{-1}$. Using a statistical analysis of average values from nineteen publications, Hoffman and Baes (1979) reported a lognormal distribution of the average with a geometric mean of $1.0 \times 10^{-2} \mathrm{~d} \mathrm{~L}^{-1}$, a geometric standard deviation of 1.73 , and a $95 \%$ confidence interval ranging from $3.4 \times 10^{-3}$ to $2.9 \times 10^{-2} \mathrm{~d} \mathrm{~L}^{-1}$. As a result of environmental monitoring in the aftermath of the Chernobyl accident, Köhler et al. (1991) reported values of $F_{m}$ from eight locations in the Northern Hemisphere ranging from $1.0 \times 10^{-3}$ to $7.3 \times 10^{-3} \mathrm{~d} \mathrm{~L}^{-1}$.

For the Hanford Environmental Dose Reconstruction, involving the investigation of releases of ${ }^{131}$ I from 1944 to 1956 at the Hanford site in eastern Washington, a lognormal distribution was assigned to $F_{m}$ for individual backyard cows with a geometric mean of $9.2 \times 10^{-3} \mathrm{~d} \mathrm{~L}^{-1}$, a geometric standard deviation of 2.1, and a range from $1.6 \times 10^{-3}$ to $5.2 \times 10^{-2} \mathrm{~d} \mathrm{~L}^{-1}$. For a herd of commercial cows, a normal distribution was assigned with a mean of $1.2 \times 10^{-2} \mathrm{~d} \mathrm{~L}^{-1}$, a standard deviation of $0.002 \mathrm{~d} \mathrm{~L}^{-1}$, and a range extending from $7.3 \times 10^{-3}$ to $1.6 \times 10^{-2}$ (Snyder et al., 1994).

The National Cancer Institute (NCI, 1997) reviewed 81 reported values and described the variability in $F_{m}$ for ${ }^{131} \mathrm{I}$ as a log-normal distribution with a geometric mean of $4.4 \times 10^{-3} \mathrm{~d} \mathrm{~L}^{-1}$, a geometric standard deviation of 2.1 , and a range of $4 \times 10^{-4}$ to $2.1 \times 10^{-2} \mathrm{~d} \mathrm{~L}^{-1}$.

The results of the NRC/CEC expert elicitation are similar to those presented in previous literature reviews. Based on equal weighting of cumulative probability distributions that were fit to the values given by the experts at the $5^{\text {th }}, 50^{\text {th }}$, and $95^{\text {th }}$ percentiles, NRC/CEC obtained an aggregated $90 \%$ subjective confidence range of $5.3 \times 10^{-4}$ to $3.7 \times 10^{-2} \mathrm{~d} \mathrm{~L}^{-1}$ (Table 6.2). They assumed that every value in the given range would have an equal likelihood of being the correct value. The same data set was analyzed in the present study by assigning equal weighting of the $\mathrm{F}_{\mathrm{m}}$ values submitted by the ten experts at the $5^{\text {th }}, 50^{\text {th }}$, and $95^{\text {th }}$ percentiles. The result was an overall averaged $90 \%$ subjective confidence interval of $1.6 \times 10^{-3}$ to $3.0 \times 10^{-2} \mathrm{~d} \mathrm{~L}^{-1}$ assuming that the distributions given by the experts were of a log-triangular shape. Averages of the reported $5^{\text {th }}, 50^{\text {th }}$, and $95^{\text {th }}$ percentile values were also estimated. The $90 \%$ confidence interval for this case is $1.25 \times 10^{-3}$ to $3.1 \times 10^{-2} \mathrm{~d} \mathrm{~L}^{-1}$.

The most relevant data for this study were obtained from Dr. J.K. Miller at the University of Tennessee, Knoxville (1996), who investigated the transfer of ${ }^{131} \mathrm{I}$ into the milk of dairy cows representative of farm areas in Tennessee. A set of 77 measurements on 29 cows of various 
breeds (Holstein, Jersey, Guernsey, and Brown Swiss) was performed from 1962 to 1970. Experiments took place in all seasons of the year. Radioiodine was administrated orally, in capsules containing sodium iodide. Milk was then collected for seven days after the administration. After seven days, the daily concentration of radioiodine in milk was low enough to be neglected for estimating a time-integrated concentration over all time.

Analysis of the measurements made by Dr. Miller showed no significant difference between different cow breeds (Figure 6.2). Based on these data, no substantial conclusions could be reached about seasonal effects in the amount of radioiodine transferred to milk.

Table 6.2 Subjective cumulative probability levels for values of the milk transfer coefficient for ${ }^{131}$ I recommended by individual experts.

\begin{tabular}{cccc}
\hline & \multicolumn{3}{c}{$\mathbf{F}_{\mathbf{m}}\left(\mathbf{d} \mathbf{L}^{-\mathbf{1}}\right)$} \\
Individual & Subjective cumulative probability \\
NRC/CEC Expert & $\mathbf{5 \%}$ & $\mathbf{5 0 \%}$ & $\mathbf{9 5 \%}$ \\
\hline H & $1.0 \times 10^{-3}$ & $1.0 \times 10^{-2}$ & $4.0 \times 10^{-2}$ \\
I & $1.0 \times 10^{-3}$ & $7.0 \times 10^{-3}$ & $3.0 \times 10^{-2}$ \\
J & $1.0 \times 10^{-3}$ & $1.0 \times 10^{-2}$ & $4.0 \times 10^{-2}$ \\
K & $1.0 \times 10^{-3}$ & $1.0 \times 10^{-2}$ & $3.5 \times 10^{-2}$ \\
L & $1.0 \times 10^{-3}$ & $1.1 \times 10^{-2}$ & $3.4 \times 10^{-2}$ \\
M & $1.0 \times 10^{-3}$ & $5.0 \times 10^{-3}$ & $1.0 \times 10^{-2}$ \\
N & $5.0 \times 10^{-4}$ & $5.0 \times 10^{-3}$ & $5.0 \times 10^{-2}$ \\
O & $2.0 \times 10^{-3}$ & $4.0 \times 10^{-3}$ & $2.0 \times 10^{-2}$ \\
P & $2.0 \times 10^{-3}$ & $4.0 \times 10^{-3}$ & $1.8 \times 10^{-2}$ \\
Q & $2.0 \times 10^{-3}$ & $1.0 \times 10^{-2}$ & $2.0 \times 10^{-2}$ \\
\hline Overall Mean & $1.25 \times 10^{-3}$ & $8.1 \times 10^{-3}$ & $3.1 \times 10^{-2}$ \\
NRC/CEC aggregated & $5.3 \times 10^{-4}$ & $7.6 \times 10^{-3}$ & $3.7 \times 10^{-2}$ \\
results & & & \\
Project Team & $1.6 \times 10^{-3}$ & $7.1 \times 10^{-3}$ & $3.0 \times 10^{-2}$ \\
aggregated results & & & \\
\hline a Brown et al. (1997) & &
\end{tabular}


TASK 1 REPORT

July 1999

Page 6-12

${ }^{131}$ I Releases from X-10 Radioactive Lanthanum ProcessingTransfer of ${ }^{131}$ I from Pasture to Various Food Products

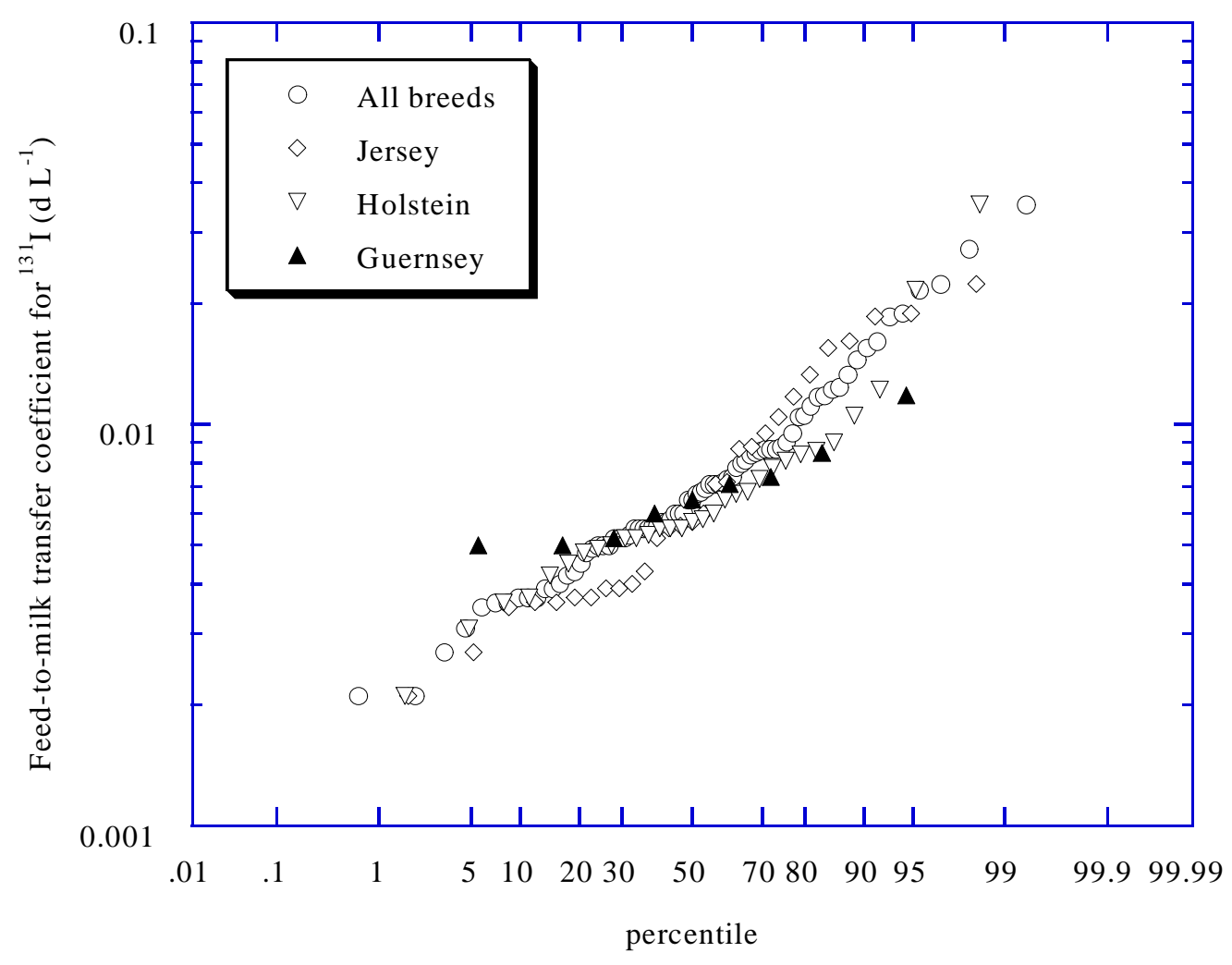

Figure 6.2 Feed-to-milk transfer factors for ${ }^{131}$ I administered to different breeds of dairy cows during 1962 to 1970 at the University of Tennessee, Knoxville (Miller, 1996). 
The data show an inverse relationship between milk yield and iodine concentration in milk (Figures 6.3 and 6.4). This finding is important for the present study because backyard cows are considered to be low-producers in terms of a daily yield of milk. Values of $F_{m}$ for cows whose milk production was less than $10 \mathrm{~L} \mathrm{~d}^{-1}$ were investigated, and the distribution of these values is lognormal with a geometric mean of $9 \times 10^{-3} \mathrm{~d} \mathrm{~L}^{-1}$ and a geometric standard deviation of 1.9.

According to $\mathrm{Ng}$ (1982), various chemical forms of iodine administered to cows are absorbed to blood by different mechanisms, influencing the amount transferred to milk. The NCI (1997) concluded, after a review of literature data, that higher values should occur for encapsulated feeding experiments than for experiments in which cows ingested contaminated forage. The NCI conclusions were drawn from plotting results from disparate references, the values of which show considerable overlap at the upper end of their range. The NCI conclusions are not drawn from specific experiments designed to determine the difference in the milk transfer coefficient between the ingestion by cows of encapsulated ${ }^{131} \mathrm{I}$ and ${ }^{131} \mathrm{I}$ contaminated forage.

For controlled conditions in which cows were fed various soluble forms of iodine, Bretthauer et al. (1972) found no significant difference in the transfer to milk. Furthermore, Miller (1996) has suggested from his experience that if ${ }^{131} \mathrm{I}$ is ingested in a soluble form, the feed-to-milk transfer coefficient should not depend on the mode of administration (e.g., encapsulated feeding versus feeding of contaminated forage).

Since the deposition onto pasture of ${ }^{131} \mathrm{I}$ from the X-10 RaLa operations was likely a soluble vapor or aerosol (see Sections 3, 4, and 5), no distinction in this report is made between the results obtained from feeding of encapsulated ${ }^{131}$ I and the ingestion of soluble ${ }^{131}$ I deposited on forage. For the purposes of this study, the experimental milk transfer coefficients obtained by Miller (1996) for various breeds of dairy cows used in East Tennessee during the 1960s are considered to be the most relevant data sets for quantifying the distribution of the milk transfer coefficient prevailing during the RaLa operation at X-10 (1944 to 1956). 

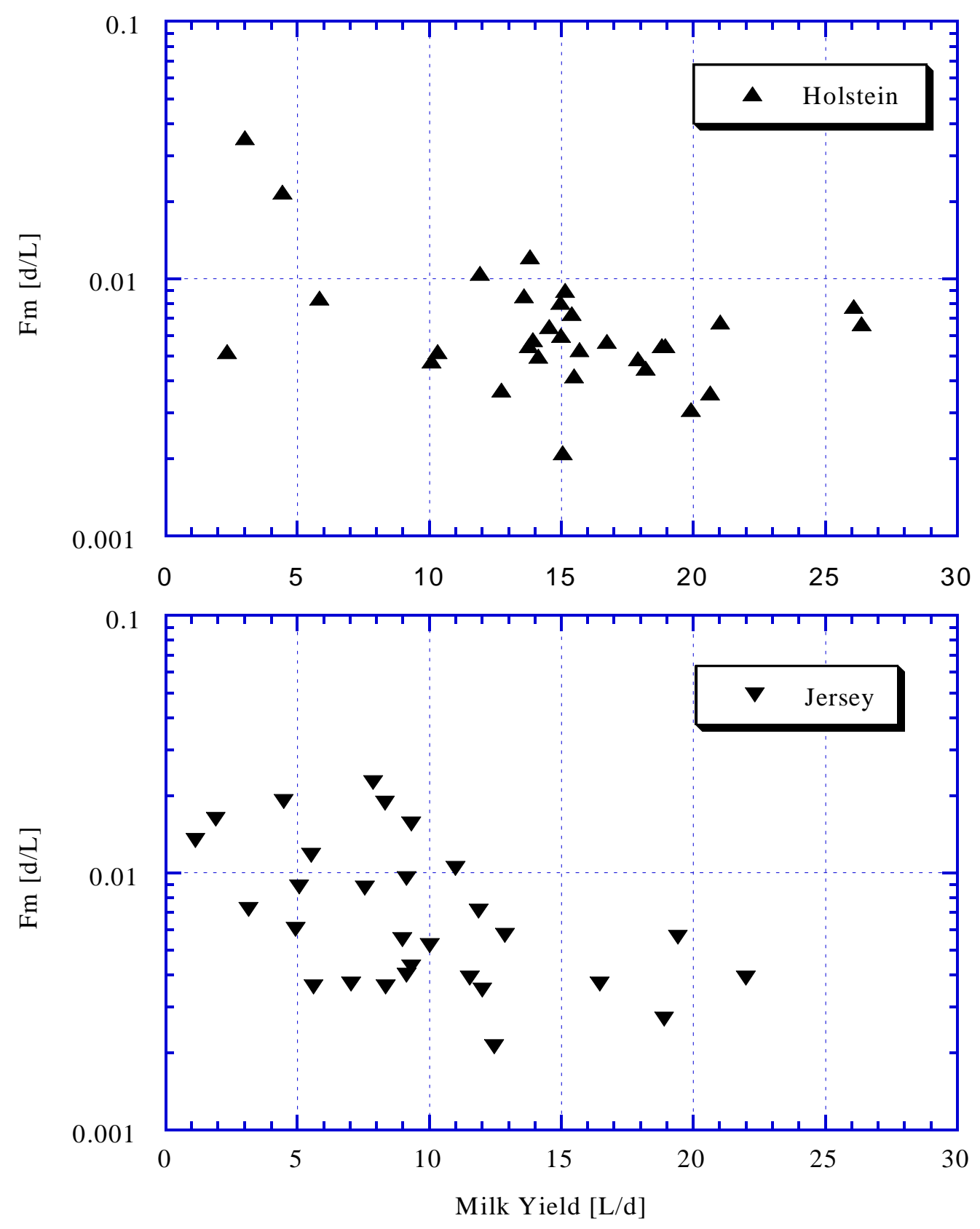

Figure 6.3 Effect of milk yield on ${ }^{131}$ I transfer from feed to milk in different breeds of dairy cows (Miller, 1996). 


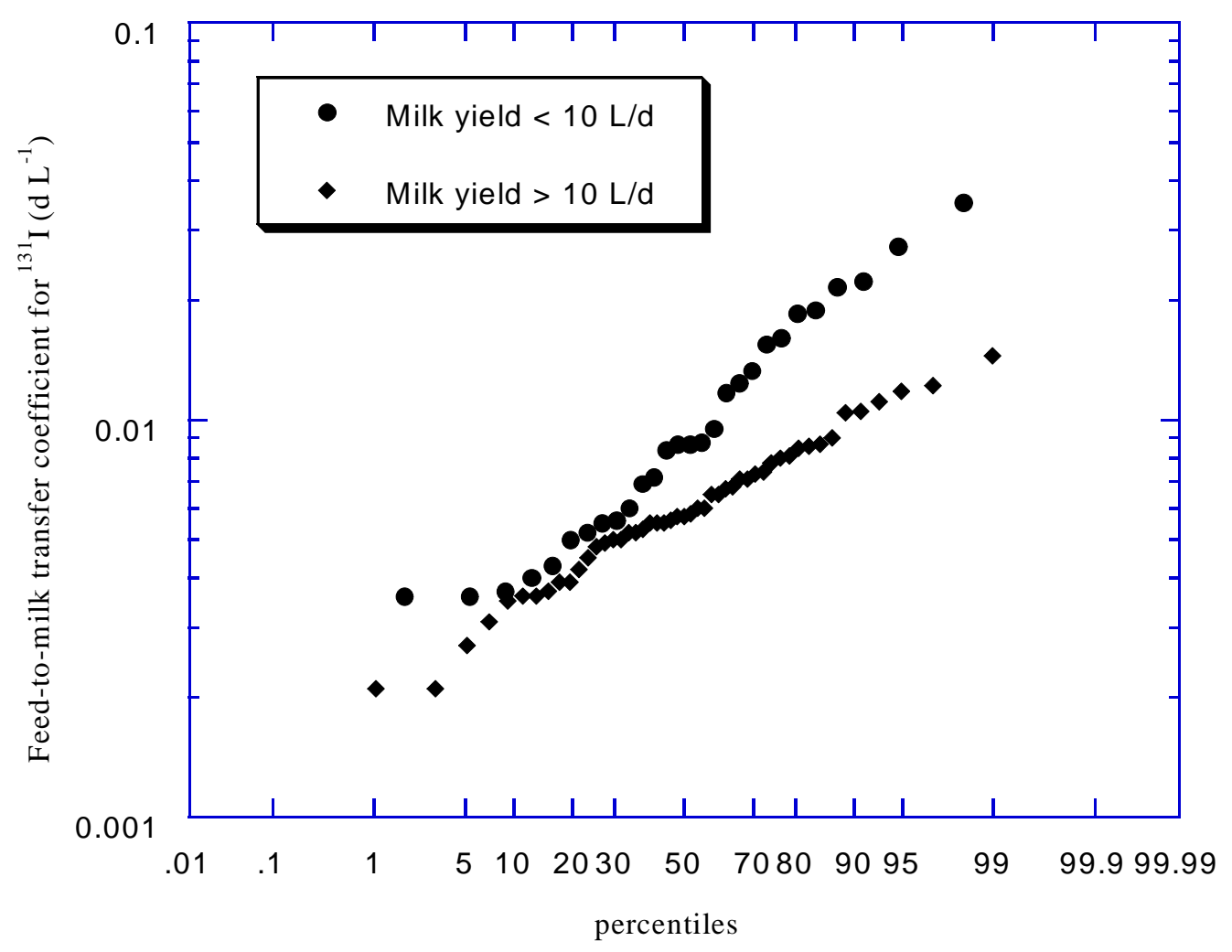

Figure 6.4 Effect of milk yield on ${ }^{131}$ I transfer from feed to milk in dairy cows (all breeds) (Miller, 1996). 


\subsubsection{Assignment of distributions}

The transfer of radioiodine to milk is influenced by many interrelated factors, most of which have been discussed above. This report distinguishes between "backyard" and "commercial" cows in terms of cow management and milk yield. The influence of the other factors is included in the distributions describing the variability of the feed-to-milk transfer coefficient. In the present study, a distinction is made between the distributions selected to represent annual averages and the distributions selected to represent the April 29, 1954, accident situation. The data on which the distribution for $F_{m}$ is based (Miller, 1996) were obtained by examining the ${ }^{131}$ I concentration in milk after a single oral administration of the radionuclide. Consequently, the data are more relevant for the accident situation, when the intake of iodine occurred during a relatively short period of time. For routine releases, ${ }^{131}$ I intake occurred over extended periods of time, and a distribution representing the annual average value of $F_{m}$ must be used. The uncertainty in the annual average is smaller than the uncertainty in the $F_{m}$ values after a single intake. This effect is taken into consideration in the selection of the distributions for the feed-to-milk transfer coefficient.

For the April 29, 1954, accident, the selected subjective probability distribution for the feed-tomilk transfer coefficient in backyard cows is lognormal with a geometric mean of $9.0 \times 10^{-3} \mathrm{~d} \mathrm{~L}^{-1}$ and a geometric standard deviation of 1.9. For routine releases, the distribution has the same geometric mean, but a geometric standard deviation of 1.8. The values for these distributions come from 77 measurements performed on 19 lactating dairy cows (Appendix 6-A). These distributions are applied in the present study to describe the current state of knowledge about $F_{m}$ for the transfer of ${ }^{131} \mathrm{I}$ into the milk of backyard cows. These distributions are not dissimilar to those described in previous reports (Table 6.3 and Figure 6.5), including the results of the recent NRC/CEC expert elicitation (Brown et al., 1997).

A different distribution is used for describing the uncertainty in the milk transfer coefficient for transfer of ${ }^{131}$ I into the milk of commercial dairy cows because these animals typically produce more than $10 \mathrm{~L}$ of milk per day, and the milk is pooled from a large population of animals, considerably reducing the effect of uncertainty due to inter-cow variability of $F_{m}$. The selected subjective probability distribution for the feed-to-milk transfer coefficient in commercial cows is lognormal with a geometric mean of $6.0 \times 10^{-3} \mathrm{~d} \mathrm{~L}^{-1}$ and a geometric standard deviation of 1.5 for the 1954 accidental release. For routine releases, the geometric standard deviation of the distribution was set to 1.4. 
Table 6.3 Summary of literature documenting the variability of the feed-to-milk transfer coefficient $\left(F_{m}\right)$ for ${ }^{131} \mathrm{I}$ in dairy cows.

\begin{tabular}{|c|c|c|c|c|c|}
\hline & $\begin{array}{c}\text { Central } \\
\text { value }\end{array}$ & GSD $^{\mathrm{a}}$ & $\begin{array}{l}\text { Lower } \\
\text { Bound }\end{array}$ & $\begin{array}{l}\text { Upper } \\
\text { Bound }\end{array}$ & Comments \\
\hline Hoffman (1978) & $1.4 \times 10^{-2}$ & 2.1 & $4.2 \times 10^{-3}$ & $3.5 \times 10^{-2}$ & \\
\hline $\begin{array}{l}\text { Hoffman and Baes } \\
\text { (1979) }\end{array}$ & $1.0 \times 10^{-2}$ & 1.73 & $3.4 \times 10^{-3}$ & $2.9 \times 10^{-2}$ & $\begin{array}{l}\text { 95\% C.I. of a lognormal } \\
\text { distribution }\end{array}$ \\
\hline Köhler et al. (1991) & & & $1.0 \times 10^{-3}$ & $7.3 \times 10^{-3}$ & $\begin{array}{l}\text { Chernobyl fallout (range of } 5 \\
\text { values) }\end{array}$ \\
\hline Snyder et al. (1994) & $9.2 \times 10^{-3}$ & 2.1 & $1.6 \times 10^{-3}$ & $5.2 \times 10^{-2}$ & $\begin{array}{l}\text { Hanford site study - Backyard } \\
\text { cows (lognormal distribution) }\end{array}$ \\
\hline Snyder et al. (1994) & $1.2 \times 10^{-2}$ & $(0.002)^{\mathrm{b}}$ & $7.3 \times 10^{-3}$ & $1.6 \times 10^{-2}$ & $\begin{array}{l}\text { Hanford site study - } \\
\text { Commercial cows (normal } \\
\text { distribution) }\end{array}$ \\
\hline NCI (1997) & $4.4 \times 10^{-3}$ & 2.1 & $4.0 \times 10^{-4}$ & $2.1 \times 10^{-2}$ & Lognormal distribution \\
\hline $\begin{array}{l}\text { Whicker and Kirchner } \\
\text { (1987) }\end{array}$ & $8.4 \times 10^{-3}$ & & & & \\
\hline Brown et al. (1997) & $7.1 \times 10^{-3}$ & 2.4 & $5.3 \times 10^{-4}$ & $3.7 \times 10^{-2}$ & $\begin{array}{l}\text { Aggregated results of } 10 \\
\text { experts' opinions }\end{array}$ \\
\hline Miller (1996) & $9.0 \times 10^{-3}$ & 1.9 & $4.0 \times 10^{-3}$ & $3.5 \times 10^{-2}$ & $\begin{array}{l}\text { Dairy cows with milk } \\
\text { production less than } 10 \mathrm{~L} \mathrm{~d}^{-1} \\
\text { (1960s Tennessee data) }\end{array}$ \\
\hline Miller (1996) & $6.0 \times 10^{-3}$ & 1.5 & $2.0 \times 10^{-3}$ & $1.5 \times 10^{-2}$ & $\begin{array}{l}\text { Dairy cows with milk } \\
\text { production greater than } 10 \mathrm{~L} \mathrm{~d}^{-1} \\
\text { (1960s Tennessee data) }\end{array}$ \\
\hline This study & $9.0 \times 10^{-3}$ & 1.8 & $2.6 \times 10^{-3}$ & $3.2 \times 10^{-2}$ & $\begin{array}{l}\text { Backyard cows - lognormal } \\
\text { distribution (routine releases) }\end{array}$ \\
\hline This study & $6.0 \times 10^{-3}$ & 1.4 & $2.7 \times 10^{-3}$ & $1.3 \times 10^{-2}$ & $\begin{array}{l}\text { Commercial cows - lognormal } \\
\text { distribution (routine releases) }\end{array}$ \\
\hline
\end{tabular}

${ }^{a}$ Geometric standard deviation.

${ }^{\mathrm{b}}$ Standard deviation of the normal distribution. 


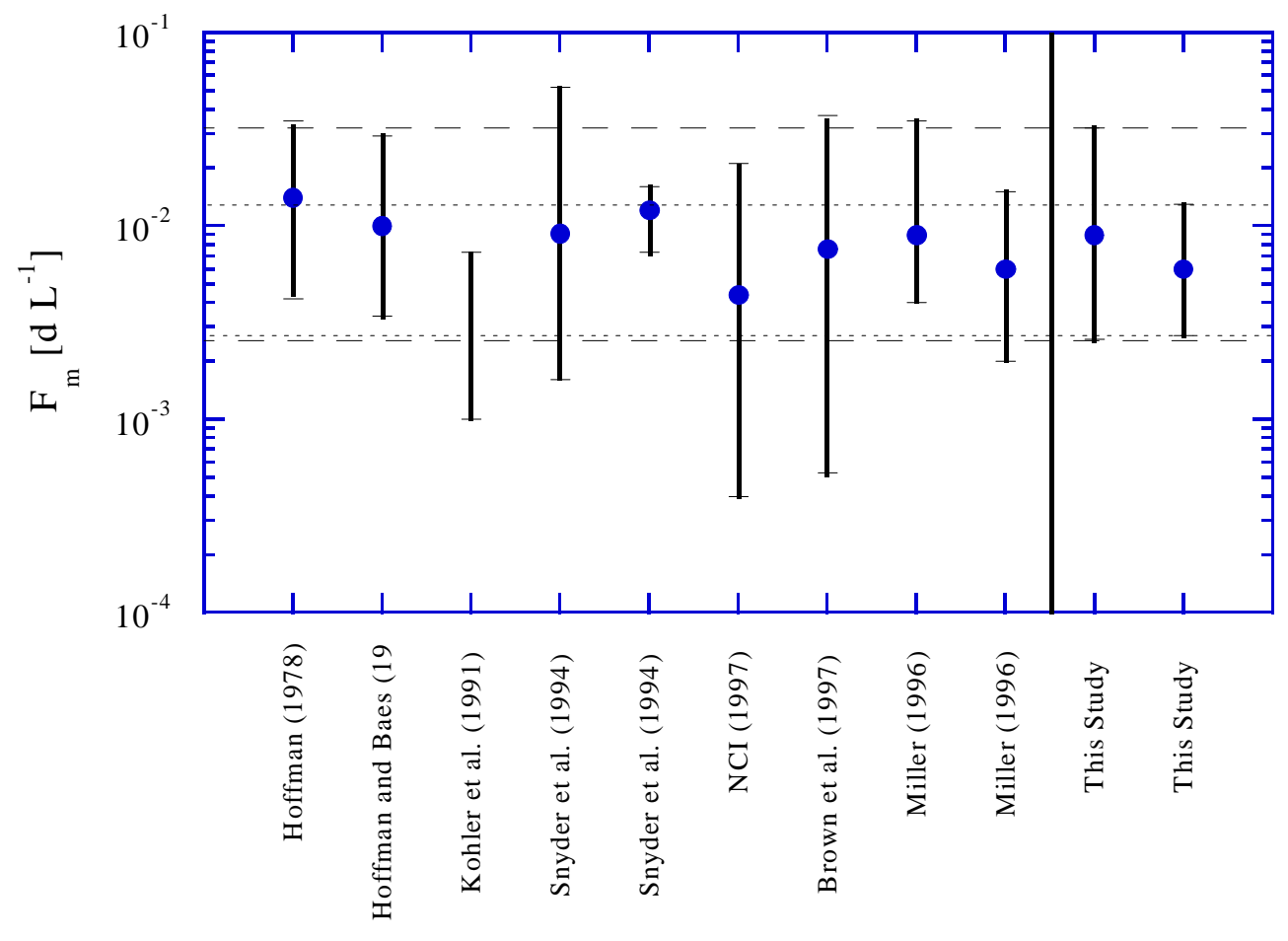

Figure 6.5 Comparison between various studies that document variability of the ${ }^{131}$ I feed-to-milk transfer coefficient. The heavy and light dashed lines indicate the 95\% subjective confidence intervals on the distributions selected in this study for backyard cows and commercial cows, respectively. 


\subsubsection{Transfer of Iodine from Feed to Milk of Goats}

The fraction of the total amount of ingested radioiodine that is transferred to the entire release of milk produced by goats is larger than the fraction transferred to cow's milk by a factor of about 5 (NCI, 1997), because the mammary gland of goats is a very efficient trap for iodine. In addition, the milk yield of a goat is about 10 times less than that of a dairy cow. After a daily intake of the same activity, radioiodine in the milk of goats will be about 50 times more concentrated than in the milk produced by dairy cows. Individuals drinking contaminated goat's milk receive a much higher dose than do those consuming similar amounts of contaminated cow's milk, even accounting for the fact that goats ingest much less vegetation on a daily basis than do dairy cows. On the other hand, the number of people consuming goat milk on a regular basis is far less than the number of people who drink cow's milk.

Hoffman (1978) summarized experimental values of the feed-to-goat's milk transfer coefficient from 10 different studies. The reported values range from $0.06 \mathrm{~d} \mathrm{~L}^{-1}$ to $0.65 \mathrm{~d} \mathrm{~L}^{-1}$ with a geometric mean of $0.34 \mathrm{~d} \mathrm{~L}^{-1}$. Snyder et al. (1994) reviewed various experimental studies and assigned a lognormal distribution with an average of $0.27 \mathrm{~d} \mathrm{~L}^{-1}$ and a range of 0.04 to $1.15 \mathrm{~d} \mathrm{~L}^{-1}$. The research performed by the National Cancer Institute (NCI, 1997) added 11 more studies to those summarized by Hoffman (1978), summarized as a lognormal distribution for the goat's milk transfer coefficient with a geometric mean of $0.22 \mathrm{~d} \mathrm{~L}^{-1}$ and a geometric standard deviation of 2.5 , which produces a range of $0.037 \mathrm{~d} \mathrm{~L}^{-1}$ to $1.33 \mathrm{~d} \mathrm{~L}^{-1}$.

By using a lognormal distribution as indicated by the summary of the literature data points, the upper limit for the transfer coefficient exceeds $1.0 \mathrm{~d} \mathrm{~L}^{-1}$, which is an unrealistic value. Thus, a log-triangular (instead of a lognormal) distribution is used to limit the upper bound of the distribution. Th mode of the distribution was set to $0.22 \mathrm{~d} \mathrm{~L}^{-1}$. The minimum and maximum values of this distribution are 0.02 and $0.9 \mathrm{~d} \mathrm{~L}^{-1}$, respectively. This distribution applies for the accidental release situation in 1954, when the intake of iodine occurred for a shorter period of time. As discussed for feed-to-milk transfer in diary cows, the distribution for routine releases represents an annual average, and thus, it should exhibit a lower uncertainty range. Therefore, for routine releases, the subjective probability distribution for the feed-to-milk transfer coefficients for goats was selected to be log-triangular with the same mode of $0.22 \mathrm{~d} \mathrm{~L}^{-1}$ (as for the 1954 accident), but with minimum and maximum values of 0.03 and $0.8 \mathrm{~d} \mathrm{~L}^{-1}$, respectively.

\subsubsection{Transfer of Iodine from Feed to Beef}

Radioiodine has a lower transfer to skeletal muscle than to other tissues (Miller et al., 1975). Because of the short half-life of ${ }^{131} \mathrm{I}$ (8.04 days), most of the ${ }^{131} \mathrm{I}$ decays away during the time elapsed from ingestion to slaughtering. Only the ${ }^{131} \mathrm{I}$ consumed two or three weeks before slaughtering will be found in the muscle tissue. Experimental evidence (Miller et al., 1975; Miller and Swanson, 1973) on Jersey and Holstein cows shows a transfer factor $\left(F_{f}\right)$ with an average varying between 0.0008 and $0.0026 \mathrm{~d} \mathrm{~kg}^{-1}$. However, in a review of transfer factors for assessing doses from radionuclides in agricultural products, $\mathrm{Ng}$ (1982) reports values ranging from 0.007 to $0.02 \mathrm{~d} \mathrm{~kg}^{-1}$ for ${ }^{131} \mathrm{I}$ in beef. The two ranges do not overlap. There is no reasonable explanation for these results, but one can generally speculate that the differences are produced by 
the use of different measurement techniques. $\mathrm{Ng}$ (1982) values seem biased towards the higher side. In his report, he actually recommends a value of $0.007 \mathrm{~d} \mathrm{~kg}^{-1}$ (which is the lower bound of his range) to be used for "food contamination by routine emissions from a nuclear facility."

A range of 0.0005 to $0.02 \mathrm{~d} \mathrm{~kg}^{-1}$ is used for the feed-to-meat transfer factor in this report; the shape of the subjective probability distribution is chosen to be log-triangular with a mode of $0.003 \mathrm{~d} \mathrm{~kg}^{-1}$.

\subsection{Validation of the Modeling Approaches Used to Estimate the ${ }^{131}$ I Concentrations in Milk}

Average concentrations of ${ }^{131} \mathrm{I}$ in raw milk collected from 8 different milk sampling stations around X-10 and the amount of ${ }^{131}$ I released from X-10 were reported for 1962 and 1964 (Morgan et al., 1963; Morgan and Davis, 1965). About $102 \mathrm{Ci}\left(3.8 \times 10^{12} \mathrm{~Bq}\right)$ of gaseous wastes were released from X-10 operations during May and October of 1962 , a large percentage of which was identified as ${ }^{131} \mathrm{I}$. Release data for other months for 1962 are not available. Since the fraction of ${ }^{131} \mathrm{I}$ in the total release was not known, $85 \%$ of the total release was ascribed as the lower bound for the fraction ${ }^{131} \mathrm{I}$. A factor of 1.05 was applied to the reported amount of gaseous releases to set the upper bound value for the amount of ${ }^{131} \mathrm{I}$ released, primarily to account for measurement errors. A triangular distribution between $3.2 \times 10^{12} \mathrm{~Bq}$ and $4.0 \times 10^{12} \mathrm{~Bq}$ was used with a most likely value of $3.8 \times 10^{12} \mathrm{~Bq}$ to describe the source term. During this period, the average weekly concentration of ${ }^{131} \mathrm{I}$ in milk was estimated from the graph provided in Morgan et al. (1963) to be $41.2 \mathrm{pCi} \mathrm{L}-1\left(1.5 \mathrm{~Bq} \mathrm{~L}^{-1}\right)$.

A total of roughly $61 \mathrm{Ci}\left(2.3 \times 10^{12} \mathrm{~Bq}\right)$ of ${ }^{131} \mathrm{I}$ was estimated to have been released between late February and late April of 1964 from the graph provided by Morgan and Davis (1965). To account for uncertainties in measurements and in interpreting the graph, an uncertainty of $\pm 5 \%$ was assigned to the amount of ${ }^{131} \mathrm{I}$ released during this period. As before, a triangular distribution between $2.1 \times 10^{12} \mathrm{~Bq}$ and $2.4 \times 10^{12} \mathrm{~Bq}$ was used with a most likely value of $2.3 \times$ $10^{12} \mathrm{~Bq}$ to describe the source term. The average weekly concentration of ${ }^{131} \mathrm{I}$ in milk during this period was estimated to be approximately $30 \mathrm{pCi} \mathrm{L}^{-1}\left(1.1 \mathrm{~Bq} \mathrm{~L}^{-1}\right)$.

The data on milk concentrations described above are representative of weekly measurements from 8 milk sampling stations surrounding the X-10 facility, as shown in Figure 6.6. In other words, these data represent crude pooled averages. The original data from these stations could not be located. Hence, large uncertainty, arising from possible zero concentrations in milk at one or more locations and from other sources of error, may occur. To account for the uncertainty in the representativeness of these data, an error of \pm 30 was assigned to the single-valued data. Some locations of interest for which milk concentrations were estimated and which are closest to the milk sampling stations are presented in Table 6.4. Since the milk concentrations in Morgan and Davis (1965) and Morgan et al. (1963) represented the average of the 8 sampling stations, it was decided to validate these concentrations using average of predictions made at the locations presented in Table 6.4. 


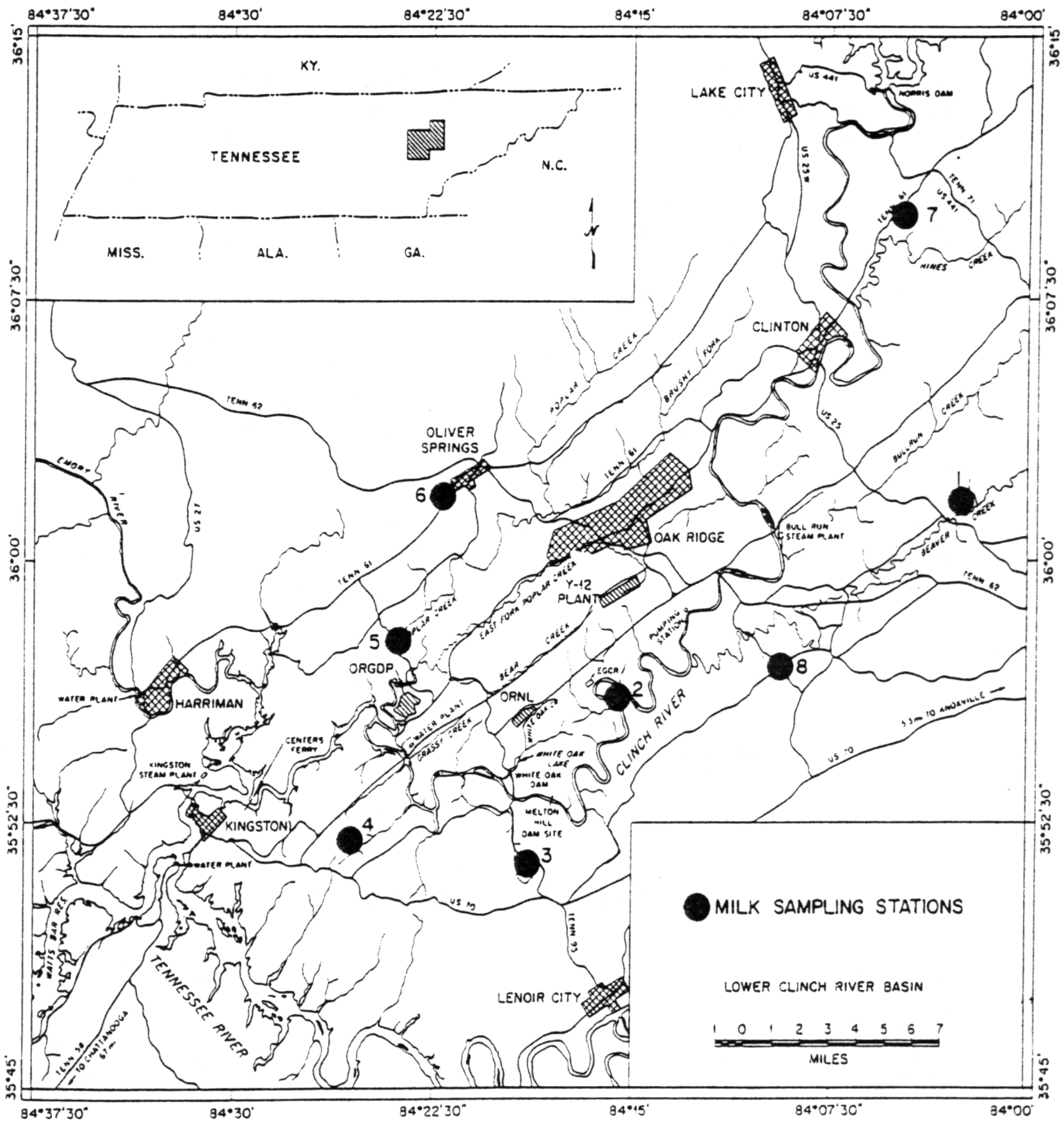

Figure 6.6 Locations of milk sampling stations around the X-10 facility ( reproduced from Morgan and Davis, 1965). 
Table 6.4 Locations of interest where concentrations of ${ }^{131} \mathrm{I}$ in milk were estimated and which are closest to the 8 milk sampling stations identified in Figure 6.6.

\begin{tabular}{cl}
\hline $\begin{array}{c}\text { Milk sampling } \\
\text { station no. }\end{array}$ & Closest location, distance and direction from X-10* \\
\hline 1 & Claxton, $22.7 \mathrm{~km}, 54^{\circ}$ \\
2 & Gallaher Bend, $6.3 \mathrm{~km}, 89^{\circ}$ \\
3 & Bradbury, $6.0 \mathrm{~km}, 228^{\circ}$ and Hines Valley, $14.5 \mathrm{~km}, 185^{\circ}$ \\
4 & Lawnville $/$ Gallaher, $9.3 \mathrm{~km}, 245^{\circ}$ \\
5 & Dyllis, $10.1 \mathrm{~km}, 293^{\circ}$, and Jonesville $9.0 \mathrm{~km}, 312^{\circ}$ \\
6 & Oliver Springs, $13.3 \mathrm{~km}, 350^{\circ}$ \\
7 & Cedar Grove, $36.2 \mathrm{~km}, 54^{\circ}$ and Lake City, $35.0 \mathrm{~km}, 27^{\circ}$ \\
8 & Hardin Valley, $12.0 \mathrm{~km}, 85^{\circ}$ \\
\hline$*$ &
\end{tabular}

Using the source terms for the 13 years of release between 1944 and 1956 and the predicted milk concentrations for each of these years at the locations of interest identified in Table 6.4, a 13-year regional average of the ratio of predicted milk concentration to the amount released was estimated to range from $2.4 \times 10^{-13}$ to $1.4 \times 10^{-12} \mathrm{~L}^{-1}$ at the $95 \%$ subjective confidence level, with a central estimate of $5.9 \times 10^{-13} \mathrm{~L}^{-1}$ for backyard cow milk, and it was estimated to range from $1.4 \times 10^{-13} \mathrm{~L}^{-1}$ to $7.2 \times 10^{-13} \mathrm{~L}^{-1}$ at the $95 \%$ subjective confidence level, with a central estimate of $3.4 \times 10^{-13} \mathrm{~L}^{-1}$ for the commercial milk. Using the amount of ${ }^{131}$ I released in 1962 and 1964, the concentrations of ${ }^{131} \mathrm{I}$ in milk during 1962 and 1964 were then predicted, as shown in Table 6.5. Table 6.5 also shows the measured concentrations, as discussed earlier for the periods of interest during 1962 and 1964. 
Table 6.5 Predicted and measured average ${ }^{131}$ I concentrations in milk $\left(\mathrm{Bq} \mathrm{L}^{-1}\right)$ for the selected periods of interest in 1962 and 1964.

\begin{tabular}{|c|c|c|c|c|c|c|}
\hline \multirow[b]{2}{*}{ Year } & \multicolumn{3}{|c|}{$\begin{array}{c}\text { Predicted average concentration } \\
\text { (95\% subjective confidence } \\
\text { interval) }\end{array}$} & \multicolumn{3}{|c|}{$\begin{array}{c}\text { Average concentration from } 8 \\
\text { sampling stations } \\
\text { (95\% subjective confidence } \\
\text { interval) }\end{array}$} \\
\hline & $\begin{array}{l}\text { Lower } \\
\text { bound }\end{array}$ & $\begin{array}{c}\text { Central } \\
\text { value }\end{array}$ & $\begin{array}{l}\text { Upper } \\
\text { bound }\end{array}$ & $\begin{array}{l}\text { Lower } \\
\text { bound }\end{array}$ & $\begin{array}{l}\text { Central } \\
\text { value }\end{array}$ & $\begin{array}{l}\text { Upper } \\
\text { bound }\end{array}$ \\
\hline & \multicolumn{6}{|c|}{ Backyard cow milk } \\
\hline 1962 & 0.85 & 2.1 & 5.2 & 0.60 & 1.5 & 2.4 \\
\hline \multirow[t]{2}{*}{1964} & 0.56 & 1.3 & 3.2 & 0.77 & 1.1 & 1.8 \\
\hline & \multicolumn{6}{|c|}{ Commercial milk } \\
\hline 1962 & 0.54 & 1.2 & 2.7 & 0.60 & 1.5 & 2.4 \\
\hline 1964 & 0.32 & 0.75 & 1.6 & 0.77 & 1.1 & 1.8 \\
\hline
\end{tabular}

The modeling approaches used in this study to simulate the processes from the release of ${ }^{131}$ I to the accumulation of ${ }^{131} \mathrm{I}$ in cows' milk provide sufficiently accurate predictions of the concentrations of ${ }^{131} \mathrm{I}$ in milk (Table 6.5). The range of the measured average concentrations is almost completely contained within the 95\% subjective confidence interval of the model predictions for both backyard cow milk and commercial milk. It is noted, however, that because of insufficient sampling data on milk for other years and because the original data for the individual sampling stations for the two years that were studied could not be located, more definitive validation of the predicted down-wind milk concentrations could not be completed at this time.

\subsection{Predicted Milk Concentrations}

Concentrations of ${ }^{131} \mathrm{I}$ in backyard cow and commercial milk were estimated for each year of release and for all locations of interest within the $38 \mathrm{~km}$ from X-10 (Equation 2-A.4). Thirteenyear average concentrations of ${ }^{131} \mathrm{I}$ in milk were then estimated. The lower bound ( 2.5 percentile) of the ${ }^{131} \mathrm{I}$ concentration in backyard cow milk is given in Figure 6.7. It is highly likely the actual concentration at a given location is greater than the value plotted in Figure 6.7 for that location. The upper bound (97.5 percentile) of the ${ }^{131}$ I concentration in milk is given in Figure 6.8. It is highly likely that the concentration at a given location is less than the value plotted in Figure 6.8 for that location. 
An important observation is that there were no dairy cows within the Oak Ridge Reservation or in the City of Oak Ridge (Appendix 11A), thus, the concentrations plotted for those locations are not relevant. At boundary of the Oak Ridge Reservation (the closest locations where dairy cows were grazing the concentration in backyard cow milk ranges from 30 to $300 \mathrm{~Bq} \mathrm{~L}^{-1}$.

\subsection{Modeling the Transfer of ${ }^{131}$ I to Food Products other than Milk and Meat}

Radioiodine accumulates in various other food products (e.g., cottage cheese and eggs) regularly consumed by people. A special case is contamination of mother's milk for lactating women breast-feeding their infants. The modeling approach for these exposure pathways is described in detail in Appendix 2A. This section investigates the transfer of radioiodine to these food products and defines the distributions for the parameter values necessary for dose and risk estimates.

\subsubsection{Accumulation of Iodine in Cottage Cheese}

Radioiodine present in milk is transferred to cottage cheese during the preparation process. The transfer depends on the type of preparation process and on the source of milk (cows or goats) (Table 6.6). The fraction transferred to cottage cheese varies between $10 \%$ and $30 \%$ of the total amount in milk (IAEA, 1992). The amount of cottage cheese produced is always lower than the starting amount of milk, and thus the concentration of iodine in cottage cheese can be even larger than the concentration in milk (Table 6.6). However, because of the long storage time which leads to a significant loss of ${ }^{131}$ I from radioactive decay and because of the low rate of consumption of cottage cheese with respect to that of milk, the risk of radiation-induced cancer from ingestion of contaminated cottage cheese is much lower than from ingestion of contaminated milk for the same deposition of ${ }^{131} \mathrm{I}$.

The geometric mean of the five independent studies reported in Table 6.6 is $1.4 \mathrm{~Bq} \mathrm{~kg}^{-1}$ cheese per $\mathrm{Bq} \mathrm{L}^{-1}$ milk, while the minimum and maximum values are 0.2 and $5.8 \mathrm{~Bq} \mathrm{~kg}^{-1}$ cheese per $\mathrm{Bq} \mathrm{L}^{-1}$ milk, respectively. A log-triangular distribution was assigned to represent the uncertainty in the milkto-cottage cheese transfer coefficients. The distribution has a mode given by the geometric mean of the five independent studies, and a range dictated by the minimum and maximum values (Figure 6.9). 
TASK 1 REPORT

${ }^{131}$ I Releases from X-10 Radioactive Lanthanum Processing July 1999 Transfer of ${ }^{131}$ I from Pasture to Various Food Products

Page 6-25
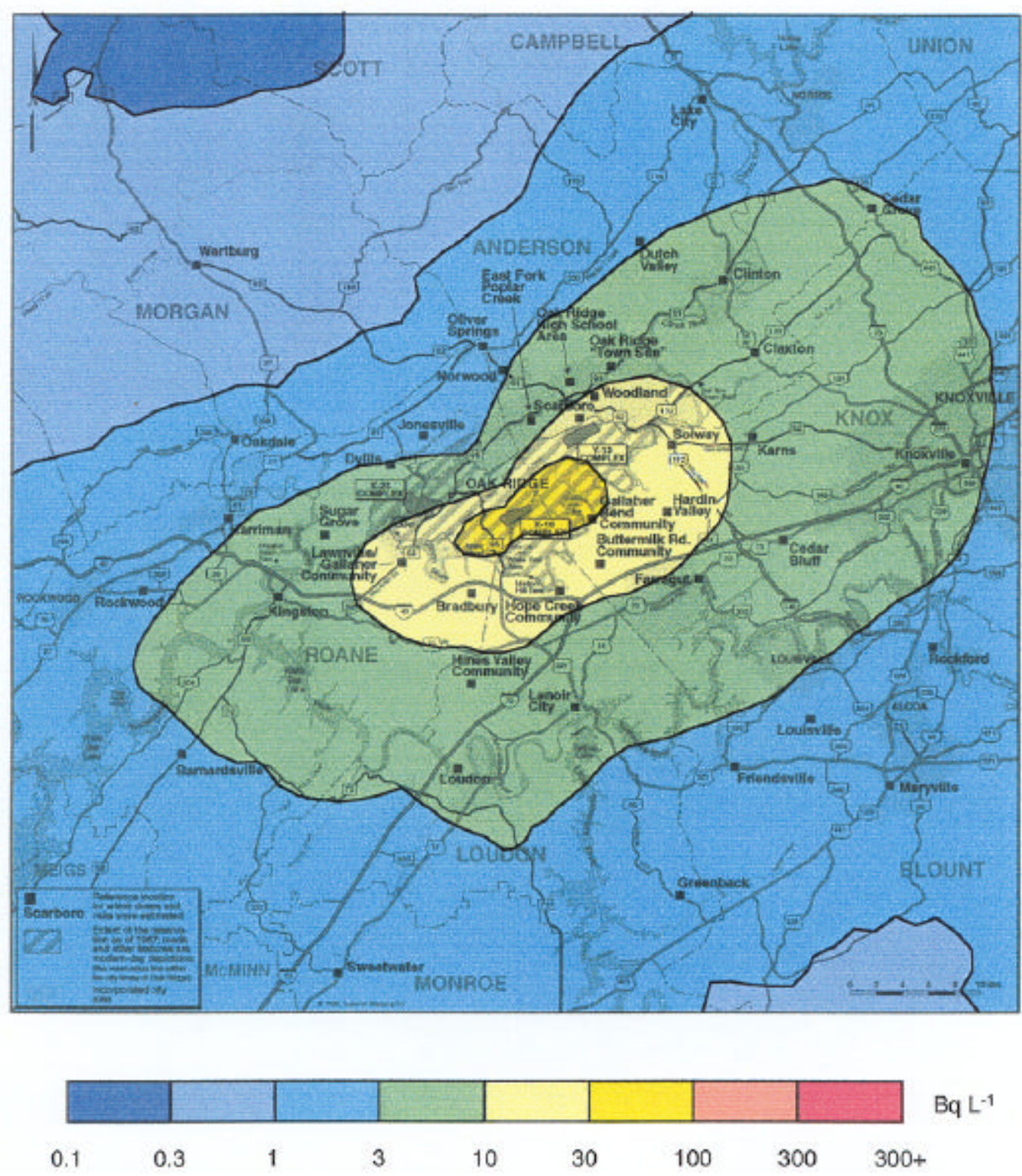

Figure 6.7 Estimated lower limit (2.5 percentile) of the thirteen-year (1944-1956) average concentration of ${ }^{131} \mathrm{I}$ in backyard cow milk $\left(\mathrm{Bq} \mathrm{L}^{-1}\right)$. It is highly likely that the actual concentration at a given location is greater than the concentration shown here. Note that there were no dairy cows within the Oak Ridge Reservation or in the City of Oak Ridge (see Appendix 11-A for details). Therefore, the concentrations at those locations are not relevant. 
TASK 1 REPORT

${ }^{131}$ I Releases from X-10 Radioactive Lanthanum Processing July 1999

Page 6-26
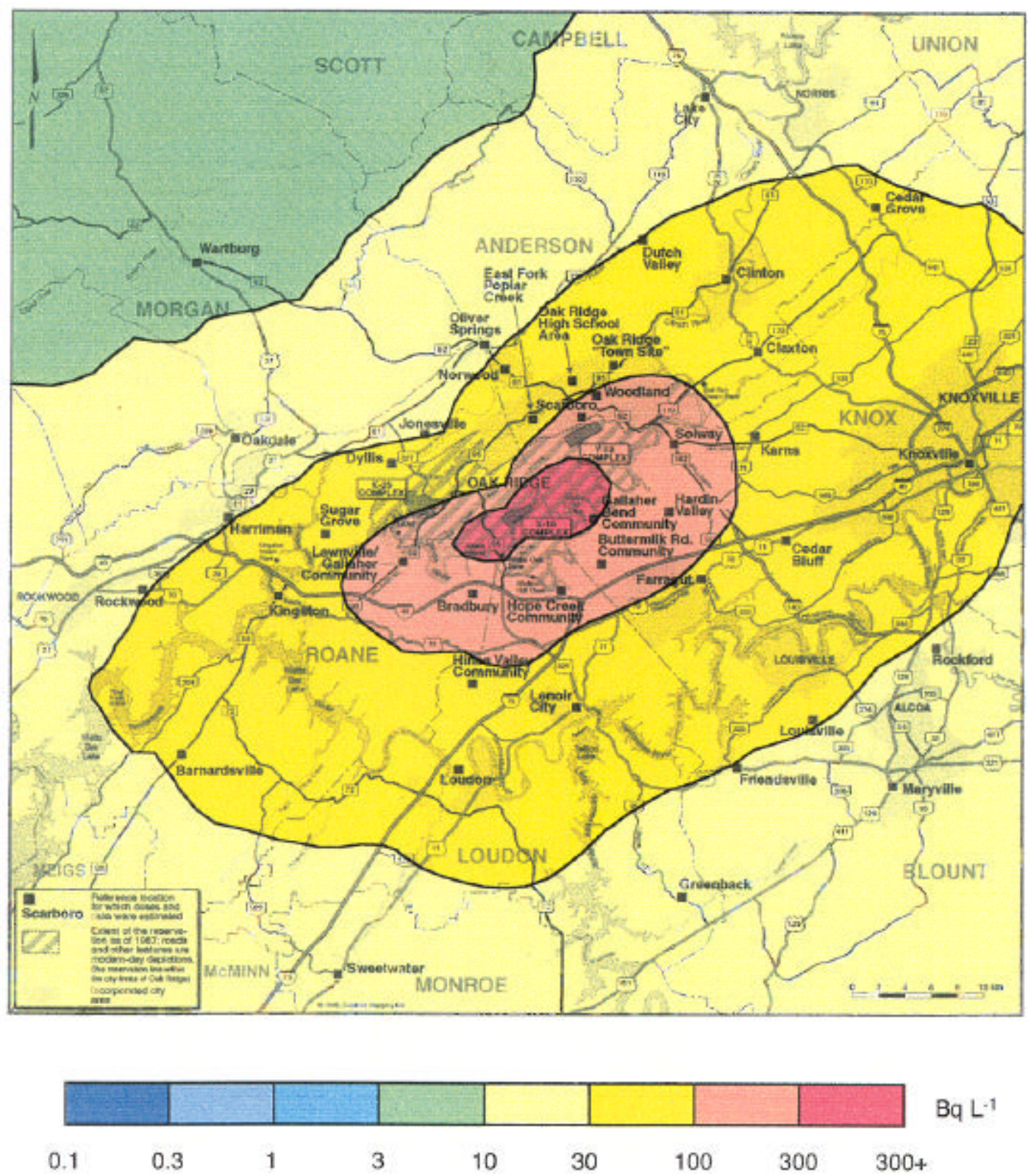

Figure 6.8 Estimated upper limit (97.5 percentile) of the thirteen-year (1944-1956) average concentration of ${ }^{131} \mathrm{I}$ in backyard cow milk $\left(\mathrm{Bq} \mathrm{L}^{-1}\right)$. It is highly likely that the actual concentration at a given location is less than the concentration shown here. Note that there were no dairy cows within the Oak Ridge Reservation or in the City of Oak Ridge (see Appendix 11-A for details). Therefore, the concentrations at those locations are not relevant. 


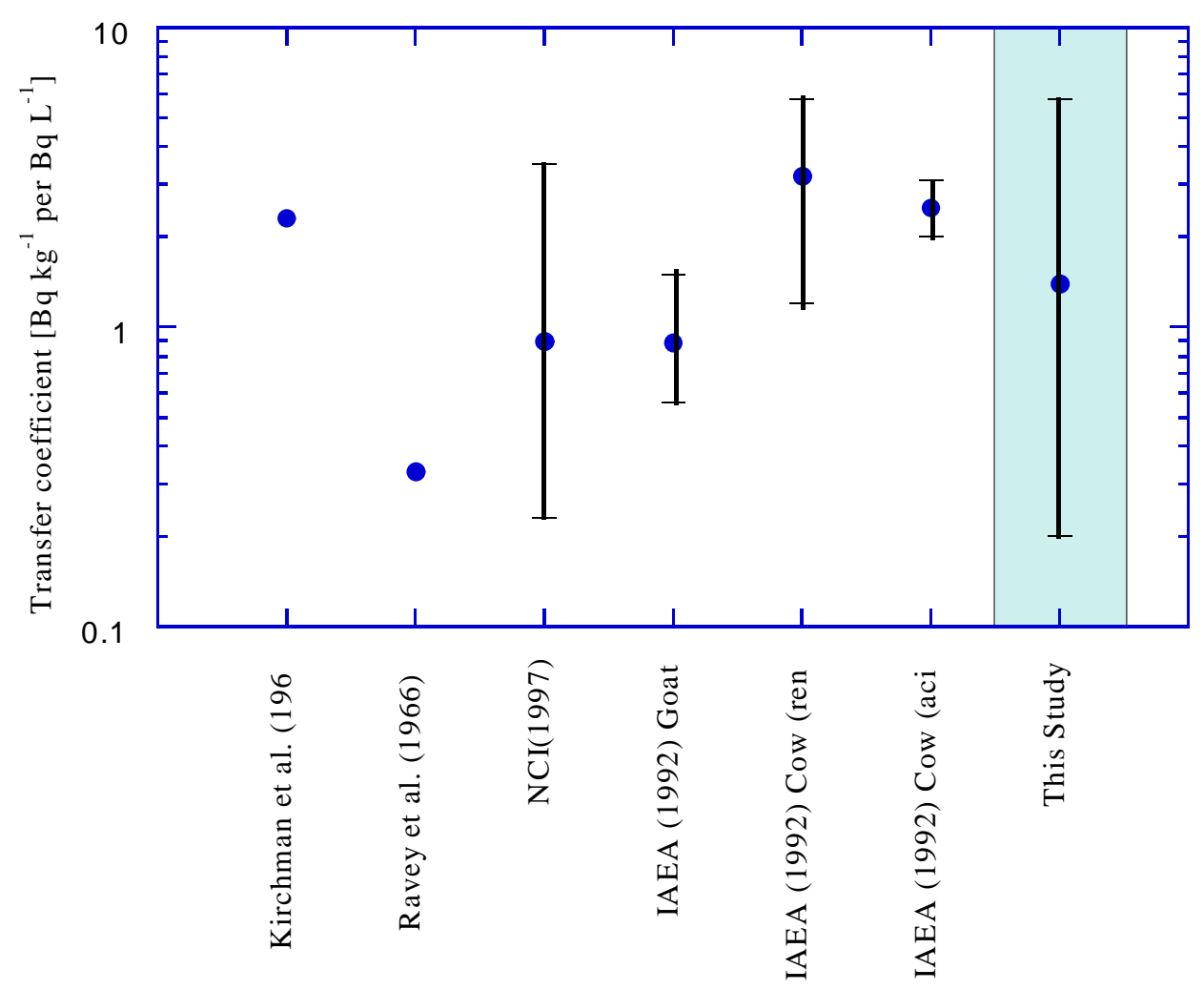

Figure 6.9 Milk-to-cottage cheese transfer coefficients reported by different studies. 
Table 6.6 Summary of milk-to-cheese transfer coefficients reported by different studies.

\begin{tabular}{|c|c|c|c|c|}
\hline \multirow[t]{3}{*}{ Type of cheese } & \multicolumn{3}{|c|}{$\begin{array}{c}\text { Transfer of }{ }^{131} \text { I from milk to cheese } \\
{\left[\mathrm{Bq} \mathrm{kg}{ }_{\text {cheese }} \text { per } \mathrm{Bq} \mathrm{L}^{-1}{ }_{\text {milk }}\right]}\end{array}$} & \multirow[t]{3}{*}{ Source } \\
\hline & \multirow[t]{2}{*}{ Central Value } & \multicolumn{2}{|c|}{ Range } & \\
\hline & & $\min$ & $\max$ & \\
\hline Goat cheese & 0.89 & 0.56 & 1.5 & $\operatorname{IAEA}(1992)^{\mathrm{a}}$ \\
\hline Cow rennet ${ }^{\mathrm{b}}$ cheese & 3.2 & 1.2 & 5.8 & $\operatorname{IAEA}(1992)^{\mathrm{a}}$ \\
\hline Cow acid ${ }^{\mathrm{b}}$ cheese & 2.5 & 2.0 & 3.1 & IAEA $(1992)^{\mathrm{a}}$ \\
\hline Not specified & 2.3 & & & Kirchman et al. (1966) \\
\hline Not specified & 0.33 & & & Ravey et al. $(1966)^{\mathrm{c}}$ \\
\hline Not specified & $0.9^{\mathrm{d}}$ & 0.23 & 3.5 & NCI (1997) \\
\hline
\end{tabular}

${ }^{a}$ derived by the authors from the data provided by IAEA (1992), the range is the $95 \%$ confidence interval obtained in the calculation.

${ }^{\mathrm{b}}$ different methods of preparing cheese.

${ }^{c}$ cited by NCI (1997).

${ }^{d}$ the central value is the geometric mean of the previous two studies cited by NCI (1997); the range is the $95 \%$ confidence interval of a lognormal distribution having a geometric mean equal to the central value and a GSD of 2.0.

\subsubsection{Accumulation of Iodine in Eggs}

The approach used in this analysis is based on the observation that, for a given ${ }^{131} \mathrm{I}$ ground deposition density, the concentration of ${ }^{131} \mathrm{I}$ in eggs is similar to the concentration of ${ }^{131} \mathrm{I}$ in milk from a backyard cow at the time of milking. Explicit equations for accumulation of iodine in eggs are presented in Appendix 2-A. NCI (1997) summarized the experimental evidence collected after some of the nuclear tests. At a farm affected by ${ }^{131}$ I fallout from the shot "Pin Stripe" conducted at the Nevada Test Site (NTS) in 1966, the time-integrated concentration in eggs was found to be twice the time-integrated concentration in milk (Barth et al., 1969). In another case, ${ }^{131}$ I from 1961 tests in the Soviet Union was measured near the New York City area. In this case, the concentration in eggs was substantially lower than the concentration in milk (Eisenbud and Wrenn, 1963). A parameter $\left(F_{\text {egg }}\right)$ is defined as the ratio between the average concentration in eggs and the average concentration in milk for the same amount of deposition on pasture vegetation $\left(\mathrm{Bq} / \mathrm{kg}_{\text {egg }}\right.$ per $\mathrm{Bq} / \mathrm{L}_{\text {milk }}$ or $\left.\mathrm{L}_{\text {milk }} \mathrm{kg}_{\text {egg }}{ }^{-1}\right)$. The values for $\left(F_{\text {egg }}\right)$ can be either above or below 1. The distribution proposed by NCI (1997), with a median of 1 and a geometric standard deviation of 1.4, was selected for this assessment.

\subsubsection{Accumulation of Iodine in Mother's Milk}

Lactating mothers consuming ${ }^{131}$ I-contaminated food will transfer ${ }^{131} \mathrm{I}$ to their milk, resulting in ${ }^{131}$ I exposure of their breast-fed infants. The accumulation of radioiodine in mother's milk is estimated using a diet-to-milk transfer coefficient $\left(F_{m m}\right)$ (Section 2). NCI (1997) presented a summary of transfer coefficients for 43 cases (Weaver et al., 1960; Karjalainen et al., 1971; Wyburn, 1973; Miller and Weetch, 1955; Nurnberger and Lipscom, 1952). The geometric mean 
of the reported data was $0.12 \mathrm{~d} \mathrm{~L}^{-1}$. This value is larger than the feed-to-milk transfer coefficient for backyard cows $\left(0.009 \mathrm{~d} \mathrm{~L}^{-1}\right)$ or commercial cows $\left(0.006 \mathrm{~d} \mathrm{~L}^{-1}\right)$. However, for the same ground deposition, the intake of ${ }^{131} \mathrm{I}$ by women is much lower than the amount ingested by grazing animals. Consequently, the concentration of ${ }^{131} \mathrm{I}$ in mother's milk is significantly lower than the concentration in milk of grazing animals. The subjective probability distribution for the diet-to-mother's milk transfer coefficient adopted for this assessment is based on the study performed by NCI (1997). The same geometric mean $\left(0.12 \mathrm{~d} \mathrm{~L}^{-1}\right)$ is used in this assessment, but a log-triangular distribution (instead of lognormal) was selected to represent the uncertainty. The minimum and maximum values of the subjective probability distribution are 0.01 and $0.6 \mathrm{~d} \mathrm{~L}^{-1}$, respectively. This range represents the reported values, and the shape of the distribution eliminates sampling of unrealistic values.

\subsection{References}

Barth, D.S. Engel, R.E. Black, S.C. and Shimoda, W. 1969. Dairy farm radioiodine studies following the Pin Stripe event. Southwestern Radiological Health Laboratory report SWRHL41r, Las Vegas, NV. 1969.

Bretthauer, E.W., Mullen, A.L., and Moghissi, A.A. 1972. Milk transfer comparisons of different chemical forms of radioiodine. Health Physics 22:257. 1972.

Brown, J., Goossens, L.H.J., Kraan, B.C.P., Cooke, R.M., Harper, F.T., Haskin, F.E., Abbott, M.L., Young, M.L., Hora, S.C., and Rood, A. 1997. Probabilistic Accident Consequence Uncertainty Analysis. Food Chain Uncertainty Analysis. NUREG/CR-6523. EUR 16771. SAND97-0335. 1997.

Eisenbud, M. and Wrenn, M.E. 1963. Biological disposition of radioiodine - A review. Health Physics 9:1133-1139. 1963.

Faw, R.E., and Shultis, J.K. 1993. Radiological Assessment. Sources and Exposures. PTR Prentice-Hall, Engelwood Cliffs, New Jersey 07632. 1993.

Hoffman, F.O. 1978. A review of measured values of the milk transfer coefficient $\left(\mathrm{F}_{\mathrm{m}}\right)$ for iodine. Health Physics 35:413. 1978. (ChemRisk Repository No. 2178)

Hoffman, F. O., and Baes, C.F.III. 1979. A Statistical Analysis of Selected Parameters for Predicting Food Chain Transport and Internal Dose of Radionuclides. Oak Ridge National Laboratory. NUREG/CR-1004, ORNL/NUREG/TM-282. October, 1979. (ChemRisk Repository No. 2171)

Hoffman, F.O., Amaral, E., Mohrbacher, D.A., and Deming, E.J. 1988. The Comparison of Generic Model Predictions with Chernobyl Fallout Data on the Transfer of Radioiodine over the Air-Pasture-Cow-Milk Pathway. J. Environ. Radioactivity 8:53-71. 1988. (ChemRisk Repository No. 2165). 
IAEA. International Atomic Energy Agency. 1992. Modelling of resuspension, seasonality and losses during food processing. First report of the VAMP Terrestrial Working Group. IAEATECDOC-647. Vienna, Austria. May, 1992. (ChemRisk Repository No. 4014)

Karjaleinen, P., Pentilla, I.M., and Pystynen, P. 1971. The amount and form of radioactivity in human milk after lung scanning, renography and placental localization by ${ }^{131}$ I labelled tracers. Acta Obstet. Gynec. Scand. 50:357-361. 1971.

Kirchmann, R., Adam, V., and van Puymbraeck, S. 1966. Radiocontamination des denies du lait de vache. In: Radioisotopes and Radiation in Dairy Science and Technology; Proc. FAO-IAEA Seminar 12-15 July, 1966; pp. 189-198. IAEA, Vienna. 1966.

Kohler, H., Peterson, S.R., and Hoffman, F.O. 1991. Multiple Model Testing using Chernobyl Fallout Data of ${ }^{131}$ I in Forage and Milk and ${ }^{137}$ Cs in Forage, Milk, Beef and Grain. Stockholm: National Institute of Radiation Protection; Biospheric Model Validation Study (BIOMOVS) Technical Report 13. 1991. (ChemRisk Repository No. 2186)

Koranda, J.J. 1965. Agricultural Factors Affecting the Daily Intake of Fresh Fallout by Dairy Cows. UCRL-12470. Biology and Medicine, UC-48. TID-4500 (38 ${ }^{\text {th }}$ Ed.). University of California, Lawrence Radiation Laboratory, Livermore, California. March, 1965.

Miller, J.K. 1996. Private Communication. The University of Tennessee, Knoxville, Tennessee. 1996.

Miller, J.K., Swanson, E.W. 1973. Metabolism of Ethylenediaminedihydriodide and Sodium or Potassium Iodide by Dairy Cows. Journal of Dairy Science 56(3):378-384. March, 1973.

Miller, J.K., Swanson, E.W., and Spalding, G.E. 1975. Iodine Absorption, Excretion, Recycling, and Tissue Distribution in the Dairy Cow. Journal of Dairy Science, 58:1578-1593. October, 1975.

Miller, H., and Weetch, R.S. 1955. The excretion of radioactive iodine in human milk. Lancet 269:1013. 1955.

Morgan, K.Z., Davis, D.M., and Hart, J.C. 1963. Applied Health Physics Annual Report for 1962. ORNL-3490. Oak Ridge National Laboratory, Oak Ridge, TN. 1963.

Morgan, K.Z., and Davis, D.M. 1965. Applied Health Physics Annual Report for 1964. ORNL3820. Oak Ridge National Laboratory, Oak Ridge, TN. 1965.

NCI. National Cancer Institute. 1997. Estimated Exposure and Thyroid Doses Received by the American People from Iodine-131 Fallout Following Nevada Atmospheric Nuclear Bomb Tests. U.S. Department of Health and Human Services. National Institute of Health. National Cancer Institute, October, 1997.

Ng, Y.C. 1982. A Review of Transfer Factors for Assessing the Dose from Radionuclides in Agricultural Products. Nuclear Safety. 23(1). Jan.-Feb. 1982. 
Nurnberger, C.E., and Lipscomb, A. 1952. Transmission of radioiodine $\left({ }^{131} \mathrm{I}\right)$ to infants through human maternal milk. J.A.M.A. 150:1398-1400. 1952.

Reavey, T.C., and Baratta, E.J. 1966. Comparison of strontium-90, iodine-131, and cesium-137 in milk and milk products. Radiological Health Data and Reports 7:215-218. 1966.

Schwartz, G., and Hoffman, F.O. 1980. Imprecision of Dose Predictions for Radionuclides Released to the Environment: An Application of a Monte Carlo Simulation Technique. Environment International 4:289-297. 1980. (ChemRisk Repository No. 2177)

Snyder, S.F., Farris, W.T., Napier, B.A., Ikenberry, T.A., and Gilbert, R.O. 1994. Parameters Used in the Environmental Pathways and Radiological Dose Modules (DESCARTES, CIDER, AND CRD Codes) of the Hanford Environmental Dose Reconstruction Integrated Codes (HEDRIC). Battelle Pacific Northwest Laboratories, Richland, Washington. PNWD-2033 HEDR Rev. 1, 1994. (ChemRisk Repository No. 3076)

Weaver, J.C., Kamm, M.L., and Dobson, R.L. 1960. Excretion of radioiodine in human milk. J.A.M.A. 173:872-875. 1960.

Whicker, F.W. and Kirchner, T.B. 1987. PATHWAY: A dynamic food-chain model to predict radionuclide ingestion after fallout deposition. Health Physics 52(6):717-737. 1987.

Wyburn, J.R. 1973. Human Breast Milk: Breast Excretion of Radionuclides Following Administration of Radiopharmaceuticals. J. Nucl. Med. 14:115-117. 1973. 
TASK 1 REPORT

July 1999

Page 6-32

${ }^{131}$ I Releases from X-10 Radioactive Lanthanum Processing Transfer of ${ }^{131}$ I from Pasture to Various Food Products

This page intentionally left blank. 


\subsection{DISTRIBUTION SYSTEM FOR VARIOUS FOOD PRODUCTS}

Distribution of food products from various producers to potential consumers is a complex process that is difficult to reproduce with high accuracy. Contaminated food products produced in the affected area can be distributed in other areas not reached by the radioactive plume. Conversely, individuals in the affected area can consume uncontaminated products imported from unaffected areas. This section addresses the effect of the distribution of contaminated food products (milk, cottage cheese, meat, vegetables, and eggs) to individual members of the public who resided in the vicinity of the X-10 facility during the time of the RaLa operation (1944 through 1956).

\subsection{Background}

During the 1940s and 1950s, about 50\% of the milk produced in the United States was consumed by humans as fresh fluid milk (Dreicer et al., 1990), about 3\% was used on farms to feed livestock, and the remainder was used to manufacture dairy products. The milk produced at a given farm is assumed to be consumed locally (backyard cow scenario) or distributed to the local population for consumption (commercial cow scenario). Milk was often exchanged between counties to cover the consumption needs in milk-deficient areas. Statistics for 1954 (NCI, 1997) show that the counties around the X-10 facility (Morgan, Anderson, Roane, Loudon, Blount and Knox) were generally milk-deficient and imported milk from other areas. The processes of mixing the milk from a number of sources (the commercial cow scenario) and the fact that not all the milk consumed was contaminated are taken into consideration explicitly by the term $\left(F_{c m}\right)$, which represents the fraction of milk that is contaminated. The concentration of ${ }^{131} \mathrm{I}$ in milk consumed by an individual is affected by the radioactive decay of ${ }^{131}$ I during the time between milking and human consumption. This process is taken into account by an exponential decay term in Equation 7.1 (next section).

Similar assumptions can be made regarding the consumption of beef and leafy vegetables. For beef, the holdup time between harvest and human consumption is longer than that for milk, and it applies year-round. Production of leafy vegetables is seasonal; fresh leafy vegetables are available for harvest only during certain months of the year. In addition, washing can reduce the contamination of leafy vegetables.

Chickens that are allowed to range freely after a deposition event could ingest small amounts of contaminated soil and grass. Eggs laid by such chickens will show an elevated concentration of radioactive iodine. Cottage cheese prepared from contaminated milk will contain $10 \%$ to $30 \%$ of the amount of radioiodine in milk (IAEA, 1992). Compared to fresh milk, both cottage cheese and eggs are stored longer before consumption and are consumed in smaller quantities. The entire amount of eggs and cottage cheese consumed by an individual is assumed to be produced locally. This assumption is conservative because it does not take into consideration the likelihood that the diet contains uncontaminated eggs and cottage cheese produced outside the region affected by ${ }^{131} \mathrm{I}$ contamination from $\mathrm{X}-10$. 


\subsection{Modeling Approach}

The effects of the distribution system for a given food type are considered by defining a bias correction factor that relates the concentration of ${ }^{131} \mathrm{I}$ in a food type at the time of consumption to the concentration of ${ }^{131} \mathrm{I}$ in the same food type at harvest or collection. This "distribution" factor is modeled by a radioactive decay term multiplied by a factor representing the fraction of food that is contaminated. For leafy vegetables, a factor is added to account for the reduction of contamination due to washing. For meat, the reduction of contamination due to cooking is taken into account by a multiplicative factor. The values for these parameters apply to all release years. Eggs and cottage cheese are assumed to be produced and consumed locally, and only the reduction of contamination due to radioactive decay during the time between production and consumption is considered.

The equations that relate the concentration in milk at milking, the concentration in beef at slaughter, and the concentration in leafy vegetables at harvest to the respective concentrations at the time of consumption are given below. These equations apply both for routine releases and for the 1954 accident. The parameter values, however, are estimated separately for each type of release (routine or accident).

\section{$\underline{\text { Dairy Cows or Goats }}$}

$$
M D=\left(\frac{C_{m}}{C_{m m}}\right)=\exp \left(-\lambda_{R} \cdot T_{d, m}\right) \cdot F_{c m}
$$

where

$$
\begin{aligned}
& M D=\text { milk distribution factor [unitless]; } \\
& C_{m} \quad=\text { average concentration of }{ }^{131} \mathrm{I} \text { in milk at consumption }\left[\mathrm{Bq} \mathrm{L}{ }^{-1} \text { milk }\right] \text {; } \\
& C_{m m}=\text { average concentration of }{ }^{131} \mathrm{I} \text { in milk at milking }\left[\mathrm{Bq} \mathrm{L}^{-1}{ }_{\text {milk }}\right] \text {; } \\
& \lambda_{R} \quad=\text { radioactive decay constant }\left[\mathrm{d}^{-1}\right] \\
& T_{d, m} \quad=\text { delay time between milking and consumption [d]; and } \\
& F_{c m}=\text { fraction of milk consumed by an individual that is obtained from }
\end{aligned}
$$

$\underline{\text { Beef Cattle }}$

$$
F D=\left(\frac{C_{f}}{C_{f s}}\right)=\exp \left(-\lambda_{R} \cdot T_{d f}\right) \cdot F_{c f} \cdot F_{r f}
$$

where 
$F D=$ beef distribution factor [unitless];

$C_{f} \quad=$ average concentration of ${ }^{131} \mathrm{I}$ in beef at consumption $\left[\mathrm{Bq} \mathrm{kg}^{-1}\right]$;

$C_{f s} \quad=$ average concentration of ${ }^{131} \mathrm{I}$ in beef at slaughtering $\left[\mathrm{Bq} \mathrm{kg}^{-1}\right]$;

$\lambda_{R} \quad=$ radioactive decay constant $\left[\mathrm{d}^{-1}\right]$

$T_{d, f} \quad=$ delay time between slaughtering and consumption [d];

$F_{c f} \quad=$ fraction of meat consumed by an individual that is obtained from contaminated sources [unitless]; and

$F_{r f} \quad=$ fraction of radioiodine that remains in the meat after food preparation.

\section{$\underline{\text { Leafy Vegetables }}$}

$$
L D=\left(\frac{C_{v}}{C_{v h}}\right)=\exp \left(-\lambda_{R} \cdot T_{d, v}\right) \cdot F_{c v} \cdot F_{w}
$$

where

$$
\begin{aligned}
& L D \quad=\text { leafy vegetables distribution factor; } \\
& C_{v} \quad=\underset{\substack{1 \\
\text { fresh }]}}{\text { average concentration of }}{ }^{131} \mathrm{I} \text { in leafy vegetables at consumption }\left[\mathrm{Bq} \mathrm{kg}^{-}\right. \\
& C_{v h} \quad=\text { average concentration of }{ }^{131} \mathrm{I} \text { in leafy vegetables at harvesting }\left[\mathrm{Bq} \mathrm{kg}^{-1} \text { fresh }\right] \text {; } \\
& \lambda_{R} \quad=\text { radioactive decay constant }\left[\mathrm{d}^{-1}\right] \\
& T_{d, v} \quad=\text { delay time between harvesting and consumption [d]; } \\
& F_{c v} \quad=\text { fraction of vegetables consumed by an individual that is contaminated } \\
& \text { [unitless]; and } \\
& F_{w} \quad=\text { factor accounting for reduction of the contamination of vegetables by } \\
& \text { washing [unitless]. }
\end{aligned}
$$

\subsection{Input Parameters}

The input parameter ranges and the shapes of the subjective probability distributions used to express the state of knowledge about true but partially known values (Table 7.1) were selected using professional judgment based on experience and a review of published data. The rationales for the choice of specific parameter values are presented below. For all pathways, $\lambda_{R}$ (the radioactive decay constant) for ${ }^{131} \mathrm{I}=8.04 \mathrm{~d}$. 


\subsubsection{Holdup Time from Collection or Harvest to Consumption}

\section{Milk from various sources}

For people drinking milk from backyard cows or goats, a minimum holdup time between milking and human consumption of milk is usually about 8 hours (0.33 days). This time is required for the fresh milk to cool down (Simon et al., 1990). The upper limit of the holdup time was chosen to be about 2 days, based on the assumption that storage and refrigeration systems were not generally available to the population in the late 1940s and early 1950s. For the delay between milking and consumption for backyard cow's milk or goat's milk, a uniform distribution between 0.33 and 2 days was chosen.

For milk from commercial dairies, at least one day is necessary for the transportation of milk from the producer to the consumer. Commercial milk was kept in grocery stores for at most 3 to 4 days, and then consumed within 1 or 2 days from the day of purchase. As a result, some individuals might have consumed milk up to 6 days after milking.

When estimating effects from routine releases of ${ }^{131} \mathrm{I}$, an annual average approach is used in the present study. For routine releases, it is assumed that, on average, commercial milk is consumed after a period of up to 4 days after milking. For the April 29, 1954 accident, a follow-up period of about two months is considered. Given the shorter follow-up period, the use of an annual average is inappropriate. Thus, an upper limit of 6 days is used for the delay between milking of commercial dairy cows and consumption for the 1954 accident case. Therefore, a triangular distribution between 1 and 4 days was chosen for routine releases, with a mode at 3 days. A triangular distribution between 1 and 6 days was chosen for 1954 accident, with a mode at 3 days.

The rationale for choosing the values for the holdup time for milk of goats is similar to that for the backyard cows. The range for the delay time between milking and consumption for goat milk was set from 0.33 to 2 days using a uniform distribution.

\section{$\underline{\text { Meat }}$}

Part of the meat obtained when an animal was slaughtered was consumed soon after the sacrifice of the animal because refrigeration systems were not widely available during 1940s and 1950s. However, amounts of meat could have been treated with salt or smoked in a smokehouse, in which case it could have been stored for longer periods of time, during which ${ }^{131}$ I decayed away. For the meat not treated for long time storage, the holdup time between slaughter and consumption of meat was judged to be at least one week, but no longer than five weeks. 
Table 7.1 Parameter distributions used for the uncertainty analysis for various food products.

\begin{tabular}{|c|c|c|c|c|c|c|c|}
\hline \multirow[t]{2}{*}{ Parameter } & \multirow[t]{2}{*}{ Footnote Codes } & \multirow[t]{2}{*}{ Units } & \multicolumn{4}{|c|}{ Distribution } & \multirow[t]{2}{*}{ Type of release } \\
\hline & & & $\min$. & $\max$. & mode & Shape & \\
\hline \multicolumn{8}{|c|}{ Milk from backyard cows } \\
\hline$T_{d}$ & $\mathrm{a}$ & [d] & 0.33 & 2 & & Uniform & Accidental and routine \\
\hline$F_{c m}-$ ages $0-5$ & $\mathrm{~b}, \mathrm{c}$ & [unitless ] & 0.6 & 1 & 0.9 & Triangular & Routine \\
\hline$F_{c m}-$ ages $0-5$ & $\mathrm{~b}, \mathrm{c}$ & [ unitless] & 0.5 & 1 & 0.75 & Triangular & Accidental \\
\hline$F_{c m}$-ages 6 - teenagers & $\mathrm{c}$ & [ unitless] & 0.4 & 0.9 & 0.8 & Triangular & Routine \\
\hline$F_{c m}$-ages 6 - teenagers & c & [ unitless] & 0.5 & 0.9 & & Uniform & Accidental \\
\hline$F_{c m-}$ adults & $\mathrm{c}$ & [ unitless] & 0.6 & 1 & 0.9 & Triangular & Routine \\
\hline$F_{c m-a d u l t s}$ & $\mathrm{c}$ & [ unitless] & 0.5 & 1 & & Uniform & Accidental \\
\hline \multicolumn{8}{|c|}{ Milk from commercial sources } \\
\hline$T_{d}$ & $\mathrm{a}$ & [d] & 1 & 4 & 3 & Triangular & Routine \\
\hline$T_{d}$ & $\mathrm{a}$ & {$[\mathrm{d}]$} & 1 & 6 & 3 & Triangular & Accidental \\
\hline$F_{c m}-$ all ages & $\mathrm{c}$ & {[]} & 0.1 & 0.9 & 0.6 & Triangular & Routine \\
\hline$F_{c m}-$ all ages & $\mathrm{c}$ & [ unitless] & 0 & 1 & & Uniform & Accidental \\
\hline \multicolumn{8}{|c|}{ Milk from goats } \\
\hline$T_{d}$ & $\mathrm{a}$ & [d] & 0.33 & 2 & & Uniform & Accidental and routine \\
\hline$F_{c m}-$ all ages & $\mathrm{c}$ & [ unitless] & 0.6 & 0.9 & 0.8 & Triangular & Routine \\
\hline$F_{c m}-$ all ages & $\mathrm{c}$ & [ unitless] & 0.5 & 1 & & Uniform & Accidental \\
\hline
\end{tabular}


TASK 1 REPORT

July 1999

${ }^{131}$ I Releases from X-10 Radioactive Lanthanum Processing -

Page 7-6 Distribution System for Various Food Products

\begin{tabular}{|c|c|c|c|c|c|c|c|}
\hline \multirow[t]{2}{*}{ Parameter } & \multirow[t]{2}{*}{ Footnote Codes } & \multirow[t]{2}{*}{ Units } & \multicolumn{4}{|c|}{ Distribution } & \multirow[t]{2}{*}{ Type of release } \\
\hline & & & $\min$. & $\max$. & Mode & Shape & \\
\hline \multicolumn{8}{|c|}{ Meat } \\
\hline$T_{d, f}$ & $\mathrm{~d}$ & {$[\mathrm{~d}]$} & 7 & 35 & & Uniform & Routine \\
\hline$F_{c f}$ - rural lifestyle & $\mathrm{e}$ & [ unitless] & 0.3 & 0.9 & & Uniform & Routine \\
\hline$F_{c f}-$ urban lifestyle & $\mathrm{e}$ & [ unitless] & 0.1 & 0.6 & & Uniform & Routine \\
\hline$F_{r f}$ & $\mathrm{f}$ & [ unitless] & 0.2 & 0.9 & & Uniform & Routine \\
\hline \multicolumn{8}{|c|}{ Vegetables } \\
\hline$T_{d, v}$ & $\mathrm{~g}$ & {$[\mathrm{~d}]$} & 0 & 7 & \multirow{4}{*}{1.0} & Uniform & Accidental and routine \\
\hline$F_{c v}$ & $\mathrm{~h}$ & [unitless ] & 0.08 & 0.25 & & Uniform & \multirow{3}{*}{$\begin{array}{c}\text { Routine } \\
\text { Accidental (spring) } \\
\text { Accidental and routine }\end{array}$} \\
\hline$F_{c v}$ & $\mathrm{~h}$ & [ unitless] & -- & -- & & Constant & \\
\hline$F_{w}$ & $\mathrm{i}$ & [ unitless] & 0.2 & 0.7 & & Uniform & \\
\hline \multicolumn{8}{|c|}{ Eggs } \\
\hline$T_{g g}$ & $\mathrm{j}$ & [d] & 3 & 7 & & Uniform & Accidental and routine \\
\hline \multicolumn{8}{|c|}{ Cottage cheese } \\
\hline$T_{c c}$ & $\mathrm{k}$ & [d] & 2 & 7 & & Uniform & Accidental and routine \\
\hline
\end{tabular}

Delay time between milking and consumption.

Ages refer to the age of the individual consuming the indicated product.

Fraction of milk consumed by an individual that is obtained from contaminated sources.

Delay time between slaughtering and consumption.

Fraction of meat consumed by an individual that is obtained from contaminated sources.

Fraction of radioiodine that remains in the meat after food preparation.

Delay time between harvesting and consumption.

Fraction of vegetables consumed by an individual that is contaminated.

Factor accounting for reduction of the contamination of vegetables by washing.

Delay time between gathering and consumption.

Delay time between milking and consumption. 
A uniform distribution between 7 and 35 days was chosen for the delay time between slaughtering and consumption. This distribution is consistent with assumptions employed for the dose reconstruction at Hanford, Washington (Snyder et al., 1994).

\section{$\underline{\text { Vegetables }}$}

Leafy vegetables are assumed to be consumed fresh during the harvest months of the year, and the storage time for "fresh" vegetables is assumed not to exceed one week. The distribution of the delay time between harvesting and consumption of leafy vegetables is assumed to be uniform between 0 and 7 days. This distribution applies for both routine releases and the 1954 accident.

\section{$\underline{\text { Eggs and Cottage cheese }}$}

Eggs are usually kept for at least 2-3 days before consumption. NCI (1997) assumes that eggs are kept between 3 and 18 days after collection. However, during the early 1950s, refrigeration systems were not widely available, so it is unlikely that eggs were kept more than one week. A uniform distribution between 3 and 7 days was assigned for the holdup time of eggs.

The storage time for cheese depends on the type of cheese. In the absence of a refrigeration system, cottage cheese was probably consumed within a week after production. In a refrigerator, the "hard" cheese can be kept much longer (at least one month). During this time, most of ${ }^{131}$ I will decay away. This study focuses on contaminated cottage cheese for which a holdup time of 2 to 7 days was assumed. A uniform probability distribution was assigned to this parameter.

\subsubsection{Fraction of Food that is Contaminated}

\section{Milk from various sources}

The milk provided commercially during the late 1940s and early 1950s in Morgan, Anderson, Roane, Loudon, Blount and Knox counties was not entirely from local production (NCI, 1997), since these areas were considered milk-deficient. A study for 1954 (NCI, 1997) estimated that in the above counties, milk demand was about $5.1 \times 10^{7} \mathrm{~L} \mathrm{y}^{-1}$, while the amount available locally was only $3.2 \times 10^{7} \mathrm{~L} \mathrm{y}^{-1}$ (about $63 \%$ of the total necessary for consumption). Part or all of the milk deficit was covered by supplies from areas remote from the contaminated locations and provided essentially uncontaminated milk. All milk produced locally is assumed to be contaminated.

For an individual consuming commercial milk during the accidental release, a uniform distribution and a range of values from 0 to 1 was chosen for the fraction of milk that is contaminated. The lower bound expresses the possibility that the entire milk supply in the store is obtained from a source not contaminated by releases of ${ }^{131} \mathrm{I}$ from $\mathrm{X}-10$. The upper bound represents the case in which the entire amount of milk bought at a store was obtained from local dairy farms. For routine releases, a triangular distribution with a mode of 0.6 and a range of values from 0.1 to 0.9 was chosen. The lower bound indicates that the annual average fraction of contaminated milk consumed would be no less than $10 \%$ for the area surrounding the Oak Ridge 
Reservation (ORR), while the upper bound would be no more than $90 \%$, averaged on an annual basis.

For a preschool child consuming milk from a backyard cow during the 1954 accident, most of the milk was assumed to be contaminated. For children under the age of 6 , the distribution for the fraction of milk that is contaminated is assumed to be triangular with the upper bound being equal to 1. The lower bound of the triangular distribution was set to 0.5 and should be interpreted as the case in which the locally produced milk was supplemented by milk brought from farms located outside the contaminated plume. A triangular distribution with a mode of 0.75 was used for accidental releases affecting preschool children.

Since the counties surrounding the ORR were considered milk-deficient, the range for the fraction of contaminated milk consumed by children under the age of 6 was considered to vary on an annual basis between 0.6 and 0.9. The lower bound represents the amount of milk that was locally available, while the upper bound accounts for decreased milk production (due to nursing calves or nonproduction periods) averaged over a one-year period. A triangular distribution with a mode of 0.9 was selected for this age group.

According to Dreicer et al. (1990) and Downen (1955; 1956), school-aged children received milk for lunch as part of the School Milk Program. The amount of milk received in school was estimated (Dreicer et al., 1990) to be about one-third of the total milk consumed by a child daily. The milk provided by schools was assumed to be commercial milk (about $66 \%$ of the total milk ingested daily, 180 days per year, 5 days per week), while the milk consumed at home was assumed to be collected from a backyard cow. The total amount of milk ingested daily by a school-aged child was based on consumption rates expected for backyard cow's milk, but the fraction of milk that is contaminated was modified to a triangular distribution with a mode of 0.8 and a range of values from 0.4 to 0.9 for routine releases.

The 1954 accident occurred in April, during the school year. For this situation, the fraction of contaminated milk consumed was considered to have a uniform distribution that ranged from 0.5 to 0.9 . The lower bound indicates that at least $50 \%$ of the milk consumed was contaminated because it was obtained from a backyard cow, as well as from commercial sources that were only partially contaminated during the release. The upper bound indicates that no more than $90 \%$ of the commercial milk supply obtained by the schools was contaminated during the accident release.

Adults living in rural areas around the ORR obtained milk from backyard cows on their own farms. It is assumed that such individuals did not normally obtain milk from commercial sources. The subjective probability distribution for the fraction of milk that is contaminated is assumed to be triangular with a mode of 0.9 and an upper bound of 1 . As with the case of preschool children, the lower bound of the triangular distribution was set to 0.6 for routine releases and should be interpreted as the case in which locally produced milk was supplemented by uncontaminated milk bought from farms located outside the contaminated plume. The distribution for the fraction of milk that is contaminated during the accidental release was assumed to be uniform with a range of 0.5 to 1.0 . Since most of the milk that adults consume is 
considered to come from their homes, the upper bound was set to 1.0. The lower bound indicates that at least half of the milk consumed was contaminated from ${ }^{131}$ I releases from $\mathrm{X}-10$, because a backyard cow was the source of the majority of the milk available.

The rationale for choosing the subjective probability distribution of values for the fraction of goat's milk that is contaminated is similar to that for the fraction of backyard cow's milk that is contaminated. The difference is that the distribution is used for all age groups, because there is no evidence of the consumption of goat's milk in school. Thus, the distribution for the fraction of goat's milk that is contaminated was assumed to be triangular with a mode of 0.8 and an upper bound of 0.9. The lower bound of the triangular distribution was set to 0.6 for routine releases. The distribution for the fraction of goat's milk contaminated during the accidental release was assumed to be uniform with a range of 0.5-1.0. The upper bound indicates that all of the goat milk consumed during the accidental release could have been contaminated due to limited availability from noncontaminated sources (goat's milk was not sold locally in stores, and very few individuals milked goats). The lower bound indicates that at least $50 \%$ of the milk produced by goats grazing on land in the path of the plume was contaminated.

\section{$\underline{\text { Meat }}$}

In this study, only the concentration of ${ }^{131} \mathrm{I}$ accumulated in the muscle of beef cattle was estimated, and beef was considered a surrogate for all other types of meat (e.g., pork, chicken, or meat of game animals). This assumption, although a simplification, is not of critical importance because ingestion of meat contributes minimally to the total thyroid dose from ${ }^{131}$ I (NCI, 1997).

People living in rural areas surrounding the ORR raised most of the meat they consumed on their own farms. Beef cattle, pigs, and chickens were raised for family consumption in this area, and meat was not regularly purchased from a grocery store. Since all animals consumed products potentially contaminated by ${ }^{131} \mathrm{I}$, all types of animal products were considered to be contaminated. It is assumed that contaminated meat was consumed at least $30 \%$ of the time, but no more than $90 \%$. A uniform distribution was selected to represent the true but unknown fraction of meat contaminated by routine releases and consumed by individuals living in a rural setting.

People living in urban areas surrounding the ORR could have raised their own meat or obtained a portion of their meat from local farms. Beef, pork, and chickens were raised for family consumption even in urban areas; however, many grocery stores were convenient for residents of Knoxville, Oak Ridge, and Kingston, and meat was regularly purchased from the stores. At least $10 \%$ of the meat consumed is assumed to be contaminated, but no more than $60 \%$, due to the many places to buy and the various types available. For routine releases, a uniform distribution was chosen to represent the uncertainty associated with the fraction of contaminated meat consumed by various individuals with a variety of meat purchase options.

The majority of cattle were slaughtered for meat in the fall (i.e., late November) and not in the spring, when the 1954 accident release occurred. Slaughtering of cattle was not a frequent activity in the spring because the farmers used the cold weather as a means to ensure that the 
meat did not spoil. Smokehouses and curing houses were used to cure the meat so that it was available year-round (Loveday, personal communication with C. Lewis, 1998). Meat stored for very long after slaughter would not have contained ${ }^{131} \mathrm{I}$, due to its short half-life.

Part of the radioiodine accumulated in meat is lost during its preparation for consumption. IAEA (1992) states that $20-90 \%$ of the radionuclide remains in the meat after cooking. The range applies for various methods of preparing meat: baking, boiling, frying, roasting, grilling, or mincing for cow, pig, deer, bird, and rabbit meat. The mean value reported by IAEA (1992) is consistent with a removal of $40 \%$ of the radioiodine from meat $60 \%$ remains in meat and is available for consumption). The fraction of radioactive material remaining in the food after preparation has been described by a uniform distribution, and this distribution encompasses all methods used to cook meat.

\section{$\underline{\text { Vegetables }}$}

Consumption of fresh leafy vegetables was assumed to occur mostly during the harvest period, lasting about three months a year (25\% of the year). However, consumption depends on the size of the garden and on the amount of home-produced leafy vegetables. As a minimum value, fresh vegetables were assumed to be available to the target individual at least 1 month per year (about $8 \%$ of the year). Therefore, a uniform distribution between 0.08 and 0.25 was chosen for the amount of leafy vegetables that are contaminated. This distribution is suitable for analysis of the effects of routine releases. The accidental release of April 1954 occurred in early spring when fresh vegetables usually become available. For the accidental release, it was assumed that $100 \%$ of the leafy vegetables available for consumption were contaminated.

Washing the vegetables removes about $30 \%$ to $80 \%$ of the contamination (Thiessen et al., 1996; IAEA, 1992; IAEA, 1994). A review of the literature indicates that the amount of contamination remaining on the plant after washing is larger than $20 \%$, but lower than $70 \%$. A uniform distribution between 0.2 and 0.7 was considered for estimating the uncertainty in the fraction of contamination remaining on the plants.

\section{Eggs and Cottage cheese}

The entire amount of eggs consumed locally was assumed to be produced locally, and therefore to be contaminated. It is assumed that cottage cheese was produced from commercial milk, and thus, it will be contaminated to the same degree as milk from commercial sources. 


\subsection{References}

Downen, M.L. 1955. Milk Consumption in Tennessee Schools, Rural Research Series, Monograph No. 271, Department of Agricultural Economics and Rural Sociology, August 1, 1955.

Downen, M.L. 1956. Consumption of Milk in Tennessee Schools, Rural Research Series, Monograph No. 274. Department of Agricultural Economics and Rural Sociology, March 31, 1956.

Dreicer, M., Bouville, A., and Wachholz, B.W. 1990. Pasture Practices, Milk Distribution, and Consumption in the Continental U.S. in the 1950s. Analyses and Modeling for Internal Dose Estimates, Health Physics 59(5):627-636, 1990.

IAEA (International Atomic Energy Agency). 1992. Modelling of resuspension, seasonality and losses during food processing. First report of the VAMP Terrestrial Working Group. IAEATECDOC-647. Vienna, Austria. May 1992. (ChemRisk Repository No. 4014)

IAEA (International Atomic Energy Agency). 1994. Handbook of Parameter Values for the Prediction of Radionuclide Transfer in Temperate Environments. Technical Report Series No. 364. Vienna, Austria. 1994. (ChemRisk Repository No. 4008)

Loveday, D. 1998. Food technology professor. University of Tennessee, Knoxville, Tennessee. Telephone interview with C. Lewis, March 4, 1998.

NCI (National Cancer Institute). 1997. Estimated Exposure and Thyroid Doses Received by the American People from Iodine-131 Fallout Following Nevada Atmospheric Nuclear Bomb Tests. U.S. Department of Health and Human Services. National Institutes of Health. National Cancer Institute, October 1997.

Simon, S.L., Lloyd, R.D., and Till, J.E. 1990. Development of a Method to Estimate Thyroid Dose from Fallout Radioiodine in a Cohort Study. Analyses and Modeling for Internal Dose Estimates. Health Physics 59(5):669-691.

Snyder, S.F., Farris, W.T., Napier, B.A., Ikenberry, T.A., and Gilbert, R.O. 1994. Parameters Used in the Environmental Pathways and Radiological Dose Modules (DESCARTES, CIDER, AND CRD Codes) of the Hanford Environmental Dose Reconstruction Integrated Codes (HEDRIC). Battelle Pacific Northwest Laboratories, Richland, WA. PNWD-2033 HEDR Rev. 1, 1994. (ChemRisk Repository \# 3076).

Thiessen, K.M., Hammonds, J.S., Lewis, C.J., Hoffman, F.O., and White, E.I. 1996. Task 7 Report: Screening Method for the Oak Ridge Dose Reconstruction. Oak Ridge Health Studies. April 1996. 
TASK 1 REPORT

July 1999

Page 7-12

${ }^{131}$ I Releases from X-10 Radioactive Lanthanum Processing Distribution System for Various Food Products

This page intentionally left blank. 


\subsection{FOOD CONSUMPTION AND INHALATION PARAMETERS}

Airborne radioiodine released during RaLa processing was transferred from the air to vegetation, which was then consumed by animals and humans. Contaminated human foodstuffs included vegetables, meat and milk obtained from cattle or other animals consuming contaminated pasture and grains, and eggs obtained from chickens consuming small amounts of contaminated grain, vegetation, and soil while roaming freely. Breast milk consumption is also considered for infants (0-1 year old) due to the fact that mothers may have consumed foods contaminated with radioiodine or breathed contaminated air. The most important individuals in this study are children because of their greater radiosensitivity and higher consumption of milk as compared to adults. This section investigates the food consumption patterns of Tennessee residents of both genders and all age groups, as well as the factors affecting the inhalation of contaminated air.

\subsection{Food Consumption Patterns}

Several studies provide information on food consumption patterns. Dreicer et al. (1990) reported gender- and age-specific per capita consumption rates of fresh milk from cows. In addition, the USDA $(1965 ; 1980)$ provided age- and gender- specific information for the major food groups (meat, vegetables, dairy, etc.). To ensure that the subjective probability distributions selected for actual food consumption rates for the Oak Ridge, Tennessee, area provide a reasonable representation of the habits of local populations, data reported in other studies (Schwartz and Hoffman, 1980; Hoffman and Baes, 1979; Anderson et al., 1993; Rupp, 1980; Simon et al., 1990; USDA, 1941; 1944; 1949; 1955a; 1955b; 1965; 1966; 1980; NCI, 1997) were reviewed. Interviews with individuals who have expertise in dietary intakes and animal sciences were conducted as a source of additional information. The literature review and interviews confirmed that the subjective probability distributions for each age group selected in this report encompassed values estimated by other researchers for the same age groups.

The consumption rates of the major food groups were established through expert elicitation and by two nationwide U.S. Department of Agriculture (USDA) surveys conducted in the spring of 1965 and in April-June 1977. The USDA studies included individuals residing in the 48 contiguous states. The values reported were separated into age groups for males and females. The younger age groups (less than 1 year up to 8 years of age) were not separated by gender, but considered to be one group because the evidence did not show marked differences between males and females. The older age groups ( 9 years and older) were divided by gender. The values selected are based on a one-day food intake by a "user." A "user" is classified as an individual who consumes a particular food item on the day that the survey was taken. "User" information is considered to be relevant for the reference individual, who is defined as an individual (adult or child, male or female) who consumes a potentially contaminated food item (i.e., meat, vegetables, milk, or eggs).

The subjective probability distributions provided for the consumption rates represent our current state of knowledge about these parameters. Tables $8.1-8.10$ provide the probability distributions and supporting rationales. 


\subsubsection{Milk Consumption}

For the milk ingestion pathway, both children and adults are studied. Children are the primary target individuals for this pathway due to their higher consumption rate of milk and their greater sensitivity to radiation exposures as compared to adults. For infants, cow's milk, breast milk, formula, or a combination of the three may be provided during the first year of life (Mount, personal communication with C. Lewis, 1997; Eisenberg et al., 1994). The reference infant considered in this study is assumed to consume breast milk and cow's milk, but no formula. Formula is excluded in this study because the formula powder (available in the 1940s and 50s) was generally mixed with water, which was not contaminated with radioiodine. For the average infant, cow's milk consumption constitutes approximately half of the total milk intake, while breast milk constitutes the other half. For infants fed solely breast milk or solely cow's milk, the consumption rates are identical. However, iodine transfer rates from cow to milk and mother to breast milk are different. Breast milk is considered only for infants ( $0-1$ year old), since breast milk consumption is considered to decrease on average after the first year of life.

\subsubsection{Consumption of Cow's Milk}

Contaminated milk could have been obtained from a backyard cow or from a local commercial dairy. Contaminated commercial cow's milk is considered to be milk from local contaminated commercial sources or a mixture of milk obtained from remote sources and local contaminated sources. The areas surrounding the Oak Ridge Reservation (ORR) (Anderson, Blount, Knox, Loudon, Morgan, and Roane counties) were considered "milk deficient" (Dreicer et al., 1990); therefore, the possibility existed that milk could have been obtained from outside the region affected by the releases of ${ }^{131}$ I from X-10. In the 1950s, Anderson, Knox, Loudon, and Roane counties received milk from the Chattanooga area, Middle Tennessee, and Alabama (NCI, 1997).

The fraction of contaminated milk available to children depends on their activities and their age. Milk consumption rate differences by gender are not significant for children between the ages of 0 and 14. However, at age 15, marked differences exist between males and females. Therefore, gender-specific consumption rates are not provided for children under the age of 15 .

Infants require a specific amount of milk regardless of the source (breast, cow, goat, formula); therefore, the consumption rate for infants is the same for both release scenarios and for all types of milk. During the first few months of life, infants are fed every 3 to 4 hours, with each serving consisting of 4-6 oz. (0.12-0.18 L) of milk (Eisenberg et al., 1994). As the infant develops, the number of feedings decreases, but the amount consumed increases (Eisenberg et al., 1994; Mount, personal communication with C. Lewis, 1997). Although infants have a variety of milk sources, the average situation for the East Tennessee area was assumed to be consumption of one-half cow's milk or goat's milk and one-half breast milk. For this study, reference infants are considered to consume at least one bottle of cow's or goat's milk every day of the year, regardless of their method of feeding. Studies have shown that the number of infants consuming breast milk decreases as a function of age, while the number of infants consuming cow's milk increases continuously as a function of age (NCI, 1997). However, it was not uncommon for 
mothers to breast feed their children for up to a year or longer (Eisenberg et al., 1994), so an infant's consumption of cow's or goat's milk may have been minimal for at least a year.

Children less than 6 years old were generally kept at home. These children would consume the majority of their milk at home; this milk could be obtained from a backyard cow or from a store. For children between the ages of 1 and 4, the annual average milk consumption rate was based on data reported by the NCI (1997) for children who consume milk. Non-milk drinkers were excluded from this study. The NCI (1997) study concluded that approximately $17 \%$ of the children between 1 and 4 years (both genders) did not consume milk. For children 3-11 years, Pao and Burke (1975) determined that $1.33 \mathrm{~L} \mathrm{~d}^{-1}$ was exceeded by fewer than $2.5 \%$ of the population (Rupp, 1980). The most likely value $\left(0.53 \mathrm{~L} \mathrm{~d}^{-1}\right)$ for the annual average milk consumption rate is consistent with the per capita consumption rate for rural farm residents and the median per capita milk consumption rate for milk drinkers in the State of Tennessee in 1954 (NCI, 1997).

For a child older than 6 years, school was generally an integral part of life. If the child consumed milk at school, one-half of the total milk consumed could be contaminated (that obtained from home) and the other half could have been obtained from an uncontaminated source. In Tennessee, school-aged children received milk for lunch as part of the School Milk Program (Downen, 1955; 1956; Dreicer et al., 1990). Since the counties surrounding the ORR were milk deficient (NCI, 1997), a portion of the milk obtained from schools or grocery stores may not have been contaminated from ${ }^{131}$ I released from the X-10 facility. The school milk program affects all children aged 6 to approximately 18 years. For children aged 5-9 years, an average of $22 \%$ of the total population does not consume milk (NCI, 1997). The percentage of non-milk drinkers increases to $29 \%$ for children between 10 and 14 years (NCI, 1997). However, the annual average milk consumption patterns for milk drinkers remain constant for both age groups.

When children reach the age of 15 , gender differences in milk consumption patterns become apparent. Males consume more milk on average than females (Dreicer et al., 1990). For males between the ages of 15 and 19, approximately $29 \%$ of the total population does not consume milk (NCI, 1997). Approximately $39 \%$ of the females in this age group do not consume fresh fluid cow milk. Not only does gender become a factor for this age group, it is also within this age range that children continue their education, enter the work force, get married, or return to the farm to work. Children who continue their education through high school are provided milk through the school milk program. Children who enter the work force, get married, or return to the farm to work will not be getting milk from school. Therefore, two different milk consumption patterns are provided in the following tables. The first pattern is for children who remain in school, while the latter is for those not continuing their education for various reasons.

Adults are also considered to obtain the majority of their milk at home. However, in Oak Ridge and the surrounding area, milk could be obtained from a home-raised animal or from a local dairy. Since milk was readily available to most individuals, two different consumption patterns for adults were developed: backyard milk consumers and individuals with a variety of milk sources. Regardless of the source of milk, the consumption rate of milk by adults is expected to 
be less than that of children because adults have access to other fluids than milk (e.g., water, coffee, soft drinks, tea). Approximately $39 \%$ of the males and $44 \%$ of the females between the ages of 20 and 64 did not consume fresh fluid milk as determined through dietary intake studies conducted over a short period of time (NCI, 1997).

Pregnant females and lactating mothers are also considered in the milk consumption pathway. The intake of fluid milk by pregnant or lactating women is assumed to be higher than for nonpregnant or non-lactating women because of the additional nutritional requirements of the baby. In this study, on average, the pregnant or lactating female was assumed to double her intake of milk if consuming a mixture of commercial and backyard cow's milk. If she was consuming milk only from a backyard cow, her intake on average was assumed to increase by approximately $33 \%$. A triangular distribution with a mode of $0.8 \mathrm{~L} \mathrm{~d}^{-1}$ was selected to represent the consumption rate of cow's milk by pregnant or lactating women. The NCI's reported value $(0.8$ $\left.\mathrm{L} \mathrm{d}^{-1}\right)$ is within the range selected for this study. The milk consumption distribution for pregnant or nursing mothers is the same for the routine and accidental releases. During the accidental release, ${ }^{131} \mathrm{I}$ was discharged for approximately 2.5 hours. However, the aftermath of the accidental release lasted approximately one month, with the majority of the dose delivered in the initial two weeks following the release. The range used for pregnant or nursing mothers was made sufficiently broad to encompass variations in the milk consumption habits that occur over a one-month period.

The parameters associated with the milk ingestion pathway are provided in Table 8.1 for routine releases and Table 8.2 for the accidental release. The ranges for the routine releases are based on the average consumption rate of a particular item over a one-year period. Over this period, the average is not expected to vary dramatically; therefore, a narrow distribution is used. For the accidental releases, for which consumption is averaged over a one-month period, the variability in the eating patterns can fluctuate widely. Since the variability is larger during the accidental releases, the distributions are broader to encompass varied eating habits during short time periods. The ranges for the average annual milk consumption rates provided in the following tables for both types of release are consistent with values reported by USDA (1965), Cole and Ronning (1974), USDA (1980), Rupp (1980), Dreicer et al. (1990), Simon et al. (1990), and NCI (1997). 
Table 8.1 Values for parameters used in the cow's milk ingestion pathway for routine releases.

\begin{tabular}{|c|c|c|c|c|c|}
\hline Parameter & Symbol & Range & $\begin{array}{l}\text { Mean or } \\
\text { (Mode) }\end{array}$ & Distribution & Rationale \\
\hline $\begin{array}{l}\text { Average daily } \\
\text { consumption of breast, } \\
\text { cow's, or goat's milk by } \\
\text { infants (males and } \\
\text { females) age } 0-1 \text { year } \\
\left(\mathrm{L} \mathrm{d}^{-1}\right)\end{array}$ & $\mathrm{U}_{\text {milk, infant }}$ & $0.12-0.98$ & 0.55 & Uniform & $\begin{array}{l}\text { The range represents the average milk consumption rates of a } \\
\text { child from birth to the age of twelve months for any milk type. } \\
\text { The range indicates that an infant who is being breast fed, but } \\
\text { is occasionally provided fresh cow's milk or goat's milk, will } \\
\text { consume no less than } 4 \text { oz. (0.12 L) of fresh milk per day, } \\
\text { which is the lower limit (Mount, personal communication with } \\
\text { C. Lewis, 1997). An infant who is being fed only fresh milk } \\
\text { will consume no more than } 32 \mathrm{oz} \text {. ( } 0.98 \mathrm{~L} \text { ) of cow's milk per } \\
\text { day (feeding every } 3 \text { hours). The average value indicates that } \\
\text { an infant consumes approximately one-half breast milk and } \\
\text { one-half cow's milk or goat's milk (four and one-half } 4 \text {-oz. } \\
\text { servings as an infant or two 8-oz. servings as an older infant, } \\
\text { who is usually given juice in addition to milk). A uniform } \\
\text { distribution was chosen to represent the range within which all } \\
\text { values for the true but unknown intake of fresh cow's milk } \\
\text { (averaged over the first year of life) have equal probability. }\end{array}$ \\
\hline $\begin{array}{l}\text { Average daily } \\
\text { consumption of cow's } \\
\text { milk by children (males } \\
\text { and females) ages 1-4 } \\
\text { years }\left(\mathrm{L} \mathrm{d}^{-1}\right)\end{array}$ & $\begin{array}{c}\mathrm{U}_{\text {cow milk, }} \\
1-4 \mathrm{yrs}\end{array}$ & $0.24-1.2$ & $(0.53)$ & Triangular & $\begin{array}{l}\text { According to the NCI (1997) study, } 73 \% \text { of the male } \\
\text { population and } 71 \% \text { of the female population within the ages } \\
\text { of } 1-4 \text { years are milk drinkers, who consume milk within this } \\
\text { range. The lower limit is indicative of approximately one } 8 \text {-oz. } \\
\text { glass of milk per day. The upper limit indicates at most } 5 \\
\text { glasses ( } 8 \text { oz. each) of milk consumed per day. A triangular } \\
\text { distribution with a mode of } 0.53 \mathrm{~L} \mathrm{~d}^{-1} \text { was chosen to represent } \\
\text { the uncertainty associated with the annual average daily } \\
\text { consumption rate of milk (averaged over a four- year period) } \\
\text { by children aged } 1-4 \text { years. }\end{array}$ \\
\hline
\end{tabular}


Table 8.1 (continued)

\begin{tabular}{|c|c|c|c|c|c|}
\hline Parameter & Symbol & Range & $\begin{array}{l}\text { Mean or } \\
\text { (Mode) }\end{array}$ & Distribution & Rationale \\
\hline $\begin{array}{l}\text { Average daily } \\
\text { consumption of cow's } \\
\text { milk by children (males } \\
\text { and females) ages 5-9 } \\
\text { years }\left(\mathrm{L} \mathrm{d}^{-1}\right)\end{array}$ & $\begin{array}{c}\mathrm{U}_{\text {cow milk }} \\
5-9 \mathrm{yrs}\end{array}$ & $0.24-1.2$ & $(0.6)$ & Triangular & $\begin{array}{l}\text { The range encompasses the } 69 \% \text { of males and } 65 \% \text { of females } \\
\text { in this age group who consume milk (NCI, 1997) for time } \\
\text { periods covered by short-term dietary surveys. The lower limit } \\
\text { indicates on average that children between the ages of } 5 \text { and } 9 \\
\text { drink at least one } 8 \text {-oz. }(0.24 \text { L) glass of milk per day, while } \\
\text { the upper limit represents at most five } 8 \text {-oz. glasses. A } \\
\text { triangular distribution was selected to represent the uncertainty } \\
\text { associated with the true but unknown value of the annual } \\
\text { average daily consumption rate for children between the ages } \\
\text { of } 5 \text { and } 9 \text { years. }\end{array}$ \\
\hline $\begin{array}{l}\text { Average daily } \\
\text { consumption of cow's } \\
\text { milk by children (males } \\
\text { and females) ages } 10-14 \\
\text { years }\left(\mathrm{L} \mathrm{d}^{-1}\right)\end{array}$ & $\begin{array}{c}\mathrm{U}_{\text {cow milk }} \\
10-14 \mathrm{yrs}\end{array}$ & $0.24-1.2$ & $(0.6)$ & Triangular & $\begin{array}{l}\text { The range includes the } 61 \% \text { of males and } 57 \% \text { of females in } \\
\text { this age group who consume milk (NCI, 1997). The lower } \\
\text { limit indicates that children between the ages of } 10 \text { and } 14 \\
\text { consume at least one } 8 \text {-oz. }(0.24 \mathrm{~L}) \text { glass of milk per day, but } \\
\text { no more than five } 8 \text {-oz. glasses of milk }(1.2 \mathrm{~L}) \text { per day. A } \\
\text { triangular distribution was chosen to describe the uncertainty } \\
\text { associated with the annual average daily consumption rate of } \\
\text { milk by children aged } 10-14 \text { years. }\end{array}$ \\
\hline $\begin{array}{l}\text { Average daily } \\
\text { consumption of cow's } \\
\text { milk by children (males) } \\
\text { ages } 15-19 \text { years }\left(\mathrm{L} \mathrm{d}^{-1}\right)\end{array}$ & $\begin{array}{c}\mathrm{U}_{\text {cow milk, }} \\
\text { 15-19 yrs, } \\
\text { male }\end{array}$ & $0.24-1.2$ & $(0.6)$ & Triangular & $\begin{array}{l}\text { The range includes the } 64 \% \text { of males in this age group who } \\
\text { consume milk (NCI, 1997). The lower limit indicates that } \\
\text { children between the ages of } 15 \text { and } 19 \text { consume a minimum of } \\
\text { one } 8 \text {-oz. ( } 0.24 \mathrm{~L}) \text { glass of milk per day, but no more than five } \\
8 \text {-oz. glasses of milk ( } 1.2 \mathrm{~L}) \text { per day. A triangular distribution } \\
\text { was chosen to describe the uncertainty associated with the } \\
\text { annual average daily consumption rate of milk by male } \\
\text { children aged } 15-19 \text { years. }\end{array}$ \\
\hline
\end{tabular}


Table 8.1 (continued)

\begin{tabular}{|c|c|c|c|c|c|}
\hline Parameter & Symbol & Range & $\begin{array}{l}\text { Mean or } \\
\text { (Mode) }\end{array}$ & Distribution & Rationale \\
\hline $\begin{array}{l}\text { Average daily } \\
\text { consumption of cow's } \\
\text { milk by children (males) } \\
\text { ages } 15-19 \text { years who } \\
\text { drink only backyard } \\
\left.\text { cow's milk ( } \mathrm{L} \mathrm{d}^{-1}\right)\end{array}$ & $\begin{array}{c}\mathrm{U}_{\text {cow milk }} \\
\text { (backyard), } \\
15-19 \text { yrs, male }\end{array}$ & $0.48-1.2$ & $(0.85)$ & Triangular & $\begin{array}{l}\text { The range is for male children who use backyard cow's milk as } \\
\text { their only source of milk. The lower limit indicates that } \\
\text { children between the ages of } 15 \text { and } 19 \text { with immediate access } \\
\text { to backyard cow's milk consume at least two } 8 \text {-oz. glasses of } \\
\text { milk }(0.48 \mathrm{~L}) \text { per day, but no more than five } 8 \text {-oz. glasses of } \\
\text { milk }(1.2 \mathrm{~L}) \text { per day. The mode is equivalent to approximately } \\
3.5 \text { (8-oz.) glasses of milk per day. A triangular distribution } \\
\text { was chosen to describe the uncertainty associated with the } \\
\text { average annual daily consumption rate of milk by male } \\
\text { children aged } 15-19 \text { years who had access to backyard cow } \\
\text { milk. }\end{array}$ \\
\hline $\begin{array}{l}\text { Average daily } \\
\text { consumption of cow's } \\
\text { milk by children } \\
\text { (females) ages } 15-19 \\
\text { years }\left(\mathrm{L} \mathrm{d}^{-1}\right)\end{array}$ & $\begin{array}{c}\mathrm{U}_{\text {cow milk, }} \\
\text { 15-19 yrs, } \\
\text { female }\end{array}$ & $0.12-1.1$ & $(0.4)$ & Triangular & $\begin{array}{l}\text { The range includes the } 54 \% \text { of female children in this age } \\
\text { group who consume milk (NCI, 1997). The lower limit } \\
\text { indicates that females between the ages of } 15 \text { and } 19 \text { consume } \\
\text { at least one } 4-\mathrm{oz} \text {. glass of milk }(0.12 \mathrm{~L}) \text { per day, but no more } \\
\text { than four and one-half } 8-\mathrm{oz} \text {. glasses of milk }(1.1 \mathrm{~L}) \text { per day. A } \\
\text { triangular distribution was chosen to describe the uncertainty } \\
\text { associated with the annual average daily consumption rate of } \\
\text { milk by female children between the ages of } 15-19 \text { years. }\end{array}$ \\
\hline
\end{tabular}


Table 8.1 (continued)

\begin{tabular}{|c|c|c|c|c|c|}
\hline Parameter & Symbol & Range & $\begin{array}{c}\text { Mean or } \\
\text { (Mode) }\end{array}$ & Distribution & Rationale \\
\hline $\begin{array}{l}\text { Average daily } \\
\text { consumption of cow's } \\
\text { milk by children } \\
\text { (females) ages } 15-19 \\
\text { years who drink only } \\
\text { backyard cow's milk } \\
\left(\mathrm{L} \mathrm{d}^{-1}\right)\end{array}$ & $\begin{array}{c}\mathrm{U}_{\text {cow milk }} \\
\text { (backyard), } \\
15-19 \mathrm{yrs} \\
\text { female }\end{array}$ & $0.24-1.2$ & $(0.54)$ & Triangular & $\begin{array}{l}\text { The range is for female children who have immediate access to } \\
\text { backyard cow's milk. The lower limit indicates that females } \\
\text { between the ages of } 15 \text { and } 19 \text { consume a minimum of one } 8 \text { - } \\
\text { oz. glass of milk }(0.24 \mathrm{~L}) \text { per day, but no more than five } 8 \text {-oz. } \\
\text { glasses of milk }(1.2 \mathrm{~L}) \text { per day. The mode is equivalent to two } \\
\text { and one-fourth 8-oz. glasses of cow's milk per day. A } \\
\text { triangular distribution was chosen to describe the uncertainty } \\
\text { associated with the annual average daily consumption rate of } \\
\text { milk by female children between the ages of } 15-19 \text { years who } \\
\text { have immediate access to backyard cow's milk. }\end{array}$ \\
\hline $\begin{array}{l}\text { Average daily } \\
\text { consumption of cow's } \\
\text { milk by adult males } \\
\left(\mathrm{L} \mathrm{d}^{-1}\right)\end{array}$ & $\begin{array}{l}\mathrm{U}_{\text {cow milk, }} \\
\text { adult, male }\end{array}$ & $0.24-0.8$ & $(0.48)$ & Triangular & $\begin{array}{l}\text { The range is equivalent to one } 8 \text {-oz. glass and three and one- } \\
\text { half 8-oz. glasses of milk per day, respectively. The mode is } \\
\text { equivalent to two } 8 \text {-oz. glasses of milk daily }\left(0.48 \mathrm{~L} \mathrm{~d}^{-1}\right) \text {. A } \\
\text { triangular distribution was selected to account for the } \\
\text { uncertainty associated with the annual average consumption } \\
\text { rate of milk by adult males. }\end{array}$ \\
\hline $\begin{array}{l}\text { Average daily } \\
\text { consumption of cow's } \\
\text { milk by adult males who } \\
\text { drink only backyard } \\
\text { cow's milk ( } \mathrm{L} \mathrm{d}^{1} \text { ) }\end{array}$ & $\begin{array}{l}\mathrm{U}_{\text {cow milk }} \\
\text { (backyard), } \\
\text { adult, male }\end{array}$ & $0.48-0.96$ & $(0.72)$ & Triangular & $\begin{array}{l}\text { The range is for adult males who had immediate access to } \\
\text { backyard cow's milk. These individuals were considered to } \\
\text { consume at least two } 8 \text {-oz. glasses of milk per day, but no more } \\
\text { than four } 8 \text {-oz. glasses per day. For consumers of backyard } \\
\text { cow's milk, the mode represents three } 8 \text {-oz. glasses of milk per } \\
\text { day }\left(0.72 \mathrm{~L} \mathrm{~d}^{-1}\right) \text {. A triangular distribution was chosen to } \\
\text { represent the range of the true but unknown annual average } \\
\text { consumption rate of backyard cow's milk. }\end{array}$ \\
\hline
\end{tabular}


Table 8.1 (continued)

\begin{tabular}{|c|c|c|c|c|c|}
\hline Parameter & Symbol & Range & $\begin{array}{c}\text { Mean or } \\
\text { (Mode) }\end{array}$ & Distribution & Rationale \\
\hline $\begin{array}{l}\text { Average daily } \\
\text { consumption of cow's } \\
\text { milk by adult females ( } \mathrm{L} \\
\mathrm{d}^{-1} \text { ) }\end{array}$ & $\begin{array}{l}\mathrm{U}_{\text {cow milk, }} \\
\text { adult, female }\end{array}$ & $0.12-0.72$ & $(0.4)$ & Triangular & $\begin{array}{l}\text { The lower and upper limits are equivalent to one 4-oz. glass } \\
\text { and three 8-oz. glasses of milk per day, respectively. This } \\
\text { range indicates that, on average, an adult female will consume } \\
\text { at least one-half glass of milk per day, but no more than } 3 \\
\text { glasses a day. A triangular distribution with a mode of } 0.4 \mathrm{~L} \\
\mathrm{~d}^{-1} \text { was selected to account for the uncertainty associated with } \\
\text { the annual average consumption rate of milk. }\end{array}$ \\
\hline $\begin{array}{l}\text { Average daily } \\
\text { consumption of cow's } \\
\text { milk by adult females } \\
\text { who drink only backyard } \\
\text { cow's milk }\left(\mathrm{L} \mathrm{d}^{-1}\right)\end{array}$ & $\begin{array}{l}\mathrm{U}_{\text {cow milk }} \\
\text { (backyard), } \\
\text { adult, female }\end{array}$ & $0.24-0.96$ & $(0.6)$ & Triangular & $\begin{array}{l}\text { The range is for adult females who had immediate access to } \\
\text { backyard cow's milk. These individuals were considered to } \\
\text { consume at least one } 8-\mathrm{oz} \text {. glass of milk per day, but no more } \\
\text { than four } 8-\mathrm{oz} \text {. glasses per day. Two and one-half } 8-\mathrm{oz} \text {. } \\
\text { glasses per day }\left(0.6 \mathrm{~L} \mathrm{~d}^{-1}\right) \text { is the mode of the distribution. A } \\
\text { triangular distribution was chosen to describe the uncertainty } \\
\text { associated with the annual average daily consumption rate of } \\
\text { backyard cow's milk by adult females. }\end{array}$ \\
\hline $\begin{array}{l}\text { Average daily } \\
\text { consumption of cow's } \\
\text { milk by pregnant or } \\
\text { lactating females who } \\
\text { drink commercial or } \\
\text { backyard cow's milk } \\
\left(\mathrm{L} \mathrm{d}^{-1}\right)\end{array}$ & $\begin{array}{c}\mathrm{U}_{\text {cow milk, }} \\
\text { preg/lact } \\
\text { female }\end{array}$ & $0.24-1.2$ & $(0.8)$ & Triangular & $\begin{array}{l}\text { The range is for females who are pregnant or lactating. These } \\
\text { individuals were considered to consume at least one } 8 \text {-oz. glass } \\
\text { of milk per day, but no more than five } 8 \text {-oz. glasses per day. } \\
\text { Approximately three } 8-\mathrm{oz} \text {. glasses per day }\left(0.8 \mathrm{~L} \mathrm{~d}^{-1}\right) \text { is the } \\
\text { mode of the distribution. A triangular distribution was chosen } \\
\text { to describe the uncertainty associated with the annual average } \\
\text { daily consumption rate of cow's milk by pregnant or lactating } \\
\text { females. }\end{array}$ \\
\hline
\end{tabular}


Table 8.2 Values for parameters used in the cow's milk ingestion pathway for the accidental release.

\begin{tabular}{|c|c|c|c|c|c|}
\hline Parameter & Symbol & Range & $\begin{array}{c}\text { Mean or } \\
\text { (Mode) }\end{array}$ & Distribution & Rationale \\
\hline $\begin{array}{l}\text { Average daily } \\
\text { consumption of breast, } \\
\text { cow's, or goat's milk by } \\
\text { infants (males and } \\
\text { females) age } 0-1 \text { year } \\
\left(\mathrm{L} \mathrm{d}^{-1}\right)\end{array}$ & $\mathrm{U}_{\text {milk, infant }}$ & $0.12-0.98$ & 0.55 & Uniform & $\begin{array}{l}\text { No change from the routine release scenario is used in the } \\
\text { accident scenario because a growing infant requires the same } \\
\text { amount of nutrition and food regardless of the time of year. }\end{array}$ \\
\hline $\begin{array}{l}\text { Average daily } \\
\text { consumption of cow's } \\
\text { milk by children (males } \\
\text { and females) ages 1-4 } \\
\text { years }\left(\mathrm{L} \mathrm{d}^{-1}\right)\end{array}$ & $\mathrm{U}_{\text {milk, }} 1-4 \mathrm{yrs}$ & $0.24-1.4$ & $(0.75)$ & Log-triangular & $\begin{array}{l}\text { According to the NCI (1997) study, } 77 \% \text { of the male } \\
\text { population and } 75 \% \text { of the female population within the ages } \\
\text { of } 1-4 \text { years are milk drinkers who consume milk within this } \\
\text { range. The lower limit is indicative of approximately one } 8 \text {-oz. } \\
\text { glass of milk per day. The upper limit indicates that at most } \\
6 \text { glasses ( } 8 \text { oz. each) of milk are consumed per day. A log- } \\
\text { triangular distribution with a mode of } 0.75 \text { was chosen to } \\
\text { represent the uncertainty associated with the average daily } \\
\text { consumption rate of milk, averaged over a month, by children } \\
\text { aged 1-4 years. }\end{array}$ \\
\hline $\begin{array}{l}\text { Average daily } \\
\text { consumption of cow's } \\
\text { milk by children (males } \\
\text { and females) ages 5-9 } \\
\text { years }\left(\mathrm{L} \mathrm{d}^{-1}\right)\end{array}$ & $\mathrm{U}_{\text {milk, 5-9 yrs }}$ & $0.24-1.4$ & $\begin{array}{l}(0.75 \text { for } \\
\text { males }) \text { and } \\
(0.68 \text { for } \\
\text { females })\end{array}$ & Log-triangular & $\begin{array}{l}\text { The range encompasses } 73 \% \text { of males and } 68 \% \text { of females in } \\
\text { the total population who consume milk (NCI, 1997). The } \\
\text { lower limit indicates that children between the ages of } 5 \text { and } 9 \\
\text { drink at least one } 8 \text {-oz. glass of milk }(0.24 \mathrm{~L}) \text { per day. The } \\
\text { upper limit indicates that approximately six (8-oz.) glasses are } \\
\text { consumed per day. A log-triangular distribution was selected } \\
\text { to represent the uncertainty associated with the true but } \\
\text { unknown value of the average daily consumption rate for } \\
\text { children between the ages of } 5 \text { and } 9 \text { years, averaged over a } \\
\text { month. }\end{array}$ \\
\hline
\end{tabular}


Table 8.2 (continued)

\begin{tabular}{|c|c|c|c|c|c|}
\hline Parameter & Symbol & Range & $\begin{array}{c}\text { Mean or } \\
\text { (Mode) }\end{array}$ & Distribution & Rationale \\
\hline $\begin{array}{l}\text { Average daily } \\
\text { consumption of cow's } \\
\text { milk by children (males } \\
\text { and females) ages } 10-14 \\
\text { years }\left(\mathrm{L} \mathrm{d}^{-1}\right)\end{array}$ & $\begin{array}{l}\mathrm{U}_{\text {milk, }} \quad 10- \\
14 \mathrm{yrs}\end{array}$ & $0.24-1.4$ & $\begin{array}{l}(0.75 \text { for } \\
\text { males }) \text { and } \\
(0.62 \text { for } \\
\text { females })\end{array}$ & Log-triangular & $\begin{array}{l}\text { The range includes } 69 \% \text { of males and } 60 \% \text { of females of the total } \\
\text { population who consume milk (NCI, 1997). The lower limit } \\
\text { indicates that children between the ages of } 10 \text { and } 14 \text { consume a } \\
\text { minimum of one } 8 \text {-oz. glass of milk }(0.24 \mathrm{~L}) \text { per day, but do not } \\
\text { consume more than the maximum of six } 8 \text {-oz. glasses of milk }(1.4 \mathrm{~L}) \\
\text { per day. A log-triangular distribution was chosen to describe the } \\
\text { uncertainty associated with the average daily consumption rate of } \\
\text { milk by children aged } 10-14 \text { years, averaged over a one-month } \\
\text { period. }\end{array}$ \\
\hline $\begin{array}{l}\text { Average daily } \\
\text { consumption of cow’s } \\
\text { milk by children (males) } \\
\text { ages } 15-19 \text { years }\left(\mathrm{L} \mathrm{d}^{-1}\right)\end{array}$ & $\begin{array}{c}\mathrm{U}_{\text {milk, }} \quad 15- \\
19 \text { yrs, male }\end{array}$ & $0.24-1.4$ & $(0.75)$ & Log-triangular & $\begin{array}{l}\text { The range includes } 64 \% \text { of males in the total population who } \\
\text { consume milk (NCI, 1997). The lower limit indicates that male } \\
\text { children between the ages of } 15 \text { and } 19 \text { consume at least one } 8 \text {-oz. } \\
\text { glass of milk }(0.24 \mathrm{~L}) \text { per day, but no more than six } 8 \text {-oz. glasses of } \\
\text { milk ( } 1.4 \mathrm{~L}) \text { per day. A log-triangular distribution was chosen to } \\
\text { describe the uncertainty associated with the average daily } \\
\text { consumption rate of milk by male children aged } 15-19 \text { years, } \\
\text { averaged over a month. }\end{array}$ \\
\hline $\begin{array}{l}\text { Average daily } \\
\text { consumption of cow's } \\
\text { milk by children (males) } \\
\text { ages } 15-19 \text { years who } \\
\text { consume backyard } \\
\text { cow's milk only }\left(\mathrm{L} \mathrm{d}^{-1}\right)\end{array}$ & $\begin{array}{c}\mathrm{U}_{\text {milk }} \\
\text { (backyard), } \\
\text { 15-19 yrs, } \\
\text { male }\end{array}$ & $0.48-1.4$ & $(0.90)$ & Log-triangular & $\begin{array}{l}\text { The range is for males who have immediate access to backyard } \\
\text { cow's milk. The lower limit indicates that males between the ages of } \\
15 \text { and } 19 \text { consume a minimum of two } 8 \text {-oz. glasses of milk }(0.24 \mathrm{~L}) \\
\text { per day, but no more than six } 8 \text { - oz. glasses of milk }(1.4 \mathrm{~L}) \text { per day. } \\
\text { The mode is approximately four } 8 \text {-oz. glasses of cow's milk per day. } \\
\text { A log-triangular distribution was chosen to describe the uncertainty } \\
\text { associated with the average daily consumption rate of milk by males } \\
\text { between the ages of } 15-19 \text { years who have immediate access to } \\
\text { backyard cow's milk. }\end{array}$ \\
\hline
\end{tabular}


Table 8.2 (continued)

\begin{tabular}{|c|c|c|c|c|c|}
\hline Parameter & Symbol & Range & $\begin{array}{c}\text { Mean or } \\
\text { (Mode) }\end{array}$ & Distribution & Rationale \\
\hline $\begin{array}{l}\text { Average daily } \\
\text { consumption of cow's } \\
\text { milk by children } \\
\text { (females) ages } 15-19 \\
\text { years }\left(\mathrm{L} \mathrm{d}^{-1}\right)\end{array}$ & $\begin{array}{l}\mathrm{U}_{\text {milk, }} \quad 15- \\
19 \mathrm{yrs} \text {, female }\end{array}$ & $0.12-1.2$ & $(0.42)$ & Log-triangular & $\begin{array}{l}\text { The range includes } 56 \% \text { of females of the total population who } \\
\text { consume milk (NCI, 1997). The lower limit indicates that females } \\
\text { between the ages of } 15 \text { and } 19 \text { consume a minimum of one } 4-\mathrm{oz} \text {. } \\
\text { glass of milk }(0.12 \mathrm{~L}) \text { per day, but no more than five } 8-\mathrm{oz} \text {. glasses of } \\
\text { milk ( } 1.2 \mathrm{~L}) \text { per day. A log-triangular distribution was chosen to } \\
\text { describe the uncertainty associated with the average daily } \\
\text { consumption rate of milk by females between the ages of 15-19 } \\
\text { years, averaged over a month. }\end{array}$ \\
\hline $\begin{array}{l}\text { Average daily } \\
\text { consumption of cow's } \\
\text { milk by children } \\
\text { (females) ages } 15-19 \\
\text { years who consume } \\
\text { backyard cow's milk } \\
\text { only }\left(\mathrm{L} \mathrm{d}^{-1}\right)\end{array}$ & $\begin{array}{l}\mathrm{U}_{\text {milk }} \\
\text { (backyard), } \\
15-19 \text { yrs, } \\
\text { female }\end{array}$ & $0.24-1.4$ & $(0.64)$ & Log-triangular & $\begin{array}{l}\text { The lower limit indicates that females between the ages of } 15 \text { and } 19 \\
\text { with available backyard cow's milk consume at least one } 8 \text {-oz. glass } \\
\text { of milk }(0.24 \mathrm{~L}) \text { per day, but no more than six } 8 \text {-oz. glasses of milk } \\
\text { (1.4 L) per day. A log-triangular distribution was chosen to describe } \\
\text { the uncertainty associated with the average daily consumption rate of } \\
\text { backyard cow's milk by females between the ages of } 15-19 \text { years, } \\
\text { averaged over one month. }\end{array}$ \\
\hline $\begin{array}{l}\text { Average daily } \\
\text { consumption of cow's } \\
\text { milk by adult males } \\
\left(\mathrm{L} \mathrm{d}^{-1}\right)\end{array}$ & $\begin{array}{l}\mathrm{U}_{\text {milk, adult, }} \\
\text { male }\end{array}$ & $0.24-1.4$ & $(0.64)$ & Log-triangular & $\begin{array}{l}\text { The values are equivalent to one } 8 \text {-oz. glass and six } 8 \text {-oz. glasses of } \\
\text { milk per day, respectively. A log-triangular distribution was } \\
\text { selected to account for the uncertainty associated with the average } \\
\text { daily consumption rate of milk, averaged over a one-month period. }\end{array}$ \\
\hline $\begin{array}{l}\text { Average daily } \\
\text { consumption of cow's } \\
\text { milk by adult males who } \\
\text { consume backyard } \\
\text { cow's milk only }\left(\mathrm{L} \mathrm{d}^{-1}\right)\end{array}$ & $\begin{array}{l}\mathrm{U}_{\text {milk }} \\
\text { (backyard), } \\
\text { adult, male }\end{array}$ & $0.48-1.4$ & $(0.90)$ & Log-triangular & $\begin{array}{l}\text { The range is equivalent to two } 8 \text {-oz. glasses and six } 8 \text {-oz. glasses of } \\
\text { milk per day, respectively. A log-triangular distribution was } \\
\text { selected to represent the uncertainty associated with the average } \\
\text { consumption rate of milk by adult males with immediate access to } \\
\text { backyard cow's milk. }\end{array}$ \\
\hline
\end{tabular}


Table 8.2 (continued)

\begin{tabular}{|c|c|c|c|c|c|}
\hline Parameter & Symbol & Range & $\begin{array}{l}\text { Mean or } \\
\text { (Mode) }\end{array}$ & Distribution & Rationale \\
\hline $\begin{array}{l}\text { Average daily } \\
\text { consumption of cow's } \\
\text { milk by adult females } \\
\left(\mathrm{L} \mathrm{d}^{-1}\right)\end{array}$ & $\begin{array}{l}\mathrm{U}_{\text {milk, adult, }} \\
\text { female }\end{array}$ & $0.12-1.2$ & $(0.41)$ & Log-triangular & $\begin{array}{l}\text { The range is equivalent to one-half of an 8-oz. glass and five 8-oz. } \\
\text { glasses of milk per day, respectively. A log-triangular distribution } \\
\text { was selected to account for the uncertainty associated with the } \\
\text { average consumption rate of milk, averaged over a month. }\end{array}$ \\
\hline $\begin{array}{l}\text { Average daily } \\
\text { consumption of cow's } \\
\text { milk by adult females } \\
\text { who consume backyard } \\
\text { cow's milk only }\left(\mathrm{L} \mathrm{d}^{-1}\right)\end{array}$ & $\begin{array}{c}\mathrm{U}_{\text {milk }} \\
\text { (backyard), } \\
\text { adult, female }\end{array}$ & $0.24-1.4$ & $(0.60)$ & Log-triangular & $\begin{array}{l}\text { The values are equivalent to one } 8 \text {-oz. glass and six } 8 \text {-oz. glasses of } \\
\text { milk per day, respectively. A log-triangular distribution with a mode } \\
\text { of } 0.60 \mathrm{~L} \mathrm{~d}^{-1} \text { was selected to account for the uncertainty associated } \\
\text { with the average consumption rate of backyard cow's milk by adult } \\
\text { females, averaged over one month. }\end{array}$ \\
\hline $\begin{array}{l}\text { Average daily } \\
\text { consumption of cow's } \\
\text { milk by pregnant or } \\
\text { lactating females who } \\
\text { drink commercial or } \\
\text { backyard cow's milk } \\
\left(\mathrm{L} \mathrm{d}^{-1}\right)\end{array}$ & $\begin{array}{c}\mathrm{U}_{\text {cow milk, }} \\
\text { preg/lact } \\
\text { female }\end{array}$ & $0.24-1.2$ & $(0.8)$ & Triangular & $\begin{array}{l}\text { No change from the routine release scenario is used in the accident } \\
\text { scenario because the range used for pregnant or nursing mothers was } \\
\text { made sufficiently broad to encompass variations in milk } \\
\text { consumption habits that occur over a one-month period. }\end{array}$ \\
\hline
\end{tabular}




\subsubsection{Consumption of Goat's Milk}

Goat's milk is consumed by more individuals on a worldwide basis than the milk of any other animal (ADGA, 1998). The opposite is true for the United States (UCI, 1998), as most major U.S. cities do not have fluid goat's milk available in local retail outlets (Kapture, 1998). Within the $38-\mathrm{km}$ radius of $\mathrm{X}-10$, the use of goat's milk was not extensive. However, area residents have attested to the use of goat's milk by themselves, a family member, or a neighbor (Clark, personal communication with C. Lewis, 1997; Prichard, personal communication with C. Lewis, 1997; Hackett, personal communication with C. Lewis, 1998; Hudson, personal communication with C. Lewis, 1998), due partly to allergies to cow's milk. Goat's milk is similar to cow's milk nutritionally, but goat's milk contains smaller fat globules, which are easier for some people to digest (UCI, 1998). Another reason for the production and consumption of goat's milk is the limited amount of care required for goats, as compared to cattle. A goat will eat little, occupy a small area, and produce enough milk for the average family (Haenlein and Caccese, 1998). An average milk goat will produce approximately 1-1/1/2 gallons (4-6 L) of milk per day (Leuchs Farm, 1998), which is a considerably smaller amount of milk than is produced by an average dairy cow. In the areas surrounding the ORR, goat's milk was not available commercially; therefore, all goat's milk consumed was of local origin. The milk that was produced was consumed by a limited number of individuals. Nonetheless, this pathway is considered in the analysis.

Goat's milk ingestion parameters for various age groups and both genders are provided in Table 8.3 for estimating long-term averages for exposure to routine releases. Since the consumption of goat's milk was limited to a few households and the information about goat's milk consumption is rare, the same distributions are used for the accident scenario. The ranges are considered to be sufficiently broad to encompass the uncertainty associated with a monthly average consumption rate.

The ranges provided are for goat's milk drinkers and are based on information provided by the National Cancer Institute (NCI) (1997) for cow's milk. As with cow's milk, the average daily consumption of goat's milk by females is less on average than that of males (Dreicer et al., 1990); gender differences were not considered significant until the age of 15 . 
${ }^{131}$ I Releases from X-10 Radioactive Lanthanum Processing -

TASK 1 REPORT

Table 8.3 Values for parameters used in the goat's milk ingestion pathway for routine and accidental releases.

\begin{tabular}{|c|c|c|c|c|c|}
\hline Parameter & Symbol & Range & $\begin{array}{l}\text { Mean or } \\
\text { \{Central } \\
\text { Value }\end{array}$ & Distribution & Rationale \\
\hline $\begin{array}{l}\text { Average daily consumption } \\
\text { of goat's milk by infants } \\
\text { (males and females) }\left(\mathrm{L} \mathrm{d}^{-1}\right)\end{array}$ & $\begin{array}{l}\mathrm{U}_{\text {goat milk, }} \\
\text { infant }\end{array}$ & $0.12-0.98$ & 0.55 & Uniform & $\begin{array}{l}\text { The distribution for the annual average milk consumption rate is the } \\
\text { same for all types of milk because an infant requires the same } \\
\text { amount of milk regardless of the source (see Table 8.1). }\end{array}$ \\
\hline $\begin{array}{l}\text { Average daily consumption } \\
\text { of goat's milk by children } \\
\text { (males and females) ages 1- } \\
4 \text { years }\left(\mathrm{L} \mathrm{d}^{-1}\right)\end{array}$ & $\begin{array}{c}\mathrm{U}_{\text {goat milk, }} \\
1-4 \mathrm{yrs}\end{array}$ & $0.12-1.2$ & $\{0.38\}$ & Log-uniform & $\begin{array}{l}\text { According to the NCI (1997) study, } 78 \% \text { of the male and female } \\
\text { populations within the ages of } 1-4 \text { years are milk drinkers, who } \\
\text { consume milk within this range. The lower limit is indicative of one } \\
\text { 4-oz. glass of milk per day. The upper limit indicates that at most } 5 \\
\text { glasses ( } 8 \text {-oz. each) of milk are consumed per day. The central } \\
\text { value is approximately one and one-half } 8 \text {-oz. glass per day. A log- } \\
\text { uniform distribution with a central value of } 0.38 \mathrm{~L} \mathrm{~d}^{-1} \text { was chosen to } \\
\text { represent the uncertainty associated with the annual average daily } \\
\text { consumption rate of goat's milk (averaged over a four-year period) } \\
\text { by children aged } 1-4 \text { years old. }\end{array}$ \\
\hline $\begin{array}{l}\text { Average daily consumption } \\
\text { of goat's milk by children } \\
\text { (males and females) ages 5- } \\
9 \text { years }\left(\mathrm{L} \mathrm{d}^{-1}\right)\end{array}$ & $\begin{array}{l}\mathrm{U}_{\text {goat milk, }} \\
\text { 5-9 yrs }\end{array}$ & $0.12-1.2$ & $\{0.38\}$ & Log-uniform & $\begin{array}{l}\text { The range encompasses } 74 \% \text { of males and } 72 \% \text { of females in this } \\
\text { age group who consume cow's milk (NCI, 1997). The lower limit } \\
\text { indicates that children between the ages of } 5 \text { and } 9 \text { drink at least one- } \\
\text { half of an } 8 \text {-oz. glass of goat's milk ( } 0.12 \mathrm{~L}) \text { per day, but no more } \\
\text { than } 5 \text { glasses per day. The central value of } 0.38 \mathrm{~L} \mathrm{~d}^{-1} \text { is equivalent } \\
\text { to one and one-half } 8 \text {-oz. glasses of goat's milk per day for this age } \\
\text { group. A log-uniform distribution was selected to represent the } \\
\text { uncertainty associated with the true but unknown value of the annual } \\
\text { average daily consumption rate for goat's milk by children between } \\
\text { the ages of } 5 \text { and } 9 \text { years. }\end{array}$ \\
\hline
\end{tabular}


Table 8.3 (continued)

\begin{tabular}{|c|c|c|c|c|c|}
\hline Parameter & Symbol & Range & $\begin{array}{l}\text { Mean or } \\
\text { \{Central } \\
\text { Value }\end{array}$ & Distribution & Rationale \\
\hline $\begin{array}{l}\text { Average daily } \\
\text { consumption of goat's } \\
\text { milk by children (males } \\
\text { and females) ages } 10-14 \\
\text { years }\left(\mathrm{L} \mathrm{d}^{-1}\right)\end{array}$ & $\begin{array}{l}\mathrm{U}_{\text {goat milk, }} \\
10-14 \text { yrs }\end{array}$ & $0.24-1.2$ & $\{0.54\}$ & Log-uniform & $\begin{array}{l}\text { The range includes } 61 \% \text { of males and } 57 \% \text { of females in this } \\
\text { age group who consume cow's milk (NCI, 1997). The lower } \\
\text { limit indicates that children between the ages of } 10 \text { and } 14 \\
\text { consume a minimum of one 8-oz. glass of goat's milk }(0.24 \mathrm{~L}) \\
\text { per day, but no more than five } 8 \text {-oz. glasses of goat's milk }(1.2 \\
\text { L) per day. The central value is equivalent to two and one- } \\
\text { fourth 8-oz. glasses of goat's milk per day for this age group, } \\
\text { or approximately one glass of milk each with breakfast and } \\
\text { lunch. A log-uniform distribution was chosen to describe the } \\
\text { uncertainty associated with the annual average daily } \\
\text { consumption rate of goat's milk by children aged } 10-14 \text { years. }\end{array}$ \\
\hline $\begin{array}{l}\text { Average daily } \\
\text { consumption of goat's } \\
\text { milk by children (males) } \\
\text { ages } 15-19 \text { years }\left(\mathrm{L} \mathrm{d}^{-1}\right)\end{array}$ & $\begin{array}{l}\mathrm{U}_{\text {goat milk, }} \\
\text { 15-19 yrs, } \\
\text { male }\end{array}$ & $0.24-1.2$ & $\{0.54\}$ & Log-uniform & $\begin{array}{l}\text { The range includes } 53 \% \text { of males in this age group who } \\
\text { consume cow's milk (NCI, 1997). The lower limit indicates } \\
\text { that male children between the ages of } 15 \text { and } 19 \text { consume at } \\
\text { least one } 8 \text {-oz. glass of goat's milk }(0.24 \mathrm{~L}) \text { per day, but no } \\
\text { more than five } 8 \text {-oz. glasses of goat's milk }(1.2 \mathrm{~L}) \text { daily on } \\
\text { average. The central value is equivalent to two and one-fourth } \\
\text { 8-oz. glasses of goat's milk per day. A log-uniform } \\
\text { distribution was chosen to describe the uncertainty associated } \\
\text { with the annual average daily consumption rate of goat's milk } \\
\text { by male children aged } 15-19 \text { years. }\end{array}$ \\
\hline
\end{tabular}


Table 8.3 (continued)

\begin{tabular}{|c|c|c|c|c|c|}
\hline Parameter & Symbol & Range & $\begin{array}{c}\text { Mean or } \\
\text { \{Central } \\
\text { Value\} }\end{array}$ & Distribution & Rationale \\
\hline $\begin{array}{l}\text { Average daily } \\
\text { consumption of goat's } \\
\text { milk by children } \\
\text { (females) ages } 15-19 \\
\text { years }\left(\mathrm{L} \mathrm{d}^{-1}\right)\end{array}$ & $\begin{array}{c}\mathrm{U}_{\text {goat milk, }} \\
\text { 15-19 yrs, } \\
\text { female }\end{array}$ & $0.12-1.2$ & $\{0.38\}$ & Log-uniform & $\begin{array}{l}\text { The range includes } 55 \% \text { of females in this age group who consume } \\
\text { cow's milk (NCI, 1997). The lower limit indicates that female } \\
\text { children between the ages of } 15 \text { and } 19 \text { consume a minimum of one } \\
\text { 4-oz. glass of goat's milk }(0.12 \mathrm{~L}) \text { daily, but no more than five } 8-\mathrm{oz} \text {. } \\
\text { glasses of goat's milk ( } 1.2 \mathrm{~L}) \text { per day. The central value is } \\
\text { equivalent to approximately one and one-half } 8 \text {-oz. glass per day on } \\
\text { average. A log-uniform distribution was chosen to describe the } \\
\text { uncertainty associated with the annual average daily consumption } \\
\text { rate of goat's milk by female children between the ages of } 15-19 \\
\text { years. }\end{array}$ \\
\hline $\begin{array}{l}\text { Average daily } \\
\text { consumption of goat's } \\
\text { milk by adult males } \\
\left(\mathrm{L} \mathrm{d}^{-1}\right)\end{array}$ & $\begin{array}{l}\mathrm{U}_{\text {goat milk, }} \\
\text { adult, male }\end{array}$ & $0.24-0.96$ & $\{0.48\}$ & Log-uniform & $\begin{array}{l}\text { The range encompasses at least } 34 \% \text { of adult male individuals } \\
\text { between the ages of } 20 \text { and } 64 \text { years of age who consume fresh fluid } \\
\text { milk (NCI, 1997). The values are equivalent to one and four (8-oz.) } \\
\text { glasses of goat's milk per day, respectively. A log-uniform } \\
\text { distribution was selected to account for the uncertainty associated } \\
\text { with the annual average consumption rate of goat's milk by adults. }\end{array}$ \\
\hline $\begin{array}{l}\text { Average daily } \\
\text { consumption of goat's } \\
\text { milk by adult females } \\
\left(\mathrm{L} \mathrm{d}^{-1}\right)\end{array}$ & $\begin{array}{l}\mathrm{U}_{\text {goat milk, }} \\
\text { adult, female }\end{array}$ & $0.12-0.96$ & $\{0.34\}$ & Log-uniform & $\begin{array}{l}\text { The range includes at least } 38 \% \text { of adult female individuals between } \\
\text { the ages of } 20 \text { and } 64 \text { years of age who consume fresh fluid milk } \\
\text { (NCI, 1997). The values are equivalent to one-half glass and four ( } 8 \text { - } \\
\text { oz.) glasses of goat's milk per day, respectively. A log-uniform } \\
\text { distribution was selected to account for the uncertainty associated } \\
\text { with the annual average daily consumption rate of goat's milk by } \\
\text { adult females. }\end{array}$ \\
\hline
\end{tabular}




\subsubsection{Consumption of Meat}

Radioiodine is deposited on pasture and grain crops; therefore, chickens, cattle, and swine consuming these products transfer radioactivity to their eggs, meat, and milk, which are then consumed by humans. In this study, beef is considered the surrogate for all meat, including poultry and swine. Infants consume insignificant amounts of meat due to their substantial intake of milk. As children age, their consumption rate of meat increases. Ranges are provided for 1-8 year olds (both genders combined), 9-14 year olds (males and females individually), and adults (males and females individually).

The amount of contaminated meat consumed varied with an individual's residence. Rural residents are considered to consume contaminated meat more often than are their urban counterparts. Raising animals on contaminated crops was more likely to result in a significant exposure than buying meat from a grocery store, which obtained meat from both non-local and local sources. Urban residents are considered to consume contaminated meat on a limited basis. The contaminated meat could have been obtained from a neighbor, a friend, or a local meat market.

The parameters associated with the meat ingestion pathway are provided in Table 8.4 for routine releases. The accident scenario does not include meat ingestion because farm-fresh meat was generally not available in the spring, when the accident occurred. Slaughtering is generally conducted in the late fall to ensure that the meat does not spoil (Loveday, personal communication with C. Lewis, 1998).

The ranges provided for children are consistent with the average daily consumption rate of beef, pork, and poultry reported by the USDA $(1965 ; 1980)$ and the "best estimate" reported by Rupp (1980). For children 1 year old and younger, large quantities of milk are being consumed, so their intake of meat, if any, will be minimal (Mount, personal communication with $\mathrm{C}$. Lewis, 1997). For all age groups, the range represents children who live in a rural setting and obtain the majority of their meat from home-raised animals (USDA, 1965), but also children who obtain a limited amount of their meat from a local grocery store.

For adults aged 15 to 75 plus, the ranges are consistent with consumption rates of meat reported by ATSDR (1992), Cochrane (1945), Rupp (1980), and USDA (1944; 1949; 1965; 1980). Again, the range represents individuals who live in a rural setting and obtain the majority of their meat from home-raised animals (USDA, 1965), while also representing individuals who obtain a limited portion of their meat from local grocery stores. 
Table 8.4 Values for parameters used in the meat ingestion pathway for routine releases.

\begin{tabular}{|c|c|c|c|c|c|}
\hline Parameter & Symbol & Range & Mean & Distribution & Rationale \\
\hline $\begin{array}{l}\text { Average daily } \\
\text { consumption of meat by } \\
\text { infants (males and } \\
\text { females) age } 0-1 \text { year } \\
\left(\mathrm{kg} \mathrm{d}^{-1}\right)\end{array}$ & $\mathrm{U}_{\text {meat, infants }}$ & $0.005-0.015$ & 0.01 & Uniform & $\begin{array}{l}\text { The average daily consumption rate of meat by infants ranges } \\
\text { between } 5 \text { and } 15 \mathrm{~g}(0.2-0.5 \mathrm{oz} \text {.) per day. The mean value is } \\
\text { equivalent to } 0.35 \mathrm{oz} \text {. daily. A uniform distribution was } \\
\text { selected to describe the uncertainty associated with the true but } \\
\text { unknown average daily consumption rate of meat by infants } \\
\text { (averaged over a one-year period). }\end{array}$ \\
\hline $\begin{array}{l}\text { Average daily } \\
\text { consumption of meat by } \\
\text { children (males and } \\
\text { females) ages 1-8 years } \\
\left(\mathrm{kg} \mathrm{d}^{-1}\right)\end{array}$ & $\mathrm{U}_{\text {meat, } 1-8 \mathrm{yrs}}$ & $0.05-0.11$ & 0.08 & Uniform & $\begin{array}{l}\text { The range for the average daily consumption of meat by } \\
\text { children between the ages of } 1 \text { and } 8 \text { years is equivalent to } \\
\text { approximately } 2 \text { and } 4 \text { oz. of meat per day. The mean value is } \\
\text { equivalent to approximately } 3 \text { oz. of meat per day. A uniform } \\
\text { distribution was selected to represent the uncertainty } \\
\text { associated with the average daily consumption rate of meat by } \\
\text { children between the ages of } 1 \text { and } 8 \text { because every value } \\
\text { within the range has equal probability of being the annual } \\
\text { average consumption rate of meat. }\end{array}$ \\
\hline $\begin{array}{l}\text { Average daily } \\
\text { consumption of meat by } \\
\text { male children ages 9-14 } \\
\left(\mathrm{kg} \mathrm{d}^{-1}\right)\end{array}$ & $\begin{array}{l}\mathrm{U}_{\text {meat, }} \quad 9- \\
14 \mathrm{yrs}, \text { males }\end{array}$ & $0.08-0.14$ & 0.11 & Uniform & $\begin{array}{l}\text { The consumption rate of meat by males in this age group varies } \\
\text { between } 3 \text { and } 5 \text { oz. per day, with a mean of } 4 \text { oz. per day. } \\
\text { Since every value within the range has equal probability, a } \\
\text { uniform distribution was chosen to represent the annual } \\
\text { average daily consumption rate of meat by } 9-14 \text { year-old } \\
\text { males. }\end{array}$ \\
\hline $\begin{array}{l}\text { Average daily } \\
\text { consumption of meat by } \\
\text { female children ages } \\
9-14\left(\mathrm{~kg} \mathrm{~d}^{-1}\right)\end{array}$ & $\begin{array}{l}\mathrm{U}_{\text {meat, }} \quad 9- \\
14 \mathrm{yrs} \text {, females }\end{array}$ & $0.07-0.13$ & 0.10 & Uniform & $\begin{array}{l}\text { Females ages } 9 \text { to } 14 \text { consume approximately } 2.5 \text { to } 4.5 \mathrm{oz} \text {. of } \\
\text { meat daily. The mean value for this age group is equivalent to } \\
3.5 \text { oz. per day. A uniform distribution was chosen to } \\
\text { represent the annual average daily consumption rate of meat by } \\
9-14 \text { year-old females. }\end{array}$ \\
\hline
\end{tabular}


Table 8.4 (continued)

\begin{tabular}{|c|c|c|c|c|c|}
\hline Parameter & Symbol & Range & Mean & Distribution & Rationale \\
\hline $\begin{array}{l}\text { Average daily } \\
\text { consumption of meat by } \\
\text { adult males }\left(\mathrm{kg} \mathrm{d}^{-1}\right)\end{array}$ & $\underset{\text { male }}{\mathrm{U}_{\text {meat, adult }}}$ & $0.15-0.32$ & 0.24 & Uniform & $\begin{array}{l}\text { For males aged } 15 \text { to } 75 \text { plus, the upper limit is equivalent to } \\
\text { approximately } 12 \mathrm{oz} \text {. of meat daily, while the lower limit is } \\
\text { equivalent to approximately } 5 \mathrm{oz} \text {. of meat per day. The mean } \\
\text { value is approximately } 8 \mathrm{oz} \text {. of meat daily. A uniform } \\
\text { distribution was used to describe the uncertainty associated } \\
\text { with the annual average meat ingestion rate for adult males. }\end{array}$ \\
\hline $\begin{array}{l}\text { Average daily } \\
\text { consumption of meat by } \\
\text { adult females }\left(\mathrm{kg} \mathrm{d}^{-1}\right)\end{array}$ & $\begin{array}{l}\mathrm{U}_{\text {meat, adult }} \\
\text { female }\end{array}$ & $0.10-0.20$ & 0.15 & Uniform & $\begin{array}{l}\text { The lower limit is equivalent to approximately } 4 \mathrm{oz} \text {. of meat } \\
\text { per day, while the upper limit is equivalent to approximately } 8 \\
\text { oz. of meat daily for female adults. The true but unknown } \\
\text { annual average meat consumption rate was described by a } \\
\text { uniform distribution for this group of females. }\end{array}$ \\
\hline
\end{tabular}




\subsubsection{Consumption of Eggs}

Another possible source of contaminated food was eggs from chickens that had access to contaminated feed, vegetation, and soil. The information used to obtain the age-specific egg consumption ranges was obtained from rural farm and urban area household food consumption studies (USDA, 1965: 1980) and from the National Cancer Institute (NCI, 1997). Parameters associated with the egg consumption pathway are provided in Tables 8.5 and 8.6 for routine releases and for the accident scenario, respectively.

The ranges are consistent with those reported by the USDA for all urban areas (USDA, 1980) and for rural farm residents (USDA, 1965). The mean values are consistent with the median values for egg consumption for children in the various age groups reported by the National Cancer Institute (NCI; 1997). The range for infants was considered to include children (males and females) between the ages of 6 months and one year. Children less than 6 months were not included due to their limited intake $\left[0 \mathrm{~g} \mathrm{~d}^{-1}\right.$ median value reported for the intake rate of eggs for 0-2 months and $0.005 \mathrm{~g} \mathrm{~d}^{-1}$ median value reported for 3-5 month olds (NCI, 1997)]. According to the USDA (1980), eggs were eaten by only one-tenth of the infants surveyed in the spring of 1977. Children between the ages of 1 and 8 years are considered without distinction by gender. At the age of 9 , differences between males and females become apparent in rates of consumption of various food types. 
Table 8.5 Values for parameters used in the egg ingestion pathway for routine releases.

\begin{tabular}{|c|c|c|c|c|c|}
\hline Parameter & Symbol & Range & Mean & Distribution & Rationale \\
\hline $\begin{array}{l}\text { Average daily } \\
\text { consumption of eggs by } \\
\text { infants (males and } \\
\text { females) age } 6 \text { months to } \\
1 \text { year }\left(\mathrm{kg} \mathrm{d}^{-1}\right)\end{array}$ & $\mathrm{U}_{\text {eggs, infants }}$ & $0.01-0.04$ & 0.025 & Uniform & $\begin{array}{l}\text { The lower limit is indicative of one-fourth of an egg per day, } \\
\text { while the maximum value is approximately one egg per day } \\
\text { (assumed to be scrambled for this age group). The mean value } \\
\text { was selected to represent children between the ages of } 9 \text { and } 11 \\
\text { months (NCI, 1997), as these children were expected to } \\
\text { consume more eggs than younger children. A uniform } \\
\text { distribution was chosen to describe the uncertainty associated } \\
\text { with the annual average egg consumption rate for infants. }\end{array}$ \\
\hline $\begin{array}{l}\text { Average daily } \\
\text { consumption of eggs by } \\
\text { children ages } 1-8 \text { years } \\
\text { (males and females) } \\
\left(\mathrm{kg} \mathrm{d}^{-1}\right)\end{array}$ & $\mathrm{U}_{\text {eggs, } 1-8 \mathrm{yrs}}$ & $0.02-0.08$ & 0.05 & Uniform & $\begin{array}{l}\text { The consumption rate of eggs by children ages } 1-8 \text { (both } \\
\text { genders) is representative of a child who consumes at least } \\
\text { one-half of an egg (weighing approximately } 50 \mathrm{~g} \text {, edible } \\
\text { portion only) per day (lower limit), with the maximum being } \\
\text { approximately } 1 \frac{1}{2} \text { eggs per day. A uniform distribution was } \\
\text { chosen to represent the uncertainty about the true but unknown } \\
\text { annual average egg consumption rate for children between the } \\
\text { ages of } 1 \text { and } 8 \text { years. }\end{array}$ \\
\hline $\begin{array}{l}\text { Average daily } \\
\text { consumption of eggs by } \\
\text { male children } 9-14 \text { years } \\
\left(\mathrm{kg} \mathrm{d}^{-1}\right)\end{array}$ & $\begin{array}{l}\mathrm{U}_{\text {eggs, }} \\
14 \text { yrs, }\end{array}$ & $0.03-0.10$ & 0.065 & Uniform & $\begin{array}{l}\text { The egg consumption rate for males between the ages of } 9 \text { and } \\
14 \text { varied between approximately one-half an egg to } 2 \text { eggs per } \\
\text { day. The true but unknown value of the egg consumption rate } \\
\text { for male children between the ages of } 9 \text { and } 14 \text { was described } \\
\text { by a uniform distribution because all values within the range } \\
\text { are equally probable. }\end{array}$ \\
\hline
\end{tabular}


Table 8.5 (continued)

\begin{tabular}{|c|c|c|c|c|c|}
\hline Parameter & Symbol & Range & Mean & Distribution & Rationale \\
\hline $\begin{array}{l}\text { Average daily } \\
\text { consumption of eggs by } \\
\text { female children ages } \\
9-14 \text { years }\left(\mathrm{kg} \mathrm{d}^{-1}\right)\end{array}$ & $\begin{array}{l}U_{\text {eggs, }} \quad 9-14 \\
\text { yrs, female }\end{array}$ & $0.02-0.08$ & 0.05 & Uniform & $\begin{array}{l}\text { The lower limit is equivalent to one-half an egg, while the } \\
\text { upper limit is equivalent to } 1 \frac{1}{2} \text { eggs. A uniform distribution } \\
\text { was selected to represent the uncertainty associated with the } \\
\text { annual average egg consumption rate for females in this age } \\
\text { group. }\end{array}$ \\
\hline $\begin{array}{l}\text { Average daily } \\
\text { consumption of eggs by } \\
\text { adult males }\left(\mathrm{kg} \mathrm{d}^{-1}\right)\end{array}$ & $\begin{array}{c}\mathrm{U}_{\text {eggs, adult, }} \\
\text { male }\end{array}$ & $0.03-0.10$ & 0.065 & Uniform & $\begin{array}{l}\text { The egg consumption rate for males } 15 \text { years and older ranges } \\
\text { from approximately one-half to } 2 \text { eggs per day. Since all } \\
\text { values within the range are equally likely, a uniform } \\
\text { distribution was chosen to describe the uncertainty associated } \\
\text { with this parameter. }\end{array}$ \\
\hline $\begin{array}{l}\text { Average daily } \\
\text { consumption of eggs by } \\
\text { adult females }\left(\mathrm{kg} \mathrm{d}^{-1}\right)\end{array}$ & $\begin{array}{l}\mathrm{U}_{\text {eggs, adult, }} \\
\text { female }\end{array}$ & $0.02-0.10$ & 0.06 & Uniform & $\begin{array}{l}\text { The lower limit is approximately one-half an egg, while the } \\
\text { upper limit is equivalent to } 2 \text { eggs. The mean value is } \\
\text { approximately } 1 \text { egg for this age group. A uniform distribution } \\
\text { was selected to describe the true but unknown egg } \\
\text { consumption rate for females because every value within the } \\
\text { range is equally probable. }\end{array}$ \\
\hline
\end{tabular}


Table 8.6 Values for parameters used in the egg ingestion pathway for the accidental release.

\begin{tabular}{|c|c|c|c|c|c|}
\hline Parameter & Symbol & Range & Mean & Distribution & Rationale \\
\hline $\begin{array}{l}\text { Average daily } \\
\text { consumption of eggs by } \\
\text { infants (males and } \\
\text { females) age } 6 \text { moths to } \\
1 \text { year }\left(\mathrm{kg} \mathrm{d}^{-1}\right)\end{array}$ & $\mathrm{U}_{\text {eggs, infants }}$ & $0.01-0.04$ & 0.025 & Uniform & $\begin{array}{l}\text { No change from the routine release scenario is used in the } \\
\text { accident scenario because a growing infant requires the same } \\
\text { amount of nutrition and food regardless of the time of year. }\end{array}$ \\
\hline $\begin{array}{l}\text { Average daily } \\
\text { consumption of eggs by } \\
\text { children ages } 1-8 \text { years } \\
\text { (males and females) } \\
\left(\mathrm{kg} \mathrm{d}^{-1}\right)\end{array}$ & $\mathrm{U}_{\text {eggs, } 1-8 \mathrm{yrs}}$ & $0.03-0.10$ & 0.065 & Uniform & $\begin{array}{l}\text { The consumption rate of eggs by children ages 1-8 (both } \\
\text { genders) is representative of a child who consumes at least } \\
\text { one-half of an egg (weighing approximately } 50 \mathrm{~g} \text {, edible } \\
\text { portion only) per day (lower limit), with the maximum being } \\
\text { approximately } 2 \text { eggs per day. A uniform distribution was } \\
\text { chosen to represent the uncertainty of the true but unknown } \\
\text { egg consumption rate for children between the ages of } 1 \text { and } 8 \\
\text { years averaged over a one-month period. }\end{array}$ \\
\hline $\begin{array}{l}\text { Average daily } \\
\text { consumption of eggs by } \\
\text { children } 9-14 \text { years } \\
\text { (males) }\left(\mathrm{kg} \mathrm{d}^{-1}\right)\end{array}$ & $\begin{array}{c}\mathrm{U}_{\text {eggs, }} \quad 9-14 \\
\text { yrs, male }\end{array}$ & $0.05-0.15$ & 0.10 & Uniform & $\begin{array}{l}\text { The egg consumption rate for males between the ages of } 9 \text { and } \\
14 \text { varied between approximately one and } 3 \text { eggs per day. The } \\
\text { true, but unknown value of the egg consumption rate by male } \\
\text { children between the ages of } 9 \text { and } 14 \text { was described by a } \\
\text { uniform distribution because all values are equally probable. }\end{array}$ \\
\hline
\end{tabular}


Table 8.6 (continued)

\begin{tabular}{|c|c|c|c|c|c|}
\hline Parameter & Symbol & Range & Mean & Distribution & Rationale \\
\hline $\begin{array}{l}\text { Average daily } \\
\text { consumption of eggs by } \\
\text { children ages } 9-14 \text { years } \\
\text { (females) }\left(\mathrm{kg} \mathrm{d}^{-1}\right)\end{array}$ & $\begin{array}{c}\mathrm{U}_{\text {eggs, }} 9-14 \\
\text { yrs, female }\end{array}$ & $0.03-0.13$ & 0.08 & Uniform & $\begin{array}{l}\text { The lower limit is equivalent to one-half an egg, while the } \\
\text { upper limit is equivalent to } 2 \frac{1}{2} \text { eggs. A uniform distribution } \\
\text { was selected to represent the uncertainty associated with the } \\
\text { egg consumption rate by females in this age group. }\end{array}$ \\
\hline $\begin{array}{l}\text { Average daily } \\
\text { consumption of eggs by } \\
\text { adults (males) }\left(\mathrm{kg} \mathrm{d}^{-1}\right)\end{array}$ & $\begin{array}{c}\mathrm{U}_{\text {eggs, adult, }} \\
\text { male }\end{array}$ & $0.05-0.20$ & 0.125 & Uniform & $\begin{array}{l}\text { The egg consumption rate for males } 15 \text { years and older ranges } \\
\text { from approximately one to } 4 \text { eggs per day. Since all values } \\
\text { within the range are equally likely, a uniform distribution was } \\
\text { chosen to describe the uncertainty associated with this } \\
\text { parameter. }\end{array}$ \\
\hline $\begin{array}{l}\text { Average daily } \\
\text { consumption of eggs by } \\
\text { adults (females) }\left(\mathrm{kg} \mathrm{d}^{-1}\right)\end{array}$ & $\begin{array}{l}\mathrm{U}_{\text {eggs, adult, }} \\
\text { female }\end{array}$ & $0.05-0.15$ & 0.10 & Uniform & $\begin{array}{l}\text { The lower limit is approximately one egg, while the upper limit } \\
\text { is equivalent to } 3 \text { eggs. The mean value is approximately } 2 \\
\text { eggs. A uniform distribution was selected to describe the true } \\
\text { but unknown egg consumption rate by females because every } \\
\text { value within the range is equally probable. }\end{array}$ \\
\hline
\end{tabular}




\subsubsection{Consumption of Vegetables}

Ingestion of vegetables from gardens grown in areas surrounding the ORR could have resulted in the exposure of humans to radioiodine. Leafy vegetables are of primary concern due to direct fallout of iodine onto their leaves. Leafy vegetables are considered to include lettuce, broccoli, cabbage, celery, and spinach, which are the most frequently consumed leafy vegetables (NCI, 1997). Consumption rates are provided for the following groups: infants (6 months to 1 year) and children (1-4 years) as one group; children ages 5-14 years (both genders); and adults (both genders). The average daily leafy vegetables consumption rates did not vary between males and females. Tables 8.7 and 8.8 provide the values for vegetable consumption. The ranges listed are consistent with values reported by the USDA $(1965 ; 1980)$ and the NCI (1997).

The children in the youngest age group (6 months - 4 years) are considered as one group due to their similar vegetable consumption rates, which do not vary dramatically over this time period (USDA, 1965; 1980). Children younger than 6 months do not consume substantial quantities of leafy vegetables (NCI, 1997) and are not considered reference individuals. Children in the 0-1 year range are consuming large quantities of milk in addition to any solid foods (Mount, personal communication with C. Lewis, 1997). Children older than 1 year are beginning to eat or are eating more solid foods; however, broccoli, cabbage, celery, lettuce, and spinach are not a substantial portion of their diets.

\subsubsection{Consumption of Cottage Cheese}

Cottage cheese is a soft, unripened, white cooked curd that is obtained from skim milk. According to the USDA $(1944 ; 1949)$ and the NCI (1997), cottage cheese is not a typical food source. However, dairies in East Tennessee (Mayfield Dairy Farms, etc.) were making this type of cheese available for sale as early as the 1900s (Sampson, personal communication with C. Lewis, 1997). Since contaminated milk could have been used to make this dairy product, cottage cheese is considered to be a contaminated foodstuff.

The average daily consumption rate of cottage cheese is not reported in the literature on an agespecific basis or on a gender basis. Per capita information per year is available in USDA (1949). The NCI (1997) study reported values ranging from 3-5 grams per day for children between the ages of 6 months to 19 years, based on per capita consumption rates for the entire U.S. population. The average daily consumption rate of cottage cheese by adults (both genders) is consistent with values reported by USDA (1944; 1949) and NCI (1997). The data cover the entire United States and included residents of households who lived in non-farming areas, urban areas, rural non-farming areas, and rural farming areas (USDA, 1944). For rural areas (both farming and non-farming), the consumption of cottage cheese is approximately $0.02 \mathrm{~kg} \mathrm{~d}^{-1}$ 
Table 8.7 Values for parameters used in the vegetable ingestion pathway for routine releases.

\begin{tabular}{|c|c|c|c|c|c|}
\hline Parameter & Symbol & Range & Mean & Distribution & Rationale \\
\hline $\begin{array}{l}\text { Average daily } \\
\text { consumption of leafy } \\
\text { vegetables by children } \\
\text { ages } 6 \text { months to } 4 \text { years } \\
\text { (males and females) } \\
\left(\mathrm{kg} \mathrm{d}^{-1}\right)\end{array}$ & $\begin{array}{l}\mathrm{U}_{\mathrm{veg}, 6 \text { mon- }} \\
4 \text { yrs }\end{array}$ & $0.004-0.014$ & 0.009 & Uniform & $\begin{array}{l}\text { The lower limit is approximately } 0.15 \mathrm{oz} \text {., while the upper } \\
\text { limit is approximately } 0.50 \mathrm{oz} \text {. A uniform distribution was } \\
\text { selected to represent the uncertainty associated with the } \\
\text { consumption of leafy vegetables by infants and young children } \\
\text { because every value within the range has an equal probability } \\
\text { of being the true but unknown annual average consumption } \\
\text { rate. }\end{array}$ \\
\hline $\begin{array}{l}\text { Average daily } \\
\text { consumption of leafy } \\
\text { vegetables by children } \\
\text { ages } 5-14 \text { years }(\text { males } \\
\text { and females })\left(\mathrm{kg} \mathrm{d}^{-1}\right)\end{array}$ & $\mathrm{U}_{\mathrm{veg}, 5-14 \mathrm{yrs}}$ & $0.01-0.04$ & 0.025 & Uniform & $\begin{array}{l}\text { The lower limit is equivalent to approximately } 0.35 \mathrm{oz} \text {., while } \\
\text { the upper limit is equivalent to approximately } 1.5 \mathrm{oz} \text {. of leafy } \\
\text { vegetables per day. The uncertainty associated with this } \\
\text { parameter was described by a uniform distribution. }\end{array}$ \\
\hline $\begin{array}{l}\text { Average daily } \\
\text { consumption of leafy } \\
\text { vegetables by adults } \\
\text { (males and females) } \\
\left(\mathrm{kg} \mathrm{d}^{-1}\right)\end{array}$ & $\mathrm{U}_{\mathrm{veg}, \text { adult }}$ & $0.02-0.06$ & 0.04 & Uniform & $\begin{array}{l}\text { The lower limit is equivalent to } 0.70 \mathrm{oz} \text {., while the upper limit } \\
\text { is approximately } 2 \mathrm{oz} \text {. of leafy vegetables per day. A uniform } \\
\text { distribution was selected to describe the uncertainty associated } \\
\text { with the annual average consumption of leafy vegetables } \\
\text { because every value is equally likely. }\end{array}$ \\
\hline
\end{tabular}


Table 8.8 Values for parameters used in the vegetable ingestion pathway for the accidental release.

\begin{tabular}{|c|c|c|c|c|c|}
\hline Parameter & Symbol & Range & Mean & Distribution & Rationale \\
\hline $\begin{array}{l}\text { Average daily } \\
\text { consumption of leafy } \\
\text { vegetables by children } \\
\text { ages } 6 \text { months to } 4 \text { years } \\
\text { (males and females) } \\
\left(\mathrm{kg} \mathrm{d}^{-1}\right)\end{array}$ & $\begin{array}{l}\mathrm{U}_{\text {veg, } 6 \text { mon- }} \\
4 \text { yrs }\end{array}$ & 0.004-0.014 & 0.009 & Uniform & $\begin{array}{l}\text { No change from the routine release scenario is used in the } \\
\text { accident scenario because a growing infant or child requires } \\
\text { the same amount of nutrition and food regardless of the time of } \\
\text { year. }\end{array}$ \\
\hline $\begin{array}{l}\text { Average daily } \\
\text { consumption of leafy } \\
\text { vegetables by children } \\
\text { ages } 5-14 \text { years }(\text { males } \\
\text { and females) }\left(\mathrm{kg} \mathrm{d}^{-1}\right)\end{array}$ & $\mathrm{U}_{\mathrm{veg}, 5-14 \mathrm{yrs}}$ & $0.02-0.11$ & 0.065 & Uniform & $\begin{array}{l}\text { The lower limit is equivalent to approximately } 1 \mathrm{oz} . \text {, while the } \\
\text { upper limit is equivalent to approximately } 4 \mathrm{oz} \text {. of leafy } \\
\text { vegetables per day. The uncertainty associated with this } \\
\text { parameter was described by a uniform distribution because all } \\
\text { values have equal probability. }\end{array}$ \\
\hline $\begin{array}{l}\text { Average daily } \\
\text { consumption of leafy } \\
\text { vegetables by adults } \\
\text { (males and females) } \\
\left(\mathrm{kg} \mathrm{d}^{-1}\right)\end{array}$ & $\mathrm{U}_{\text {veg, adult }}$ & $0.02-0.23$ & 0.125 & Uniform & $\begin{array}{l}\text { The lower limit is equivalent to } 1 \mathrm{oz} \text {., and the upper limit is } \\
\text { approximately } 8 \mathrm{oz} \text {. of leafy vegetables daily. A uniform } \\
\text { distribution was selected to describe the uncertainty associated } \\
\text { with the annual average consumption of leafy vegetables } \\
\text { because every value is equally likely. }\end{array}$ \\
\hline
\end{tabular}


(assuming one person consumes all cottage cheese available) (USDA, 1944). Rural residents were considered to be the reference individuals since milk was easily accessible if dairy cattle were raised. The range for adults was based on the per capita cottage cheese consumption rates for the years 1944 to 1948 (USDA, 1949). During these four years, consumption varied between 0.003 and $0.004 \mathrm{~kg} \mathrm{~d}^{-1}$ (USDA, 1949). The range is significantly broad to account for varied consumption habits as it encompasses both urban residents, who have limited availability to cottage cheese, and rural residents, who have immediate access to this food source. The distributions associated with cottage cheese consumption are provided in Table 8.9 for routine releases and Table 8.10 for the accident scenario.

\subsection{Inhalation Pathway}

\subsubsection{Description and Modeling Approach}

Although ingestion of cow's milk is the most important route for human exposure to ${ }^{131} \mathrm{I}$, the inhalation pathway involves every individual in the population. Inhalation can become an important route of exposure if other pathways are not relevant. Exposure to ${ }^{131} \mathrm{I}$ through the inhalation pathway depends on the concentration of ${ }^{131} \mathrm{I}$ in air, on the breathing rate of the specific individual, on the ability of each physico-chemical form of iodine to deposit in the respiratory system, and on the metabolism of the specific individual.

The modeling approach chosen for the inhalation pathway is based on the following major assumptions:

- $\quad$ The target individual spends a fraction of time $\left(f_{o}\right)$ outdoors;

- $\quad$ The indoor concentration of ${ }^{131} \mathrm{I}$ in air is lower than the outdoor concentration of ${ }^{131} \mathrm{I}$ by a specified factor $\left(r_{i o}\right)$;

- The amount of ${ }^{131}$ I inhaled is only partially deposited in the respiratory system; the fraction deposited $\left(D_{k}\right)$ is different for each physico-chemical form $k$ of iodine; and

- $\quad$ The ${ }^{131}$ I deposited in the respiratory system is totally absorbed and rapidly transferred to the bloodstream, from where it is metabolized in a manner similar to that of the ingested iodine. 
Table 8.9 Values for parameters used in the cottage cheese ingestion pathway for routine releases.

\begin{tabular}{lccccc}
\hline \multicolumn{1}{c}{ Parameter } & Symbol & Range & Mean & Distribution & Rationale \\
\hline $\begin{array}{l}\text { Average daily consumption of } \\
\text { cottage cheese by children } \\
\text { (males and females) }\left(\mathrm{kg} \mathrm{d}^{-1}\right)\end{array}$ & $\mathrm{U}_{\text {cheese, child }}$ & $0.001-0.01$ & 0.0055 & Uniform & $\begin{array}{l}\text { The lower limit represents a 1-oz. serving every month, while the } \\
\text { upper limit is equivalent to a 2.5 oz. serving per week. A uniform } \\
\text { distribution was chosen to describe the lack of knowledge about a } \\
\text { child's annual average cottage cheese consumption rate. }\end{array}$ \\
$\begin{array}{l}\text { Average daily consumption of } \\
\text { cottage cheese by adults (males } \\
\text { and females) }\left(\mathrm{kg} \mathrm{d}^{-1}\right)\end{array}$ & $\mathrm{U}_{\text {cheese, adult }}$ & $0.001-0.02$ & 0.01 & Uniform & $\begin{array}{l}\text { A uniform distribution with a mean of 0.01 kg d } \mathrm{d}^{-1} \text { was selected to } \\
\text { represent the uncertainty associated with the annual average } \\
\text { consumption rate of cottage cheese by adults (males and females). }\end{array}$ \\
\hline
\end{tabular}

Table 8.10 Values for parameters used in the cottage cheese ingestion pathway for the accidental release.

\begin{tabular}{|c|c|c|c|c|c|}
\hline Parameter & Symbol & Range & Mean & Distribution & Rationale \\
\hline $\begin{array}{l}\text { Average daily consumption of } \\
\text { cottage cheese by children } \\
\text { (males and females) }\left(\mathrm{kg} \mathrm{d}^{-1}\right)\end{array}$ & $\mathrm{U}_{\text {cheese, child }}$ & $0.001-0.01$ & 0.0055 & Uniform & $\begin{array}{l}\text { No change from the routine release scenario is used in the accident } \\
\text { scenario because the range is sufficiently large to account for } \\
\text { variations in the monthly consumption habits of cottage cheese by } \\
\text { children. }\end{array}$ \\
\hline $\begin{array}{l}\text { Average daily consumption of } \\
\text { cottage cheese by adults (males } \\
\text { and females) }\left(\mathrm{kg} \mathrm{d}^{-1}\right)\end{array}$ & $\mathrm{U}_{\text {cheese, adult }}$ & $0.001-0.03$ & 0.02 & Uniform & $\begin{array}{l}\text { The lower limit is equivalent to a } 1-\mathrm{oz} \text {. serving per month. The } \\
\text { upper limit is equivalent to approximately one } 8-\mathrm{oz} \text {. serving per } \\
\text { week. A uniform distribution with a mean of } 0.02 \mathrm{~kg} \mathrm{~d}^{-1} \text { was } \\
\text { selected to represent the uncertainty associated with the monthly } \\
\text { average consumption rate of cottage cheese by adults. }\end{array}$ \\
\hline
\end{tabular}


The total intake of ${ }^{131}$ I from inhalation of contaminated air is given by the following equation:

$$
T I_{I N H, i}=\left(\sum_{k=1}^{3}\left(f_{o}+\left(1-f_{o}\right) \cdot r_{i o}\right) \cdot C_{a, o, j, k} \cdot B R_{i} \cdot D_{k}\right) \cdot \Delta t
$$

where

$$
\begin{aligned}
& T I_{I N H, i} \quad=\text { total intake of }{ }^{131} \mathrm{I} \text { from inhalation at age } i[\mathrm{~Bq}] \text {; } \\
& f_{o} \quad=\text { fraction of time spent outdoors [unitless]; } \\
& r_{i o}=\text { ratio of the indoor to outdoor concentrations of iodine in air [unitless]; } \\
& C_{a, o, j, k}=\text { concentration of iodine in form } k \text { in outside air in year of release } j \\
& {\left[\mathrm{~Bq} \mathrm{~m}^{-3} \text { air }\right] \text { (Section 4); }} \\
& B R_{i} \quad=\text { breathing rate for an individual at age } i\left[\mathrm{~m}^{3} \text { air } \mathrm{d}^{-1}\right] \text {; } \\
& D_{k} \quad=\text { fraction of the total amount inhaled that deposits and is absorbed in } \\
& \text { different parts of the respiratory system for each physico-chemical form } \\
& k \text { (see Section 9.3.1.1); } \\
& i \quad=\text { age of the individual; } \\
& j \quad=\quad \text { year of release }(j=1944+i \text { for an individual born in } 1944, j=1952+i \\
& \text { for an individual born in 1952, etc.); } \\
& k \quad=\text { physico-chemical forms of iodine in air }(k=1 \text {, elemental; } k=2 \text {, } \\
& \text { particulate; } k=3 \text {, organic); and } \\
& \Delta t \quad=\text { the time period corresponding to year } j \text { [365 days]. }
\end{aligned}
$$

\subsubsection{Input Parameters}

The concentration in air of each physico-chemical form of iodine is calculated as described in Section 4. The rationales for the choice of the parameter values (Table 8.11) are presented below. 


\subsubsection{Fraction of the day spent outdoors}

The amount of time that an individual spends outdoors is highly dependent on gender, age, and lifestyle. Statistics on this parameter are sparse (Snyder et al., 1994) and practically nonexistent for the late 1940s and early 1950s; thus, professional judgment was used to define the values for this parameter. For children less than 2 years of age, the distribution for the fraction of time spent outdoors was chosen to be a triangular distribution with a most likely value of 0.05 . This represents an average of 1.2 hours per day. The values for this age group range from 0.02 (half an hour a day) to 0.2 (about 5 hours a day). For children 3 to 17 years old, the distribution is also triangular, with a most likely value of 0.21 (about 5 hours a day) and a range of values from 0.063 (about one and a half hour a day) to 0.29 (about 7 hours a day). Adults spend time outdoors or indoors according to their occupation. Workers in rural areas spend at most 10 hours outdoors every day (a fraction equal to 0.42 ). On the other hand, elderly people or housewives taking care of small children spend at least one hour and a half outside (a fraction equal to 0.063 of the year). For adults, a triangular distribution was selected with a minimum of 0.063, a maximum of 0.42 and a mode of 0.33 (eight hours a day). The distributions described above are intended to represent annual averages; they are not representative for a given individual season of the year (Table 8.11).

For an accidental release, the time spent outside will depend on the season of the year. This study investigates the consequences of the accidental release of April 29, 1954. The temperature during that day varied from 63 to $78^{\circ} \mathrm{F}$, which is within normal ranges for late spring. The assumptions on the parameter values for the fraction of time spent outdoors during the day of the 1954 accident are based on professional judgment and on the values recommended by Snyder et al. (1994) for the spring to summer season (Table 8.11).

The duration of the 1954 accidental release was only 2.5 hours (4:30 p.m. to 7:00 p.m.). Since this time period covers the normal dinner time, and since heavy rain was recorded in the last hour $\left(1.7 \mathrm{~mm} \mathrm{~h}^{-1}\right)$, it is conceivable that many individuals were indoors during the passage of the plume. Thus, the lower limits of the distributions selected for the accident case were set to zero. On the other hand, it is possible that other individuals spent at least 2 hours outdoors, since the accident began at 4:30 p.m., and the rain started only after 6:00 p.m.

For children older than 3 and for adults in both rural and urban areas, the upper limit of the distributions have been set to 2 hours for the day of the accident. This gives a daily fraction of time equal to 0.083. For children under the age of two, a maximum of 1 hour was selected, representing the possibility that they were taken out for a walk or for play activities during the afternoon of April 29, 1954. To equally cover all the possibilities, uniform distributions were selected. 
Table 8.11 Summary of the input parameters used in the estimation of dose and excess lifetime risk due to inhalation of ${ }^{131} \mathrm{I}$.

\begin{tabular}{|c|c|c|c|c|c|c|}
\hline \multirow[t]{2}{*}{ Parameter } & \multirow[t]{2}{*}{ Units } & \multicolumn{4}{|c|}{ Distribution } & \multirow[b]{2}{*}{$\begin{array}{l}\text { Type of } \\
\text { release }\end{array}$} \\
\hline & & lower & upper & mode & shape & \\
\hline \multicolumn{7}{|c|}{ Fraction of time spent outdoors } \\
\hline \multicolumn{7}{|c|}{ (hours per day) } \\
\hline$f_{O}-$ age $0-2$ & [unitless] & $\begin{array}{c}0.0 \\
(0.0)\end{array}$ & $\begin{array}{c}0.042 \\
(1.0)\end{array}$ & & uniform & Accidental \\
\hline$f_{0}-$ age 3 or older & [unitless] & $\begin{array}{c}0.0 \\
(0.0)\end{array}$ & $\begin{array}{c}0.083 \\
(2.0)\end{array}$ & & uniform & Accidental \\
\hline$f_{O}$ - age $0-2$ & [unitless] & $\begin{array}{l}0.02 \\
(0.5)\end{array}$ & $\begin{array}{l}0.21 \\
(5.0)\end{array}$ & $\begin{array}{l}0.05 \\
(1.2)\end{array}$ & triangular & Routine \\
\hline$f_{0}-$ age $3-17$ & [unitless] & $\begin{array}{c}0.063 \\
(1.5)\end{array}$ & $\begin{array}{l}0.29 \\
(7.0)\end{array}$ & $\begin{array}{l}0.21 \\
(5.0)\end{array}$ & triangular & Routine \\
\hline$f_{0}-$ age $17+$ & [unitless] & $\begin{array}{c}0.063 \\
(1.5)\end{array}$ & $\begin{array}{c}0.42 \\
(10.0)\end{array}$ & $\begin{array}{l}0.33 \\
(8.0)\end{array}$ & triangular & Routine \\
\hline \multicolumn{7}{|c|}{ Ratio of indoor to outdoor concentrations } \\
\hline$r_{i o}$ - (gases) & [unitless] & 0.3 & 1 & & uniform & Routine \\
\hline$r_{i o}-($ particles $)$ & [unitless] & 0.4 & 1 & & uniform & Routine \\
\hline$r_{i o}-$ - (gases) & [unitless] & 0.3 & 0.9 & 0.8 & triangular & Accidental \\
\hline$r_{i o}-$ (particles) & [unitless] & 0.4 & 0.9 & 0.8 & triangular & Accidental \\
\hline
\end{tabular}




\subsubsection{Indoor to outdoor concentration ratio}

The air inside a building is expected to have a different concentration of ${ }^{131}$ I than the air outside the building, unless free air exchange occurs through open windows or doors. When windows and doors are closed, some air is still exchanged between indoors and outdoors, either naturally through openings due to imperfect sealing or by a ventilation system. For rural areas, the ventilation systems were not well developed during the late 1940s and early 1950s. Air exchange by window or door opening was probably a common practice, especially during warm weather. For a given outdoor concentration of a contaminant, the indoor concentration of a contaminant is expected to have been larger during the 1940s and 1950s than in present times.

The concentration of a contaminant in indoor air is a function of the rate at which the contaminant is entering the building from the outdoor air, the rate of indoor production of the contaminant (not an issue for ${ }^{131} \mathrm{I}$ ), and the rate at which the contaminant is leaving the building. In the first approximation (for environmental levels of air contamination), the outdoor air can be considered an infinite source of contaminant at an ever-changing concentration (e.g., the outdoor concentration is not changed by the air exchange with the air in the building, but only by the movement of the outside air). Because of the dynamic exchange process, the concentration inside a building may be higher than the outside concentration at a given moment of time. An explanation for such a situation is that the air inside reaches a peak concentration after the contaminated plume passes the building. However, on a time-averaged basis, the inside concentration is lower than the outside concentration.

Various studies have been performed to determine a relationship between outdoor and indoor air concentrations. In this report, a simple relationship, defined as a long-term average ratio between the indoor and outdoor concentrations, is used. Three literature reviews of experimental measurements have been used to support values selected for the indoor-to-outdoor concentration ratio: Andersen (1972), Benson et al. (1972), and Snyder et al. (1994).

Andersen (1972) summarized 11 studies investigating indoor/outdoor ratios for sulfur dioxide $\left(\mathrm{SO}_{2}\right)$ and for suspended particle matter. The investigations took place from 1954 to 1969 in various part of the world, including Cincinnati, Ohio, and Hartford, Connecticut. For gaseous $\mathrm{SO}_{2}$, the indoor/outdoor ratios varied from $20 \%$ to $100 \%$. For suspended matter, a low range of values $(20-60 \%)$ was observed in Tokyo, Japan, while for other locations, the ratio varied from $40 \%$ to $95 \%$. Andersen (1972) also reports his own set of measurements performed in Denmark: $51 \%$ for $\mathrm{SO}_{2}$ and $83 \%$ for suspended particulate matter.

Benson et al. (1972) compiled many indoor/outdoor ratios for gases $\left[\mathrm{SO}_{2}\right.$, carbon monoxide (CO), and gaseous substances other than $\mathrm{SO}_{2}$ and $\mathrm{CO}$ ], for "viable" particles (spores, pollen, and bacteria), and for particulate matter. A number of the reported measurements of the indoor/outdoor ratio are larger than $100 \%$. As argued before, these values may be valid in a single measuring event, but they do not apply for a long-term average of the indoor/outdoor ratio. For gases, the values below $100 \%$ ranged from $20 \%$ to $75 \%$ for $\mathrm{SO}_{2}$, from $59 \%$ to $100 \%$ for $\mathrm{CO}$, and from $34 \%$ to $80 \%$ for other gases. For particulate matter, a minimum value of $16 \%$ was 
observed in a 1971 measurement in Hartford, Connecticut, during wintertime. However, most of the measurements indicated values larger than $40 \%$, with a maximum of $100 \%$.

Two more recent studies (Hawley, 1985; Christensen and Mustonen, 1987) are cited by Snyder et al. (1994) in a review performed for the reconstruction of ${ }^{131}$ I doses for people living around the Hanford Nuclear Facility in the state of Washington. The reported experimental measurements of the indoor/outdoor ratio for Norwegian houses built in 1954 range from $40 \%$ to $86 \%$ (Christensen and Mustonen, 1987). In another set of measurements, a minimum value of 35\% was reported by Hawley (1985).

Snyder et al. (1994) made no difference between different species of iodine (gases versus particulate matter), and they assigned a uniform distribution from 35\% to $100 \%$ for the indoor-tooutdoor ratio. This range of values appears appropriate because it eliminates very low values, which are probably artifacts of special measurement conditions (such as a cold wintertime when the exchange of air between indoors and outdoors is deliberately limited). The maximum value $(100 \%)$ takes into account the situation when there is free airflow between indoors and outdoors.

Data collected for the present study indicate that the indoor-to-outdoor ratios for gases are slightly lower than for particulate matter. For gases, the distribution for this parameter was chosen to be uniform, with a minimum value of $0.3(30 \%)$ and a maximum value of $1(100 \%)$. For particulate matter, a uniform distribution between 0.4 and 1.0 (40\% to $100 \%$ ) was selected. These distributions are applied for routine releases.

The plume from the 1954 accident (April 29, 1954), released from both the RaLa building and the stack, covered the area of interest in few hours. The temperature during that day had a maximum of $78^{\circ} \mathrm{F}$ in the early afternoon. The minimum temperature $\left(63^{\circ} \mathrm{F}\right)$ was registered around 6 a.m. the next day, while most of the time during the night of April 29-30, the temperature was about $64^{\circ} \mathrm{F}$. Given the relatively warm weather, it is highly probable that some individuals would have kept their windows open, and free exchange between outdoor and indoor air was possible in many homes. Normally, the windows might have been closed during the rain event, but the rain started only after 6:00 p.m., while most of the ${ }^{131}$ I was released between 4:30 p.m. and 6:00 p.m. (Table 3.18). Thus, for this particular situation, the rain event should have had little influence on the indoor/outdoor exchange of air.

Triangular distributions with a mode of $0.8(80 \%)$ and upper limits of $0.9(90 \%)$ have been chosen for the indoor-to-outdoor ratio for the accidental release. The same lower limits as in the case of routine releases have been preserved for the accident case, since some of the houses might have had their windows closed, thus allowing for much less air exchange. 


\subsubsection{Age-dependent breathing rate}

Age-dependent breathing rates are reported by Roy and Courtay (1991). These values were also recommended by the NCI (1997) as adequate for the general population. The values have been linearly interpolated between different age groups. The values in Table 8.12 are medians of lognormal distributions for each age group. A geometric standard deviation of 1.3, applicable for all age groups, was chosen based on the work of Roy and Courtay (1991) and on recommendations from the NCI (1997).

Table 8.12 Age-dependent breathing rates for exposed individuals.

\begin{tabular}{|c|c|c|c|c|c|}
\hline \multirow[t]{2}{*}{ Parameter } & \multirow[t]{2}{*}{ Units } & \multicolumn{4}{|c|}{ Distribution } \\
\hline & & $\begin{array}{c}\text { Geometric } \\
\text { Mean }\end{array}$ & $\begin{array}{l}\text { Geometric } \\
\text { Standard } \\
\text { Deviation }\end{array}$ & Shape & Type of release \\
\hline BR - age $0-1$ & {$\left[\mathrm{~m}^{3} \mathrm{~d}^{-1}\right]$} & 3.5 & 1.3 & Lognormal & accidental and routine \\
\hline $\mathrm{BR}$ - age $1-4$ & {$\left[\mathrm{~m}^{3} \mathrm{~d}^{-1}\right]$} & 7.0 & 1.3 & Lognormal & accidental and routine \\
\hline BR - age 5 - 9 & {$\left[\mathrm{~m}^{3} \mathrm{~d}^{-1}\right]$} & 12.0 & 1.3 & Lognormal & accidental and routine \\
\hline $\mathrm{BR}$ - age $10-14$ & {$\left[\mathrm{~m}^{3} \mathrm{~d}^{-1}\right]$} & 17.0 & 1.3 & Lognormal & accidental and routine \\
\hline $\begin{array}{l}\mathrm{BR} \text { - age } 15-19 \\
\quad \text { (females) }\end{array}$ & {$\left[\mathrm{m}^{3} \mathrm{~d}^{-1}\right]$} & 18.0 & 1.3 & lognormal & accidental and routine \\
\hline $\begin{array}{l}\mathrm{BR} \text { - age } 15-19 \\
\quad \text { (males) }\end{array}$ & {$\left[\mathrm{m}^{3} \mathrm{~d}^{-1}\right]$} & 19.0 & 1.3 & lognormal & accidental and routine \\
\hline $\begin{array}{l}\text { BR - adult } \\
\text { (females) }\end{array}$ & {$\left[\mathrm{m}^{3} \mathrm{~d}^{-1}\right]$} & 18.0 & 1.3 & lognormal & accidental and routine \\
\hline $\begin{array}{l}\text { BR -adult } \\
\text { (males) }\end{array}$ & {$\left[\mathrm{m}^{3} \mathrm{~d}^{-1}\right]$} & 23.0 & 1.3 & lognormal & accidental and routine \\
\hline
\end{tabular}




\subsection{References}

ADGA (American Dairy Goat Association). 1998. Did you know? Dairy goat facts. http://www.adga.org/facts.htm. August 6, 1998.

Andersen I. 1972. Relationship Between Outdoor and Indoor Air Pollution. Atmospheric Environment 6: 275-278.

Anderson, D.M., Bates, D. J., and Marsh, T.L. 1993. Estimation of 1945 to 1957 food consumption. Hanford Environmental Dose Reconstruction Project, Battelle Pacific Northwest Laboratories. PNWD-2113 HEDR UC-000.

ATSDR (Agency for Toxic Substances and Disease Registry). 1992. Public health assessment guidance manual. U.S. Department of Health and Human Services. Atlanta, Georgia.

Benson F.B., Henderson J.J., and Caldwell, D.E. 1972. Indoor-Outdoor Air Pollution Relationships: A Literature Review. Environmental Protection Agency. National Environmental Research Center. Research Triangle Park, North Carolina.

Christensen, G.C., and Mustonen, R. 1987. The Filtering Effect of Buildings on Airborne Particles. Radiation Protection Dosimetry 21(1-3):125-128.

Clark, C.M. 1997. Resident of the Claxton, Tennessee, community who raised and consumed milk and beef products from backyard cattle. Telephone interview with C. Lewis. April 25, 1997.

Cochrane, W.W. 1945. High-level food consumption in the United States. Bureau of Agricultural Economics, U.S. Department of Agriculture. Miscellaneous Publication No. 581. Washington, DC.

Cole, H.H., and Ronning, M. (editors). 1974. Animal Agriculture: The Biology of Domestic Animals and Their Use by Man. W.H. Freeman and Company. San Francisco, California.

Downen, M.L. 1955. Milk consumption in Tennessee schools. Rural research series. Department of Agricultural Economics and Rural Sociology. Monograph No. 271.

Downen, M.L. 1956. Milk consumption in Tennessee schools. Rural research series. Department of Agricultural Economics and Rural Sociology. Monograph No. 274.

Dreicer, M., Bouville, A., and Wachholz, B.W. 1990. Pasture practices, milk distribution, and consumption in the continental U.S. in the 1950s. Analyses and modeling for internal dose estimates. Health Physics 59(5):627-636.

Eisenberg, A., Murkoff, H.E., and Hathaway, S.E. 1994. What to expect when you're expecting. Revised Edition. Workman Publishing Company. 
Hackett, B. 1998. Resident of Hickory Creek/Gallaher Bend Community who consumed goat milk as a child. Telephone interview with C. Lewis. May 29, 1998.

Haenlein, G.F.W., and Caccese, R. 1998. Goat milk versus cow milk. Goat Handbook. http://www.inform.umd.edu:8080/EdRes/Topic/AgrEnv/ndd/goat/. May 27, 1998.

Hawley, J.K. 1985. Assessment of Health Risk from Exposure to Contaminated Soil. Risk Analysis 5(4):289-302.

Hoffman, F.O., and Baes, C. 1979. A Statistical Analysis of Selected Parameters for Predicting Food Chain Transport and Internal Dose of Radionuclides. Oak Ridge National Laboratory. NUREG/CR-1004, ORNL/NUREG/TM-282.

Hudson, J. 1998. Resident of Hickory Creek/Gallaher Bend Community who consumed goat milk as a child. Telephone interview with C. Lewis. May 19, 1998.

Kapture, J. 1998. Goats. Goat Handbook. http://www.inform.umd.edu/ EdRes/Topic/AgrEnv/ndd/goat/GOATS.html. August 6, 1998.

Leuchs Farm. 1998. Operating dairy goat farm in rural Jackson, New Jersey. http://members.aol.com/MLeuchs/index.html. September 10, 1998.

Loveday, D. 1998. Professor in Food Technology at the University of Tennessee. Knoxville, Tennessee. Telephone interview with C. Lewis, March 4, 1998.

Mount, K. 1997. Registered dietitian, licensed nutritionist, and dietitian at East Tennessee Children's Hospital. Telephone interview with C. Lewis, November 4, 1997.

National Cancer Institute (NCI). 1997. Estimated Exposures and Thyroid Doses Received by the American People from Iodine-131 in Fallout Following Nevada Atmospheric Nuclear Bomb Tests. A report from the National Cancer Institute. U.S. Department of Health and Human Services. National Institutes of Health. National Cancer Institute, Washington DC.

Pao, E.M., and Burke, M.C. 1975. Portion sizes and days intake of selected foods. U.S. Department of Agriculture, Agricultural Resource Services Northeast. ARS-NE-67. Cited in Rupp, 1980.

Prichard, D. 1997. Long-time resident and farmer in Roane County, Tennessee. Personal communication with C. Lewis. April 8, 1997.

Roy, M., and Courtay, C., 1991. Daily Activities and Breathing Parameters for Use in Respiratory Tract Dosimetry. Radiation Protection Dosimetry 35(3):179-186.

Rupp, E.M. 1980. Age dependent values of dietary intake for assessing human exposures to environmental pollutants. Health Physics 39:151-163. 
Sampson, S. 1997. Consumer Affairs Delegate for Mayfield Dairy Farms, Inc. Telephone interview with C. Lewis. December 1, 1997.

Schwartz, G., and Hoffman, F.O., 1980. Imprecision of Dose Predictions for Radionuclides Released to the Environment: An Application of a Monte Carlo Simulation Technique. Environment International 4:289-297.

Simon, S.L., Lloyd, R.D., and Till, J.E. 1990. Development of a method to estimate thyroid dose from fallout radioiodine in cohort study analyses and modeling internal dose estimates. Health Physics 59(5):669-691.

Snyder, S.F., Farris, W.T., Napier, B.A., Ikenberry, T.A., and Gilbert, R.O. 1994. Parameters Used in the Environmental Pathways and Radiological Dose Modules (DESCARTES, CIDER, AND CRD Codes) of the Hanford Environmental Dose Reconstruction Integrated Codes (HEDRIC). Battelle Pacific Northwest Laboratories, Richland, Washington. PNWD-2033 HEDR Rev. 1.

USDA (U.S. Department of Agriculture). 1941. Family Food Consumption and Dietary Levels Five Regions, Miscellaneous Publication No. 405. Washington, D.C.

USDA (U.S. Department of Agriculture). 1944. Family food consumption in the United States, Spring 1942. Bureau of Human Nutrition and Home Economics. Report No. 550. Washington, DC.

USDA (U.S. Department of Agriculture). 1949. Consumption of Food in the United States, 1909-48. Bureau of Agricultural Economics. Miscellaneous Publication 691. Washington, DC.

USDA (U.S. Department of Agriculture). 1955a. Dietary Levels of Households in the South, Household Food Consumption Survey 1955, Report No. 9. Washington, D.C.

USDA (U.S. Department of Agriculture). 1955b. Dietary Levels of Households in the United States, Household Food Consumption Survey 1955, Report No. 6. Washington, D.C.

USDA (U.S. Department of Agriculture). 1965. Food and nutrient intake of individuals in the United States. Household food consumption survey 1965-1966. Agricultural Research Service. Report No. 11. Washington, DC.

USDA (U.S. Department of Agriculture). 1966. Food Consumption of Households in the United States, Seasons and Year 1965-1966, Report No. 12. Washington, D.C.

USDA (U.S. Department of Agriculture). 1980. Food and nutrient intakes of individuals in one day in the United States, Spring 1977. Nationwide food consumption survey 1977-78. Report No. 2. Washington, DC.

University of California, Irvine (UCI). 1998. Dairy goats. http://www.ics.uci.edu/ $\sim$ pazzani/4H/DairyGoats.html. August 6, 1998. 
TASK 1 REPORT

July 1999

Page 8-40
${ }^{131}$ I Releases from X-10 Radioactive Lanthanum Processing Food Consumption/Inhalation Parameters

This page intentionally left blank. 


\subsection{INTERNAL DOSIMETRY FOR IODINE-131}

Radiogenic thyroid cancer increases with the radiation dose to the thyroid gland, depending on the gender and age at exposure (see Section 10). To estimate the thyroid dose, a quantitative evaluation of the behavior of radioiodine in the human body is necessary. After ingestion of contaminated food, iodine is completely absorbed into the blood stream (ICRP, 1989). On the other hand, during inhalation of contaminated air, a fraction of the inhaled iodine is deposited in the lungs and transferred to blood (Section 9.3.1.1). Radioiodine absorbed into the bloodstream is metabolized similarly to stable iodine. Usually, less than one-fourth of the iodine in the bloodstream is absorbed by the thyroid gland, while about three-fourths is collected by the kidneys and excreted in urine (Guyton, 1991).

This section describes the biological basis and the methodology for estimating the dose delivered to the thyroid by the ${ }^{131} \mathrm{I}$ accumulated in the interior of the gland. The dose to the thyroid per unit intake of ${ }^{131} \mathrm{I}$ is called the "dose factor" or "dose coefficient." Such coefficients have been estimated by Dunning and Schwartz (1981), Killough and Eckerman (1986), and the International Commission on Radiological Protection (ICRP, 1989). Calculation of the dose factors for the thyroid gland has been repeated in this report to make use of the most recent data on the mass of the thyroid obtained by ultrasonography and to identify the most important contributors to the uncertainty in the dose factor.

\subsection{Biokinetics of ${ }^{131} I$ in the Human Body}

The thyroid gland is regulated by thyroid-stimulating hormone (TSH), which is produced by the anterior pituitary gland. The passage of iodine from the extracellular fluid (supplied by blood) into the thyroid cells and follicles is controlled by the basal membrane of the thyroid cell, which traps iodide. The concentration of iodine in the cells of a thyroid functioning at normal levels is about 30 times larger than the concentration in blood. Iodine is used in production of two significant thyroid hormones, thyroxine (T4) and triiodothyronine (T3). These hormones, which are secreted to blood, have very important roles in controlling the metabolic rate of the body. Complete lack of thyroid secretion usually causes the basal metabolic rate to fall to about $40 \%$ below normal, and extreme excess of thyroid hormones can cause the basal metabolic rate to rise as high as 60 to $100 \%$ above normal (Guyton, 1991).

After formation, the major thyroid hormones are stored inside the thyroid gland, in follicular colloids, outside the cells where the hormones are produced. For an adult, the total amount of thyroid hormone stored is sufficient to supply the body with its normal requirement for 2 to 3 months (Guyton, 1991). The storage period of hormones in the gland defines the biological halflife of iodine in the thyroid, which is estimated at about 80 days in adults (ICRP, 1979). This long storage time allows most of the ${ }^{131}$ I to decay before the hormones are released to blood, thus irradiating the thyroid gland.

Once the hormones are released to the blood, they are transported to cells all over the body, where they are metabolized. Metabolization of the hormones releases the iodine, which again enters the bloodstream. However, due to the long storage time of the hormones in the thyroid 
gland as compared to the short half-life of ${ }^{131} \mathrm{I}$, very little ${ }^{131} \mathrm{I}$ is actually recycled in this manner. As a result, recycling contributes little to the radiological dose for a given intake of ${ }^{131} \mathrm{I}$.

Certain characteristics of the thyroid gland, such as its mass, are dependent on the age of the individual. Similarly, the metabolic parameters, such as the uptake of iodine by the thyroid or the storage time of the hormones in the gland, are also age-dependent. For the same amount of iodine ingested, the uptake of iodine by the thyroid is greater in children than in adults. Children, however, have a much lower mass of the thyroid than adults. They store iodine for a shorter period of time than adults, but the lower thyroid mass during childhood makes the dose to a child's thyroid appreciably higher than the dose to an adult's thyroid. Children are the critical population subgroup for exposure to ${ }^{131} \mathrm{I}$, not only because of the higher dose per unit intake, but also because their thyroids are more radiosensitive than the thyroid of an adult (see Section 10).

\subsection{Fraction of energy absorbed in the thyroid}

The largest energy from an electron emission from ${ }^{131} \mathrm{I}$ decay is approximately $0.35 \mathrm{MeV}$ and occurs in less than $0.2 \%$ of the disintegrations of ${ }^{131} \mathrm{I}$ (Figure 9.1). Given that the density of the thyroid is $1.036-1.064 \mathrm{~g} \mathrm{~cm}^{-3}$ (ICRP, 1979), the distance traveled by this electron in the thyroid tissue is about $1 \mathrm{~mm}$ (Turner, 1986). Iodine is not uniformly distributed in the thyroid itself, accumulating primarily in the thyroid follicles. The follicles are surrounded by follicular cells, connective tissue, and blood vessels and are located further than $1 \mathrm{~mm}$ deep inside the thyroid gland. As a consequence, the electrons emitted by each nuclear transformation of ${ }^{131} \mathrm{I}$ are assumed to be completely absorbed by the thyroid gland.

Part of the energy of the penetrating radiation emitted during decay of ${ }^{131} \mathrm{I}$ (gamma and X-rays) escapes from the thyroid. The amount of energy deposited in the thyroid gland, along with its dependence on the size of the gland, is described by the absorbed energy fraction (Section 9.3.1).

\subsection{Modeling Approach}

The dose to the thyroid can be calculated using the methodology described by the ICRP (Eckerman, 1994; Killough and Eckerman, 1986). The amount of radioactivity present in the thyroid depends on the age and gender of the individual and can be estimated using a twocompartment biokinetic model (Figure 9.2). 
TASK 1 REPORT

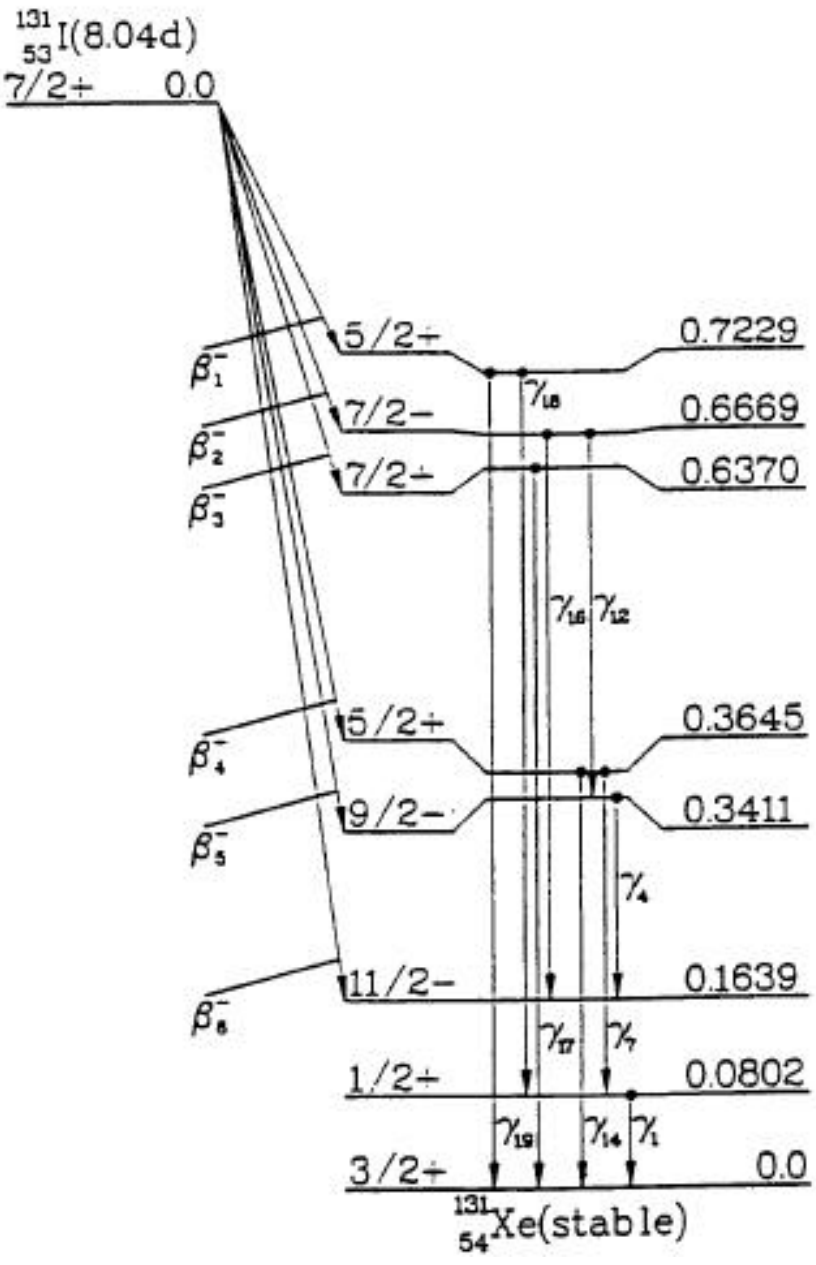

3a - IODINE-131

MALFLIFE DEM DAYS
DELAY MODEIST: $J^{\circ}$

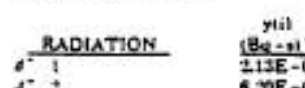

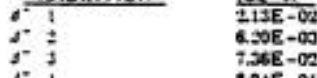

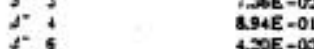

$+16 \quad 2.62 E-02$

$0-k,+1$ 2.6JE -02

$+1,+120 \mathrm{E}-09$

$\div \div$

a $-\mathrm{K}, \mathrm{I}$

+12 , 12 (2)

$\rightarrow 14$

E*K. $>14 \quad 1.58-02$

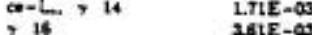

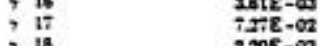

$?$ is $220 \mathrm{E}-\infty$

i ${ }^{12} \mathrm{X}-\mathrm{nay}$

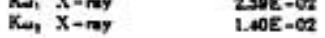

1. $.0 E-62$

LESTED $X$, Y AND II RADLATIONS

OMITED $\mathrm{X}$ + AND TI RNDUTIONS

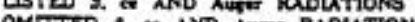

LISTED RUDUTEONS

OMTTED RADLATIONS:-

- avernaE ENERCY (Mov)

- EACH OMrtTre transtrton contrmutres

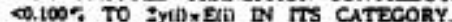
XENON-I3:M DAUCHTER, YEID t.1LE-02

IS RNDIOACTIVE

XENON-1TI DACCHTER, YLELD 2ASE-OL.

is STABLE.

Figure 9.1 Nuclear decay scheme for ${ }^{131}$ I (ICRP, 1983) 
TASK 1 REPORT

July 1999

${ }^{131}$ I Releases from X-10 Radioactive Lanthanum Processing-

Page 9-4 Internal Dosimetry for Iodine-131

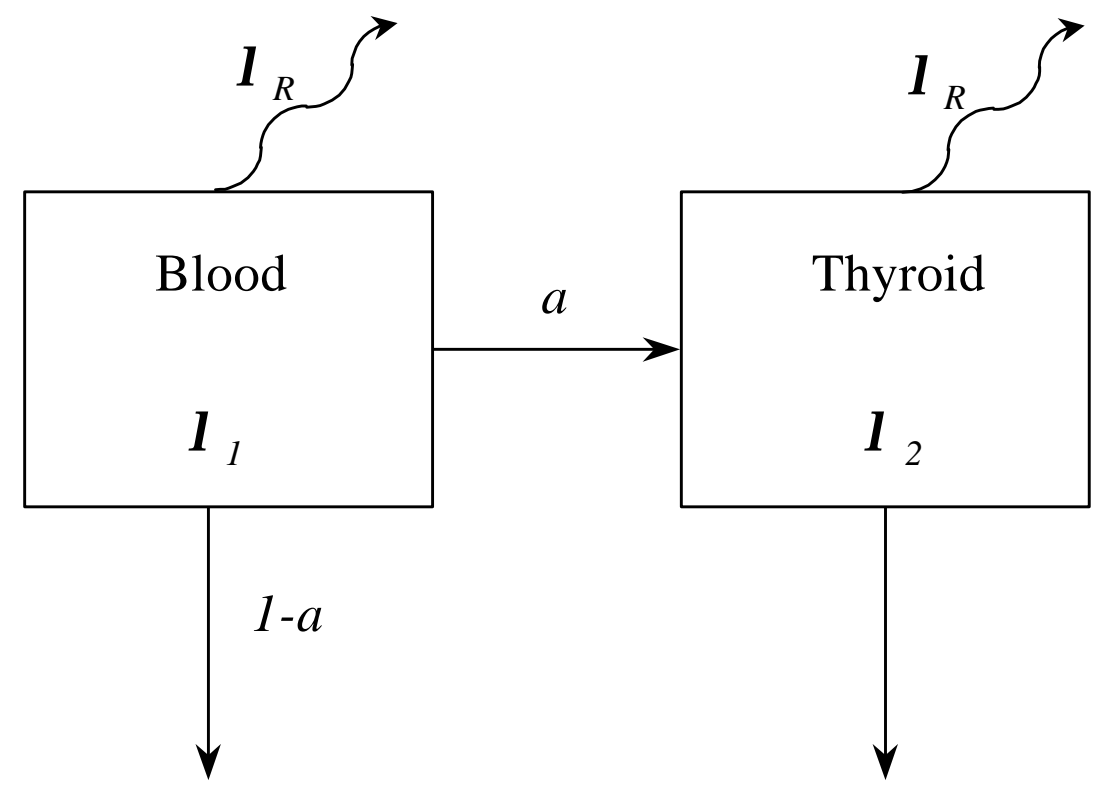

Figure 9.2 Two-compartment biokinetic model for iodine (Killough and Eckerman, 1986). $8_{1}$ and $8_{2}$ are the biological rate constants for each compartment, $8_{R}$ is the radioactive decay rate of ${ }^{131} \mathrm{I}$, and $a$ is the fraction of ${ }^{131}$ I transferred from blood to the thyroid. 
The dose factor is given by the following equations:

$$
D F=I A \cdot S E E
$$

and

$$
I A=\frac{\left(a \cdot \lambda_{1}\right)}{\left(\lambda_{R}+\lambda_{1}\right)\left(\lambda_{R}+\lambda_{2}\right)}
$$

where

$$
\begin{aligned}
& D F=\quad \text { the dose factor }\left(\mathrm{Gy} \mathrm{Bq}^{-1}\right) ; \\
& I A=\quad \text { the integrated activity burden of }{ }^{131} \mathrm{I} \text { in the thyroid for a unit intake }(\mathrm{d}) ; \\
& S E E=\quad \text { specific effective energy }\left(\mathrm{Gy} \mathrm{Bq}^{-1} \mathrm{~d}^{-1}\right) ; \\
& a=\quad \text { the fractional uptake of }{ }^{131} \mathrm{I} \text { from the blood to the thyroid (unitless); } \\
& \lambda_{1}=\quad \text { rate constant for the transfer of iodine from blood to thyroid }\left(\mathrm{d}^{-1}\right) ; \\
& \lambda_{2}=\quad \text { rate constant for the transfer of iodine from thyroid to blood }\left(\mathrm{d}^{-1}\right) ; \text { and } \\
& \lambda_{R}=\quad \text { iodine-131 radioactive decay rate }\left(\mathrm{d}^{-1}\right) .
\end{aligned}
$$

The specific effective energy ( $S E E$ ) is the energy deposited per unit mass of the thyroid from the nuclear transformations given by an activity of $1 \mathrm{~Bq}$ stored in the thyroid gland for 1 day. SEE depends on the fraction of energy absorbed in the gland. The largest contribution to the SEE is the nonpenetrating radiation ( $\beta$ radiation, conversion electrons, and Auger electrons), which is completely absorbed in the thyroid. The contribution of the penetrating radiation (gamma and $\mathrm{X}$-radiation) is much less important. The $S E E$ can be empirically expressed as a function of thyroid mass $\left(M_{t h}\right)$ :

$$
S E E=\frac{g}{M_{t h}}+h \cdot\left(M_{t h}\right)^{p}
$$


If the mass of the thyroid is expressed in grams, the fitted parameters are $g=0.406, h=0.01014$, and $p=-0.666$ (Killough and Eckerman, 1986). By using these parameter values, the resulting $S E E$ is expressed in units of $\mathrm{rad} \mathrm{mCi}^{-1} h^{-1}$. Before using the calculated SEE, a unit transformation is performed to obtain the values expressed in $G y B q^{-1} d^{-1}$. The unit transformation factor is $1.54 \times 10^{8} \mathrm{~Gy} \mathrm{~Bq}^{-1} d^{-1}$ per $\mathrm{rad} \mathrm{mCi}^{-1} h^{-1}$.

Derivation of the rate constants in the blood $\left(\lambda_{1}\right)$ and thyroid $\left(\lambda_{2}\right)$ is based on measurements of iodine levels in the thyroid after an I.V. injection of a known amount of radioiodine. Normally, the maximum activity in the thyroid is observed about 24 hours $\left(t_{\max }\right)$ after injection, after which it decreases slowly. The activity level is measured at (at least) two moments of time $\left(t_{1}\right.$ and $\left.t_{2}\right)$ after the injection. Based on this, an "apparent" half-life $\left(T^{*}\right)$ describing the decline of the activity of iodine in the thyroid can be determined. The measurements are usually performed 24 hours (for the peak activity) and 15 days after the injection. Based on the measured "apparent" half-life $\left(T^{*}\right)$, the rate constants of iodine in the blood $\left(\lambda_{1}\right)$ and thyroid $\left(\lambda_{2}\right)$ can be derived as follows (Killough and Eckerman, 1986):

$$
\begin{aligned}
& \lambda_{1}=\frac{s}{1-r}-\lambda_{R} \\
& \lambda_{2}=\frac{(s \cdot r)}{1-r}-\lambda_{R}
\end{aligned}
$$

where " $r$ " is defined by the equation:

$$
\frac{\left[1-r^{\frac{t_{2}}{t_{\max }}}\right] \cdot r^{\left(\frac{r}{1-r} \cdot \frac{t_{2}}{t_{\max }}\right)}}{\left[1-r^{\frac{t_{1}}{t_{\max }}}\right] \cdot r^{\left(\frac{r}{1-r} \cdot \frac{t_{1}}{t_{\max }}\right)}}=\exp \left[-\frac{0.693}{T^{*}} \cdot\left(t_{2}-t_{1}\right)\right]
$$


In equations 9.4 through 9.6, the index "1" represents the blood compartment, and the index "2" represents the thyroid compartment. The equations were derived by Killough and Eckerman (1986) on the basis of measurements of the "apparent" biological half-life of ${ }^{131} \mathrm{I}$ in the human body $\left(T^{*}\right)$ as a function of age and sex. The parameter $t_{\max }=t_{1}=1$ day represents the constraint that the activity in the thyroid has its maximum 1 day after the intake. Killough and Eckerman (1986) showed that a variation in this parameter from 0.5 to 2 days produces a deviation in the results of less than $11 \%$. The parameters $t_{1}=1$ day and $t_{2}=15$ days define the interval in which the "apparent" biological half-life is usually measured.

\subsubsection{Biokinetic Model Parameters}

Three parameters must be determined to completely solve the system of equations (Eq. 9.1 to Eq. 9.6). The first is the measured "apparent" biological half-life $\left(T^{*}\right)$, and the second is the fraction of iodine taken up by the thyroid from the blood $(a)$. Dunning and Schwartz (1981) reviewed the measurements of $(a)$ and $\left(T^{*}\right)$ (Table 9.1). They grouped the data in four age groups: (a) newborns, (b) children ages 0.5 to 2 years, (c) adolescents ages 6 to 16, and (d) adults (age > 18 years). Because of this peculiar categorization, the change of the apparent half-life and of the uptake with age does not appear to be monotonic.

Dunning and Schwartz (1981) fitted normal and lognormal distributions to observed values. For the apparent half-life, the lognormal distribution has been chosen because it offers a better fit. However, a normal or lognormal distribution for the uptake fraction (a) can produce values larger than 1 (100\%), which do not have any physical significance. To avoid values larger than 1, a log-triangular distribution has been used. The median reported by Dunning and Schwartz (1981) was chosen as the mode of the log-triangular distribution. The minimum and the maximum values of the log-triangular distribution match the 2.5 and 97.5 percentiles of the lognormal distribution derived by Dunning and Schwartz (1981). The data were interpolated between age groups using a piecewise linear interpolation technique. The interpolation was performed by assigning the reported data (Table 9.1) to ages 0 (newborn), 1, 11, 15 and 20 years. Table 9.1 presents the $95 \%$ confidence intervals of the selected distributions.

The third parameter is the mass of the thyroid as a function of age. Numerous studies report measurements of the thyroid mass obtained by autopsy (Mochizuki et al., 1963; Kereiakes et al., 1965; Kay et al., 1966; Dunning and Schwartz, 1981; Killough and Eckerman, 1986). However, most of the sets of measurements are incomplete, either because some age groups are not covered or because few measurements are available for a specific age group. Modern ultrasonography methods are currently used to determine the thyroid volumes in large populations. The masses of the thyroids can be calculated from the measured volumes using the density of the thyroid tissue. As a result, better distributions of the thyroid masses in the population are becoming available. 
Table 9.1 Distributions for the biological half-life $\left(T^{*}\right)$ and the fractional uptake $(a)$ based on the data from Dunning and Schwartz (1981).

\begin{tabular}{lcccccl}
\hline Parameter & & \multicolumn{3}{c}{ Units } & \multicolumn{3}{c}{ 95\% subjective confidence interval } & Shape \\
& & & $\begin{array}{c}\text { Lower } \\
\text { limit }\end{array}$ & $\begin{array}{c}\text { Central } \\
\text { value }\end{array}$ & $\begin{array}{c}\text { Upper } \\
\text { Limit }\end{array}$ & \\
\hline Newborn & $T^{*}$ & $\mathrm{~d}$ & $4.0^{\mathrm{a}}$ & 13.0 & $44.0^{\mathrm{a}}$ & Lognormal \\
& $a$ & unitless & 0.17 & 0.37 & 0.81 & Log-triangular \\
Child & $T^{*}$ & $\mathrm{~d}$ & $3.0^{\mathrm{a}}$ & 10.0 & $42.0^{\mathrm{a}}$ & Lognormal \\
& $a$ & unitless & 0.17 & 0.37 & 0.81 & Log-triangular \\
\multirow{2}{*}{ Adolescent } & $T^{*}$ & $\mathrm{~d}$ & $16.0^{\mathrm{a}}$ & 44.0 & $119.0^{\mathrm{a}}$ & Lognormal \\
& $a$ & unitless & 0.2 & 0.43 & 0.94 & Log-triangular \\
\multirow{2}{*}{ Adult } & $T^{*}$ & $\mathrm{~d}$ & $24.0^{\mathrm{a}}$ & 72.0 & $220.0^{\mathrm{a}}$ & Lognormal \\
& $a$ & unitless & 0.08 & 0.17 & 0.37 & Log-triangular \\
\hline
\end{tabular}

a "Lower" and "upper" represent the 2.5 percentile and the 97.5 percentile of the lognormal distribution, respectively.

In some cases, ultrasonography has shown smaller thyroid masses than those usually obtained by autopsy (Gutenkunst et al., 1986; Likhtarev et al., 1993). Such a result will produce larger dose estimates. In other cases, such as the measurements performed in the former Soviet Union in areas affected by deposition from the Chernobyl accident, ultrasonographic measurements do not conclusively indicate a potential bias of the thyroid mass towards lower values (Yureiva et al., 1994; Derzhitsky et al., 1994; Averichev et al., 1994; Avramenko et al., 1994; Danilyuk, 1994).

A recent review of a large body of available ultrasound data (Table 9.2) was performed by Bier (1996). He found a clear trend towards lower values of the thyroid mass obtained by modern ultrasonography as compared to similar data obtained from autopsy (Figure 9.3). The values reported by Bier (1996) are weighted values (based on the numbers used in each study) across several reviewed studies. For a child of age 6, the thyroid mass from Bier (1996) is lower compared with values for children ages 5 and 7 . This effect is probably an artifact obtained due to different numbers of children being measured at different ages. Therefore, in this study, the observed thyroid mass for age 6 was replaced by the value obtained by linear interpolation between values for ages 5 and 7 . 
Table 9.2 Thyroid mass (grams) obtained by ultrasonography (Bier, 1996).

\begin{tabular}{cccc}
\hline $\begin{array}{c}\text { Age } \\
\text { [Years] }\end{array}$ & $\begin{array}{c}\text { Number of } \\
\text { measurements }\end{array}$ & $\begin{array}{c}\text { Mean } \\
\text { Thyroid Mass }\end{array}$ & Standard Deviation \\
\hline Newborn & 33 & 1.89 & 0.40 \\
1 & 30 & 2.05 & 0.60 \\
2 & 12 & 2.10 & 0.50 \\
3 & 16 & 2.50 & 0.70 \\
4 & 65 & 2.70 & 0.20 \\
& & & \\
5 & 18 & 3.40 & 1.00 \\
6 & 56 & 3.10 & 1.25 \\
7 & 223 & 4.70 & 0.21 \\
8 & 400 & 5.60 & 0.42 \\
9 & 266 & 5.70 & 0.17 \\
& & & \\
10 & 289 & 6.90 & 0.60 \\
11 & 236 & 7.80 & 0.69 \\
12 & 261 & 8.80 & 0.24 \\
13 & 6740 & 9.10 & 1.62 \\
14 & 209 & 11.6 & 0.89 \\
15 & 415 & 11.5 & 0.29 \\
\hline
\end{tabular}



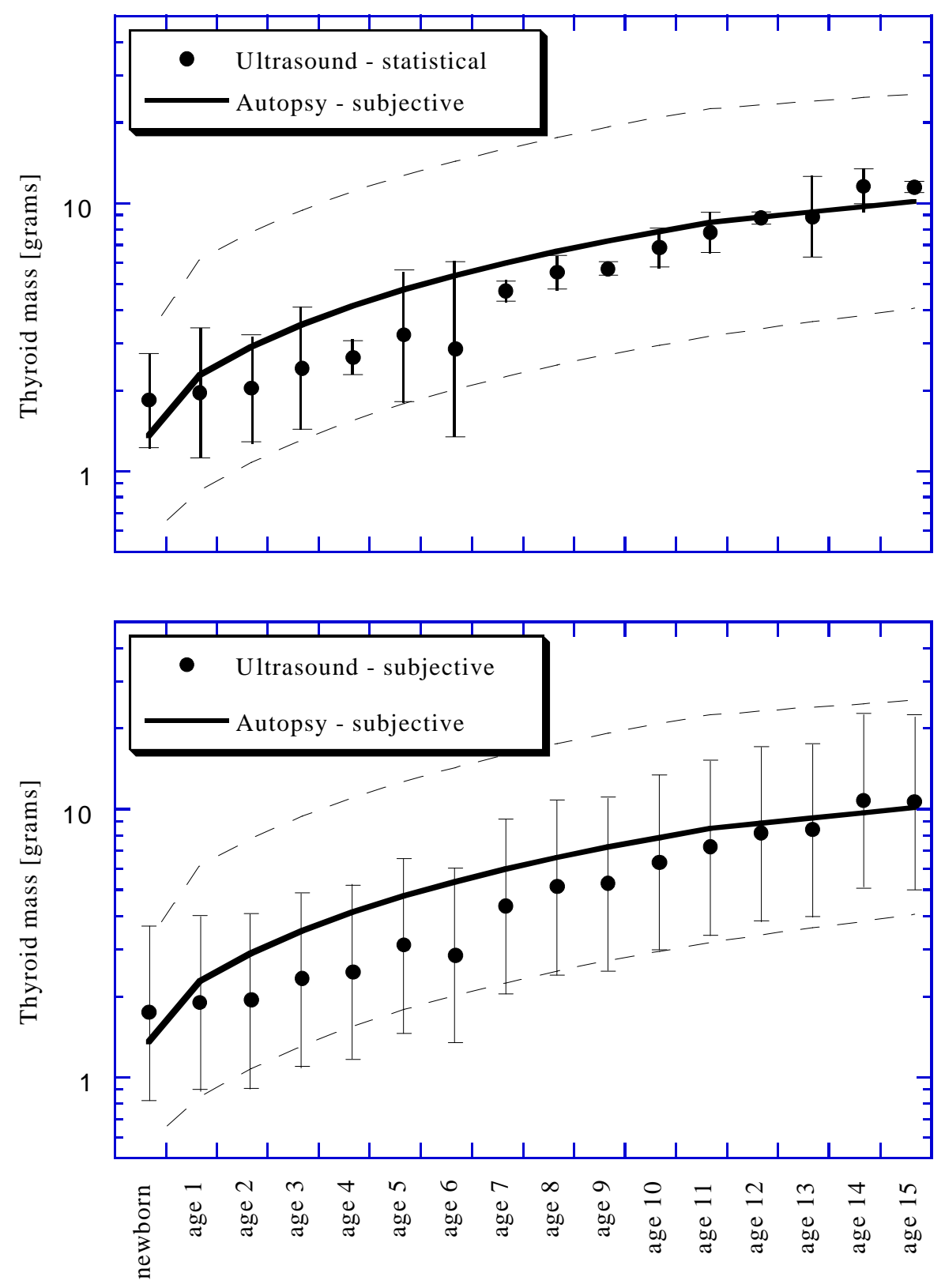

Figure 9.3 Comparison of thyroid masses obtained by autopsy investigation (Killough and Eckerman, 1996) and by ultrasound investigations (Bier, 1996). The 95\% confidence intervals are included. For autopsy data, the dashed lines represent the $95 \%$ subjective confidence interval. For ultrasound data, the vertical lines represent the $95 \%$ confidence interval from statistical analysis of data, and the dashed lines represent the $95 \%$ confidence interval induced by the inter-individual variability. 
The issue of interindividual variability of the thyroid mass was not addressed by Bier (1996). To investigate interindividual variability, the data from Bier (1996) were compared to data from the countries of the former Soviet Union (FSU; Yureiva et al., 1994; Derzhitsky et al., 1994; Averichev et al., 1994; Avramenko et al., 1994; Danilyuk, 1994; Figures 9.3, 9.4, 9.5, and 9.6). The data from the FSU, obtained by ultrasound measurements, report the distributions of thyroid mass in the population of children from areas affected by the Chernobyl accident. These distributions show a trend toward higher values of thyroid mass, but their interindividual variability, normalized for a central value of thyroid mass, should be representative for any population of children. A geometric standard deviation was obtained from the comparison of FSU data with Bier (1996), data for which lognormal distributions with different GSDs were applied (Figures 9.3, 9.4, 9.5, and 9.6). A GSD of 1.5 was determined to be the best representation of uncertainty in thyroid mass due to interindividual variability of the thyroid mass. In conclusion, the uncertainty in the thyroid mass is expressed using lognormal distributions having average values from Bier (1996; Table 9.2) and a geometric standard deviation of 1.5 applied to all age groups.

Because children are the critical group for exposure to ${ }^{131} \mathrm{I}$, ultrasound measurements of the thyroid mass had been reviewed only for children under the age of 15 . Therefore, the dose factor for adults (age 20 and older) was obtained from ICRP Publication 56 (ICRP, 1989). For individuals 15 to 20 years of age, a linear interpolation was performed between the estimated dose factor for age 15 and the dose factor from ICRP Publication 56 for age 20. A geometric standard deviation of 1.5 was applied to the dose factors for individuals older than 15 years.

\subsubsection{Fraction of ${ }^{131}$ I Deposited and Absorbed in the Respiratory System}

Part of the ${ }^{131}$ I inhaled by a person is deposited in his or her respiratory system, from where it is rapidly absorbed and transferred to body fluids and the gastrointestinal tract. Deposition and absorption of ${ }^{131} \mathrm{I}$ depend on its physico-chemical form. Different rates of deposition and absorption occur for gases than for aerosols. To estimate the inhalation doses, we use a parameter $\left(D_{k}\right.$, Section 8.2.1) that accounts for both deposition and absorption in the respiratory tract.

The classical ICRP model (ICRP, 1979) divides the respiratory system into three major parts the nasal passage (NP), the trachea and the bronchial tree (BT), and the pulmonary parenchyma (P). According to this model, a fraction of about 0.63 of the inhaled iodine is deposited and absorbed to the lungs, if iodine is attached to particles having an activity median aerodynamic diameter (AMAD) of $1 \mu \mathrm{m}$. If the particle diameter is different from $1 \mu \mathrm{m}$ (e.g., 0.2, 0.5, 2 or 5 $\mu \mathrm{m})$, the deposition fractions are $0.63,0.58,0.75$ and 0.87 , respectively. 

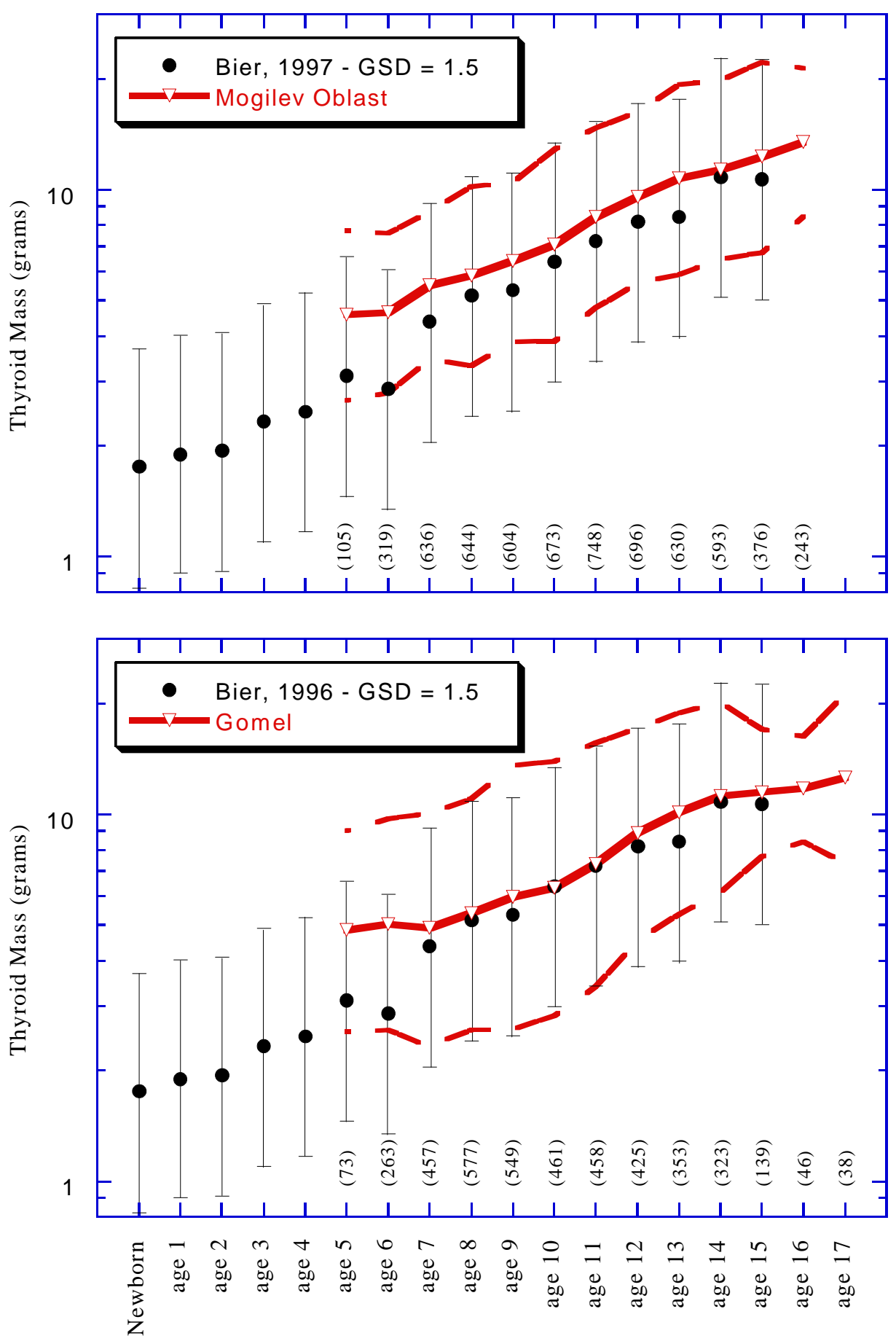

Figure 9.4 Comparison of thyroid masses from Bier (1996) and from the Mogilev and Gomel Regions (former Soviet Union), which were affected by the Chernobyl accident. A lognormal distribution with a geometric standard deviation of 1.5 is assigned to the average thyroid masses from Bier (1996). The upper and lower bounds delimit a 95\% confidence interval. The numbers in parentheses represent the number of samples for the FSU data. 

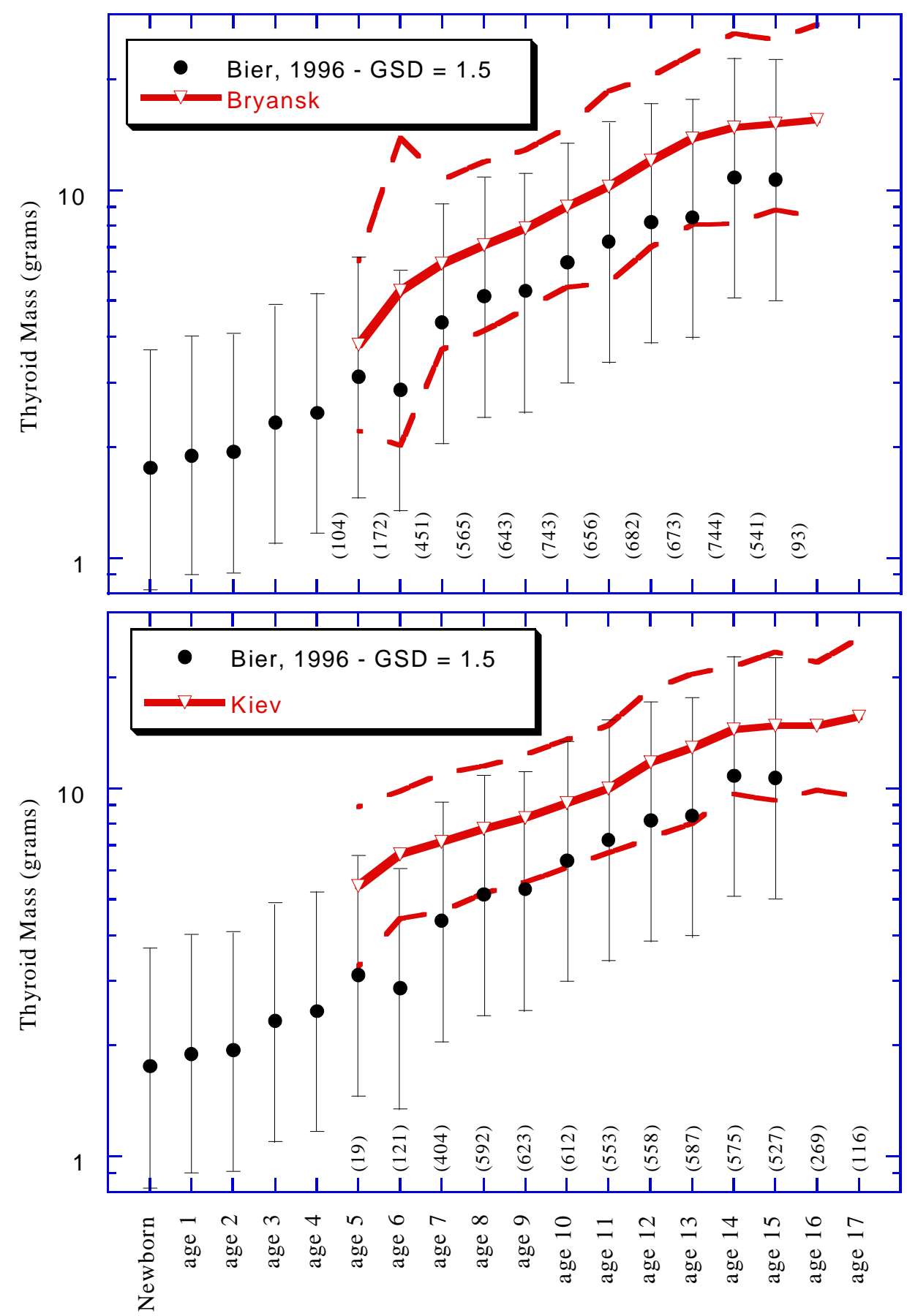

Figure 9.5 Comparison of thyroid masses from Bier (1996) and from the Bryansk and Kiev Regions (former Soviet Union), which were affected by the Chernobyl accident. A lognormal distribution with a geometric standard deviation of 1.5 is assigned to the average thyroid masses from Bier (1996). The upper and lower bounds delimit a 95\% confidence interval. The numbers in parentheses represent the number of samples for the FSU data. 


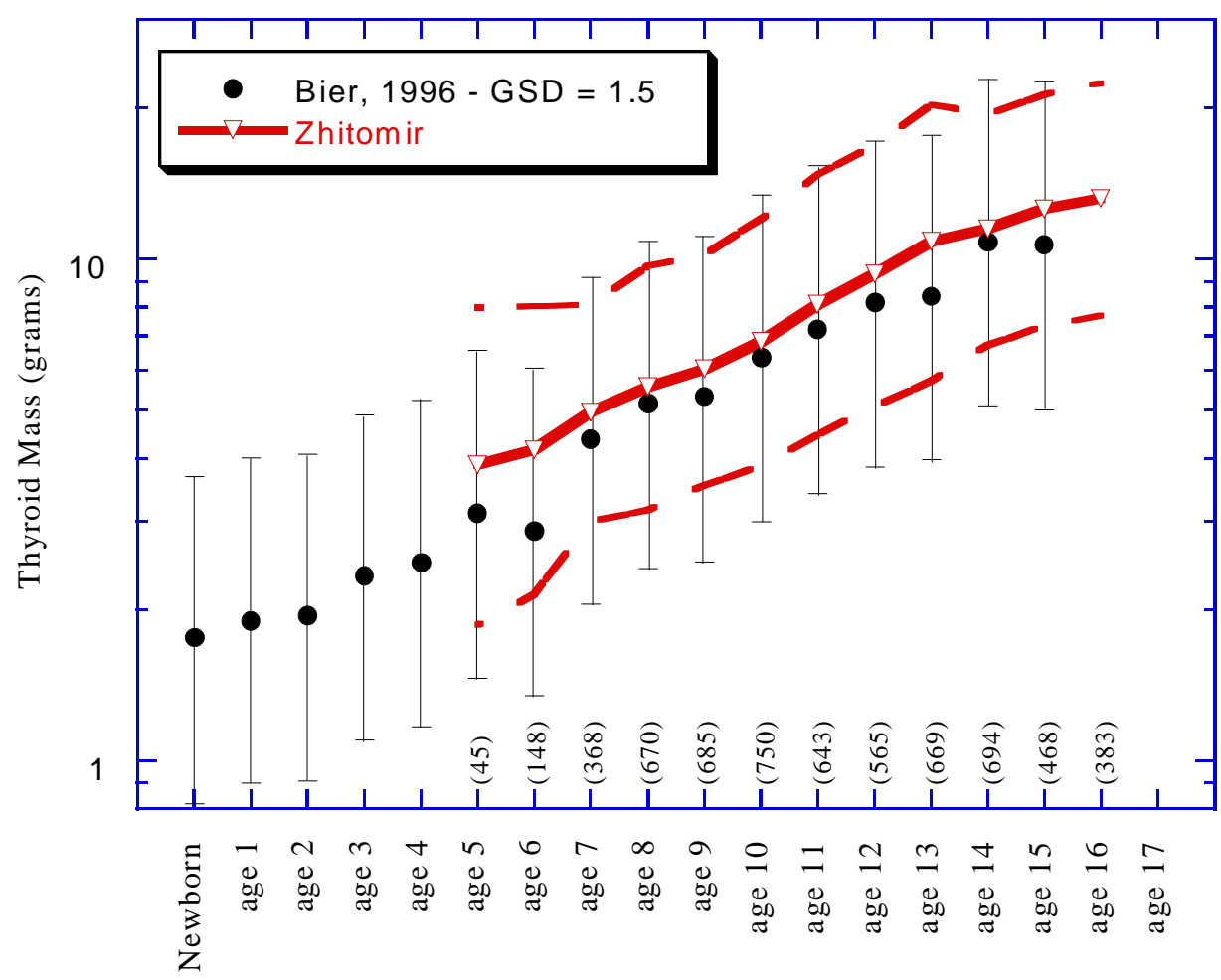

Figure 9.6 Comparison of the thyroid mass from Bier (1996) and from Zhytomir Region (former Soviet Union), which were affected by the Chernobyl accident. A lognormal distribution with a geometric standard deviation of 1.5 is assigned to the average thyroid masses from Bier (1996). The upper and lower bounds delimit a 95\% confidence interval. The numbers in parentheses represent the number of samples for the FSU data. 
More recent studies (Raabe, 1987) distinguish between nasal and oral inhalation, which lead to different depositions in different areas of the respiratory system. However, the total deposition of particles is similar, being between 0.6 to 0.8 for particles varying in size between 0.01 and 0.1 $\mu \mathrm{m}$, between 0.3 and 0.6 for particles with sizes between 0.1 and $1 \mu \mathrm{m}$, and between 0.3 and 1.0 for particles between 1 and $10 \mu \mathrm{m}$. The larger the particle size, the more deposition occurs in the nasopharyngeal and oropharyngeal parts of the respiratory system.

The most advanced model is described by ICRP Publication 66 (ICRP, 1994), which distinguishes 5 primary regions of the respiratory tract based on structure and function. Also, nasal and oral inhalation are treated separately. Deposition of particles and gases is regarded as a passage through a series of 6 filters. There are five filters corresponding to the five defined regions of the respiratory tract, and an additional "filter," called "inhalability," which accounts for the complex flow of particles around the human head and torso.

Based on the new respiratory tract model, ICRP published age-dependent effective inhalation dose factors for many radioactive elements, including ${ }^{131}$ I (ICRP Publication 72; 1996). In this publication, ${ }^{131} \mathrm{I}$ is classified as a "soluble and reactive" substance; that is, deposition may occur throughout the entire respiratory tract. This classification emphasizes the difference between iodine and other substances (e.g., hydrogen and carbon), considered to be "highly soluble and reactive"; that is, they are deposited and absorbed in the extrathoracic portions of the respiratory tract (without reaching the lungs).

Deposition of gases is driven by different physical laws than is deposition of particles. ICRP Publication 72 (1996) uses a 100\% deposition for elemental iodine, and only a 70\% deposition for organic iodine.

Absorption of iodine deposited within the respiratory tract is also a function of the physicochemical form of iodine. For particles, ICRP (1996) differentiates between three broad absorption classes (Types $F, M$, and $S$, for Fast, Moderate, and Slow), which correspond to absorption fractions $\left(f_{1}\right)$ of $1.0,0.1$ and 0.01 respectively. Iodine in gaseous forms (both elemental and organic) is assumed to be totally absorbed once deposited.

In the case of releases from $\mathrm{X}-10$, iodine was released mostly in gaseous form (elemental and organic), but also a small amount was released in particulate form (Section 3). During atmospheric transport, some of the elemental iodine attaches to particles already existing in the atmosphere. By the time the plume arrives at the downwind location where iodine is inhaled, the fraction in particulate form will be appreciable (Section 4). Most particles will have small or very small sizes $(<1 \mu \mathrm{m})$ and are more likely to be of type $F$, rather than type $M$ or type $S$.

We performed a comparison between the thyroid dose factors for ingestion presented in ICRP Publication 67 (1993) and the thyroid dose factors for inhalation derived from ICRP Publication 72 (1996), which are based on the new ICRP lung model and contain the effect of partial deposition and absorption of ${ }^{131} \mathrm{I}$ in the respiratory tract. ICRP Publication 67 (1993) reports ingestion dose factors based on a $100 \%$ absorption of iodine. 
The comparison was performed by taking the ratios between the dose factors based on the new lung model and the ingestion dose factors (no deposition or absorption in the respiratory tract). Once iodine reaches the blood, the metabolism and dosimetry is similar in the two ICRP Publications. Thus, the estimated ratios are an indicator of the overall effect of the deposition and absorption of ${ }^{131} \mathrm{I}$ as incorporated in the new lung model. For elemental iodine the ratio was 0.9 and for organic iodine the ratio was 0.7 . For the fast absorbing particles $\left(f_{l}=1\right)$, the ratio was 0.4 , and for the medium absorbing particles $\left(f_{l}=0.1\right)$, the ratio was 0.1 .

Based on the information presented above, the following distributions were used for the fraction of iodine deposited and absorbed in the respiratory tract $\left(D_{k}\right)$ :

(a) for elemental iodine - a uniform distribution between 0.8 and 1.0 (central value 0.9 );

(b) for particulate iodine - a triangular distribution between 0.1 and 0.8 with a mode of 0.4 ; and

(c) for organic iodine - a uniform distribution between 0.6 and 0.8 (central value 0.7 ).

The same distributions were used for both the routine releases and the 1954 accident.

\subsection{Results and Conclusions}

The estimates of the $95 \%$ subjective confidence intervals for the age-specific dose conversion factors are listed in Table 9.3. The central values of the ingestion dose factors estimated in this study are in good agreement with the factors recommended by ICRP (1989; Figure 9.7).

The central values of the distributions produced in this study are comparable to the ICRP's recommended values for children exposed as newborns or at age 1. In order to use the new thyroid masses and to ensure a correct uncertainty analysis by taking into account the correlation between the biokinetic parameters, the metabolic model for iodine (Figure 9.2) was solved explicitly. The biological half-lives of ${ }^{131} \mathrm{I}$ in the thyroid gland obtained from Equation 9.5 were lower than the ICRP (1989) recommended values for different ages. This is equivalent to a faster clearance by the thyroid gland, and thus a bias towards lower doses. The increase in dose introduced by lower thyroid masses is balanced by the decrease introduced by the faster clearance rate, and the net effect is minimal. 
Table 9.3 Age-specific dose factors $\left(\mathrm{Gy} \mathrm{Bq}^{-1}\right)$ for ingestion of ${ }^{131} \mathrm{I}$, derived for use in this study.

\begin{tabular}{|c|c|c|c|}
\hline \multirow[b]{2}{*}{ Age in years } & \multicolumn{3}{|c|}{ 95\% Subjective Confidence Interval } \\
\hline & Lower Limit & Central Estimate $^{a}$ & Upper Limit \\
\hline Fetus ${ }^{\mathrm{b}}$ & $1.7 \times 10^{-7}$ & $5.5 \times 10^{-7}$ & $1.8 \times 10^{-6}$ \\
\hline Newborn & $1.0 \times 10^{-6}$ & $3.0 \times 10^{-6}$ & $1.1 \times 10^{-5}$ \\
\hline 1 & $7.1 \times 10^{-7}$ & $2.4 \times 10^{-6}$ & $7.2 \times 10^{-6}$ \\
\hline 2 & $8.3 \times 10^{-7}$ & $2.5 \times 10^{-6}$ & $7.1 \times 10^{-6}$ \\
\hline 3 & $6.1 \times 10^{-7}$ & $2.1 \times 10^{-6}$ & $7.3 \times 10^{-6}$ \\
\hline 4 & $7.0 \times 10^{-7}$ & $2.2 \times 10^{-6}$ & $5.4 \times 10^{-6}$ \\
\hline 5 & $6.1 \times 10^{-7}$ & $1.8 \times 10^{-6}$ & $5.2 \times 10^{-6}$ \\
\hline 6 & $4.8 \times 10^{7}$ & $1.6 \times 10^{-6}$ & $5.0 \times 10^{-6}$ \\
\hline 7 & $5.1 \times 10^{-7}$ & $1.5 \times 10^{-6}$ & $3.9 \times 10^{-6}$ \\
\hline 8 & $4.5 \times 10^{-7}$ & $1.4 \times 10^{-6}$ & $3.7 \times 10^{-6}$ \\
\hline 9 & $5.3 \times 10^{-7}$ & $1.4 \times 10^{-6}$ & $3.9 \times 10^{-6}$ \\
\hline 10 & $4.8 \times 10^{-7}$ & $1.3 \times 10^{-6}$ & $3.6 \times 10^{-6}$ \\
\hline 11 & $4.7 \times 10^{-7}$ & $1.3 \times 10^{-6}$ & $3.6 \times 10^{-6}$ \\
\hline 12 & $3.7 \times 10^{-7}$ & $1.1 \times 10^{-6}$ & $3.1 \times 10^{-6}$ \\
\hline 13 & $3.5 \times 10^{-7}$ & $9.8 \times 10^{-7}$ & $2.7 \times 10^{-6}$ \\
\hline 14 & $2.7 \times 10^{-7}$ & $7.3 \times 10^{-7}$ & $2.0 \times 10^{-6}$ \\
\hline 15 & $2.6 \times 10^{-7}$ & $7.0 \times 10^{-7}$ & $1.8 \times 10^{-6}$ \\
\hline 16 & $2.4 \times 10^{-7}$ & $6.5 \times 10^{-7}$ & $1.7 \times 10^{-6}$ \\
\hline 17 & $2.2 \times 10^{-7}$ & $5.9 \times 10^{-7}$ & $1.6 \times 10^{-6}$ \\
\hline 18 & $2.0 \times 10^{-7}$ & $5.4 \times 10^{-7}$ & $1.4 \times 10^{-6}$ \\
\hline 19 & $1.8 \times 10^{-7}$ & $4.9 \times 10^{-7}$ & $1.3 \times 10^{-6}$ \\
\hline 20 and older & $1.6 \times 10^{-7}$ & $4.4 \times 10^{-7}$ & $1.2 \times 10^{-6}$ \\
\hline $\begin{array}{l}\text { verage for the } \\
-14 \text { age group }\end{array}$ & $1.5 \times 10^{-6}$ & $2.0 \times 10^{-6}$ & $2.9 \times 10^{-6}$ \\
\hline
\end{tabular}

\footnotetext{
${ }^{\mathrm{a}}$ median

bother's ingestion
} 
TASK 1 REPORT

July 1999

${ }^{131}$ I Releases from X-10 Radioactive Lanthanum Processing-

Page 9-18 Internal Dosimetry for Iodine-131

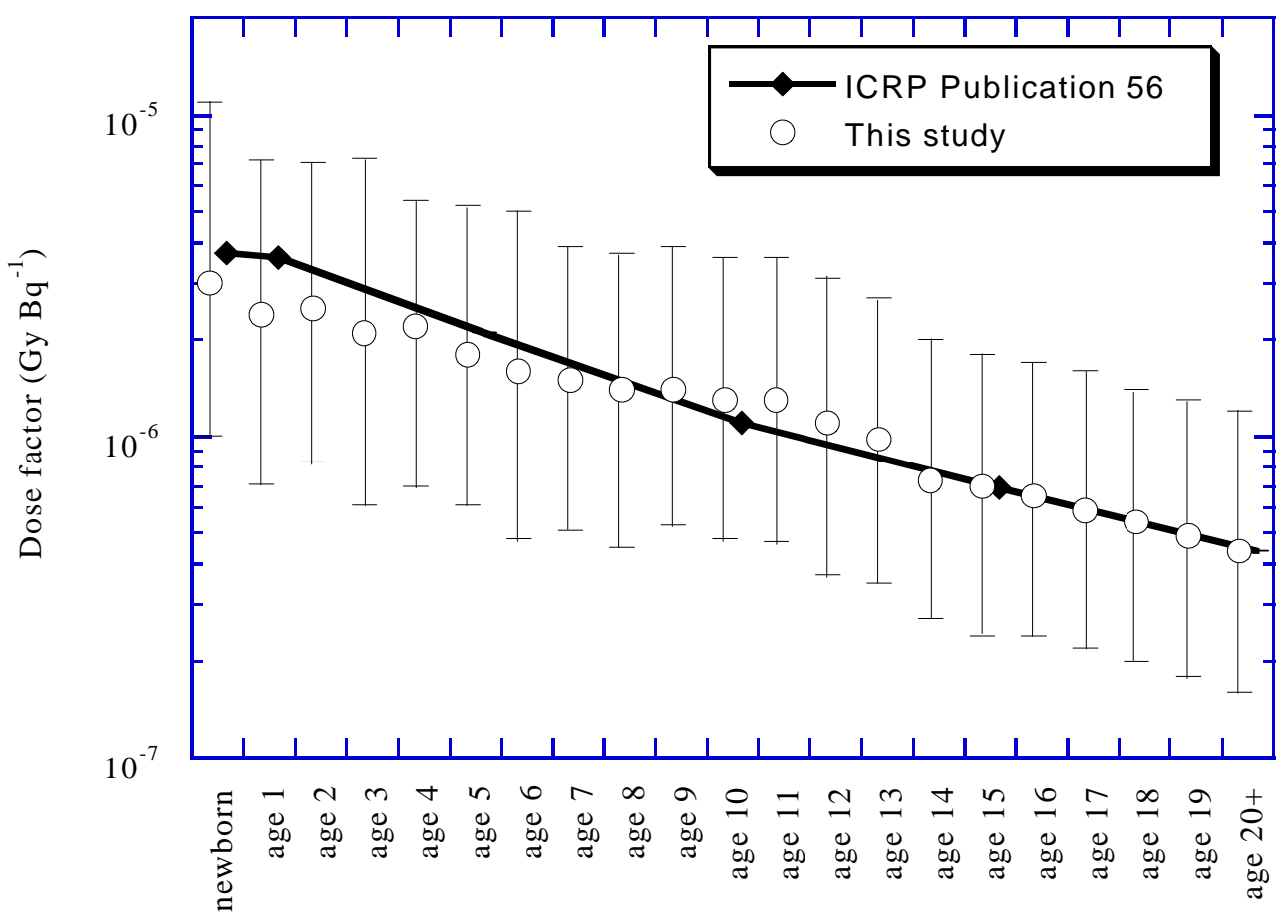

Figure 9.7 Comparison of the ingestion dose factors for ${ }^{131} \mathrm{I}$ calculated in this study to the most recent recommended values from ICRP (1989). The doses are estimated for the thyroid gland. 


\section{Average dose factor for estimation of the total number of cases in the affected region}

Estimation of the total number of cancers possibly induced by exposure to ${ }^{131}$ I released from X10 (Section 2.0) is based on the doses to children under the age of 15 (the critical group of the population). This dose is calculated using a dose factor for children $0-14$, obtained as an average of the dose factors for each age between age 0 and 14, weighted for the fraction of population of that age. According to the Census of the Population (Brunsman, 1952), in the counties affected by the ${ }^{131}$ I releases from X-10, there were 31,946 children between 0 and 14 in 1950, with 12,479 between 0 and 4 (39\%), 10,586 between 5 and $9(33 \%)$, and 8,881 between 10 and $14(28 \%)$.

\section{Dose factor for the fetus}

Recently, the National Cancer Institute produced a report (NCI, 1997) on the estimated exposures received by the American people from ${ }^{131} \mathrm{I}$ in fallout following Nevada atmospheric nuclear bomb tests in Nevada. This report summarizes the doses to a fetus at various stages of development from ingestion of ${ }^{131} \mathrm{I}$ by the pregnant mother (Table 9.4). The average dose factor

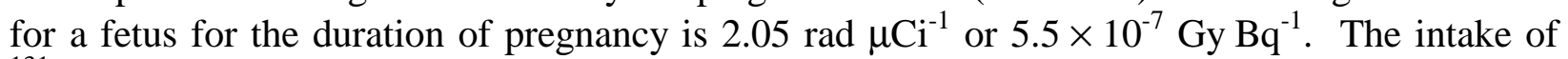
${ }^{131} \mathrm{I}$ by an exposed individual is estimated usually on an annual basis. However, for pregnant women, the intake is reduced to nine out of twelve month; that is, the intake is multiplied by (9/12). NCI (1997) estimates the uncertainty due to interindividual variability in the dose factor for the fetus as a lognormal distribution having a geometric standard deviation of 1.8. This value is consistent with the geometric standard deviation estimated in this study for the dose factor for post-natal exposures, and thus, was adopted for our calculations.

Table 9.4 Dose to the thyroid of a fetus per unit activity of ${ }^{131}$ I ingested by a pregnant woman (NCI, 1997).

\begin{tabular}{ccc}
\hline Age of the fetus $[\mathbf{r a d} / \mathbf{m C i}]$ & {$[\mathbf{G y} / \mathbf{B q}]$} \\
\hline $0-10 \mathrm{wks}$ & 0.0 & 0.0 \\
$11-20 \mathrm{wks}$ & 2.7 & $7.3 \times 10^{-7}$ \\
$21-30 \mathrm{wks}$ & 3.8 & $1.0 \times 10^{-6}$ \\
$31-40 \mathrm{wks}$ & 1.7 & $4.6 \times 10^{-7}$ \\
\hline average & $\mathbf{2 . 0 5}$ & $\mathbf{5 . 5} \times \mathbf{1 0}^{-7}$ \\
\hline
\end{tabular}




\subsection{Sensitivity Analysis}

The sensitivity analysis identifies the variables that are major contributors to the uncertainty in the estimates of the dose factors. Sensitivity analysis results are shown in Figure 9.8. The analysis was performed only for selected age groups because of the relatively low variation of the parameters with increasing age. For all ages, the major contributors to the uncertainty in the dose per unit intake are the thyroid mass followed by the fractional uptake $(a)$. Due to the strong dependencies between the biological elimination rates from blood $\left(\lambda_{1}\right)$ and from the thyroid $\left(\lambda_{2}\right)$, both are shown as having equal contributions to the overall uncertainty in the dose factor. Their contribution becomes lower as the age increases. Therefore, to estimate the thyroid dose for a given individual, one should obtain specific values for the thyroid mass and the fractional uptake for that individual, and concentrate less on the retention time of the iodine in the thyroid gland. The thyroid mass for a given individual can be obtained by direct measurement of thyroid volume using ultrasound techniques, or by using relationships between thyroid mass and other easy to measure characteristics such as the body mass or body height. Further research is necessary to identify a method for assessing the fractional uptake of iodine by the thyroid gland as a function of individual characteristics. 

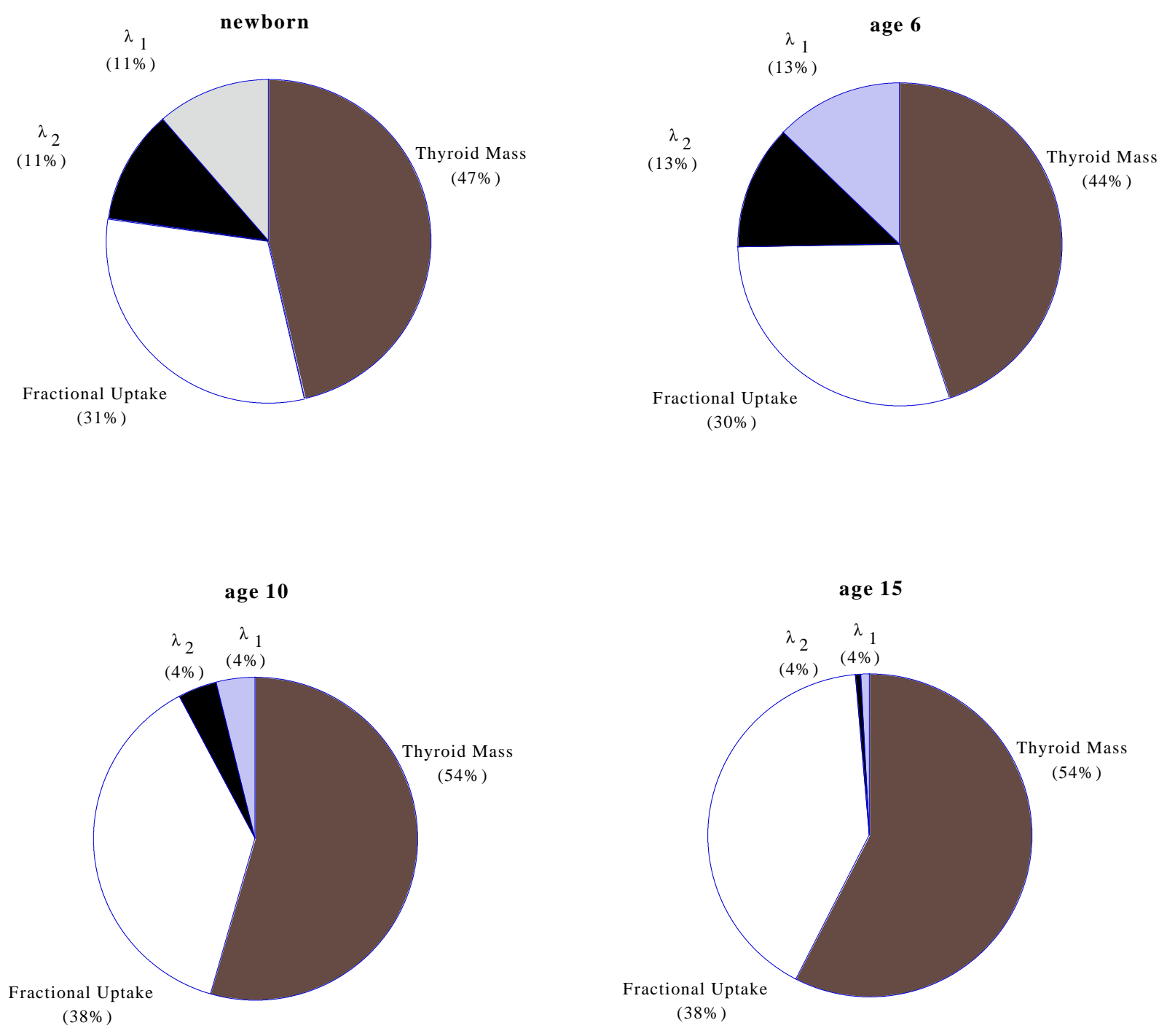

Figure 9.8 Contributors to the uncertainty in the ${ }^{131} \mathrm{I}$ ingestion dose factor for various age groups. 


\subsection{References}

Averichev, A.A., Karevskaya, I.V., Steputin, L.A., Korobkova, L.P., Fokina, M.M., Kovalev, A.I., Aksyonov, A.S., and Troyanova, N.N. 1994. Results of the Examination of the Health Status of Children in the Southern-Western Rayons of Bryansk Oblast. Klincy City Children's Hospital. A Report on the 1994 Chernobyl Sasakawa Project Workshop. Moscow. May 16-17, 1996.

Avramenko, A.I., Elagin, V.V., Nikiforova, N.V., Semoushina, S.V., Grinko, V.I., Nedozhdy, A.V., and Kochubey, S.S. 1994. Results of the Examination of Children in Kiev Oblast. Kiev Regional Hospital No. 2, "Sasakawa-Chernobyl" Diagnostic Center. A Report on the 1994 Chernobyl Sasakawa Project Workshop. Moscow. May 16-17, 1996.

Bier, S.G. 1996. Thyroid mass in children: A comparison of autopsy and ultrasound data. Masters Thesis. Nuclear Engineering Department, University of Tennessee, Knoxville.

Brunsman, H.G. 1952. Census of Population: 1950. A Report of the Seventeenth Decennial Census of the United States. Volume II: Characteristics of the Population, part 42, Tennessee. Bureau of the Census. U.S. Dept. of Commerce. U.S. Government Printing Office. Washington, DC.

Danilyuk, V.V, Petrova, A.A., Saiko, A.S., Danilyuk, L.V., Stotskaya, L.P., Rudnitskii, S.S., Sokolovskii, I.N., and Goncharenko, O.E. 1994. Results of the Examination of Children Residing in the Northern Rayons of Zhitomir Oblast. Korosten Inter-Area Medical Diagnostic Center. A Report on the 1994 Chernobyl Sasakawa Project Workshop. Moscow. May 16-17, 1996.

Derzhitskiy, V.E., Panasyuk, G.D., Derzhitskaya, N.K., Demidenko, A.N., Kalimullin, V.A., Anikina, I.V., Cot, V.A., and Masyakin, V.B. 1994. Results of the Examination of the Health Status of Children in Gomel Oblast 1991-1993. Chernobyl Sasakawa Project. Gomel Specialized Medical Dispensary. A Report on the 1994 Chernobyl Sasakawa Project Workshop. Moscow. May 16-17, 1996.

Dunning, D.E., Jr., and Schwartz, G. 1981. Variability of human thyroid characteristics and estimates of dose from ingested I-131. Health Physics 40:661-675.

Eckerman, K.F. 1994. Dosimetric methodology of the ICRP. Chapter 13 in Internal Radiation Dosimetry. Health Physics Society Summer School, 1994.

Gutenkunst, R., Smolarek, H., Hasenpusch, U., Stubbe, P., Friedrich, H.J., Wood, W.G., and Scriba, P.C. 1986. Goitre epidemiology: Thyroid volume, iodine excretion, thyroglobulin and thyrotropin in Germany and Sweden. Acta Endocrinologica 112:494-501.

Guyton, A.C. 1991. Textbook of Medical Physiology. W.B. Saunders Company.

ICRP (International Commission on Radiological Protection). 1979. Limits for Intake of Radionuclides by Workers. ICRP Publication 30, Pergamon Press, Oxford. 
ICRP (International Commission on Radiological Protection). 1983. Radionuclide Transformations. Energy and Intensity of Emissions. ICRP Publication 38. Annals of the ICRP Vols. 11-13. Pergamon Press, New York.

ICRP (International Commission on Radiological Protection). 1989. Age Dependent Doses to Members of the Public from Intake of Radionuclides: Part 1. ICRP Publication 56, Pergamon Press, New York.

ICRP (International Commission on Radiological Protection). 1993. Age-dependent Doses to Members of the Public from Intake of Radionuclides: Part 2, Ingestion Dose Coefficients. ICRP Publication 67. Annals of the ICRP Vol. 23 (3-4). Pergamon Press, New York.

ICRP (International Commission on Radiological Protection). 1994. Human respiratory tract model for radiological protection. ICRP Publication 66. Annals of the ICRP Vol. 24 (1-3). Pergamon Press, New York.

ICRP (International Commission on Radiological Protection). 1996. Age-dependent Doses to Members of the Public from Intake of Radionuclides: Part 5 Compilation of Ingestion and Inhalation Coefficients. ICRP Publication 72. Annals of the ICRP Vol. 26. Pergamon Press, New York.

Kay, C., Abrahams, S., and McClain, P. 1966. The Weight of Normal Thyroid Glands in Children. Arch. Path. 82:349-352.

Kereiakes, J.G., Seltzer, R.A., Blackburn, B., and Saenger, E.L. 1965. Radionuclide doses to infants and children: A plea for a standard child. Health Physics 11:999-1004.

Killough, G.G., and Eckerman, K.F. 1986. Age- and sex-specific estimation of dose to a normal thyroid from clinical administration of ${ }^{131}$ I. ORNL/TM-9800, NUREG/CR-3955. Oak Ridge National Laboratory. Oak Ridge, Tennessee.

Likhtarev, I.A., Gulko, G.M., Kairo, I.A., Sobolev, B.G., Chepurnoy, N.I., Cheban, A.K., Nikonov, D.A., Djachkov, I.A., Proehl, P., and Roth, P. 1993. Reliability and accuracy of the ${ }^{131}$ I thyroid activity measurements performed in the Ukraine after the Chernobyl accident in 1986. GSF-Institut fur Strahlenschutz.

Mochizuki, Y., Mowafy, R., and Pasternak, B. 1963. Weights of human thyroids in New York City. Health Physics 9:1299-1301.

NCI (National Cancer Institute). 1997. Estimated Exposure and Thyroid Doses Received by the American People from Iodine-131 Fallout Following Nevada Atmospheric Nuclear Bomb Tests. U.S. Department of Health and Human Services. National Institute of Health. National Cancer Institute, October, 1997. 
Raabe, O.G. 1987. Experimental Dosimetry: Introduction. in "Extrapolation of Dosimetric Relationships for Inhaled Particles and Gases." Edited by Crapo, J.D., Smolko, E.D., Miller F.J., Graham, J.A., and Hayes, A.W. Academic Press, Inc.

Turner, J.E. 1986. Atoms, Radiation, and Radiation Protection. Pergamon Press, New York.

Yureiva, N.D., Rafeenko, S.M., Sharipov, V.F., Krupnik, T.A., Dolbeshkin, N.K., and Kovalev, V.M. 1994. Results of the Investigation of the Health Status of Children in Mogilev Oblast. A Report on the 1994 Chernobyl Sasakawa Project Workshop. Moscow. May 16-17, 1996. 


\subsection{EXCESS LIFETIME RISK OF THYROID CANCER PER UNIT ABSORBED DOSE FOR INDIVIDUALS EXPOSED TO IODINE-131}

\subsection{Introduction}

Several studies have established that X-and gamma irradiation of the thyroid will result in an increased incidence of thyroid carcinomas and adenomas in people exposed as children under 15 years of age (NRC, 1990; Ron et al., 1995). Radiation might also induce thyroid conditions not involving cancer, such as hypothyroidism, benign thyroid nodules, or Hashimoto's thyroiditis. An increased number of cases of hypothyroidism was found among patients treated with highdose radiation for Hodgkin's disease and among female Japanese A-bomb survivors (Spengler, 1997). To induce cancer, only a small number of cells be affected; these cells can then evolve into cancerous cells. In non-cancer effects, it is thought that radiation must induce functional changes to a large number of cells, so that the function of the thyroid is impaired.

Although fatal thyroid cancers are rare $^{1}$, the thyroid gland in children has one of the highest radiogenic risk coefficients of any organ. Convincing evidence for increased risk occurs at just below $0.1 \mathrm{~Gy}$, and linearity best describes the dose-response relationship (Ron et al., 1995). This section describes the methods used to derive the excess lifetime risk of thyroid cancer incidence from a unit absorbed dose of ${ }^{131} \mathrm{I}$, for individuals of various ages and both genders. Also, it describes the necessary steps for estimation of the relative risk of thyroid cancer, and of the probability that an existing thyroid cancer was caused by exposure to ${ }^{131} \mathrm{I}$. Definitions of the terms are presented in Section 10.2.

\subsubsection{Radiation-induced thyroid cancer}

A list of epidemiological studies regarding effects of radiation on the thyroid gland is presented in Table 10.1 for exposure to gamma and X-rays, in Table 10.2 for exposure to X-rays only, and in Table 10.3 for exposure to ${ }^{131}$ I. Selected studies are discussed below.

A pooled analysis of seven studies on thyroid cancer after childhood exposure to external radiation (Ron et al., 1995) produced an excess relative risk per Gy (ERR Gy ${ }^{-1}$ ) of 7.7 (95\% C. I. $=2.1$ to 28.7), and an excess absolute risk per 10,000 persons per year per Gy $(\mathrm{EAR} / 10,000 \mathrm{PYGy})$ of $4.4(95 \%$ C. I. $=1.9$ to 10.1$)$. The excess relative risk (ERR) is the relative increase in the rate of cancer incidence above that expected in an unexposed population. The excess absolute risk (EAR) is the average number of cases of thyroid cancer observed above the expected amount per 10,000 person years of follow-up examination.

\footnotetext{
${ }^{1}$ Thyroid cancer has an overall 5-year survival rate of $95 \%$.
} 
Table 10.1 Results of epidemiological studies for exposure to gamma and X-rays.

\begin{tabular}{|c|c|c|c|c|c|}
\hline Who was exposed & $\begin{array}{l}\text { How and why they were } \\
\text { exposed }\end{array}$ & $\begin{array}{c}\text { Mean thyroid dose } \\
\text { Gy } \\
\text { (range) }\end{array}$ & $\begin{array}{l}\text { Excess relative risk for } \\
\text { thyroid cancer } \\
\text { ERR/Gy } \\
(95 \% \text { C.I. })\end{array}$ & $\begin{array}{l}\text { Excess absolute risk } \\
\text { of thyroid cancer } \\
\text { EAR/10,000PYGy } \\
(95 \% \text { C.I. })\end{array}$ & Reference \\
\hline $\begin{array}{l}\text { 41,234 Japanese of all ages; mean age at } \\
\text { time of exposure was } 27 \text { years; } 60 \% \text { were } \\
\text { females; } 24 \text { years of follow-up. } \\
\text { [ }<15 \text { years old at time of exposure }]\end{array}$ & $\begin{array}{l}\text { Gamma rays and neutrons } \\
\text { from bombings of Hiroshima } \\
\text { and Nagasaki. }\end{array}$ & $\begin{array}{l}0.27(0.01 \text { to } 3.99) \\
\text { based on average dose } \\
\text { to the entire body. }\end{array}$ & $4.7(1.7,10.9)$ & $2.7(1.2,4.6)$ & $\begin{array}{l}\text { Ron et al. } \\
\text { (1995) }\end{array}$ \\
\hline \multicolumn{6}{|l|}{ [> 15 years at time of exposure] } \\
\hline & & & $0.4(-0.1,1.2)$ & $0.4(-0.1,1.4)$ & \\
\hline $\begin{array}{l}2,475 \text { newborn children in Rochester, NY; } \\
\text { mean age } 0.1 \mathrm{yr} \text {; } 42 \% \text { females; } 35 \text { years of } \\
\text { follow-up. }\end{array}$ & $\begin{array}{l}\text { X-ray therapy for enlarged } \\
\text { thymus gland; } 15 \% \text { received } \\
3 \text { to } 11 \text { multiple exposures. }\end{array}$ & $\begin{array}{c}1.36(0.03 \text { to } 11) \\
{[<1.0 \mathrm{yr} \text { at time of }} \\
\text { exposure }]\end{array}$ & $9.1(3.6,28.8)$ & $2.6(1.7,3.6)$ & $\begin{array}{l}\text { Ron et al. } \\
\text { (1995) }\end{array}$ \\
\hline $\begin{array}{l}\text { 10,834 Israeli children; mean age } 7 \text { years; } \\
51 \% \text { females; } 30 \text { years of follow-up. }\end{array}$ & $\begin{array}{l}\text { X-ray therapy for tinea capitis } \\
\text { (ringworm of the scalp); } 5 \\
\text { treatments given daily, with } \\
9 \% \text { having repeat treatments } \\
\text { beyond one year after initial } \\
\text { treatment. }\end{array}$ & $\begin{array}{l}0.09(0.04 \text { to } 0.5) \\
{[<15 \text { yr at time of }} \\
\text { exposure }]\end{array}$ & $32.5(14.0$ to 57.1$)$ & $7.6(2.7,13.0)$ & $\begin{array}{l}\text { Ron et al. } \\
\text { (1995) }\end{array}$ \\
\hline $\begin{array}{l}\text { 2,634 children in Chicago, IL; mean age } 4 \\
\text { yrs.; } 40 \% \text { females; } 33 \text { years of follow-up. }\end{array}$ & $\begin{array}{l}\text { X-ray therapy for enlarged } \\
\text { tonsils and adenoids; } 3 \\
\text { treatments given weekly, with } \\
12 \% \text { having treatments more } \\
\text { than } 6 \text { months after first } \\
\text { treatment. }\end{array}$ & $\begin{array}{c}0.59(0.01 \text { to } 5.8) \\
{\left[\begin{array}{c}0 \text { to } 15 \text { yrs at time of } \\
\text { exposure }]\end{array}\right.}\end{array}$ & $2.5(0.6,26.0)$ & $3.0(0.5,17.1)$ & $\begin{array}{l}\text { Ron et al. } \\
(1995)\end{array}$ \\
\hline $\begin{array}{l}22 \text { cases and } 82 \text { controls in a case control } \\
\text { study of childhood cancer of } 9,170 \text { patients; } \\
\text { mean age } 7 \text { years; } 45 \% \text { females. }\end{array}$ & $\begin{array}{l}\text { X-ray therapy; large variation } \\
\text { in the number of treatments } \\
\text { given; doses were sufficient to } \\
\text { suppress cancer through cell } \\
\text { killing. }\end{array}$ & $\begin{array}{c}12.5(1 \text { to } 76) \\
\text { [age } 0 \text { to } 18 \text { yrs. at } \\
\text { time of exposure] }\end{array}$ & $1.1(0.4,29.4)$ & not applicable & $\begin{array}{l}\text { Ron et al. } \\
(1995)\end{array}$ \\
\hline $\begin{array}{l}\text { Pooled analysis of the first four studies plus } \\
\text { additional data on young patients treated for } \\
\text { lymphoid hyperplasia in Boston, MA; } \\
\text { almost } 58,000 \text { exposed. }\end{array}$ & $\begin{array}{l}\text { Atomic bomb gamma-rays } \\
\text { and neutrons, and X-ray } \\
\text { therapy. }\end{array}$ & $\begin{array}{l}\text { (0.01 to 11) } \\
\text { [persons } 0-14 \text { at time } \\
\text { of exposure] }\end{array}$ & $\begin{array}{c}\mathbf{7 . 7}(\mathbf{2 . 1}, \mathbf{2 8 . 8}) ; \\
\text { children under } 14 \text { at time of } \\
\text { exposure receiving a single } \\
\text { exposure }\end{array}$ & $4.4(1.9,10.1)$ & $\begin{array}{l}\text { Ron et al. } \\
(1995)\end{array}$ \\
\hline $\begin{array}{l}\text { 14,351 Swedish infants exposed to } \beta \\
\text { radiation, gamma and X rays between } 1920 \\
\text { and } 1959 \text {. }\end{array}$ & $\begin{array}{l}\text { Radiotherapy for skin } \\
\text { hemangioma. }\end{array}$ & $\begin{array}{c}0.26 \\
(<0.01 \text { to } 28.5)\end{array}$ & $\begin{array}{c}4.92 \\
(1.26,10.2)\end{array}$ & 0.9 & $\begin{array}{l}\text { Lundell et } \\
\text { al. (1994) }\end{array}$ \\
\hline
\end{tabular}


Table 10.2 Results of epidemiological studies for exposure to X-rays.

\begin{tabular}{|c|c|c|c|c|c|}
\hline Who was exposed & $\begin{array}{c}\text { How and why they were } \\
\text { exposed }\end{array}$ & $\begin{array}{c}\text { Mean thyroid } \\
\text { dose } \\
\text { Gy } \\
\text { (range) }\end{array}$ & $\begin{array}{c}\text { Excess relative risk for } \\
\text { thyroid cancer } \\
\text { ERR/Gy } \\
(95 \% \text { C.I. }) \\
\end{array}$ & $\begin{array}{c}\text { Excess absolute risk of } \\
\text { thyroid cancer } \\
\text { EAR/10,000PYGy } \\
(95 \% \text { C.I. }) \\
\end{array}$ & Reference \\
\hline $\begin{array}{l}2,227 \text { New York City children } \\
\text { under the age of } 18 \text { who were } \\
\text { followed up for an average of } \\
35 \text { years. }\end{array}$ & $\mathrm{X}$-ray therapy for tinea capitis. & $\begin{array}{l}0.06 \\
\text { [children under the } \\
\text { age of } 18 \text { at time of } \\
\text { treatment] }\end{array}$ & 7.7 (90\% C.I. $0,48.2)$ & $1.5(90 \%$ C.I $0,9.4)$ & Shore (1992) \\
\hline $\begin{array}{l}1,677 \text { mixed juveniles and } \\
\text { adults ages } 2 \text { to } 82 \text {, followed up } \\
\text { for an average of } 10 \text { years. }\end{array}$ & $\begin{array}{l}\text { X-ray therapy for Hodgkin's } \\
\text { disease. } 52 \% \text { received } \\
\text { chemotherapy as well. }\end{array}$ & $\begin{array}{c}34 \\
\text { (dose was } \\
\text { sufficiently high to } \\
\text { produce cell } \\
\text { killing) } \\
\text { [mixed juveniles } \\
\text { and adults at time } \\
\text { of treatment] }\end{array}$ & $0.4(90 \%$ C.I. $0.2,0.8)$ & $0.2(90 \%$ C.I. $0.1,0.3)$ & Shore (1992) \\
\hline $\begin{array}{l}43 \text { females who had thyroid } \\
\text { cancer were identified from a } \\
\text { cohort of } 150,000 \text { cervical } \\
\text { cancer patients treated in } 14 \\
\text { countries. } 81 \text { matched controls } \\
\text { were also selected. The mean } \\
\text { age at diagnosis was } 53 \text { years. }\end{array}$ & $\begin{array}{l}\text { Treatment consisted of } \\
\text { combinations of intracavity } \\
\text { radium and external beam } \\
\text { X-rays. } 50 \% \text { treated for } 5 \text { days } \\
\text { per week for } 30 \text { days. }\end{array}$ & $\begin{array}{c}0.11 \\
(0.01 \text { to } 0.24) \\
\text { [adults at time of } \\
\text { treatment] }\end{array}$ & $34.9(-2.2, \infty)$ & not applicable & $\begin{array}{c}\text { Ron et al. } \\
\text { (1995) }\end{array}$ \\
\hline
\end{tabular}


Table 10.3 Results of epidemiological and experimental studies for exposure to ${ }^{131} \mathrm{I}$.

\begin{tabular}{|c|c|c|c|c|c|}
\hline Who was exposed & $\begin{array}{l}\text { How and why they } \\
\text { were exposed }\end{array}$ & $\begin{array}{c}\text { Mean thyroid dose } \\
\text { Gy } \\
\text { (range) }\end{array}$ & $\begin{array}{c}\text { Excess relative risk for } \\
\text { thyroid cancer } \\
\text { ERR/Gy } \\
(95 \% \text { C.I. })\end{array}$ & $\begin{array}{c}\text { Excess absolute risk of thyroid } \\
\text { cancer } \\
\text { EAR/10,000PYGy } \\
(95 \% \text { C.I. }) \\
\end{array}$ & Reference \\
\hline $\begin{array}{l}3,503 \text { individuals in the US } \\
\text { under the age of } 20,27 \text { years of } \\
\text { follow-up, }\end{array}$ & $\begin{array}{l}\text { Diagnostic treatment } \\
\text { with }{ }^{131} \mathrm{I}\end{array}$ & $\begin{array}{c}\text { approx. } 0.6 \mathrm{~Gy} \\
\text { [ages } 0 \text { to } 20 \text { years at } \\
\text { time of treatment] }\end{array}$ & $3.1(90 \%$ C.I. $<0,23.0)$ & $0.5(90 \%$ C.I. $<0,3.5)$ & $\begin{array}{l}\text { Shore } \\
(1992)\end{array}$ \\
\hline $\begin{array}{l}13,896 \text { mostly adult German } \\
\text { patients, followed up for about } \\
17 \text { years. }\end{array}$ & $\begin{array}{l}\text { Diagnostic treatment } \\
\text { with }{ }^{131} \mathrm{I}\end{array}$ & $\begin{array}{c}1.0 \\
\text { [mostly adults at time of } \\
\text { treatment] }\end{array}$ & 0.3 (90\% C.I. $0,1.4)$ & 0.9 (90\% C.I. 0.0,1.4) & $\begin{array}{l}\text { Shore } \\
(1992)\end{array}$ \\
\hline $\begin{array}{l}1,764 \text { Swedish patients under the } \\
\text { age of } 20 \text { at time of exposure, } \\
\text { followed up for } 40 \text { years. }\end{array}$ & $\begin{array}{l}\text { Diagnostic treatment } \\
\text { with }{ }^{131} 1\end{array}$ & $\begin{array}{c}1.5 \\
{[<20 \mathrm{y} ; \text { it is likely that }} \\
\text { the majority of these } \\
\text { individuals were over } \\
15 \text { at time of exposure }]\end{array}$ & $\begin{array}{c}0.25 \\
(3 \text { cancers observed; } \\
1.8 \text { expected })\end{array}$ & not given & $\begin{array}{l}\text { Hall et al. } \\
\text { (1996a) }\end{array}$ \\
\hline $\begin{array}{l}\text { 20,911 Swedish adults, followed } \\
\text { up for } 40 \text { years. }\end{array}$ & $\begin{array}{l}\text { Diagnostic treatment } \\
\text { with }{ }^{131} 1\end{array}$ & $\begin{array}{c}0.9 \\
{\left[\begin{array}{c}20 \text { years at time of } \\
\text { exposure }]\end{array}\right.} \\
\end{array}$ & 0 & 0 & $\begin{array}{c}\text { Hall et al. } \\
(1996 a)\end{array}$ \\
\hline $\begin{array}{l}1,005 \text { Swedish females of mixed } \\
\text { ages, } 83 \% \text { were over the age of } \\
20 \text { at time of exposure. }\end{array}$ & $\begin{array}{l}\text { Diagnostic treatment } \\
\text { with }{ }^{131} \mathrm{I}\end{array}$ & $\begin{array}{c}0.49 \text { to } 0.7 \\
\text { [most over the age of } 20 \\
\text { at time of exposure] }\end{array}$ & $\begin{array}{c}0.9(0.3,2.3) \\
\text { thyroid nodules } \\
\text { determined by palpation } \\
\end{array}$ & not determined & $\begin{array}{l}\text { Hall et al. } \\
\text { (1996b) }\end{array}$ \\
\hline $\begin{array}{l}2,473 \text { Utah residents under the } \\
\text { age of } 8 \text { at time of first exposure. } \\
\text { Approximately } 35 \text { years of } \\
\text { follow-up. }\end{array}$ & $\begin{array}{l}\text { Nevada Test Site } \\
\text { fallout }{ }^{131} \mathrm{I}\end{array}$ & $\begin{array}{c}0.09 \\
\left(10^{-5} \text { to about } 3\right) \\
\text { [all under the age of } 8 \text { at } \\
\text { time of exposure] }\end{array}$ & $\begin{array}{c}7.0(0.7,33) \\
\text { all neoplasms } \\
7.9(0.0,100) \\
\text { all carcinomas }\end{array}$ & $\begin{array}{c}- \\
3.1^{*} \\
(0-6.9) \\
\text { *estimated } \\
\end{array}$ & $\begin{array}{l}\text { Stevens et } \\
\text { al. (1992) }\end{array}$ \\
\hline $\begin{array}{l}\text { Geographical epidemiological } \\
\text { analysis of } 2,328,000 \text { children } \\
\text { under the age of } 15 \text { at time of } \\
\text { exposure in Ukraine, Belarus, } \\
\text { and Russia ( } 5 \text { y follow-up } \\
\text { period). }\end{array}$ & Chernobyl ${ }^{131} \mathrm{I}$ & $\begin{array}{l}(0.05-0.92) \\
\text { range of average doses } \\
\text { in } 9 \text { study areas }\end{array}$ & $\begin{array}{c}46 \\
\min =21 \text { (Zhytomir } \\
\text { county, Ukraine) } \\
\max =85 \text { (city of Kiev, } \\
\text { Ukraine) }\end{array}$ & $\begin{array}{c}2.4 \\
(1.4-3.8) \\
\min =1.0(\text { Zhytomir county, Ukraine }) \\
\max =3.5 \text { (Gomel county, Belarus) }\end{array}$ & $\begin{array}{c}\text { Jacob et al. } \\
(1998)\end{array}$ \\
\hline $\begin{array}{l}1,800 \text { prepubescent female } \\
\text { Long-Evans rats randomly } \\
\text { assigned to } 6 \text { equal treatment } \\
\text { groups. All surviving animals }\end{array}$ & $\begin{array}{l}3 \text { groups injected } \\
\text { intraperitoneally with } \\
{ }^{131} \mathrm{I}\end{array}$ & $\begin{array}{c}\text { mean doses of } \\
0.80,3.3 \text {, and } 8.5\end{array}$ & not estimated & $\begin{array}{l}2.3,1.1,0.90 \times 10^{-4} \text { lifetime risk per cGy } \\
\text { [equivalent to a human EAR of } 4.3 \text { to } 1.7 \text { ] }\end{array}$ & $\begin{array}{l}\text { Lee et al. } \\
(1982)\end{array}$ \\
\hline $\begin{array}{l}\text { sacrificed after } 2 \text { years; cancers } \\
\text { mostly follicular; cancers are } \\
\text { mostly papillary in humans. }\end{array}$ & $\begin{array}{l}3 \text { groups irradiated } \\
\text { with localized } X \text { rays }\end{array}$ & $\begin{array}{c}\text { mean doses of } \\
0.94,4.1 \text {, and } 10.6\end{array}$ & & $\begin{array}{l}1.5,1.3,0.74 \times 10^{-4} \text { lifetime risk per cGy } \\
\text { [equivalent to a human EAR of } 2.9 \text { to } 1.1 \text { ] }\end{array}$ & \\
\hline
\end{tabular}


The ERR was highest for females exposed to a single treatment before the age of 5 and lowest for males over the age of 10 who received more than a single treatment, with the ratios of relative risks between these two groups being a factor of about 14 (Ron et al., 1995). The pooled analysis by Ron et al. (1995) included 5 cohort studies and two case-control studies totaling approximately 120,000 persons (58,000 exposed subjects and 61,000 nonexposed subjects), in which nearly 700 thyroid cancers were observed during 3,000,000 person years of follow-up examinations.

Unlike the evidence for X-rays and gamma rays, the effectiveness of ${ }^{131} \mathrm{I}$ in producing thyroid cancer is a subject associated with considerable uncertainty (Laird, 1987; Van Middlesworth, 1989; Shore, 1992). Epidemiological studies of 2,473 Utah school children exposed to ${ }^{131}$ I from atmospheric weapons testing at the Nevada Test Site show a significant dose response for all categories of thyroid neoplasms with an ERR per Gy of about 7.0 (90\% C. I. $=0.7$ to 33), but the number of carcinomas (8) and adenomas (11) in this cohort is small (Stevens et al., 1992; Kerber et. al., 1993).

The most convincing evidence of the link between ${ }^{131} \mathrm{I}$ exposure and thyroid cancer is still emerging from reports and preliminary results from the follow-up of children in the aftermath of the Chernobyl accident of 1986. Since 1990, about 1,000 cases of childhood thyroid cancers have been identified within Belarus, Russia and Ukraine, and it is apparent that this observed increase in thyroid cancer is the result of exposure to ${ }^{131} \mathrm{I}$. A preliminary dose-response relationship for children under the age of 15 (EAR of about 4 cases per 10,000 individuals per Gy per year) for exposures to Chernobyl ${ }^{131} \mathrm{I}$ in Belarus, Russia and Ukraine was reported by Jacob et al. (1998). Jacob et al. (1998) found an excess absolute risk for children under the age of 15 of 2.4 (95\% confidence interval of 1.4 to 3.8 ) per $10^{4}$ person-year Gy for the three countries. The differences between the derived excess absolute risks are quite small: from 1.0 per $10^{4}$ person year Gy in Zhytomyr county (Ukraine) to 3.5 per $10^{4}$ person year Gy in Gomel county (Belarus). The excess relative risk from the same set of studies (Jacob et al., 1998) was 46 ERR Gy ${ }^{-1}$, with values ranging from $21 \mathrm{ERR} \mathrm{Gy}^{-1}$ in Zhytomyr county (Ukraine) to 85 ERR $\mathrm{Gy}^{-1}$ in the city of Kiev (Ukraine). The large values of the excess relative risk per unit dose from exposure to ${ }^{131}$ I released from Chernobyl are probably explained by the short follow-up period of the exposed cohort (1991 to 1995), and by the young age of the people in the cohort. At this young age, the natural incidence of thyroid cancer is low. As a population ages, the background incidence of thyroid cancer increases, while the radiosensitivity of the thyroid decreases. If this hypothesis is correct, it is expected that the excess lifetime relative risk per unit dose from the Chernobyl cohort will be much lower than the current observed values. In conclusion, the values derived from the epidemiological studies of the Chernobyl cohort cannot be used directly to estimate the lifetime risk of thyroid cancer, but they are useful in shedding some light on the issue of ${ }^{131}$ I effectiveness in producing thyroid cancer (Section 10.2.4). 
Induction of thyroid carcinomas was also observed in experimental exposure to X-rays and ${ }^{131} \mathrm{I}$ of prepubescent female Long-Evans rats (Lee et al., 1982); these effects were observed regardless of the dose rate at which the animals were exposed. For rats, Lee et al. (1982) estimated a lifetime (24 mo.) excess risk of cancer of $1.9 \times 10^{-2}$ per Gy. This value is similar to a value of $2.1 \times 10^{-2}$ per Gy estimated by the same authors for children exposed at age 10 to X-rays and gamma rays, assuming a human lifetime of 73 years.

\subsubsection{Non-Neoplastic Radiogenic Thyroid Disease}

At high levels of exposure, there is a significant association between ionizing radiation exposure and the occurrence of nonmalignant thyroid disease (IOM/NAS, 1998). At radiation doses of 50 Gy and above, the thyroid gland is destroyed, resulting in hypothyroidism. High radiation doses clearly cause direct follicular cell injury, but there is considerable evidence that this is not the only factor involved in radiation-related non-neoplastic thyroid disease. According to the IOM/NAS (1998), various forms of primary thyroiditis have an autoimmune origin. Both hypothyroidism (including Hashimoto's and atrophic thyroiditis) and some forms of hyperthyroidism (Grave's disease) are caused by closely related genetic and immunologic disturbances, although they are distinctly different diseases. Autoimmune thyroiditis leading to hypothyroidism is related to the presence of antibodies directed against components of the thyroid such as thyroid globulin or thyroid cell microsomes. In Grave's disease, there is diffusely hyperplastic goiter from production of antibody directed against the TSH receptors, which causes excessive stimulation of the follicular cells. It is well known that both autoimmune hypo- and hyperthyroidism can be induced by exposure to ionizing radiation. Thyroid autoimmunity has been observed in individuals (children and adolescents at time of exposure) exposed to Chernobyl ${ }^{131}$ I (Pacini et al., 1998).

The risk of radiogenic autoimmune thyroiditis could plausibly extend down into the range of doses below 1 Gy (100 rad), but the evidence reviewed to date by the IOM/NAS (1998) indicates that hypothyroidism from ${ }^{131} \mathrm{I}$ would be unlikely at doses below 0.1 to $0.2 \mathrm{~Gy}$ (10 to $20 \mathrm{rad}$ ). The current Hanford Thyroid Disease Study (1998) is evaluating all thyroid diseases, including autoimmune thyroiditis and hypothyroidism as well as thyroid antibody concentration, in association with radiation dose. It is expected that the data from this study will be extremely valuable in the estimation of radiogenic risk of non-malignant thyroid disease.

\subsubsection{Other Neoplasms}

Thyroid cancer and benign nodules are both classified as neoplasms. Although thyroid cancer is usually clinically manifested as a nodule on the gland, most thyroid nodules are benign (IOM/NAS, 1998). Larger thyroid nodules $(\geq 1.5 \mathrm{~cm})$ are more likely to be associated with clinically significant thyroid cancer. About $10 \%$ of all nodules larger than $1.5 \mathrm{~cm}$ in size and found to be carcinomas. For nodules less than $1.5 \mathrm{~cm}$, the frequency of carcinomas drops to $3 \%$. Palpable thyroid nodules, those that can be felt through the neck by a trained physician, increase in frequency with age. They are more common in women than in men. Approximately 5\% of women over the age of 50 and $1 \%$ of men over 50 have palpable thyroid nodules. Ultrasound detects thyroid nodules that are less than $1 \mathrm{~cm}$ in size, a size sufficiently small to escape 
detection through palpation. The prevalence of thyroid nodules detected by ultrasonagraphy is a factor of 7 to 10 times greater than that for palpable thyroid nodules.

The radiogenic relative risk of noncancerous neoplasms appears to be similar to that of thyroid cancer (Kerber et al., 1993; Schneider et al., 1993; Stevens et al., 1992). Thus, the calculated excess lifetime risk of neoplasms will be strongly influenced by the method used to determine the background incidence of thyroid nodules.

\subsection{Methodology Used for Estimation of Various Risk-Related Quantities}

\section{Estimation of the Risk Factors}

The risk of acquiring thyroid cancer from exposure to a unit radiation dose (the risk factor) can be determined using a relative or an absolute risk model. A relative risk model seems to be a better representation of the true dose-response relationship for radiation-induced thyroid cancer (Ron et al., 1995). However, an absolute risk model cannot be totally discarded. The risk factors based on a relative risk model are therefore compared to those obtained using an absolute risk model. In this study, the risk of thyroid cancer is estimated for an average individual in the population having an average life expectancy of 70 years.

When a relative risk model is used, the excess lifetime risk per unit dose from a ${ }^{131}$ I exposure at age $\boldsymbol{i}$ (the risk factor) is obtained as follows:

$$
R F_{i}=B_{i} \cdot \beta_{i}=B_{i} \cdot\left(\beta \cdot g \cdot \varepsilon_{i} / \delta\right)
$$

where

$R F_{i}=$ the excess lifetime risk of thyroid cancer per unit dose from exposure at age " $i$ " per unit absorbed dose $\left[\mathrm{Gy}^{-1}\right]$ (risk factor);

$B_{i}=\quad$ the background lifetime risk of thyroid cancer for an exposure at age " $i$ " [unitless];

$\beta_{i}=$ the age- and gender-dependent excess relative risk of thyroid cancer per unit dose for exposure to ${ }^{131} \mathrm{I}_{\left[\mathrm{Gy}^{-1}\right] \text {; }}$

$\beta=$ the excess relative risk (ERR) per unit dose for children of both genders $0-4$ years of age $\left[\mathrm{Gy}^{-1}\right]$;

$g \quad=\quad$ a modifying factor accounting for differences between genders [unitless];

$\varepsilon_{i} \quad=\quad$ a modifying factor accounting for age at time of exposure [unitless]; and

$\delta \quad=\quad$ a modifying factor accounting for the relative effectiveness of ${ }^{131} \mathrm{I}$ in producing thyroid cancer as compared with $\mathrm{X}$ and gamma rays [unitless]. 
When an absolute risk model is used, the excess lifetime risk of radiation-induced thyroid cancer from a unit dose of ${ }^{131} \mathrm{I}$ can be estimated as follows:

$$
R F=E A R \cdot\left(\frac{1}{\delta}\right) \cdot 70
$$

where

$$
\begin{aligned}
& E A R=\quad \text { the excess absolute risk per unit dose for children under the age of } 15 \\
& \text { exposed to gamma and X-rays [per 10,000 person year Gy]. }
\end{aligned}
$$

An estimate of the excess absolute risk is readily available for children under the age of 15 exposed to X-rays and gamma rays (Ron et al., 1995). Since risk estimates based on the absolute risk model are used in this report for comparison purposes only, a breakdown by age or gender was not performed. However, application of the dose-response relationship derived for external radiation to that for internal exposure to ${ }^{131}$ I requires a similar effectiveness factor $(\delta)$ as defined above. The average life expectancy is taken to be 70 years.

Estimation of the Relative Risk and of the Probability of Thyroid Cancer Causation

The relative risk (RR) represents the factor by which the background risk of cancer is increased after exposure to a carcinogen (in this case ${ }^{131} \mathrm{I}$ ). By definition, the relative risk is obtained as

$$
R R_{i}=1+\beta_{i} \cdot D_{i}=1+\left(\beta \cdot g \cdot \varepsilon_{i} / \delta\right) \cdot D_{i}
$$

where $D_{i}$ is the thyroid dose received by an individual of age " $i$," and the rest of the parameters are the same as defined for Equation 10.1.

A relative risk of 2.0 means that exposure to ${ }^{131} \mathrm{I}$ doubled the background lifetime risk of thyroid cancer for the exposed individual. A relative risk equal to 3.0 means that the lifetime risk has been increased by a factor of three because of exposure, and so on.

Another useful quantity is the probability of causation $(\boldsymbol{P C})$, which is defined as the probability that a present or future malignancy may have been caused by an individual's exposure to radiation (NCRP, 1993). The probability of causation is usually expressed in percent. For exposure at age $i$, the probability of causation $P c_{i}$ can be estimated as

$$
P C_{i}=\frac{R R_{i}-1}{R R_{i}} \times 100 \%=\frac{\beta_{i}}{1+\beta_{i}} \times 100 \%
$$

The quantities in Equation 10.4 are those defined above. Estimates of the relative risk and the probability of causation for selected thyroid doses and ages at exposure are presented in Section 11 . 


\section{Estimation of a risk factor for the 0-14 age group}

Estimation of the total number of cancers possibly induced by exposure to ${ }^{131}$ I released from X10 (Section 2.0) is based on the critical group of the population with respect to exposure to ${ }^{131} \mathrm{I}$, that is, children under the age of 15. The risk factor for children 0-14 is obtained as an average of the dose factors for each age group between ages of 0 and 14, weighted by the fraction of the number of people in that age-group. According to the Census of the Population (Brunsman, 1952), in the counties affected by the ${ }^{131}$ I releases from X-10 there were 31,946 children between 0 and 15 in 1950 (15,842 males and 16,104 females), with 12,479 between 0 and 4 (6,126 males and 6,353 females), 10,586 between 5 and 9 (5,281 males and 5,305 females), and 8,881 between 10 and 14 (4,435 males and 4,446 females). For, both males and females, the percentage of people in each age group is $39 \%$ for $0-4,33 \%$ for $5-9$, and $28 \%$ for $10-14$. The estimated risk factor for the 0-14 age group is presented in Section 10.3.

\subsubsection{The Lifetime Background Risk of Thyroid Cancer}

An individual of a given age " $i$," who has never been exposed to ${ }^{131}$ I has a finite risk of getting thyroid cancer over the duration of his or her remaining life. This risk is called "lifetime background risk" $\left(B_{i}\right)$; it depends on the age (" $i$ ") and on the life expectancy of the individual. In this study, the exposed individual is an average individual having an average lifetime of 70 years.

If an individual is exposed at age " $i$," the lifetime background incidence of thyroid cancer for that individual at age $i$ can be estimated as shown in Equation 10.5. The derivation of this relationship is presented in Appendix 10A.

$$
B_{i}=\sum_{k=i}^{\text {lifetime }}\left(R_{0, k} \cdot \Delta t\right)
$$

where

$$
\begin{aligned}
& R_{0, k}=\text { age-specific thyroid cancer incidence rate }\left[\mathrm{yr}^{-1}\right], \text { and } \\
& \Delta t \quad=\quad 1 \text { year. }
\end{aligned}
$$

The age-specific thyroid cancer incidence rates for Tennessee were provided by the Tennessee Department of Health in Nashville (Figure 10.1; Turri, 1998). The data were collected between 1988 and 1995. These data are gender-specific and represent mixed races; they are separated into three geographical groups: (1) incidence rates for all Tennessee counties, (2) incidence rates for Anderson, Roane, Loudon and Knox counties, (3) incidence rates for all counties in Tennessee other than Anderson, Roane, Loudon and Knox. The third group of data was used for estimation of the background risk of thyroid cancer because they are considered to be specifically for Tennessee areas and because they represent people that most probably had not been exposed to ${ }^{131} \mathrm{I}$ released from X-10. Appendix $10 \mathrm{~A}$ presents a summary of the data and 


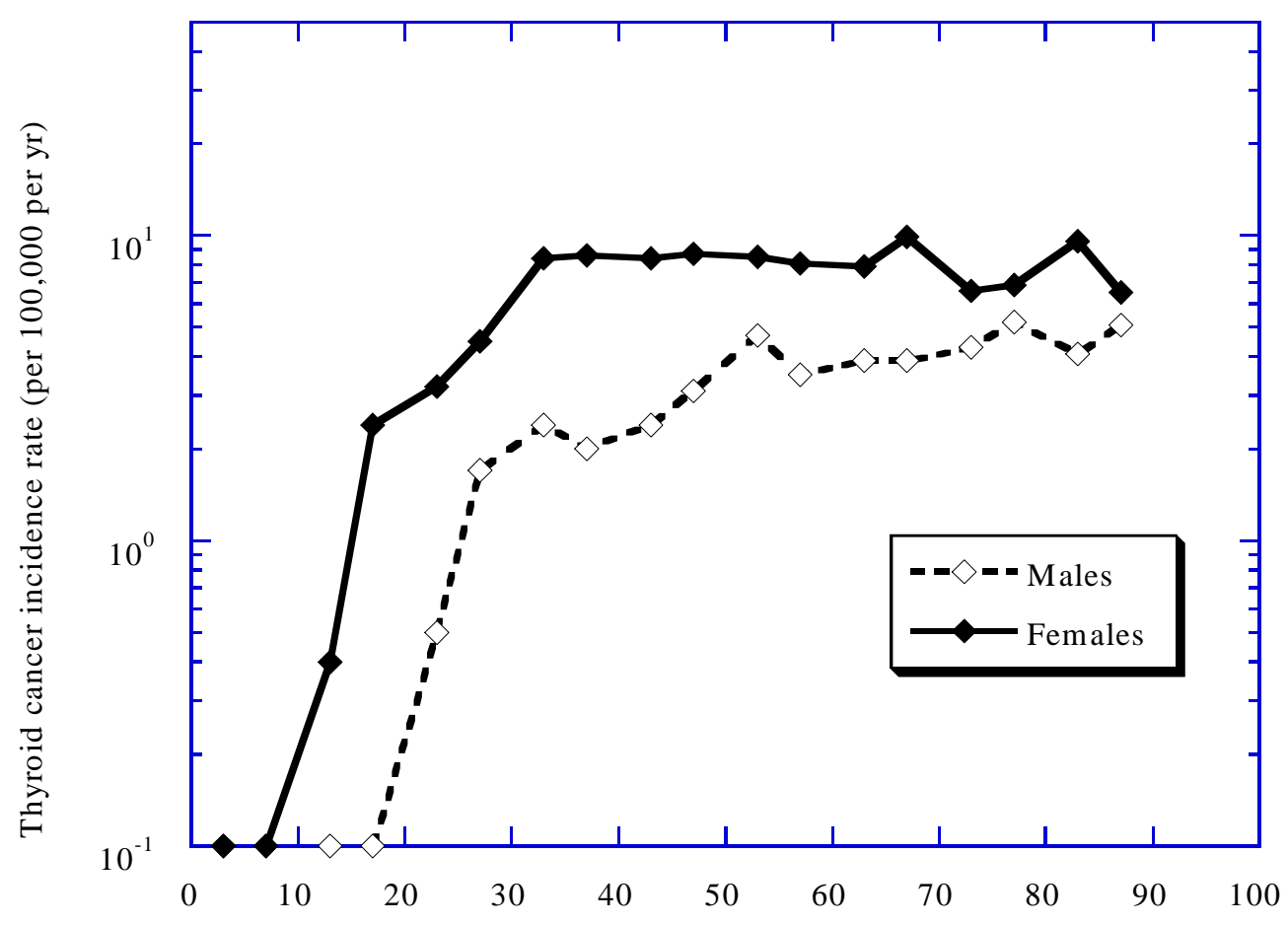

Age (yrs)

Figure 10.1 Age-specific thyroid cancer rates for males and females in Tennessee (1988-1995 diagnosis period). Source: Turri (1998). 
The summation in Equation 10.5 was done for an average lifetime of 70 years. The calculated background lifetime risk was found to be almost constant for exposures at ages less than 14 years (Figure 10.2). This effect can be explained by the low incidence rate of thyroid cancer in childhood as compared with the rates in adulthood.

For exposures at ages less than 5 years, the background lifetime risk of thyroid cancer for females for all counties in Tennessee other than Anderson, Roane, Loudon and Knox was found to be $B_{i}=3.9 \times 10^{-3}$ for an average lifetime of 70 years. For a life expectancy of 85 years or more, the calculated background lifetime risk was $5.4 \times 10^{-3}$. Because the lifetime of an exposed individual is unknown, the ratio between the background for 85 years and the background for 70 years was calculated $\left(5.4 \times 10^{-3} / 3.9 \times 10^{-3}=1.4\right)$. This ratio was used as an uncertainty factor that was applied to the background for a lifetime of 70 years. The estimated range for the background lifetime risk of thyroid cancer for females in Tennessee is $2.8 \times 10^{-3}$ to $5.4 \times 10^{-3}$. A log-uniform distribution was assigned to this range. A similar rationale was applied for all ages and for both genders, and the uncertainties in the background incidence rates were assigned accordingly (Table 10.4).

\subsubsection{The Excess Relative and Excess Absolute Risk of Thyroid Cancer per Unit Dose}

The most comprehensive source of information about radiation-induced thyroid cancer is the analysis of thyroid cancer after childhood exposure to external radiation by Ron et al. (1995). This study found an excess relative risk per Gy $\left(\right.$ ERR $\left.\mathrm{Gy}^{-1}\right)$ of $7.7(95 \%$ C. I. $=2.1$ to 28.7$)$ for childhood exposure ( $<15$ years) of children of both genders. The most at risk individuals are found to be females exposed to a single dose of external radiation of $0.1 \mathrm{~Gy}$ or more before the age of 5 .

To indicate the relative differences between the risk for other age and gender groups and the risk for individuals under the age of 5, Ron et al. (1995) present a set of age-at-first-exposure and gender modifying factors (Table 10.5) (see also Section 10.2.3). However, the study does not report an excess relative risk for individuals under the age of $5(\beta)$. This quantity $(\beta)$ is derived starting from the reported excess relative risk for childhood exposures (7.7 ERR Gy $\left.{ }^{-1}\right)$

$$
\beta=7.7 \cdot \frac{n}{\left(n_{<1} \cdot \varepsilon_{<1}+n_{1-4} \cdot \varepsilon_{1-4}+n_{5-9} \cdot \varepsilon_{5-9}+n_{10-14} \cdot \varepsilon_{10-14}\right)}
$$




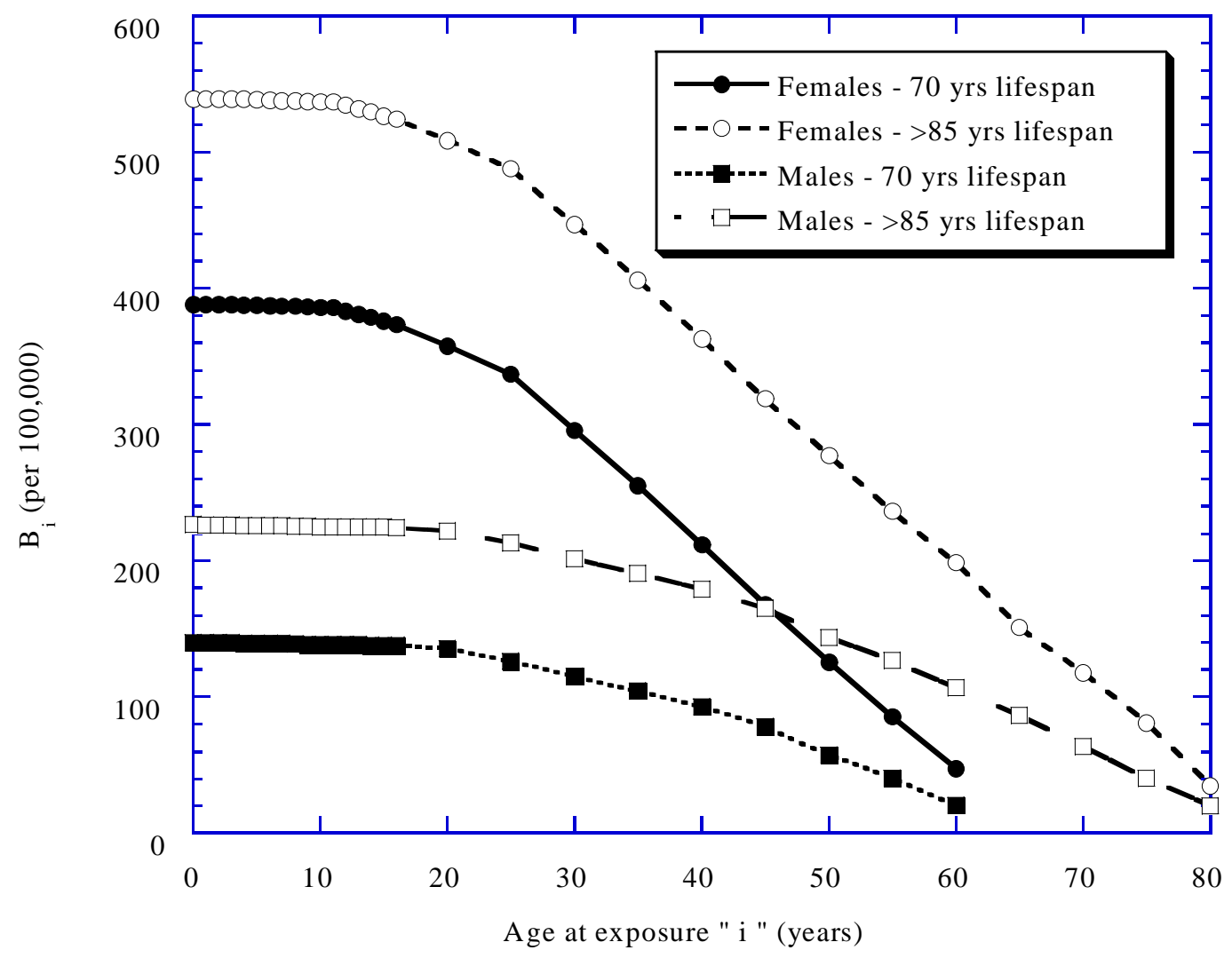

Figure 10.2 Background lifetime risk of thyroid cancer based on statistics for all counties in the State of Tennessee other than Anderson, Roane, Loudon, and Knox counties (1988 -1995 diagnosis period). 
Table 10.4 Background lifetime risk of cancer for all counties in the State of Tennessee other than Anderson, Roane, Loudon and Knox, determined for an average life expectancy of 70 years.

\begin{tabular}{|c|c|c|c|c|c|}
\hline \multirow{3}{*}{$\begin{array}{c}\text { Age at } \\
\text { exposure }^{\mathrm{a}} \\
{[\mathrm{yr}]}\end{array}$} & \multicolumn{5}{|c|}{ Background lifetime risk of cancer } \\
\hline & \multirow[t]{2}{*}{$\begin{array}{l}\text { Duration } \\
\text { of life }^{b}\end{array}$} & \multirow[t]{2}{*}{$\begin{array}{l}\text { Determined value for a } \\
\text { lifetime up to age } 70^{c}\end{array}$} & \multirow[t]{2}{*}{$\begin{array}{l}\text { Uncertainty } \\
\text { factor }^{d}\end{array}$} & \multicolumn{2}{|c|}{$95 \%$ subjective confidence interval } \\
\hline & & & & lower limit & upper limit \\
\hline \multicolumn{6}{|l|}{ Females } \\
\hline $0-4$ & $66-70$ & $3.88 \times 10^{-3}$ & 1.4 & $2.8 \times 10^{-3}$ & $5.4 \times 10^{-3}$ \\
\hline $5-9$ & $61-65$ & $3.87 \times 10^{-3}$ & 1.4 & $2.8 \times 10^{-3}$ & $5.4 \times 10^{-3}$ \\
\hline $10-14$ & $56-60$ & $3.84 \times 10^{-3}$ & 1.4 & $2.7 \times 10^{-3}$ & $5.4 \times 10^{-3}$ \\
\hline $15-19$ & $51-55$ & $3.74 \times 10^{-3}$ & 1.4 & $2.7 \times 10^{-3}$ & $5.2 \times 10^{-3}$ \\
\hline $20-29$ & $41-50$ & $3.37 \times 10^{-3}$ & 1.4 & $2.4 \times 10^{-3}$ & $4.7 \times 10^{-3}$ \\
\hline $30-39$ & $31-60$ & $2.56 \times 10^{-3}$ & 1.6 & $1.6 \times 10^{-3}$ & $4.1 \times 10^{-3}$ \\
\hline \multicolumn{6}{|l|}{ Males } \\
\hline $0-4$ & $66-70$ & $1.40 \times 10^{-3}$ & 1.6 & $8.7 \times 10^{-4}$ & $2.2 \times 10^{-3}$ \\
\hline $5-9$ & $61-65$ & $1.39 \times 10^{-3}$ & 1.6 & $8.7 \times 10^{-4}$ & $2.2 \times 10^{-3}$ \\
\hline $10-14$ & $56-60$ & $1.38 \times 10^{-3}$ & 1.6 & $8.7 \times 10^{-4}$ & $2.2 \times 10^{-3}$ \\
\hline $15-19$ & $51-55$ & $1.38 \times 10^{-3}$ & 1.6 & $8.6 \times 10^{-4}$ & $2.2 \times 10^{-3}$ \\
\hline $20-29$ & $41-50$ & $1.27 \times 10^{-3}$ & 1.7 & $7.4 \times 10^{-4}$ & $2.2 \times 10^{-3}$ \\
\hline $30-39$ & $31-60$ & $1.05 \times 10^{-3}$ & 1.8 & $5.8 \times 10^{-4}$ & $1.9 \times 10^{-3}$ \\
\hline \multirow{2}{*}{\multicolumn{6}{|c|}{$\begin{array}{l}{ }^{\mathrm{a}} \text { People age } 40 \text { and above are not included in this table because the risks are zero (see Table 10.5). } \\
{ }^{\mathrm{b}} \text { Expected average duration of life from the age at first exposure to }{ }^{131} \mathrm{I} \text {. }\end{array}$}} \\
\hline & & & & & \\
\hline \multicolumn{6}{|c|}{${ }^{\mathrm{c}}$ Three digits are presented here to emphasis the slight variation in childhood years. } \\
\hline \multicolumn{6}{|c|}{$\begin{array}{l}\text { d The uncertainty factor is obtained as a ratio between the background lifetime risks for an average life } \\
\text { expectancy of more than } 85 \text { years and an average life expectancy of } 70 \text { years. The uncertainty is described } \\
\text { as a log-uniform distribution between the lower and the upper limits obtained applying the uncertainty } \\
\text { factor to the central value calculated for an average life expectancy of } 70 \text { years. Numbers are rounded to } \\
\text { two significant digits. }\end{array}$} \\
\hline
\end{tabular}

In Equation 10.6, the quantities denoted by $\varepsilon$ are the age modifying factors reported by Ron et al. (1995) (Table 10.5). The quantities denoted by $n$ are the number of cases for the different age groups, respectively. The numbers of cases $\left(n_{\text {age-group }}\right)$ in each age group are as follows: 64 children exposed as newborns ( $<1$ year old), 251 children exposed between 1 and 4 years of age, 111 children exposed between 5 and 9 years old, and 47 children exposed between 10 and 14 years of age, with a total of $n=473$ (Table 10.5).

The "central estimate" of $7.7\left(\mathrm{ERR} \mathrm{Gy}^{-1}\right)$ from Ron et al. (1995) is used as the geometric mean of a lognormal distribution, and the $95 \%$ confidence interval of 2.1 to 28.7 is used to obtain a geometric standard deviation of 1.95 . The excess relative risk for children of both genders exposed before the age of 5 was found to be 9.7 (ERR Gy ${ }^{-1}$ ), with a $95 \%$ confidence interval of 2.5 to 35.4 . 
Table 10.5 Pooled analysis of cohort studies of persons exposed before age 15 years: modifiers of excess relative risk per Gy (ERR Gy ${ }^{-1}$ ) (Ron et al., 1995).

\begin{tabular}{|c|c|c|c|c|}
\hline Variable & Categories & Number of cases & $\begin{array}{l}\text { Modifying } \\
\text { factor }(\varepsilon)\end{array}$ & $P$ value \\
\hline \multirow[t]{2}{*}{ Gender } & Male & 202 & 0.5 & 0.07 \\
\hline & Female & 271 & 1.0 & \\
\hline \multirow[t]{7}{*}{ Age at first exposure (years) } & $<1$ & 64 & $1.0^{\mathrm{a}}$ & 0.004 \\
\hline & $1-4$ & 251 & $1.0^{\mathrm{a}}$ & \\
\hline & $5-9$ & 111 & $0.5^{\mathrm{a}}$ & \\
\hline & $10-14$ & 47 & $0.2^{\mathrm{a}}$ & \\
\hline & $15-19$ & & $1.0^{\mathrm{b}}$ & \\
\hline & $20-39$ & & $0.6^{\mathrm{b}}$ & \\
\hline & 40 & & $0.0^{\mathrm{c}}$ & \\
\hline \multirow[t]{2}{*}{ Number of treatments } & 1 & 373 & 1.0 & 0.18 \\
\hline & $2+$ & 63 & 0.7 & \\
\hline
\end{tabular}

${ }^{a}$ For children under the age of 15, the ERR per Gy derived from Ron et al. (1995) is used (9.7 ERR Gy ${ }^{-1}$ ).

${ }^{b}$ For adults, the ERR per Gy from the Japanese Atomic Bomb Survivors cohort as reported by Ron et al. (1995) is used (0.4 ERR Gy $\left.{ }^{-1}\right)$.

${ }^{\mathrm{c}}$ No dose-response relationship for thyroid cancer can be found for people exposed after the age of 40 (Thompson et al., 1994).

Adolescents older than 15 years of age and adults are less susceptible to radiation-induced thyroid cancer (Ron, 1996; Ron et al., 1995). The excess relative risk per unit dose for people in this group was estimated at $0.4\left(\mathrm{ERR} \mathrm{Gy}^{-1}\right)$, with a $95 \%$ confidence interval of $(-0.1,1.2)$ (Ron et al., 1995). A lognormal distribution with an arithmetic mean of 0.4 and a standard deviation of 0.41 was assigned to describe the uncertainty in the ERR per unit dose. The standard deviation was obtained from the reported $95 \%$ confidence interval.

The analysis of the Japanese Atomic Bomb Survivors shows a negative dose-response relationship for adults exposed after age 40 (Thompson et al., 1994). Thus, no risk associated with ${ }^{131} \mathrm{I}$ exposure for this age group is assumed in this report for individuals over the age of 40 at the time of exposure.

For children under the age of 15 exposed to x-rays and gamma rays, the excess absolute risk per $10^{4}$ PY Gy from the pooled epidemiological study by Ron et al. $(1995)$ is $4.4(95 \%$ C.I. $=1.9$, 10.1). For teenagers and adults (age > 15), the excess absolute risk per $10^{4} \mathrm{PY}-\mathrm{Gy}$ is $0.4(95 \%$ C.I. $=-0.1,1.4$ ) (from Ron et al., 1995, based on the A-Bomb Survivors study). 


\subsubsection{Age and gender modifying factors}

Ron et al. (1995) also present a number of age modifying factors (Table 10.5) that can result in a net increase or decrease in the excess relative risk per unit dose (ERR Gy ${ }^{-1}$ ). These modifying factors were applied to the excess relative risk per unit dose for individuals of both genders under the age of 5 at the time of exposure as follows: a modifying factor of 1 is used for the age group of 0 to 4 years; for the age groups of 5 to 9 years and 10 to 14 years, modifying factors are 0.5 and 0.2 , respectively. An example of the effect of age at the time of exposure on the annual risk of thyroid cancer is shown in Figure 10.3.

For adolescents, a specific excess relative risk per unit dose was used (see Section 10.2.2), and the modifying factor related to the age at exposure for this age-group is 1.0. For adults under the age of 40, a modifying factor of 0.6 was used, as reported by Ron et al. (1995) from an analysis of the Japanese Atomic Bomb Survivors. For adults exposed after 40 years of age, no positive dose-response relationship is found (Thompson et al., 1994).

Gender is another important factor influencing the estimation of the radiation-induced risk of thyroid cancer. In the pooled analysis of the available epidemiological study, Ron et al. (1995) found a tendency for females to be twice as sensitive as males in acquiring thyroid cancer from exposure to external radiation, but this finding was only of only marginal statistical significance $(\mathrm{P}=0.07)$. However, the relative difference between females and males is different from study to study. For instance, males were found to be more sensitive than females in the cohort of Abomb surviving children (by a factor of 2.9), and in the cohort of children treated with X-rays for inflamed tonsils (by a factor of 1.8). In contrast, females were found to be more sensitive than males in the Israel tinea capitis study (by a factor of 5).

To account for the influence of gender in risk estimation, the relative difference between females and males [i.e., the ratio $(R F M)$ between the ERR for females and the ERR for males in a given age group] was treated as an uncertain variable. For instance, RFM $=5$ means that females are 5 times more sensitive than males, and RFM $=0.5$ means males are twice as sensitive as females. The factor $g$ in equation 10.1 is estimated as:

$$
\begin{array}{cc}
g=\sqrt{R F M} & \text { for females, and } \\
g=\frac{1}{\sqrt{R F M}} & \text { for males. }
\end{array}
$$




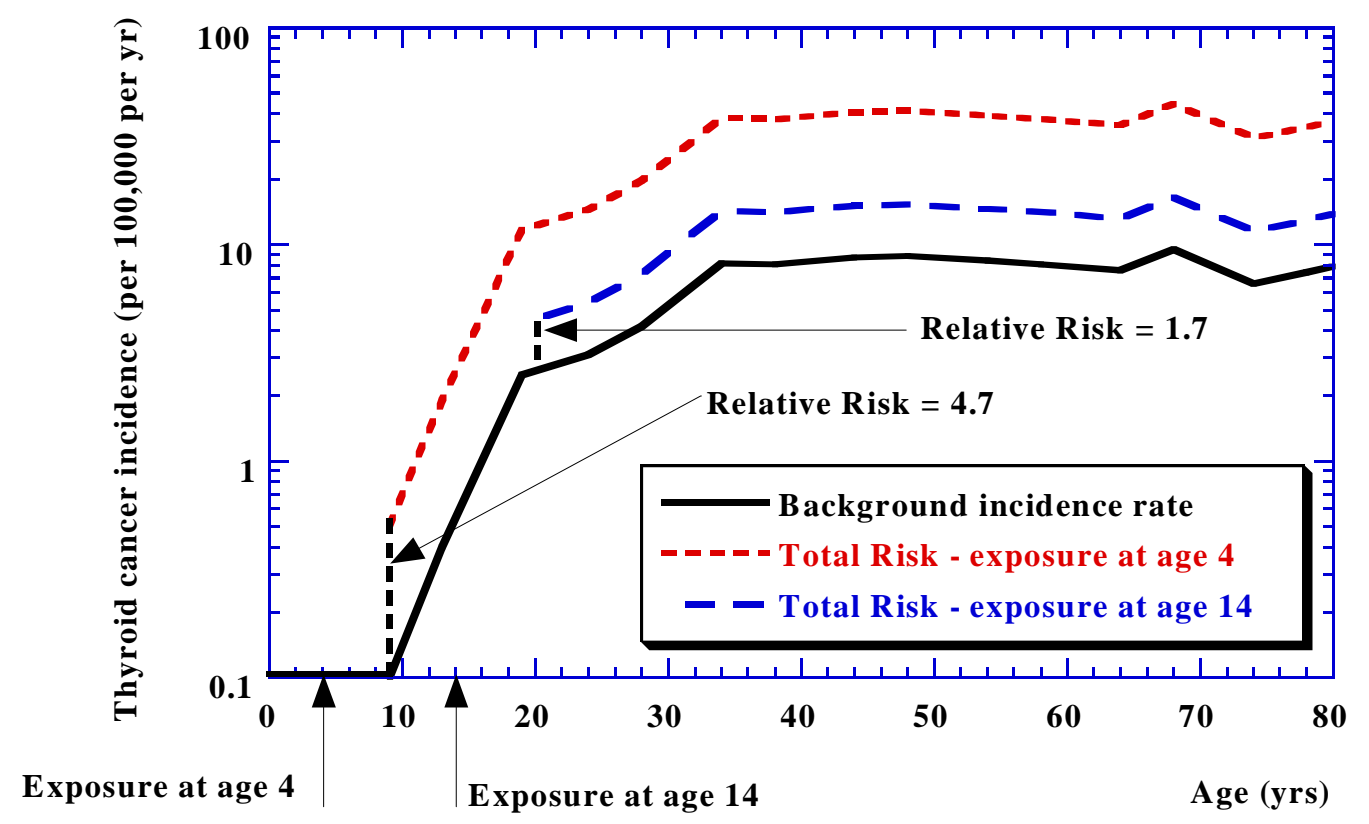

Figure 10.3 Example of the effect of age at time of exposure on the annual risk of thyroid cancer for a single dose of $0.3 \mathrm{~Gy}$ (external exposure). The relative risk is calculated according to Eq. 10.3 where $\beta^{*} g=12.3 \mathrm{~Gy}^{-1}, \delta=1.0$, and $\varepsilon$ is given in Table 10.5. 
The ratio between the risk for females and the risk for males will always be numerically equal to RFM. The relative difference between the radiosensitivity of females and males was defined as a discrete probability distribution function having the following values for children under age 15 .

$$
R F M=\left\{\begin{array}{cc}
5.0 & p=5 \% \\
3.0 & p=10 \% \\
2.0 & p=40 \% \\
1.0 & p=30 \% \\
0.7 & p=10 \% \\
0.2 & p=5 \%
\end{array}\right.
$$

where $p$ is the discrete probability associated with each individual value of RFM.

For adolescents and adults (age > 15), the difference between the radiosensitivity of females and males is larger than for children. Information about adolescents and adults is taken from the ABomb survivors study, which indicate that males are 5 times less sensitive than females. However, as seen in the case of children, differences induced by gender in the observed excess relative risk may vary substantially from one study to another. Thus, the results of a single study can not be treated as information directly applicable to the conditions of the present analysis. To account for this variation in the case of adolescents and adults, the same distribution for RFM as for children was used.

This distribution offers a large spectrum of possible values for this parameter, including a relative difference of a factor of 5 as found by the A-Bomb survivor study.

\subsubsection{Iodine-131 Effectiveness Factor}

The ${ }^{131}$ I effectiveness factor $(\delta)$ represents the value by which the risk from external exposure to $\mathrm{X}$-rays and gamma rays must be divided to obtain the risk from exposure to ingested or inhaled ${ }^{131}$ I. National and international organizations have stated that an absorbed dose (Gy) of ${ }^{131} \mathrm{I}$ is at least a factor of three less effective than a similar absorbed dose of shorter-lived isotopes of iodine or of X-rays and gamma rays (ICRP, 1991; UNSCEAR, 1994; NCRP, 1985; USEPA, 1994). These statements appear to be supported by the publications of Shore (1992) and Hall et al. (1996a). The problem is that most of the epidemiological data on ${ }^{131} \mathrm{I}$ is for diagnostic and therapeutic doses applied to adolescents and adults. In adults, especially those exposed after 40 years of age, the effect of exposure to ${ }^{131} \mathrm{I}$ is similar to that for X-rays and gamma rays: No conclusive dose-response relationship for thyroid cancer has been demonstrated.

At the 1996 annual meeting of the National Council on Radiation Protection and Measurements (NCRP), it was concluded that data were insufficient to support past claims that a given dose of ${ }^{131} \mathrm{I}$ is less effective than the same dose of X-rays and gamma rays (Ron, 1996). Similar concerns have been expressed by Laird (1987) and Van Middlesworth (1989). The few 
epidemiological data that are available on the effects of ${ }^{131} \mathrm{I}$ doses to the thyroid when individuals are exposed in childhood show a dose response that is quite similar to those reported for X-rays and gamma rays.

After review of the literature (Ron, 1996; Jacob et al. 1998; Hall et al., 1996a; Ron et al., 1995; Shore, 1992; Laird, 1987; Lee et al., 1982; ICRP, 1991; UNSCEAR, 1994; NCRP, 1985; USEPA, 1994; Johnson and Myers, 1983) and after discussions with outside experts, the authors of this study believe that the state of knowledge for this parameter is currently described by a range from 1.0 to 5.0. The weight of evidence now seems to support values much closer to unity than to 5.0 (IOM/NAS, 1998).

To capture the uncertainty associated with this parameter, two approaches are used. The first assumes no difference between the effectiveness of external radiation and of ${ }^{131} \mathrm{I}$. The support for this assumption comes from recent observations of the incidence of thyroid cancer in children exposed to ${ }^{131} \mathrm{I}$ in the aftermath of the Chernobyl accident (Beral, 1997; Jacob et al., 1998), the dose response observed in Utah residents exposed in childhood to ${ }^{131} \mathrm{I}$ from weapons testing at the Nevada Test Site (Stevens et al., 1992; Kerber et al., 1995), and the animal study performed by Lee et al. (1982).

The second approach treats the effectiveness of ${ }^{131} \mathrm{I}$ as an uncertain variable with subjective weight given to values of 1.0, 1.5, 2.0, 3.0, and 5.0 (Figure 10.4). In this approach, a subjective weight of $35 \%$ is assigned to the value of 1.0 because strong evidence exists that there may not be a difference between exposure to external radiation and exposure to ${ }^{131} \mathrm{I}$. A subjective weight of $40 \%$ is assigned to the value of 1.5 because of a tendency in the data on fractionated external exposures to show a slightly lower effect over time than when the dose is delivered as a single unit exposure (Ron et al., 1995). It should be noted, however, that this effect was not statistically significant.

Subjective weights of $15 \%, 7 \%$, and $3 \%$ are assigned to values of 2.0,3.0, and 5.0, respectively. The possibilities of these high values are given much lower subjective weights due to the absence of direct supportive evidence. However, these values acknowledge the recommendations of national and international committees for a reduction of the effectiveness of radiation-induced cancer when exposure involves a low dose rate, as is the case with exposures to prolonged releases of ${ }^{131}$ I to the air from the X-10 facility (UNSCEAR, 1994; ICRP, 1991; NCRP, 1985, 1997; USEPA, 1994).

The possibility of a low ${ }^{131} \mathrm{I}$ effectiveness is included because there is limited epidemiological evidence demonstrating an increase in thyroid cancer resulting from long-term, low dose-rate exposures to children spanning a period of several years to a decade or more. One such case is the Utah study (Kerber et al., 1993) in which children were exposed to ${ }^{131}$ I from Nevada Test Site fallout over a period of years. Another is the outcome of cohorts of children exposed to multiple X-ray treatments, extending over a period of several years (Ron et al., 1995). Additional information may be forthcoming in early 1999 with the completion of the Hanford Thyroid Disease Study, which is a case study of children in eastern Washington exposed over a period of years to ${ }^{131}$ I releases to the atmosphere from fuel reprocessing operations at Hanford. 


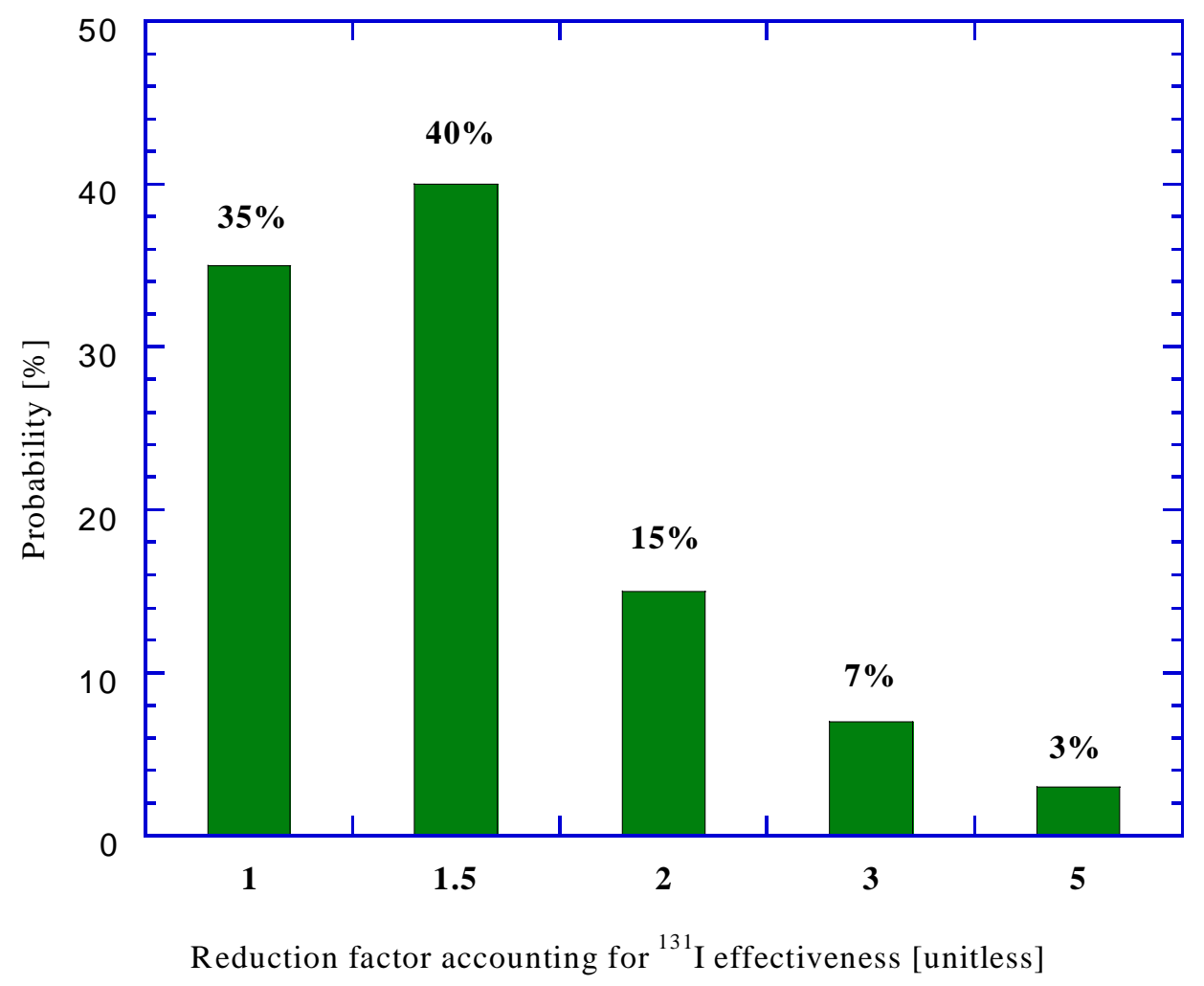

Figure 10.4 Discrete probability distribution function that describe the uncertainty in the reduction factor accounting for ${ }^{131} \mathrm{I}$ effectiveness with respect to an acute dose of $\mathrm{X}$ rays or gamma rays. 


\subsection{Estimates of ${ }^{131}$ I Risk Factors}

The excess lifetime risk of thyroid cancer per unit dose for females exposed under the age of 5 was found to range between $4.5 \times 10^{-3} \mathrm{~Gy}^{-1}$ and $1.6 \times 10^{-1} \mathrm{~Gy}^{-1}$, with a central estimate of $3.0 \times$ $10^{-2} \mathrm{~Gy}^{-1}$ or $3.0 \times 10^{-4} \mathrm{rad}^{-1}$ (Table 10.6; Figure 10.5). The risk at older ages is still high for exposures occurring during childhood, but the risk decreases substantially (about two orders of magnitude) for exposure as an adult. Males have a lower risk of developing thyroid cancer by about a factor of 3 to 4 (Figure 10.5), although the upper bounds for males are almost as high as those for females.

Table 10.6 Estimates of the excess lifetime risk per unit dose (risk factors) [ $\left.\mathrm{Gy}^{-1}\right]$, assuming an uncertain ${ }^{131}$ I effectiveness factor.

\begin{tabular}{|c|c|c|c|}
\hline \multirow[t]{3}{*}{ Age at exposure } & \multicolumn{3}{|c|}{$\begin{array}{l}\text { Excess Lifetime Risk per unit dose } \\
\text { (Risk Factor) }\left[\mathrm{Gy}^{-1}\right]\end{array}$} \\
\hline & \multicolumn{3}{|c|}{$95 \%$ subjective confidence interval } \\
\hline & Lower limit & Central estimate $^{a}$ & Upper limit \\
\hline \multicolumn{4}{|l|}{ Females } \\
\hline $0-4$ & $4.5 \times 10^{-3}$ & $3.0 \times 10^{-2}$ & $1.6 \times 10^{-1}$ \\
\hline $5-9$ & $2.2 \times 10^{-3}$ & $1.5 \times 10^{-2}$ & $7.9 \times 10^{-2}$ \\
\hline $10-14$ & $9.4 \times 10^{-4}$ & $6.7 \times 10^{-3}$ & $2.9 \times 10^{-2}$ \\
\hline $15-19$ & $9.3 \times 10^{-5}$ & $8.8 \times 10^{-4}$ & $5.0 \times 10^{-3}$ \\
\hline $20-29$ & $5.4 \times 10^{-5}$ & $4.7 \times 10^{-4}$ & $2.9 \times 10^{-3}$ \\
\hline $30-39$ & $4.2 \times 10^{-5}$ & $3.4 \times 10^{-4}$ & $2.2 \times 10^{-3}$ \\
\hline \multicolumn{4}{|l|}{ Males } \\
\hline $0-4$ & $1.3 \times 10^{-3}$ & $7.6 \times 10^{-3}$ & $8.2 \times 10^{-2}$ \\
\hline $5-9$ & $6.5 \times 10^{-4}$ & $3.9 \times 10^{-3}$ & $5.4 \times 10^{-2}$ \\
\hline $10-14$ & $2.7 \times 10^{-4}$ & $1.5 \times 10^{-3}$ & $1.8 \times 10^{-2}$ \\
\hline $15-19$ & $2.6 \times 10^{-5}$ & $2.2 \times 10^{-4}$ & $4.1 \times 10^{-3}$ \\
\hline $20-29$ & $1.2 \times 10^{-5}$ & $1.2 \times 10^{-4}$ & $2.9 \times 10^{-3}$ \\
\hline $30-39$ & $1.0 \times 10^{-5}$ & $1.0 \times 10^{-4}$ & $2.3 \times 10^{-3}$ \\
\hline $0-14^{b}$ & $5.8 \times 10^{-3}$ & $1.6 \times 10^{-2}$ & $4.5 \times 10^{-2}$ \\
\hline
\end{tabular}




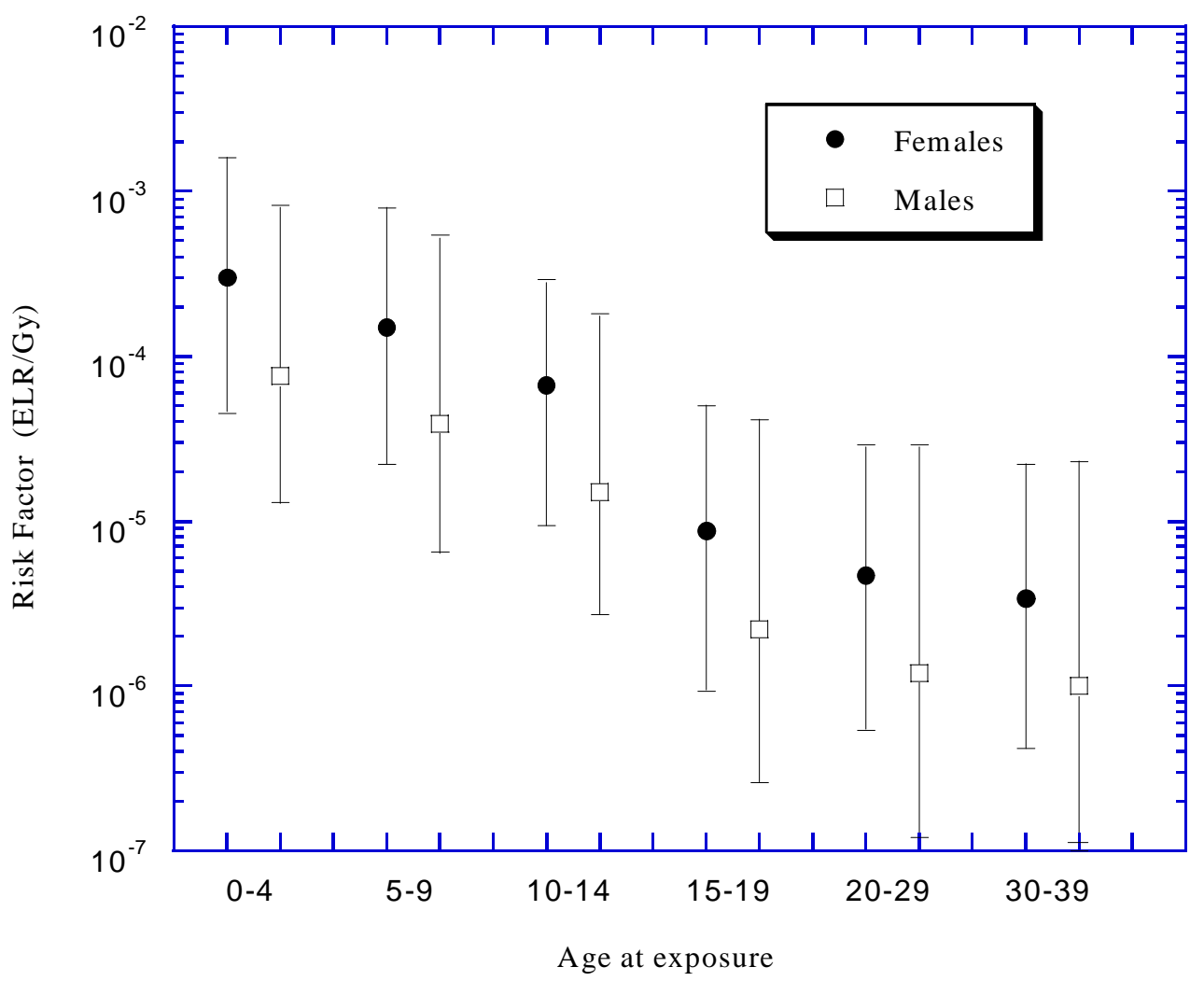

Figure 10.5 Excess lifetime risk of thyroid cancer per Gy (estimated Risk Factors). Vertical bars represent $95 \%$ subjective confidence intervals obtained through propagation of all known sources of uncertainty in the ${ }^{131} \mathrm{I}$ dose-response function. 
The risk factors derived in the present study are obtained by expressing the differences in thyroid cancer induction between internal exposure to ${ }^{131} \mathrm{I}$ and external exposure to $\mathrm{X}$ rays and gamma rays as an uncertain effectiveness factor having a distribution as described in Section 10.2.4. The weighted average value of the ${ }^{131}$ I effectiveness factor from this distribution is 1.6. The extreme situation, as indicated by the recent studies of thyroid cancer incidence in children exposed to ${ }^{131} \mathrm{I}$ released during the Chernobyl accident (Jacob et al. 1998), is that ${ }^{131} \mathrm{I}$ is as effective as X-rays and gamma rays in inducing thyroid cancer; that is, the ${ }^{131}$ I effectiveness factor is 1.0. If this is the case, the average risk estimates will be larger by a factor of 1.6. A re-estimation of the risk factors has been performed assuming that the effectiveness factor is 1.0 (Table 10.7), a comparison of the two sets of results is shown in Figure 10.6. Since an effectiveness factor equal to 1.0 is not yet totally confirmed, and in the presence of the high uncertainty associated with the preliminary results from investigations in Russia, Belarus, and Ukraine, the risk estimates in this report are based on the probability distribution defined for the effectiveness factor in Section 10.2.4.

The risk of acquiring thyroid cancer can be obtained using a relative or an absolute risk model. A relative risk model seems to be a better representation of the true dose-response relationship for radiation-induced thyroid cancer (Ron et al., 1995). However, an absolute risk model cannot be totally discarded. For children below the age of 15 at the time of exposure, the risk factor based on a relative risk model is compared to the risk factor obtained from an absolute risk model (Table 10.8). The central values of the two risk factors are identical $\left(2.1 \times 10^{-2} \mathrm{~Gy}^{-1}\right)$. The absolute risk model provides a tighter confidence interval because of the smaller statistical uncertainties obtained from the pooled epidemiological studies. Data from Ron et al. (1995) indicate a geometric standard deviation of 1.96 for the excess relative risk per unit dose (ERR $\mathrm{Gy}^{-1}$ ), while a geometric standard deviation of 1.53 is observed for the excess absolute risk per $10^{4}$ PY Gy. Also, in estimation of the risk factors based on the relative risk model, additional uncertainty is introduced by the background incidence of cancer. No equivalent source of uncertainty influences the risk factors based on an absolute risk model. In conclusion, even though the absolute risk model provides less uncertain risk estimates, the relative risk model is preferred in this study because it is currently accepted as the better representation of the true dose-response relationship (Ron et al., 1995). No bias in the final risk estimates is introduced by this choice (Table 10.8).

The relative risk (the factor by which the background incidence of cancer is increased because of irradiation) and the probability of causation (the probability that a thyroid cancer may have been caused by a given ${ }^{131}$ I thyroid dose) were also estimated. We found that for thyroid doses larger than $0.1 \mathrm{~Gy}$, the probability of causation for cases of thyroid cancer in children exposed to ${ }^{131} \mathrm{I}$ before the age of 15 is at least $10 \%$. The relative risks and the probability of causation estimated for 41 selected locations around X-10 are presented in Appendix 11B. 
Table 10.7 Estimates of the excess lifetime risk per unit dose (risk factors) [ $\mathrm{Gy}^{-1}$ ], assuming that ${ }^{131} \mathrm{I}$ is equally effective as $\mathrm{X}$-rays and gamma rays in inducing thyroid cancer $(\delta=1.0)$.

\begin{tabular}{cccc}
\hline Age at exposure & \multicolumn{3}{c}{$\begin{array}{c}\text { Excess Lifetime Risk per unit dose } \\
\text { (Risk Factor) }\left[\mathbf{G y}^{-1} \text { ] }\right.\end{array}$} \\
\hline \multicolumn{4}{c}{$\mathbf{9 5 \%}$ subjective confidence interval } \\
& Lower limit & $\begin{array}{c}\text { Central estimate }{ }^{\mathbf{a}} \\
\text { Upper limit }\end{array}$ \\
Females & $9.3 \times 10^{-3}$ & $4.6 \times 10^{-2}$ & $2.3 \times 10^{-1}$ \\
$0-4$ & $4.1 \times 10^{-3}$ & $2.3 \times 10^{-2}$ & $9.4 \times 10^{-2}$ \\
$5-9$ & $1.8 \times 10^{-3}$ & $9.0 \times 10^{-3}$ & $3.3 \times 10^{-2}$ \\
$10-14$ & $1.7 \times 10^{-4}$ & $1.2 \times 10^{-3}$ & $7.0 \times 10^{-3}$ \\
$15-19$ & $1.2 \times 10^{-4}$ & $6.6 \times 10^{-4}$ & $3.9 \times 10^{-3}$ \\
$20-29$ & $9.7 \times 10^{-5}$ & $5.1 \times 10^{-4}$ & $2.8 \times 10^{-3}$ \\
$30-39$ & & & \\
Males & $2.2 \times 10^{-3}$ & $1.1 \times 10^{-2}$ & $6.0 \times 10^{-2}$ \\
$0-4$ & $1.3 \times 10^{-3}$ & $5.6 \times 10^{-3}$ & $2.9 \times 10^{-2}$ \\
$5-9$ & $5.4 \times 10^{-4}$ & $2.2 \times 10^{-3}$ & $1.1 \times 10^{-2}$ \\
$10-14$ & $5.1 \times 10^{-5}$ & $3.2 \times 10^{-4}$ & $1.8 \times 10^{-3}$ \\
$15-19$ & $2.4 \times 10^{-5}$ & $1.7 \times 10^{-4}$ & $1.1 \times 10^{-3}$ \\
$20-29$ & $1.8 \times 10^{-5}$ & $1.5 \times 10^{-4}$ & $1.1 \times 10^{-3}$ \\
$30-39$ & & \\
& & &
\end{tabular}




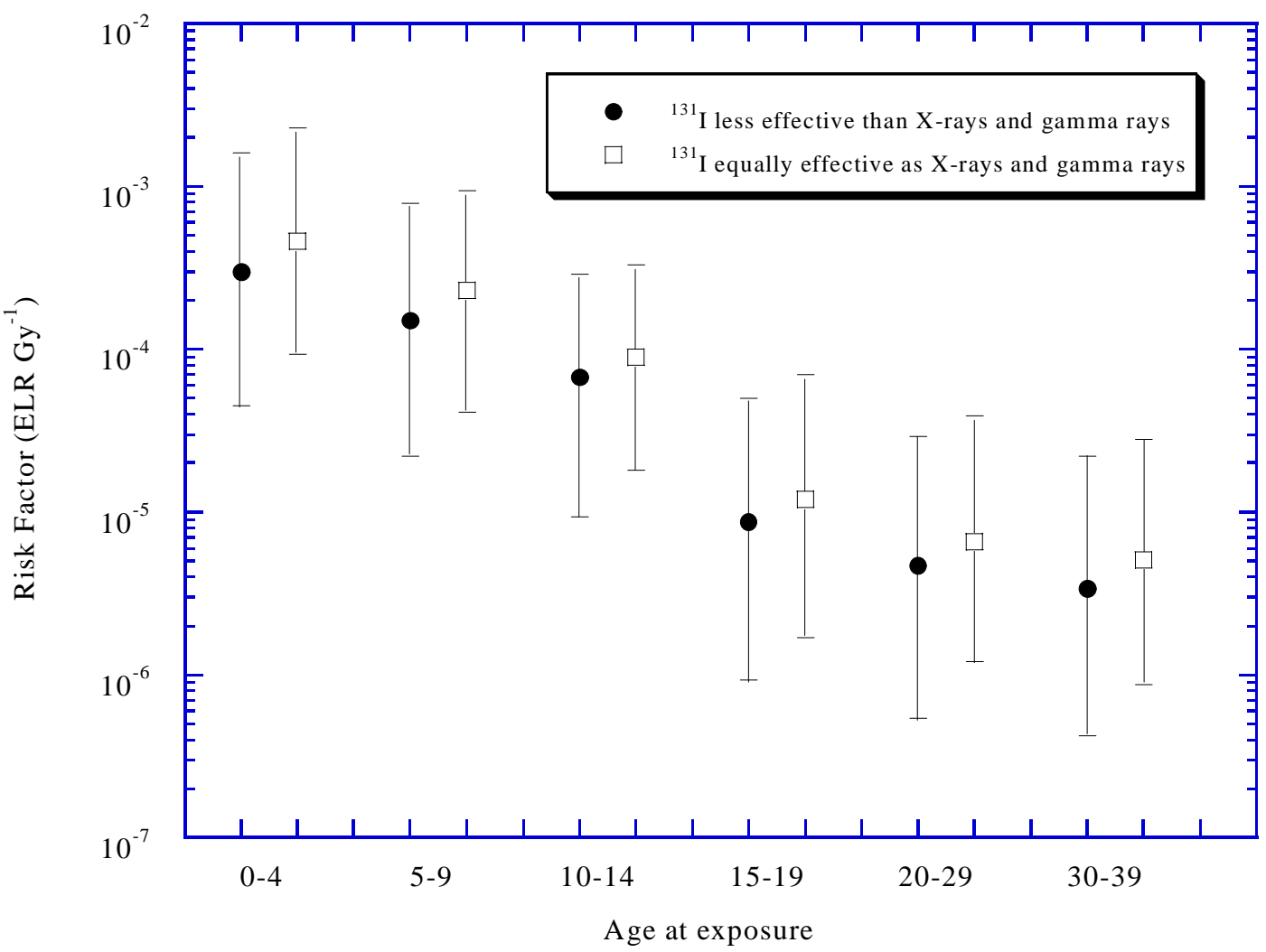

Figure 10.6 Comparison between different assumptions about ${ }^{131}$ I effectiveness in the estimation of excess lifetime risks of thyroid cancer per Gy for females. 
Table 10.8 Comparison between the excess lifetime risk per unit dose (risk factors) obtained using two types of dose-response relationships (models). The risk factors are estimated for children of both genders (ages 0 to 14 ) exposed to ${ }^{131} \mathrm{I}$.

\begin{tabular}{lccc}
\hline & \multicolumn{3}{c}{${\text { Risk factor }\left[\mathbf{G y}^{-1}\right]}$} \\
& $\mathbf{9 5 \%}$ subjective confidence interval \\
\hline Type of model & Lower limit & Central estimate ${ }^{\mathrm{a}}$ & Upper limit \\
\hline Absolute Risk & $5.5 \times 10^{-3}$ & $2.1 \times 10^{-2}$ & $5.9 \times 10^{-2}$ \\
Relative Risk & $3.9 \times 10^{-3}$ & $2.1 \times 10^{-2}$ & $9.3 \times 10^{-2}$ \\
\hline${ }^{a}$ median & &
\end{tabular}

\subsection{Sensitivity Analysis}

To identify the most important contributors to the uncertainty in the risk factors, a sensitivity analysis was performed for females under the age of 5. The excess relative risk per Gy $(\beta)$ is the most important contributor to the uncertainty $(69.5 \%)$, followed by the ${ }^{131}$ I effectiveness factor $(\delta, 13.7 \%)$ and the gender modifying factor $(\mathrm{g}, 11.8 \%)$. The lowest contribution is given by the background incidence of cancer $(\mathrm{Bi}, 5.0 \%)$. These findings apply to males also, because the uncertainty associated with the gender-modifying factor is the same for both genders. Similar results are expected for all age groups, because there is little change in the uncertainty associated with the excess relative risk per Gy for a given age, or with the background incidence of cancer for a given age. Also, the ${ }^{131} \mathrm{I}$ effectiveness factor applies for all ages.

The use of an absolute risk model would reduce the uncertainty in the results because the uncertainty in the $E R R_{1 G y}$ is lower than the uncertainty in the $E A R_{1 G y}$, and because of the elimination of the background incidence of cancer (see Equations 10.1, and 10.2). However, as argued before, epidemiological evidence favors a relative risk model over an absolute risk model as being a more representative description of the true dose-response relationship (Ron et al. 1995). 
Reduction of uncertainty could be obtained by using data from epidemiological studies of cohorts of people specifically exposed to ${ }^{131} \mathrm{I}$ (as opposed to people exposed to X- and gamma rays). If dose-response relationships were available for ${ }^{131} \mathrm{I}$, then the uncertainty introduced by the ${ }^{131}$ I effectiveness factor would be eliminated. Such studies are currently ongoing in parts of Belarus, Russia and Ukraine affected by the radioactive fallout from the 1986 Chernobyl accident, but only preliminary results are available to date (Table 10.3). Additional studies are planned for the cohorts of individuals downwind of the Mayak facility in the Ural Mountains, and for individuals in Kazakhstan and the Marshall Island exposed to ${ }^{131} \mathrm{I}$ in weapons testing fallout.

\subsection{References}

Beral, V. 1997. Thyroid Cancer Around Chernobyl. In: Proceedings of the Thirty-Second Annual Meeting of the National Council on Radiation Protection and Measurements. Proceedings No. 18. Implications of New Data on Radiation Cancer Risk. Boice, J.D., Jr. (ed.). NCRP, Bethesda, MD. 1997.

Brunsman, H.G. 1952. Census of Population: 1950. A Report of the Seventeenth Decennial Census of the United States. Volume II: Characteristics of the Population, part 42, Tennessee. Bureau of the Census. U.S. Dept. of Commerce. U.S. Government Printing Office. Washington, DC. 1952

Hall, P., Mattsson, A., and Boice, J.D., Jr. 1996a. Thyroid Cancer after Diagnostic Administration of Iodine-131. Radiation Research 145:86-92. 1996a.

Hall, P., First, C.J., Mattson, A., Holm, L.E., Boice, J.D., and Inskip, P.D. 1996b. Thyroid Nodularity after Diagnostic Administration of Iodine-131. Radiation Research 146:673-682. 1996b.

Hanford Thyroid Disease Study (HTDS). 1998. Newsletter: Plan for Communicating the Study Results. September, 1998.

ICRP, (International Commission on Radiological Protection). 1991. 1990 Recommendations of the International Commission on Radiological Protection. ICRP 60. Annals of the ICRP Vol. 21, No. 1-3. Oxford, England. 1991. (ChemRisk Repository No. 3078)

IOM/NAS. (Institute of Medicine/National Academy of Sciences). 1998. Exposure of the American People to Iodine-131 from Nevada Nuclear-Bomb Tests: Review of the National Cancer Institute Report and Public Health Implications. National Academy Press. Washington, DC. 1998

Jacob, P., Goulko, G., Heidenreich, W., Likhtarev, I., Kairo, I., Tronko, N., Bogdanova, T., Kenigsberg, J., Buglova, E., Drozdovich, V., Golovneva, A., Demidchik, E., Balanov, M., Zvonova, I., and Beral, V. 1998. Thyroid cancer risk to children calculated. Nature, 392:31-32. 1998. 
Johnson J.R. and Myers D.K. 1983. Is ${ }^{131}$ I Less Efficient than External Irradiation at Producing Thyroid Cancers? IAEA-SM-166/14, International Atomic Energy Agency, Vienna. 1983.

Kerber, R.A., Till, J.E., Simon, S., Lyon, J.L., Thomas, D.C., Preston-Martin, S., Rallison, M.L., Lloyd, R.D., and Stevens, W.A. 1993. A Cohort Study of Thyroid Disease in Relation to Fallout From Nuclear Weapons Testing. JAMA 270:2076-2082. 1993.

Laird, N.M. 1987. Thyroid Cancer Risk from Exposure to Ionizing Radiation: A Case Study in the Comparative Potency Model. Risk Analysis 7(3):299-309. 1987.

Lee, W., Chiacchierini, R.P., Shleien, B., and Telles, N.C. 1982. Thyroid Tumors Following ${ }^{131}$ I or Localized X Irradiation to the Thyroid and Pituitary Glands in Rats. Radiation Research 92:307-319. 1982.

Lundell M., Hakulinen T., Holm, L-E. 1994. Thyroid Cancer after Radiotherapy for Skin Hemangioma in Infancy. Radiation Research 140:334-339. 1994.

NCRP (National Council on Radiation Protection and Measurements). 1985. Induction of thyroid cancer by ionizing radiation. NCRP Report No. 80. Bethesda, Maryland. 1985.

NCRP (National Council on Radiation Protection and Measurements). 1993. The Probability That a Particular Malignancy May Have Been Caused by a Specific Irradiation. A commentary on the NCRP Statement No. 7 issued September 30, 1992. Radiation Research 134:394-397. 1993.

NCRP (National Council on Radiation Protection and Measurements). 1997. Proceedings of the Thirty-Second Annual Meeting of the National Council on Radiation Protection and Measurements. Proceedings No. 18. Implications of New Data on Radiation Cancer Risk. Boice, J.D., Jr. (ed.).NCRP, Bethesda, MD. 1997.

NRC. (National Research Council). 1990. Health Effects of Exposure to Low Levels of Ionizing Radiation, BEIR V. National Academy Press. Washington, DC. 1990.

Pacini, F., Vorontsova, T., Molinaro, E., Kuchinskaya, E., Agate, L., Shavrova, E., Astachova, L., Chiovato, L., and Pinchera, A. 1998. Prevalence of thyroid autoantibodies in children and adolescents from Belarus exposed to the Chernobyl radioactive fallout. Lancet 352:763-766. 1998.

Ron, E. 1996. Cancer Risk following Radioactive Iodine-131 Exposures in Medicine. In: Proceedings of the Thirty-second Annual Meeting of the National Council on Radiation Protection and Measurements. Proceedings No. 18. Implications of New Data on Radiation Cancer Risk. National Council on Radiation Protection and Measurements, Bethesda, MD. 1996. 
Ron, E., Lubin, J.H., Shore, R.E., Mabuchi, K., Modan, B., Pottern, L.M., Schneider, A.B., Tucker, M.A., and Boice, J.D., Jr. 1995. Thyroid Cancer after Exposure to External Radiation: A Pooled Analysis of Seven Studies. Radiation Research 141:259-277. 1995.

Schneider, A.B., Ron, E., Lubin, J., Stovall, M., Geirlowski, T.C. 1993. Dose-response relationships for radiation-induced thyroid cancer and thyroid nodules: Evidence for prolonged effects of radiation on the thyroid. Journal of Clinical Endocrinology and Metabolism 82:40204027. 1993.

Shore, R. 1992. Issues and Epidemiological Evidence Regarding Radiation-Induced Thyroid Cancer. Radiation Research 111. 1992.

Spengler, R.F. (ed.). 1997. Hanford Medical Monitoring Program: Background Consideration Document and ATSDR Decision. U.S. Department of Health and Human Services. Agency for Toxic Substances and Disease Registry. Atlanta, Georgia. July, 1997.

Stevens, W., Till, J.E., Thomas, D.C., Lyon, J.L., Kerber, R.A., Preston-Martin, S., Simon, S.L., Rallison, M.L., and Lloyd, R.D., 1992. Assessment of Leukemia and Thyroid Disease in Relation to Fallout in Utah. Report of a Cohort Study of Thyroid Disease and Radioactive Fallout from the Nevada Test Site. The University of Utah. Salt Lake City, UT. July, 1992.

Thompson, D.E., Mabuchi K., Ron, E., Soda, M., Tokunaga, M., Ochikubo, S., Sugimoto, S., Ikeda, T., Terasaky, M., Izumi, S., and Preston, D.L. 1994. Cancer Incidence in Atomic Bomb Survivors. Part II: Solid Tumors, 1958-1987. Radiation Research 137:S17-S67. 1994.

Turri, P. 1998. Personal Communication. Tennessee Department of Health, Tennessee Towers, Cordell Hull Building, 425 5th Avenue North, Nashville, TN 37247-5261. 1998.

UNSCEAR (United Nations Scientific Committee on the Effects of Atomic Radiation). 1994. Sources and Effects of Ionizing Radiation. UNSCEAR 1994 Report to the General Assembly, with Annexes. New York, United Nations. 1994.

USEPA (United States Environmental Protection Agency). 1994. Estimating Radiogenic Cancer Risks. Washington, DC. EPA 402-R-93-076. June, 1994. (ChemRisk Repository No. 3085).

Van Middlesworth, L. 1989. Effects of Radiation on the Thyroid Gland. Adv. Intern. Med. 34:265-284. 1989. 


\subsection{RESULTS}

Estimates of the absorbed dose to the thyroid gland and the excess risk of thyroid cancer from the 13 years of continuous exposure are obtained by combining the intermediate processes described in previous sections of this report: (a) the release of approximately 8,800 to 42,000 $\mathrm{Ci}$ ( 0.3 to 1.6 PBq; $1 \mathrm{PBq}=10^{15} \mathrm{~Bq}$ ) of ${ }^{131} \mathrm{I}$ between 1944 and 1956 (releases from the April 29, 1954, accident are included in this estimate) from the RaLa processing facility at X-10 (Section 3); (b) the atmospheric transport, dispersion, and chemical transformation of ${ }^{131} \mathrm{I}$ over a radius of $38 \mathrm{~km}$ from the facility (Section 4); (c) ${ }^{131}$ I deposition onto vegetation surfaces (Section 5) and its subsequent transfer to milk, beef, eggs, leafy vegetables, cottage cheese, and human breast milk (Sections 6 and 7); (d) the ingestion and inhalation of ${ }^{131}$ I by individuals of both genders and of different ages at time of exposure (Section 8); and (e) the subsequent doses per unit intake and risks of thyroid cancer per unit dose (Sections 9 and 10). This section provides a summary of the historic releases of ${ }^{131} \mathrm{I}$, the off-site concentrations in various environmental media, and the dose and risk to individuals by gender, age, diet and location. More detailed information is provided in the Appendices (11A, 11B, and 11C). In addition to the evaluation of public exposures from releases of ${ }^{131} \mathrm{I}$ from RaLa processing at X-10, a brief analysis is made of the combined exposure to ${ }^{131}$ I from the X-10 releases and from regional ${ }^{131}$ I fallout that occurred primarily from 1952 through 1957 from atmospheric testing of nuclear weapons at the Nevada Test Site.

\subsection{A Summary of Estimates of the Historical Releases of ${ }^{131}$ I from X-10}

From 1944 to 1956 , releases of ${ }^{131}$ I occurred from the X-10 facility from the dissolution of freshly spent uranium fuel slugs in nitric acid to obtain radioactive lanthanum, which was used for weapons design, production, and testing at Los Alamos. During this period, approximately 8,800 to $42,000 \mathrm{Ci}(0.3$ to $1.6 \mathrm{PBq})$ of ${ }^{131} \mathrm{I}$ was released, of which 6,300 to $36,000 \mathrm{Ci}(0.23$ to 1.3 $\mathrm{PBq}$ ) was in the reactive (elemental) form and the remainder was in the nonreactive volatile organic form. The largest releases occurred between 1952 and 1956, during which period the freshly spent uranium fuel slugs were supplied by Hanford reactors in the state of Washington. Complete details of the estimation of the amount of ${ }^{131}$ I released from the radioactive lanthanum processing facility at X-10 are presented in Section 3 of this report.

\subsection{A Summary of Off-site Concentrations of ${ }^{131}$ I in Environmental Media}

Estimates of the 13-year time averaged concentrations of ${ }^{131} \mathrm{I}$ in air, pasture, milk, eggs, beef, leafy vegetables, cottage cheese, and mother's milk have been made for 41 representative locations within $38 \mathrm{~km}$ of the X-10 facility (Table 11.1 and Figure 1.1). The rationale for the selection of these locations and the exposure pathways considered at each location are given in Appendix 11A. The 13-year, time-averaged concentrations for each environmental medium are provided in Appendix 11B. The average concentrations in milk are estimated for a family or "backyard" cow, for commercially distributed milk from a local dairy, for commercially distributed milk from major regional dairies, and for milk from goats. Although Appendix 11B provides information on the 13-year, time-averaged concentrations, the actual estimates of exposure, dose, and risk are based on annual averages calculated for each year of release. 
Table 11.1 Locations of concern for which the analysis was conducted.

\begin{tabular}{ccc}
\hline Location of Concern & $\begin{array}{c}\text { Distance from } \\
\mathrm{X}-10(\mathrm{~km})\end{array}$ & $\begin{array}{c}\text { Angle from North } \\
\text { direction (degrees) }\end{array}$
\end{tabular}

\begin{tabular}{|c|c|c|}
\hline Bradbury & 6.0 & 228 \\
\hline Gallaher Bend & 6.3 & 89 \\
\hline EFPC & 7.6 & 7 \\
\hline Hope Creek & 7.6 & 119 \\
\hline Buttermilk Rd. & 8.4 & 106 \\
\hline Jonesville & 9.0 & 312 \\
\hline OR Scarboro & 9.3 & 32 \\
\hline Lawnville/Gallaher & 9.3 & 245 \\
\hline Dyllis & 10.1 & 293 \\
\hline OR High School Area & 11.2 & 27 \\
\hline Norwood & 11.3 & 355 \\
\hline Woodland & 11.5 & 35 \\
\hline Hardin Valley & 12 & 85 \\
\hline Oliver Springs & 13.3 & 350 \\
\hline Solway & 13.5 & 61 \\
\hline Sugar Grove & 13.6 & 263 \\
\hline OR Townsite & 13.8 & 31 \\
\hline Hines Valley & 14.5 & 185 \\
\hline Farragut & 15.1 & 108 \\
\hline Lenoir City & 15.5 & 155 \\
\hline Kingston & 19 & 250 \\
\hline Karns & 19.5 & 73 \\
\hline Loudon & 19.5 & 192 \\
\hline Harriman & 20.5 & 271 \\
\hline Cedar Bluff & 21.1 & 95 \\
\hline Oakdale & 21.1 & 286 \\
\hline Claxton & 22.7 & 54 \\
\hline Dutch Valley & 24 & 27 \\
\hline Clinton & 25.4 & 38 \\
\hline Friendsville & 26.4 & 138 \\
\hline Wartburg & 27 & 317 \\
\hline Rockwood & 28 & 258 \\
\hline Louisville & 28.2 & 125 \\
\hline Barnardville & 31.4 & 232 \\
\hline Greenback & 32.3 & 156 \\
\hline Rockford & 33.3 & 104 \\
\hline Lake City & 35 & 27 \\
\hline Sweetwater & 35.1 & 204 \\
\hline Knoxville & 35.8 & 80 \\
\hline Maryville & 36.2 & 136 \\
\hline Cedar Grove & 36.2 & 54 \\
\hline
\end{tabular}


The highest concentrations of ${ }^{131} \mathrm{I}$ in all environmental media occurred for the agricultural communities of Gallaher Bend and Bradbury, which are located $6.3 \mathrm{~km} \mathrm{E}$ and $6.0 \mathrm{~km} \mathrm{SW}$, respectively, of the X-10 site. At these locations, the 13-year, time-averaged air concentrations of ${ }^{131} \mathrm{I}$ ranged from about 0.074 to $0.2 \mathrm{~Bq} \mathrm{~m}^{-3}$ (the lowest and highest limits estimated for the $95 \%$ subjective confidence intervals). The ${ }^{131}$ I concentrations in air estimated for the 5 sites in the city of Oak Ridge $\left(0.018\right.$ to $\left.0.089 \mathrm{~Bq} \mathrm{~m}^{-3}\right)$ are a factor of 2.6 to 4 times less than the concentrations estimated for the two off-site locations with the highest concentrations.

In general, for most of the other locations, the averaged ${ }^{131} \mathrm{I}$ air concentrations are still within a factor of ten of those for the two near-in communities of Gallaher Bend and Bradbury. More than a factor of ten reduction in ${ }^{131} \mathrm{I}$ concentrations occurs at distances beyond $25 \mathrm{~km}$ to the south of the X-10 facility and at distances beyond $15 \mathrm{~km}$ to the north and northwest. At these northern distances, the plumes from X-10 approach Walden's Ridge and the Cumberland Mountains (see Figs. 4.8 and 4.9 in Section 4).

In the general southwest to northeast direction of the ridges and valleys surrounding the Oak Ridge Reservation, the 13-year, time-averaged air concentrations do not diminish substantially with increasing downwind distance. Air concentrations in these directions, even at distances of up to $20 \mathrm{~km}$, remain within a factor of 4 to 5 of those estimated for the communities of Gallaher Bend and Bradbury. The city of Knoxville, for example, at a distance of $35.8 \mathrm{~km}$ ENE of the X10 facility, is estimated to have a time-averaged ${ }^{131} \mathrm{I}$ air concentration of 0.015 to $0.036 \mathrm{~Bq} \mathrm{~m}^{-3}$, only 6 times less than that estimated at Gallaher Bend.

Differences in air concentrations with downwind distance determine the spatial distinction in local concentrations of ${ }^{131} \mathrm{I}$ for pasture grass and food products. Thus, at any location, the 13year, time-averaged ${ }^{131}$ I concentrations for pasture and food products will be a multiplicative factor above the 13-year, time-averaged value for air. For example, the concentration of ${ }^{131} \mathrm{I}$ in pasture grass $\left(\mathrm{Bq} \mathrm{kg}{ }^{-1}\right.$ dry wt $)$ is approximately a factor of 6,000 to 20,000 greater than the concentration in air $\left(\mathrm{Bq} \mathrm{m}^{-3}\right)$. The concentration in milk obtained from a backyard cow $\left(\mathrm{Bq} \mathrm{L}^{-1}\right)$, and the concentration in locally produced commercial milk, are about 250 to 1600 and 200 to 750 times the local concentration of ${ }^{131} \mathrm{I}$ in air, respectively. The average concentrations of ${ }^{131} \mathrm{I}$ in goat's milk will be about 3 to 5 times higher than that for a backyard cow, or about 1000 to 8100 times higher than the average concentration in air at the same location. Higher concentrations in goat's milk are due to the higher efficiency with which goats transfer iodine from their diet into milk (Section 6).

For commercial milk that is distributed from large regional dairies, the 13-year, time-averaged ${ }^{131}$ I concentration is virtually independent of location, due to the regional mixing of milk obtained over a large area. The average concentration of ${ }^{131} \mathrm{I}$ in this milk is estimated to range from 2.2 to $16 \mathrm{~Bq} \mathrm{~L}^{-1}$ for all locations. This is the milk concentration that is ascribed to all locations within the city of Oak Ridge, except for the East Fork Poplar Creek site (EFPC), where there were small farms that kept a small number of dairy cows for family use. 
Since $1 \mathrm{~L}$ of milk weighs approximately $1 \mathrm{~kg}$, the concentration in milk expressed in units of $\mathrm{Bq}$ $\mathrm{L}^{-1}$ can be directly compared with concentrations in other food products expressed in units of $\mathrm{Bq}$ $\mathrm{kg}^{-1}$. The 13-year, time-averaged concentration of ${ }^{131} \mathrm{I}$ in leafy vegetables on a fresh weight basis is about the same as that in backyard cow's milk, while those for locally produced cottage cheese and eggs are nearly the same as for locally produced commercial milk. The estimated timeaveraged concentrations of ${ }^{131} \mathrm{I}$ in beef are about 0.25 to 1 times the concentrations in locally produced commercial milk at a given location. The concentration of ${ }^{131} \mathrm{I}$ in mother's milk depends on the amount of iodine released in the year when a child was born. Appendix 11B reports concentrations of ${ }^{131} \mathrm{I}$ in mother's milk for the case in which a child is born in 1952, and the lactating mother consumes regionally mixed commercial milk

\subsection{Estimates of Dose and Risk from the Combined Effect of Routine and Accidental Emissions from RaLa Processing at X-10 from 1944 to 1956.}

The absorbed radiation dose to the thyroid gland is determined by the amount of ${ }^{131}$ I ingested and inhaled, the age of the individual at the time of exposure, the location of the individual, and, to a certain extent, the gender. The amount of ${ }^{131} \mathrm{I}$ taken into the body is determined primarily by the source and makeup of the individual's diet, with most of an individual's exposure coming from the consumption of contaminated fresh milk.

For the purpose of estimating the excess risk of thyroid cancer, four reference diets are considered: diet 1, which is a rural diet composed entirely of local produce and milk from a backyard cow; diet 2, which is a rural diet composed of local produce and milk from a local commercial dairy; diet 3 , which is dominated by the intake of regionally mixed retail milk; and diet 4, which is dominated by the intake of goat's milk. The highest doses and risks occur with diet 4. Most residents in the city of Oak Ridge would have been exposed according to the assumptions used for diet 3 .

\subsubsection{The importance of food type and source of milk.}

Differences in the absorbed dose as a function of different food types and dietary composition for a female born in 1952 in Solway are shown in Fig. 11.1. This figure clearly shows that for a child, the highest doses result from the consumption of goat's milk, followed by milk from a backyard cow, milk from a local commercial dairy, and commercial milk that is regionally. Since regionally mixed retail milk is about the same within the domain of this assessment regardless of location, its importance will vary from location to location with respect to the consumption of local produce (or to inhalation). For example, the dose from the consumption of locally produced eggs at Solway is approximately the same as the dose from the consumption of regionally mixed retail milk. 


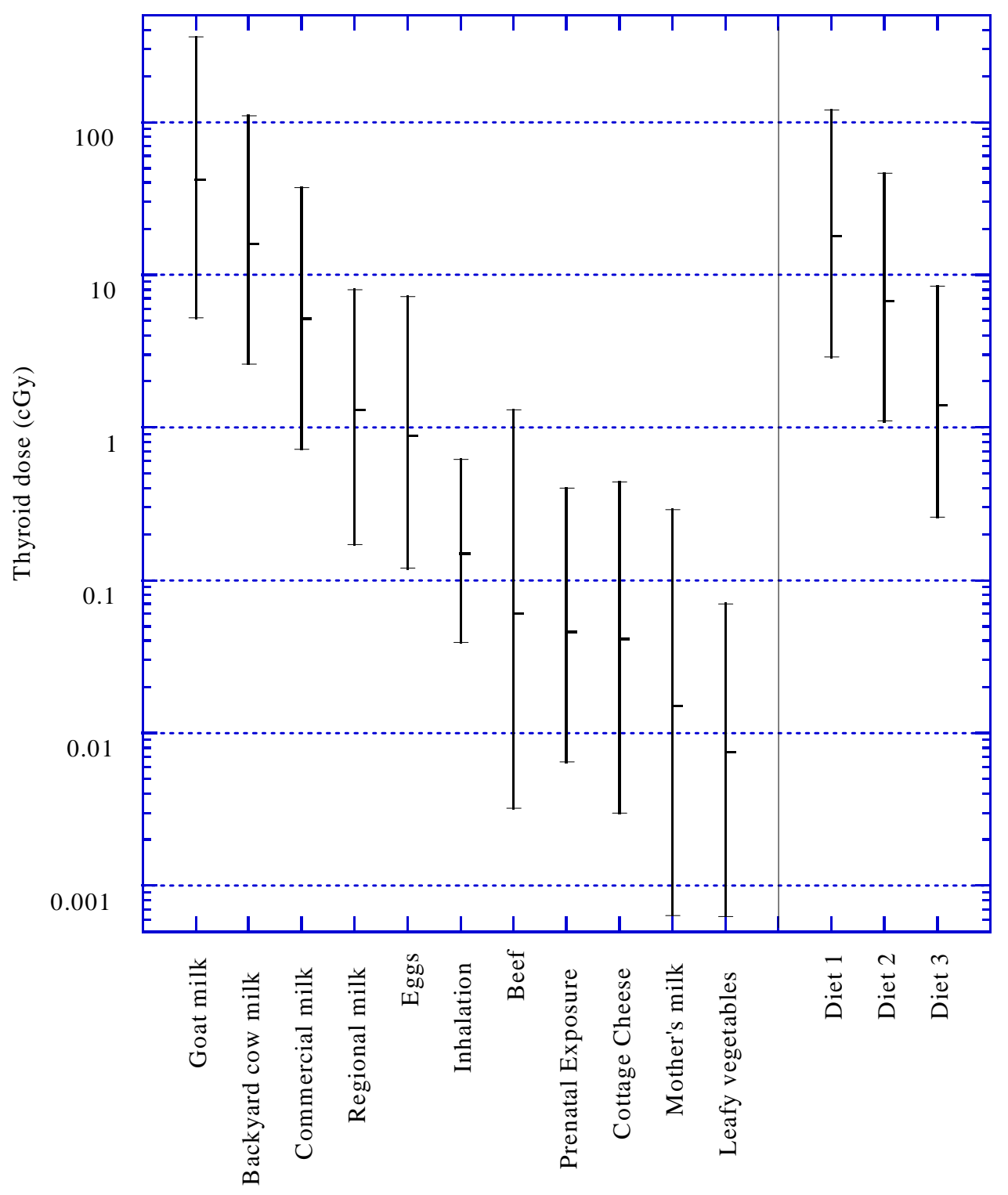

Figure 11.1 Thyroid doses (cGy) from various exposure pathways and diets for a female born in 1952 who lived in Solway, Tennessee. The composition of the various diets is described in Section 11.3. The vertical lines indicate the 95\% subjective confidence intervals on the dose estimates; the central values $\left(50^{\text {th }}\right.$ percentiles $)$ are also indicated. 
Lower doses are obtained from inhalation or from the consumption of locally produced beef, cottage cheese, mother's milk (with the mother assumed to be on a diet composed of local produce and milk from a backyard cow, i.e., diet 1), or leafy vegetables. The doses from inhalation and the consumption of these food types for a child under the age of 5 at the time of exposure are several hundred to more than 1000 times less important than the dose from the consumption of backyard cow's milk. The thyroid dose from prenatal exposure during the first part of 1952 (assuming the mother to be on a rural diet, i.e., diet 1) is about equal to the 5-year total thyroid dose obtained from the consumption of beef or cottage cheese.

An example of the dose and risk estimates provided as a function of location, birth year, gender and diet is given in Table 11.2 for a female born in 1952 and residing in Solway. The risk estimates include the chance of getting a radiogenic thyroid cancer during one's lifetime (Excess Lifetime Risk), the multiplicative effect of the exposure to ${ }^{131} \mathrm{I}$ in increasing the risk of thyroid cancer above the background incidence rate for an unexposed population of similar age and gender (Relative Risk), and the probability that a specific diagnosed thyroid carcinoma could have been caused by one's exposure to ${ }^{131}$ I released from X-10 production of radioactive lanthanum (Probability of Causation). Detailed results, which are presented in a format identical to Table 11.2 for 41 locations, nine birth years $(1920,1930,1935,1940,1944,1950$, 1952, 1954, 1956), and both genders, are included in Appendix 11C. Note that the results in Appendix $11 \mathrm{C}$ apply to the general vicinity of any specified location.

\subsubsection{The effect of birth year and gender on dose and risk.}

Assuming the same dietary sources for ingestion of ${ }^{131} \mathrm{I}$ at a specific location, differences in gender account for only minor differences in the estimation of the thyroid doses (Table 11.3). More significant differences are determined by the year of birth, with the lowest doses being for individuals born in 1920, 1930, and 1956; these doses are about one-fourth to one fifth of the highest doses received by individuals born within the years of 1944 to 1952 . Individuals born in 1954 have about the same doses as those born in 1940, or about $65 \%$ of the doses for those born between 1944 and 1953 .

Much larger differences are observed in the estimates of the excess lifetime risk of thyroid cancer (Table 11.4). Depending on the year of birth, the excess lifetime risk to females is from 3 to 4 times larger than the risk to males. The lowest risk is for a male born in 1920, who has an excess lifetime risk of thyroid cancer almost 1000 times less than the highest risk for females born in 1952. A female born in 1920 has a risk about 350 times lower than that for a female born in 1952. Individuals of the same gender born in 1944 have about 50 to $60 \%$ of the risk for those born in 1952, while individuals born in 1940 or 1956 have risks about 5 times lower than those for individuals born in 1952. However, a male born in 1940 or 1956 has a risk almost 20 times less than that for a female born in 1952. For this reason, most of the examples of results given in Section 11.3.3 focus on the doses and risks to females born in 1952. 
Table 11.2 Thyroid doses (cGy), excess lifetime risk of thyroid cancer, relative risk, and probability of causation for a female born in 1952 who lived in Solway, Tennessee, from 1952 to 1956.

\begin{tabular}{|c|c|c|c|}
\hline \multirow[b]{2}{*}{ Exposure Pathway } & \multicolumn{3}{|c|}{ 95\% Subjective Confidence Interval } \\
\hline & lower limit & $\begin{array}{r}\text { central estimate } \\
\text { Thyroid Dose }[\mathrm{cGy}]\end{array}$ & upper limit \\
\hline Backyard Cow's Milk & 2.6 & 16 & 110 \\
\hline Commercial Milk (locally produced) & 0.72 & 5.1 & 37 \\
\hline Commercial Milk (regionally mixed) & 0.17 & 1.3 & 8 \\
\hline Goat’s Milk (locally produced) & 5.2 & 42 & 360 \\
\hline Beef (locally produced) & 0.0032 & 0.06 & 1.3 \\
\hline Leafy Vegetables (locally produced) & 0.00063 & 0.0075 & 0.07 \\
\hline Eggs (locally produced) & 0.12 & 0.89 & 7.2 \\
\hline Cottage Cheese (locally produced) & 0.003 & 0.041 & 0.44 \\
\hline Inhalation & 0.039 & 0.15 & 0.62 \\
\hline Mother's milk (mother on Diet 1) & 0.00064 & 0.015 & 0.29 \\
\hline Prenatal exposure (mother on Diet 1) & 0.0065 & 0.046 & 0.4 \\
\hline Diet 1 & 2.9 & 18 & 120 \\
\hline Diet 2 & 1.1 & 6.7 & 46 \\
\hline \multirow[t]{2}{*}{ Diet 3} & 0.26 & 1.4 & 8.4 \\
\hline & \multicolumn{3}{|c|}{ Excess Lifetime Risk [ ] } \\
\hline Diet 1 & $3.2 \times 10^{-4}$ & $5.2 \times 10^{-3}$ & $7.4 \times 10^{-2}$ \\
\hline Diet 2 & $1.5 \times 10^{-4}$ & $2.0 \times 10^{-3}$ & $2.5 \times 10^{-2}$ \\
\hline Diet 3 & $3.9 \times 10^{-5}$ & $4.1 \times 10^{-4}$ & $4.9 \times 10^{-3}$ \\
\hline \multirow[t]{2}{*}{ Diet 4} & $8.0 \times 10^{-4}$ & $1.4 \times 10^{-2}$ & $2.1 \times 10^{-1}$ \\
\hline & \multicolumn{3}{|c|}{ Relative Risk [ ] } \\
\hline Diet 1 & 1.13 & 2.3 & 16 \\
\hline Diet 2 & 1.05 & 1.5 & 7.4 \\
\hline Diet 3 & 1.012 & 1.1 & 2.3 \\
\hline \multirow[t]{2}{*}{ Diet 4} & 1.31 & 4.1 & 52 \\
\hline & \multicolumn{3}{|c|}{ Probability of Causation [\%] } \\
\hline Diet 1 & 12 & 56 & 94 \\
\hline Diet 2 & 4.8 & 33 & 86 \\
\hline Diet 3 & 1.2 & 9.4 & 56 \\
\hline Diet 4 & 24 & 76 & 98 \\
\hline
\end{tabular}

Diet $1 \quad$ Backyard cow's milk + all other locally produced non-milk exposure pathways

Diet 2 Locally produced commercial milk + all other locally produced non-milk exposure pathways

Diet 3 Regionally mixed commercial milk + inhalation (other regionally mixed food items are minor contributors to the total)

Diet 4 Goat's milk (all other exposure pathways are of negligible importance compared to goat's milk) 
Table 11.3 Thyroid doses (cGy) from ingestion of commercial milk produced at Solway and contaminated with ${ }^{131}$ I from routine releases at X-10.

\begin{tabular}{|c|c|c|c|c|c|c|c|c|}
\hline \multirow[t]{2}{*}{$\begin{array}{l}\text { Year of } \\
\text { Birth }\end{array}$} & \multicolumn{2}{|c|}{$\begin{array}{l}\text { Age at time of } \\
\text { exposure }\end{array}$} & \multicolumn{3}{|c|}{$\begin{array}{c}\text { 95\% Subjective Confidence Interval } \\
\text { Males }\end{array}$} & \multicolumn{3}{|c|}{$\begin{array}{l}\text { 95\% Subjective Confidence Interval } \\
\text { Females }\end{array}$} \\
\hline & $(1944)^{\mathrm{a}}$ & $(1956)^{b}$ & lower limit & central estimate & upper limit & lower limit & central estimate & upper limit \\
\hline 1920 & 24 & 36 & 0.18 & 1.1 & 6.8 & 0.15 & 0.86 & 5.9 \\
\hline 1930 & 14 & 26 & 0.22 & 1.3 & 8.4 & 0.17 & 1.1 & 6.8 \\
\hline 1935 & 9 & 21 & 0.33 & 2.0 & 13 & 0.29 & 1.8 & 12 \\
\hline 1940 & 4 & 16 & 0.51 & 3.4 & 22 & 0.49 & 3.2 & 22 \\
\hline 1944 & Newborn & 12 & 0.76 & 5.0 & 34 & 0.76 & 4.9 & 34 \\
\hline 1950 & --- & 6 & 0.7 & 4.9 & 37 & 0.68 & 5.0 & 36 \\
\hline 1952 & --- & 4 & 0.69 & 5.2 & 39 & 0.72 & 5.1 & 37 \\
\hline 1954 & --- & 2 & 0.41 & 3.3 & 22 & 0.41 & 3.3 & 26 \\
\hline 1956 & --- & newborn & 0.068 & 0.99 & 9.0 & 0.073 & 0.94 & 9.5 \\
\hline
\end{tabular}

${ }^{a}$ Beginning of exposure

${ }^{\mathrm{b}}$ End of exposure 
Table 11.4 Excess Lifetime Risk of thyroid cancer from ingestion of local produce and locally produced commercial milk (diet 2) for individuals living in Solway. The milk and produce are contaminated with ${ }^{131}$ I from routine releases at X-10.

\begin{tabular}{|c|c|c|c|c|c|c|c|c|}
\hline \multirow[t]{2}{*}{$\begin{array}{l}\text { Year of } \\
\text { Birth. }\end{array}$} & \multicolumn{2}{|c|}{$\begin{array}{l}\text { Age at time of } \\
\text { exposure }\end{array}$} & \multicolumn{3}{|c|}{$\begin{array}{c}\text { 95\% Subjective Confidence Interval } \\
\text { Males }\end{array}$} & \multicolumn{3}{|c|}{$\begin{array}{c}\text { 95\% Subjective Confidence Interval } \\
\text { Females }\end{array}$} \\
\hline & $(1944)^{\mathrm{a}}$ & $(\mathbf{1 9 5 6})^{b}$ & lower limit & central estimate & upper limit & lower limit & central estimate & upper limit \\
\hline 1920 & 24 & 36 & $4.7 \times 10^{-8}$ & $2.2 \times 10^{-6}$ & $8.1 \times 10^{-5}$ & $3.7 \times 10^{-7}$ & $5.7 \times 10^{-6}$ & $7.0 \times 10^{-5}$ \\
\hline 1930 & 14 & 26 & $1.0 \times 10^{-7}$ & $4.4 \times 10^{-6}$ & $1.5 \times 10^{-4}$ & $1.0 \times 10^{-6}$ & $1.4 \times 10^{-5}$ & $1.8 \times 10^{-4}$ \\
\hline 1935 & 9 & 21 & $8.8 \times 10^{-7}$ & $2.7 \times 10^{-5}$ & $6.4 \times 10^{-4}$ & $9.1 \times 10^{-6}$ & $1.0 \times 10^{-4}$ & $1.3 \times 10^{-3}$ \\
\hline 1940 & 4 & 16 & $3.6 \times 10^{-6}$ & $1.1 \times 10^{-4}$ & $2.7 \times 10^{-3}$ & $3.7 \times 10^{-5}$ & $4.2 \times 10^{-4}$ & $5.2 \times 10^{-3}$ \\
\hline 1944 & Newborn & 12 & $1.1 \times 10^{-5}$ & $2.9 \times 10^{-4}$ & $6.2 \times 10^{-3}$ & $9.3 \times 10^{-5}$ & $1.1 \times 10^{-3}$ & $1.5 \times 10^{-2}$ \\
\hline 1950 & --- & 6 & $1.7 \times 10^{-5}$ & $4.3 \times 10^{-4}$ & $9.7 \times 10^{-3}$ & $1.5 \times 10^{-4}$ & $1.6 \times 10^{-3}$ & $2.0 \times 10^{-2}$ \\
\hline 1952 & --- & 4 & $2.2 \times 10^{-5}$ & $5.1 \times 10^{-4}$ & $1.1 \times 10^{-2}$ & $1.5 \times 10^{-4}$ & $2.0 \times 10^{-3}$ & $2.5 \times 10^{-2}$ \\
\hline 1954 & --- & 2 & $1.2 \times 10^{-5}$ & $3.3 \times 10^{-4}$ & $7.4 \times 10^{-3}$ & $7.7 \times 10^{-5}$ & $1.2 \times 10^{-3}$ & $1.8 \times 10^{-2}$ \\
\hline 1956 & --- & newborn & $2.8 \times 10^{-6}$ & $8.8 \times 10^{-5}$ & $2.4 \times 10^{-3}$ & $1.9 \times 10^{-5}$ & $3.7 \times 10^{-4}$ & $6.4 \times 10^{-3}$ \\
\hline
\end{tabular}

\footnotetext{
${ }^{\mathrm{a}}$ Beginning of exposure

${ }^{\mathrm{b}}$ End of exposure
} 
The doses and risks for other birth years, for males or females, can be roughly estimated by multiplying the doses or risks for the female born in 1952 (Section 11.3.3) by the values presented in Table 11.5. Similarly, doses for single exposure pathways for an individual born in 1952 can be estimated from the doses provided in Section 11.3.3 for a female born in 1952 and on diet 1 (local produce and backyard cow's milk) using the values provided in Table 11.6 (except for doses from consumption of commercial milk or goat's milk, which are listed separately in Section 11.3.3). Note, however, that the results in Appendix 11C have been produced from calculations explicit for each location, dietary category, gender, and birth year; therefore, they provide more accurate estimates than those obtained from the use of Tables 11.5 and 11.6.

\subsubsection{Doses and risks to a female born in 1952 for different dietary sources of milk and different locations within a $38-\mathrm{km}$ radius of $\mathrm{X}-10$.}

\section{Estimates of Thyroid Dose}

Estimated thyroid doses for each of the 41 selected locations for a female born in 1952 on a rural diet consisting of backyard cow's milk and all other local produce (diet 1) are presented in Table 11.7 and in Figures 11.2, 11.3 and 11.4 for the entire region within a radius of $38 \mathrm{~km}$ of the X-10 facility. Among the 41 selected locations, the highest doses (6.1 to 250 cGy) occur at Gallaher Bend, a little more than $5 \mathrm{~km}$ to the east of X-10, while the lowest doses (0.1 to 5.7 cGy) occur at Wartburg, $27 \mathrm{~km} \mathrm{NW}$ of X-10.

A map of contour plots of the lower limit of the 95\% subjective confidence intervals (Fig. 11.2) of thyroid doses for females born in 1952 on diet 1 indicates that it is highly likely that the thyroid doses exceed $1.0 \mathrm{cGy}$ at distances out to $20 \mathrm{~km}$ to the SW and $25 \mathrm{~km}$ to the NE of X-10. A dose of $3 \mathrm{cGy}$ is exceeded with very high confidence at distances of $10 \mathrm{~km}$ to the $\mathrm{SW}$ and up to $15 \mathrm{~km}$ to the NE. The contour map of the central (50\%-ile) doses (Fig. 11.3) indicates that values above $10 \mathrm{cGy}$ are likely out to $15 \mathrm{~km}$ to the $\mathrm{SW}$ and $20 \mathrm{~km}$ to the NE of X-10. Doses above 30 cGy are likely near Jones Island and Gallaher Bend. The contour map of the upper limit of the $95 \%$ subjective confidence intervals is provided in Fig. 11.4. This map indicates that doses exceeding $100 \mathrm{cGy}$ are highly unlikely at distances beyond $10 \mathrm{~km}$ from X-10 and that doses exceeding $30 \mathrm{cGy}$ are highly unlikely at distances beyond $25 \mathrm{~km}$ to the $\mathrm{SW}$ and $30 \mathrm{~km}$ to the NE of X-10. 
Table 11.5 Factors for estimating doses and risks for individuals of any birth year from those estimated for females born in $1952^{\mathrm{a}}$.

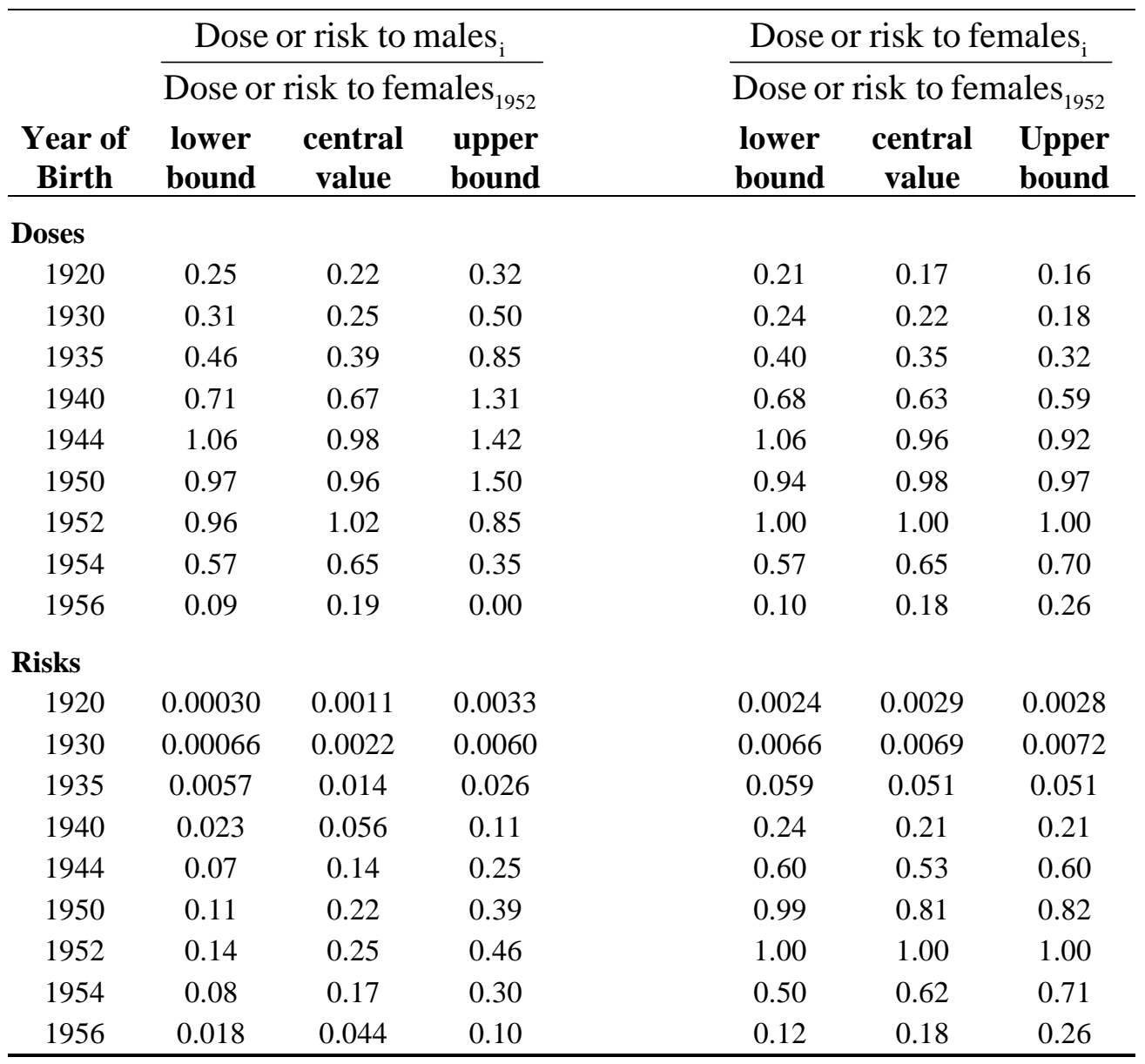

a The lower bound, central value, and upper bound in this table represent the factors to be multiplied by the lower bound, central value, and upper bound, respectively, of the estimated dose or risk for a female born in 1952. 
Table 11.6 Factors for estimating doses for single exposure pathways from the estimated dose from diet 1 (backyard cow's milk and all other non-milk exposure pathways) for a female born in $1952^{\mathrm{a}}$.

\begin{tabular}{lccc}
\hline Exposure Pathway & lower bound & central value & upper bound \\
\hline Backyard cow's milk & 0.90 & 0.89 & 0.92 \\
Eggs & 0.041 & 0.049 & 0.060 \\
Inhalation & 0.013 & 0.0083 & 0.0052 \\
Beef & 0.0011 & 0.0033 & 0.011 \\
Prenatal Exposure & 0.0022 & 0.0026 & 0.0033 \\
Cottage Cheese & 0.0010 & 0.0023 & 0.0037 \\
Mother's milk & 0.00022 & 0.00083 & 0.0024 \\
Leafy vegetables & 0.00022 & 0.00042 & 0.00058 \\
\hline
\end{tabular}

${ }^{a}$ The lower bound, central value, and upper bound in this table represent the factors to be multiplied by the lower bound, central value, and upper bound, respectively, of the estimated dose from diet 1 for a female born in 1952 (Section 11.3.3). 
Table 11.7 Thyroid doses (cGy) to a female born in 1952 from a diet consisting of local produce and backyard cow's milk (diet 1).

\begin{tabular}{|c|c|c|c|}
\hline \multirow[b]{2}{*}{ Location } & \multicolumn{3}{|c|}{ 95\% Subjective Confidence Interval } \\
\hline & lower limit & central estimate & upper limit \\
\hline Gallaher Bend & 6.1 & 39 & 250 \\
\hline Bradbury & 4.9 & 30 & 220 \\
\hline Buttermilk Rd. & 3.4 & 22 & 150 \\
\hline Hope Creek & 3.5 & 22 & 150 \\
\hline Lawnville/Gallaher & 3.7 & 20 & 130 \\
\hline Hardin Valley & 3.0 & 19 & 130 \\
\hline Solway & 2.9 & 18 & 120 \\
\hline Karns & 1.7 & 11 & 68 \\
\hline Farragut & 1.6 & 9.9 & 70 \\
\hline Hines Valley & 1.4 & 8.7 & 61 \\
\hline Cedar Bluff & 1.3 & 8.2 & 54 \\
\hline EFPC & 1.2 & 8.0 & 54 \\
\hline Kingston & 1.3 & 7.8 & 51 \\
\hline Sugar Grove & 1.1 & 7.4 & 51 \\
\hline Claxton & 1.2 & 6.9 & 44 \\
\hline Lenoir City & 1.1 & 6.2 & 37 \\
\hline Loudon & 1.0 & 5.8 & 41 \\
\hline Clinton & 0.9 & 5.5 & 36 \\
\hline Dyllis & 0.6 & 4.1 & 30 \\
\hline Knoxville & 0.6 & 3.9 & 27 \\
\hline Norwood & 0.6 & 3.9 & 32 \\
\hline Cedar Grove & 0.6 & 3.7 & 23 \\
\hline Barnardville & 0.6 & 3.6 & 24 \\
\hline Rockwood & 0.6 & 3.5 & 24 \\
\hline Harriman & 0.6 & 3.5 & 22 \\
\hline Dutch Valley & 0.5 & 3.4 & 21 \\
\hline Louisville & 0.5 & 3.3 & 20 \\
\hline Rockford & 0.5 & 3.2 & 21 \\
\hline Friendsville & 0.5 & 3.1 & 20 \\
\hline Jonesville & 0.5 & 3.0 & 23 \\
\hline Oliver Springs & 0.5 & 2.9 & 22 \\
\hline Sweetwater & 0.4 & 2.4 & 14 \\
\hline Greenback & 0.3 & 2.0 & 15 \\
\hline Oakdale & 0.3 & 2.0 & 14 \\
\hline Maryville & 0.3 & 1.9 & 13 \\
\hline Lake City & 0.3 & 1.9 & 12 \\
\hline Wartburg & 0.1 & 0.9 & 5.7 \\
\hline
\end{tabular}


Doses to females born in 1952 consuming milk from a local commercial dairy are approximately 3 to 4 times less (Table 11.8) than doses associated with a contaminated rural diet and milk obtained from a backyard cow (Table 11.7). Doses from the consumption of regionally mixed commercial retail milk are about the same as those from the consumption of milk from a local commercial dairy for the entire region, ranging from 0.18 to $8.0 \mathrm{cGy}$ (95\% subjective confidence interval) regardless of location. Doses from the consumption of goat's milk (Table 11.9), however, are about 1.6 to 3 times higher than doses from a rural diet of locally grown produce and milk from a backyard cow (Table 11.7). At Gallaher Bend, the consumption of goat's milk on a regular basis could result in a thyroid dose ranging from 10 to $760 \mathrm{cGy}$, with a central estimate of $88 \mathrm{cGy}$.

\section{Estimates of the Lifetime Risk of Thyroid Cancer.}

The highest risk for a female born in 1952 on diet 1 (local produce and milk from a backyard cow) occurs at the agricultural communities of Bradbury and Gallaher Bend (Table 11.10), where estimates are confidently above one chance in one thousand $\left(1 \times 10^{-3}\right)$ but less than one chance in ten $\left(1 \times 10^{-1}\right)$, with central estimates approaching or exceeding one chance in one hundred $(1 \times$ $10^{-2}$ ). At these locations the central estimate of the probability of causation approaches or exceeds $50 \%$, meaning that a diagnosed thyroid carcinoma has more than an even chance of being due to exposure for either rural diet ( 1 or 2 ) or from diet 4 , the consumption of goat's milk (see Appendix 11C).

The contour map of the lower limit of the $95 \%$ subjective confidence interval of the risk to a female born in 1952 on diet 1 (Fig. 11.5) shows that an excess lifetime risk confidently above one chance in ten thousand $\left(1 \times 10^{-4}\right)$ extends from $25 \mathrm{~km}$ to the SW to $30 \mathrm{~km}$ to the NE of X-10. The central estimate of risk (Fig. 11.6) shows a risk of more than one chance in one thousand (1 $\times 10^{-3}$ ) is likely out to distances of $35 \mathrm{~km}$ to the $\mathrm{SW}$ of $\mathrm{X}-10$ and more than $38 \mathrm{~km}$ to the NE. A risk of more than one chance in ten thousand $\left(1 \times 10^{-4}\right)$ will occur with a subjective confidence level of $>50 \%$ at all locations. The upper limit of the $95 \%$ subjective confidence intervals of risk are provided in Fig. 11.7. This figure indicates that risks exceeding one chance in ten $(1 \times$ $10^{-1}$ ) are highly unlikely beyond $6 \mathrm{~km}$ to the SW and $8 \mathrm{~km}$ to the NE of X-10. Risks exceeding one chance in one hundred $\left(1 \times 10^{-2}\right)$ are highly unlikely beyond $15 \mathrm{~km}$ to the $\mathrm{N}, 20 \mathrm{~km}$ to the $\mathrm{NW}$, and $38 \mathrm{~km}$ to the $\mathrm{S}$ of $\mathrm{X}-10$.

The estimates of excess lifetime risk at each of the 41 selected locations for local commercial cow's milk (Table 11.11) and diet 4 (local goat's milk, Table 11.12) indicate the same trends among locations and from one milk source to another as was indicated in the results for doses, with the highest risks occurring for females in early childhood at the time of exposure who consumed goat's milk on a regular basis. The general regional estimate of risk from the consumption of retail commercial milk mixed from many dairies throughout the region ranges from three chances in one hundred thousand $\left(3 \times 10^{-5}\right)$ up to almost 5 chances in a thousand $(5 \times$ $\left.10^{-3}\right)$, with a central estimate of almost 4 chances in ten thousand $\left(4 \times 10^{-4}\right)$. 
TASK 1 REPORT

${ }^{131}$ I Releases from X-10 Radioactive Lanthanum ProcessingJuly 1999
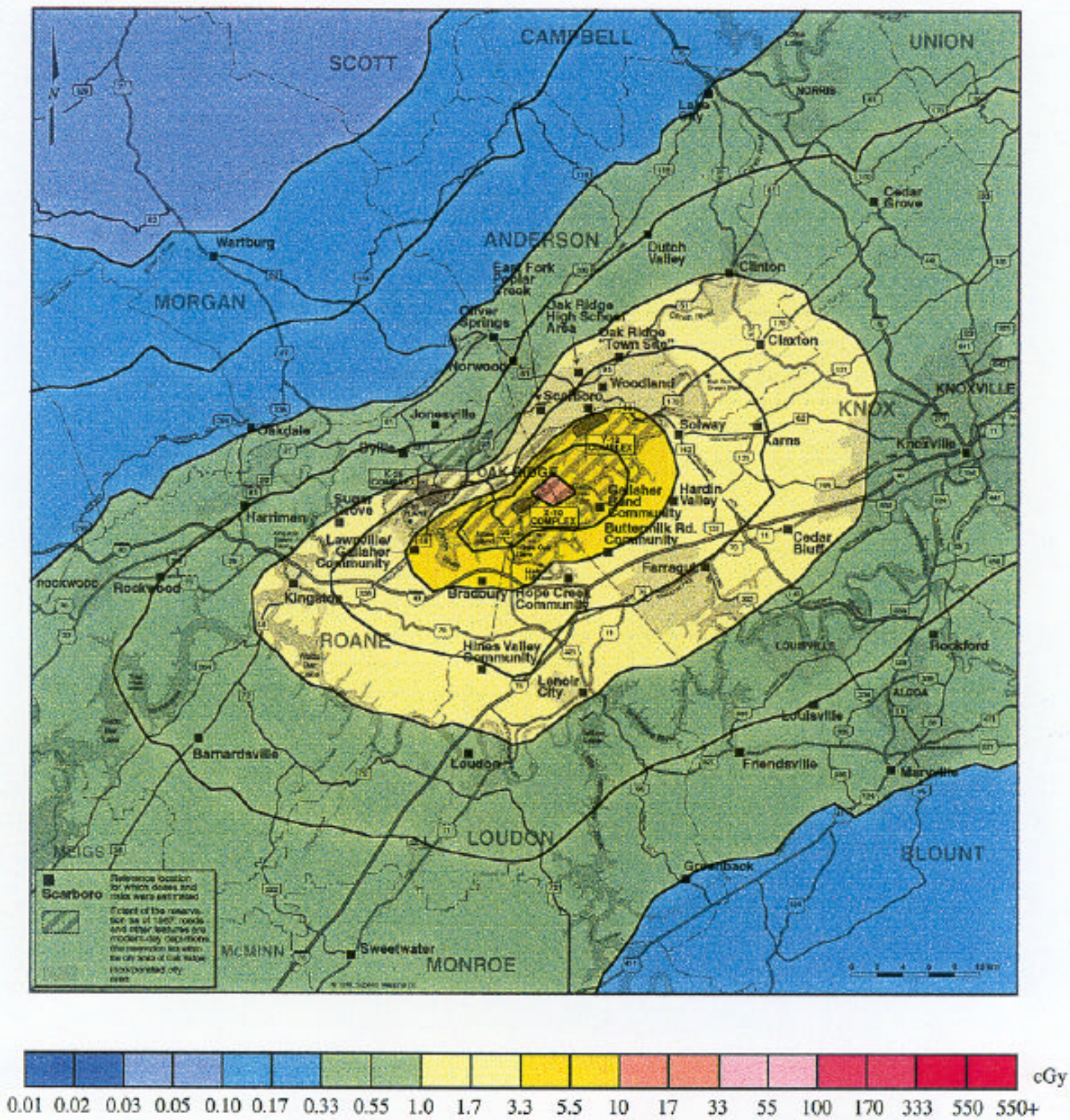

Figure 11.2 Contours for the lower bound (2.5th percentile) of the 95\% subjective confidence interval of the dose to a female born in 1952 from a diet consisting of local produce and backyard cow's milk (diet 1). It is highly likely that the actual dose at a given location is greater than the value shown for that location in this figure. 
TASK 1 REPORT

${ }^{131}$ I Releases from X-10 Radioactive Lanthanum ProcessingJuly 1999 Results

Page 11-16
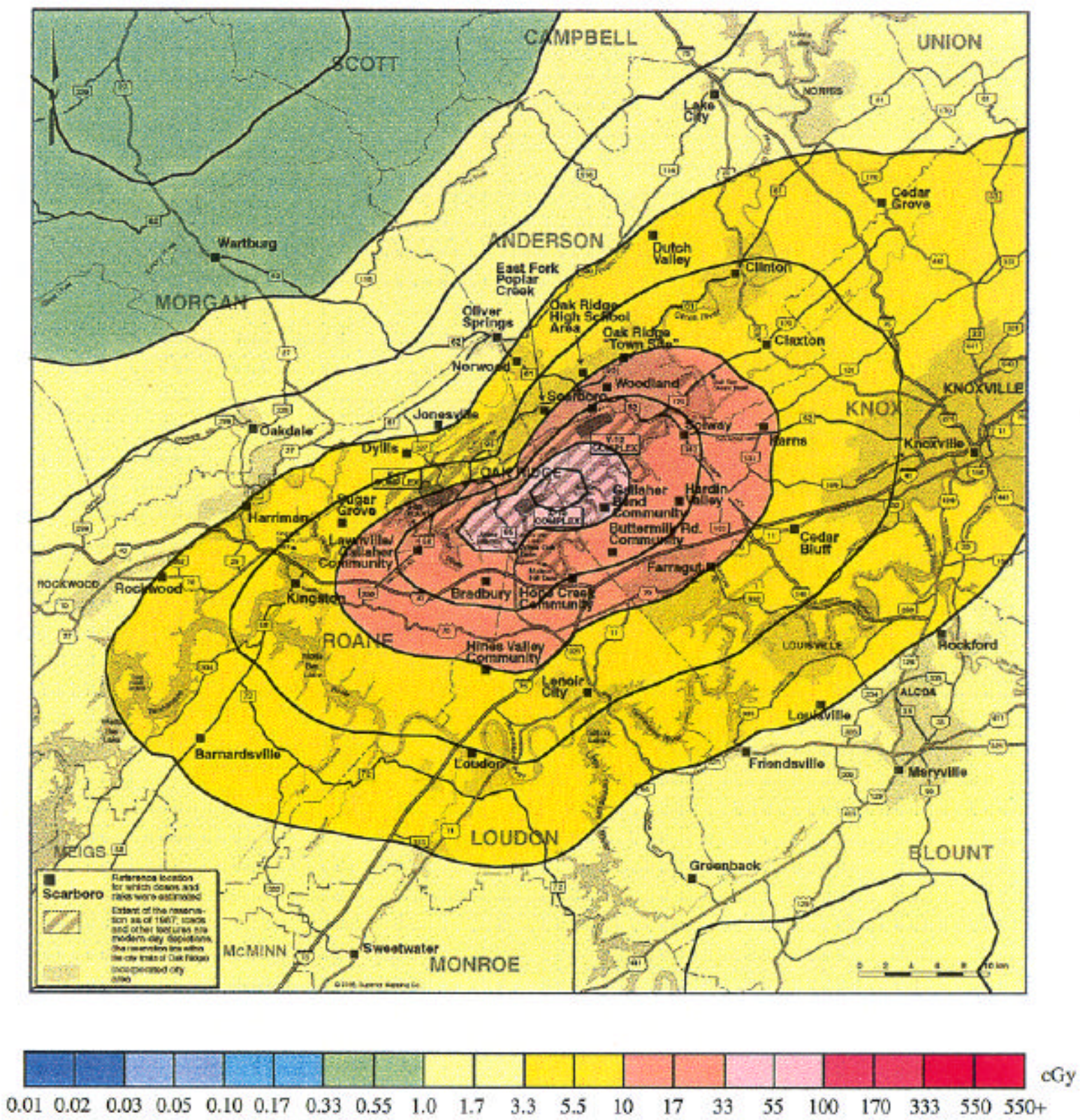

Figure 11.3 Contours for the central estimate $\left(50^{\text {th }}\right.$ percentile) of the $95 \%$ subjective confidence interval of the dose to a female born in 1952 from a diet consisting of local produce and backyard cow's milk (diet 1). 
TASK 1 REPORT
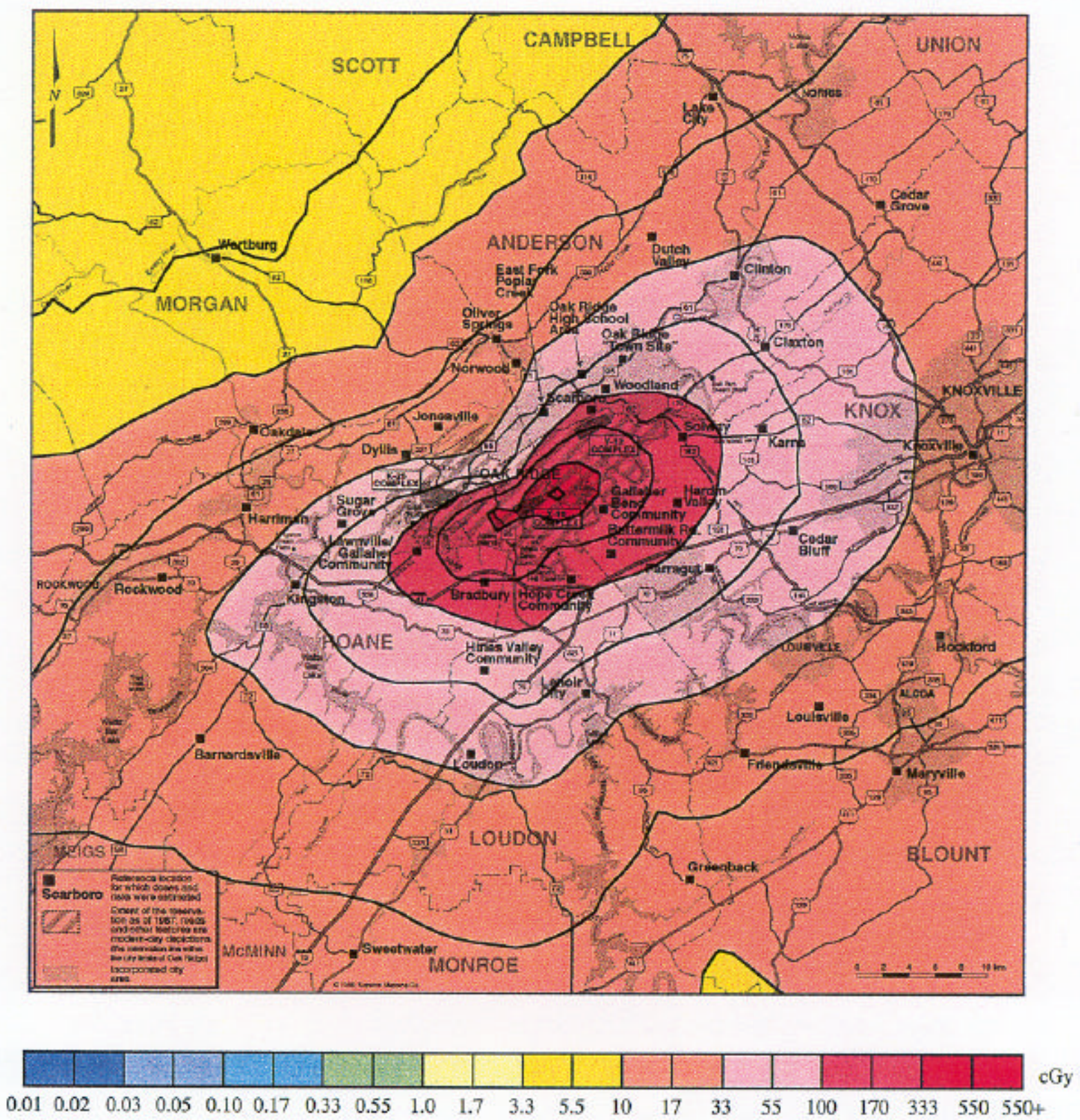

Figure 11.4 Contours for the upper bound (97.5th percentile) of the $95 \%$ subjective confidence interval of the dose to a female born in 1952 from a diet consisting of local produce and backyard cow's milk (diet 1). It is highly unlikely that the actual dose at a given location exceeds the value shown for that location in this figure. 
TASK 1 REPORT
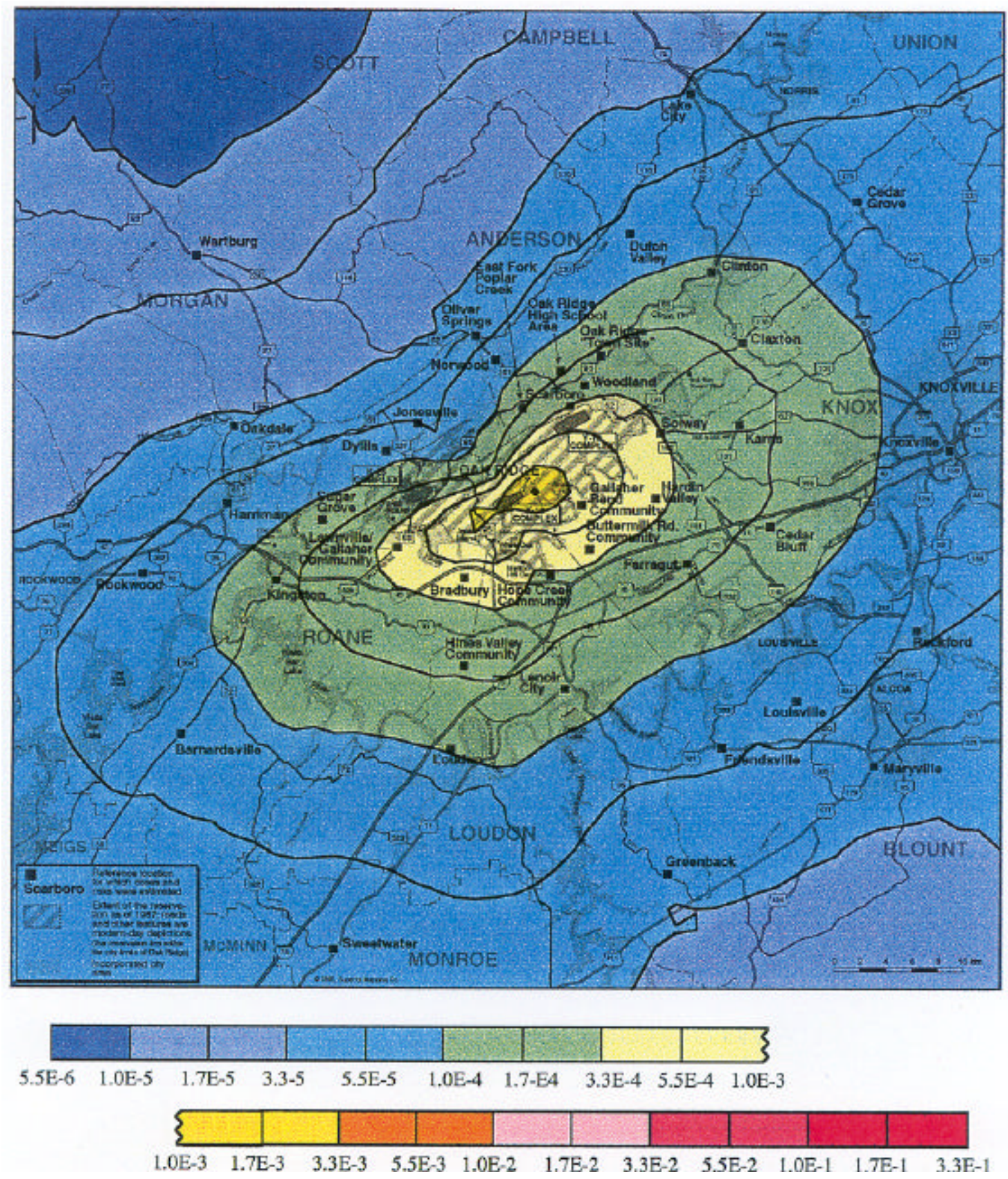

Figure 11.5 Contours for the lower bound (2.5th percentile) of the $95 \%$ subjective confidence interval of the excess lifetime risk of developing thyroid cancer for a female born in 1952 on a diet consisting of local produce and backyard cow's milk (diet 1). It is highly likely that the actual risk at a given location is greater than the value shown for that location in this figure. 
Table 11.8 Thyroid doses (cGy) to a female born in 1952 consuming locally produced commercial milk.

\begin{tabular}{|c|c|c|c|}
\hline \multirow[b]{2}{*}{ Location } & \multicolumn{3}{|c|}{ 95\% Subjective Confidence Interval } \\
\hline & lower limit & central estimate & upper limit \\
\hline Gallaher Bend & 1.6 & 11 & 84 \\
\hline Bradbury & 1.2 & 9.2 & 65 \\
\hline Buttermilk Rd. & 0.88 & 6.4 & 48 \\
\hline Hope Creek & 0.88 & 6.4 & 49 \\
\hline Lawnville/Gallaher & 0.86 & 5.9 & 46 \\
\hline Hardin Valley & 0.80 & 5.8 & 42 \\
\hline Solway & 0.72 & 5.1 & 37 \\
\hline Karns & 0.45 & 3.1 & 21 \\
\hline Farragut & 0.42 & 3.0 & 20 \\
\hline Hines Valley & 0.37 & 2.6 & 19 \\
\hline Cedar Bluff & 0.36 & 2.5 & 18 \\
\hline EFPC & 0.30 & 2.4 & 17 \\
\hline Kingston & 0.35 & 2.3 & 16 \\
\hline Sugar Grove & 0.30 & 2.2 & 17 \\
\hline Claxton & 0.31 & 2.1 & 15 \\
\hline Loudon & 0.23 & 1.8 & 13 \\
\hline Lenoir City & 0.26 & 1.7 & 14 \\
\hline Clinton & 0.23 & 1.6 & 11 \\
\hline Knoxville & 0.17 & 1.2 & 8.2 \\
\hline Dyllis & 0.17 & 1.2 & 10 \\
\hline Norwood & 0.16 & 1.1 & 10 \\
\hline Cedar Grove & 0.16 & 1.1 & 7.6 \\
\hline Barnardville & 0.16 & 1.1 & 8.1 \\
\hline Rockwood & 0.15 & 1.1 & 7.5 \\
\hline Harriman & 0.15 & 1.0 & 7.8 \\
\hline Dutch Valley & 0.14 & 0.99 & 7.5 \\
\hline Louisville & 0.13 & 0.96 & 6.9 \\
\hline Rockford & 0.13 & 0.94 & 6.5 \\
\hline Friendsville & 0.12 & 0.92 & 6.2 \\
\hline Jonesville & 0.11 & 0.89 & 7.3 \\
\hline Oliver Springs & 0.12 & 0.88 & 7.4 \\
\hline Sweetwater & 0.09 & 0.72 & 5.1 \\
\hline Oakdale & 0.08 & 0.59 & 4.4 \\
\hline Lake City & 0.09 & 0.58 & 4.1 \\
\hline Maryville & 0.07 & 0.58 & 4.5 \\
\hline Greenback & 0.08 & 0.58 & 4.5 \\
\hline Wartburg & 0.032 & 0.25 & 2.0 \\
\hline All locations ${ }^{\mathrm{a}}$ & 0.18 & 1.3 & 8.0 \\
\hline
\end{tabular}


Table 11.9 Thyroid doses (cGy) to a female born in 1952 consuming goat's milk (diet 4).

\begin{tabular}{|c|c|c|c|}
\hline \multirow[b]{2}{*}{ Location } & \multicolumn{3}{|c|}{ 95\% Subjective Confidence Interval } \\
\hline & lower limit & central estimate & upper limit \\
\hline Gallaher Bend & 10 & 88 & 758 \\
\hline Bradbury & 8.9 & 75 & 653 \\
\hline Buttermilk Rd. & 5.8 & 51 & 440 \\
\hline Lawnville/Gallaher & 5.9 & 49 & 402 \\
\hline Hardin Valley & 5.5 & 46 & 394 \\
\hline Solway & 5.2 & 42 & 360 \\
\hline Karns & 3.0 & 25 & 219 \\
\hline Farragut & 2.9 & 24 & 212 \\
\hline Hines Valley & 2.7 & 21 & 177 \\
\hline Cedar Bluff & 2.6 & 20 & 165 \\
\hline Kingston & 2.4 & 19 & 168 \\
\hline Sugar Grove & 2.2 & 18 & 159 \\
\hline Claxton & 2.1 & 16 & 138 \\
\hline Loudon & 1.7 & 14 & 121 \\
\hline Clinton & 1.6 & 13 & 107 \\
\hline Dyllis & 1.1 & 9.8 & 94 \\
\hline Norwood & 1.0 & 9.7 & 96 \\
\hline Knoxville & 1.2 & 9.5 & 80 \\
\hline Barnardville & 1.1 & 8.7 & 77 \\
\hline Cedar Grove & 1.1 & 8.6 & 74 \\
\hline Rockwood & 1.1 & 8.5 & 72 \\
\hline Harriman & 1.0 & 8.3 & 69 \\
\hline Dutch Valley & 1.0 & 8.0 & 67 \\
\hline Louisville & 0.9 & 7.9 & 70 \\
\hline Rockford & 0.9 & 7.5 & 65 \\
\hline Friendsville & 0.9 & 7.4 & 68 \\
\hline Jonesville & 0.8 & 7.3 & 73 \\
\hline Oliver Springs & 0.8 & 7.1 & 68 \\
\hline Sweetwater & 0.6 & 5.7 & 55 \\
\hline Greenback & 0.6 & 5.0 & 44 \\
\hline Maryville & 0.6 & 4.7 & 42 \\
\hline Oakdale & 0.5 & 4.7 & 42 \\
\hline Lake City & 0.6 & 4.7 & 40 \\
\hline Wartburg & 0.2 & 2.1 & 19 \\
\hline
\end{tabular}


Table 11.10 Excess Lifetime Risk for a female born in 1952 on a diet consisting of local produce and backyard cow's milk (diet 1).

\begin{tabular}{|c|c|c|c|}
\hline \multirow[b]{2}{*}{ Location } & \multicolumn{3}{|c|}{ 95\% Subjective Confidence Interval } \\
\hline & lower limit & central estimate & upper limit \\
\hline Gallaher Bend & $7.1 \times 10^{-4}$ & $1.1 \times 10^{-2}$ & $1.7 \times 10^{-1}$ \\
\hline Bradbury & $6.0 \times 10^{-4}$ & $9.3 \times 10^{-3}$ & $1.4 \times 10^{-1}$ \\
\hline Hope Creek & $4.2 \times 10^{-4}$ & $6.6 \times 10^{-3}$ & $9.7 \times 10^{-2}$ \\
\hline Buttermilk Rd. & $4.0 \times 10^{-4}$ & $6.5 \times 10^{-3}$ & $9.2 \times 10^{-2}$ \\
\hline Lawnville/Gallaher & $3.9 \times 10^{-4}$ & $6.0 \times 10^{-3}$ & $9.1 \times 10^{-2}$ \\
\hline Hardin Valley & $3.7 \times 10^{-4}$ & $5.7 \times 10^{-3}$ & $8.3 \times 10^{-2}$ \\
\hline Solway & $3.2 \times 10^{-4}$ & $5.2 \times 10^{-3}$ & $7.4 \times 10^{-2}$ \\
\hline Karns & $2.0 \times 10^{-4}$ & $3.2 \times 10^{-3}$ & $4.9 \times 10^{-2}$ \\
\hline Farragut & $1.9 \times 10^{-4}$ & $3.1 \times 10^{-3}$ & $4.9 \times 10^{-2}$ \\
\hline Hines Valley & $1.7 \times 10^{-4}$ & $2.7 \times 10^{-3}$ & $4.2 \times 10^{-2}$ \\
\hline Cedar Bluff & $1.6 \times 10^{-4}$ & $2.5 \times 10^{-3}$ & $4.0 \times 10^{-2}$ \\
\hline Kingston & $1.4 \times 10^{-4}$ & $2.4 \times 10^{-3}$ & $3.5 \times 10^{-2}$ \\
\hline EFPC & $1.5 \times 10^{-4}$ & $2.4 \times 10^{-3}$ & $3.6 \times 10^{-2}$ \\
\hline Sugar Grove & $1.4 \times 10^{-4}$ & $2.2 \times 10^{-3}$ & $3.3 \times 10^{-2}$ \\
\hline Claxton & $1.4 \times 10^{-4}$ & $2.1 \times 10^{-3}$ & $3.2 \times 10^{-2}$ \\
\hline Lenoir City & $1.1 \times 10^{-4}$ & $1.8 \times 10^{-3}$ & $3.0 \times 10^{-2}$ \\
\hline Loudon & $1.1 \times 10^{-4}$ & $1.8 \times 10^{-3}$ & $2.9 \times 10^{-2}$ \\
\hline Clinton & $1.0 \times 10^{-4}$ & $1.6 \times 10^{-3}$ & $2.4 \times 10^{-2}$ \\
\hline Norwood & $7.3 \times 10^{-5}$ & $1.3 \times 10^{-3}$ & $1.9 \times 10^{-2}$ \\
\hline Dyllis & $8.2 \times 10^{-5}$ & $1.2 \times 10^{-3}$ & $2.0 \times 10^{-2}$ \\
\hline Knoxville & $7.3 \times 10^{-5}$ & $1.2 \times 10^{-3}$ & $1.8 \times 10^{-2}$ \\
\hline Cedar Grove & $7.0 \times 10^{-5}$ & $1.1 \times 10^{-3}$ & $1.6 \times 10^{-2}$ \\
\hline Rockwood & $6.6 \times 10^{-5}$ & $1.1 \times 10^{-3}$ & $1.7 \times 10^{-2}$ \\
\hline Barnardville & $6.7 \times 10^{-5}$ & $1.1 \times 10^{-3}$ & $2.1 \times 10^{-2}$ \\
\hline Harriman & $6.7 \times 10^{-5}$ & $1.1 \times 10^{-3}$ & $1.7 \times 10^{-2}$ \\
\hline Dutch Valley & $6.1 \times 10^{-5}$ & $1.0 \times 10^{-3}$ & $1.6 \times 10^{-2}$ \\
\hline Louisville & $5.6 \times 10^{-5}$ & $9.6 \times 10^{-4}$ & $1.6 \times 10^{-2}$ \\
\hline Rockford & $5.9 \times 10^{-5}$ & $9.6 \times 10^{-4}$ & $1.4 \times 10^{-2}$ \\
\hline Friendsville & $6.1 \times 10^{-5}$ & $9.2 \times 10^{-4}$ & $1.5 \times 10^{-2}$ \\
\hline Oliver Springs & $5.2 \times 10^{-5}$ & $9.1 \times 10^{-4}$ & $1.6 \times 10^{-2}$ \\
\hline Jonesville & $5.6 \times 10^{-5}$ & $9.0 \times 10^{-4}$ & $1.4 \times 10^{-2}$ \\
\hline Sweetwater & $4.2 \times 10^{-5}$ & $7.0 \times 10^{-4}$ & $1.2 \times 10^{-2}$ \\
\hline Greenback & $3.8 \times 10^{-5}$ & $6.1 \times 10^{-4}$ & $1.0 \times 10^{-2}$ \\
\hline Maryville & $3.9 \times 10^{-5}$ & $6.1 \times 10^{-4}$ & $1.2 \times 10^{-2}$ \\
\hline Oakdale & $3.3 \times 10^{-5}$ & $6.0 \times 10^{-4}$ & $9.1 \times 10^{-3}$ \\
\hline Lake City & $3.5 \times 10^{-5}$ & $5.9 \times 10^{-4}$ & $8.7 \times 10^{-3}$ \\
\hline Wartburg & $1.3 \times 10^{-5}$ & $2.6 \times 10^{-4}$ & $4.8 \times 10^{-3}$ \\
\hline
\end{tabular}


Table 11.11 Excess Lifetime Risk for a female born in 1952 consuming locally produced commercial milk.

\begin{tabular}{|c|c|c|c|}
\hline \multirow[b]{2}{*}{ Location } & \multicolumn{3}{|c|}{ 95\% Subjective Confidence Interval } \\
\hline & lower limit & central estimate & upper limit \\
\hline Gallaher Bend & $2.7 \times 10^{-4}$ & $3.3 \times 10^{-3}$ & $4.9 \times 10^{-2}$ \\
\hline Bradbury & $2.1 \times 10^{-4}$ & $2.7 \times 10^{-3}$ & $3.6 \times 10^{-2}$ \\
\hline Hope Creek & $1.5 \times 10^{-4}$ & $1.9 \times 10^{-3}$ & $2.7 \times 10^{-2}$ \\
\hline Buttermilk Rd. & $1.5 \times 10^{-4}$ & $1.8 \times 10^{-3}$ & $2.6 \times 10^{-2}$ \\
\hline Lawnville/Gallaher & $1.6 \times 10^{-4}$ & $1.8 \times 10^{-3}$ & $2.5 \times 10^{-2}$ \\
\hline Hardin Valley & $1.2 \times 10^{-4}$ & $1.7 \times 10^{-3}$ & $2.3 \times 10^{-2}$ \\
\hline Solway & $1.3 \times 10^{-4}$ & $1.5 \times 10^{-3}$ & $2.1 \times 10^{-2}$ \\
\hline Karns & $8.0 \times 10^{-5}$ & $9.2 \times 10^{-4}$ & $1.3 \times 10^{-2}$ \\
\hline Farragut & $6.8 \times 10^{-5}$ & $8.9 \times 10^{-4}$ & $1.2 \times 10^{-2}$ \\
\hline Hines Valley & $6.3 \times 10^{-5}$ & $7.6 \times 10^{-4}$ & $1.2 \times 10^{-2}$ \\
\hline Cedar Bluff & $6.0 \times 10^{-5}$ & $7.2 \times 10^{-4}$ & $1.1 \times 10^{-2}$ \\
\hline EFPC & $5.2 \times 10^{-5}$ & $7.1 \times 10^{-4}$ & $1.0 \times 10^{-2}$ \\
\hline Kingston & $5.5 \times 10^{-5}$ & $6.6 \times 10^{-4}$ & $9.6 \times 10^{-3}$ \\
\hline Sugar Grove & $4.8 \times 10^{-5}$ & $6.5 \times 10^{-4}$ & $8.7 \times 10^{-3}$ \\
\hline Claxton & $5.1 \times 10^{-5}$ & $6.0 \times 10^{-4}$ & $8.5 \times 10^{-3}$ \\
\hline Loudon & $4.0 \times 10^{-5}$ & $5.2 \times 10^{-4}$ & $7.2 \times 10^{-3}$ \\
\hline Lenoir City & $4.5 \times 10^{-5}$ & $5.1 \times 10^{-4}$ & $7.3 \times 10^{-3}$ \\
\hline Clinton & $3.8 \times 10^{-5}$ & $4.7 \times 10^{-4}$ & $6.4 \times 10^{-3}$ \\
\hline Dyllis & $2.2 \times 10^{-5}$ & $3.7 \times 10^{-4}$ & $5.0 \times 10^{-3}$ \\
\hline Knoxville & $2.7 \times 10^{-5}$ & $3.5 \times 10^{-4}$ & $4.9 \times 10^{-3}$ \\
\hline Norwood & $2.6 \times 10^{-5}$ & $3.4 \times 10^{-4}$ & $5.7 \times 10^{-3}$ \\
\hline Barnardville & $2.6 \times 10^{-5}$ & $3.3 \times 10^{-4}$ & $4.4 \times 10^{-3}$ \\
\hline Cedar Grove & $2.6 \times 10^{-5}$ & $3.2 \times 10^{-4}$ & $4.3 \times 10^{-3}$ \\
\hline Rockwood & $2.4 \times 10^{-5}$ & $3.1 \times 10^{-4}$ & $4.5 \times 10^{-3}$ \\
\hline Harriman & $2.7 \times 10^{-5}$ & $3.0 \times 10^{-4}$ & $4.2 \times 10^{-3}$ \\
\hline Dutch Valley & $2.5 \times 10^{-5}$ & $2.8 \times 10^{-4}$ & $4.4 \times 10^{-3}$ \\
\hline Louisville & $2.2 \times 10^{-5}$ & $2.8 \times 10^{-4}$ & $4.1 \times 10^{-3}$ \\
\hline Rockford & $2.2 \times 10^{-5}$ & $2.7 \times 10^{-4}$ & $3.8 \times 10^{-3}$ \\
\hline Friendsville & $2.1 \times 10^{-5}$ & $2.7 \times 10^{-4}$ & $3.7 \times 10^{-3}$ \\
\hline Jonesville & $1.9 \times 10^{-5}$ & $2.6 \times 10^{-4}$ & $3.8 \times 10^{-3}$ \\
\hline Oliver Springs & $2.0 \times 10^{-5}$ & $2.5 \times 10^{-4}$ & $4.1 \times 10^{-3}$ \\
\hline Sweetwater & $1.6 \times 10^{-5}$ & $2.1 \times 10^{-4}$ & $3.2 \times 10^{-3}$ \\
\hline Maryville & $1.2 \times 10^{-5}$ & $1.8 \times 10^{-4}$ & $2.4 \times 10^{-3}$ \\
\hline Oakdale & $1.3 \times 10^{-5}$ & $1.7 \times 10^{-4}$ & $2.5 \times 10^{-3}$ \\
\hline Greenback & $1.4 \times 10^{-5}$ & $1.7 \times 10^{-4}$ & $2.6 \times 10^{-3}$ \\
\hline Lake City & $1.3 \times 10^{-5}$ & $1.7 \times 10^{-4}$ & $2.4 \times 10^{-3}$ \\
\hline Wartburg & $5.2 \times 10^{-6}$ & $6.9 \times 10^{-5}$ & $1.1 \times 10^{-3}$ \\
\hline All locations ${ }^{\mathrm{a}}$ & $3.2 \times 10^{-5}$ & $3.6 \times 10^{-4}$ & $4.7 \times 10^{-3}$ \\
\hline
\end{tabular}

${ }^{a}$ Based on ingestion of regionally mixed commercial milk 
TASK 1 REPORT

${ }^{131}$ I Releases from X-10 Radioactive Lanthanum ProcessingJuly 1999
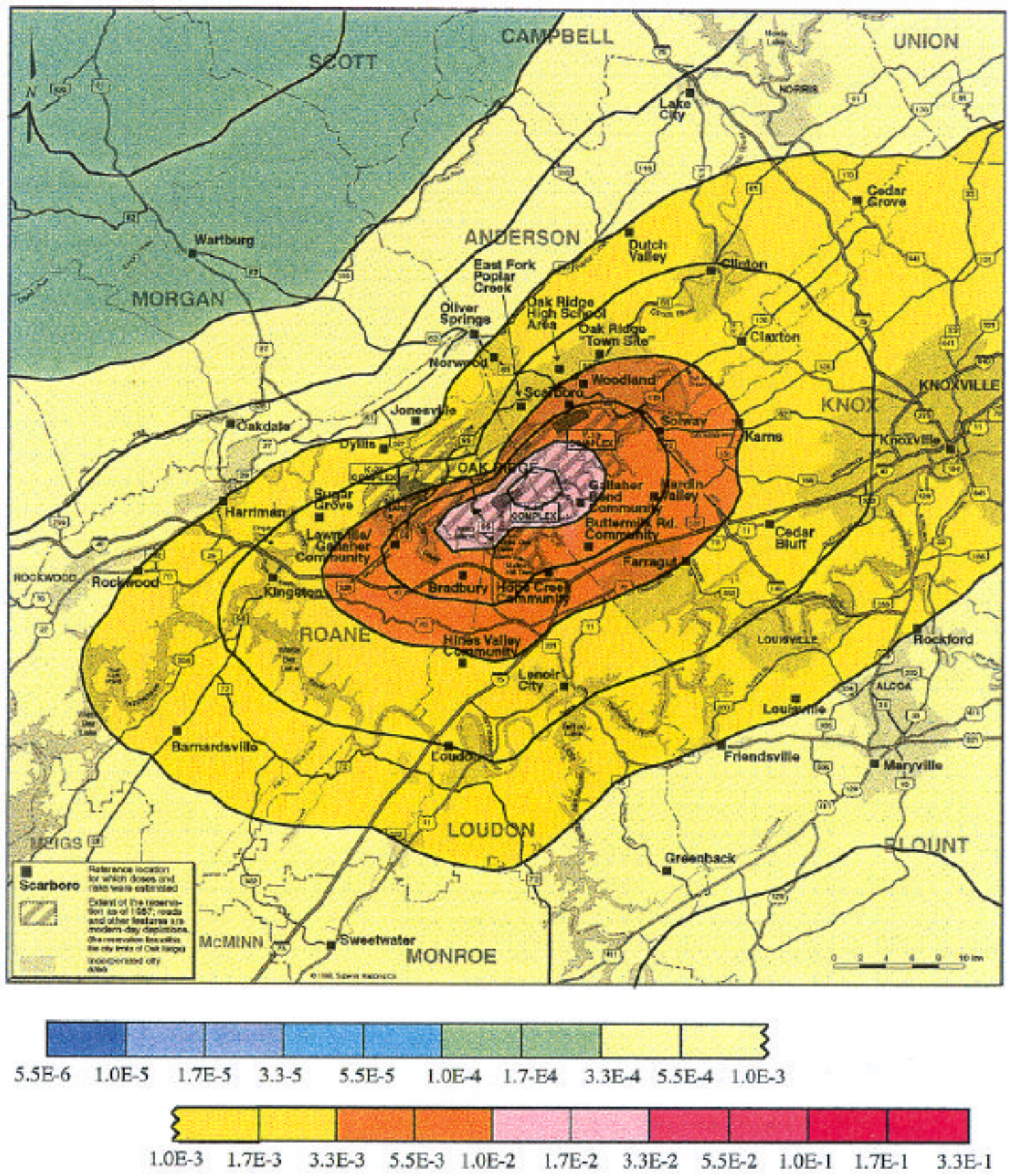

Figure 11.6 Contours for the central estimate ( $50^{\text {th }}$ percentile) of the $95 \%$ subjective confidence interval of the excess lifetime risk of developing thyroid cancer for a female born in 1952 on a diet consisting of local produce and backyard cow's milk (diet 1). 
TASK 1 REPORT
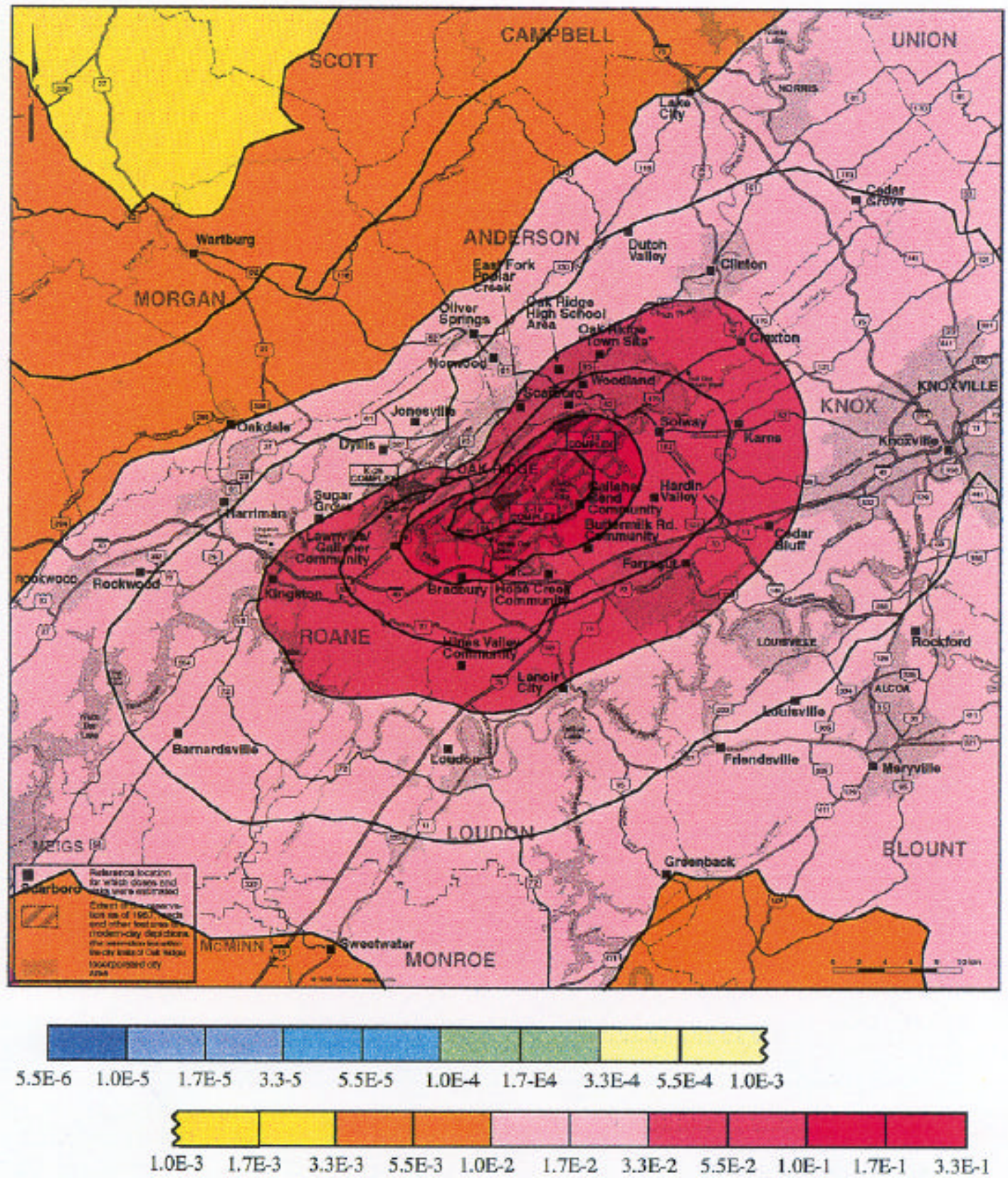

Figure 11.7 Contours for the upper bound (97.5th percentile) of the $95 \%$ subjective confidence interval of the excess lifetime risk of developing thyroid cancer for a female born in 1952 on a diet consisting of local produce and backyard cow's milk (diet 1). It is highly unlikely that the actual risk at a given location exceeds the value shown for that location in this figure. 
Table 11.12 Excess Lifetime Risk for a female born in 1952 consuming goat's milk (diet 4).

\begin{tabular}{|c|c|c|c|}
\hline \multirow[b]{2}{*}{ Location } & \multicolumn{3}{|c|}{ 95\% Subjective Confidence Interval } \\
\hline & lower limit & central estimate & upper limit \\
\hline Gallaher Bend & $1.8 \times 10^{-3}$ & $3.1 \times 10^{-2}$ & $4.3 \times 10^{-1}$ \\
\hline Bradbury & $1.3 \times 10^{-3}$ & $2.5 \times 10^{-2}$ & $3.6 \times 10^{-1}$ \\
\hline Buttermilk Rd. & $9.4 \times 10^{-4}$ & $1.7 \times 10^{-2}$ & $2.5 \times 10^{-1}$ \\
\hline Hardin Valley & $8.6 \times 10^{-4}$ & $1.6 \times 10^{-2}$ & $2.3 \times 10^{-1}$ \\
\hline Lawnville/Gallaher & $1.0 \times 10^{-3}$ & $1.6 \times 10^{-2}$ & $2.7 \times 10^{-1}$ \\
\hline Solway & $8.0 \times 10^{-4}$ & $1.4 \times 10^{-2}$ & $2.1 \times 10^{-1}$ \\
\hline Karns & $5.1 \times 10^{-4}$ & $8.7 \times 10^{-3}$ & $1.2 \times 10^{-1}$ \\
\hline Farragut & $4.7 \times 10^{-4}$ & $8.2 \times 10^{-3}$ & $1.2 \times 10^{-1}$ \\
\hline Hines Valley & $3.8 \times 10^{-4}$ & $7.2 \times 10^{-3}$ & $1.0 \times 10^{-1}$ \\
\hline Cedar Bluff & $3.7 \times 10^{-4}$ & $6.8 \times 10^{-3}$ & $9.6 \times 10^{-2}$ \\
\hline Kingston & $3.6 \times 10^{-4}$ & $6.2 \times 10^{-3}$ & $9.2 \times 10^{-2}$ \\
\hline Sugar Grove & $3.3 \times 10^{-4}$ & $5.9 \times 10^{-3}$ & $9.1 \times 10^{-2}$ \\
\hline Claxton & $3.2 \times 10^{-4}$ & $5.8 \times 10^{-3}$ & $8.1 \times 10^{-2}$ \\
\hline Loudon & $2.7 \times 10^{-4}$ & $4.8 \times 10^{-3}$ & $7.5 \times 10^{-2}$ \\
\hline Clinton & $2.3 \times 10^{-4}$ & $4.3 \times 10^{-3}$ & $6.0 \times 10^{-2}$ \\
\hline Dyllis & $1.7 \times 10^{-4}$ & $3.4 \times 10^{-3}$ & $5.3 \times 10^{-2}$ \\
\hline Knoxville & $1.8 \times 10^{-4}$ & $3.3 \times 10^{-3}$ & $4.7 \times 10^{-2}$ \\
\hline Norwood & $1.8 \times 10^{-4}$ & $3.2 \times 10^{-3}$ & $5.6 \times 10^{-2}$ \\
\hline Barnardville & $1.7 \times 10^{-4}$ & $3.0 \times 10^{-3}$ & $4.2 \times 10^{-2}$ \\
\hline Cedar Grove & $1.7 \times 10^{-4}$ & $3.0 \times 10^{-3}$ & $4.3 \times 10^{-2}$ \\
\hline Rockwood & $1.5 \times 10^{-4}$ & $2.9 \times 10^{-3}$ & $4.0 \times 10^{-2}$ \\
\hline Harriman & $1.8 \times 10^{-4}$ & $2.7 \times 10^{-3}$ & $4.8 \times 10^{-2}$ \\
\hline Dutch Valley & $1.6 \times 10^{-4}$ & $2.7 \times 10^{-3}$ & $4.3 \times 10^{-2}$ \\
\hline Rockford & $1.5 \times 10^{-4}$ & $2.5 \times 10^{-3}$ & $3.9 \times 10^{-2}$ \\
\hline Louisville & $1.6 \times 10^{-4}$ & $2.5 \times 10^{-3}$ & $4.2 \times 10^{-2}$ \\
\hline Friendsville & $1.7 \times 10^{-4}$ & $2.5 \times 10^{-3}$ & $3.8 \times 10^{-2}$ \\
\hline Jonesville & $1.4 \times 10^{-4}$ & $2.3 \times 10^{-3}$ & $4.4 \times 10^{-2}$ \\
\hline Oliver Springs & $1.4 \times 10^{-4}$ & $2.3 \times 10^{-3}$ & $4.0 \times 10^{-2}$ \\
\hline Sweetwater & $1.2 \times 10^{-4}$ & $1.9 \times 10^{-3}$ & $3.2 \times 10^{-2}$ \\
\hline Maryville & $8.1 \times 10^{-5}$ & $1.6 \times 10^{-3}$ & $2.4 \times 10^{-2}$ \\
\hline Lake City & $8.8 \times 10^{-5}$ & $1.6 \times 10^{-3}$ & $2.4 \times 10^{-2}$ \\
\hline Greenback & $9.9 \times 10^{-5}$ & $1.5 \times 10^{-3}$ & $3.0 \times 10^{-2}$ \\
\hline Oakdale & $8.4 \times 10^{-5}$ & $1.5 \times 10^{-3}$ & $2.4 \times 10^{-2}$ \\
\hline Wartburg & $3.4 \times 10^{-5}$ & $6.2 \times 10^{-4}$ & $1.1 \times 10^{-2}$ \\
\hline
\end{tabular}




\subsubsection{Sensitivity analysis}

A sensitivity analysis has been performed for a female born in 1952 who resided at any location and who consumed milk from a backyard cow and obtained produce grown locally. This analysis indicates that the uncertainty in the dose estimate is dominated by the estimate of the concentration of ${ }^{131} \mathrm{I}$ in milk (45\%), followed by the uncertainty in the internal dose conversion factor for the thyroid gland $(40 \%)$. The uncertainty in the estimate of the milk concentration is given by the uncertainty in the transfer of ${ }^{131} \mathrm{I}$ from air to pasture $(71 \%)$, the uncertainty in the concentration of ${ }^{131} \mathrm{I}$ in air $(21 \%)$, and the uncertainty in the milk transfer coefficient $(8 \%)$. The uncertainty in the internal dose conversion factor is dominated by the uncertainty in determining the actual mass of the thyroid for any individual of a given gender and age (Section 9). This source of uncertainty could be reduced substantially if the endpoint of the calculation were to be organ burden (the amount of energy deposited in the entire organ), rather than organ dose (the amount of energy deposited per unit mass of the organ), and if the estimation of risk were to be developed from organ burden-response relationships instead of dose-response relationships.

Most of the uncertainty in the risk estimate is due to the uncertainty in the dose itself (55\%), followed by the uncertainty in the dose response $(45 \%)$. The uncertainty in the relative biological effectiveness for ${ }^{131} \mathrm{I}$ with respect to external exposure to an equivalent dose of X-rays and gamma rays is only a minor contributor to the uncertainty in the overall dose response for ${ }^{131} \mathrm{I}$ (see Section 10).

\subsection{The April 29, 1954, Accident}

The previous sections summarized the combined effects from both the routine operations and accidental releases from the RaLa process from 1944 to 1956 . This section addresses the doses and risks resulting from the specific exposure to ${ }^{131}$ I released from the April 29, 1954, accident that took place in the afternoon, beginning sometime between 4:30 and 5 p.m. It is estimated that the duration of releases of radioactivity from this accident was about 2 and one-half hours, with between $105 \mathrm{Ci}$ and $500 \mathrm{Ci}$ (3.9 to $21 \mathrm{TBq}$ ) escaping to the atmosphere from the RaLa Stack and the building (706-D) containing the RaLa processing facility (see Section 3.15). Most of the releases are reported to have occurred during the first ten minutes to 2 hours.

The winds were to the $\mathrm{N}$ and NW directions during the first hour of the accident and to the $\mathrm{N}$ during the last 1.5 hours. Figures 4.10 through 4.12 in Section 4 of this report present the groundlevel air concentrations of ${ }^{131} \mathrm{I}$ within the $38-\mathrm{km}$ radius around the $\mathrm{X}-10$ facility. As discussed in Section 4.4.2, the uncertainty assigned to the fluctuation for the measured half-hourly wind direction results in a wide range of values for the $95 \%$ subjective confidence interval of the predicted time-integrated concentrations of ${ }^{131} \mathrm{I}$ in air. This is the dominant contributor to the uncertainties in the predicted concentrations of ${ }^{131} \mathrm{I}$ in air, which in turn affect the uncertainty in the predicted concentrations of ${ }^{131} \mathrm{I}$ in milk and in the predicted doses and risks. For example, at Jonesville, the time-integrated ${ }^{131} \mathrm{I}$ air concentration is estimated to have ranged from less than 1 to more than $1000 \mathrm{~Bq} \mathrm{~h} \mathrm{~m}^{-3}$, with a central estimate above $100 \mathrm{~Bq} \mathrm{~h} \mathrm{~m}^{-3}$. 
The primary locations affected by this release are those situated to the NW and N of X-10, such as Jonesville, Norwood, EFPC in Oak Ridge, Oliver Springs and Wartburg. Under the most extreme conditions the outer edge of the plume may have impacted the Scarboro and Woodland areas, and portions of Oak Ridge near the high school. Exposures at these locations would have been much lower than those experienced at EFPC, both because of the path of the plume and the fact that only the EFPC area associated with milk cows or local produce.

The doses for Jonesville, Norwood, EFPC, and Oliver Springs are not appreciably different. For a female who was either a newborn, or who was up to age 4 at the time of the accident, the doses from ingesting backyard cow's milk and other local produce and from inhaling contaminated air ranged from less than $0.023 \mathrm{cGy}$ to no more than 3 to $5.5 \mathrm{cGy}(95 \%$ subjective confidence interval), with central estimates of about 0.20 to $0.25 \mathrm{cGy}$ (Table 11.13). Doses to those in their late teens on a similar rural diet would be about one-half the doses for those who were in early childhood at the time of the accident (Table 11.14). At larger distances downwind, such as at Wartburg, the doses were about 20 times less than the doses estimated for the above four communities.

For those females exposed in early childhood and who were on a rural diet that included milk from a backyard cow at either Jonesville, Norwood, EFPC, or Oliver Springs, the excess lifetime risk of thyroid cancer (Table 11.13) ranged from a few chances in 10 million $\left(3\right.$ or $\left.4 \times 10^{-7}\right)$ to nearly one chance in a thousand $\left(1 \times 10^{-3}\right)(95 \%$ subjective confidence interval), with central estimates of from 1.5 to 2 chances in one hundred thousand $\left(1.5\right.$ to $\left.2 \times 10^{-5}\right)$.

For those females on a rural diet exposed at the age of 10 at these locations, the risks would be about 10 times less than the risks for those exposed in early childhood (Table 11.14). For those females exposed as late teenagers, the risks would be almost 100 times less than for those exposed in early childhood. The risks for males exposed at age 10 and 19 who had similar diets would be about 3.5 times less than those for females of the respective age groups.

In general, the total doses and risks from exposure to the April 29, 1954, accident are much lower than those resulting from exposure to the accident and the routine emissions reported in the previous sections (and presented for 41 locations in Appendix 11C). Therefore, exposure to the routine emissions would have dominated over the exposure to the accidental release of ${ }^{131} \mathrm{I}$. This finding is a marked change from previous results reported in earlier drafts of this Task.

\subsection{Influence of ${ }^{131}$ I Deposited in Tennessee from the Atmospheric Testing of Nuclear Weapons in Nevada}

A significant additional source of ${ }^{131} \mathrm{I}$ exposure to the residents of east Tennessee was fallout from the atmospheric testing of nuclear weapons. Atmospheric testing of atomic weapons occurred at the Nevada Test Site (NTS) from 1945 until 1962 (NCI, 1997). Fallout also occurred, even in later years, from atmospheric testing by other countries (UNSCEAR, 1977; WHO, 1983), but the majority of the deposition of ${ }^{131} \mathrm{I}$ in east Tennessee occurred from tests conducted at the Nevada Test Site in the years of 1952, 1953, 1955, and 1957. These years overlap the final years of RaLa processing at X-10. 
Table 11.13 Thyroid doses and excess lifetime risks of thyroid cancer for females of various ages exposed to ${ }^{131}$ I released from X10 during the April 29, 1954, accident. Exposure consists of ingestion of contaminated backyard cow's milk, ingestion of other non-milk food products, and inhalation of contaminated air (diet 1). The locations listed are those most affected by the contaminated plume.

\begin{tabular}{|c|c|c|c|c|c|c|c|c|c|}
\hline \multirow[b]{4}{*}{ Location } & \multicolumn{9}{|c|}{ Thyroid Dose (cGy) } \\
\hline & \multirow{2}{*}{\multicolumn{3}{|c|}{$\begin{array}{c}\text { Newborn female } \\
\text { 95\% Subjective Confidence Interval }\end{array}$}} & \multirow{2}{*}{\multicolumn{3}{|c|}{$\begin{array}{c}\text { age } 2 \\
\text { 95\% Subjective Confidence Interval }\end{array}$}} & \multirow{2}{*}{\multicolumn{3}{|c|}{$\begin{array}{c}\text { age } 4 \\
\text { 95\% Subjective Confidence Interval }\end{array}$}} \\
\hline & & & & & & & & & \\
\hline & Lower limit & $\begin{array}{l}\text { Central } \\
\text { estimate }\end{array}$ & upper limit & lower limit & central estimate & upper limit & lower limit & central estimate & upper limit \\
\hline Jonesville & 0.00056 & 0.23 & 5.48 & 0.00077 & 0.27 & 5.7 & 0.00062 & 0.25 & 5.49 \\
\hline Norwood & 0.018 & 0.23 & 3.96 & 0.021 & 0.26 & 3.8 & 0.023 & 0.23 & 3.34 \\
\hline EFPC & 0.012 & 0.22 & 4.17 & 0.021 & 0.23 & 3.3 & 0.015 & 0.21 & 3.49 \\
\hline Oliver Springs & 0.017 & 0.20 & 3.27 & 0.014 & 0.23 & 3.8 & 0.019 & 0.21 & 2.87 \\
\hline \multirow[t]{4}{*}{ Wartburg } & $<<0.0001$ & 0.01 & 0.63 & $<<0.0001$ & 0.02 & 0.6 & $<<0.0001$ & 0.01 & 0.61 \\
\hline & \multicolumn{9}{|c|}{ Excess Lifetime Risk of Thyroid Cancer } \\
\hline & \multirow{2}{*}{\multicolumn{3}{|c|}{$\begin{array}{c}\text { Newborn female } \\
\text { 95\% Subjective Confidence Interval }\end{array}$}} & \multirow{2}{*}{\multicolumn{3}{|c|}{$\begin{array}{c}\text { age } 2 \\
\text { 95\% Subjective Confidence Interval }\end{array}$}} & \multicolumn{3}{|c|}{ age 4} \\
\hline & & & & & & & $95 \%$ Sub & jective Confidenc & ce Interval \\
\hline Location & Lower limit & $\begin{array}{l}\text { Central } \\
\text { estimate }\end{array}$ & upper limit & lower limit & central estimate & upper limit & lower limit & central estimate & upper limit \\
\hline Jonesville & $2.2 \times 10^{-8}$ & $1.7 \times 10^{-5}$ & $9.8 \times 10^{-4}$ & $3.0 \times 10^{-8}$ & $2.1 \times 10^{-5}$ & $1.1 \times 10^{-3}$ & $2.8 \times 10^{-8}$ & $1.8 \times 10^{-5}$ & $9.1 \times 10^{-4}$ \\
\hline Norwood & $5.6 \times 10^{-7}$ & $1.7 \times 10^{-5}$ & $9.9 \times 10^{-4}$ & $4.7 \times 10^{-7}$ & $1.9 \times 10^{-5}$ & $1.1 \times 10^{-3}$ & $4.8 \times 10^{-7}$ & $1.8 \times 10^{-5}$ & $7.6 \times 10^{-4}$ \\
\hline EFPC & $3.6 \times 10^{-7}$ & $1.7 \times 10^{-5}$ & $8.4 \times 10^{-4}$ & $3.9 \times 10^{-7}$ & $1.9 \times 10^{-5}$ & $8.5 \times 10^{-4}$ & $4.1 \times 10^{-7}$ & $1.7 \times 10^{-5}$ & $6.4 \times 10^{-4}$ \\
\hline Oliver Springs & $4.7 \times 10^{-7}$ & $1.5 \times 10^{-5}$ & $8.3 \times 10^{-4}$ & $4.1 \times 10^{-7}$ & $1.7 \times 10^{-5}$ & $9.0 \times 10^{-4}$ & $3.8 \times 10^{-7}$ & $1.6 \times 10^{-5}$ & $7.9 \times 10^{-4}$ \\
\hline Wartburg & $6.6 \times 10^{-12}$ & $8.1 \times 10^{-7}$ & $1.1 \times 10^{-4}$ & $6.1 \times 10^{-12}$ & $9.5 \times 10^{-7}$ & $1.1 \times 10^{-4}$ & $5.5 \times 10^{-12}$ & $8.1 \times 10^{-7}$ & $9.8 \times 10^{-5}$ \\
\hline
\end{tabular}


Table 11.14 Thyroid doses and excess lifetime risks of thyroid cancer for females of different ages exposed to ${ }^{131}$ I released from X10 during the April 29, 1954, accident. Exposure consists of ingestion of contaminated backyard cow's milk, ingestion of other non-milk food products, and inhalation of contaminated air (diet 1). The locations listed are those most affected by the contaminated plume.

\begin{tabular}{|c|c|c|c|c|c|c|c|}
\hline \multirow[b]{3}{*}{ Year of birth } & \multirow[b]{3}{*}{$\begin{array}{c}\text { Age at } \\
\text { exposure }\end{array}$} & \multicolumn{6}{|c|}{ Thyroid dose (cGy) } \\
\hline & & \multicolumn{3}{|c|}{ Jonesville } & \multicolumn{3}{|c|}{ Norwood } \\
\hline & & $\begin{array}{r}95 \% \text { Sub } \\
\text { lower limit }\end{array}$ & $\begin{array}{l}\text { jective Confidence } \\
\text { central estimate }\end{array}$ & $\begin{array}{l}\text { e Interval } \\
\text { upper limit }\end{array}$ & $\begin{array}{r}95 \% \text { Sub } \\
\text { lower limit }\end{array}$ & $\begin{array}{l}\text { jective Confidenc } \\
\text { central estimate }\end{array}$ & $\begin{array}{l}\text { Interval } \\
\text { upper limit }\end{array}$ \\
\hline 1954 & Newborn & 0.00056 & 0.23 & 5.5 & 0.018 & 0.23 & 4.0 \\
\hline 1952 & 2 & 0.00077 & 0.27 & 5.7 & 0.021 & 0.26 & 3.8 \\
\hline 1944 & 10 & 0.00041 & 0.17 & 3.1 & 0.012 & 0.16 & 2.1 \\
\hline \multirow[t]{3}{*}{1935} & 19 & 0.00017 & 0.090 & 1.5 & 0.0070 & 0.074 & 1.1 \\
\hline & & \multicolumn{6}{|c|}{ Excess Lifetime Risk } \\
\hline & & \multicolumn{3}{|c|}{ Jonesville } & \multicolumn{3}{|c|}{ Norwood } \\
\hline Year of birth & $\begin{array}{c}\text { Age at } \\
\text { exposure }\end{array}$ & $\begin{array}{r}95 \% \text { Sub } \\
\text { lower limit }\end{array}$ & $\begin{array}{l}\text { jective Confidence } \\
\text { central estimate }\end{array}$ & $\begin{array}{l}\text { e Interval } \\
\text { upper limit }\end{array}$ & $\begin{array}{r}95 \% \text { Sub } \\
\text { lower limit }\end{array}$ & $\begin{array}{l}\text { jective Confidenc } \\
\text { central estimate }\end{array}$ & $\begin{array}{l}\text { Interval } \\
\text { upper limit }\end{array}$ \\
\hline 1954 & Newborn & $2.2 \times 10^{-8}$ & $1.7 \times 10^{-5}$ & $9.8 \times 10^{-4}$ & $5.6 \times 10^{-7}$ & $1.7 \times 10^{-5}$ & $9.9 \times 10^{-4}$ \\
\hline 1952 & 2 & $3.0 \times 10^{-8}$ & $2.1 \times 10^{-5}$ & $1.1 \times 10^{-3}$ & $4.7 \times 10^{-7}$ & $1.9 \times 10^{-5}$ & $1.1 \times 10^{-3}$ \\
\hline 1944 & 10 & $3.4 \times 10^{-9}$ & $2.1 \times 10^{-6}$ & $1.6 \times 10^{-4}$ & $6.0 \times 10^{-8}$ & $2.1 \times 10^{-6}$ & $1.1 \times 10^{-4}$ \\
\hline 1935 & 19 & $1.9 \times 10^{-9}$ & $1.7 \times 10^{-7}$ & $1.4 \times 10^{-5}$ & $3.7 \times 10^{-9}$ & $1.7 \times 10^{-7}$ & $1.3 \times 10^{-5}$ \\
\hline
\end{tabular}


A detailed analysis of ${ }^{131} \mathrm{I}$ in Nevada Test Site fallout has recently been published by the National Cancer Institute (NCI, 1997). Specific estimates of thyroid doses by gender and age at time of exposure for all 3100 counties in the coterminous United States can be obtained at the NCI Web site at [http://rex.nci.nih.gov], or more specifically, at [http://ww2.nci.nih.gov/fallout/html/]. From this NCI Web site, thyroid doses, along with estimates of uncertainty (expressed as geometric standard deviations, GSD), were obtained for females born on January 1, 1952, in Anderson, Roane, Loudon, and Knox counties and whose primary source of milk was from backyard cows, goats, or commercial milk purchased from the store (Table 11.15).

Table 11.15 Estimates of average thyroid doses, with associated uncertainties from exposure to

${ }^{131} \mathrm{I}$ in fallout from atmospheric nuclear bomb testing in Nevada, for females born on January 1, 1952, in counties adjacent to X-10, shown by milk source.

\begin{tabular}{lcccccc}
\hline & \multicolumn{2}{c}{ Backyard Cow } & \multicolumn{2}{c}{ Commercial Sources } & \multicolumn{2}{c}{ Goat } \\
\multicolumn{1}{c}{ Location } & GM $^{*}(\mathbf{c G y})$ & GSD $^{* *}$ & GM (cGy) & GSD & GM (cGy) & GSD \\
\hline $\begin{array}{l}\text { Anderson Co. } \\
\text { (Norwood) }\end{array}$ & 21 & 1.7 & 6.5 & 1.9 & 120 & 1.9 \\
$\begin{array}{l}\text { Roane Co. } \\
\text { (Lawnville/Gallaher) }\end{array}$ & 19 & 1.9 & 6.3 & 1.8 & 110 & 2.2 \\
$\begin{array}{l}\text { Loudon Co. } \\
\text { (Bradbury and Lenoir City) }\end{array}$ & 15 & 1.8 & 5.4 & 1.8 & 90 & 2.0 \\
$\begin{array}{l}\text { Knox Co. } \\
\text { (Solway) }\end{array}$ & 15 & 1.6 & 5.0 & 1.6 & 86 & 1.7 \\
\hline$* \quad \begin{array}{l}\text { Geometric mean } \\
\text { Geometric standard deviation }\end{array}$ & & & & & \\
$\begin{array}{l}\text { Source: http://rex.nci.nih.gov } \\
\text { http://www2.nci.nih.gov/fallout.html }\end{array}$ & & & & &
\end{tabular}

In general, the magnitude of the doses from ${ }^{131}$ I from Nevada Test Site fallout in these counties is determined more by diet and age at the time of exposure than by location of residence (NCI, 1997). For a child born in 1952 who primarily consumed milk from a backyard cow, central estimates of thyroid doses vary from 15 to 20 cGy. These dose estimates decrease by approximately a factor of 3 if the child's primary source of milk was from the grocery store. On the other hand, for a child who consumed milk from a goat, the central estimates at all locations approach or exceed 100 cGy (Table 11.15).

The uncertainties on the NTS dose estimates obtained from the National Cancer Institute vary depending on whether fallout was estimated from gross beta activity collected at a county monitoring station or through interpolation from samples collected in adjacent counties (NCI, 1997). Thus, differences in the reported NCI dose estimates among the four local counties should not be considered significant. The uncertainty (upper bound of the $95 \%$ subjective confidence interval) on the NTS fallout dose estimates varies from about a factor of 2.5 to 3.5 times the 
central value for a child who consumed cow's milk to more than 4.5 for a child who consumed goat's milk.

Because individuals living at the locations of interest considered for releases of ${ }^{131} \mathrm{I}$ from X-10 were also were exposed to NTS fallout, we have conducted an analysis of the overall dose and risk to selected individuals in the communities of Norwood, Lawnville/Gallaher, Bradbury, Lenoir City, and Solway. Doses and risks were calculated for females who were born in 1952 and lived in that community through 1957 . These individuals would have received combined exposures to ${ }^{131}$ I from routine and accidental releases from the RaLa operation at X-10 (from 1952 through 1956) and from NTS weapons fallout (from 1952 through 1957). At Bradbury, the combined doses are dominated by releases from X-10 for an individual who drank milk from a backyard cow (Table 11.16). At Lawnville/Gallaher and Solway, the doses and risks for the combined exposures to contaminated cow's milk were approximately twice those from X-10 alone (Tables 11.16 and 11.17). At locations beyond $15 \mathrm{~km}$ from X-10 or that are closer to X10 but less frequented by winds from the X-10 site, the overall doses and risks from combined exposures are dominated by NTS fallout (e.g., Norwood in Tables 11.15 through 11.18).

For milk obtained from a backyard cow or from the grocery store, the central estimates of risk from combined exposures exceed one chance in one thousand $\left(1 \times 10^{-3}\right)$ at all locations. The upper bound of the $95 \%$ subjective confidence interval for the excess lifetime risk of thyroid cancer exceeds one chance in one hundred $\left(1 \times 10^{-2}\right)$ at all locations. At the communities of Bradbury and Gallaher Bend, however, the upper bound of risk exceeds one chance in ten $(1 \times$ $10^{-1}$ ), due primarily to releases from the X-10 RaLa operations.

As expected, the highest estimates of dose and risk are for individuals who consumed goat's milk (Table 11.18). For a female born in 1952 who consumed goat's milk, central estimates of the thyroid dose from combined exposures to X-10 releases and NTS fallout ranged from 140 to $200 \mathrm{cGy}$, with upper bounds of the $95 \%$ subjective confidence interval approaching or exceeding $500 \mathrm{cGy}$. The combined thyroid cancer risk estimates at all locations for a female child consuming goat's milk are at least one chance in one hundred $\left(1 \times 10^{-2}\right)$, with upper bounds of 3 to 5 chances in ten $\left(3\right.$ to $\left.5 \times 10^{-1}\right)$.

We conclude that weapons fallout was a significant contributor to the total ${ }^{131} \mathrm{I}$ exposure for individuals located within $38 \mathrm{~km}$ of the X-10 site. Beyond this distance, ${ }^{131}$ I from NTS fallout was clearly the dominant source of exposure. The contribution of ${ }^{131} \mathrm{I}$ in global fallout during the early 1960 s to the total exposure received by populations in the vicinity of the X-10 facility has not been examined as a part of this study, but indications are that thyroid doses from global fallout could have been as much as $50 \%$ of those estimated from the Nevada Test Site (NCI, 1997). 
Table 11.16 Thyroid doses and risks from combined exposure to ${ }^{131}$ I from NTS fallout and X10 releases (routine and accidental) for females born in 1952 and on a diet of backyard cow's milk.

\begin{tabular}{|c|c|c|c|c|c|c|}
\hline \multirow[b]{2}{*}{ Location } & \multicolumn{3}{|c|}{$\begin{array}{c}\text { Dose (cGy) } \\
\text { 95\% Subjective Confidence }\end{array}$} & \multicolumn{3}{|c|}{$\begin{array}{c}\text { Lifetime Risk } \\
\text { 95\% Subjective Confidence }\end{array}$} \\
\hline & $\begin{array}{l}\text { lower } \\
\text { bound }\end{array}$ & $\begin{array}{c}\text { central } \\
\text { estimate }\end{array}$ & $\begin{array}{l}\text { upper } \\
\text { bound }\end{array}$ & $\begin{array}{l}\text { lower } \\
\text { bound }\end{array}$ & $\begin{array}{c}\text { central } \\
\text { estimate }\end{array}$ & $\begin{array}{l}\text { upper } \\
\text { bound }\end{array}$ \\
\hline \multicolumn{7}{|c|}{ Norwood (Anderson Co.) } \\
\hline NTS (1952-1957) & 7.4 & 21 & 59 & $8.1 \times 10^{-4}$ & $6.2 \times 10^{-3}$ & $4.5 \times 10^{-2}$ \\
\hline X-10 (1952-1956) & 0.51 & 3.5 & 29 & $8.1 \times 10^{-5}$ & $1.3 \times 10^{-3}$ & $1.8 \times 10^{-2}$ \\
\hline combined exposure $^{\mathrm{a}}$ & 11 & 27 & 72 & $1.1 \times 10^{-3}$ & $8.0 \times 10^{-3}$ & $6.2 \times 10^{-2}$ \\
\hline \multicolumn{7}{|c|}{ Lawnville/Gallaher (Roane Co.) } \\
\hline NTS (1952-1957) & 5.6 & 19 & 64 & $7.4 \times 10^{-4}$ & $5.8 \times 10^{-3}$ & $4.7 \times 10^{-2}$ \\
\hline X-10 (1952-1956) & 3.3 & 19 & 120 & $5.2 \times 10^{-4}$ & $5.8 \times 10^{-3}$ & $6.4 \times 10^{-2}$ \\
\hline combined exposure $^{a}$ & 13 & 47 & 150 & $1.8 \times 10^{-3}$ & $1.4 \times 10^{-2}$ & $1.1 \times 10^{-1}$ \\
\hline \multicolumn{7}{|l|}{ Bradbury (Loudon Co.) } \\
\hline NTS (1952-1957) & 4.7 & 15 & 48 & $6.7 \times 10^{-4}$ & $4.6 \times 10^{-3}$ & $3.2 \times 10^{-2}$ \\
\hline X-10 (1952-1956) & 4.4 & 28 & 200 & $5.8 \times 10^{-4}$ & $9.3 \times 10^{-3}$ & $8.9 \times 10^{-2}$ \\
\hline combined exposure $^{\mathrm{a}}$ & 15 & 48 & 225 & $1.6 \times 10^{-3}$ & $1.5 \times 10^{-2}$ & $1.1 \times 10^{-1}$ \\
\hline \multicolumn{7}{|l|}{ Solway (Knox Co.) } \\
\hline NTS (1952-1957) & 6.0 & 15 & 38 & $6.0 \times 10^{-4}$ & $4.5 \times 10^{-3}$ & $3.1 \times 10^{-2}$ \\
\hline X-10 (1952-1956) & 2.6 & 16 & 110 & $4.6 \times 10^{-4}$ & $4.7 \times 10^{-3}$ & $7.6 \times 10^{-2}$ \\
\hline Combined exposure $^{\mathrm{a}^{a}}$ & 13 & 35 & 124 & $1.6 \times 10^{-3}$ & $1.0 \times 10^{-2}$ & $9.4 \times 10^{-2}$ \\
\hline
\end{tabular}

a The combined exposure is obtained through summation over the full distributions of values; therefore, the results will differ from simple summations of the values in the columns. 
Table 11.17 Thyroid doses and risks from combined exposure to ${ }^{131}$ I from NTS fallout and X10 releases (routine and accidental) for females born in 1952 and on a diet of milk from commercial sources.

\begin{tabular}{|c|c|c|c|c|c|c|}
\hline \multirow[b]{2}{*}{ Location } & \multicolumn{3}{|c|}{ Dose (cGy) } & \multicolumn{3}{|c|}{$\begin{array}{c}\text { Lifetime Risk } \\
\text { 95\% Subjective Confidence }\end{array}$} \\
\hline & $\begin{array}{l}\text { lower } \\
\text { bound }\end{array}$ & $\begin{array}{c}\text { central } \\
\text { estimate }\end{array}$ & $\begin{array}{l}\text { upper } \\
\text { bound }\end{array}$ & $\begin{array}{l}\text { lower } \\
\text { bound }\end{array}$ & $\begin{array}{c}\text { central } \\
\text { estimate }\end{array}$ & $\begin{array}{l}\text { upper } \\
\text { bound }\end{array}$ \\
\hline \multicolumn{7}{|c|}{ Norwood (Anderson Co.) } \\
\hline NTS (1952-1957) & 1.8 & 6.5 & 22 & $2.3 \times 10^{-4}$ & $1.9 \times 10^{-3}$ & $1.4 \times 10^{-2}$ \\
\hline $\mathrm{X}-10(1952-1956)$ & 0.16 & 1.1 & 10 & $2.6 \times 10^{-5}$ & $4.1 \times 10^{-4}$ & $6.1 \times 10^{-3}$ \\
\hline Combined exposure $^{\mathrm{a}^{-}}$ & 2.9 & 8.8 & 26 & $2.9 \times 10^{-4}$ & $2.5 \times 10^{-3}$ & $2.0 \times 10^{-2}$ \\
\hline \multicolumn{7}{|c|}{ Lawnville/Gallaher (Roane Co.) } \\
\hline NTS (1952-1957) & 2.0 & 6.3 & 20 & $2.7 \times 10^{-4}$ & $1.9 \times 10^{-3}$ & $1.5 \times 10^{-2}$ \\
\hline $\mathrm{X}-10(1952-1956)$ & 0.86 & 5.9 & 46 & $1.4 \times 10^{-4}$ & $1.8 \times 10^{-3}$ & $2.4 \times 10^{-2}$ \\
\hline Combined exposure $\mathrm{a}^{-}$ & 4.2 & 15 & 56 & $5.7 \times 10^{-4}$ & $4.6 \times 10^{-3}$ & $3.8 \times 10^{-2}$ \\
\hline \multicolumn{7}{|l|}{ Lenoir City (Loudon Co.) } \\
\hline NTS (1952-1957) & 1.7 & 5.4 & 17 & $2.4 \times 10^{-4}$ & $1.7 \times 10^{-3}$ & $1.1 \times 10^{-2}$ \\
\hline $\mathrm{X}-10(1952-1956)$ & 0.26 & 1.7 & 14 & $3.5 \times 10^{-5}$ & $6.1 \times 10^{-4}$ & $6.0 \times 10^{-3}$ \\
\hline Combined exposure $^{\mathrm{a}^{-}}$ & 3.0 & 8.1 & 24 & $3.8 \times 10^{-4}$ & $2.6 \times 10^{-3}$ & $1.5 \times 10^{-2}$ \\
\hline \multicolumn{7}{|l|}{ Solway (Knox Co.) } \\
\hline NTS (1952-1957) & 2.0 & 5.0 & 12 & $2.0 \times 10^{-4}$ & $1.5 \times 10^{-3}$ & $1.0 \times 10^{-2}$ \\
\hline X-10 (1952-1956) & 0.72 & 5.1 & 37 & $1.3 \times 10^{-4}$ & $1.4 \times 10^{-3}$ & $2.5 \times 10^{-2}$ \\
\hline Combined exposure ${ }^{\mathrm{a}}$ & 4.1 & 11 & 42 & $4.9 \times 10^{-4}$ & $3.4 \times 10^{-3}$ & $3.1 \times 10^{-2}$ \\
\hline
\end{tabular}

${ }^{\mathrm{a}}$ The combined exposure is obtained through summation over the full distributions of values; therefore, the results will differ from simple summations of the values in the columns. 
Table 11.18 Thyroid doses and risks from combined exposure to ${ }^{131}$ I from NTS fallout and X10 releases (routine and accidental) for females born in 1952 drinking goat's milk.

\begin{tabular}{|c|c|c|c|c|c|c|}
\hline \multirow[b]{2}{*}{ Location } & \multicolumn{3}{|c|}{$\begin{array}{c}\text { Dose (cGy) } \\
\text { 95\% Subjective Confidence }\end{array}$} & \multicolumn{3}{|c|}{$\begin{array}{c}\text { Lifetime Risk } \\
\text { 95\% Subjective Confidence }\end{array}$} \\
\hline & $\begin{array}{l}\text { lower } \\
\text { bound }\end{array}$ & $\begin{array}{c}\text { central } \\
\text { estimate }\end{array}$ & $\begin{array}{l}\text { upper } \\
\text { bound }\end{array}$ & $\begin{array}{l}\text { Lower } \\
\text { bound }\end{array}$ & $\begin{array}{l}\text { Central } \\
\text { estimate }\end{array}$ & $\begin{array}{l}\text { upper } \\
\text { bound }\end{array}$ \\
\hline \multicolumn{7}{|c|}{ Norwood (Anderson Co.) } \\
\hline NTS (1952-1957) & 34 & 120 & 410 & $4.2 \times 10^{-3}$ & $3.6 \times 10^{-2}$ & $2.6 \times 10^{-1}$ \\
\hline$X-10(1952-1956)^{a}$ & 1 & 9.7 & 96 & $1.8 \times 10^{-4}$ & $3.2 \times 10^{-3}$ & $5.6 \times 10^{-2}$ \\
\hline Combined exposure $^{\mathrm{b}^{-}}$ & 42 & 140 & 460 & $4.9 \times 10^{-3}$ & $4.1 \times 10^{-2}$ & $3.0 \times 10^{-1}$ \\
\hline \multicolumn{7}{|c|}{ Lawnville/Gallaher (Roane Co.) } \\
\hline NTS (1952-1957) & 23 & 110 & 500 & $3.2 \times 10^{-3}$ & $3.3 \times 10^{-2}$ & $3.1 \times 10^{-1}$ \\
\hline$X-10(1952-1956)^{a}$ & 5.9 & 49 & 400 & $1.0 \times 10^{-3}$ & $1.6 \times 10^{-2}$ & $2.7 \times 10^{-1}$ \\
\hline combined exposure $^{\mathrm{b}}$ & 44 & 200 & 660 & $6.8 \times 10^{-3}$ & $5.7 \times 10^{-2}$ & $5.2 \times 10^{-1}$ \\
\hline \multicolumn{7}{|l|}{ Bradbury (Loudon Co.) } \\
\hline NTS (1952-1957) & 23 & 90 & 340 & $3.6 \times 10^{-3}$ & $2.8 \times 10^{-2}$ & $2.2 \times 10^{-1}$ \\
\hline$X-10(1952-1956)^{a}$ & 8.9 & 75 & 650 & $1.3 \times 10^{-3}$ & $2.5 \times 10^{-2}$ & $3.6 \times 10^{-1}$ \\
\hline Combined exposure $^{\mathrm{b}}$ & 55 & 190 & 870 & $7.2 \times 10^{-3}$ & $5.9 \times 10^{-2}$ & $4.1 \times 10^{-1}$ \\
\hline \multicolumn{7}{|l|}{ Solway (Knox, Co.) } \\
\hline NTS (1952-1957) & 30 & 86 & 240 & $3.3 \times 10^{-3}$ & $2.6 \times 10^{-2}$ & $1.8 \times 10^{-1}$ \\
\hline$X-10(1952-1956)^{a}$ & 5.2 & 42 & 360 & $8.0 \times 10^{-4}$ & $1.4 \times 10^{-2}$ & $2.1 \times 10^{-1}$ \\
\hline Combined exposure $^{\mathrm{b}}$ & 54 & 150 & 460 & $5.8 \times 10^{-3}$ & $4.5 \times 10^{-2}$ & $3.4 \times 10^{-1}$ \\
\hline
\end{tabular}

${ }^{a}$ Numbers reflect the doses and risks estimated for "diet 4" (Appendix 11C).

${ }^{\mathrm{b}}$ The combined exposure is obtained through summation over the full distributions of values; therefore, the results will differ from simple summations of the values in the columns.

\subsection{References}

NCI (National Cancer Institute). 1997. Estimated Exposure and Thyroid Doses Received by the American People from Iodine-131 Fallout Following Nevada Atmospheric Nuclear Bomb Tests. U.S. Department of Health and Human Services. National Institutes of Health. National Cancer Institute. October, 1997.

UNSCEAR (United Nations Scientific Committee on the Effects of Atomic Radiation). 1977. Sources and effects of ionizing radiation. New York. 1977.

WHO (World Health Organization). 1983. Environmental Health Criteria 25. Selected radionuclides: Tritium, Carbon-14, Krypton-85, Strontium-90, Iodine, Caesium-137. Radon, Plutonium. Geneva. 1983. 


\subsection{ESTIMATION OF THE HEALTH IMPACT OF X-10 IODINE-131 RELEASES}

The health impact on the communities surrounding the X-10 facility from the release of radioiodine can vary from thyroid nodules and hypothyroidism to thyroid cancer. As a global measure of the health impact, it is important to determine the number of thyroid cancers that may have occurred from exposure to the releases of ${ }^{131}$ I between 1944 and 1956. The purpose of this section is to estimate the total number of cancers resulting from the iodine releases at X-10 due to the contamination of milk produced within the study domain of $38 \mathrm{~km}$. To obtain an approximate estimate of the additional excess cancers produced from contamination occurring outside this range, an extrapolation has been made from $38 \mathrm{~km}$ to $200 \mathrm{~km}$, based on the amount of ${ }^{131}$ I expected to have been deposited on pasture land in this region.

\subsection{Estimation of the Number of Excess Thyroid Cancers within the Study Domain}

The number of excess cancers in the 38-km area of interest was determined using the time- and space-averaged concentrations of iodine in milk, the volume of milk produced in the area, and the dose and risk conversion factors.

$$
N C=C_{\text {milk }} \cdot V_{\text {milk }} \cdot D C F_{0-14} \cdot R F_{0-14}
$$

where

$\mathrm{NC} \quad=\quad$ the number of excess thyroid cancers that could have occurred in individuals exposed as children under the age of 15 between 1944 and 1956 [cancers];

$C_{\text {milk }}=\quad$ the time- and space-averaged concentration of ${ }^{131} \mathrm{I}$ in milk $\left[\mathrm{Bq} \mathrm{L}^{-1}\right]$;

$V_{\text {milk }}=$ the volume of milk produced in the area of interest between 1944 and 1956 $[\mathrm{L}]$

$D C F_{0-14}=$ the dose factor for children under the age of 15 at the time of exposure [Gy $\left.\mathrm{Bq}^{-1}\right]$; and

$R F_{0-14}=$ the risk factor for children under the age of 15 at the time of exposure [excess lifetime risk $\mathrm{Gy}^{-1}$, or excess cancer per Gy]. 


\subsubsection{Time- and space-averaged concentration of Iodine-131 in milk}

The average concentration of ${ }^{131} \mathrm{I}$ in milk decreases as a function of increasing distance from the RaLa facility, as shown in Section 6. For the purpose of estimating the total number of thyroid cancers induced by exposure to ${ }^{131} \mathrm{I}$, a spatial and temporal average over the region of interest was needed. The region of interest was defined as a circle with a radius of $38 \mathrm{~km}$ around X-10. As there were no dairy cows within the Oak Ridge Reservation during the period of interest, the area covered by the Oak Ridge Reservation is eliminated from the $38-\mathrm{km}$ circle.

As discussed in Sections 4 through 6, the concentrations of ${ }^{131} \mathrm{I}$ in milk were estimated for the nodes of a radial grid (Figure 12.1). The radius of the grid increases by $1.5 \mathrm{~km}$ per step from 2 to $38 \mathrm{~km}$. The angular interval is 22.5 degrees. Thus, there are 400 nodes in the grid ( 25 radial and 16 angular).

To obtain a spatial average for the region of interest, the estimated concentration of ${ }^{131}$ I in milk at each node location was used. However, because of the design of the grid, there are more locations closer to X-10 (where the concentrations are higher) and fewer locations further away from X-10 (where the concentrations are lower). Thus, a direct average of the concentrations at each node location would produce an overestimate of the regional average. Given that the production of milk per unit area of land is constant over large areas of land (Section 12.1.2), a better estimate of the regional average concentration can be obtained by weighting the average concentration in milk in a sector by the area of the sector.

If points A, B, C, and D define a sector of land in the radial grid (Figure 12.1), the average concentration in milk in that sector is

$$
C_{S}=\frac{1}{4}\left(C_{A}+C_{B}+C_{C}+C_{D}\right)
$$

where $C_{A}, C_{B}, C_{C}$, and $C_{D}$ are the estimated concentrations of ${ }^{131} \mathrm{I}$ in milk at the four nodes of the sector.

The area of the sector is

$$
A_{S}=\frac{22.5 \cdot \pi}{360}\left(r_{B C}^{2}-r_{A D}^{2}\right)
$$




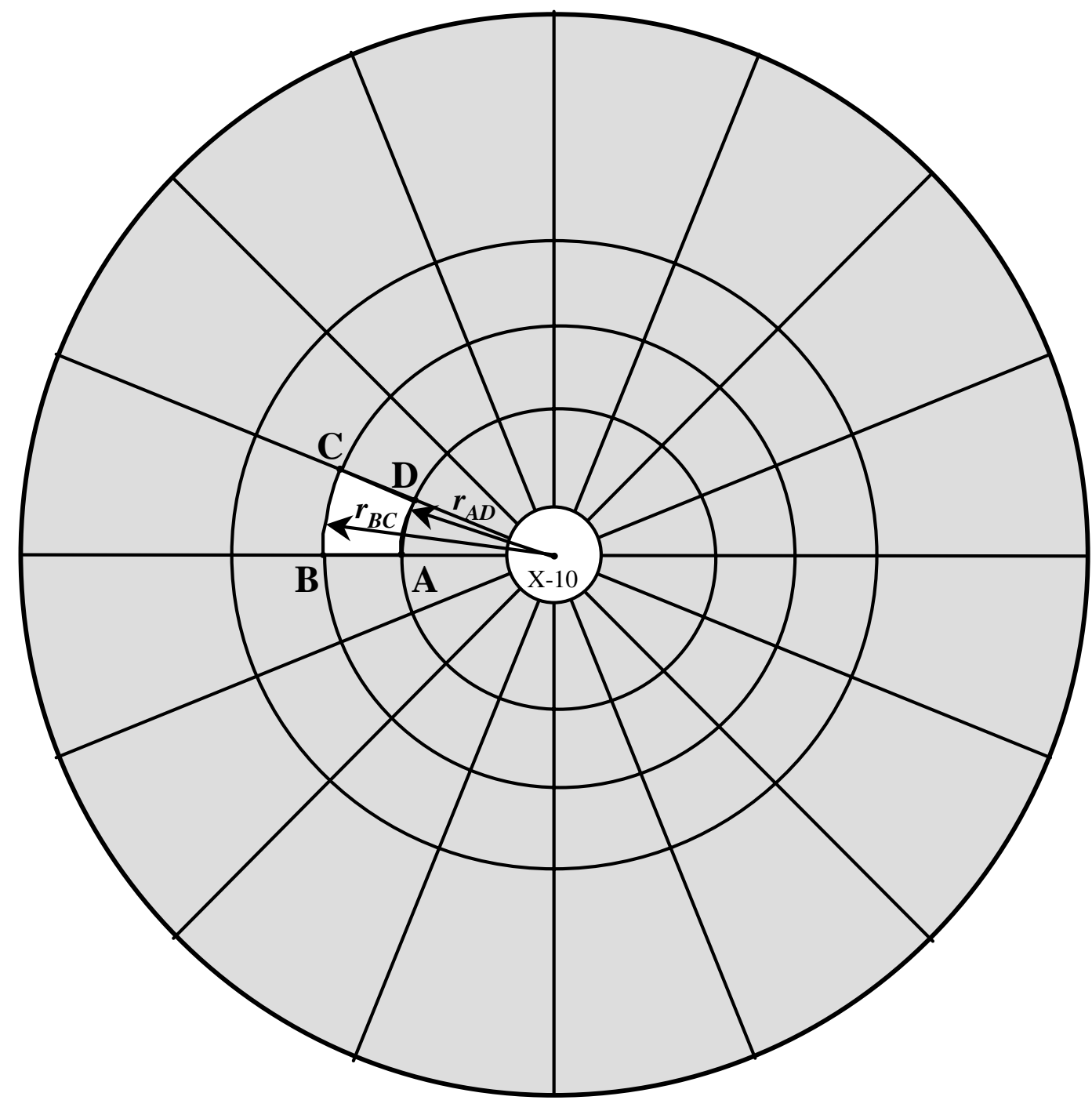

Figure 12.1. Schematic showing the locations at which the ${ }^{131}$ I concentrations in milk were calculated. Concentrations were estimated at the intersection of each circle and each ray from the center of the circle. 
The regional average concentration in milk in a given year of release $(t)$ is calculated as a weighted average, with the weights given by the area of land for which the concentration is representative:

$$
C_{\text {reg }}(t)=\frac{\sum_{S}^{N_{S}} A_{S} \cdot C_{S}(t)}{\sum_{S}^{N_{S}} A_{s}}
$$

Such regional averages are calculated for each year of release from X-10, for milk produced by backyard cows and for milk produced for commercial purposes. The summation in the above equation is performed only for sectors that lie outside the Oak Ridge Reservation. The reservation boundaries are at about $5.0 \mathrm{~km}$ from X-10 in most directions (Figure 1.1). Farther boundary points are found towards the west (about 8 to $10 \mathrm{~km}$ ). However, the farthest boundary points are toward the northeast $(6.5 \mathrm{~km}$ for 22.5 degrees northeast, $16 \mathrm{~km}$ for 45 degrees northeast, and $9.5 \mathrm{~km}$ for 90 degrees northeast). The closest boundary points are oriented to the north (about $3.5 \mathrm{~km}$ ). The sectors that are totally contained in the reservation were eliminated from the summation in equation 12.4. The sectors that have a significant part of their area within the reservation were also eliminated. Judgment was used for the sectors that have smaller areas of the reservation within their boundaries. For instance, the sector located to the northeast at more than $16 \mathrm{~km}$ was included in the averaging process, while the sector containing Jones Island on the Clinch River was excluded from averaging, even though a small portion of this sector does not belong to the reservation. This method is a good approximation for the purpose of estimating the total number of cancers, since most of the milk is actually produced further away from the reservation.

Finally, the time- and space-averaged concentration of ${ }^{131} \mathrm{I}$ in milk in the region of interest was estimated using the following equation.

$$
C_{\text {milk }}=\frac{\sum_{t}^{N_{t}} C_{\text {reg }}(t)}{N_{t}}
$$

The best estimates of the time- and space-averaged concentrations in milk were 3.3 to $29.6 \mathrm{~Bq} \mathrm{~L}^{-}$ ${ }^{1}\left(95 \%\right.$ C.I.; $50^{\text {th }}$ percentile $\left.=10.1 \mathrm{~Bq} \mathrm{~L}^{-1}\right)$ for backyard cow's milk, and 2.2 to $16.4 \mathrm{~Bq} \mathrm{~L}^{-1}(95 \%$ C.I.; $50^{\text {th }}$ percentile $=6.0 \mathrm{~Bq} \mathrm{~L}^{-1}$ ) for commercial milk. The confidence intervals were described by lognormal distributions. 


\subsubsection{Milk production}

Milk production estimates for the 38-km region of interest were based on data obtained from the National Agricultural Statistical Service (NASS, 1998a; 1998b) for the state of Tennessee. The estimates were determined using the milk production records for the state and the number of cows in each of the six counties located within the $38-\mathrm{km}$ radius. The milk production estimates for the $38-\mathrm{km}$ area of interest were compared to milk production records reported for various types of cattle that were raised around the ORR (Jersey, Guernsey, and Holstein). The estimates developed for milk production were consistent with those reported in the literature (Reaves and Pegram, 1956; Eckles, 1939; Spreckley, 1979; O’Neal, 1964; NCI, 1997b; TDHIA, 1998; USDA, 1950; 1951; 1952a; 1952b; 1953; 1954; 1955; 1956; 1957; 1958; 1959) and cited by a dairy expert (Montgomery, personal communication with C. Lewis, 1998).

\subsubsection{Volume of milk}

The volume of milk available for consumption in the area of interest during the thirteen years of

${ }^{131}$ I releases from X-10 was determined separately for commercial milk and backyard cow's milk. The general equation used to determine the volume of milk produced by commercial and backyard cows is as follows,

$$
V_{\text {milk }}=M_{\text {produced }} \cdot f_{1} \cdot f_{2} \cdot f_{3}
$$

where

$V_{\text {milk }}=$ the volume of fluid milk used for consumption that was produced either commercially or by backyard cows in the area of interest between 1944 and 1956 [L];

$M_{\text {produced }}=$ the total volume of milk produced by either commercial cows or backyard cows in each county between 1944 and 1956 [L];

$f_{1}=\quad$ the fraction of milk that remains fluid (i.e., milk not processed into cheese or butter) [unitless];

$f_{2}=\quad$ the fraction of fluid milk that is consumed by children under the age of 15 [unitless]; and

$f_{3}=\quad$ the fraction of Anderson, Blount, Knox, Loudon, Morgan, and Roane counties included in the $38-\mathrm{km}$ area of interest [unitless].

Using data obtained for the state of Tennessee during the period between 1944 and 1956 (NASS, 1998b), milk production was determined for each year in each county. "Commercial milk" was considered to be fluid milk and cream sold to dealers or individuals who operated commercial dairies (Mayfield Dairy, Broadacre Dairy, Weigel's Dairy, etc.). "Backyard cow” production was considered to include fluid milk and cream consumed on the farm and milk and cream sold to 
consumers or neighbors by farmers (door to door delivery). The volume of milk produced in each county of interest was not available, but using state statistical records, it was determined from the following relationship:

$$
M_{\text {produced }}=F_{c, 1944-1956} \cdot P_{\text {state }}
$$

where

$$
\begin{aligned}
& M_{\text {produced }} \quad=\quad \text { the total volume of milk produced by either commercial cows or } \\
& \text { backyard cows in each county between } 1944 \text { and } 1956 \text { [L]; }
\end{aligned}
$$

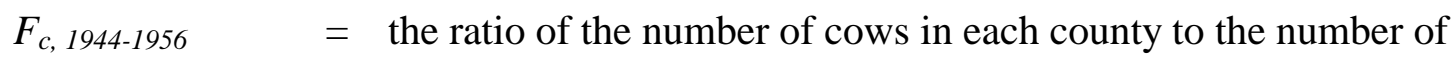

$$
\begin{aligned}
& \text { cows in the state of Tennessee [unitless]; and } \\
& P_{\text {state }} \quad=\quad \text { the volume of milk produced by either commercial cows or } \\
& \text { backyard cows in the state of Tennessee for the period between } \\
& 1944 \text { and } 1956 \text { [L]. }
\end{aligned}
$$

\subsubsection{State milk production}

As previously mentioned, the total production of milk in the state was estimated for commercial and backyard milk production. The total milk production for the state of Tennessee was determined as

$$
P_{\text {state }}=P_{c} \text { or } P_{\text {state }}=P_{b}
$$

where

$P_{\text {state }} \quad=\quad$ the volume of milk produced in the state of Tennessee for the period between 1944 and 1956 [L];

$P_{c} \quad=\quad$ the volume of milk produced commercially in the state of Tennessee [L]; and

$P_{b} \quad=\quad$ the volume of milk produced by backyard cows in the state of Tennessee [L].

The equations for each specific form of production (commercial or backyard) are provided below. The equations refer to milk production between 1944 and 1956.

$$
P_{c}=M_{\text {fluid }(c)}+M_{\text {cream }(c)}
$$

where

$P_{c} \quad=\quad$ the volume of milk produced commercially in the state of Tennessee [L];

$M_{\text {fluid (c) }}=$ the volume of fluid milk sold to dealers or other dairies in the state of Tennessee [L]; and 
$M_{\text {cream (c) }}=\quad$ the volume of cream sold to dealers or other dairies in Tennessee [L].

$$
P_{b}=M_{\text {fluid (f) }}+M_{\text {cream (f) }}+M_{\text {delivered }}
$$

where

$P_{b} \quad=$ the volume of milk produced by backyard cows in Tennessee [L];

$M_{\text {fluid }(f)} \quad=$ the volume of fluid milk consumed on farms where it was produced [L];

$M_{\text {cream }(f)}=$ the volume of cream consumed on farms where it was produced [L]; and

$M_{\text {delivered }}=\quad$ the volume of milk and cream delivered door to door or to neighbors by farmers [L].

\subsubsection{County milk production}

The total volume of milk produced in the 13 years of interest in the $38-\mathrm{km}$ area surrounding X-10 was based on the number of cows in this area during the period of interest. The number of cows present within a $38-\mathrm{km}$ radius of $\mathrm{X}-10$ was not directly available. Therefore, a ratio was developed to determine the number of cows in the area of interest. This ratio is comprised of the average number of cows present in each of the six counties (NASS, 1998a) potentially affected by ${ }^{131}$ I releases and the average number of cows in the state (NASS, 1998b).

$$
F_{c, 1944-1956}=\frac{C_{\text {countyavg }, 1944-1956}}{C_{\text {stateavg }, 1944-1956}}
$$

where

$F_{c, 1944-1956}=$ the ratio of the number of cows in each county to the number of cows in the state of Tennessee [unitless];

$C_{\text {county avg, 1944-1956 }}=$ the average number of cows in each county between the years 1944 and 1956 [cows]; and

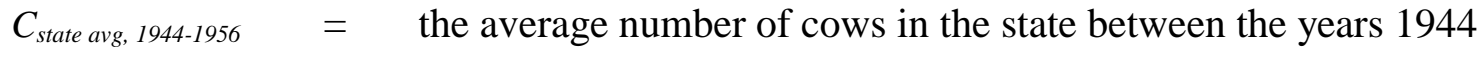
and 1956 [cows].

The 1944-1956 data provided information for the state of Tennessee and not for the specific counties or area of interest. No data were available on the number of cows per county for the time period of interest (1944-1956). Therefore, to determine the number of cows in each county between 1944 and 1956, a ratio of the average number of cows in 1944-1956 and the average amount of milk produced during 1944-1956 was used and equated to the ratio of the average 
number of cows and the average milk production from recent years (1975-1991). The following equation describes this relationship.

$$
C_{\text {countyavg }, 1944-1956}=\frac{\left(P_{\text {stateavg }, 1975-1991} \cdot C_{\text {countyavg }, 1975-1991}\right)}{P_{\text {stateavg }, 1944-1956}}
$$

where

$C_{\text {county avg, 1944-1956 }}=$ the average number of cows in each county between the years 1944 and 1956 [cows];

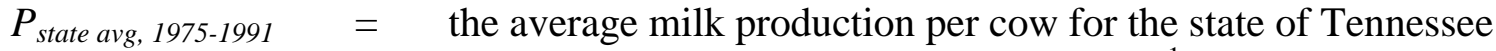
between the years 1975 and 1991 [ $\mathrm{L} \mathrm{yr}^{-1}$;

$P_{\text {state avg, 1944-1956 }}=\quad$ the average milk production per cow for the state of Tennessee between the years 1944 and 1956 [ $\left.\mathrm{L} \mathrm{yr}^{-1}\right]$; and

$C_{\text {county avg, 1975-1991 }}=$ the average number of cows in each county between the years 1975 and 1991 [cows].

The ratio of the average number of cows and the production per cow was considered appropriate because milk production records between 1929 and 1996 for the state of Tennessee indicate a steady milk production trend, regardless of the average number of cows producing the milk. Over the last three decades, there has been a reduction in the number of dairy cows in the United States. Although the number of cows has been decreasing, the average amount of milk produced per cow has increased (Majeskie, 1998). Specifically, there has been a continuous reduction in the number of cows from 1940 until the early 1980s when the U.S. cattle population leveled off (Majeskie, 1998). The reduction in the number of cows can also been seen in Tennessee, and more specifically in the six counties surrounding the Oak Ridge Reservation (Anderson, Blount, Knox, Loudon, Morgan, and Roane). Although the number of cows has decreased since 1944, milk production per cow has more than doubled since 1958, as a result of improved dairy cattle feeding, breeding, and management (Majeskie and Eastwood, 1998). Figure 12.2 depicts the relationship between the number of cows and the volume of milk produced in the state of Tennessee between 1929 and 1996.

The ratio of cows $\left(F_{c}, 1944-1956\right)$ in the county was then used in conjunction with the milk production records for the state for the time period of interest to calculate the milk production in Anderson, Blount, Knox, Loudon, Morgan, and Roane counties.

The milk production records for the state of Tennessee were obtained from the National Agricultural Statistical Service (NASS, 1998) and are provided in Appendix 12-A. The volume of milk produced $\left(M_{\text {produced }}\right)$ for each type of milk (commercial or backyard) was summed for 
the six counties. The range for backyard cow's milk production between 1944 and 1956 was 3.5 $\times 10^{7}$ to $8.5 \times 10^{7} \mathrm{~L}$, with a mean value of $6.0 \times 10^{7} \mathrm{~L}$. Commercial milk production averaged $1.7 \times 10^{8} \mathrm{~L}$ for the thirteen-year period. The range for commercial milk was $1.3 \times 10^{8}$ to $2.3 \times$ $10^{8} \mathrm{~L}$. A uniform distribution was selected to describe the uncertainty associated with the volume of each type of milk produced in each county because milk production for the state of Tennessee appears to be stable over a thirteen-year period (see Figure 12.2).

The distribution for the total volume of milk produced ( $M_{\text {produced }}$ ) (Eq. 12.7) was multiplied by the other three fractions $\left(f_{1}, f_{2}\right.$, and $\left.f_{3}\right)$ and their distributions described in the next sections to obtain the total volume of fluid milk used for consumption (Eq. 12.6).

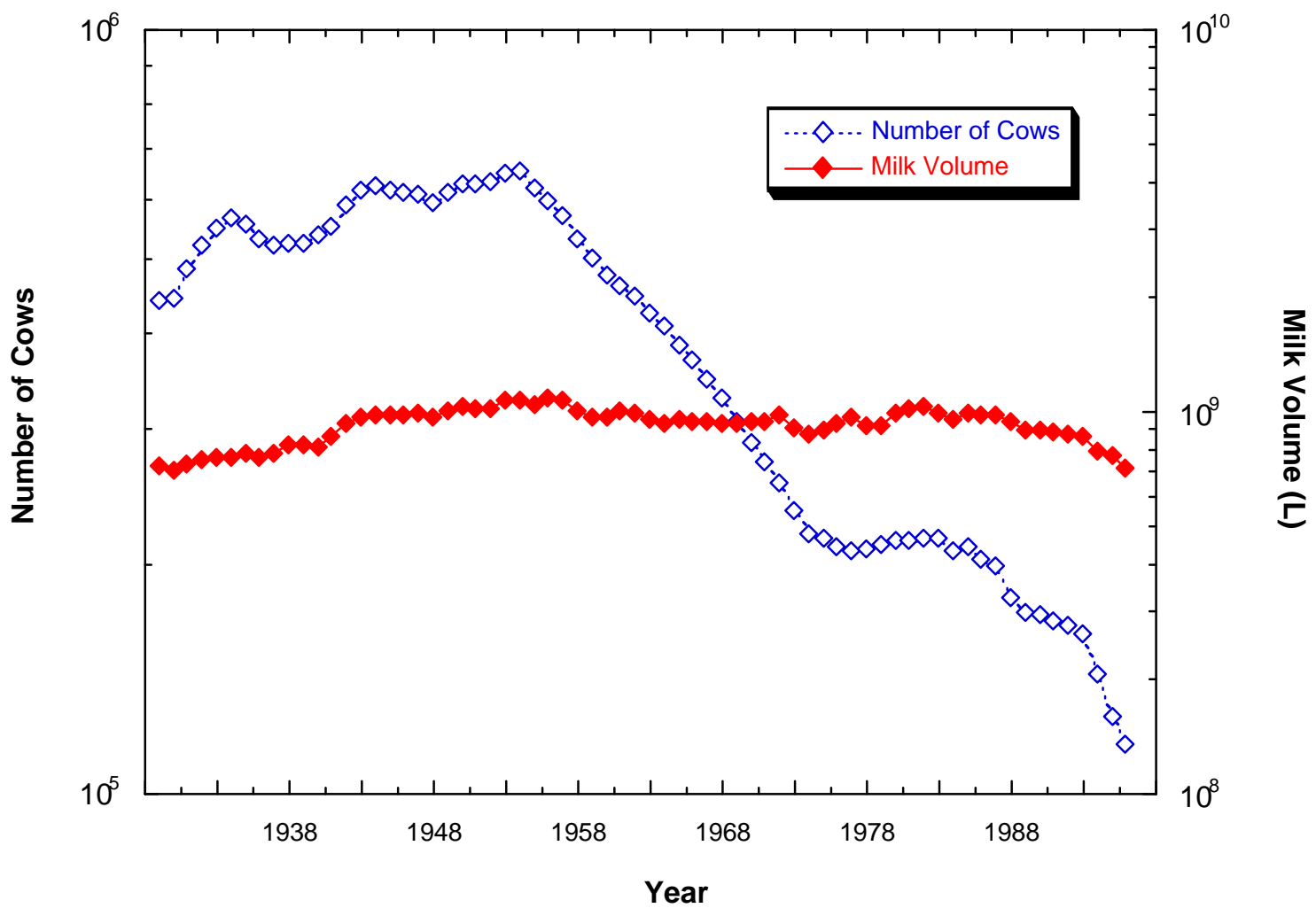

Figure 12.2 The number of cows and the annual milk volumes for the state of Tennessee. 


\subsubsection{Fraction of fluid milk}

The fraction of milk consumed by humans that was fluid $\left(f_{l}\right)$ was determined using the annual milk production records and the amount of milk marketed in the State of Tennessee (NASS, 1998b). The total volume of milk marketed and available for human use was determined by subtracting the volume of milk used to feed calves and the volume of milk used for cream and butter from the volume of milk produced during the year. The ratio of the volume of milk marketed and the volume of milk produced was used as the fraction of fluid milk consumed by humans.

Data on the volume of milk fed to calves and used for butter and cream were not available for the early years (1944-1950), but were available for sporadic years that followed. The lower limit of the distribution was determined using the years when the data were available (1950-1956) during the period of interest (1944-1956) when more milk was used to produce butter and cheese than for resale through distributors. The upper limit was determined using all of the relevant data available between the period of 1950-1996. The data indicate that in later years (1975-1996), more milk was sold to distributors than used to make butter and cheese on the farm.

The range for the fraction of fluid milk was 0.69 to 0.88 , with a mean of 0.79 . A uniform distribution was selected to describe the uncertainty associated with this parameter. ${ }^{1}$

\subsubsection{Fraction of milk consumed by children under 15 years of age}

The fraction of milk consumed by children 0-14 years $\left(f_{2}\right)$ was determined using data from the NCI report (1997a). The NCI provides a study group comprised of individuals who drink milk and those who do not consume milk. In the age group of $0-14$, an average of $73 \%$ of the male and female children surveyed consume milk. The upper limit for the fraction of milk consumed by children under 15 years of age was determined using the smallest percentage of children who did not consume milk in this survey group. According to the NCI (1997a), 15.9\% of males aged 1-4 years old did not consume milk. The lower limit for the fraction of milk consumed by children between the ages of 0 and 14 was determined by the largest percentage of children who did not consume milk (41.2\%), which represented females under the age of 1 . The resulting range for the fraction of milk consumed by children in this age group was $60-85 \%$, with an average of $73 \% .^{2}$ The fraction of milk consumed by children under the age of 15 was described

\footnotetext{
${ }^{1}$ Additional information obtained since the performance of this assessment could be used to refine the estimates of average fraction of milk consumed by humans that was fluid. Data from 1944-1956 indicate that the average fraction of fluid milk available was $66 \%$, as opposed to the $79 \%$ assumed in this assessment. Considering the new information, it appears that a uniform distribution for $f_{l}$ between 0.52 and 0.80 with a mean of 0.66 would be more defensible. If this change were made, it would result in a decrease in the total number of cancers expected.

${ }^{2}$ Additional information obtained since the performance of this assessment could be used to refine the estimates of the fraction of milk consumed by children under $15 \mathrm{y}$. When combined with data from the 1950 Tennessee population census, information from the 1977 NCI report indicate that children in the 0-14 age group consume about 30 to $60 \%$ of the volume of milk consumed by a Tennessee population typical of 1950 . Based on the information currently available, it appears that a distribution for $f_{2}$ between 0.30 and 0.60 with a mean of 0.45 would be more defensible. If this change were made, it would result in a decrease in the total number of cancers expected.
} 
by a triangular distribution with a mode of 0.73. A triangular distribution was selected because more children in the rural setting are expected to have access to milk than not. Therefore, with a mode of 0.73 , approximately three-fourths of the children are expected to consume milk.

\subsubsection{Fraction of milk produced within the area of interest}

The fraction of milk produced within the $38-\mathrm{km}$ radius of $\mathrm{X}-10\left(f_{3}\right)$ was determined using the area of a circle of $38-\mathrm{km}$ in radius and the area of the counties surrounding X-10. Six counties lie within the 38-km vicinity of the X-10 facility: Anderson, Blount, Knox, Loudon, Morgan, and Roane (Figure 1.1). The ratio describes the relationship between the area of the 38-km circle to the total area of the six counties. The ratio was representative of the fraction of milk produced within the area of interest.

Since the boundaries of the counties did not change, the fraction of milk produced within the 38$\mathrm{km}$ of interest was considered a point estimate. The fraction of milk produced within the $38-\mathrm{km}$ radius of X-10 was $68 \%$. The data concerning the county areas were obtained from the 1950 Agricultural Census for the State of Tennessee (USDOC, 1952), which is directly applicable to the time frame of interest (1944-1956).

\subsubsection{Dose and risk factors}

To determine the total number of cancers in the $38-\mathrm{km}$ area surrounding the $\mathrm{X}-10$ facility, the dose conversion factors and the risk factors for children under the age of 15 were needed. Sections 9 and 10 present the methods used to determine the dose $\left(D C F_{0-14}\right)$ and risk factors $\left(R F_{0-14}\right)$, respectively.

For the age group between 0-14 years, the dose conversion factor was described by a lognormal distribution with a $95 \%$ confidence interval of $1.5 \times 10^{-6}$ to $2.9 \times 10^{-6} \mathrm{~Gy} \mathrm{~Bq}^{-1}$. The $50^{\text {th }}$ percentile value was $2.0 \times 10^{-6} \mathrm{~Gy} \mathrm{~Bq}^{-1}$ (Table 9.3).

For 0-14 year olds, the risk factor was $1.6 \times 10^{-2}$ excess lifetime risk (ELR) $\mathrm{Gy}^{-1}$. A lognormal distribution with a $95 \%$ confidence interval of $5.8 \times 10^{-3}$ to $4.5 \times 10^{-2} \mathrm{ELR} \mathrm{Gy}^{-1}$ was selected to describe this parameter (Table 10.3).

\subsection{Results}

For the area within $38-\mathrm{km}$ of X-10, the estimates of the $95 \%$ subjective confidence interval for the total number of excess thyroid cancers resulting from the consumption of cow's milk (commercial and backyard combined) range between 6 and 84 cancers, with a central estimate $\left(50^{\text {th }}\right.$ percentile) of 21 cancers. For the backyard cow scenario, the $95 \%$ confidence interval ranged between 1 and 33 cancers. The central estimate for this scenario was approximately 7 cancers. Commercial cow's milk contributed more excess cancers to the total due to the larger quantity of commercial milk than backyard milk produced in the area around X-10. This increased contribution was expected because the number of individuals exposed via the commercial milk consumption pathway is greater than the number exposed via the backyard cow 
milk pathway. The $95 \%$ subjective confidence interval for the milk scenario involving the commercial cow was 3-53 excess thyroid cancers, with a central estimate of 13 . These cancers should be expected to manifest between 1950 and 2020, which is a 70-year period from when the releases occurred (including a six-year latency period during which the cancers will manifest).

\subsection{Sensitivity Analysis}

The most significant contributor to the uncertainty in the total number of excess thyroid cancers resulting from the consumption of cow's milk was the risk conversion factor. This factor alone contributed approximately $62 \%$ of the uncertainty. The average concentrations of ${ }^{131} \mathrm{I}$ in the commercial and backyard milk contributed approximately 15 and $13 \%$, respectively, to the overall uncertainty in this estimate. Figure 12.3 provides the uncertainties associated with the estimation of the total number of cancers resulting from the consumption of cows milk in the 38$\mathrm{km}$ area surrounding the $\mathrm{X}-10$ facility.

\subsection{Estimation of the Incidence of Thyroid Cancer beyond $38 \mathrm{~km}$}

This study estimates in detail the thyroid doses and thyroid cancer incidence within $38 \mathrm{~km}$ from X-10 (Sections 12.1-12.3). This region includes areas of Anderson, Knox, Blount, Loudon, Roane and Morgan counties. However, ${ }^{131}$ I was dispersed further than $38 \mathrm{~km}$ from X-10. A detailed estimation of the effects of ${ }^{131} \mathrm{I}$ beyond this limit is particularly difficult for a variety of reasons: (a) modeling of the atmospheric transport and dispersion has been conducted only up to $38 \mathrm{~km}$, and (b) adequate information about the milk production and distribution in a larger area is currently unavailable. This section presents an approach for an approximate estimation of the number of thyroid cancers beyond $38 \mathrm{~km}$.

The approach is based on the following assumptions:

(a) the total number of cancers for any region is directly proportional to the total amount of ${ }^{131} \mathrm{I}$ deposited in that region;

(b) the milk production per unit area is constant for the entire area; and

(c) the dose-response relationship for thyroid cancer is linear, with no threshold.

With these assumptions, the variation in the total number of cancers with distance from X-10 should follow the same trend as the inventory of ${ }^{131} \mathrm{I}$ deposited on the ground as a function of distance. 


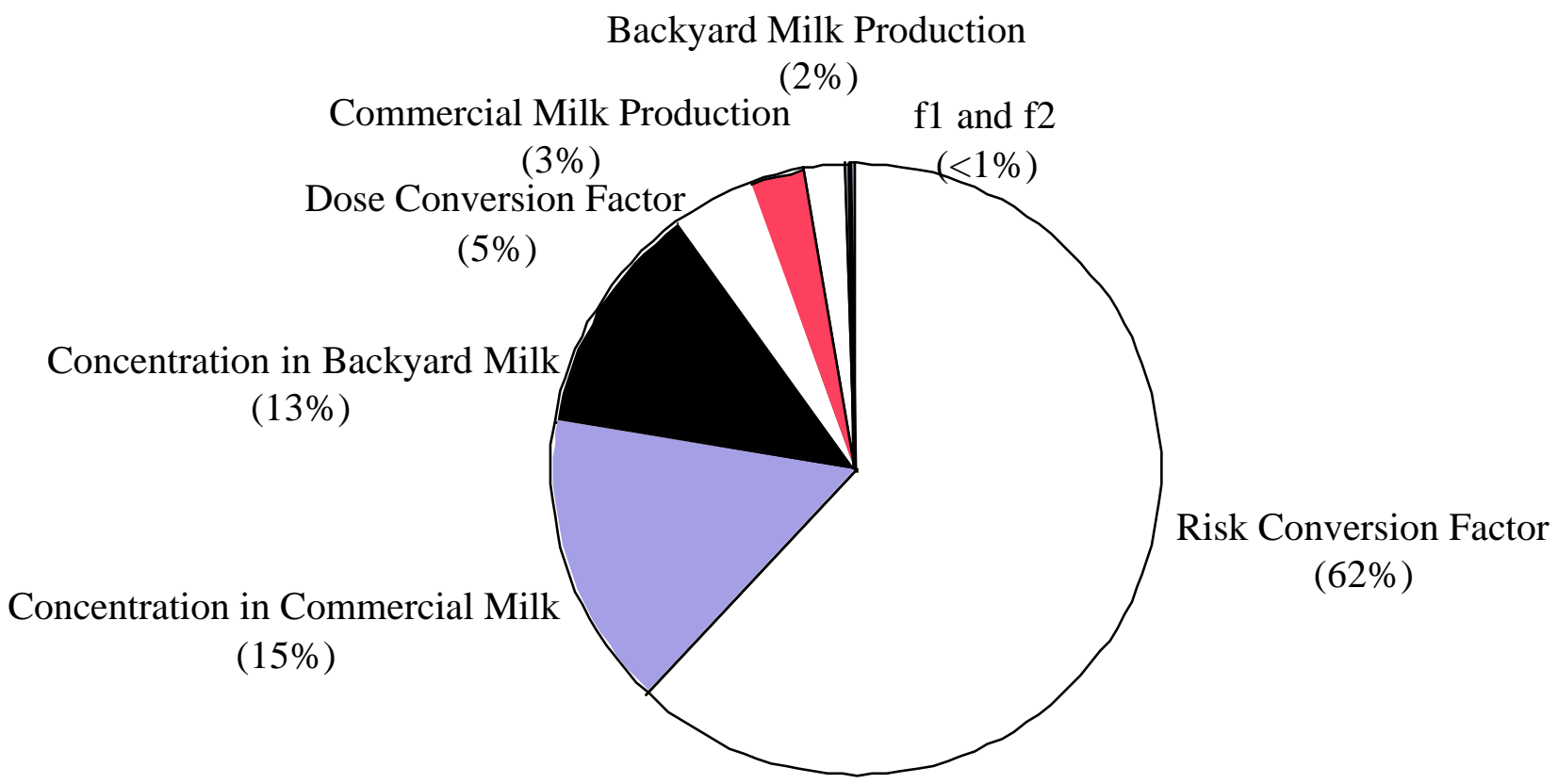

Figure 12.3 Contributors to the uncertainty in the total number of cancers estimate.

The inventory of ${ }^{131} \mathrm{I}$ deposited to the ground within a circle of radius $R$ around X-10 in a given year of releases is:

$$
I(R, t)=\sum_{S}^{N_{S}} A_{S} \cdot\left[\sum_{k=1}^{3}\left(C_{a, k}(t) \cdot\left(V_{d, k}+V_{w, k}\right)\right)\right] \cdot 365
$$

where

$$
\begin{array}{ll}
I(R, t) \quad=\quad \begin{array}{l}
\text { the amount of }{ }^{131} \mathrm{I} \text { deposited within a circle of radius } R \text { from } \mathrm{X}-10 \text { in a given } \\
\text { year of release } t[\mathrm{~Bq}] ;
\end{array} \\
A_{S} \quad \quad \text { the area of a sector in the polar grid (Figure 12.1, Eq. 12.3); } \\
N_{S} \quad \text { the number of sectors within a radius } R \text { from } \mathrm{X}-10 ; \\
k \quad \quad \begin{array}{l}
\text { the index for the physico-chemical form of iodine: } k=1 \text { for the elemental } \\
\text { form, } k=2 \text { for the organic form, and } k=3 \text { for the particulate form; }
\end{array} \\
C_{a, k} \quad \text { concentration of physico-chemical form } k \text { of }{ }^{131} \mathrm{I} \text { in air in a given sector }
\end{array}
$$




$$
\begin{array}{lll}
V_{d, k} & = & \text { total dry deposition velocity }\left[\mathrm{m} \mathrm{d}^{-1}\right] \text { for form } k(\operatorname{Section} 5) ; \\
V_{w, k} & = & \text { total wet deposition velocity }\left[\mathrm{m} \mathrm{d}^{-1}\right] \text { for form } k(\text { Section } 5) ; \text { and } \\
365 & = & \text { the number of days in year } t \text { [days]. }
\end{array}
$$

The total inventory deposited from 1944 to 1956 within a circle of radius $R$ is

$$
I(R)=\sum_{t=1944}^{1956} I(R, t)
$$

The total inventory deposited within a circle of radius $R$ increases as a function of distance, and it reaches a region of constant (linear) increase after about $20 \mathrm{~km}$. Figures 12.4 and 12.5 present the lower bound, central estimate and the upper bound of the $95 \%$ confidence interval of the inventory deposited as a function of distance. For uncertainty analysis purposes, a curve for the inventory deposited was obtained for each of 400 Monte Carlo runs, and a probability distribution was obtained for the derived set of 400 slopes. This probability distribution represents the uncertainty in the slope of the total inventory deposited. The $95 \%$ subjective confidence interval for the slope is $3.3 \times 10^{9}$ to $16 \times 10^{9} \mathrm{~Bq} \mathrm{~km}^{-1}$, with a central estimate of $8.1 \times$ $10^{9} \mathrm{~Bq} \mathrm{~km}^{-1}$.

The $95 \%$ subjective confidence interval of the amount of ${ }^{131} \mathrm{I}$ deposited within $38 \mathrm{~km}$ is $1.3 \times 10^{11}$ to $5.9 \times 10^{11} \mathrm{~Bq}$ (central estimate, $3.0 \times 10^{11} \mathrm{~Bq}$ ). This amount is much lower than the estimated amount of ${ }^{131}$ I released, $3.3 \times 10^{14}$ to $1.6 \times 10^{15} \mathrm{~Bq}\left(95 \%\right.$ C.I.; $50^{\text {th }}$ percentile $=7.8 \times$ $\left.10^{14} \mathrm{~Bq}\right)$. This finding is very important from many points of view. First, the ${ }^{131} \mathrm{I}$ plume is hardly depleted of iodine within $38 \mathrm{~km}$. Secondly, one should note that out of the total amount of iodine released, $5.2 \times 10^{14}$ to $9.6 \times 10^{14} \mathrm{~Bq}\left(95 \% \mathrm{CI}\right.$; $50^{\text {th }}$ percentile $\left.=7.1 \times 10^{14} \mathrm{~Bq}\right)$ was in elemental form, $1.1 \times 10^{14}$ to $3.4 \times 10^{14} \mathrm{~Bq}\left(95 \% \mathrm{CI}\right.$; $50^{\text {th }}$ percentile $\left.=1.9 \times 10^{14} \mathrm{~Bq}\right)$ was in organic form, and only $1.7 \times 10^{11}$ to $1.3 \times 10^{12} \mathrm{~Bq}\left(95 \% \mathrm{CI}\right.$; $50^{\text {th }}$ percentile $\left.=3.5 \times 10^{11} \mathrm{~Bq}\right)$ was in particulate form. In addition, as presented in Section 4.2, one must take into account that about a third of the iodine released in elemental form stays in elemental form after its release to the atmosphere, while another third is transformed to particulate iodine and the rest is transformed to organic iodine. Iodine in elemental form has the highest deposition rate, while deposition of iodine as particles is also important. Iodine in organic form has a very low deposition rate, meaning that it probably decays away before being deposited. Thus, beyond 38 $\mathrm{km}$, the plume released from X-10 can still be considered a large reservoir of ${ }^{131} \mathrm{I}$ available for deposition. 


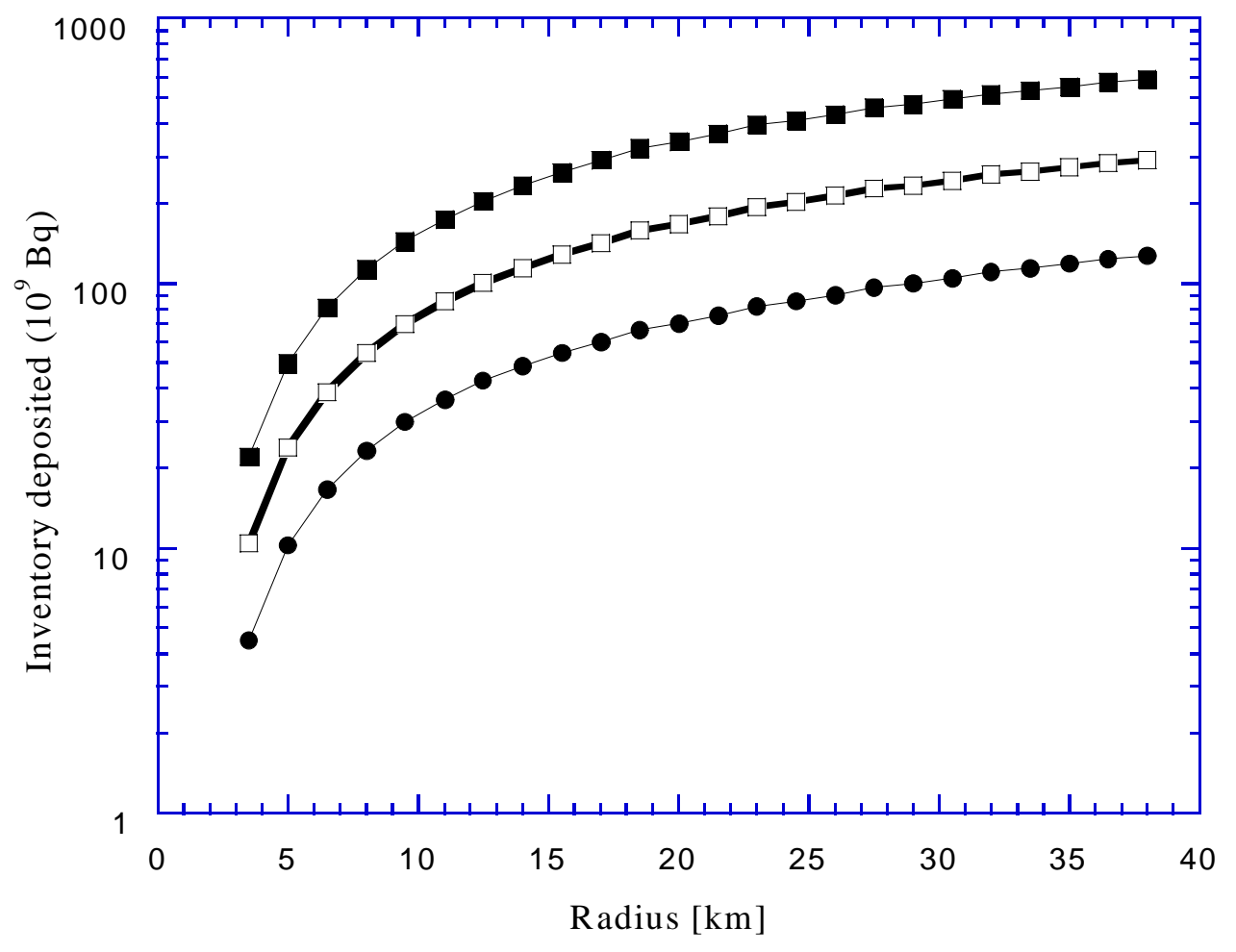

Figure 12.4 Amount of ${ }^{131}$ I deposited within a circle of a given radius around X-10. The figure presents the lower limit, central estimate, and upper limit of the $95 \%$ confidence interval of the inventory deposited as a function of radius. 


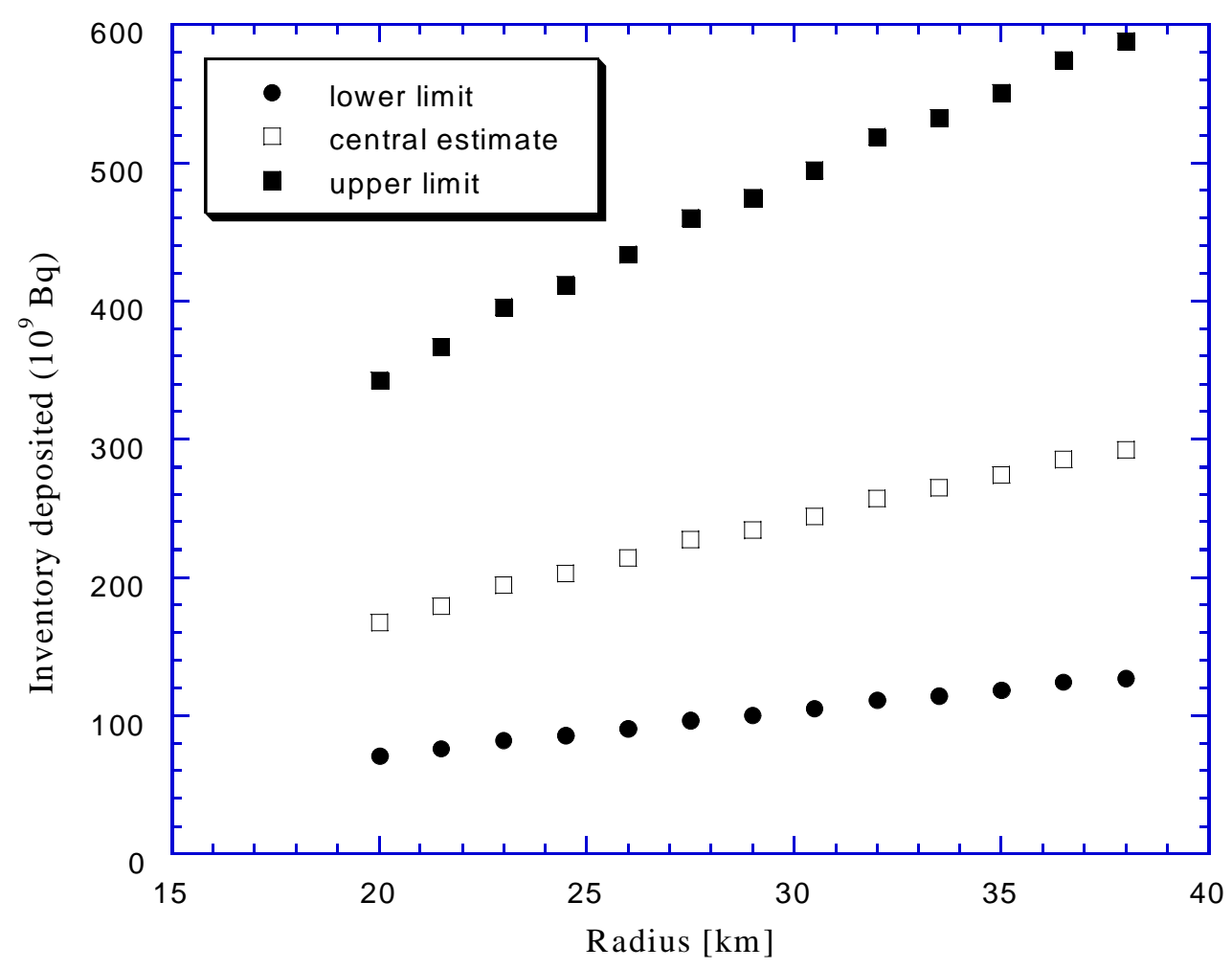

Figure 12.5 Amount of ${ }^{131}$ I deposited within a circle of a given radius around X-10. The figure presents the lower limit, central estimate, and upper limit of the $95 \%$ confidence interval of the inventory deposited as a function of radius. For radii larger than 20 $\mathrm{km}$, the amount deposited increases linearly with distance. 
Assuming that the inventory deposited increases linearly for some distance after $38 \mathrm{~km}$, a linear extrapolation can be developed to calculate inventories beyond $38 \mathrm{~km}$. If the rate of increase stays the same up to $100 \mathrm{~km}$, the estimated total amount of ${ }^{131} \mathrm{I}$ deposited within $100 \mathrm{~km}$ would be $3.3 \times 10^{11}$ to $1.6 \times 10^{12} \mathrm{~Bq}\left(95 \% \mathrm{CI}\right.$; $50^{\text {th }}$ percentile $\left.=8.0 \times 10^{11} \mathrm{~Bq}\right)$. Similarly, if the rate of increase is the same up to $200 \mathrm{~km}$, the estimated amount of ${ }^{131} \mathrm{I}$ deposited would be $6.7 \times 10^{11}$ to $3.2 \times 10^{12} \mathrm{~Bq}\left(95 \% \mathrm{CI} ; 50^{\text {th }}\right.$ percentile $\left.=1.6 \times 10^{12} \mathrm{~Bq}\right)$.

The total number of cancers was estimated for the region within a circle of radius $38 \mathrm{~km}$ from X10, as described in Sections 12.1-12.3. However, a functional dependency of the number of cancers with distance cannot be determined for distances beyond $38 \mathrm{~km}$. Thus, we used the assumption that the number of cancers is directly proportional to the inventory deposited; that is, the slopes of the curves in Figure 12.5 represent both the increase in curies deposited per kilometer, and the increase in the number of thyroid cancers per kilometer. In this case, an additional 6-27 cancers (95\% C.I.; 50 ${ }^{\text {th }}$ percentile $=13$ ) may be induced by the ${ }^{131}$ I deposited between 38 and $100 \mathrm{~km}$, and an additional 15-71 thyroid cancers $\left(95 \%\right.$ C.I.; $50^{\text {th }}$ percentile $\left.=35\right)$ may be induced between 38 and $200 \mathrm{~km}$.

Given the estimated number of thyroid cancers within $38 \mathrm{~km}\left(95 \%\right.$ C.I. $=6-84 ; 50^{\text {th }}$ percentile $=$ 21 ), the estimated total number of thyroid cancers induced within $100 \mathrm{~km}$ from X-10 is $14-103$ $\left(95 \%\right.$ C.I.; $50^{\text {th }}$ percentile $\left.=35\right)$, and the estimated number of cancers induced within $200 \mathrm{~km}$ from $\mathrm{X}-10$ is $25-149\left(95 \%\right.$ C.I.; $50^{\text {th }}$ percentile $\left.=58\right)$.

The reader should keep in mind that these numbers are based on the following assumptions: (a) the total number of cancers for any region is directly proportional to the total amount of ${ }^{131} \mathrm{I}$ deposited in that region, (b) the milk production per unit area is constant for the entire area, (c) the dose-response relationship for thyroid cancer is linear, with no threshold, and (d) a linear extrapolation of the inventory of ${ }^{131}$ I deposited to the ground is valid from 38 to $200 \mathrm{~km}$. If any of these assumptions are invalidated by future research, then the total number of cancers should be re-estimated.

The total number of cancers within 38 or $200 \mathrm{~km}$ of X-10 was estimated from a baseline of thyroid cancer diagnoses within the regional population assuming that individuals in this population were unexposed. According to the Institute of Medicine and the National Academy of Sciences (IOM/NAS, 1998), only about 28\% (39 to 140) of the total number of thyroid cancers in a population are diagnosed and reported. Therefore, it is likely that the total baseline rate used in this report may understate the total risk of radiogenic thyroid cancer in the population. In addition, for every cancer detected, there are approximately 8-10 cases of benign nodules of at least $1.5 \mathrm{~cm}$ in diameter. About $75 \%$ of such nodules are generally detected through medical examination using palpation. The number of benign nodules identified can be increased by another factor of 7 by the use of ultrasound, which can detect nodules less than $0.5 \mathrm{~cm}$ in size. 


\subsection{References}

Eckles, C.H. 1939. Dairy cattle and milk production. Third edition. Macmillan Company. New York, New York.

IOM/NAS. (Institute of Medicine/National Academy of Sciences). 1998. Exposure of the American People to Iodine-131 from Nevada Nuclear-Bomb Tests: Review of the National Cancer Institute Report and Public Health Implications. National Academy Press. Washington, DC.

Majeskie, J.L. 1998. Status of United States dairy cattle. National Cooperative Dairy Herd Improvement Program. http://www.inform.umd.edu/EdRes/Topic/AgrEnv/ndd/ dairy/. August 17, 1998.

Majeskie, J.L., and Eastwood, B.R. 1998. Status of United States dairy cattle. II. Dairy herd improvement 1988. NCDHIP Handbook. http://www.inform.umd.edu/EdRes/ Topic/AgrEnv/ndd/dairy/. August 17, 1998.

Montgomery, M. 1998. University of Tennessee professor and dairy specialist; also a member of the Tennessee Dairy Herd Improvement Association. Telephone interview with C. Lewis. August 25, 1998.

NASS (National Agricultural Statistical Service). 1998a. Data on the number of cows in each county in the State of Tennessee between 1975 and 1991. Excel file. Nashville, TN. July 30, 1998.

NASS (National Agricultural Statistical Service). 1998b. Data on the volume of milk produced for the State of Tennessee between 1929 and 1996. Excel file. Nashville, TN. July 30, 1998.

NCI (National Cancer Institute). 1997a. Estimated exposures and thyroid doses received by the American people from Iodine-131 in fallout following Nevada atmospheric nuclear bomb tests. U.S. Department of Health and Human Services. Washington, D.C. October, 1997.

NCI (National Cancer Institute). 1997b. Estimated exposures and thyroid doses received by the American people from Iodine-131 in fallout following Nevada atmospheric nuclear bomb tests. Appendices to the report from the National Cancer Institute. U.S. Department of Health and Human Services. Washington, D.C. October, 1997.

O’Neal, J.G. 1964. Crop production and feeding programs as related to milk production on 20 Grade A dairy farms in Anderson County, Tennessee. A problem in lieu of thesis presented to the Graduate Council of the University of Tennessee. University of Tennessee. March, 1964.

Reaves, P.M., and Pegram, C.W. 1956. Southern dairy farming. The Interstate. Danville, Illinois. 
Spreckley, V. 1979. Keeping a cow. David \& Charles. London, England.

TDHIA (Tennessee Dairy Herd Improvement Association, Inc.). 1998. Twenty-fourth annual meeting. Quality Inn. Sweetwater, Tennessee. February 20, 1998.

USDA (U.S. Department of Agriculture). 1950. Farm production, disposition, and income from milk, 1948-1949. Bureau of Agricultural Economics, Crop Reporting Board. Washington, DC. April 1950.

USDA (U.S. Department of Agriculture). 1951. Farm production, disposition, and income from milk, 1949-50. Bureau of Agricultural Economics, Crop Reporting Board. Washington, DC. April, 1951.

USDA (U.S. Department of Agriculture). 1952a. Farm production, disposition, and income from milk, 1950-51. Bureau of Agricultural Economics, Crop Reporting Board. Washington, DC. April, 1952.

USDA (U.S. Department of Agriculture). 1952b. Farm production, disposition, and income from milk, 1940-1949, revised estimates by states. Bureau of Agricultural Economics, Crop Reporting Board. Washington, DC. April, 1952.

USDA (U.S. Department of Agriculture). 1953. Farm production, disposition, and income from milk, 1951-52. Bureau of Agricultural Economics, Crop Reporting Board. Washington, DC. April, 1953.

USDA (U.S. Department of Agriculture). 1954. Farm production, disposition, and income from milk, 1952-53. Agricultural Marketing Service, Crop Reporting Board. Washington, DC. April, 1954.

USDA (U.S. Department of Agriculture). 1955. Farm production, disposition, and income from milk, 1953-54. Agricultural Marketing Service, Crop Reporting Board. Washington, DC. April, 1955. AM-30.

USDA (U.S. Department of Agriculture). 1956. Milk: Farm production, disposition, and income, 1954-55. Agricultural Marketing Service, Crop Reporting Board. Washington, DC. April, 1956. AMS-30 (1954-55).

USDA (U.S. Department of Agriculture). 1957. Milk: Farm production, disposition, and income, 1955-56. Agricultural Marketing Service, Crop Reporting Board. Washington, DC. April, 1957.

USDA (U.S. Department of Agriculture). 1958. Milk: Farm production, disposition, and income, 1956-57. Agricultural Marketing Service, Crop Reporting Board. Washington, DC. April, 1958. 
USDA (U.S. Department of Agriculture). 1959. Milk: Farm production, disposition, and income, 1957-58. Agricultural Marketing Service, Crop Reporting Board. Washington, DC. April, 1959.

USDOC (U.S. Department of Commerce). 1952. United States census of agriculture: 1950. Counties and state economic areas, Tennessee. Volume 1, Part 20. Farms, farm characteristics, livestock and products, crops, fruits, and values. Bureau of the Census. Washington, DC. 


\section{DISCUSSION}

This section provides a detailed discussion to support proper interpretation of the results of this study. Most of this discussion is devoted to the identification of future opportunities to reduce remaining uncertainty in the estimates of dose and risk for specific individuals. In addition, the estimated excess number of carcinomas produced from the deposition of ${ }^{131} \mathrm{I}$ within the $38-\mathrm{km}$ radius around the X-10 facility is compared with actual cases of thyroid cancer incidence reported to have occurred in this region from 1988 to 1995. The results of this study are also compared with other investigations of populations exposed to ${ }^{131} \mathrm{I}$ and to results reported during previous iterations of the present dose reconstruction.

\subsection{Differences in Individual Risk}

For an individual who resided in the study domain (within 38-km of X-10) of this investigation, the main determinants of risk are age at time of exposure, gender, and diet, with location of residence being a secondary factor. The highest doses occurred for males and females born between the years of 1940 and 1954 who consumed milk from backyard cows or goats. In this age group, the highest excess lifetime risks for thyroid cancer occurred in females born between 1944 and 1954, with males having risks that were about 8 to $46 \%$ of those for females. In addition, a male born in 1920 would have a risk of about $0.2-0.7 \%$ of that for a male born in 1952 , given that both were consuming food produced locally.

Given the importance of age, gender, and diet in determining risk, some individuals residing more than $25 \mathrm{~km}$ from X-10 could have doses and risks higher than those residing in communities near the Oak Ridge Reservation. For example, a male residing near the community of Gallaher Bend (6 km E of X-10) who was born in 1920 and who consumed regionally mixed commercial milk would have had a dose about $50 \%$, and an excess lifetime risk of thyroid cancer of about $1 \%$, of that for a female born between 1944 and 1954 who resided in the vicinity of Wartburg ( $27 \mathrm{~km}$ to the $\mathrm{NW}$ ) who consumed backyard cow's milk and local produce.

At any given location, the uncertainty in the estimate of thyroid dose for a given individual ranges over a factor of 30 to 40 (or a factor of 5.5 to 6.3 either side of the central estimate), while the uncertainty in the excess lifetime risk of thyroid cancer covers a range of about a factor of 230 to 1000 (or a factor of about 15 to 33 either side of a central estimate. The larger uncertainty in the risk estimate is due to the uncertainty in the dose response for radiogenic thyroid cancer obtained from pooled dose-response relationships for childhood exposures to external radiation (see Section 10). These large uncertainties, although comparable to results in other dose reconstruction studies where individuals have been exposed to ${ }^{131} \mathrm{I}$ (Section 13.4), have profound implications for distinguishing geographic differences in dose and risk.

It is entirely plausible that specific individuals living far downwind from X-10 (25 to $38 \mathrm{~km}$ in any direction) may have actual doses and risks that exceed those for individuals of the same gender and of similar age and dietary habits who reside in communities adjacent to the Oak Ridge Reservation. The probability of this occurring depends on the proportion of the 
uncertainty that is random and independent among individuals as opposed to shared by all individuals. As the proportion of uncertainty that is shared increases, the probability for individuals residing at distances far downwind to have higher doses and risks than those (of the same gender and similar age and dietary habits) residing close to X-10 decreases.

If the proportion of uncertainty that is not shared is large, it could impede distinction of geographic differences among individual doses and risks. Large uncertainty in dose and risk that is not shared among exposed individuals will affect the feasibility and design of epidemiological investigations. The determination of the amount of uncertainty that is shared among exposed individuals versus that which is independent is a subject for future research.

\subsection{Opportunities to Reduce Uncertainty in Dose and Risk Estimates}

In any follow-up work, it will be useful to investigate opportunities to reduce the uncertainty in dose and risk and to identify and eliminate any remaining sources of possible bias. A sensitivity analysis has identified the major sources of uncertainty as the transfer of ${ }^{131}$ I from air to milk, the uncertainty in the mass of the thyroid gland, and the uncertainty in the dose-response function for ingested ${ }^{131}$ I. Some additional uncertainty may be due to the time scale of the analysis, which is on an annual average basis for the source term, and the transfer of ${ }^{131} \mathrm{I}$ from the atmosphere to the individual person. It is expected that some fraction of the present extent of uncertainty will be reducible through further investigation and analysis, but a major portion of uncertainty is likely to remain.

\subsubsection{Reduce the scale of time averaging to account for releases occurring for each dissolution run}

The present analysis provides source term estimates on an annual average basis. Improved estimates could be made by reducing the scale of time averaging to 6- to 12-hour time blocks and matching the atmospheric dispersion modeling to the meteorological conditions prevailing during that period. Differences in the transport and chemical transformation of ${ }^{131} \mathrm{I}$ in the atmosphere and in its transfer from air to vegetation could then account for differences in humidity, precipitation, temperature, and diurnal versus nocturnal conditions. A reduction in the scale of time averaging would be particularly useful if most of the releases during the year occurred during the day versus the night, or summer versus winter.

\subsubsection{Estimation of the transfer of ${ }^{131}$ I from pasture to milk}

Some reduction in overall uncertainty may occur if the specific season of the year could be taken into account and if the specific farming and dairy management practices were known for milk consumed by a specific person. Some of the uncertainty, however, is contributed by the milk transfer coefficient for ${ }^{131} \mathrm{I}$, and much of this uncertainty is not likely to be reducible with further research. The ${ }^{131}$ I milk transfer coefficient has been intensively investigated in the literature (see Section 6). However, studies could be undertaken to identify the components of uncertainty in the milk transfer coefficient that are random due to inter-cow and inter-dairy variability versus 
the components that are nonrandom, i.e., shared among cows in a dairy and shared among dairies in a region.

\subsubsection{Assumptions about diet and consumption of milk}

Some uncertainty could be reduced through knowledge of an individual's diet and consumption of milk. These variables, however, although random and independent among individuals in a given age and dietary category, are only minor contributors to the overall uncertainty in dose and risk. A potentially major source of uncertainty would be individual patterns of diet and milk consumption that departed markedly from the assumptions made in this report for reference individuals. One example would be those individuals who resided only a small fraction of the time within the overall domain of this study.

\subsubsection{Organ burden versus absorbed dose}

A major source of uncertainty in the estimation of the thyroid dose is the dose conversion factor for ${ }^{131} \mathrm{I}$. Within the dose conversion factor, the dominant source of uncertainty is the lack of knowledge about the actual mass of the thyroid gland for a given individual. Information on the inter-individual variability of the size of the thyroid for individuals of like age and gender is available, but it is difficult to determine thyroid mass for a specific individual or for specific times in the past. Some improvements might be realized if dependencies between body mass or height and organ mass are considered (see Section 9).

Significant improvements might be realized, however, if the endpoint of the calculation were organ burden instead of organ dose. This improvement is possible because the fractional uptake of ${ }^{131}$ I from blood to the thyroid is relatively independent of the size of the gland. The utility of the organ burden instead of organ dose would be especially evident if epidemiological information were available on the risk as a function of organ burden. Such information is not yet widely available for populations of mixed age and gender exposed to ${ }^{131} \mathrm{I}$.

Because of the likelihood that ${ }^{131}$ I induction of thyroid cancer is linear with either organ dose or organ burden, without a threshold (an organ dose or burden below which there is no effect), we believe that organ burden, in addition to being less uncertain than organ dose, would be a more meaningful indicator of risk. This is because at the same dose (energy absorbed per mass of tissue), more cells are at risk in a large thyroid than in a small thyroid for individuals of similar gender and age at time of exposure.

\subsubsection{Estimation of the dose-response relationship for ${ }^{131}$ I}

The second most important source of uncertainty in risk, after the uncertainty in the thyroid dose, is the uncertainty in the dose response for radiogenic thyroid cancer from exposure in childhood to external X-rays and gamma rays (see Section 10). The additional uncertainty due to lack of knowledge about the relative biological effectiveness of cancer induction by exposure to ${ }^{131}$ I was considered, but it did not contribute substantially to the overall risk per unit dose for ${ }^{131}$ I taken 
into the gland from ingestion or inhalation. With the information currently available, we doubt that further analysis will be successful in reducing uncertainty in the dose-response relationship.

Some refinements can be made in specifying risk as a function of ethnic origin, with Americans of Asian origin being at highest risk and Americans of African origin being at lowest risk (see Section 10). More research is needed to determine whether differences in the background incidence of thyroid cancer observed in various ethnic groups are due to socio-economic discrepancies in terms of access to and quality of medical care. For published data on the dose response from exposure in childhood to external radiation, more advanced analyses could be initiated to attempt to identify and partition the portion of uncertainty that is shared among individuals and the portion that is random and independent.

Major improvements in the estimation of risk can be anticipated either from the initiation of epidemiological investigations on individuals actually exposed to ${ }^{131}$ I released from X-10 during 1944 to 1956 , or from epidemiological research already underway on populations exposed to ${ }^{131}$ I in Ukraine, Russia, and Belarus (downwind of Chernobyl), in Russia (downwind of the Mayak facility in the Ural Mountains), and in Kazakstan (downwind of the Semipalatinsk Test Site of the former Soviet Union). Epidemiological research has been completed by the Fred Hutchison Cancer Research Center for cohorts of individuals exposed in childhood to ${ }^{131}$ I released at Hanford between 1944 and 1956, when spent uranium fuel slugs were dissolved in nitric acid to extract plutonium (HTDS, 1998); the results of this study, which includes dose-response analyses for numerous disease endpoints, are expected to be released to the public in early 1999.

Additional studies are planned by the Centers for Disease Control and Prevention for residents of the Marshall Islands exposed to ${ }^{131}$ I from U.S. testing of nuclear weapons in the Pacific. It is expected that each of these studies will contribute extensively to the present state of knowledge about the dose-response for exposure to ${ }^{131} \mathrm{I}$ for both neoplastic and non-neoplastic thyroid disease, including autoimmune thyroiditis.

\subsubsection{Estimation of the total cases of radiogenic thyroid disease}

In this report it is estimated that from 6 to 84 excess cases of thyroid cancer will be diagnosed within the lifetimes of individuals who were below the age of 15 between 1944 and 1956 and who consumed fresh milk from a backyard cow or locally and regionally mixed commercial milk produced within the $38-\mathrm{km}$ radius of X-10. Extrapolating out to $200 \mathrm{~km}$ from X-10, the estimated excess cancer range increases to 25 to 149 . Improvement in these estimates will be directly affected by either producing specific dose estimates for each individual in the exposed cohort combined with an epidemiological investigation of the dose response that is specific to this cohort, or from the use of refined ${ }^{131}$ I dose-response relationships obtained from numerous ongoing or recently completed epidemiological investigations of other exposed population groups.

The total number of excess thyroid cancers, as well as the excess lifetime risk of thyroid cancer for an individual, is dependent on the estimate of the background incidence rate in an unexposed population. Disease registries contain information only on the disease incidence determined 
through medical diagnosis. It is estimated that the number of actual thyroid cancers in an unexposed population may be as much as 3 to 4 times larger than the number diagnosed (IOM/NAS, 1998). These cancers are usually occult, i.e., without symptoms. Increasing the background incidence rate by a factor three to four would increase both the estimate of the total excess cases of thyroid cancer and the excess lifetime risk of thyroid cancer for an individual by the same amount.

In addition, for every cancer detected, there are approximately 8 to 10 cases of benign nodules of at least $1.5 \mathrm{~cm}$ in diameter. About $75 \%$ of such nodules can generally be detected through medical examination using palpation. The number of benign nodules identified can be increased by another factor of 7 by the use of ultrasonography, which can detect nodules less than $1.0 \mathrm{~cm}$ in size. Physician palpation of a patient's neck usually cannot detect thyroid nodules under $1.0 \mathrm{~cm}$.

Although the dose response (in terms of excess relative risk per Gy) of radiogenic benign neoplasms is not substantially different from the dose response for thyroid cancer (see Section 10 ), the clinical significance of excess benign nodules induced from radiation exposure is not evaluated in this study. About $9 \%$ of nodules diagnosed through palpation and about $28 \%$ diagnosed through ultrasound are subject to surgical removal (lobectomy), even though they are identified after surgery as benign (IOM/NAS, 1998).

Exposure to ${ }^{131} \mathrm{I}$ may induce non-neoplastic thyroid disease in addition to thyroid cancer and benign nodules. Because of the absence of definitive dose-response functions for non-neoplastic thyroid disease, only semi-quantitative analysis has been given. At doses below $20 \mathrm{cGy}$ there is no present evidence that exposure induces non-neoplastic thyroid disease. Above 20 to $30 \mathrm{cGy}$ up to about $100 \mathrm{cGy}$, radiogenic autoimmune thyroiditis is a possibility. Above $100 \mathrm{cGy}$, radiogenic autoimmune thyroiditis is plausible. Additional insights on a possible dose response for non-neoplastic thyroid disease from exposure to releases of ${ }^{131} \mathrm{I}$ at Hanford is expected in early 1999 from the Fred Hutchison Cancer Research Center and the Centers for Disease Control and Prevention (HTDS, 1998).

\subsubsection{Estimation of the probability of causation for diagnosed thyroid carcinomas}

Appendix 11C presents the probability of causation (PC) for a diagnosed carcinoma. These values are given for males and females at 41 selected locations, nine different years of birth, and 4 different types of diet. These values are generic. They may not represent the true probability of causation for any specific person. They are estimated in a manner described in Section 10, the calculational procedure being similar to approaches recommended by the National Council on Radiation Protection and Measurements (NCRP, 1993).

The estimates of the probability of causation in this study are based on a pooled analysis of epidemiological investigations on children exposed to external radiation (Ron et al., 1995) and consider uncertainty in the relative biological effectiveness of ${ }^{131} \mathrm{I}$ with respect to external radiation and the uncertainty in radiosensitivity due to differences in gender (see Section 10). Differences in individual radiosensitivity due to differences in genetic make-up or ethnic origin 
are not considered. A bias towards underestimation of the probability of causation is possible when radiation exposure (a) induces carcinomas in individuals who, without exposure, would normally be free of the disease, and (b) induces the early appearance of carcinomas in other individuals who, without exposure, would normally acquire the disease later in life due to high personal susceptibility to cancer ( for additional discussion on this topic see Greenland and Robins, 1988; Robins and Greenland, 1989a; 1989b; and Greenland and Rothman, 1998).

In this study, central estimates of the probability of causation exceeding $50 \%$ occur for both males and females under the age of 5 at time of exposure when the dose to the thyroid exceeds 15 to $20 \mathrm{rad}$. For a female or male born in 1952 on a rural diet composed of backyard cow's milk and local produce (diet 1), a central estimate of the probability of causation of $50 \%$ extends from about $12 \mathrm{~km}$ to the SW up to $15 \mathrm{~km}$ to the NE of X-10. A central estimate of PC of $50 \%$ encompasses a larger area for individuals on a diet of goat's milk and born between the years of 1944 and 1954.

\subsection{Comparison with 1988-1995 Incidence Data for Thyroid Carcinoma}

For children under the age of 15 consuming milk produced within the $38-\mathrm{km}$ radius of $\mathrm{X}-10$, it is estimated that from 6 to 84 excess thyroid cancers will occur over the lifespan of this exposed cohort. The central value of this estimate is 21 excess thyroid cancers. At the present time it is not possible to verify this number. Comparison of this range of values with incidence rates in the disease registry maintained by the Tennessee Department of Health may provide an apparent corroboration of our estimates, but interpretation of data from disease registries must be made with extreme caution because of the large number of uncontrolled confounding factors that may have a marked influence on the results.

Analysis of the reported cases of thyroid cancer for Loudon, Knox, Roane, and Anderson Counties indicates that for the period of 1988 to 1995 there was an average of 4.5 cases per 100,000 persons per year (Turri, 1998).

Given a population in these four counties of about 500,000 persons, the total number of cancers observed during this 8-year period is

$$
5 \times 10^{5} \text { persons } \times 8 \mathrm{y} \times 4.5 \times 10^{-5} \text { persons }^{-1} \mathrm{y}^{-1}=180 \text { cases of thyroid cancers. }
$$

The average for the state of Tennessee, excluding the above four counties, is 3.8 cases per 100,000 persons per year. Thus the estimated number of cases of thyroid cancers in these counties using this averaged incidence rate is

$$
5 \times 10^{5} \text { persons } \times 8 \mathrm{y} \times 3.8 \times 10^{-5} \text { persons }^{-1} \mathrm{y}^{-1}=152 \text { cases of thyroid cancers. }
$$


The difference is

$$
180 \text { cases }-152 \text { cases }=28 \text { excess cases }\left( \pm 25 \text { cases }^{1}\right)
$$

over an 8-year reporting period. The direction of this difference is consistent with the direction indicated by the results of this study, but the absolute value is much larger. Given that the manifestation of radiogenic thyroid cancer should follow the age-specific incidence rates of thyroid cancer for an unexposed population, the majority of the 6 to 84 excess thyroid cancers predicted from exposure to X-10 releases would be manifested between 1970 and 2020.

Assuming that the entire number of predicted excess cases would occur during this 50-year time period, an excess of about 1 case $([6$ cases $/ 50 \mathrm{y}] \times 8 \mathrm{y}=0.96)$ to 13 cases $([84$ cases $/ 50 \mathrm{y}] \times 8 \mathrm{y}$ $=13$ ) would have been expected during the reporting period of 1988 to 1995. These numbers are from about 4 to $50 \%$ of the 28 excess cases estimated from the disease registry.

As stated previously, disease registries can be affected by numerous uncontrolled confounding factors. Some of these factors are related to differences between races. The analysis presented above indicates that if one compares thyroid cancer incidence rates from the four counties surrounding the ORR to those in Tennessee excluding those four counties, the numbers of excess cases of thyroid cancer forecast by this study due to ${ }^{131}$ I releases from X-10 are about 4 to $50 \%$ of the excess cases estimated using the disease registry. However, as shown below, if one compares incidence rates among whites in the local counties to whites in the rest of the state, the difference is much lower, and is not statistically significant.

For the period of 1988 to 1995 , there was an average of 4.7 thyroid cancer cases per 100,000 persons per year among whites (Turri, 1998). Given a population in these four counties of about 500,000 persons, the total number of cancers observed during this 8 -year period among whites is

$$
5 \times 10^{5} \text { persons } \times 8 \mathrm{y} \times 4.7 \times 10^{-5} \text { persons }^{-1} \mathrm{y}^{-1}=188 \text { cases of thyroid cancers. }
$$

The average for whites in the state of Tennessee, excluding the above four counties, is 4.55 cases per 100,000 persons per year. Thus the estimated number of cases of thyroid cancers in these counties using this averaged incidence rate is

$$
5 \times 10^{5} \text { persons } \times 8 \mathrm{y} \times 4.55 \times 10^{-5} \text { persons }^{-1} \mathrm{y}^{-1}=182 \text { cases of thyroid cancers. }
$$

The difference is

$$
188 \text { cases }-182 \text { cases }=6 \text { excess cases }\left( \pm 27 \text { cases }^{2}\right)
$$

\footnotetext{
${ }^{1}$ The $95 \%$ confidence interval, $28 \pm 25$, is stated as $28 \pm 2$ standard deviations, where the standard deviation is estimated as the square root of 152 .

${ }^{2}$ The $95 \%$ confidence interval, $6 \pm 27$, is stated as $6 \pm 2$ standard deviations, where the standard deviation is estimated as the square root of 182 .
} 
Non-whites make up a significantly smaller fraction of the population in the four local counties than they do in the remainder of Tennessee, and non-whites have lower age-specific thyroid cancer incidence rates than whites. Because of the presence of these racial differences and other uncontrolled confounding factors affecting disease registries, extreme caution must be used when interpreting the possible significance of these observations. For this reason, the numbers of excess thyroid cancers estimated using the disease registry should not be used to either confirm or refute the scientific validity of the risk estimates made in this report.

\subsection{Comparison with Dose Reconstruction Results for Atmospheric Releases of ${ }^{131} I$ at Other Sites}

To help put the results of this analysis for Oak Ridge into perspective, comparisons are made with other dose reconstruction studies involving populations exposed to atmospheric releases of

${ }^{131}$ I. This comparison indicates that the range of doses for a child born in 1952 and consuming fresh milk from a backyard cow located in the vicinity of X-10 is similar to the doses estimated for either reference or actual children at other sites (Table 13.1). Furthermore, doses estimated for children consuming local sources of milk at $22.7 \mathrm{~km} \mathrm{NE}$ of X-10 as well as for those consuming regionally mixed commercial retail milk are comparable with the doses estimated for a median or typical child for other studies.

This comparison includes a study of Utah school children exposed to fallout ${ }^{131} \mathrm{I}$ from atmospheric testing at the Nevada Test Site (NTS) (Till et al., 1995; Stevens et al., 1992), the nationwide dose reconstruction of NTS ${ }^{131}$ I fallout in the 3100 counties of the coterminous USA (NCI 1997), and a study of both reference and actual children exposed to ${ }^{131} \mathrm{I}$ released from Hanford (Farris et al., 1994; Kopecky, 1995).

The similarity between the magnitude of thyroid doses estimated for individuals downwind of X10 and those from other sites is somewhat counterintuitive because the releases of ${ }^{131} \mathrm{I}$ at the other sites were much greater than at Oak Ridge. For example, the release of ${ }^{131} \mathrm{I}$ from the NTS was 3500 to almost 19,000 times greater than at Oak Ridge. However, most of the ${ }^{131} \mathrm{I}$ released from the NTS was injected into the upper atmosphere and transported for long distances. The small

fraction of NTS ${ }^{131}$ I deposited in southern Utah was associated with relatively insoluble large particles. These particles were not retained effectively by vegetation surfaces and were not readily taken up by dairy cows and secreted into milk (Stevens et al., 1992), in contrast to the situation assumed in this analysis for RaLa ${ }^{131} \mathrm{I}$ released from X-10. 
Table 13.1 Comparison of uncertainty statements for estimated doses from other ${ }^{131} \mathrm{I}$ dose reconstruction studies.

\begin{tabular}{|c|c|c|c|c|c|}
\hline \multirow[b]{2}{*}{ Location } & \multirow[b]{2}{*}{$\begin{array}{c}\text { Release } \\
{[\mathrm{Ci}]^{\mathrm{a}}} \\
\end{array}$} & \multicolumn{4}{|c|}{$\begin{array}{l}\text { 95\% subjective confidence } \\
\text { interval }\end{array}$} \\
\hline & & Reference & $\begin{array}{c}\text { Lower bound } \\
{[c G y]}\end{array}$ & $\begin{array}{c}\text { Upper bound } \\
{[\mathbf{c G y}]^{\text {b }}}\end{array}$ & $\begin{array}{c}\mathbf{U F}^{\mathrm{c}} \\
\text { [unitless] }\end{array}$ \\
\hline $\begin{array}{c}\text { Nevada Test Site } \\
\text { (3545 Utah school children) }\end{array}$ & $\begin{array}{c}1.5 \times 10^{8} \\
(5600 \mathrm{PBq})\end{array}$ & $\begin{array}{l}\text { Till et al. } \\
\text { (1995) }\end{array}$ & & & \\
\hline Maximum person & & & 22 & 2300 & 10 \\
\hline Median individual & & & 0.33 & 19 & 7.6 \\
\hline $\begin{array}{l}\text { Nationwide study } \\
(3,100 \text { counties })\end{array}$ & & $\begin{array}{c}\mathrm{NCI} \\
(1997)\end{array}$ & & & \\
\hline Meagher Co., MT $^{\mathrm{d}}$ & & & 3.8 & 792 & 14.4 \\
\hline Anderson $\mathrm{Co}, \mathrm{TN}^{\mathrm{e}}$ & & & 1.8 & 23 & 3.6 \\
\hline Washington, $\mathrm{DC}^{\mathrm{e}}$ & & & 1.2 & 21 & 4.2 \\
\hline Hanford & $\begin{array}{l}7.3 \times 10^{5} \\
(27 \mathrm{PBq})\end{array}$ & & & & \\
\hline Maximum reference child & & $\begin{array}{l}\text { Farris et al. } \\
\quad(1994)\end{array}$ & 49 & 1,100 & 4.7 \\
\hline "typical child" & & & 1.9 & 52 & 5.2 \\
\hline $\begin{array}{c}\text { HTDS } \\
\max \text { of } 841\end{array}$ & & $\begin{array}{l}\text { Kopecky } \\
\text { (1995) }\end{array}$ & 33 & 477 & 3.9 \\
\hline $\begin{array}{c}\text { HTDS } \\
\text { median of } 841\end{array}$ & & & 2.7 & 20 & 3.7 \\
\hline $\begin{array}{l}\text { Oak Ridge Dose } \\
\text { Reconstruction }\end{array}$ & $\begin{array}{c}0.88 \text { to } 4.2 \times \\
10^{4} \\
\text { (0.3 to } 1.6 \\
\text { PBq) }\end{array}$ & This study & & & \\
\hline $\begin{array}{l}\text { Gallaher Bend } \\
\qquad(6 \mathrm{~km})^{\mathrm{f}}\end{array}$ & & & 10 & 760 & 8.6 \\
\hline $\begin{array}{l}\text { Gallaher Bend } \\
\qquad(6 \mathrm{~km})^{\mathrm{d}}\end{array}$ & & & 5.5 & 230 & 6.6 \\
\hline $\begin{array}{c}\text { Claxton } \\
(22.7 \mathrm{~km})^{\mathrm{e}}\end{array}$ & & & 0.31 & 15 & 7.1 \\
\hline $\begin{array}{l}\text { Regionally mixed commercial } \\
\text { milk, all locations }\end{array}$ & & & 0.17 & 80 & 6.2 \\
\hline $1 \mathrm{Ci}$ equals $3.7 \times 10^{10} \mathrm{~Bq}$ & & & & & \\
\hline $\begin{array}{l}\text { b } 1 \text { cGy equals } 1 \text { rad } \\
\text { c } \text { UF = uncertainty factor, the rat } \\
\text { d female born in } 1952 \text { who consu } \\
\text { e female born in } 1952 \text { who consu } \\
\text { f female born in } 1952 \text { who consu }\end{array}$ & $\begin{array}{l}\text { milk from a } \\
\text { milk from cor }\end{array}$ & $\begin{array}{l}\text { bound of the } \\
\text { yard cow. } \\
\text { ercial dairies. }\end{array}$ & $\%$ subjective conf & e interval and the & th percentile. \\
\hline
\end{tabular}


At Hanford, the release of ${ }^{131}$ I was 17 to 90 times greater than for RaLa processing at X-10. The larger release at Hanford, however, was compensated for by much greater dispersion and dilution

of ${ }^{131} \mathrm{I}$ in the atmosphere prior to reaching locations where pastures were contaminated. Average wind speeds at Hanford are also substantially higher than average wind speeds for the region around Oak Ridge. East Tennessee also has much more precipitation than eastern Washington. In addition, the distance to the nearest residence at Hanford was almost $16 \mathrm{~km}$ (Ringold, Washington), whereas the nearest location of dairy animals in the vicinity of X-10 is the Gallaher Bend Community at $6 \mathrm{~km}$. The "typical" child around Hanford was located farther than $38 \mathrm{~km}$ from the site, whereas the domain for model calculations in this study extended $38 \mathrm{~km}$.

The uncertainty estimates for doses calculated for children consuming milk in the vicinity of X10 are comparable to the uncertainty estimates for representative individuals at Hanford, but somewhat larger than the uncertainties estimated for specific persons participating in the Hanford Thyroid Disease Study. The low uncertainties reported for the Hanford Thyroid Disease Study (HTDS) reflect the improved state of knowledge for specific individuals regarding their lifestyles and dietary habits and the fact that many individuals in the HTDS pilot study were on a diet of commercial as opposed to backyard cow's milk (Kopecky, 1995). The high uncertainty estimates reported for the maximum child within a cohort of 3545 school children in Utah primarily reflect the uncertain state of knowledge associated with the deposition of ${ }^{131} \mathrm{I}$, its retention on vegetation, and its transfer into goat's milk (Stevens et al., 1992). The high uncertainty estimates reported for representative children consuming milk from backyard cows in Meagher, Montana, reflect the lack of knowledge about the amount of ${ }^{131}$ I deposited and retained by vegetation and the fact that the location was at some distance from environmental monitoring stations (NCI, 1997).

\subsection{Comparison with Preliminary Results of Previous Iterations of the Task 1 Report}

The process of investigating the health impacts of the releases of ${ }^{131}$ I from the RaLa Facility extended over 4 years (1994 to 1998) and consisted of three major iterations (including the present report). In each iteration, thyroid doses and risks of thyroid cancer were estimated. The outcomes of the first two iterations were reported in April 1996 (Hoffman et al., 1996), and in March, 1998 (Apostoaei et al., 1998,). Each iteration brought an expansion of the study both in size (e.g., area of the study domain, number of locations, number of exposure pathways) and in depth (e.g., refinements of the modeling approaches, better differentiation between the exposed individuals, additional types of health outcomes, and model validation). A summary of the progress of Task 1 of the Oak Ridge Dose reconstruction is presented in Table 13.2, and a comparison of the major findings in each iteration are presented in numerical form in Table 13.3 and in graphical form in Figure 13.1.

This report provides a more detailed analysis than presented in its previous drafts, but, most importantly, it gives a better perspective of the health impacts of ${ }^{131}$ I releases which are now discussed not only in terms of thyroid doses and risks of thyroid cancer, but also in terms of relative risk, of probability of causation, and of the total number of excess cancers expected in the study area. 
Table 13.2 Progress of Task 1 of the Oak Ridge Dose Reconstruction study during the three iterations.

\begin{tabular}{|c|c|c|c|}
\hline \multirow{2}{*}{ Issue } & \multicolumn{3}{|c|}{ Date of the report } \\
\hline & April 1996 & March 1998 & July 1999 \\
\hline Study domain & $20-\mathrm{km}$ radius & $25-\mathrm{km}$ radius & $38-\mathrm{km}$ radius \\
\hline Locations & 2 & 20 & 41 \\
\hline April 29, 1954, accident & Not explicitly considered & studied separately & Studied separately \\
\hline $\begin{array}{l}\text { Atmospheric transport and } \\
\text { deposition }\end{array}$ & $\begin{array}{l}\text { annual average } \\
\text { meteorology }\end{array}$ & $\begin{array}{l}\text { hourly meteorology for both routine } \\
\text { releases and } 1954 \text { accident }\end{array}$ & $\begin{array}{l}\text { - Hourly meteorology for routine releases } \\
\text { - Half-hourly meteorology for the } 1954 \text { accident }\end{array}$ \\
\hline Plume depletion & none & $\begin{array}{l}\text { same deposition rates for pasture grass } \\
\text { and forest canopy }\end{array}$ & $\begin{array}{l}\text { - Deposition rates specific for pasture grass } \\
\text { - } \quad \text { Plume depletion rates specific for Oak Ridge area }\end{array}$ \\
\hline Atmospheric chemistry & included & Included & $\begin{array}{c}\text { Improved based on knowledge of absorption of } \\
\text { elemental iodine on aerosols }\end{array}$ \\
\hline Exposure pathways & 6 & 9 & 11 \\
\hline Diet categories & 1 & 1 & 4 \\
\hline Exposed individuals & Females under age of 15 & females and males ages 0 to 40 & $\begin{array}{c}\text { females and males ages } 0 \text { to } 40 \\
\text { (ethnic background differences discussed) }\end{array}$ \\
\hline $\begin{array}{l}\text { Endpoints of the } \\
\text { calculation }\end{array}$ & $\mathrm{TD}^{\mathrm{a}}$ and $\mathrm{TELR}^{\mathrm{b}}$ & $\mathrm{TD}^{\mathrm{a}}$ and $\mathrm{TELR}^{\mathrm{b}}$ & $\mathrm{TD}^{\mathrm{a}}, \mathrm{TELR}^{\mathrm{b}}, \mathrm{RR}^{\mathrm{c}}, \mathrm{PC}^{\mathrm{d}}, \mathrm{TEC}^{\mathrm{e}}$ \\
\hline Health impact & thyroid cancer & thyroid cancer & $\begin{array}{l}\text { - Thyroid cancer, } \\
\text { - Other neoplasms } \\
\text { - Non-neoplastic disease, including autoimmune } \\
\text { thyroiditis }\end{array}$ \\
\hline Model validation & none & $\begin{array}{l}\text { concentration of }{ }^{131} \mathrm{I} \text { in air } \\
\text { (annual average basis) }\end{array}$ & $\begin{array}{l}\text { - Concentration of }{ }^{131} \mathrm{I} \text { in air (annual average basis) } \\
\text { - Concentration of }{ }^{131} \mathrm{I} \text { in air (weekly basis) } \\
\text { - Concentration of }{ }^{131} \mathrm{I} \text { in cow's milk (long-term } \\
\text { average basis }\end{array}$ \\
\hline
\end{tabular}


Table 13.3 Summary of the changes in the major results during the three iterations of Task 1 of the Oak Ridge Dose Reconstruction.

\begin{tabular}{lcc}
\hline Results & April 1996 & \multicolumn{1}{c}{$\begin{array}{l}\text { Date of the report } \\
\text { March 1998 }\end{array}$} \\
\hline Total amount of ${ }^{131}$ I released & $0.78-1.9 \mathrm{PBq}$ & $0.74-1.4 \mathrm{PBq}$ \\
& $(21,000-51,000 \mathrm{Ci})$ & $(26,000-30,000 \mathrm{Ci})$ \\
$\begin{array}{l}\text { Amount of }{ }^{131} \mathrm{I} \text { released in elemental } \\
\text { form }\end{array}$ & $0.67-1.33 \mathrm{PBq}$ & $0.29-0.66 \mathrm{PBq}$
\end{tabular}

April 29, 1954, accident

Thyroid dose (cGy) for a female born in 1944 living 4-6 km downwind of X-10 and consuming milk from commercial sources.

Thyroid dose (cGy) for a female born in 1944 living 19-20 km downwind of X-10 and consuming milk from commercial sources

Excess Lifetime Risk of Thyroid Cancer for a female born in 1944 living 4-6 km downwind of X-10 and consuming milk from commercial sources.

Excess Lifetime Risk of Thyroid Cancer for a female born in 1944 living 19-20 $\mathrm{km}$ downwind of $\mathrm{X}-10$ and consuming milk from commercial sources not explicitly considered

$39 \%$ of the amount of iodine released

$$
\text { in } 1954
$$

$6.5 \%$ of the amount of iodine released

$$
2.3-210
$$

(4 km downwind)

$$
0.26-23
$$

(20 km downwind)

$$
6.8 \times 10^{-5}-2.1 \times 10^{-2}
$$

(4 km downwind)

$8.9 \times 10^{-6}-2.6 \times 10^{-3}$

(20 km downwind)

$$
3.0-130
$$

(Bradbury, 6 km downwind)

$$
0.53-21
$$

(Kingston, $19 \mathrm{~km}$ downwind)

$$
4.5 \times 10^{-4}-3.5 \times 10^{-2}
$$

(Bradbury, 6 km downwind)

$8.3 \times 10^{-5}-6.7 \times 10^{-3}$

(Kingston, 19km downwind) in 1954

$$
1.3-61
$$

(Bradbury, $6 \mathrm{~km}$ downwind)

$$
0.34-15
$$

(Kingston, 19 km downwind)

$$
1.1 \times 10^{-4}-2.0 \times 10^{-2}
$$

(Bradbury, $6 \mathrm{~km}$ downwind)

$$
3.0 \times 10^{-5}-5.3 \times 10^{-3}
$$

(Kingston, 19 km downwind) 


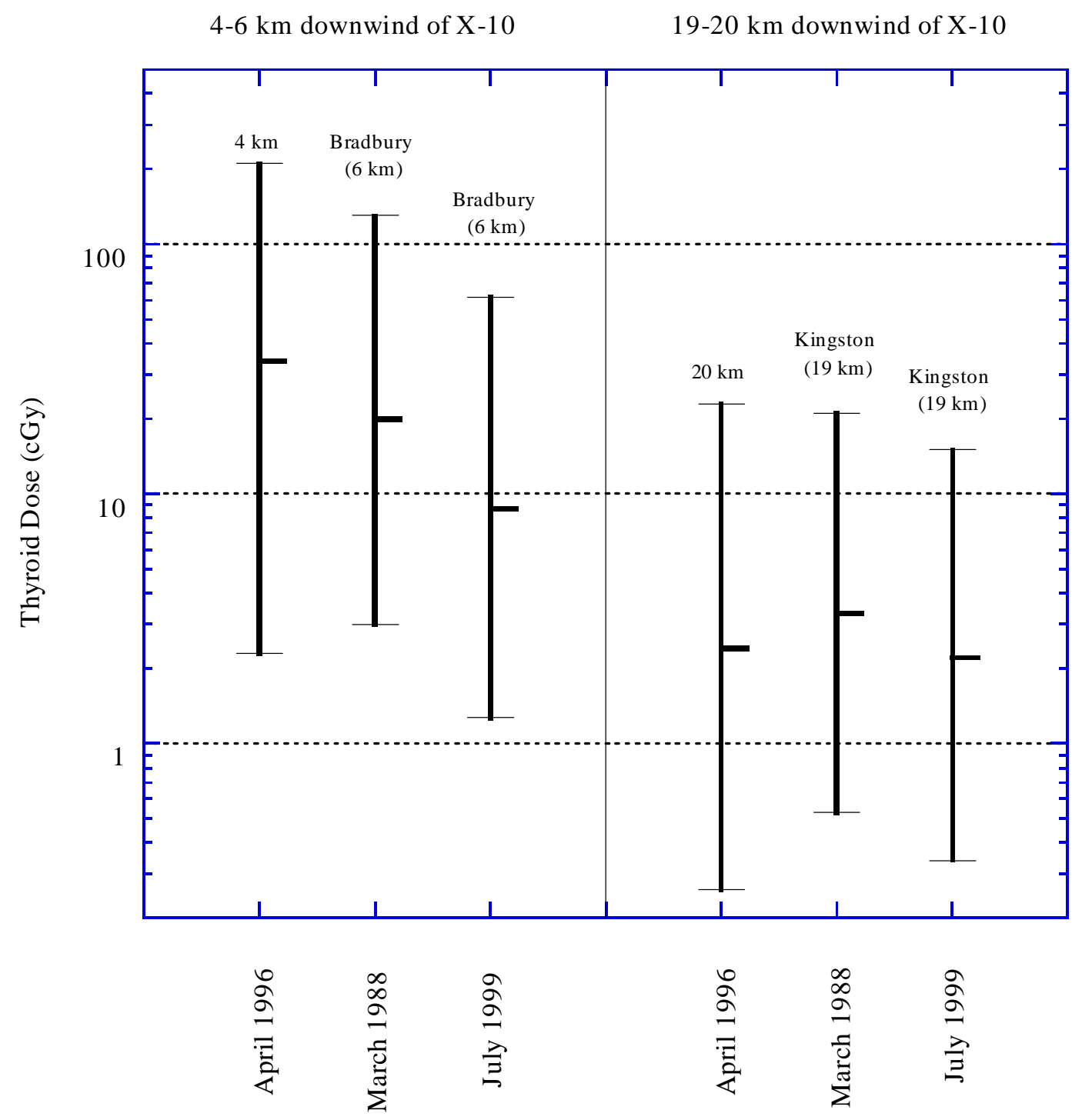

Figure 13.1 Comparison of the results produced in the three iterations of the Oak Ridge Dose Reconstruction, in terms of thyroid dose for a female born in 1944 drinking milk from commercial sources. 


\subsection{References}

Apostoaei, A.I., Burns, R.E., Hoffman, F.O., Ijaz, T., Lewis, C.J., Nair, S.K., and Widner, T.E. 1998. Iodine-131 Releases from Radioactive Lanthanum Processing at the X-10 Site in Oak Ridge, Tennessee--An Assessment of Historical Quantities Released, Off-Site Radiation Doses, and Potential Excess Risks of Thyroid Cancer. Task 1 Draft Report. Oak Ridge Health Studies, Oak Ridge Dose Reconstruction. March 1998.

Farris, W.T., Napier, B.A., Ikenberry, T.A., Simpson, J.C., Snyder, S.F., and Shipler, D.B. 1994. Atmospheric Pathway Dosimetry Report, 1944-1992. PNWD-2228 HEDR, Battelle, Pacific Northwest Laboratories, Richland, Washington.

Greenland, S. and Robins, J.M. 1988. Reviews and Commentary: Conceptual problems in the definition and interpretation of attributable fractions. Journal of Epidemiology 128(6):11851197.

Greenland, S., and Rothman, K.J. 1998. Measures of Effect and Measures of Association. Chapter 4, in Modern Epidemiology. $2^{\text {nd }}$ Edition. Rothman, K.J., and Greenland, S. (eds.) Philadelphia: Lippincott.

Hoffman, F.O., Apostoaei, A.I., Nair, S.K., Widner, T.E., and Burns, R.E. 1996. First iteration dose and health risk assessment for Iodine- ${ }^{131}$ emissions from X-10 radioactive Lanthanum processing. Task 1 preliminary report for the Oak Ridge Health Studies. Oak Ridge Dose Reconstruction Study. Oak Ridge, Tennessee.

HTDS (Hanford Thyroid Disease Study). 1998. Newsletter: Plan for Communicating the Study Results. Fred Hutchinson Cancer Research Center. September, 1998.

IOM/NAS. (Institute of Medicine/National Academy of Sciences). 1998. Exposure of the American People to Iodine-131 from Nevada Nuclear-Bomb Tests: Review of the National Cancer Institute Report and Public Health Implications. National Academy Press. Washington, DC.

Kopecky, 1995. Kopecky, K. Private Communication.

NCI (National Cancer Institute). 1997. Estimated Exposure and Thyroid Doses Received by the American People from Iodine- ${ }^{131}$ Fallout Following Nevada Atmospheric Nuclear Bomb Tests. U.S. Department of Health and Human Services. National Institutes of Health. National Cancer Institute, October 1997.

NCRP (National Council on Radiation Protection and Measurements). 1993. The Probability that a Particular Malignancy May Have Been Caused by a Specified Irradiation. NCRP Statement No. 7. Radiation Research 134:394-397.

Robins, J.M., and Greenland, S. 1989a. Estimability and estimation of excess and etiologic fractions. Statistics in Medicine 8:845-859. 
Robins, J.M., and Greenland, S. 1989b. The probability of causation under a stochastic model for individual risk. Biometrics 45:1125-1138.

Ron, E., Lubin, J.H., Shore, R.E., Mabuchi, K., Modan, B., Pottern, L.M., Schneider, A.B., Tucker, M.A., and Boice, J.D., Jr. 1995. Thyroid Cancer after Exposure to External Radiation: A Pooled Analysis of Seven Studies. Radiation Research 141:259-277.

Stevens, W., Till, J.E., Thomas, J.C., Lyon, J.L., Kerber, R.A., Preston-Martin, S., Simon, S.L., Rallison, M.L., and Lloyd, R.D. 1992. Report of A Cohort Study of Thyroid Disease and Radioactive Fallout from the Nevada Test Site. University of Utah. National Cancer Institute Contract \#NO1-CO-2391.

Till, J.E., Simon, S.L., Kerber, R., Lloyd, R.D., Stevens, W., Thomas, D.C., Lyon, J.L., and Preston-Martin, S. 1995. The Utah Thyroid Cohort Study: Analysis of the Dosimetry Results. Health Physics 68:472-483.

Turri, P. 1998. Personal Communication. Tennessee Department of Health, Cordell Hull Building, $4255^{\text {th }}$ Avenue North, Nashville, TN 37247-5261. 
TASK 1 REPORT

July 1999

${ }^{131}$ I Releases from X-10 Radioactive Lanthanum Processing -

Page 13-16

Discussion

This page intentionally left blank. 


\subsection{CONCLUSIONS}

Approximately 8,800 to $42,000 \mathrm{Ci}\left(0.3\right.$ to $\left.1.6 \mathrm{PBq} ; 1 \mathrm{PBq}=10^{15} \mathrm{~Bq}\right)$ of ${ }^{131} \mathrm{I}$ was released from the RaLa processing facility at X-10 between 1944 and 1956. An April 29, 1954, accident, which lasted a total of 2.5 hours, released 105 to $500 \mathrm{Ci}\left(3.9\right.$ to $\left.21 \mathrm{TBq} ; 1 \mathrm{TBq}=10^{12} \mathrm{~Bq}\right)$, accounting for about $6.5 \%$ of the total releases for 1954 .

To investigate the thyroid doses and potential health risks to the public exposed to these releases, a 38-km vicinity of X-10 was defined as the study domain. Within this domain, 41 representative locations were identified for estimating the doses and health impacts from exposure to the ${ }^{131}$ I released from $\mathrm{X}-10$ as a function of age, gender, and diet.

Exposures to ${ }^{131}$ I were estimated for eleven different pathways, of which the ingestion of goat's milk, backyard cow's milk, and locally and regionally produced commercial milk were the most significant pathways in terms of their contribution to total radiation exposure. In addition, four plausible diet scenarios were developed, each containing a combination of pathways, based on the dietary habits of individuals living in the study domain between 1944 and 1956. Excess risks of developing thyroid cancers were estimated for individuals of both genders and of various ages at the time of exposure under each of the diet scenarios.

Doses were calculated for males and females born in 1920, 1930, 1940, 1944, 1952, 1954, and 1956 who lived near X-10 during the period of radioactive lanthanum processing (1944-1956). These years of birth were chosen to allow comparison of doses and risks from ${ }^{131} \mathrm{I}$ intake by individuals of different ages at time of exposure. It was assumed that all members of the reference populations received no ${ }^{131} \mathrm{I}$ exposure other than that from the X-10 RaLa releases that occurred from 1944 to 1956 . This being the case, it is not important where a person was born or where they lived before 1944 or after 1956. It is important, however, where in the Oak Ridge area the person lived from 1944 through 1956.

Females born in 1952 who consumed goat's milk (from one 4-ounce to five 8-ounce glasses each day from age 1-4 y) received the highest doses and have the highest risks of contracting thyroid cancer during their lifetimes. The next highest doses result from the consumption of milk from a backyard cow (between one and five 8-ounce glasses each day), followed by milk from a local commercial dairy and milk that was regionally mixed. Lower doses result from inhalation or from the consumption of locally produced beef, cottage cheese, mother's milk (with the mother on a diet of backyard cow's milk and local produce, i.e., diet 1), or leafy vegetables. The doses from inhalation or from the consumption of one of these food types for a child under the age of 5 at the time of exposure are several hundred to more than 1000 times less important than the dose from the consumption of backyard cow's milk. The thyroid dose from prenatal exposure during the first part of 1952 (assuming the mother to be on diet 1) is about equal to the 5-year total thyroid dose obtained from the consumption of beef or cottage cheese.

Assuming the same dietary sources for ingestion of ${ }^{131} \mathrm{I}$ at a specific location, differences in gender account for only minor differences in the estimation of the thyroid doses. More significant differences are determined by the year of birth, with the lowest doses being for individuals born in 1920, 1930, and 1956. These doses are about one-fourth to one-fifth of the 
largest doses received by individuals born between the years of 1944 and 1952. Individuals born in 1954 have about the same doses as those born in 1940, which are about $65 \%$ of the doses for those born between 1944 and 1952.

Among the 41 selected locations within the $38-\mathrm{km}$ vicinity of the $\mathrm{X}-10$ facility, the highest doses occurred at Gallaher Bend, located a little more than $6 \mathrm{~km}$ to the east of X-10, while the lowest doses occurred at Wartburg, located $27 \mathrm{~km}$ northwest of X-10. For example, at Gallaher Bend the thyroid doses ranged from about 4 to $250 \mathrm{cGy}$ for individuals of each gender born between 1940 and 1952 who consumed milk from a backyard cow (between one and five 8-ounce glasses each day) and who consumed food products from a local farm. A similar group of individuals residing in Wartburg would have received doses ranging from about 0.08 to $6 \mathrm{cGy}$. Doses from the consumption of regionally mixed commercial retail milk ranged from about 0.3 to 10cGy for individuals born between 1940 and 1952 and did not vary by location.

The highest excess risks of developing thyroid cancer for a female born in 1952 on diet 1 occur at the agricultural communities of Bradbury and Gallaher Bend. For these locations, based on the assumed quantities of backyard cows' milk, beef, vegetables, eggs, and cheese consumed each day, the risk estimates are confidently above one chance in one thousand $\left(1 \times 10^{-3}\right)$ but less than 1 chance in ten $\left(1 \times 10^{-1}\right)$. At these locations, the central estimate of the probability of causation approaches or exceeds $50 \%$ for females born in 1952 on diets 1, 2, and 4, meaning that a diagnosed thyroid cancer has more than an even chance of being due to exposure to ${ }^{131} \mathrm{I}$ released from X-10. The central estimate of risk for a female born in 1952 on diet 1 is likely to exceed 1 chance in $1,000\left(1 \times 10^{-3}\right)$ at distances up to $35 \mathrm{~km}$ to the southwest or more than $38 \mathrm{~km}$ to the northeast of $X-10$. A risk of more than one chance in ten thousand $\left(1 \times 10^{-4}\right)$ is likely with a subjective confidence of over $50 \%$ at all locations in the $38-\mathrm{km}$ vicinity of $\mathrm{X}-10$.

Depending on the year of birth, the excess lifetime risk to females is 3 to 4 times larger than the risk to males. At a location such as Bradbury or Gallaher Bend, the lowest risk is for a male born in 1920, who has an excess lifetime risk of thyroid cancer almost 1000 times less than the highest risk, that for females born in 1952. A female born in 1920 has a risk about 350 times lower than that for a female born in 1952. Individuals of the same gender born in 1944 have about 50-60\% of the risk that those born in 1952 have, while individuals born in 1940 or 1956 have risks about 5 times lower than those for individuals born in 1952. However, a male born in 1940 or 1956 has a risk almost 20 times less than that for a female born in 1952.

In general, the total doses and risks from exposure to the April 29, 1954, accident are much lower than those resulting from exposure to the accident and the routine emissions combined. For the accidental release, the excess lifetime risk of thyroid cancer for females on diet 1 and exposed in early childhood at either Jonesville, Norwood, East Fork Poplar Creek, or Oliver Springs ranged from a few chances in 10 million $\left(3\right.$ or $\left.4 \times 10^{-7}\right)$ to nearly one chance in thousand $\left(1 \times 10^{-3}\right)$.

Most of the uncertainty in the estimates of risk is associated with the uncertainty in the estimates of dose $(55 \%)$, followed by the uncertainty in the dose response for cohorts exposed to external sources of radiation. Uncertainty in the dose estimates is dominated by the uncertainty in the estimated concentrations of ${ }^{131} \mathrm{I}$ in milk (45\%), followed by the uncertainty in the internal dose 
conversion factor (40\%). The uncertainty in the estimated milk concentrations is dominated by the uncertainty in the transfer of ${ }^{131} \mathrm{I}$ from air to pasture $(71 \%)$. The uncertainty in the internal dose conversion factor is dominated by the uncertainty in determining the actual mass of the thyroid for any individual of a given gender and age.

Fallout from atmospheric testing of nuclear weapons at the Nevada Test Site (NTS) during 1952, 1953, 1955, and 1957 was a significant contributor to the total ${ }^{131} \mathrm{I}$ exposure for individuals located within $38 \mathrm{~km}$ of the X-10 site. Beyond this distance, ${ }^{131} \mathrm{I}$ from NTS fallout was clearly the dominant source of exposure. For a female born in 1952 who consumed backyard cow's milk, central estimates of the thyroid dose from combined exposures from X-10 releases and NTS fallout within the $15-\mathrm{km}$ vicinity of X-10 ranged from 25 to $30 \mathrm{cGy}$, with upper bounds of the $95 \%$ subjective confidence interval exceeding $200 \mathrm{cGy}$. At all locations within $15 \mathrm{~km}$ of X10, the risk to a female born in 1952 on a rural diet (diet 1) exceeds one chance in one thousand $\left(1 \times 10^{-3}\right)$. The upper bound of the 95\% subjective confidence interval for the excess lifetime risk of thyroid cancer exceeds several cases in one hundred $\left(6-9 \times 10^{-2}\right)$ in this $15-\mathrm{km}$ region and exceeds one chance in ten $\left(1 \times 10^{-1}\right)$ at the communities of Bradbury and Gallaher Bend. The doses from the combined exposure to ${ }^{131} \mathrm{I}$ released from X-10 and ${ }^{131} \mathrm{I}$ deposited in fallout from the Nevada Test Site are sufficiently high to have possibly manifested excess cases of nonneoplastic disease, namely autoimmune thyroiditis.

Within the $38-\mathrm{km}$ vicinity of $\mathrm{X}-10$, the $95 \%$ subjective confidence interval of the number of excess thyroid cancers resulting from consumption of cow's milk (commercial and backyard milk, combined) contaminated by the releases of ${ }^{131} \mathrm{I}$ from the RaLa processing facility between 1944 and 1956 ranges from 6 to 84, with a central estimate of 21. For the consumption of backyard cow's milk alone, the 95\% subjective confidence interval ranges from 1 to 33 excess cases of cancer, with a central estimate of 7 cases. Commercial cow's milk contributed more than backyard cow's milk to the number of excess cancers because of the larger quantity of commercial milk produced and the larger number of individuals exposed via its consumption.

The 95\% subjective confidence interval of the number of excess thyroid cancers from consumption of cow's milk contaminated by X-10 RaLa releases (commercial and backyard milk, combined) ranges from 14 to 103 (central estimate of 35) within a 100-km vicinity of X10 , and from 25 to 149 (central estimate of 58) within a $200-\mathrm{km}$ vicinity of X-10. These cancers are expected to be detected between 1950 and 2020, with the majority occurring after 1970. It should be noted that these calculations of possible numbers of excess thyroid cancers are based on the linear, no threshold hypothesis. Under that hypothesis, added cancer risk is assumed to be proportional to the collective radiation dose received. This holds whether one has large doses received by a few people or low doses received by a much larger population. However, available data cannot exclude the possibility that there is a low-level threshold for causation of cancer. If this is the case, then the values presented above are likely over-estimates of true incidence rates.

These estimates were made using a baseline of thyroid cancer diagnoses within the regional population with the assumption that the individuals in this population were unexposed. Only about one-third to one-fourth of the total number of thyroid cancers in a population are diagnosed and reported. Therefore, it is likely that the total number of cases of thyroid cancer, those that are diagnosed and those that are occult, are 3 to 4 times greater than the total estimates 
given on the basis of cancers reported only through clinical diagnosis. The excess incidence of benign nodules will be about 8 to 10 times that of the excess thyroid cancer if the detection of nodules is made by palpation of the neck. An additional factor of 7 increase in benign nodules can be expected if diagnosis is determined with ultrasonography. The clinical significance of an excess incidence of benign nodules is not evaluated in this study, but it is noted that about $9 \%$ of benign nodules diagnosed through palpation and about $28 \%$ of those diagnosed through ultrasound are surgically removed.

In the 38-km vicinity of X-10, an increase in the number of cases of thyroid cancer is forecast by both this study and by the incidence rates in the disease registry maintained by the Tennessee Department of Health. The number of excess cases of thyroid cancer forecast by this study, is about 4 to $50 \%$ of the number of excess cases estimated using the disease registry.

In the 38-km vicinity of X-10, an increase in the number of cases of thyroid cancer is forecast by this study and may be reflected in the incidence rates obtained from the disease registry maintained by the Tennessee Department of Health. Unfortunately, due to the large uncertainties and numerous possible confounding factors involved, any simple comparisons at this time are speculative at best. If one compares thyroid cancer incidence rates (for 1988 to 1995) from the four counties surrounding the ORR to those in Tennessee excluding those four counties, the numbers of excess cases of thyroid cancer forecast by this study due to ${ }^{131} \mathrm{I}$ releases from X-10 are about 4 to $50 \%$ of the excess cases estimated using the disease registry, not accounting for the statistical uncertainty in the projection from statewide results. However, if one compares incidence rates among whites in the local counties to whites in the rest of the state, the difference is much lower and is not statistically significant. Non-whites make up a significantly smaller fraction of the population in the four local counties than they do in the remainder of Tennessee, and non-whites have lower age-specific thyroid cancer incidence rates than whites. Because of the presence of these racial differences and other uncontrolled confounding factors affecting disease registries, extreme caution must be used when interpreting the possible significance of these observations.

The thyroid dose estimates made in this study for individuals in the 38-km vicinity of X-10 exposed to ${ }^{131}$ I released from RaLa processing at X-10 were found to be comparable to similar studies conducted at other sites in the United States. The possibility that doses above 30 to 100 cGy may induce autoimmune thyroiditis has been discussed on the basis of the most recent reviews of the doses of radiation required to induce non-neoplastic thyroid disease.

A number of recommendations have been made in this report that can be used in follow-up work to reduce the uncertainty in dose and risk estimates and to identify and eliminate any remaining sources of possible bias. 


\section{KEY TECH NICAL REPORT SOFTHE OAK RIDGE DOSE RECON STRUCTION PROJECT}

- Volume 1 .

I odine-131 $R$ eleases from $R$ adioactive $L$ anthanum Processing at the $X-10$ Site in $O$ ak R idge, T ennessee (1944-1956)- an A ssessment of $Q$ uantities $R$ eleased, 0 ff-Site R adiation D oses, and Potential Excess $R$ isks of T hyroid C ancer

The report of project T ask 1

- Volume $1 \mathrm{~A}$.

A ppendices to the lodine-131 R eport

- Volume 2 .

Mercury R eleases from Lithium Enrichment at the 0 ak R idge $Y-12$ Planta $R$ econstruction of $H$ istorical $R$ eleases and $O$ ff-Site $D$ oses and $H$ ealth $R$ isks

The report of project T ask 2

- V olume $2 \mathrm{~A}$.

A ppendices to the Mercury R eport

- Volume 3 .

PC Bs in the Environment near the 0 ak $R$ idge $R$ eservationa R econstruction of $H$ istorical $D$ oses and $H$ ealth $R$ isks

The report of project T ask 3

- Volume 4 -

$R$ adionuclide $R$ eleases to the $C$ linch $R$ iver from $W$ hite $\mathbf{O}$ ak $C$ reek on the $O$ ak $R$ idge $R$ eservation- an $A$ ssessment of $H$ istorical $Q$ uantities $R$ eleased, O ff-Site $\mathbf{R}$ adiation $D$ oses, and $H$ ealth $R$ isks

The report of project Task 4

- $V$ olume $4 \mathrm{~A}$.

A ppendices to the $\mathrm{W}$ hite $\mathrm{O}$ ak $\mathrm{C}$ reek $\mathrm{R}$ eport

- Volume 5 .

$U$ ranium $R$ eleases from the 0 ak $R$ idge $R$ eservation-

a $R$ eview of the $Q$ uality of $H$ istorical Effluent M onitoring $D$ ata and a Screening Evaluation of Potential O ff-Site Exposures

The report of project Task 6

- V olume 6 .

Screening-L evel Evaluation of A dditional Potential M aterials of $C$ oncern The report of project T ask 7

- Volume 7 .

$\mathrm{O}$ ak R idge D ose R econstruction Project Summary R eport 